\title{
Zugang zu Agrar- \& Pharmawirkstoffen durch organische Synthese
}

\section{Design und Synthese von Spinosynanaloga und Hsp47-Inhibitoren} sowie enantioselektive Totalsynthese des Naturstoffes Linoxepin

\author{
Dissertation \\ zur Erlangung des mathematisch-naturwissenschaftlichen Doktorgrades \\ „Doctor rerum naturalium“ \\ der Georg-August-Universität Göttingen \\ im Promotionsprogramm der \\ Georg-August University School of Science (GAUSS) \\ vorgelegt von \\ Simon Biller \\ aus Lübbecke
}

Göttingen, 2015 

Betreuungsausschuss:

Prof. Dr. Dr. h.c. Lutz. F. Tietze

Institut für Organische und Biomolekulare Chemie

Georg-August-Universität Göttingen

Prof. Dr. Daniel B. Werz

Institut für Organische Chemie

Technische Universität Braunschweig

Mitglieder der Prüfungskommission:

Referent: Prof. Dr. Dr. h.c. Lutz. F. Tietze

IOBC, Georg-August-Universität Göttingen

Koreferent: Prof. Dr. Daniel B. Werz

IOC, Technische Universität Braunschweig

Weitere Mitglieder der Prüfungskommission:

Prof. Dr. Hartmut Laatsch

IOBC, Georg-August-Universität Göttingen

Dr. Alexander Breder

IOBC, Georg-August-Universität Göttingen

Prof. Dr. Sven Schneider

IAC, Georg-August-Universität Göttingen

Prof. Dr. Ulf Diederichsen

IOBC, Georg-August-Universität Göttingen

Tag der mündlichen Prüfung: 10. Juni 2015 

Die vorliegende Arbeit wurde in der Zeit von November 2011 bis Juni 2015 unter der Leitung von Prof. Dr. Dr. h.c. L. F. Tietze am Institut für Organische und Biomolekulare Chemie der Georg-August-Universität Göttingen angefertigt.

Ich danke Herrn Prof. Dr. Dr. h.c. L. F. Tietze

für die interessante Themenstellung, die Bereitstellung der hervorragenden Arbeitsbedingungen, seine stete Unterstützung sowie für die zahlreichen Diskussionen und Anregungen und sein Interesse am Fortgang dieser Arbeit. 

MEINER FAMILIE 

"WHEN EVERYTHING SEEMS TO BE AGAINST YOU, REMEMBER THAT THE AIRPLANE TAKES OFF AGAINST THE WIND, NOT WITH IT" -HENRY FORD- 



\section{INHALTSVERZEICHNIS}

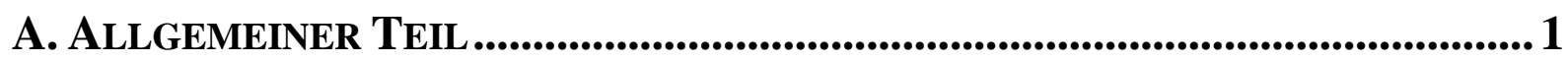

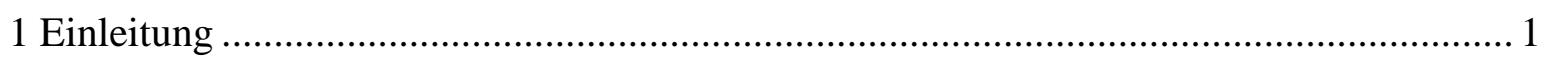

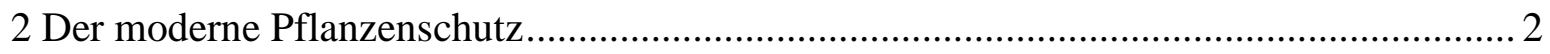

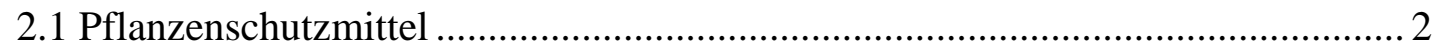

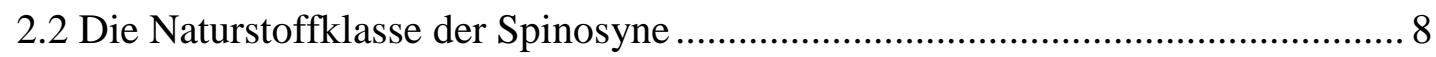

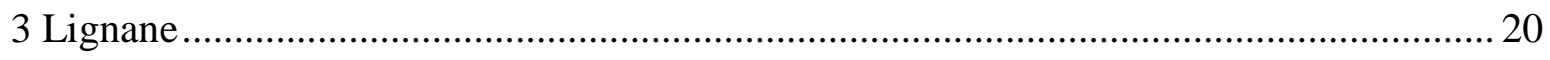

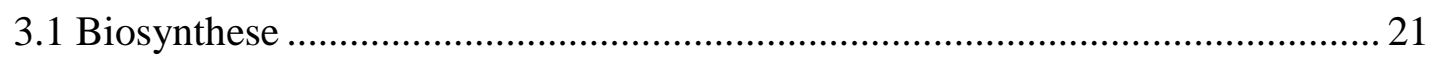

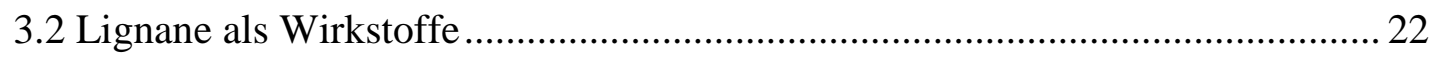

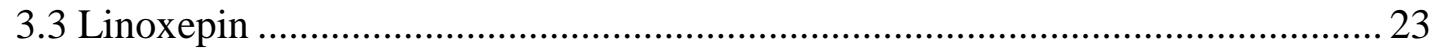

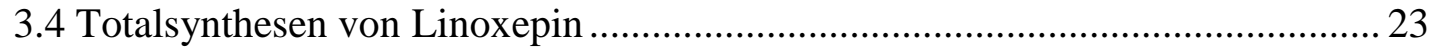

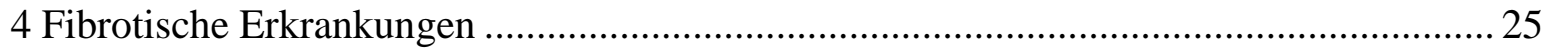

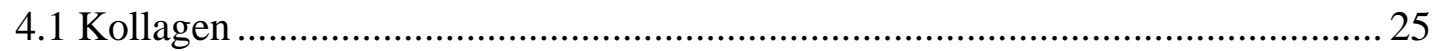

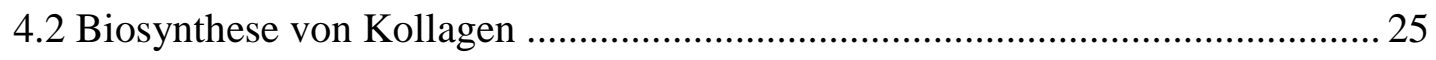

4.3 Funktion des Hsp47 in der Kollagenbildung ................................................. 27

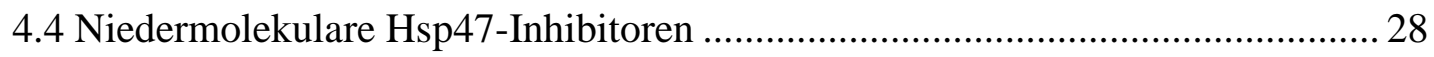

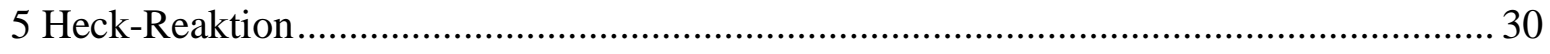

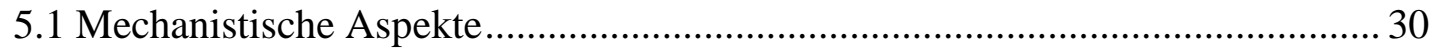

5.2 Anwendungen der Heck-Reaktion in der Organischen Synthese ....................... 37

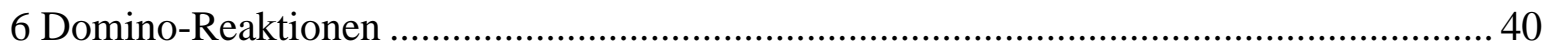

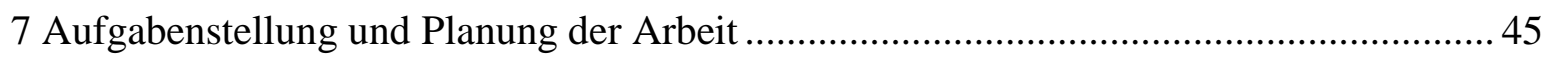

B. ERGEBNISSE \& DISKUSSION .................................................................................51

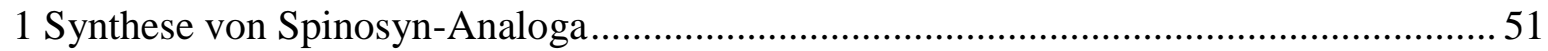

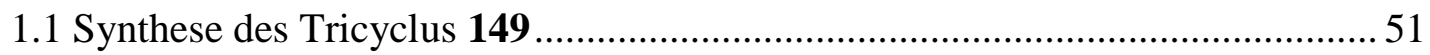

1.2 Synthese von Seitenketten des Typs 150 ................................................. 59

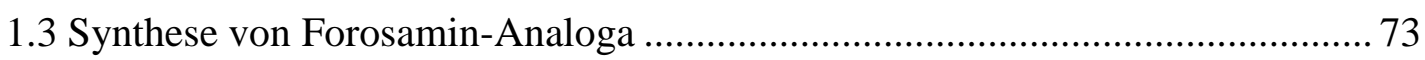

1.4 Alternative Synthesestrategien zur Kupplung der Spinosyn-Bausteine.............. 75

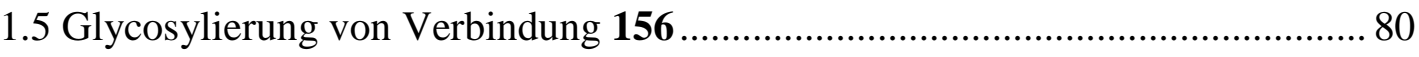

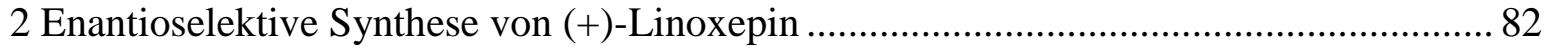

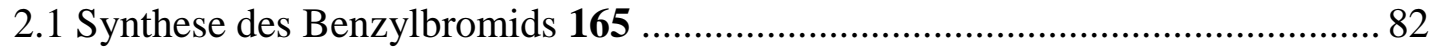

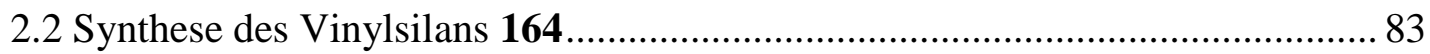

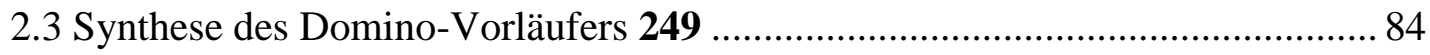


2.4 Domino-Carbopalladierung-Heck-Reaktion zu Oxepin 161 .85

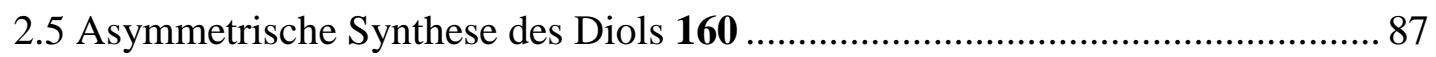

2.6 Untersuchungen zur Stabilität der chiralen Achse des Diols 160 ....................... 88

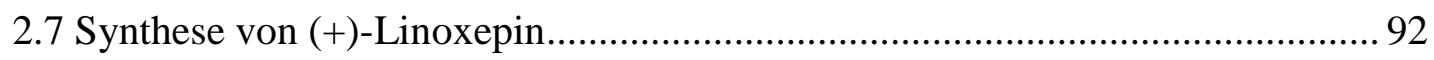

2.8 Diskussion der spektroskopischen Daten von (+)-Linoxepin ........................... 93

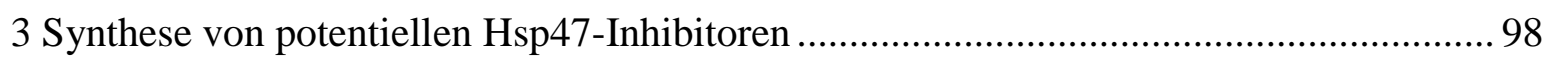

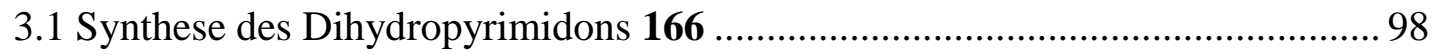

3.2 Synthese der $\alpha$-Bromacetophenone 167a-g ................................................... 98

3.3 Synthese der potentiellen Hsp47-Inhibitoren 84a-g ....................................... 100

3.4 Diskussion der spektroskopischen Daten von Verbindung 84f ....................... 102

C. ZuSAMmenfassung \& AUSBlick .........................................................106

D. EXPERIMENTELLER TEIL......................................................................................... 113

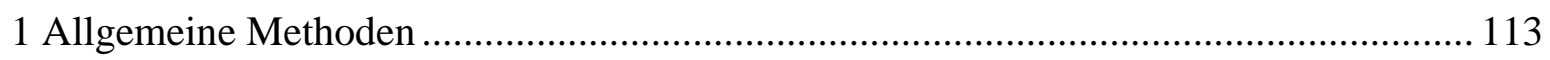

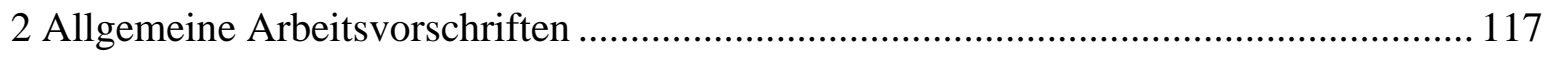

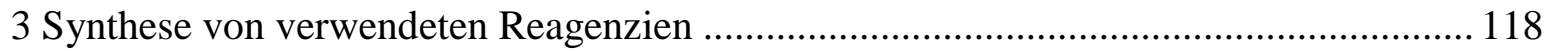

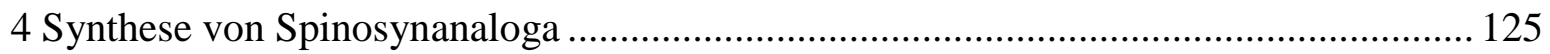

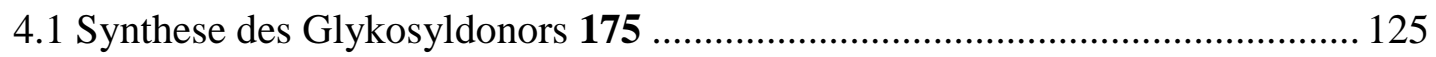

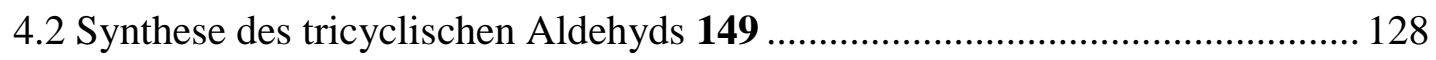

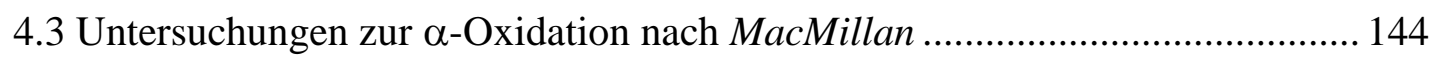

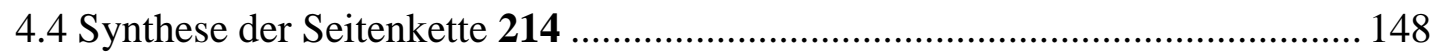

4.5 Synthese der Forosamin-Analoga 222a-d und 225...................................... 178

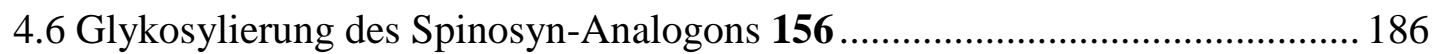

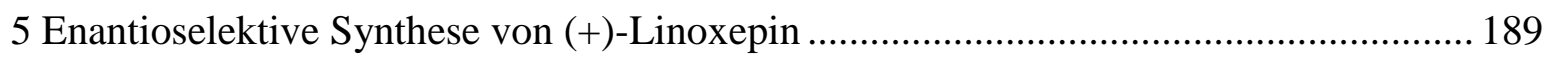

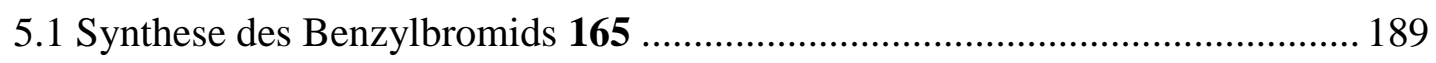

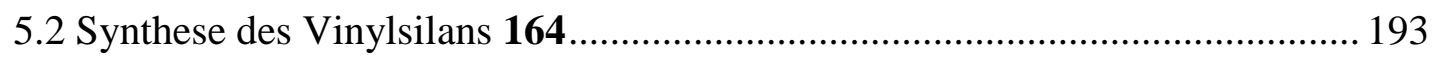

5.3 Synthese des Domino-Vorläufers 249 ......................................................... 198

5.4 Enantioselektive Synthese von (+)-Linoxepin (69) _...................................... 202

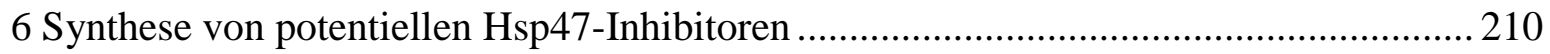

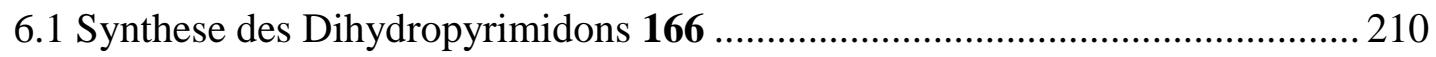

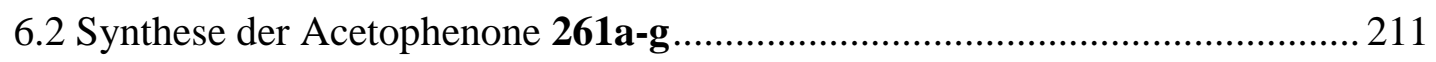

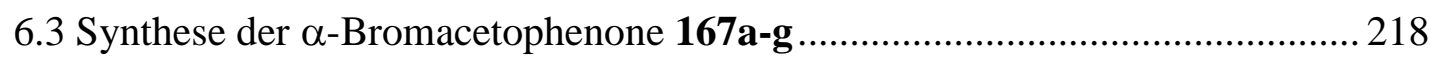

6.4 Synthese der potentiellen Hsp47-Inhibitoren 48a-g ..................................... 225

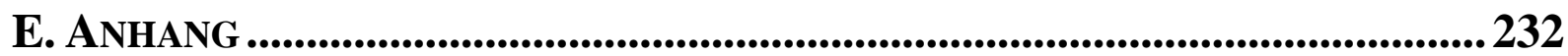




\section{A. Allgemeiner TeIL}

\section{Einleitung}

Die Bedeutung der Chemie und die Verbreitung von chemisch hergestellten Produkten in unserer heutigen Gesellschaft sind beträchtlich. So ist die Entwicklung neuer Werkstoffe, aktiver Pharmazeutika oder auch effektiver und umweltschonender Pflanzenschutzmittel vermehrt auf die organische Chemie zurückzuführen. Als Leitmotive für das Design neuer Wirkstoffe dienen häufig aufgrund ihrer strukturellen Diversität und ihres breiten Wirkspektrums aus Pflanzen, Bakterien und anderen Organismen isolierte Naturstoffe. Durch geringfügige strukturelle oder funktionelle Modifikationen des Naturstoffes wird häufig eine höhere Potenz, eine spezifischere Wirkung oder eine bessere Verträglichkeit gegenüber der bioidentischen Substanz erreicht. Auch das Auftreten von Resistenzen erfordert häufig die Modifizierung von bereits aktiven Substanzen. Acetylsalicylsäure, der Wirkstoff eines der in Deutschland meistverkauften Medikamente, das von der Bayer AG unter dem Markennamen Aspirin ${ }^{\circledR}$ vertrieben wird, ist ein Derivat des schlechter verträglichen Naturstoffes Salicin und findet unterschiedlichste Anwendung in der Medizin. Auch das von Alexander Fleming entdeckte Penicillin und dessen Derivate sind in vielfältiger Weise variiert worden um eine verbesserte Behandlung von bakteriellen Infektionen zu erzielen.

Die Isolierung eines Naturstoffes gestaltet sich trotz des technischen Fortschritts weiterhin schwierig, da die Konzentrationen häufig sehr gering sind und die Anreicherung des Wirkstoffes aufgrund der zumeist hohen benötigten Rohstoffmenge unrentabel sein kann. Auswege bietet hier die Totalsynthese, genauer gesagt die vollständige Laborsynthese des Naturstoffes oder seiner Analoga. Ein weiterer Ansatz für die Entwicklung von neuen, aktiven Arzneimitteln ist die Kombination von kleinen Bausteinen mit charakteristischen Strukturmotiven $\mathrm{zu}$ niedermolekularen Wirkstoffen. Monumentale Datenbanken mit entsprechenden Bausteinen („Building Blocks“) und hochmoderne Verfahren wie das HighThroughput-Screening sowie roboterunterstützte Reaktionsabläufe ermöglichen heutzutage die Synthese und biologische Evaluierung von unzähligen potentiellen Wirkstoffen binnen kürzester Zeit.

Die vorliegende Arbeit beschäftigt sich mit der Entwicklung neuer Synthesemethoden für den Aufbau von Analoga des Pflanzenschutzmittels Spinosyn A, einem enantioselektiven Zugang zu dem Naturstoff Linoxepin und der Synthese von niedermolekularen Verbindungen mit potentieller Inhibierung des Hitzeschockproteins 47. 


\section{Der moderne Pflanzenschutz}

Während die Weltbevölkerung immer weiter wächst, bleiben die Flächen für den Anbau von Nutzpflanzen über Jahre hinweg konstant. Ohne eine Steigerung der Flächenerträge der wichtigsten Nutzpflanzen wäre folglich eine ausreichende Ernährung der Weltbevölkerung in Zukunft nicht mehr möglich. Um diese Leistungssteigerung zu erreichen, werden vor allem neue, ertragreichere Kulturpflanzen, verbesserte Anbaumöglichkeiten, aber auch chemische Hilfsmittel wie Mineraldünger und Pflanzenschutzmittel eingesetzt. Trotz der stetigen Entwicklung von immer leistungsfähigeren Nutzpflanzen und deren Früchten sind Pflanzenschutzmittel in der heutigen Zeit von hoher Bedeutung. Neben schlechten Witterungsbedingungen sorgen Schadorganismen wie Insekten, Pilzbefall und Unkräuter für teilweise existenzbedrohende Verluste des Ernteertrags. Des Weiteren macht die naturbedingte, unaufhörliche Entwicklung von Resistenzen bei den Schadorganismen die stetige Synthese und Bereitstellung von neuen Pflanzenschutzmitteln zu einem wichtigen Ziel der organischen Wirkstoffsynthese.

\subsection{Pflanzenschutzmittel ${ }^{1,2}$}

Die ersten eingesetzten chemischen Pflanzenschutzmittel waren hauptsächlich anorganischen Ursprungs und oftmals auch für den Menschen und Nutzinsekten äußerst toxisch (vgl. Abbildung 1). Aufgrund ihrer in der Regel recht unselektiven Wirkung mussten sie außerdem in großen Mengen ausgebracht werden.

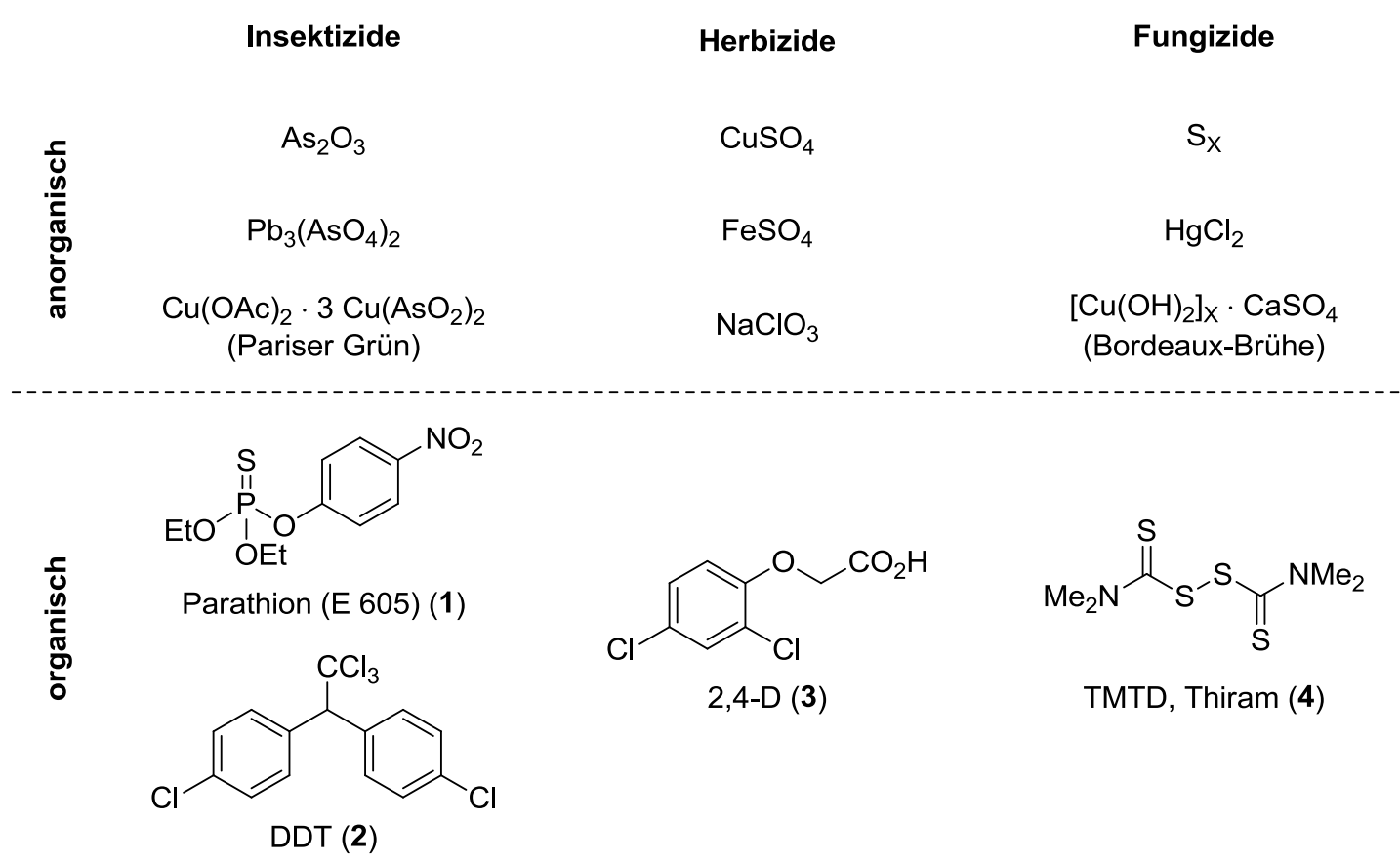

Abbildung 1: Historische Pflanzenschutzmittel nach Stetter $e t a l^{2}$ 
Durch die Erschließung synthetisch leicht zugänglicher und breit anwendbarer organischchemischer Wirkstoffe im 20. Jahrhundert wurde die Grundlage für den modernen Pflanzenschutz geschaffen. Vor allem Organophosphor- (z. B. Parathion $\left.(\mathbf{1})^{3}\right)$, Organochlor(z. B. DDT (2), ${ }^{4}$ 2,4-D (3) ${ }^{5}$ ) und Carbaminsäurederivate (z. B. TMDT (4) ${ }^{6}$ ) erwiesen sich als sehr effektive Mittel der prosperierenden agrochemischen Industrie. Neben dem Einsatz zur Produktivitätssteigerung im Agrarbereich werden Insektizide wie DDT (2) ebenfalls im Gesundheitssektor bei der Bekämpfung von Moskitos und der einhergehenden Malariaprävention in tropischen und subtropischen Gebieten eingesetzt.

Trotz der spezifischeren Wirkweise der organischen Substanzklassen birgt eine dauerhafte Anwendung in großem Maßstab auch Risiken und Gefahren. Organophosphorverbindungen werden beispielsweise wegen ihrer unspezifischen, irreversiblen Phosphorylierung der Acetylcholinesterase heutzutage mit der Entstehung von Tumoren in Zusammenhang gebracht. ${ }^{7}$ Carbamate sind hingegen in der Regel spezifischere, reversible Acetylcholinesterasehemmer, die von Säugetieren rasch detoxifiziert werden. Allerdings sind Nutzinsekten wie Bienen nicht in der Lage, Carbaminsäurederivate zu metabolisieren und werden somit zum ungewollten Target dieser Wirkstoffklasse. ${ }^{8}$

Aufgrund ihrer hohen Persistenz lagern sich Organochlorinsektizide wie DDT (2) im Wasser bzw. Erdreich an und gelangen so in die Nahrungskette des Menschen. Durch die Einlagerung der POPs (Persistant Organic Pollutants) im Fettgewebe von Menschen und Tieren kann es zu Schädigungen im Nervensystem, ${ }^{9}$ schnellerem Altern ${ }^{10}$ und Brustkrebserkrankungen ${ }^{11}$ kommen. Durch die gesteigerte öffentliche Aufmerksamkeit gegenüber teratogenen Pflanzenschutzmitteln fand das Zeitalter der POPs schließlich durch das Inkrafttreten der Stockholmer Konvention ein Ende. Unter Einbeziehung dieser Aspekte wird deutlich, weshalb eine stetige Weiterentwicklung der Pflanzenschutzmittel unabdingbar ist.

\subsubsection{Anforderungen an neue Wirkstoffe ${ }^{12}$}

Während frühere Pflanzenschutzmittelgesetze sich lediglich mit dem Schutz der Nutzpflanze beschäftigten, sind die heutigen Anforderungen an Pflanzenschutzmittel wesentlich höher und vielseitiger. Durch die Zulassungspflicht und hohen Auflagen für neue Wirkstoffe in der Agrochemie hat sich die Forschung zu einer High-Tech-Industrie entwickelt. In den hohen Qualitätsansprüchen an Pflanzenschutzmittel unterscheidet sich das Forschungsgebiet nur noch marginal von der Arzneimittelentwicklung. Neben einer selektiven Wirkung auf Schadorganismen müssen die Hersteller auch die toxikologische Unbedenklichkeit für den Anwender, den Konsumenten und Nutzorganismen wie Bienen sowie eine hervorragende Umweltverträglichkeit gewährleisten. Unter diesen Gesichtspunkten gehören Pflanzenschutz- 
mittel schon heute zu den am besten untersuchten Chemikalien, mit denen Mensch und Umwelt in Kontakt kommen.

Ein weiterer wichtiger Aspekt ist die Wirtschaftlichkeit des Produktes. Die Bereitstellung des Wirkstoffs, sei es durch Gewinnung oder Herstellung, sollte möglichst unkompliziert und mit einem geringen Kostenaufwand verbunden sein. Darüber hinaus gilt es, für die benötigte Wirkung eine nach Möglichkeit geringe Substanzmenge aufzuwenden. Um Kreuzresistenzen mit anderen Pflanzenschutzmitteln zu vermeiden, sollte ein potentieller Wirkstoff zudem idealerweise einen neuartigen Wirkmechanismus aufweisen. All diese Vorgaben führen dazu, dass der durchschnittliche Entwicklungszeitraum von neuen Pflanzenschutzmitteln in der Agrochemie mittlerweile eine Zeitspanne von acht bis zwölf Jahren umfasst.

Das Konzept des integrierten Pflanzenschutzes sieht vor, dass die ökologischen und wirtschaftlichen Aspekte auf Basis einer nachhaltigen Entwicklung miteinander verknüpft werden, um somit eine Maximierung der biologischen und mechanischen Schutzmechanismen bei gleichzeitiger Minimierung der eingesetzten Wirkstoffmenge zu erreichen. ${ }^{13}$ Um dies zu gewährleisten, wurde der integrative Pflanzenschutz im August 2011 gesetzlich verankert und ist seit 1. Januar 2014 für alle Mitgliedstaaten der Europäischen Union verpflichtend.

\subsubsection{Fungizide $^{14}$}

Fungizide sind Pflanzenschutzmittel, die die Entwicklung pathogener Pilze an Kulturpflanzen hemmen oder den Pilzbefall direkt mortifizieren. Neben Ernteausfällen sind pathogene Pilze auch für die Ausbildung toxischer Verbindungen wie beispielsweise der Mutterkornalkaloide verantwortlich. Allein in Deutschland wurden 2012 etwa 9066 t Fungizide abgesetzt. ${ }^{15}$

Da Pilze sowohl in Form von Sporen auf der Oberfläche als auch als Organismus im Inneren der Nutzpflanze zu finden sind, gibt es für Fungizide verschiedene Wirkorte und Wirkmechanismen. Strukturell betrachtet weisen Fungizide infolgedessen eine hohe Vielfältigkeit in den Leitmotiven der Wirkstoffe auf (Abbildung 2).

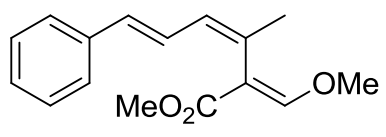

Strobilurin A (5)

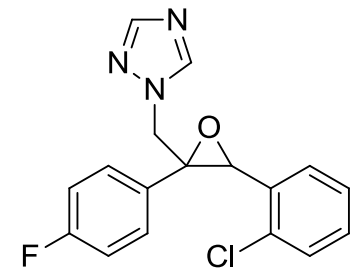

Epoxiconazol (6)

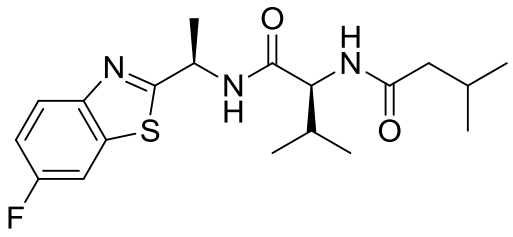

Benthiavalicarb (7)

Abbildung 2: Beispiele für moderne organische Fungizide. 
Der Großteil der Fungizide wirkt inhibitorisch auf die mitochondriale Atmungskette der pathogenen Pilze. Ein namhaftes Beispiel aus dieser Substanzklasse ist das Strobilurin A (5), welches eine ausgezeichnete fungizide Wirkung aufweist und als Namensgeber sowie Leitstruktur der Wirkstoffklasse der Strobilurine fungiert. Strobilurine bilden innerhalb des Gewebes ein Wirkstoffdepot, welches über einen längeren Zeitraum den Wirkstoff an die Nutzpflanze abgibt und somit einen permanenten Schutz bietet. Der große Vorteil dieser Substanzklasse ist, dass sie infolgedessen nicht so frequentiert auf die Agrarfläche aufgetragen werden muss und der Wirkstoff nicht durch Regen ausgewaschen wird.

Demethylierungsinhibitoren (DMIs) sind in der Lage, in die Steroidbiosynthese der pathogenen Pilze einzugreifen und so deren Wachstum und die Sporenbildung zu unterdrücken. Dabei sind die Wirkstoffe meist innerhalb der Pflanze mobil und können eine kurative und protektive Wirkung entfalten. Als wichtiger Vertreter der DMIs gilt das Triazol Epoxiconazol (6), welches von der BASF vertrieben wird. Ein weiterer potenter Wirkstoff mit anderem Strukturmotiv ist das Benthiavalicarb (7), welches effektiv den Zellwandaufbau des Schadorganismus inhibiert.

\subsubsection{Herbizide $^{16}$}

Die zweite und wirtschaftlich wichtigste Klasse der Pflanzenschutzmittel sind die Herbizide. Herbizide zeichnen sich durch ihre Aktivität gegen die unerwünschte Begleitflora der Kulturpflanzen aus und stellen mit $19907 \mathrm{t}$ und 43\% einen enormen Anteil der 2012 in Deutschland ausgetragenen Pflanzenschutzmittel dar. ${ }^{15}$

Eine Klassifizierung der Herbizide kann anhand ihres Wirkmechanismus erfolgen. Ähnlich den Fungiziden gibt es auch hier eine Vielzahl von Wirkorten. Von den 30 experimentell bestätigten Targets, die sich hauptsächlich in den Chloroplasten befinden, sind ca. 19 wirtschaftlich interessant. Zwei der bekanntesten Herbizide, die Bipyridiniumsalze Paraquat (8) und Diquat (9) (Abbildung 3) wirken beispielsweise am Photosystem I. Aufgrund ihrer unselektiven Wirkung und der dadurch hervorgerufenen toxikologischen Bedenken werden sie jedoch in vielen Ländern nur noch eingeschränkt eingesetzt oder wurden komplett verboten.

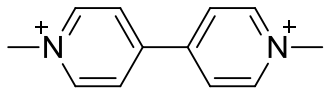

$2 \mathrm{Cl}^{-}$

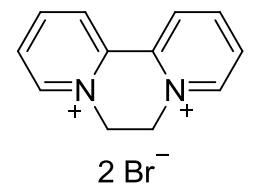

Diquat (9)<smiles>N#Cc1cc(Br)c(O)c(Br)c1</smiles>

Bromoxynil (10)<smiles>O=C(O)CNCP(=O)(O)O</smiles>

Glyphosat (11)

Abbildung 3: Beispiele für kommerzielle Herbizide. 
Während die Bipyridiniumsalze am Photosystem I wirken, beeinflusst der Großteil der Herbizide das Photosystem II. Ein wichtiger, weil sehr selektiver, Wirkstoff ist das Bromoxynil (10), das zusätzlich zu der Photosyntheseinhibition in die Atmungskette eingreift und den für die ATP-Synthese essentiellen Aufbau eines Protonengradienten an der Mitochondrienmembran verhindert.

Eines der kommerziell erfolgreichsten Pflanzenschutzmittel ist das Glyphosat (11), welches ein zentrales Enzym im Shikimisäureweg hemmt und somit die Biosynthese der aromatischen Aminosäuren Tyrosin, Phenylalanin und Tryptophan inhibiert. Glyphosat (11) wird besonders auf dem amerikanischen Kontinent und in Kombination mit gentechnisch verändertem Saatgut eingesetzt. Die Nutzpflanzen diese Saatguts - vor allem Mais, Soja, Raps und Baumwolle - besitzen ein Resistenzgen, welches eine Immunität gegen den Wirkstoff hervorruft.

Auf eine ähnliche Weise funktionieren die sogenannten Safener. ${ }^{17}$ Safener sind Zusatzstoffe, die selektiv die Nutzpflanze gegen die schädigende Wirkung des applizierten Herbizids schützen, ohne dabei ihr Erbgut zu verändern. Sie aktivieren spezifisch die Abbauenzyme der Kulturpflanze, die für die Metabolisierung des Herbizids verantwortlich sind. Der Wirkstoff wird so schneller abgebaut und das Wachstum der Nutzpflanze weniger gehemmt. Ein Beispiel eines solchen Antidots ist das Oxabetrinil (12), das den Abbau des Herbizids Metolachlor (13) durch Stimulation der Glutathion-S-Transferase beschleunigt (Abbildung 4).

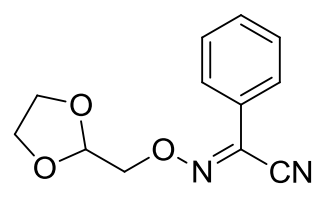

Oxabetrinil (12)

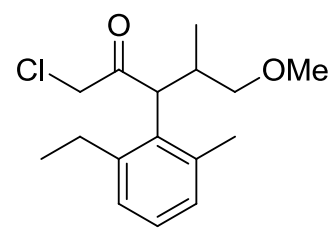

Metolachlor (13)

Abbildung 4: Oxabetrinil (12), ein Safener des Herbizids Metolachlor (13).

\subsubsection{Insektizide ${ }^{18}$}

Die dritte große Klasse der Pflanzenschutzmittel sind die Insektizide. Seit 2011 werden in Deutschland erstmals mehr Insektizide als Fungizide abgesetzt und die Tendenz ist weiter steigend. ${ }^{15}$ Im Jahr 2012 wurden deutschlandweit etwa 13359 t Insektizide ausgebracht, was einem Marktanteil von 29\% entspricht. Etwa 80\% der Insektizide sind Neurotoxine und haben Neurotransmitter wie das Acetylcholin und deren Rezeptoren als Target. Nur etwa 5\% der Wirkstoffe, unter ihnen Hormonmimetika und Inhibitoren der Chitinbiosynthese, beeinflussen die Entwicklung des Schadorganismus. Bei 15\% der Insektizide ist der Wirkmechanismus bis 
heute nicht aufgeklärt. Viele der klassischen Insektizide, wie das bereits angesprochene DDT (2), zählen zu den POPs. Sie stehen im Verdacht kanzerogen zu wirken und sind seit der Stockholmer Konvention 2001 verboten. ${ }^{19}$ Ein weiteres Problem der Insektizide ist die Bienentoxizität. Der Wirkstoff sollte nach Möglichkeit selektiv auf Schadorganismen wirken und nur geringe oder bestenfalls keine Aktivität gegenüber Nutzorganismen zeigen.

Diflubenzuron (14) ist ein Inhibitor der Chitinbiosynthese und verhindert die Ausbildung einer neuen Cuticula (Außenhaut) des Insekts, ohne dabei eine relevante Aktivität gegenüber Bienen aufzuweisen (Abbildung 5). Die betroffenen Schadorganismen sterben im Zuge ihrer nächsten regulären Häutung ab.<smiles>O=C(NC(=O)c1c(F)cccc1F)Nc1ccc(Cl)cc1</smiles>

Diflubenzuron (14)<smiles>CC1(C)C(C=C(Cl)Cl)C1C(=O)OC(C#N)c1cccc(Oc2ccccc2)c1</smiles>

Cypermethrin (rac-15)<smiles>N#Cc1ccc(C/C(=N\NC(=O)Nc2ccc(OC(F)(F)F)cc2)c2ccc(C(F)(F)F)cc2)cc1</smiles>

Metaflumizon (16)

Abbildung 5: Der Chitinbiosynthese-Inhibitor Diflubenzuron (14) und die Ionenkanal-Modulatoren Cypermethrin (rac-15) und Metaflumizon (16).

In der Klasse der Neurotoxine gibt es Ionenkanal-Modulatoren und Wirkstoffe, die den Abbau des Neurotransmitters Acetylcholin verhindern oder als Agonist am Acetylcholin-Rezeptor wirken. Das racemische Pyrethroid Cypermethrin (rac-15) sowie das Semicarbazon Metaflumizon (16) sind Antagonisten des Natriumionenkanals und rufen somit eine HyperErregung vor, die schlussendlich in der Lähmung des Insekts endet.<smiles>CNC(=O)Oc1cccc2c1OC(C)(C)C2</smiles>

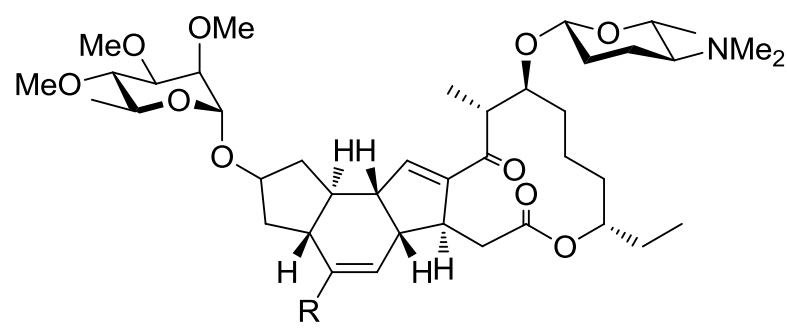

$\mathrm{R}=\mathrm{H}: \quad$ (-)-Spinosyn A (19) $\mathrm{R}=\mathrm{Me}:(-)$-Spinosyn D (20)

Abbildung 6: Beispiele für Neurotoxine.

Weitere wichtige Vertreter der Neurotoxine sind das bereits vorgestellte Organophosphat Parathion (1) und das Carbamat Carbofuran (17) (Abbildung 6). Diese beiden irreversibel wirkenden Acetylcholinesterase-Inhibitoren zeichnen sich vor allem durch ihre strukturelle 
Einfachheit und eine damit verbundene kostengünstige Herstellung aus, sind allerdings aufgrund ihrer hohen Toxizität gegen Nicht-Schadorganismen nicht mehr zulässig. Eine weitere Gruppe hochwirksamer Insektizide sind die Neonicotinoide. Diese Substanzklasse leitet sich von der Struktur des Nikotins ab und fungiert als Antagonist des AcetylcholinRezeptors, wodurch eine kontrollierte Reizweiterleitung im Schadorganismus verhindert wird. Der bekannteste Vertreter dieser Wirkstoffklasse ist das 1991 eingeführte Imidacloprid (18), das eines der weltweit meistverwendeten Insektizide ist. Neonicotinoide zeichnen sich durch eine hohe systemische Wirkung bei vergleichsweise geringer Säugertoxizität aus. Auch Spinosad, eine Mischung der beiden Naturstoffe Spinosyn A (19) und Spinosyn D (20) im Verhältnis 85:15, wirkt vermutlich als Acetylcholin-Antagonist. Anders als bei anderen Antagonisten ist der genaue Wirkort von Spinosad bislang allerdings unbekannt.

\subsection{Die Naturstoffklasse der Spinosyne ${ }^{20}$}

Einige der Wirkstoffe im modernen Pflanzenschutz sind durch Fermentation oder Extraktion gewonnene Naturstoffe oder deren Analoga. Diese Naturstoffe sind Metaboliten von Bakterien, Pilzen oder auch Pflanzen und weisen meist sehr komplexe Strukturen mit einer großen Anzahl an Stereozentren auf. In der Bereitstellung des Pflanzenschutzmittels ist dies aus finanzieller Sichtweise ein gravierender Nachteil gegenüber synthetischen Wirkstoffen.

Dennoch findet die Naturstoffklasse der Spinosyne häufigen Einsatz, da sie eine hervorragende insektizide Wirkung gegenüber zahlreichen Lepidopterenarten vorzuweisen haben. Die beiden bekanntesten Vertreter dieser Wirkstoffklasse, Spinosyn A (19) und Spinosyn D (20), wurden 1982 auf den Jungferninseln aus einer Bodenprobe vom Gelände einer stillgelegten Rumdestille isoliert. Es handelt sich um die beiden Hauptmetaboliten des aeroben, grampositiven und Mycel-bildenden Actinomycet Saccharopolyspora spinosa. Aufgrund des anspruchsvollen synthetischen Zugangs wird Spinosad hauptsächlich durch Fermentation gewonnen.

\subsubsection{Strukturmerkmale der Spinosyne}

Das Grundgerüst der Spinosyne bildet ein tetracyclisches Ringsystem, bestehend aus einem 12-gliedrigen Makrolacton und einem daran annelierten cis-anti-trans-5,6,5-Tricyclus (Abbildung 7). Dieses Strukturmotiv ist mit anderem Substitutionsmuster außerdem noch in den beiden Antibiotika Ikarugamycin ${ }^{21}$ und Capsimycin ${ }^{22}$ enthalten. Spinosyn A (19) weist insgesamt 17 Stereozentren auf, neun davon im tetracyclischen Grundkörper. Zwei dieser Stereozentren tragen Hydroxyfunktionen, die als Bindungsstellen für Zuckersubstituenten 
fungieren. An C-9-Position ist durch eine $\alpha$-Verknüpfung die 2,3,4-Tri- $O$-methyl-L-rhamnose und an C-17 durch eine $\beta$-Verknüpfung der Aminozucker D-Forosamin gebunden.

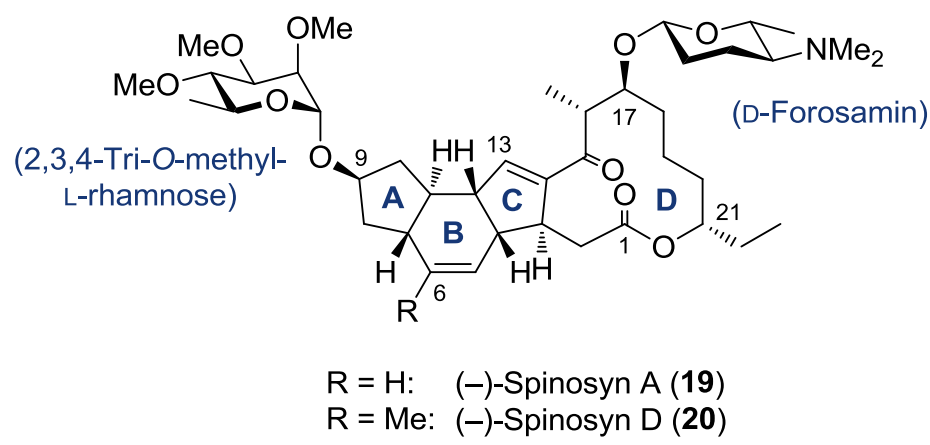

Abbildung 7: Hauptmetabolite von Saccharopolyspora spinosa.

Bis heute wurden über 20 unterschiedliche Spinosyne aus Saccharopolyspora spinosa isoliert und charakterisiert, die sich im Wesentlichen im $O$ - und $N$-Methylierungsmuster der beiden Saccharideinheiten, aber auch im Alkylierungsmuster des Grundgerüsts unterscheiden. Der Unterschied zwischen den beiden im Spinosad vorkommenden Spinosynen ist dabei eine Methylgruppe an C-6-Position bei Spinosyn D (20) gegenüber einem Wasserstoffatom in Spinosyn A (19). Zudem wurden vor einigen Jahren mehr als 30 weitere Spinosyne aus dem Stamm Saccharopolyspora pogona gewonnen. ${ }^{23}$ Viele dieser als Pogonine bezeichneten Verbindungen sind an C-21-Position anstatt der im Spinosyn enthaltenen Ethylgruppe mit einer Butenylgruppe substituiert und dadurch auch unter dem Namen Butenyl-Spinosyne bekannt.

\subsubsection{Eigenschaften und Wirkungsweise ${ }^{24}$}

Spinosyne finden heutzutage eine immer breitere Anwendung. Das durch Fermentation gewonnene Spinosad ist seit 1997 unter anderem unter den Markennamen Conserve ${ }^{\circledR}$, Success $^{\circledR}$, SpinTor ${ }^{\circledR}$ und Entrust $^{\circledR}$ als Pflanzenschutzmittel erhältlich. Auch in der Veterinärmedizin wird Spinosad mittlerweile gegen Ektoparasiten wie Flöhe oder Läuse eingesetzt. Seit 2011 ist die Wirkstoffmischung in den USA auch in Natroba ${ }^{\mathrm{TM}}$, einem Medikament zur äußerlichen Behandlung von Kopfläusen, enthalten. Zusätzlich sicherte sich Eli Lilly 2010 die Patentrechte an der Verwendung von Spinosad zur Verbesserung der Wundheilung. ${ }^{25}$

Agrochemisch betrachtet zeichnet sich Spinosad durch seine selektive Wirkung auf Schadinsekten und seine hervorragende Umweltverträglichkeit aus. Da Spinosyne vergleichsweise unpolare Verbindungen sind ( $\log P=4$.0, bei $\mathrm{pH} 7$ für Spinosyn $\mathrm{A})$ und eine schlechte Wasserlöslichkeit besitzen (235 mg/L bei pH 7), werden tiefere Bodenschichten und 
das Grundwasser durch die Verwendung kaum kontaminiert. Auf der Boden- bzw. Pflanzenoberfläche wird Spinosad durch Photolyse $\left(\mathrm{t}_{1 / 2}=0.5-2.5 \mathrm{~h}\right.$; je nach $\left.\mathrm{pH}\right)$ und in den oberen Bodenschichten durch mikrobiell gesteuerte Degradierungsprozesse vergleichsweise schnell abgebaut. ${ }^{26}$

Ein weiterer entscheidender Faktor ist die hohe Selektivität gegenüber dem Zielmechanismus. Während die Mortalität bei Schadinsekten wie dem Maiszünsler (Ostrinia nubilalis) bei 100\% liegt, zeigt Spinosad nur eine geringe bis keine toxische Wirkung für Säugetiere, Vögel und Wasserlebewesen (Tabelle 1). ${ }^{27}$ Auch gegenüber Nutzinsekten wie Raubmilben oder räuberischen Insekten ist Spinosad nicht oder nur kaum schädigend.

Tabelle 1: Toxische Wirkung von Spinosad auf ausgewählte Säugetiere, Fische und Vögel.

\begin{tabular}{cccc}
\hline Spezies & Test & Ergebnis & Einstufung \\
\hline Ratte ( $)$ & akute orale $\mathrm{LD}_{50}$ & $>5000 \mathrm{mg} / \mathrm{kg}$ Körpergewicht & nicht toxisch \\
Ratte & Inhalation $\mathrm{LC}_{50}$ & $>5 \mathrm{mg} / \mathrm{L} \mathrm{Luft}$ & nicht toxisch \\
Maus & akute orale $\mathrm{LD}_{50}$ & $>5000 \mathrm{mg} / \mathrm{kg}$ Körpergewicht & nicht toxisch \\
Kaninchen & akute dermale $\mathrm{LD}_{50}$ & $>5000 \mathrm{mg} / \mathrm{kg}$ Körpergewicht & nicht toxisch \\
Karpfen & $96 \mathrm{~h}$ akute $\mathrm{LC}_{50}$ & $5.0 \mathrm{mg} / \mathrm{L}$ Wasser & mäßig toxisch \\
Forelle & $96 \mathrm{~h}$ akute $\mathrm{LC}_{50}$ & $30.0 \mathrm{mg} / \mathrm{L}$ Wasser & leicht toxisch \\
Stockente & akute orale $\mathrm{LD}_{50}$ & $>2000 \mathrm{mg} / \mathrm{kg}$ Körpergewicht & nicht toxisch \\
Wachtel & akute orale $\mathrm{LD}_{50}$ & $>2000 \mathrm{mg} / \mathrm{kg}$ Körpergewicht & nicht toxisch \\
\hline
\end{tabular}

Ein weit verbreitetes Problem bei der Entwicklung von Insektiziden ist die Bienentoxizität. So konnte nachgewiesen werden, dass Neonicotinoide für das als „Colony Collapse Disorder“ (CCD) bekannte Phänomen des Bienensterbens verantwortlich sind. ${ }^{28}$ Die Bienentoxizität wird in vier Kategorien eingeteilt: ${ }^{29}$

- B4: nicht bienengefährlich

- B3: nicht bienengefährlich; aufgrund von Auflagen in Bezug auf die Anwendung

- B2: bienengefährlich; Anwendung nach Ende des täglichen Bienenflugs möglich

- B1: bienengefährlich.

Spinosad fällt unter Laborbedingungen in die Kategorie B1 und gilt als akut toxisch für Bienen. ${ }^{30}$ Feldstudien konnten allerdings zeigen, dass Spinosad bei sachgemäßer Anwendung nur eine geringe Bedrohung für Bienen darstellt. ${ }^{31}$ Bienen, die auf der Pflanze angetrockneten 
Wirkstoffrückständen des Spinosads ausgesetzt wurden, zeigten später beispielsweise keinerlei Vergiftungserscheinungen.

Dank der hochpotenten Wirkung des Spinosads gegenüber Zielorganismen ergibt sich eine vergleichsweise geringe Ausbringungsmenge. Je nach Art des Zielorganismus werden zur Kontrolle der Schädlingspopulation in den meisten Fällen unter $100 \mathrm{~g}$ pro Hektar benötigt (Tabelle 2).

Tabelle 2: Targetbezogene Ausbringungsmengen für Spinosad.

\begin{tabular}{ccc}
\hline Spezies & Art & Ausbringungsmenge [g/ha] \\
\hline Kohlmotte & Plutella xylostella & $15-50$ \\
Maiszünsler & Ostrinia nubilalis & $25-50$ \\
Traubenwickler & Lobesia botrana & $25-50$ \\
Tomatenschwärmer & Manduca quinquemaculata & $40-100$ \\
Baumwollkapselbohrer & Helicoverpa zea & $40-100$ \\
Baumwollkapselwurm & Helicoverpa armigera & $50-100$ \\
Kohlweißling & Pieris rapae & $50-100$ \\
\hline
\end{tabular}

Bei Kontamination eines Zielorganismus mit dem Wirkstoff kommt es zum Tremor, unkontrollierten Bewegungen der Mundwerkzeuge, Hyperventilation bis hin zur vollständigen irreversiblen Lähmung des Insekts. Die Wirkung setzt dabei meist einige Minuten bis Stunden nach der Kontamination ein.

In frühen Studien wurde angenommen, dass Spinosyn als Antagonist eines nicotinergen Acetylcholin-Rezeptors ${ }^{32}$ und des $\gamma$-Aminobuttersäure-Rezeptors ${ }^{33}$ agiert. Neuere Studien mit Tritium-basierten Austauschexperimenten zeigen jedoch keine Wechselwirkung mit bisher bei anderen Insektiziden bekannten Bindungsstellen dieses Typs. ${ }^{34}$ Lediglich an den spannungsabhängigen Calciumkanälen konnte ein geringfügig erhöhter Austausch festgestellt werden. Diese Ergebnisse legen die Schlussfolgerung nahe, dass die Bioaktivität der Spinosyne auf einem neuartigen Mechanismus beruht und sie höchstwahrscheinlich ihre Wirkung an einem bisher unbekannten nicotinergen Acetylcholin-Rezeptor entfalten. Läge ein solcher neuartiger Mechanismus vor, wäre die Gefahr des Auftretens von Kreuzresistenzen bei der Verwendung von Spinosynen im Pflanzenschutz als relativ gering einzuschätzen. 


\subsubsection{Stand gegenwärtiger Forschung}

Trotz seiner hervorragenden Eigenschaften als Pflanzenschutzmittel wurden 1998 im Labor ${ }^{35}$ und 1999 auch in Feldstudien ${ }^{36}$ Resistenzen gegenüber Spinosad beobachtet. Um den Resistenzentwicklungen entgegenzuwirken, bedarf es weiterhin intensiver Forschung auf diesem Gebiet. Die Erschließung neuer Zugangsquellen durch eine chemische Totalsynthese würde unter anderem die Bereitstellung von Derivaten und Analoga deutlich erleichtern. Von Seiten der industriellen Forschung wurden bereits mehr als 1000 semisynthetische Derivate der Spinosyne hergestellt und auf ihre biologische Aktivität getestet (Abbildung 8). ${ }^{37}$ Mit Hilfe dieser Struktur-Aktivitäts-Beziehungen (engl. structure activity relationship = SAR) konnten nützliche Informationen für die weitere Derivatisierung erlangt werden und somit der Grundstein zur Synthese noch wirkeffizienterer Spinosoide gelegt werden.

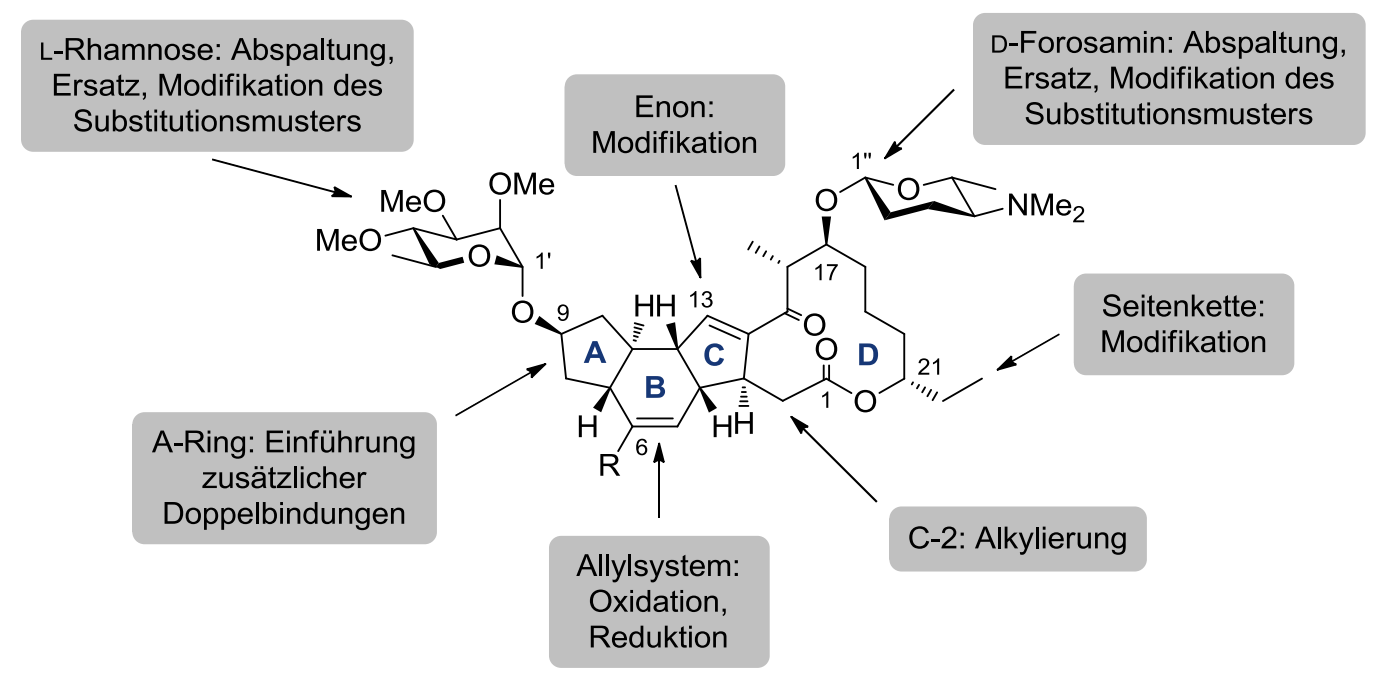

Abbildung 8: Übersicht bereits durchgeführter Derivatisierungen am Naturstoff.

So konnte beobachtet werden, dass eine Abspaltung beider Zuckereinheiten zum völligen Verlust der insektiziden Wirkung führt (vgl. Tabelle 3). ${ }^{38}$ Weitere Untersuchungen von Crouse et al. zum Substitutionsmuster des Rhamnoglycosids zeigten, dass durch Modifikation eine merkliche Aktivitätssteigerung beobachtet werden konnte. ${ }^{39}$ Die ersten Veränderungen des tetracyclischen Grundgerüsts durch Epoxidierung oder Substitution am 13,14-Enon- und 5,6-Allylsystem erwiesen sich ebenso wie eine Alkylierung an C-2-Position als aktivitätssenkend. Auch weitere Modifikationen an diesen Positionen führten zu keinem verbesserten Wirkprofil.

Die Einführung weiterer Doppelbindungen zwischen dem A- und B-Ring des Spinosyns resultierte trotz des Verlustes von stereogenen Zentren erstaunlicherweise nur in einer marginalen Änderung der letalen Konzentration. Während das 7,8-Dehydro-Spinosyn D (21) 
einen ähnlichen $\mathrm{LC}_{50}$-Wert wie der Naturstoff aufweist, zeigt das 7,11-Dehydro-Spinosyn D (22) sogar eine Aktivitätssteigerung (Abbildung 9). Bei dem Indenyl-Derivat 23 hingegen wurde ein vollständiger Wirkverlust beobachtet.

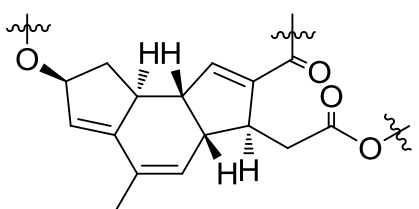

7,8-Dehydro-Spinosyn D (21)

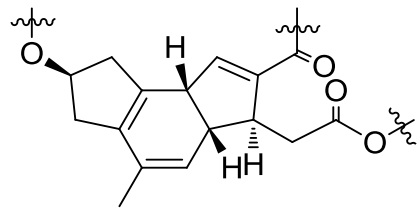

7,11-Dehydro-Spinosyn D (22)

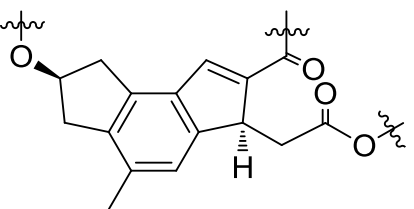

Indenyl-Spinosyn D (23)

Abbildung 9: Derivate des Spinosyn D mit zusätzlichen Doppelbindungen.

Diese Ergebnisse deuten an, dass die stereogenen Zentren in 7- und 8-Position keinen gravierenden Einfluss auf die biologische Aktivität haben, während die cis-Verknüpfung zwischen B- und C-Ring essentiell für den toxischen Effekt ist. Eine strukturvereinfachende Substitution des aliphatischen A-Rings durch ein sechsgliedriges aromatisches System könnte somit zu neuen, interessanten Spinosynanaloga führen. Durch das Aussparen von zwei stereogenen Zentren wäre der Syntheseaufwand für solche neuen Verbindungstypen im Vergleich zu den bioidentischen Verbindungen wesentlich geringer. Weitere Testergebnisse sind in Tabelle 3 aufgeführt, wobei interessante Ergebnisse in Bezug auf eine AnalogaSynthese hervorgehoben sind.

Tabelle 3: Insektizide Wirkung von ausgewählten semisynthetischen Spinosyn-Derivaten gegenüber der amerikanischen Tabakeule (Heliothis virescens).

\begin{tabular}{|c|c|c|c|c|c|}
\hline Modifikation & Spinosyn & $\mathbf{L C}_{50}[\mathrm{ppm}]$ & Modifikation & Spinosyn & $\mathbf{L C}_{50}[\mathrm{ppm}]$ \\
\hline Spinosyn A (19) & A & 0.3 & $13-\mathrm{NHOH}$ & A & 5.6 \\
\hline Spinosyn D (20) & $\mathrm{D}$ & 0.5 & 5,6- $\beta$-Ероху & $\mathrm{D}$ & 10 \\
\hline Aglycon & A & $>64$ & 5,6-Dihydro & A & 0.5 \\
\hline $2^{\prime}, 3^{\prime}, 4^{\prime}-$ Tri-O-Et & $\mathbf{A}$ & 0.02 & $5-\mathrm{OH}$ & $\mathrm{D}$ & $>64$ \\
\hline 4"-Keto & A & 3.4 & 2-Methyl & A & 4.5 \\
\hline $13,14-\alpha$-Dihydro & A & 4.7 & 7,8-Dehydro (21) & $\mathbf{D}$ & 0.6 \\
\hline $13,14-\beta$-Dihydro & A & 20 & 7,11-Dehydro (22) & D & 0.2 \\
\hline 13,14-Eроху & A & 1.4 & Indenyl (23) & $\mathrm{D}$ & $>64$ \\
\hline
\end{tabular}


Die bereits vorgestellten, an C-21 butenylsubstituierten Pogonine lieferten ebenfalls neue Erkenntnisse zu den Struktur-Aktivitäts-Beziehungen der Spinosyne. Da diese ButenylSpinosyne keine oder nur eine marginale Aktivitätssteigerung gegenüber den korrespondierenden ethylsubstituierten Spinosynen zeigten, scheint die Beschaffenheit der Seitenkette an C21-Position keinen großen Einfluss auf die biologische Aktivität zu haben.

Mit den Kenntnissen der SARs der Spinosyne wurde ein neuer Wirkstoff namens Spinetoram entwickelt und unter den Namen Delegate ${ }^{\circledR}$ und Radiant ${ }^{\circledR}$ im Pflanzenschutz zugelassen. Es handelt sich dabei um ein Gemisch aus 3'-O-Ethyl-substituierten Spinosynen mit reduzierter (24) oder vorhandener Doppelbindung (25) in 5,6-Position (Abbildung 10).

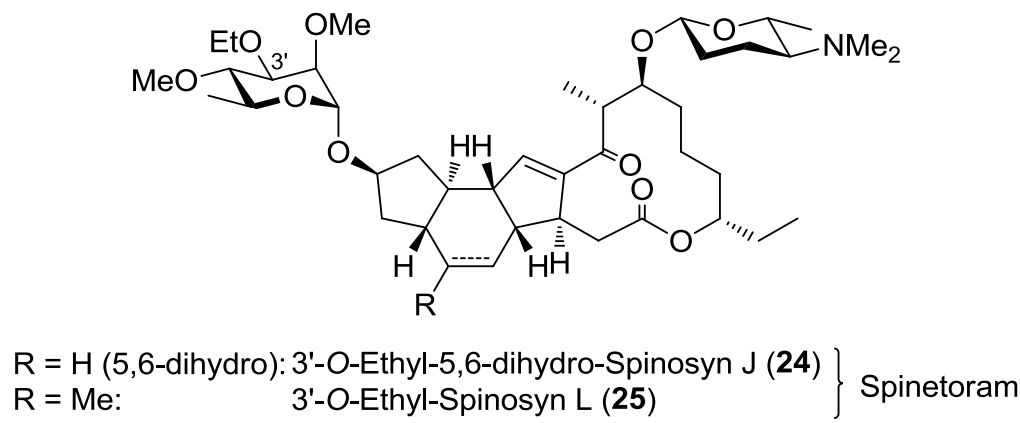

Abbildung 10: Struktur des semi-synthetischen Wirkstoffes Spinetoram.

Die Herstellung des Pflanzenschutzmittels erfolgt semi-synthetisch ausgehend von den durch Fermentation gewonnenen, natürlich vorkommenden 3'-demethylierten Spinosynen J (für 24) und L (für 25). Beide werden zunächst am Rhamnopyranosid ethyliert und im Fall von Verbindung 24 anschließend an der Doppelbindung reduziert. Das so erhaltene Spinetoram zeigt im Vergleich mit Spinosad eine höhere Wirksamkeit gegenüber Schadorganismen sowie eine verbesserte Photostabilität. ${ }^{40}$ Ein weiterer Vorteil gegenüber dem bioidentischen Wirkstoff ist die geringere Bienentoxizität. ${ }^{41}$

Neue, interessante Erkenntnisse von Worden et al. zeigen, dass auch die D-Forosaminyleinheit ohne markanten Wirkverlust gegen andere stickstoffhaltige Saccharide und sogar Substituenten, die nicht zu den Kohlenhydraten zählen, austauschbar ist. ${ }^{42}$

\subsubsection{Biosynthese}

Die Biosynthese der Spinosyne ist ein kontrovers diskutiertes Thema, gilt heutzutage aber als weitgehend aufgeklärt. So konnten nach Liu et al. die Gene SpnA E des Genclusters von Saccharopolyspora spinosa dem Aufbau des Post-Polyketid-Synthase-Makrolactons 26 zugeteilt werden (Abbildung 11). ${ }^{43}$ Im nächsten Schritt erfolgt eine selektive Oxidation des Alkohols an C-15 durch die Flavin-abhängige Dehydrogenase SpnJ, wodurch die 
nachfolgende 1,4-Dehydratisierung durch SpnM erleichtert wird. Der Schlüsselschritt der Biosynthese ist eine transannulare [4+2]-Cycloaddition, die zum tricyclischen Aglycon 28 führt. Bisher ist nicht vollständig geklärt, ob $\mathrm{SpnF}$ als Diels-Alderase fungiert oder die [4+2]-Cycloaddition schrittweise erfolgt.

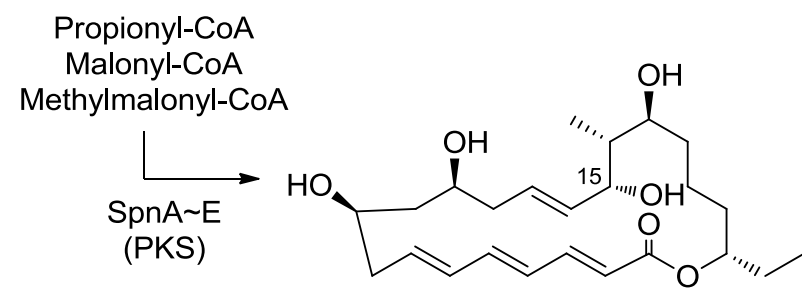

26<smiles>C/C=C\C(=O)O[C@H](CC)CCC[C@@H](O)[C@@H](C)C(=O)/C=C/[C@@H]1[C@H](C)C=C[C@@H]2C[C@H](O)C[C@@H]21</smiles>

28<smiles>[Y]C(C)([SnH2])[R7][Ba]</smiles>

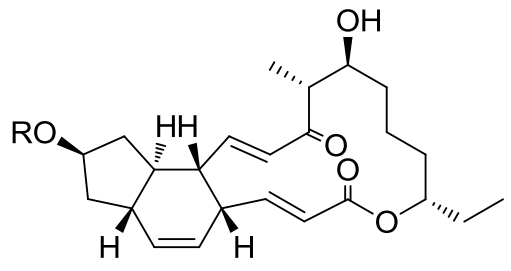

29

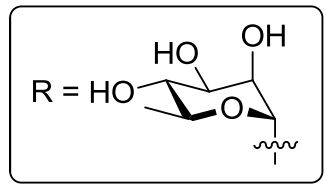

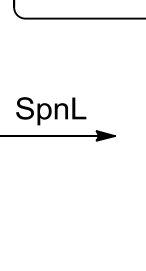

SpnL

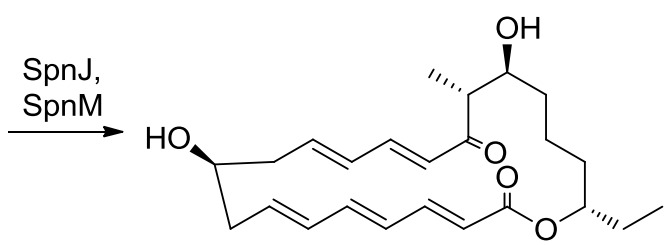

27

$\downarrow \uparrow \begin{gathered}\text { cis-trans- } \\ \text { Isomerisierung }\end{gathered}$

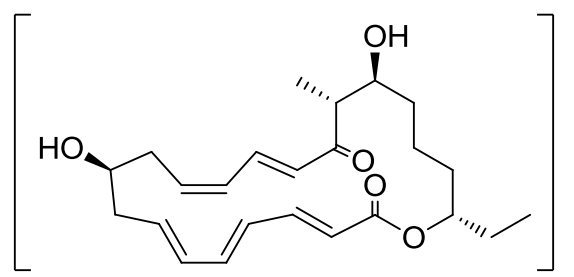

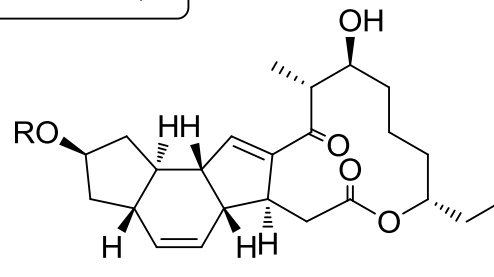

30
$\mathrm{SpnH}, \mathrm{I}, \mathrm{K}$

SpnP

(-)-Spinosyn A (19)

Abbildung 11: Postulierter Mechanismus der Biosynthese von Spinosyn A nach Liu et al.

Ausgehend von Aglycon 28 läuft als nächstes eine von der Transferase SpnG katalysierte Glycosylierung mit L-Rhamnose ab, bevor die letzte intramolekulare $\mathrm{C}-\mathrm{C}$-Bindungsknüpfung zum Tetracyclus 30 durch SpnL vollendet wird. Bei letzterer Umsetzung wird vermutet, dass es sich um eine Rauhut-Currier-Reaktion, also eine vinyloge Morita-Baylis-HillmanReaktion, handelt. ${ }^{44}$ Im nächsten Schritt der Biosynthese erfolgt die schrittweise Permethylierung der Rhamnose durch von SpnH, SpnI und SpnK exprimierte Methyltransferasen. Abgeschlossen wird die Biosynthese von Spinosyn A durch eine SpnPkatalysierte Glycosylierung mit dem relativ säurelabilen Zucker D-Forosamin. ${ }^{45}$

Die Biosynthese der beiden Zuckereinheiten erfolgt in beiden Fällen ausgehend von Glucose1-phosphat in vier (TDP-L-Rhamnose) bzw. sechs Schritten (TDP-D-Forosamin). Die Aufklärung des Biosynthesewegs kann zu einer Optimierung der Fermentationsprozesse 
führen oder neue Möglichkeiten für die Partial- oder Totalsynthese von Naturstoffen eröffnen (vgl. Kapitel A. 2.2.5).

\subsubsection{Totalsynthesen von Spinosyn A}

Chemische Totalsynthesen sind von zentraler Bedeutung, wenn ein Wirkstoff in der Natur nur in geringen Mengen vorhanden oder schwer zu isolieren ist. Auch bei der Aufklärung von Absolutkonfigurationen oder bei der Verifizierung von postulierten Strukturen spielen sie eine große Rolle. Auch wenn heutzutage ein Zugang zu Spinosynen durch Fermentation gesichert ist, ist ein chemischer Zugang in Hinblick auf die Synthese von resistenzbrechenden Analoga wünschenswert.

Bis zum heutigen Zeitpunkt sind lediglich drei Totalsynthesen und ein chemoenzymatischer Zugang zu Spinosynen bekannt. Die erste Totalsynthese von Spinosyn A wurde 1993 von Evans et al. publiziert (Abbildung 12). ${ }^{46}$

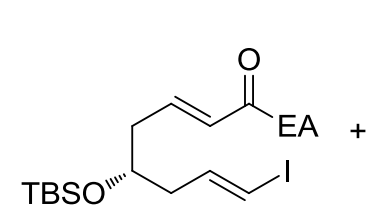

31

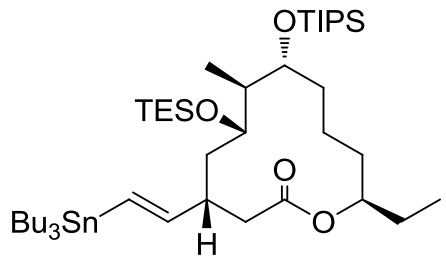

32

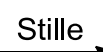

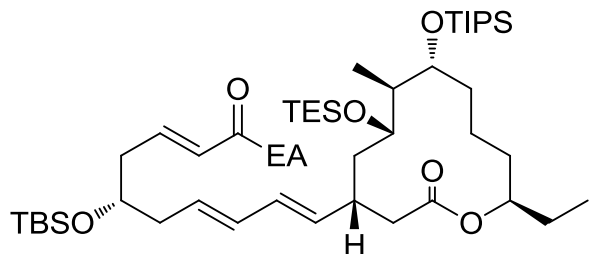

33 $\downarrow$ Diels-Alder

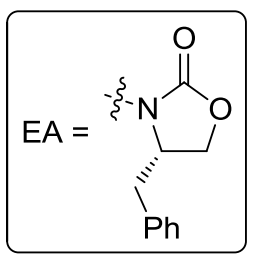

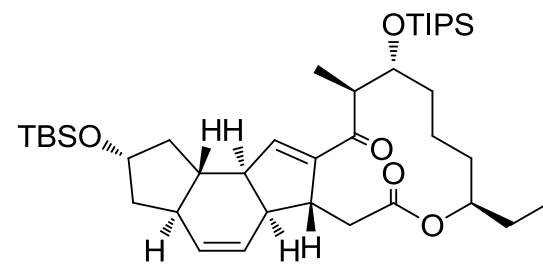

35

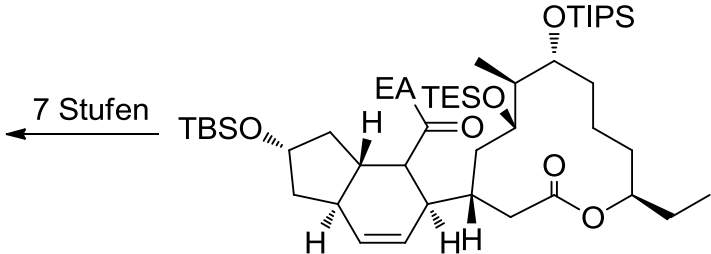

34

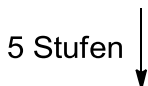

(+)-Spinosyn A (ent-19)

Abbildung 12: Schlüsselschritte der Totalsynthese von (+)-Spinosyn A nach Evans et al.

Die beiden Schlüsselschritte dieser Synthese umfassen die intermolekulare Stille-Kupplung des Vinyliodids 31 mit dem Makrolacton 32 zu 33 und die anschließende intramolekulare Diels-Alder-Reaktion zu Cyclohexen 34. In sieben weiteren Stufen, die unter anderem eine intramolekulare Aldolkondensation beinhalten, gelang der Aufbau des Tetracyclus 35, der nach Abspaltung der Schutzgruppen und Einführung der beiden Zuckereinheiten zum nicht natürlichen Isomer (+)-Spinosyn A (ent-19) führte. Da die genaue Stereochemie 1993 noch 
nicht vollständig aufgeklärt war, lieferte Evans' Synthese wichtige Erkenntnisse zur absoluten Konfiguration von Spinosyn A.

Die erste Totalsynthese von bioidentischem (-)-Spinosyn A wurde 1998 von Paquette et al. beschrieben (Abbildung 13). ${ }^{47}$

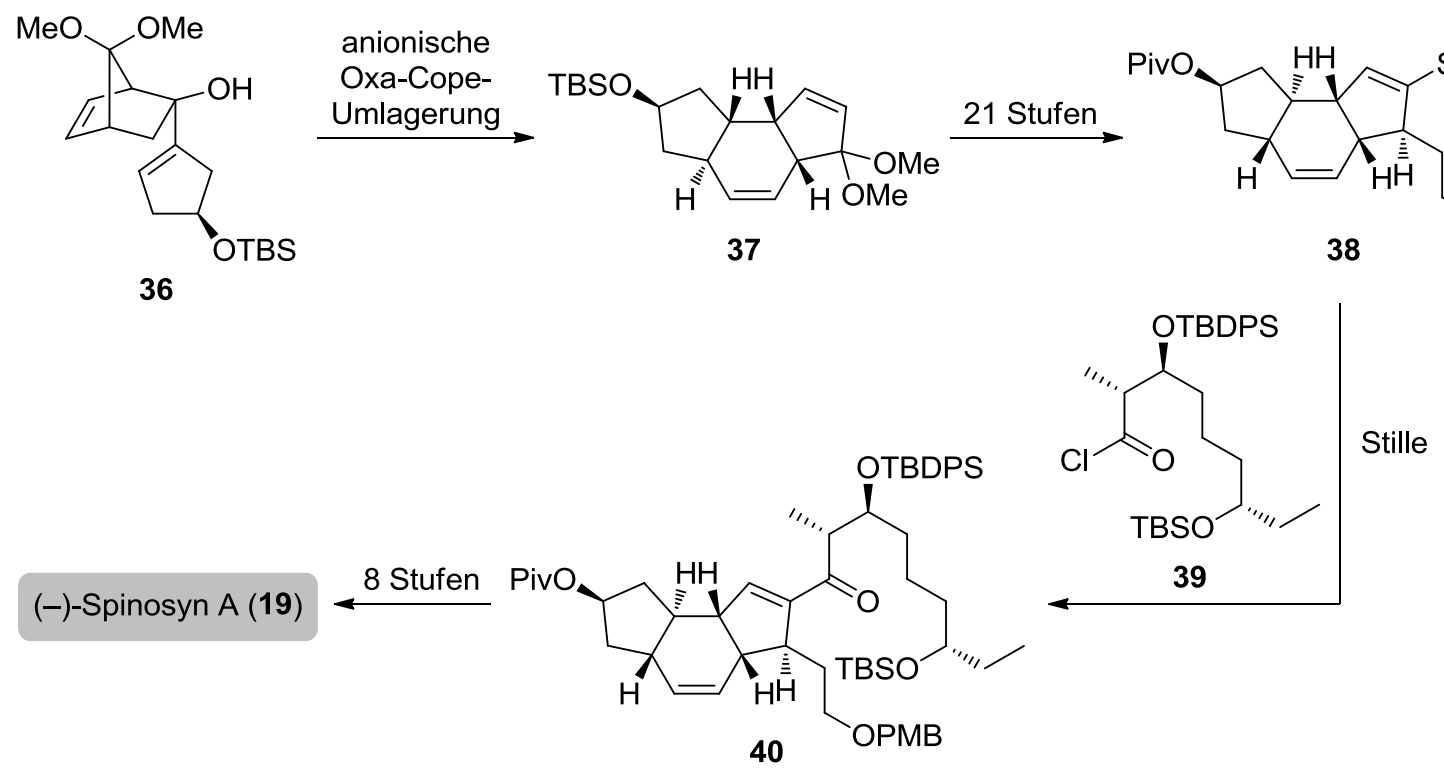

Abbildung 13: Schlüsselschritte der Totalsynthese von (-)-Spinosyn A nach Paquette et al.

Ausgehend von dem enantiomerenreinen Baustein 36 konnte das tricyclische Grundgerüst durch eine anionische Oxa-Cope-Umlagerung in inverser Stereochemie zwischen A- und B-Ring aufgebaut werden. Das annelierte System 37 konnte anschließend in 21 linearen Schritten in die natürliche Konfiguration überführt werden. Eine anschließende StilleKupplung mit dem Säurechlorid 39 sowie acht weitere Stufen, unter anderem eine Makrolactonisierung, führten zu dem natürlich vorkommenden (-)-Spinosyn A (19).

Die dritte Totalsynthese von Spinosyn A, veröffentlicht 2002 durch Roush et al., zeigt sich insbesondere durch die neuen Erkenntnisse in der Biosynthese von Spinosynen (vgl. Kapitel A. 2.2.4) inspiriert (Abbildung 14). So wurde zunächst der acyclische Vorläufer 41 hergestellt, dessen geplante Wittig-Horner-Makrolactonisierung überraschenderweise zu einer Dominoreaktion (vgl. Kapitel A. 6) mit inkludierter transannularer Diels-Alder-Reaktion, und somit direkt zum Tricyclus 42 führte. Eine anschließende vinyloge Morita-Baylis-HillmanReaktion lieferte analog zur Biosynthese Tetracyclus 43, der in sieben weiteren Stufen zu (-)-Spinosyn A (19) umgewandelt werden konnte. 


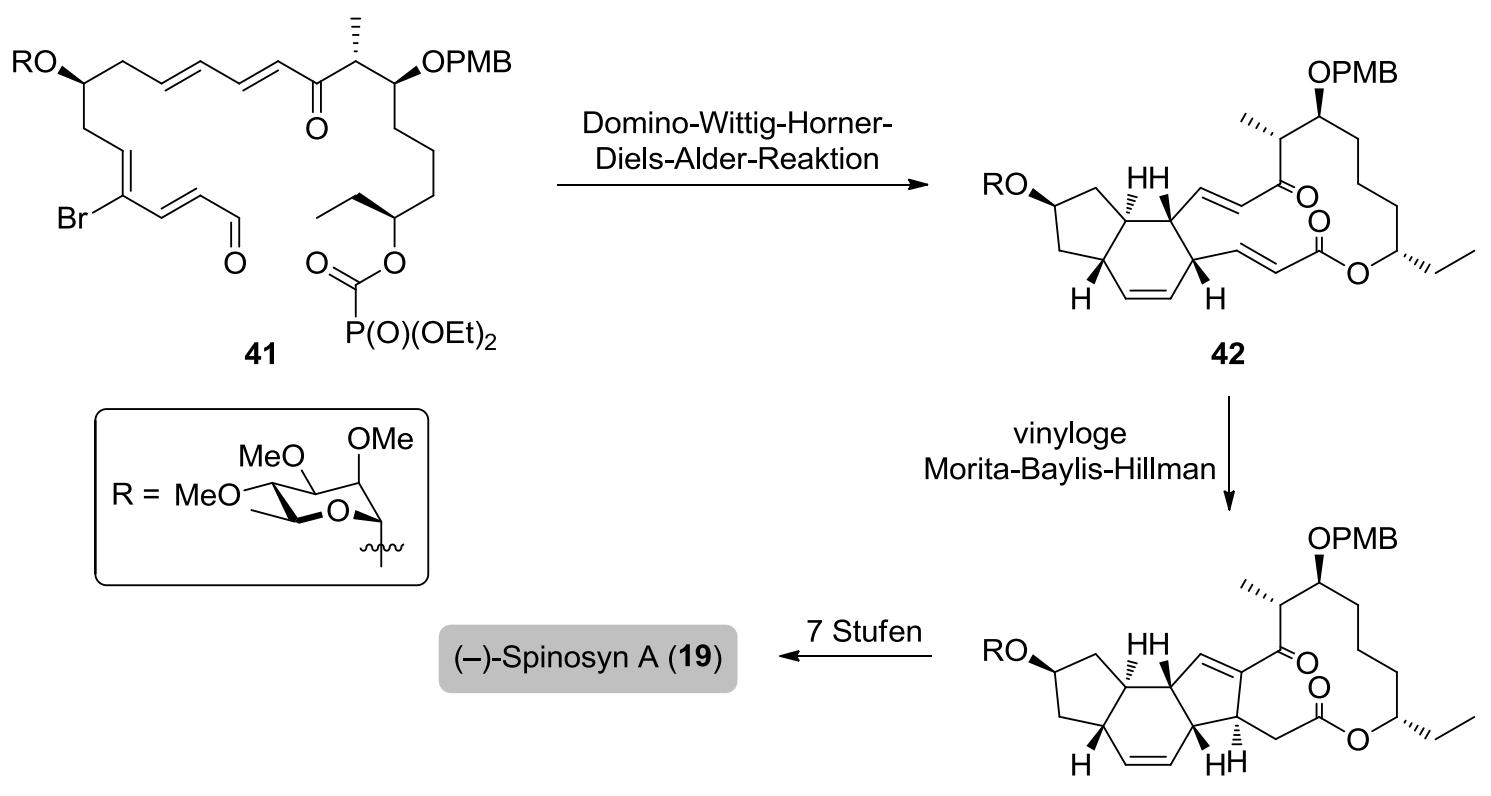

43

Abbildung 14: Schlüsselschritte der Totalsynthese von (-)-Spinosyn A nach Roush et al.

Der neueste Ansatz zum Aufbau von Spinosynen wurde 2014 von Liu et al. veröffentlicht und ist chemoenzymatischer Natur. Hierbei wurde zunächst totalsynthetisch das in Kapitel 2.2.4 vorgestellte Makrolacton 26 aufgebaut, welches anschließend in einer Eintopf-Reaktion mit Hilfe der Spn-Enzyme zu dem Pseudoaglycon 30 umgesetzt wurde (Abbildung 15).

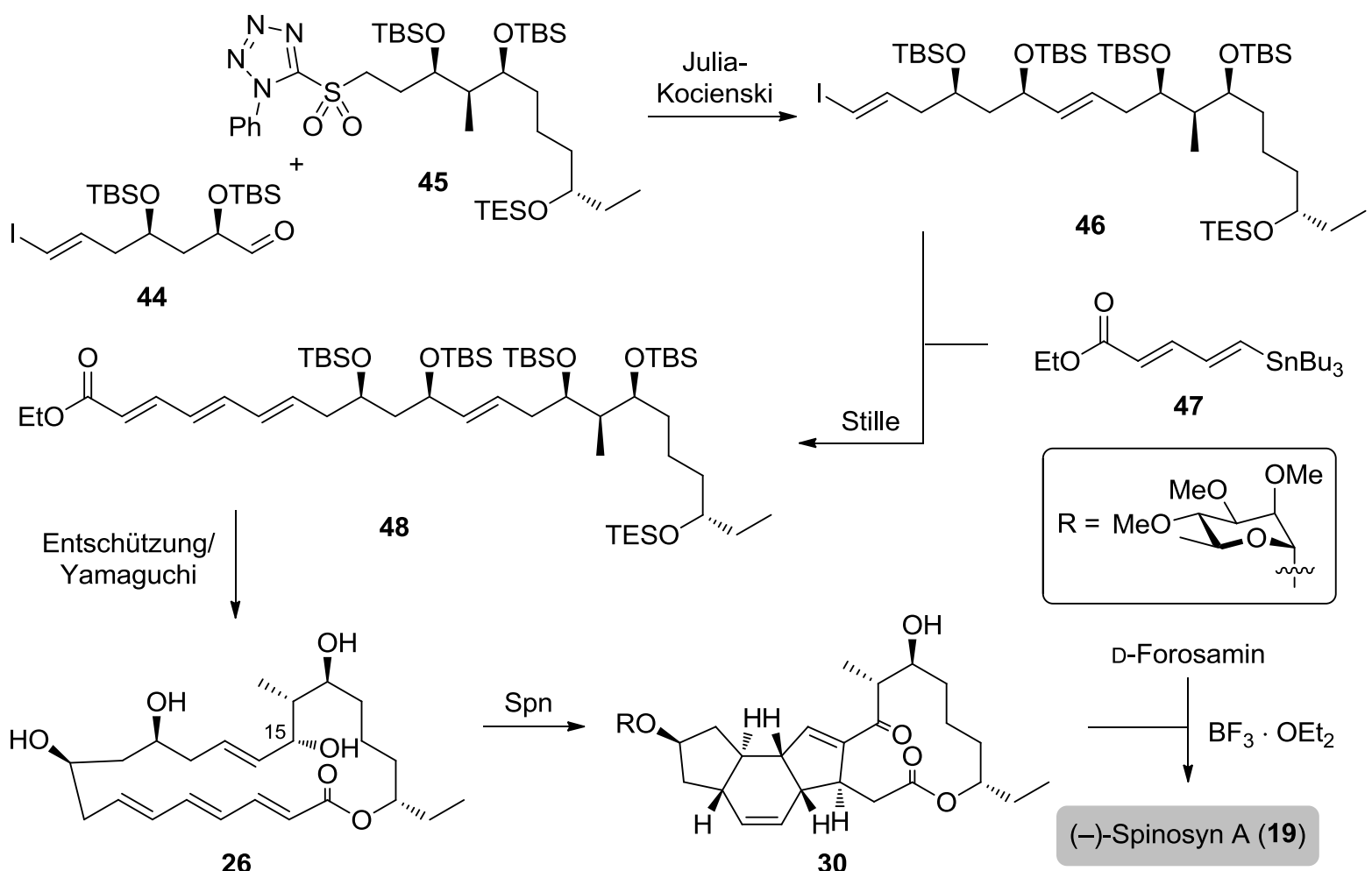

Abbildung 15: Schlüsselschritte der chemoenzymatischen Totalsynthese von (-)-Spinosyn A nach Liu et al. 
Schlüsselschritte im chemischen Teil der Synthese sind eine Julia-Kocienski-Olefinierung und eine Stille-Kupplung, welche die die drei Bausteine 44, 45 und 47 miteinander verbinden. Eine Yamaguchi-Makrolactonisierung des Polyketids 48 führt anschließend zum Vorläufer der enzymatischen Eintopf-Reaktion. Die finale Glycosylierung von Verbindung 30 mit SpnP konnte enzymatisch nicht realisiert werden und wurde wieder chemisch durchgeführt. Die Ergebnisse dieses chemoenzymatischen Ansatzes führen zu der Vermutung, dass die von SpnP exprimierte Gycosyltransferase ein weiteres, bisher unbekanntes, Hilfsprotein zur Aktivierung benötigt.

In Kapitel B. 1 dieser Doktorarbeit wird ein neuartiger Ansatz zur Synthese von SpinosynAnaloga diskutiert. 


\section{Lignane}

Die Totalsynthese kann nicht nur den Zugang zu Naturstoffen und deren Derivaten erleichtern, sondern auch die Methodik in der organischen Synthese vorantreiben.

Lignane sind eine Gruppe von Naturstoffen, die in der Wurzel oder den komestiblen Pflanzenteilen wie beispielsweise Samen und Körnern vorkommen. Aufgrund ihrer strukturellen Ähnlichkeit zu Östrogen gehören Lignane zu der Gruppe der Phytoöstrogene und weisen ein breites Spektrum an biologischen Aktivitäten auf. Neben ihren antiviralen, antiinflammatorischen, hepatoprotektiven und antineoplastischen Wirkungen treten sie häufig auch als Antioxidantien auf. $^{48}$

Strukturell betrachtet sind Lignane zwei an den 8,8'-Position verknüpfte Phenylpropanoide (Abbildung 16). Der Term „Lignan“ wurde 1936 von Haworth eingeführt ${ }^{49}$ und 1978 von Gottlieb um die sogenannten Neolignane mit 3 ',8-Verknüpfung erweitert. ${ }^{50}$

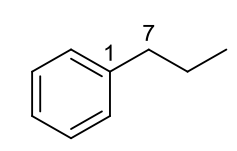

Phenylpropan (49)

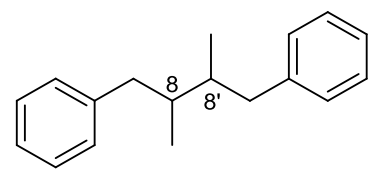

Lignan (50)

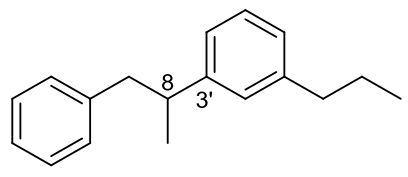

8,3'-Neolignan (51)

Abbildung 16: Struktur der Lignane und Neolignane.

Eine weitere strukturelle Unterklasse der Lignane bilden an C-9 und C-9' oxidierte Systeme, die je nach Oxidationsgrad neue Ringschlüsse ermöglichen und somit eine Vielzahl an neuartigen Strukturmotiven bieten. $\mathrm{Zu}$ den insgesamt acht Unterklassen gehören unter anderem die Dibenzylbutyrolactone (52), Aryltetraline (53) und Arylnaphthaline (54) (Abbildung 17).

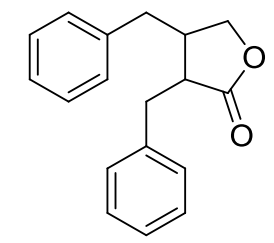

Dibenzylbutyrolacton (52)

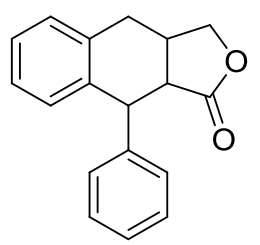

Aryltetralin (53)<smiles>O=C1OCc2cc3ccccc3c(-c3ccccc3)c21</smiles>

Arylnaphthalin (54)

Abbildung 17: Interessante Untergruppen der an C-9 und C-9' oxidierten Lignane.

In der Natur vorkommende Lignane sind ohne Ausnahme an den aromatischen Systemen substituiert. ${ }^{51}$ Häufige Variationen sind hierbei Hydroxy-, Methoxy- und MethylendioxySubstituenten. Außerdem kommen manche natürliche Lignane als racemisches Gemisch vor, während andere als reine Enantiomere auftreten. ${ }^{48}$ 
Aufgrund der strukturellen Vielfalt und des breiten Wirkspektrums sind Lignane ein in Bezug auf die Wirkstoffforschung beliebtes Target. Dieser Umstand macht natürlich vorkommende Lignane und ihre Analoga auch zu einem beliebten Ziel der organisch-chemischen Totalsynthese.

\subsection{Biosynthese ${ }^{52}$}

Aufgrund ihrer Ähnlichkeit in Strukturmotiven und Substitutionsmuster wird davon ausgegangen, dass zumindest frühe Stufen der Biosynthese von Lignanen der des Lignin entsprechen.

In diesem Biosynthesepfad wird unter E2-Eliminierung von Ammoniak die Aminosäure L-Tyrosin (57) durch eine Lyase in p-Cumarinsäure (58) überführt (Abbildung 18).<smiles>N[C@@H](Cc1ccccc1)C(=O)O</smiles>

(55)

(56)

$$
\begin{gathered}
\mathrm{O}_{2} \\
\mathrm{NADPH}
\end{gathered} \text { E3 }
$$

E1: Phenylalanin-Ammoniak-Lyase (PAL)

E2: Tyrosin-Ammoniak-Lyase (TAL)

E3: Zimtsäure-4-hydroxylase

E4: $p$-Cumarinsäure-3-hydroxylase

E5: Kaffeesäure-O-methyltransferase

E6: Ferulasäure-5-hydroxylase

SAM: S-Adenosylmethionin

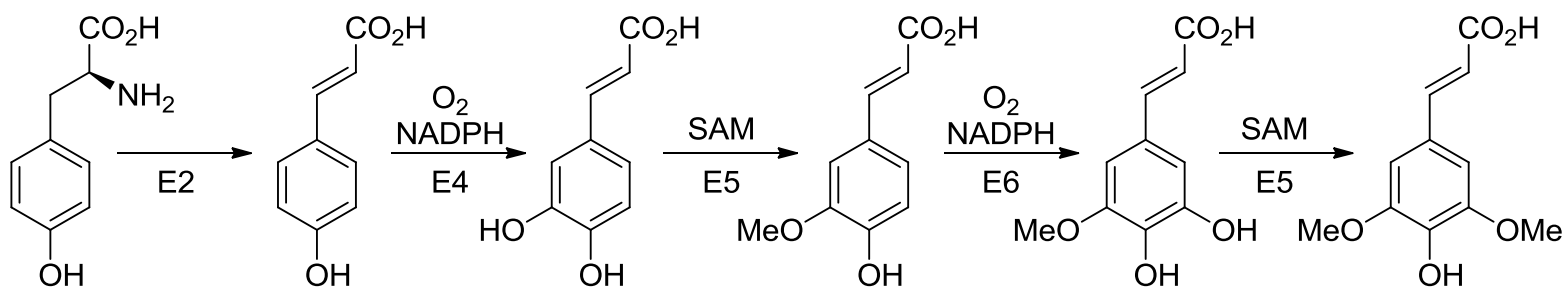<smiles>OCC=Cc1ccc(O)cc1</smiles>

p-Cumarylalkohol

(63)

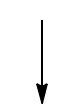<smiles>COc1cc(/C=C/CO)ccc1O</smiles>

Coniferylalkohol

(64)

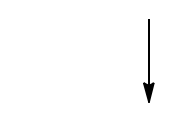<smiles>COc1cc(/C=C/CO)cc(OC)c1O</smiles>

Sinapylalkohol

(65)

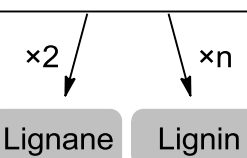

Abbildung 18: Biosynthese von Lignan-Vorläufern nach Derwick. ${ }^{52}$ 
Einen weiteren Pfad zur $p$-Cumarinsäure bildet die analoge E2-Eliminierung an L-Phenylalanin (55) mit anschließender Hydroxylierung der resultierenden Zimtsäure (56) in 4-Position. Durch nachfolgende Hydroxylierungen und Methylierungen sowie anschließender Reduktion der Carbonsäure zum korrespondierenden Alkohol sind die drei Lignan- bzw. Lignin-Vorläufer 63, 64 und 65 zugänglich.

Bei der Bildung eines Coniferylalkohol-Radikals zeigt sich durch Betrachtung der mesomeren Grenzstrukturen eine erhöhte Aufenthaltswahrscheinlichkeit des freien Elektrons an C-3 und C-8, was die Verknüpfung von Lignanen und Neolignanen erklärt.

\subsection{Lignane als Wirkstoffe ${ }^{51}$}

Eines der bedeutendsten Lignane ist das natürlich vorkommende Podophyllotoxin (66) (Abbildung 19). Es kann aus Pflanzen der Gattung Linum sowie den Wurzeln des amerikanischen Maiapfels (Podophyllum peltatum) gewonnen werden und wird aufgrund der hohen Toxizität gegen L6-Zellen $\left(\mathrm{IC}_{50}=7.7 \mathrm{ng} / \mathrm{mL}\right)$ unter dem Handelsnamen Wartec ${ }^{\circledR}$ als Medikament gegen venerische Warzen eingesetzt. Podophyllotoxin (66) ist ein Spindelgift und unterdrückt durch seine Bindung an Tubulin die Bildung von Mikrotubuli. Die Mitose wird somit verhindert, was wiederum zu einer Nekrose der Zelle führt.

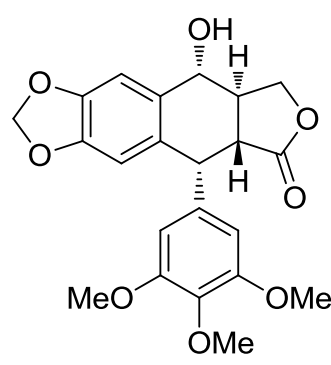

Podophyllotoxin (66)

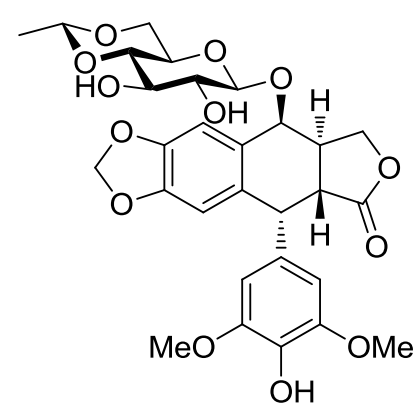

Etoposid (67)

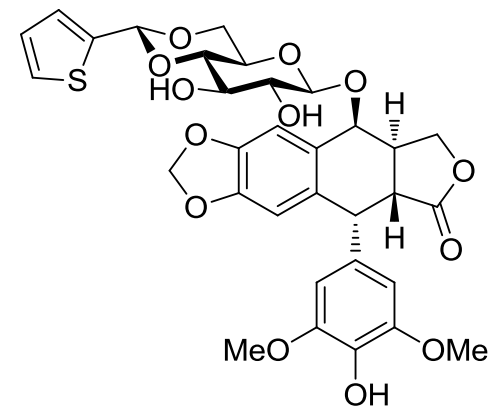

Teniposid (68)

Abbildung 19: Medizinisch bedeutungsvolle Aryltetralin-Derivate.

Weitere aktive Lignane sind die Topoisomerase II-Hemmer Etoposid (67) und Teniposid (68). Sie werden semisynthetisch durch Glycosylierung des Podophyllotoxins (66) erhalten und hauptsächlich als Zytostatika in der Chemotherapie eingesetzt. Durch die Inhibition der Topoisomerase II wird die Zytokinese während der G2-Phase unterdrückt und die Apoptose der Zelle eingeleitet. Darüber hinaus wird die Zytotoxizität der Verbindungen durch ihre Eigenschaft DNA-Einzel- und Doppelstrangbrüche $\mathrm{zu}$ verursachen weiter erhöht. Die Verbindungen sind unter den Markennamen Vepesid ${ }^{\circledR}$ und Vumon ${ }^{\circledR}$ zugelassen. 


\subsection{Linoxepin}

Im Jahr 2007 wurden von Schmidt et al. aus oberirdischen Pflanzenteilen des ausdauernden Leins (Linum perenne) vier Lignane des Arylnaphthalin-Typs isoliert (Abbildung 20). ${ }^{53}$ Darunter befand sich neben bereits bekannten Lignanen auch das Linoxepin (69), das ein bis dahin einzigartiges 2,8-Dihydro-3H-benzo[ $e]$ naphtho[1,8- $b c]$ oxepin-Ringsystem aufweist.<smiles>COc1ccc2c3c1OCc1c(ccc4c1OCO4)C3=C1C(=O)OC[C@H]1C2</smiles>

$(+)$-Linoxepin (69)

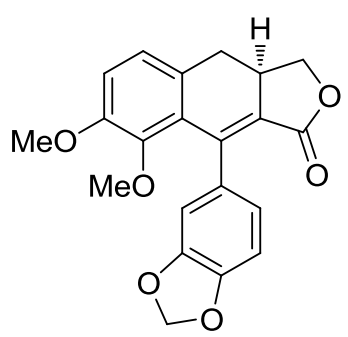

7,8-Dihydrojusticidin B (70)

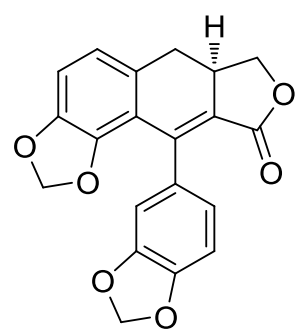<smiles>O=C1OC[C@H]2Cc3cc4c(cc3C(c3ccc5c(c3)OCO5)=C12)OCO4</smiles>

Abbildung 20: Linoxepin (69) und weitere in Linum perenne enthaltene Lignane.

Durch CD-Spektroskopie und DFT-Berechnungen konnte die absolute Konfiguration des stereogenen Zentrums am Linoxepin (69) als $R$ bestimmt werden. Eine biologische Aktivität konnte dem Naturstoff bislang allerdings noch nicht nachgewiesen werden.

Da Lignane ein breites Wirkspektrum aufweisen und Linoxepin ein bisher neuartiges Ringsystem enthält, ist ein synthetischer Zugang zu dieser Verbindung von besonderem Interesse.

\subsection{Totalsynthesen von Linoxepin}

Bislang sind zwei Totalsynthesen von Linoxepin bekannt. Die erste Synthese des Naturstoffes gelang 2013 Tietze et al. mithilfe einer Sonogashira-Reaktion zwischen Arylodid 73 und Propargylalkohol (74) und anschließender Palladium-katalysierter Domino-Reaktion (vgl. Kapitel A. 6) zum Aufbau des Oxepin- und Cyclohexadien-Rings in 75 (Abbildung 21). ${ }^{54}$ Ausgehend von Verbindung 76 konnten, nach Trennung der Enantiomere mittels HPLC, das bioidentische (+)-Linoxepin (69) sowie das (-)-Enantiomer in vier weiteren Stufen aufgebaut werden. Ein großer Vorteil dieser Totalsynthese ist der komplette Verzicht auf Schutzgruppen jeglicher Art. Die Gesamtausbeute der zehnstufigen Totalsynthese beträgt zudem hervorragende $30 \%$. 
<smiles>CC/C=C\Cc1ccc(OC)c(OCc2c(I)ccc3c2OCO3)c1Br</smiles>

73

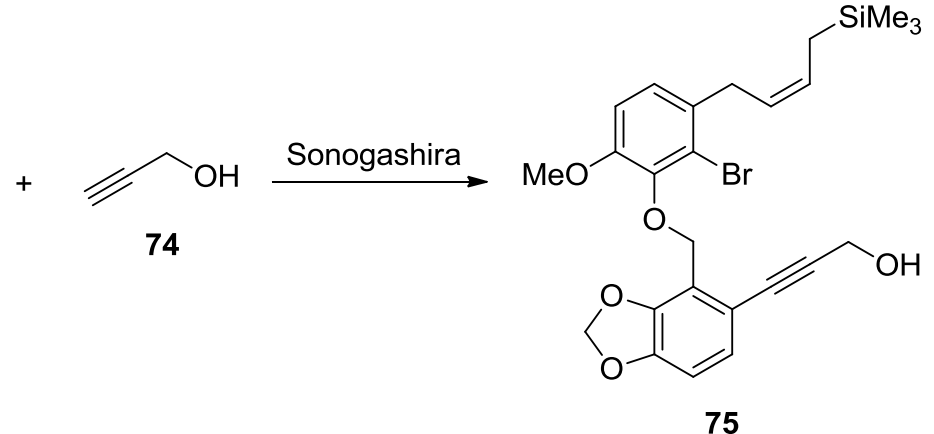

Domino-Carbopalladierung-Heck<smiles>C=CC1Cc2ccc(OC)c3c2C(=C1CO)C1=CC=CC2=C(OCO2)C13CO</smiles>

76

Abbildung 21: Schlüsselschritte in der Totalsynthese von (+)-Linoxepin nach Tietze et al.

Noch im selben Jahr wurde eine weitere Totalsynthese von (+)-Linoxepin (69) durch Lautens et al. veröffentlicht. ${ }^{55}$ Der Schlüsselschritt dieser Synthese ist eine Norbonen-unterstützte, Palladium-katalysierte Catellani-Reaktion, die Aryliodid 77 mit dem enantiomerenreinen Iodlacton 78 und tert-Butylacrylat (79) zu dem Tricyclus 80 verbindet (Abbildung 22). Der enantiomerenreine Naturstoff 69 konnte nach drei weiteren Stufen ebenfalls mit einer Gesamtausbeute von $30 \%$ isoliert werden.

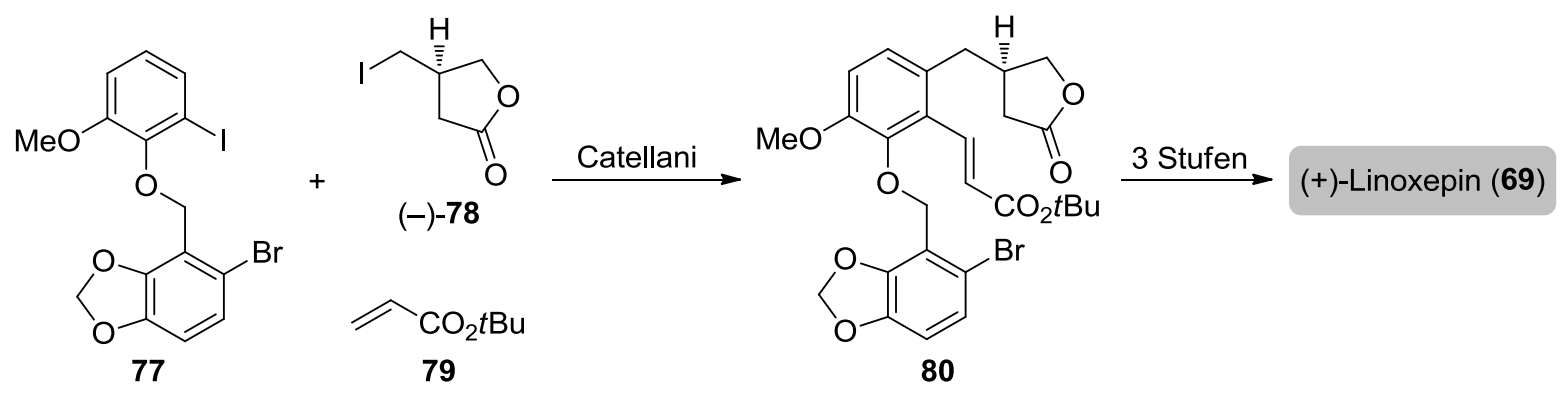

Abbildung 22: Schlüsselschritt der enantioselektiven Totalsynthese von (+)-Linoxepin nach Lautens et al.

Im Kapitel B. 2 wird eine noch effektivere enantioselektive Totalsynthese von (+)-Linoxepin diskutiert. 


\section{Fibrotische Erkrankungen}

Erkrankungen des Kreislaufsystems stellten laut Statistischem Bundesamt 2013 mit knapp $40 \%$ die häufigste Todesursache in Deutschland dar. ${ }^{56}$ Die neben koronaren Herzkrankheiten wichtigste spezifische Untergruppe der Herz-Kreislauf-Erkrankungen ist mit 54538 Verstorbenen auf akute sowie rezidivierende Myokardinfarkte zurückzuführen. Bei einem Herzinfarkt kommt es an einer arteriellen Engstelle zu einer anhaltenden Durchblutungsstörung des Herzmuskels, die häufig durch ein Blutgerinnsel ausgelöst wird. Durch die Unterbrechung der Sauerstoffzufuhr wird eine Nekrose des umliegenden Herzmuskelgewebes ausgelöst, das im Falle des Überlebens durch Narbengewebe ersetzt wird.

Etwa 30\% der Todesfälle durch einen Herzinfarkt ereignen sich unmittelbar nach dem Arterienverschluss. Da das neu gebildete Narbengewebe die Pumpleistung des Herzens verschlechtert und somit Herzrhythmusstörungen und weitere Infarkte verursachen kann, ist für die betroffenen Patienten eine dauerhafte Therapie mit aggregationshemmenden Medikamenten essentiell. ${ }^{57}$

\subsection{Kollagen}

Ein wesentlicher Bestandteil dieses Narbengewebes ist das Kollagen. Kollagen ist das im Menschen am häufigsten vorkommende Protein und macht etwa 30\% der Proteingesamtmasse aus. Es bildet einen Großteil der extrazellulären Matrix des Bindegewebes und ist verantwortlich für die Strukturgebung und Stabilität von körpereigenem Gewebe. ${ }^{58}$ Strukturell betrachtet besteht Kollagen aus einem Aggregat mehrerer quervernetzter tripelhelicaler Tropokollagenmoleküle. ${ }^{59}$ Diese Tropokollagenmoleküle umfassen wiederum drei linksgängige Polypeptid- $\alpha$-Ketten, die sich über Disulfidbrücken zu einer rechtsgängigen Tripelhelix zusammenlagern. ${ }^{60}$ Auffällig ist hierbei der hohe Anteil von Glycin und Prolin in den $\alpha-$ Ketten. Die Aminosäurezusammensetzung unterbindet größtenteils die Ausbildung von $\alpha$-Helices und $\beta$-Faltblättern und bevorzugt die etwas gestrecktere Tripelhelix, die für die große Stabilität und Zugfestigkeit des Kollagens verantwortlich ist. Je nach Verknüpfung und Zusammenlagerung der Polypeptidketten werden bislang 28 Kollagentypen unterschieden, wovon Typ I Kollagen der wichtigste und häufigste ist. ${ }^{61}$

\subsection{Biosynthese von Kollagen}

Die Biosynthese des Kollagens beginnt am rauen endoplasmatischen Retikulum (ER). Zunächst erfolgt an der durch Gentranslation erzeugten, Ribosom-gebundenen $\alpha$-Kette eine 
Vitamin C-induzierte Hydroxylierung einiger Prolin- und Lysineinheiten (Abbildung 23, Schritt 1). ${ }^{62}$ Anschließend werden einige ausgewählte Hydroxyfunktionen der Lysineinheiten mit Galactose oder Glucosylgalactose glycosyliert. (Schritt 2). Nach der Zusammenlagerung von drei dieser Polypeptidketten (Schritt 3) erfolgt die Chaperon-unterstützte Bildung der Tripelhelix, die durch Disulfidbrücken am C-Terminus und Wasserstoffbrücken der freien Hydroxyaminosäuren miteinander verbunden sind (Schritt 4).

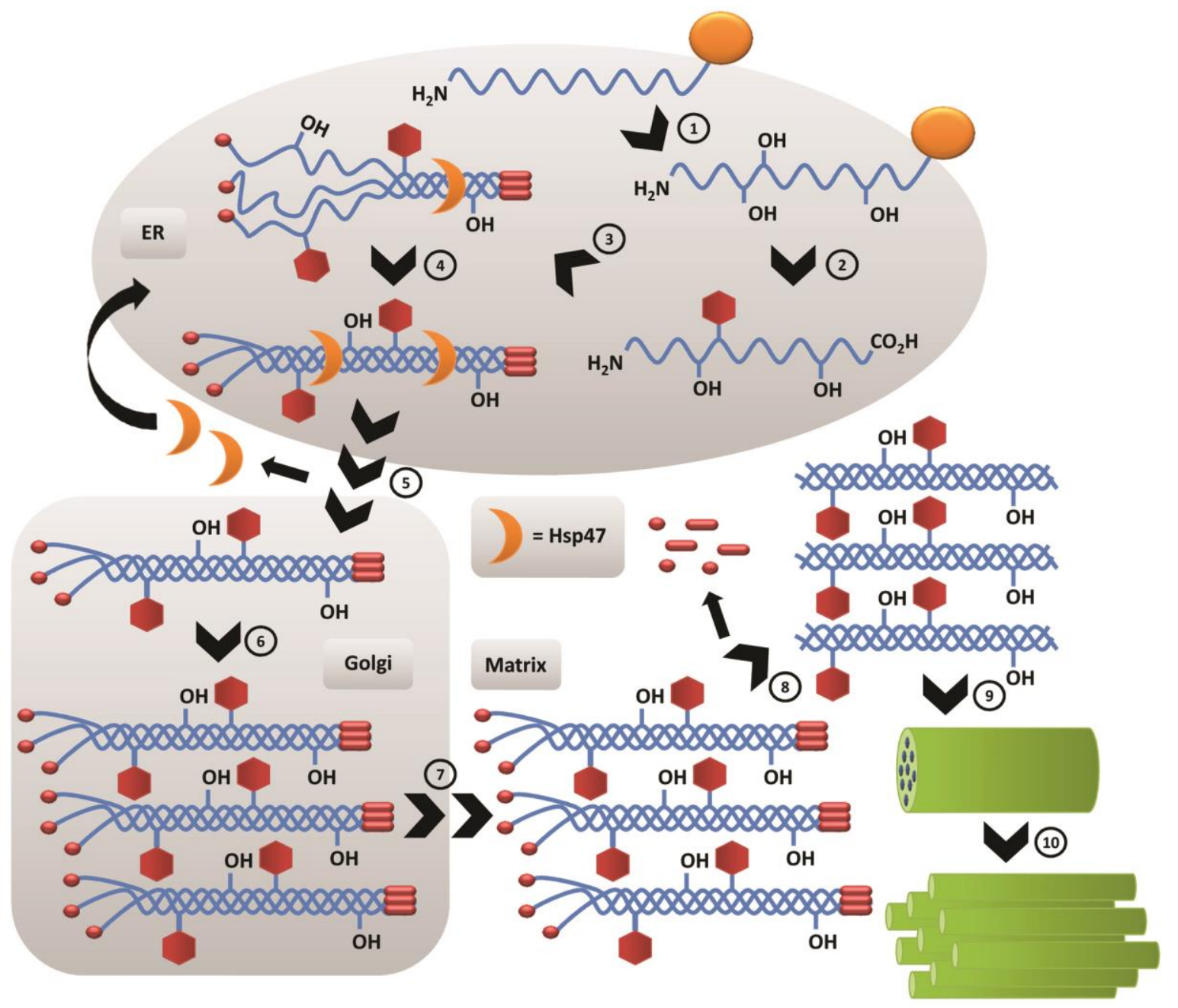

Abbildung 23: Biosynthese von Kollagen.

Die einzelnen Prokollagenmoleküle werden über Vesikel in den Golgi-Apparat transportiert (Schritt 5), wo sie nach Abspaltung der Chaperone geordnet werden (Schritt 6). Nach der Sekretion in den Extrazellularraum (Schritt 7) werden die terminalen Propeptide abgespalten (Schritt 8) und die Quervernetzung der Tropokollagenmoleküle zu Kollagenfibrillen durch Oxidation der Hydroxylysineinheiten eingeleitet (Schritt 9). Diese Kollagenfibrillen lagern sich anschließend zu einer Kollagenfaser zusammen (Schritt 10). 


\subsection{Funktion des Hsp47 in der Kollagenbildung}

Chaperone sind Hilfsproteine, die die korrekte Faltung und Entfaltung von neu synthetisierten Proteinen gewährleisten und diese vor Aggregatbildung und Denaturierung schützen. ${ }^{63}$ Eine spezifische Unterklasse der Chaperone bilden die Hitzeschockproteine (Hsps). Die Expression von Hitzeschockproteinen wird durch Hitze, ER-Stress oder auch Hypoxie eingeleitet.

Ein Chaperon, dem bei der Kollagenbiosynthese eine entscheidende Rolle zukommt, ist das Hitzeschockprotein 47 (Hsp47). ${ }^{64}$ Neben der Einleitung des Faltprozesses wird eine Aggregatbildung oder lokale Entfaltung ${ }^{65}$ durch die Dissoziation des Hsp47 an die hydrophobe Oberfläche der Tripelhelix effektiv verhindert (vgl. Abbildung 23). Neben der Stabilisierung der Tripelhelix-Form wir auch der Transport des Prokollagens in den GolgiApparat begünstigt und somit eine Akkumulation im ER verhindert. ${ }^{66}$ Aufgrund des niedrigen pH-Werts im Golgi-Apparat (Golgi: pH 6.3; ER: pH 7.0 ${ }^{67}$ ) erfolgt die Dissoziation des Hsp47, welches anschließend zurück in das ER transportiert wird. ${ }^{68}$

Um den Einfluss des Hsp47 in der Biosynthese des Kollagens genauer zu untersuchen, wurden im Jahr 2000 von Nagai et al. in vivo Studien an Hsp47-Knockout-Mäusen (Hsp47 ${ }^{-{ }^{-}}$) durchgeführt, bei denen das Hsp47-exprimierende Gen durch homologe Rekombination deaktiviert wurde. ${ }^{69}$

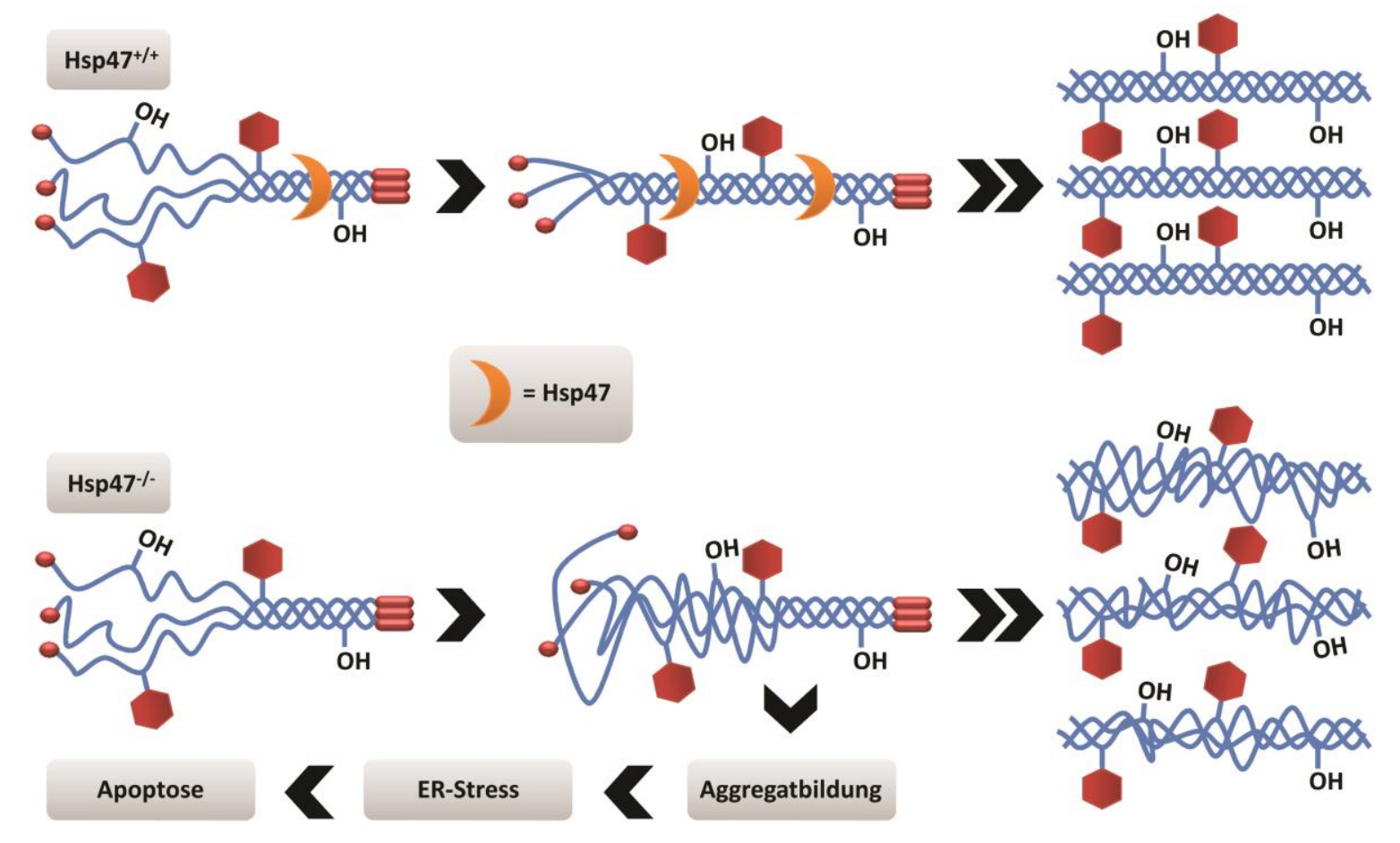

Abbildung 24: Auswirkungen von Hsp47 auf die Kollagen-Biosynthese. 
Bei diesem Experiment stellte sich heraus, dass Hsp47 essentiell für die Entwicklung der Mäuse ist. Mäuse, die nicht in der Lage waren Hsp47 zu bilden, überlebten demzufolge nicht länger als 11.5 Tage. Außerdem wurde bei Hsp47/--Mäusen beobachtet, dass eine erhöhte Konzentration von Prokollagen im ER vorliegt, während es kaum zu Kollagenanlagerungen im Gewebe kommt. Dies ist auf eine Aggregatbildung von fehlgefalteten Prokollageneinheiten zurückzuführen, da Hsp47 als Chaperon eine korrekte Faltung des Proteins sicherstellt (Abbildung 24). Die Aggregatbildung und erhöhte Akkumulation von Prokollagen führt zu ER-Stress und schließlich zur Apoptose der Zelle.

Fibrotische Erkrankungen sind häufig auf Fehlbildung oder exzessive Akkumulation von Kollagen zurückzuführen. Wie die in vivo Studien gezeigt haben, führt eine selektive Blockierung der Hsp47-Expression zu einer kontrollierten Produktion von Kollagen und somit auch zu einer Progressionsverringerung von Fibrosen. ${ }^{70}$ Da bislang kaum Ansätze für die therapeutische Behandlung von fibrotischen Erkrankungen vorhanden sind, ist die Synthese von spezifischen Hsp47-Inhibitoren im Hinblick auf ihre potentielle Anwendung als Medikamente ein erstrebenswertes Ziel der organischen Chemie.

\subsection{Niedermolekulare Hsp47-Inhibitoren}

Die neu entdeckte Rolle von Hsp47 in der Biosynthese des Kollagens nutzte Ananthanarayanan et al. 2005 für ein Screening mit über 2000 niedermolekularen Verbindungen. ${ }^{71}$ Dabei wurde der Einfluss der Small Molecules auf die Ausbildung von Kollagenfibrillen im ER untersucht.

Bei $34{ }^{\circ} \mathrm{C}$ und einem pH-Wert von 7.4 lagern sich Kollagenmonomere ohne Zusatz von Hsp47 spontan zu Fibrillen zusammen, die mithilfe eines Photospektrometers durch die Trübung der Probelösung nachgewiesen werden können. Bei Zugabe von Hsp47 wird das Einsetzen der Trübung von 30 Minuten auf etwa 4 Stunden verzögert. Die biologische Aktivität der potentiellen Inhibitoren wurde im Assay durch die Verringerung der Zeit bis zum Einsetzen der Trübung bestimmt.

Mit Hilfe dieser Methode konnten vier Substanzen identifiziert werden, die eine Inhibition des Hitzeschockproteins 47 hervorrufen und somit die Ausbildung von Kollagenfibrillen beschleunigen (Abbildung 25). Die Small Molecules, von denen besonders Verbindungen 83 und 84 eine hohe biologische Aktivität aufweisen, können als Leitstrukturen für die Synthese von neuen, noch aktiveren Inhibitoren von Hsp47 dienen. 
<smiles>COc1ccc(C(C[N+](=O)[O-])Sc2ccc(N)cc2)cc1</smiles>

81

$I C_{50}=26.6 \mu \mathrm{M}$<smiles>CC(=O)c1cc(Cl)cc2sc(=O)[nH]c12</smiles>

83

$\mathrm{IC}_{50}=3.1 \mu \mathrm{M}$<smiles>Nc1ccc(SC(C[N+](=O)[O-])c2ccc(F)cc2)cc1</smiles>

82

$\mathrm{IC}_{50}=26.6 \mu \mathrm{M}$<smiles>Cc1cc(C(=O)CSc2cc(-c3cccs3)c(C#N)c(=O)[nH]2)ccc1Cl</smiles>

84

$\mathrm{IC}_{50}=6.3 \mu \mathrm{M}$

Abbildung 25: Strukturen und Aktivitäten bisher identifizierter Small Molecules mit Hsp47-Inhibitionspotential.

Kapitel B. 3 befasst sich mit der Synthese von potentiellen Hsp47-Inhibitoren des Typs 84.

Durch Änderung der Substituenten und des Substitutionsmusters am aromatischen Benzolring sollte in einer Kooperationsarbeit mit dem Arbeitskreis Zimmermann die Auswirkung der strukturellen Veränderungen auf die biologische Aktivität untersucht werden. 


\section{Heck-Reaktion}

In der organischen Synthesechemie und besonders in der Naturstoffsynthese sind $\mathrm{C}-\mathrm{C}$-Bindungsknüpfungen von elementarer Bedeutung. So wurde der Nobelpreis für Chemie im Jahr 2010 an Heck, Negishi und Suzuki für die Entwicklung Palladium-katalysierter Kreuzkupplungen in der organischen Synthese verliehen. ${ }^{72}$ Die Heck-Reaktion, die mechanistisch gesehen eigentlich nicht zu den klassischen Kreuzkupplungen gehört, wurde von Heck und Mizoroki 1971 bzw. 1972 unabhängig voneinander veröffentlicht. ${ }^{73}$ Es handelt sich dabei um eine Palladium(0)-katalysierte C-C-Bindungsbildung zwischen Olefinen und Aryl- bzw. Vinylhalogeniden, -triflaten oder -tosylaten (Abbildung 26).

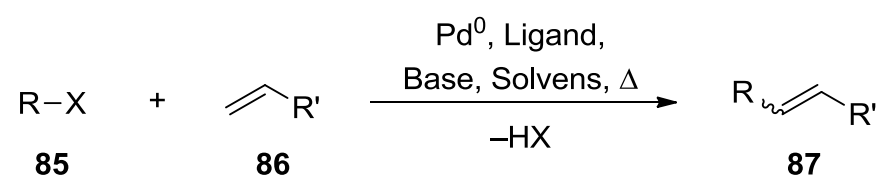

$\mathrm{R}=$ Aryl, Benzyl, Vinyl (Alkenyl), Alkyl (ohne $\beta-\mathrm{H})$ etc . $\mathrm{R}^{\prime}=$ Aryl, Alkenyl, Alkyl etc.

$\mathrm{X}=\mathrm{I}, \mathrm{Br}, \mathrm{Cl}$, OTf, OTs, $\mathrm{N}_{2}^{+}$etc.

Abbildung 26: Die Heck-Reaktion.

Die Heck-Reaktion zeichnet sich besonders durch ihre milden Reaktionsbedingungen und die hohe Toleranz von funktionellen Gruppen (z.B. Ester, Ether, Amide, Ketone, Carbonsäuren, Nitrile, Phenole, Diene) aus und findet somit breite Anwendung in der organischen Synthesechemie. ${ }^{74}$ Neben chemo- und regioselektiven Transformationen besteht auch die Möglichkeit einer enantioselektiven Heck-Reaktion. ${ }^{75}$ Erste asymmetrische Umsetzungen wurden 1989 von Shibasaki ${ }^{76}$ und Overman ${ }^{77}$ beschrieben.

Mit steigender Beliebtheit der Heck-Reaktion wurde der Anwendungsbereich um eine Vielzahl an Substratklassen erweitert und unterschiedlichste Aspekte der Transformation untersucht. ${ }^{78}$ Heutzutage gilt sie als eine der wichtigsten C-C-Bindungsknüpfungsreaktionen und ist auch hervorragend für die Einbindung in Domino-Reaktionen geeignet. Dies ist im Wesentlichen auf die stetige Weiterentwicklung alter und die Etablierung neuer leistungsfähiger Katalysatorsysteme zurückzuführen. ${ }^{79}$

\subsection{Mechanistische Aspekte}

Trotz vielfältiger Bemühungen sind bis heute nicht alle mechanistischen Details der HeckReaktion eindeutig geklärt. Der genaue mechanistische Pfad scheint sich in Abhängigkeit der Reaktionsbedingungen partiell $\mathrm{zu}$ ändern. Dennoch ist der in Abbildung 27 dargestellte 
Katalysecyclus durch zahlreiche experimentelle Befunde belegt und gilt als allgemein akzeptiert. Die katalytisch aktive Spezies bildet der koordinativ ungesättigte 14-ElektronenPalladium(0)-Komplex 89 vom Typ $\mathrm{Pd}^{0} \mathrm{~L}_{2}$.

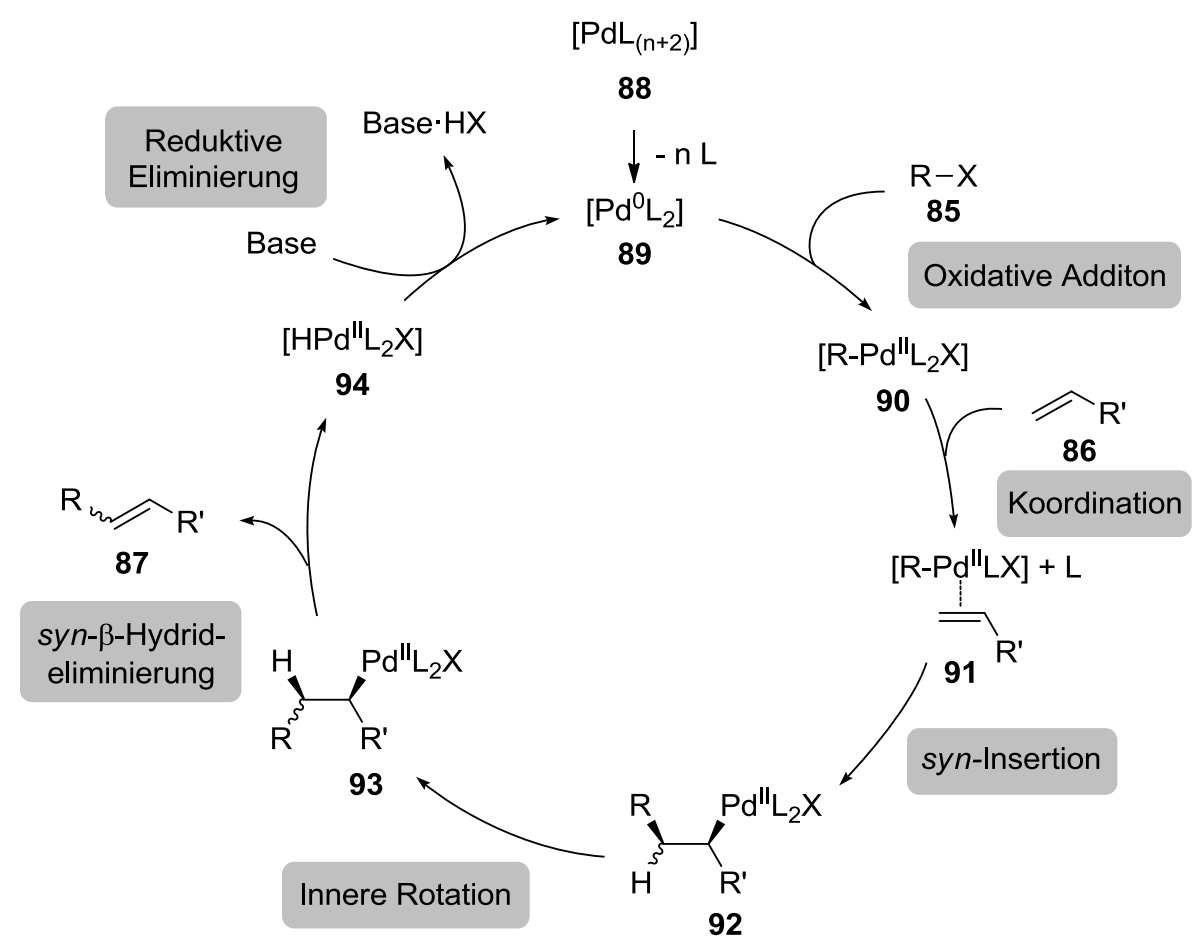

Abbildung 27: Postulierter Katalysecyclus der Heck-Reaktion.

Ungeachtet der Reaktionsbedingungen besteht die Heck Reaktion, ob sie neutral oder kationisch verläuft, immer aus den folgenden sechs Teilschritten: Oxidative Addition der aktiven Spezies in die Kohlenstoff-Halogen-Bindung, Koordination des Alkens, syn-Insertion der Palladium-Spezies in die Doppelbindung, innere Rotation um die $\mathrm{C}-\mathrm{C}$-Bindung, syn- $\beta$ Hydrid-Eliminierung und basenvermittelte reduktive Eliminierung, um die aktive KatalysatorSpezies wieder freizusetzen. Im Folgenden werden die einzelnen Abschnitte des Katalysecyclus noch einmal näher beschrieben.

\subsubsection{Bildung der katalytisch aktiven Spezies}

Für die oxidative Addition in eine Kohlenstoff-Halogen-Bindung wird ein koordinativ ungesättigter und somit reaktiver Katalysator benötigt. Die Bildung dieser katalytisch aktiven Spezies erfolgt entweder durch direkte Zugabe einer Palladium(0)-Spezies (z.B. $\left.\mathrm{Pd}\left(\mathrm{PPh}_{3}\right)_{4}\right)$ oder durch in situ-Reduktion einer geeigneten Palladium(II)-Verbindung (z.B. Pd(OAc) $)_{2}{ }^{80}$ wobei die Reduktion bei Heck-Reaktionen meist schneller verläuft. ${ }^{81}$ Üblicherweise ist die Entstehung von $\mathrm{Pd}^{0} \mathrm{~L}_{2}$ unabhängig von der Base, dem Alken oder anwesendem Wasser. ${ }^{82}$ 
Wenngleich der 14-Elektronen-Palladium(0)-Komplex 89 seit geraumer Zeit postuliert wurde, konnte dessen Existenz erst 2007 für die Stille-Reaktion mithilfe massenspektrometrischer Methoden nachgewiesen werden. ${ }^{83}$ Darüber hinaus bildet sich bei sterisch stark anspruchsvollen Liganden (z.B. $\left.\mathrm{P}(t \mathrm{Bu})_{3}\right)$ als aktive Spezies der monosubstituierte Komplex $\mathrm{Pd}^{0} \mathrm{~L}$, der aufgrund seiner 12 Valenzelektronen und der freien Koordinationsstelle als hochreaktiv gilt. ${ }^{84}$ Ebenso können auch elektronenreiche Biarylphosphin-Liganden (z.B. Buchwald-Liganden) die Ausbildung des extrem reaktiven 12-Elektronen-Komplexes $\mathrm{Pd}^{0} \mathrm{~L}$ begünstigen. ${ }^{85}$ Die Existenz der Pd $^{0}$ L-Spezies konnte indes auch mit massenspektrometrischen Analysemethoden nachgewiesen werden.

Ein beliebtes katalytisches System in Heck-Reaktionen bildet $\mathrm{Pd}(\mathrm{OAc})_{2} / \mathrm{PPh}_{3}$, da es in der Regel schneller reagiert als $\mathrm{Pd}\left(\mathrm{PPh}_{3}\right)_{4}$. Untersuchungen von Jutand et al. zufolge erfolgt die Reduktion der Palladium(II)-Spezies in situ aus dem zuvor gebildeten $\mathrm{Pd}(\mathrm{OAc})_{2}\left(\mathrm{PPh}_{3}\right)_{2}$ (Abbildung 28). ${ }^{86}$

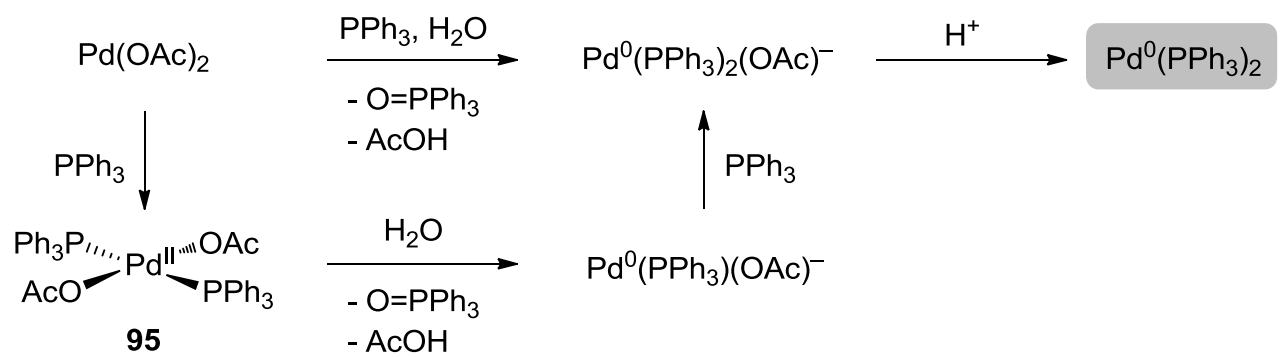

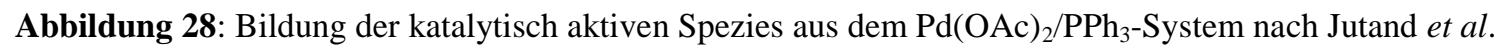

In dieser intramolekularen Reaktion wird zunächst neben Triphenylphosphinoxid die labile Palladium(0)-Spezies $\mathrm{Pd}\left(\mathrm{PPh}_{3}\right)_{2}(\mathrm{OAc})^{-}$gebildet, die im Beisein von Protonen $\mathrm{zu}$ der reaktivsten Spezies $\mathrm{Pd}\left(\mathrm{PPh}_{3}\right)_{2}$ zerfällt. ${ }^{87}$ Dieser Prozess kann durch einen Überschuss Base, der die Protonenkonzentration senkt, oder auch durch überschüssigen Phosphinliganden, der die Bildung von stabilerem, aber unreaktiverem $\mathrm{Pd}^{0}\left(\mathrm{PPh}_{3}\right)_{3}(\mathrm{OAc})^{-}$bevorzugt, verlangsamt werden. ${ }^{88}$ Auch die Anwesenheit von Alkenen, wie bei der Heck-Reaktion, wirkt sich verlangsamend auf die Reaktion aus, da das Alken einen $\eta^{2}$-Komplex mit dem $\mathrm{Pd}^{0}\left(\mathrm{PPh}_{3}\right)_{2}(\mathrm{OAc})^{-}$-Komplex bildet und somit dessen Konzentration verringert. ${ }^{89}$

\subsubsection{Oxidative Addition}

Die oxidative Addition ist ein konzertierter Ablauf, bei dem es nach Ausbildung einer 3-Zentren-2-Elektronen-Bindung zu einer cis-Insertion der aktiven Spezies in das Arylhalogenid oder -triflat kommt. Während der zunächst gebildete cis-Komplex 90 bei der Verwendung von einzähnigen Phosphinliganden $\mathrm{zu}$ dem stabilerem trans-Komplex 
isomerisieren kann, ist diese Möglichkeit bei der Verwendung bidentaler Liganden (wie z.B. Diphosphanen) nicht gegeben (Abbildung 29).

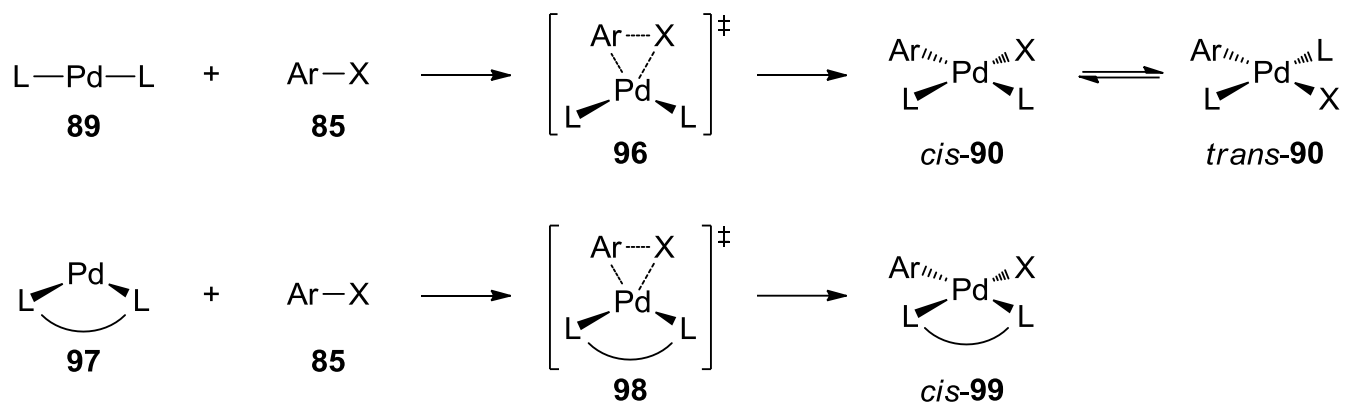

Abbildung 29: Oxidative Addition der katalytisch aktiven Spezies in das Arylhalogenid oder -triflat.

Die Reaktivität der eingesetzten Arylhalogenide und -triflate nimmt mit der Qualität von X als Abgangsgruppe und zunehmender Bindungsstärke der $\mathrm{C}\left(\mathrm{sp}^{2}\right)$-X-Bindung in folgender Reihenfolge ab:

$$
X=\mathrm{I}>\mathrm{OTf} \geq \mathrm{Br}>>\mathrm{Cl}
$$

Auch die Elektronendichte am Elektrophil hat einen Einfluss auf die Geschwindigkeit der oxidativen Addition. Da der niedervalente Palladium(0)-Komplex verhältnismäßig elektronenreich und somit auch nucleophil ist, erfolgt die Insertion in eine elektronenärmere Aryl-X-Bindung schneller. Die Reaktivität der Arylreste nimmt innerhalb der folgenden Reihe dementsprechend ab:<smiles>[X]c1ccc(COC(=O)c2ccc([X])cc2)cc1</smiles>

Auch der Einsatz von elektronenreichen Liganden (z.B. $\left.\mathrm{P}(t \mathrm{Bu})_{3}\right)$ kann durch die erhöhte Elektronendichte zu einer gesteigerten Nucleophilie am Palladium führen und folglich zu einer Beschleunigung der oxidativen Addition beitragen.

\subsubsection{Koordination des Alkens}

Für die Koordination des Alkens ist eine freie Koordinationsstelle und dementsprechend die Abspaltung eines Liganden aus dem koordinativ gesättigten Palladium(II)-Komplex erforderlich. Je nachdem ob ein neutraler Ligand oder ein Anion abgespalten wird, erfolgt die Koordination des Alkens über einen neutralen oder einen kationischen Mechanismus. Die Affinität von Palladium(II) für Halogen- und Triflatanionen nimmt in folgender Reihenfolge $\mathrm{ab}$ : 
Üblicherweise wird wie in Abbildung 27 der neutrale Mechanismus der Heck-Reaktion mit Arylhalogeniden beschrieben. In Gegenwart von Silber(I)- oder Thallium(I)-Salzen sowie bei der Verwendung von Aryltriflaten in Gegenwart schwach nucleophiler Gegenionen (z.B. Acetat, Phosphat, Carbonat) ist allerdings auch das Durchlaufen des kationischen Reaktionspfads denkbar (Abbildung 30).

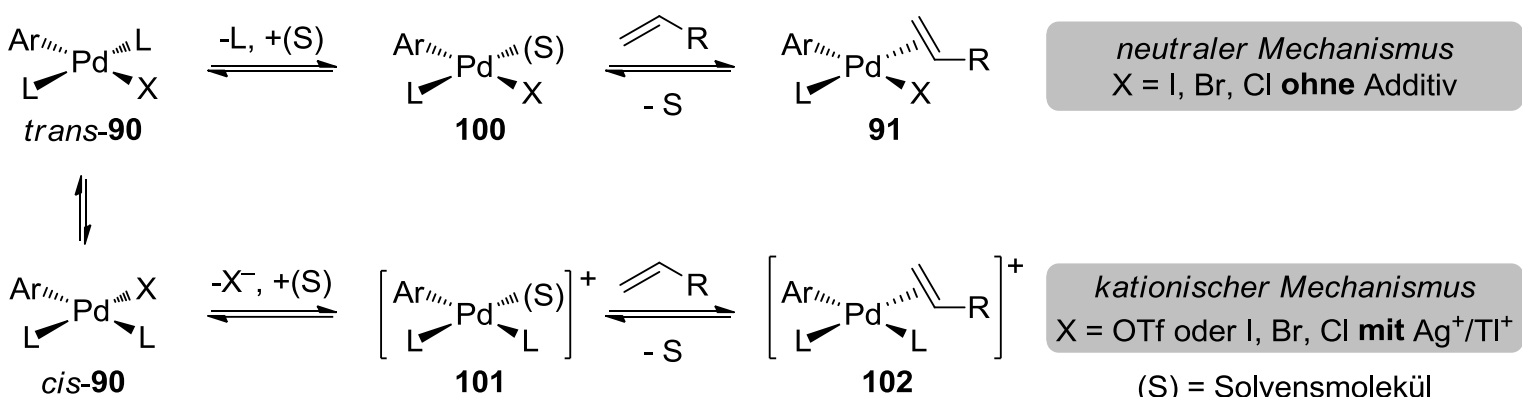

Abbildung 30: Neutraler und kationischer Reaktionsverlauf bei der Koordination des Alkens.

Da allgemein angenommen wird, dass nur der cis-Komplex (bezüglich Olefin und Arylrest) im nächsten Schritt zu einer syn-Insertion in das Alken führt, muss bei dem kationischen Mechanismus zunächst eine Isomerisierung des stabileren trans-Komplexes 90 zu cis-90 erfolgen. ${ }^{90}$

Die Bindungsverhältnisse des gebildeten Metall- $\pi$-Komplexes werden qualitativ mit dem Dewar-Chatt-Duncanson-Modell beschrieben. ${ }^{91}$ Die Bindung besteht aus einer dativen $\sigma$-Hinbindung, die aus der Überlappung eines besetzten bindenden $\pi$-Orbitals des Olefins und eines unbesetzten dsp-Hybridorbitals des Metalls entsteht sowie einer stabilisierenden retrodativen $\pi$-Rückbindung, die die Folge einer Überlappung besetzter d-Orbitale des Metalls $\left(\mathrm{d}_{\mathrm{xz}}\right.$ in der out-of-plane-Konformation, $\mathrm{d}_{\mathrm{xy}}$ in der in-plane-Konformation) mit dem unbesetzten antibindenen $\pi^{*}$-Orbital des Alkens ist (Abbildung 31). ${ }^{92}$

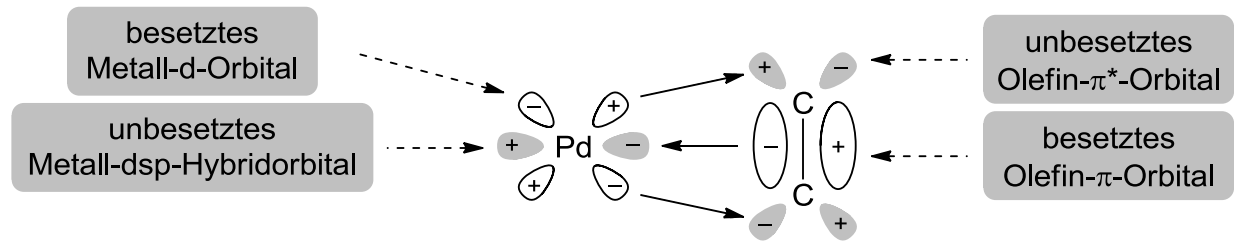

Abbildung 31: Bindungsverhältnisse im Alken-Metall-Komplex.

Da Alkene schwache $\sigma$-Donoren und $\pi$-Akzeptoren sind, bilden elektronenreiche Metallzentren mit elektronenarmen Olefinen aufgrund der resultierenden starken $\pi$-Rückbindung besonders stabile Komplexe. Als strukturelle Folge der Komplexbildung wird die Planarität am Alken aufgehoben und der Hybridisierungsgrad von $\mathrm{sp}^{2}$ in Richtung $\mathrm{sp}^{3}$ verschoben. Je 
stärker der $\pi$-Komplex desto stärker ist auch die Deformation des Alkens. Die geschwächte $\mathrm{C}-\mathrm{C}$-Doppelbindung wird dementsprechend länger und die $\mathrm{C}=\mathrm{C}$-Schwingungsfrequenz nimmt ab.

\subsection{4 syn-Insertion}

Aus dem im vorangegangenen Kapitel beschriebenen Komplex erfolgt der regio- und stereodiskriminierende Schritt der Heck-Reaktion, die syn-Insertion. Sie stellt den produktbildenden Schritt des Katalysecyclus dar und ist ebenfalls für die Substratselektivität verantwortlich.

Um eine syn-Insertion zu ermöglichen, muss das Olefin zunächst aus der nach der Bildung des $\pi$-Komplexes vorliegenden stabileren out-of-plane-Konformation 91 in die sterisch gehinderte in-plane-Position übergehen (Abbildung 32). Es wird dabei angenommen, dass die Insertion aus dem cis-Komplex (bezüglich Olefin und Arylrest) stattfindet.

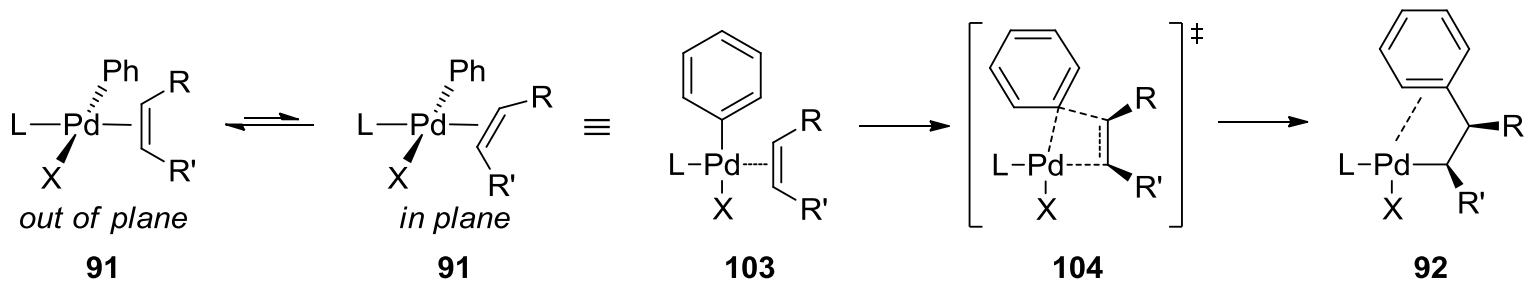

Abbildung 32: Konformationsgleichgewicht sowie Insertion des Olefins in die Pd-C-Bindung im neutralen Mechanismus.

Der Insertionsschritt erfolgt über einen 4-Elektronen-4-Zentren-Übergangszustand 104, aus dem sich unter Erhalt der stereochemischen Information die Alkylpalladiumspezies 92 bildet. $^{93}$ Enantioselektive Umsetzungen erfolgen zumeist unter Einsatz von zweizähnigen Liganden nach dem kationischen Mechanismus. Hier sind die chiralen Liganden ununterbrochen mit dem Metallzentrum verbunden, womit eine maximale asymmetrische Induktion auf das Substrat ausgeübt wird.

Bei intramolekularen Heck-Reaktionen, die unter Ausbildung eines Cyclus verlaufen, ist die Vorhersage des Produktes aufgrund der Baldwin-Regeln möglich. ${ }^{94}$ Ein Großteil der Cyclisierungen verläuft unter Ausbildung von normalen Ringgrößen (besonders fünfgliedrigen Ringen) nach dem exo-trig-Modus (Abbildung 33). Mit steigender Ringgröße wird der endo-trig-Modus bevorzugt, da sterische Wechselwirkungen sowie die Ringspannung eine untergeordnete Rolle spielen und die Bildung des endo-Produktes von elektronischen Faktoren begünstigt wird. ${ }^{95}$ 


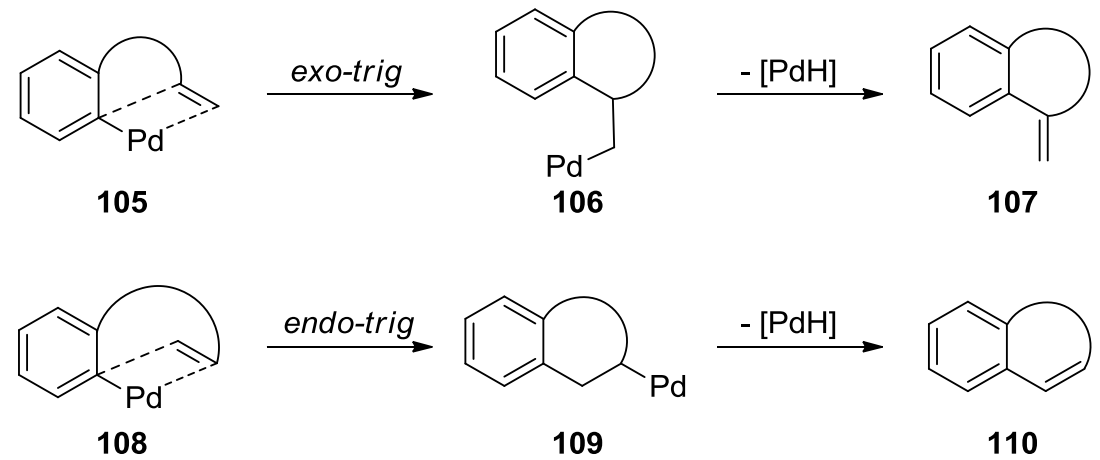

Abbildung 33: Regioselektivität der Heck-Cyclisierung.

\subsection{5 syn- $\beta$-Hydrid-Eliminierung}

Im nächsten Schritt des Katalysecyclus erfolgt eine $\beta$-Hydrid-Eliminierung, die mit einer Dissoziation des Alkens vom Palladium-Hydrid-Komplex abschließt. Die Eliminierung verläuft konzertiert und stereoselektiv aus der syn-Anordnung, da diese über agostische Wechselwirkungen stabilisiert wird. ${ }^{96}$ Um eine syn-coplanare Anordnung von PalladiumKatalysator und Wasserstoffatom $\mathrm{zu}$ erreichen, ist eine innere Rotation erforderlich (Abbildung 34).

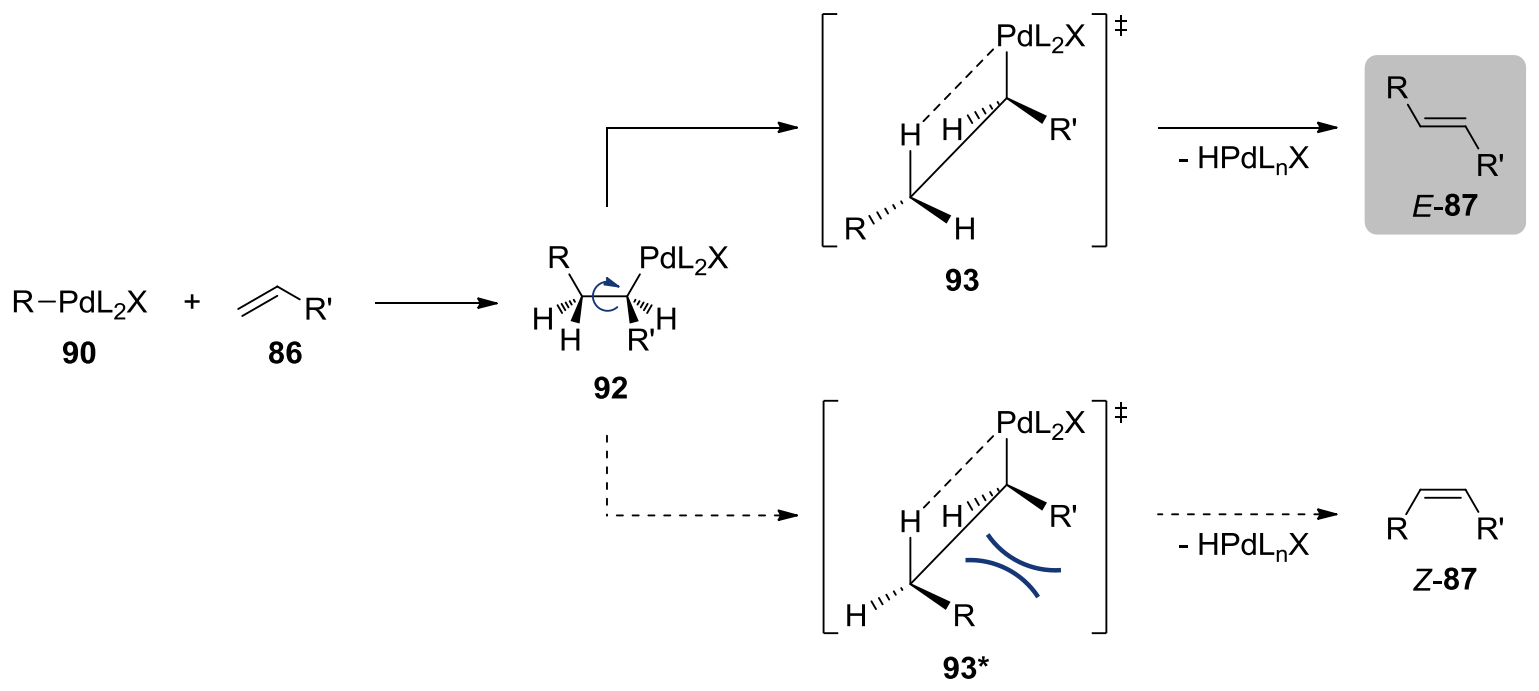

Abbildung 34: Mechanismus der syn- $\beta$-Hydrid-Eliminierung im neutralen Mechanismus.

Die Produktverteilung der Heck-Reaktion gehorcht in der Regel dem Curtin-Hammett-Prinzip der kinetischen Reaktionskontrolle. ${ }^{97}$ So spiegelt das E/Z-Verhältnis die relative Energie der Übergangszustände 93 und 93* wider. Aufgrund der sterischen Wechselwirkung der beiden Reste in Übergangszustand 93* wird hauptsächlich das E-Isomer 87 gebildet. Die hohe Stereoselektivität der Heck-Reaktion ist ein großer Vorteil gegenüber klassischen 
Olefinierungsmethoden wie der Wittig-Horner-Reaktion, bei der häufig Produktgemische entstehen.

Befindet sich an $\mathrm{R}^{\prime}$ ebenfalls ein zum Palladium $\beta$-ständiges H-Atom, kann es bei der Eliminierung zu Regioselektivitätsproblemen kommen. Ist andererseits kein syn-ständiges $\beta$-H-Atom vorhanden, so kann die Terminierung auch durch nucleophile Substitution am Palladium, Transmetallierungen oder die reduktive Eliminierung anderer $\beta$-ständiger Substituenten wie $\mathrm{SiR}_{3}$ erfolgen. Die Abwesenheit eines syn-ständigen $\beta$-H-Atoms kann entweder in einem quartären Kohlenstoffatom in $\beta$-Position begründet sein (vgl. 93 mit allen $\beta-\mathrm{H}=\mathrm{R}$ ) oder, bei einem olefinischen Substrat, mit der Anwesenheit eines trans-ständigen $\beta$-H-Atoms (vgl. 111 in Abbildung 35). Diese Tatsache ermöglicht die Verwendung der Heck-Reaktion in Domino-Reaktionen (vgl. Kapitel A. 6). Als Beispiele für nachfolgende $\mathrm{C}-\mathrm{C}$-Bindungsknüpfungen in der Folge alternativer Terminierungen in Domino-Reaktionen sind Carbonylierungen, Substitutionen sowie Kreuzkupplungen und in Abbildung 35 dargestellt.

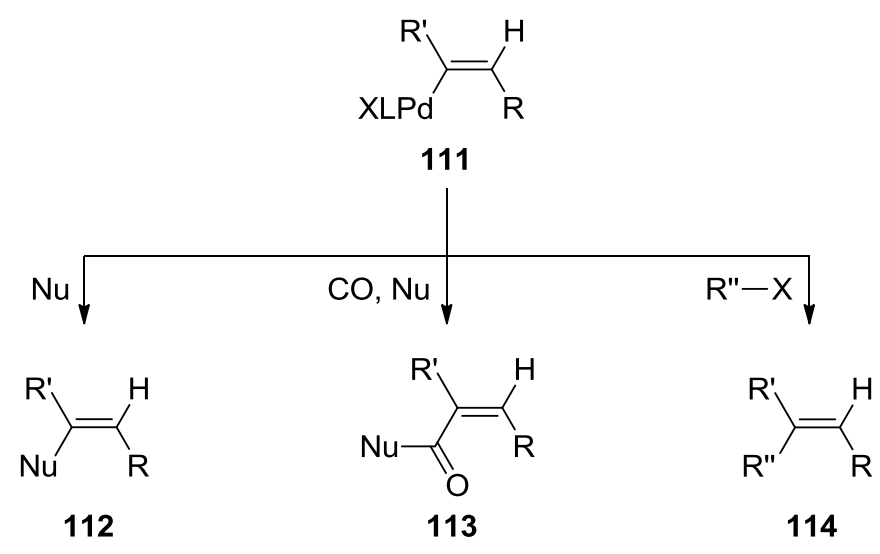

Abbildung 35: Mögliche Folge-Reaktionen von Heck Intermediat 111 als Teil einer Domino-Reaktion.

\subsubsection{Reduktive Eliminierung}

Im letzten Teilschritt des Katalysecyclus erfolgen die Dekoordination des Alkens von dem Palladiumkomplex und die baseninduzierte Reduktion des Palladium-Hydrid-Komplexes unter Rückbildung der katalytisch aktiven Palladium(0)-Spezies. Verläuft dieser Prozess nicht schnell genug, kann das zu einer Reinsertion von $\mathrm{H}-\mathrm{Pd}-\mathrm{X}$ in das Olefin mit einhergehender Veränderung der Regio- und Stereochemie führen.

\subsection{Anwendungen der Heck-Reaktion in der Organischen Synthese}

Die Heck-Reaktion ist aufgrund der in den milden Reaktionsbedingungen begründeten hohen Toleranz von funktionellen Gruppen ein beliebtes Werkzeug in der organischen 
Synthesechemie. Besonders regio- und stereoselektive Heck-Reaktionen zum Aufbau von Polyeneinheiten, bei Kupplungsreaktionen oder auch in intramolekularen Ringschlussreaktionen, finden in der Totalsynthese von komplexen Naturstoffen ein breites Anwendungsgebiet.

Die enantioselektive Variante der Heck-Reaktion verläuft in den meisten Fällen als intramolekulare Ringschlussreaktion und findet häufig Anwendung im selektiven Aufbau von tertiären oder quartären Stereozentren. Die Stereoinformation wird zumeist durch den Einsatz von chiralen Liganden (z.B. BINAP) induziert. In der von Overman und Mitarbeitern veröffentlichten enantioselektiven Synthese des Naturstoffes (+)-Minfiensin (118) führte eine enantiodifferenzierende Heck-Reaktion unter Einsatz des chiralen Pfaltz-Liganden 116 zum selektiven Aufbau des Pyrrolrings im Tricyclus 117 (Abbildung 36). ${ }^{98}$ Die Reaktion wurde bei $170{ }^{\circ} \mathrm{C}$ in einem Mikrowellenreaktor durchgeführt und lieferte eine hervorragende Enantioselektivität von $99 \%$ ee.

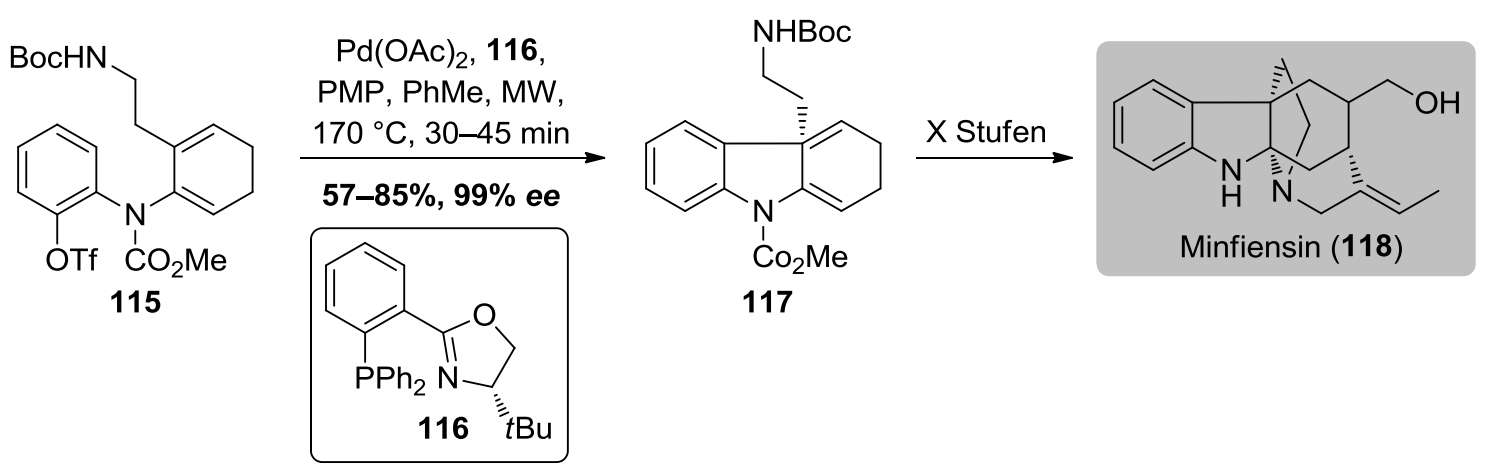

Abbildung 36: Anwendung der enantioselektiven Heck-Reaktion in der Synthese von Minfiensin (118) nach Overman et al.

Ein weiteres Beispiel einer Palladium-katalysierten Heck-Reaktion ist die von Tietze et al. entwickelte Strategie zum selektiven Aufbau des Steroidgerüsts. ${ }^{99}$ Die Bildung des steroidalen B-Rings erfolgt hierbei durch zwei sowohl regio- als auch diastereoselektive HeckReaktionen zwischen dem Vinylbromid 119 und dem Olefin 120 (Abbildung 37).

Die Regioselektivität der ersten Heck-Reaktion scheint auf den ersten Blick verwunderlich. Da beide substituierbaren Positionen des Olefins 120 vergleichsweise ähnliche elektronische Eigenschaften aufweisen, scheint eine Insertion in 5-Position aus sterischen Gründen wahrscheinlicher. Eine genauere Betrachtung der Intermediate zeigt allerdings, dass nur der Angriff an C-4 zu dem stabileren Sesselkonformer führt, über das ohne Konformationsänderungen die reduktive Eliminierung erfolgen kann. Ein Angriff des Palladiums in 5-Position würde das Inden hingegen in die energetisch ungünstigere Wannenkonformation zwingen, in der die reduktive Eliminierung gehindert ist und die Rückreaktion dominiert. Die 
Diastereoselektivität ist in der angulären Methylgruppe begründet, die zu einer Abschirmung der $\beta$-Seite führt und somit einen Angriff des Palladiums von unten induziert.<smiles>COc1ccc(Br)c(/C=C\Br)c1</smiles>

119<smiles>CCCC1CC[C@H]2C=CCC[C@]12C</smiles>

120

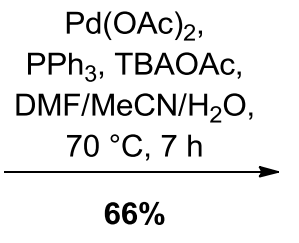

(NeO)
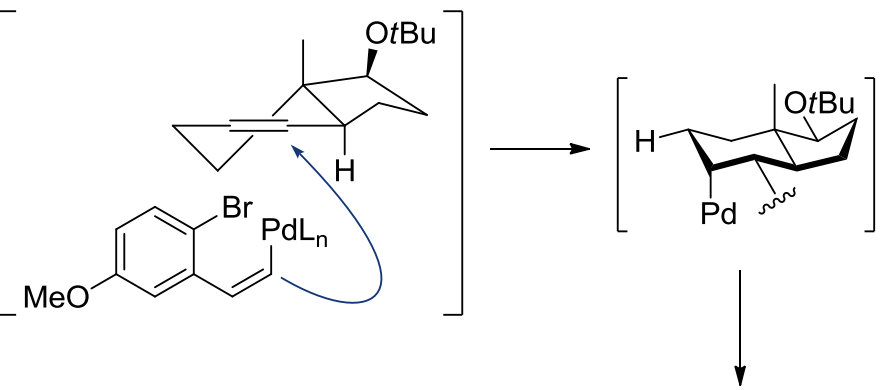

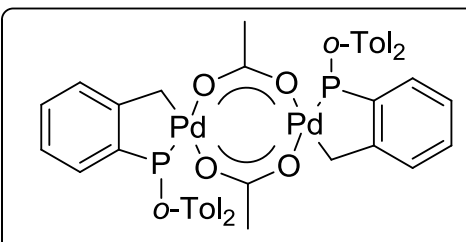

Herrmann-Beller-Katalysator (122)

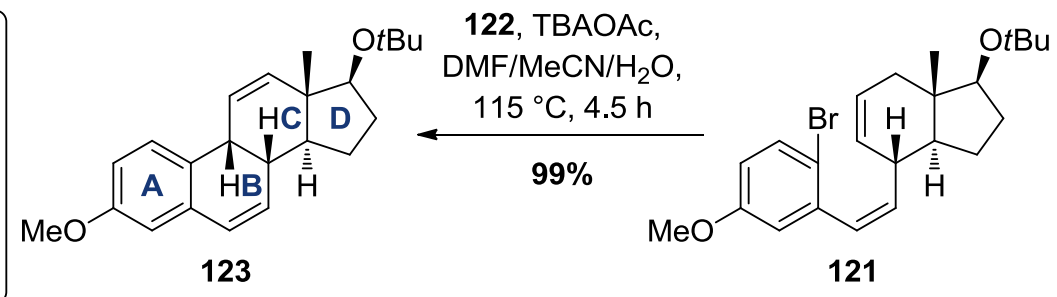

Abbildung 37: Selektive Synthese von Steroiden über eine zweifache Heck-Reaktion nach Tietze et al.

Die zweite Heck-Reaktion verläuft unter Zusatz des Herrmann-Beller-Katalysators 122 intramolekular. Die Ringschlussreaktion erfolgt nach den bereits beschriebenen BaldwinRegeln in einer 6-exo-trig-Anordnung und liefert das Steroidgrundgerüst 123 in einer exzellenten Ausbeute von 99\%. 


\section{Domino-Reaktionen}

Die Naturstoffsynthese gilt traditionell als die Königsdisziplin der organischen Chemie. Während Totalsynthesen in der Vergangenheit primär der Strukturaufklärung der biologisch aktiven und somit bedeutungsvollen Naturstoffe dienten, sind die modernen Anforderungen an die Naturstoffsynthese vielmehr eine hohe Selektivität sowie Effizienz und die einhergehende Eleganz des Prozesses. Totalsynthesen sollten aus ökonomischer und ökologischer Sicht auch eine möglichst geringe Anzahl an Synthesestufen und die damit verbundene Einsparung von Reagenzien und Lösungsmitteln sowie eine hohe Atomökonomie aufweisen.

Eine effiziente Methode für den Aufbau komplexer Strukturen aus einfachen Grundbausteinen bietet das Konzept der Domino-Reaktionen. Nach der Definition von Tietze ist eine Domino-Reaktion ,die Durchführung zweier oder mehrerer bindungsbildender Transformationen unter identischen Reaktionsbedingungen, in der die letzte Transformation jeweils an der im vorangegangenen Teilschritt entstandenen Funktionalität erfolgt.“100 Domino-Reaktionen sind herbei besonders von Tandem-Reaktionen, bei denen zeitgleiche Reaktionen lokal unabhängig verlaufen können und Eintopf-Reaktionen, die eine sequentielle Zugabe der Reagenzien erlauben, zu differenzieren. Ebenfalls wird häufig der Begriff der Kaskaden-Reaktion verwendet, wenngleich dieser deutlich weiter gefasst ist und bereits in anderen chemischen Prozessen, wie photochemischen Reaktionen, Anwendung findet. ${ }^{101}$

Die Klassifizierung von Domino-Reaktionen kann nach dem Mechanismus der einzelnen bindungsbildenden Schritte erfolgen. Hierbei wird zwischen kationischen, anionischen, radikalischen, pericyclischen, photochemischen, Übergangsmetall-katalysierten, oxidativen, reduktiven und enzymatischen Reaktionen unterschieden. Ein Großteil der DominoReaktionen erfolgt im ersten Schritt anionisch, während die nachfolgenden Schritte meist intramolekular verlaufen. Ein Schwerpunkt der aktuellen Forschung in der Arbeitsgruppe Tietze liegt in der Entwicklung neuer Palladium-katalysierter Domino-Reaktionen und ihre Anwendung in der Totalsynthese von biologisch aktiven Naturstoffen und deren Analoga.

Das Prinzip der Domino-Reaktionen ist dabei keineswegs neuartig, sondern in der Natur bei der Biosynthese von Terpenen, Steroiden und Alkaloiden weit verbreitet. Ein interessantes Beispiel ist die Biosynthese des Cholesterin-Vorläufers Lanosterin (126). ${ }^{102}$ Ausgehend von (S)-2,3-Oxidosqualen (124) erfolgt zunächst die enzymkatalysierte Öffnung des Epoxids, bei der vier neue $\mathrm{C}-\mathrm{C}$-Bindungen gebildet werden (Abbildung 38). Das Protosterolkation 125 wird anschließend nach Deprotonierung in das korrespondierende Steroid 126 umgelagert. 
Durch die Adaption dieses Domino-Konzepts auf die organische Synthese wird der Aufbau von komplexen Strukturmotiven aus weitgehend einfachen Substraten ermöglicht.

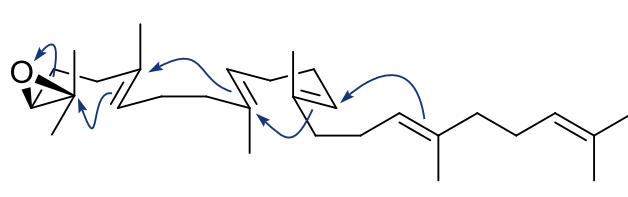

(S)-2,3-Oxidosqualen (124)

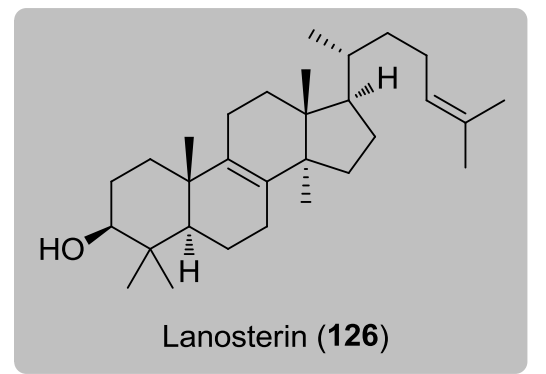

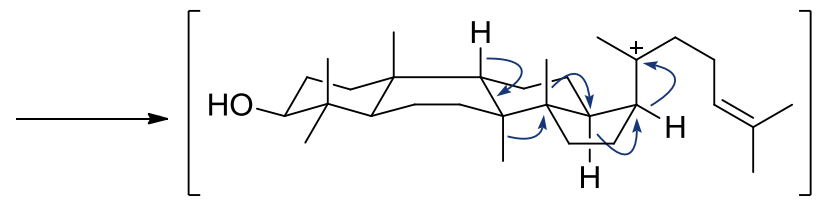

125

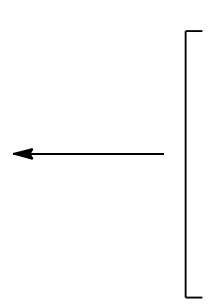

$\equiv$

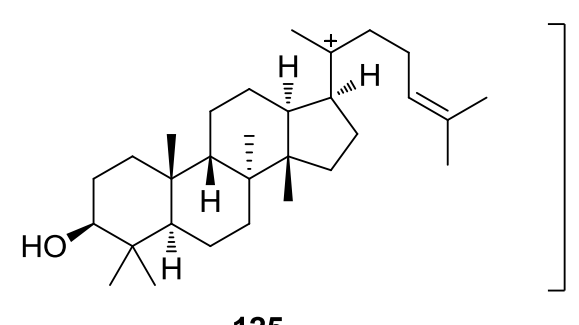

125

Abbildung 38: Biosynthese von Lanosterin (126).

Ist der genaue Mechanismus des Domino-Prozesses einer Biosynthese bekannt, kann mit Hilfe dieses Wissens eine biomimetische Totalsynthese des Naturstoffes erfolgen. So nutzten George et al. 2012 den zuvor postulierten Mechanismus der Biosynthese des polyprenylierten, polycyclischen Acylphloroglucins Garcibracteaton (129), um ausgehend von kommerziell erhältlichem Phloroglucin (127) eine Totalsynthese des Naturstoffes in vier Stufen zu bewerkstelligen (Abbildung 39). ${ }^{103}$ Der Schlüsselschritt dieser Synthese ist eine radikalische Domino-Reaktion des intermediären Weddellianon A (128). Durch Zugabe von Mangan(III)acetat entsteht zunächst das Radikal 130, das nach einer 7-endo-trig/5-exo-trig/5-exo-trig Cyclisierungssequenz mit abschließender radikalischer aromatischer Substitution Garcibracteaton (129) in 14\% Ausbeute liefert. Überdies wurden 8\% 5-epi-Garcibracteaton 129 isoliert. Infolge dieser faszinierenden Domino-Reaktion wurden vier neue $\mathrm{C}-\mathrm{C}$ Bindungen, fünf neue Stereozentren und vier neue carbocyclische Ringsysteme ausgebildet. 

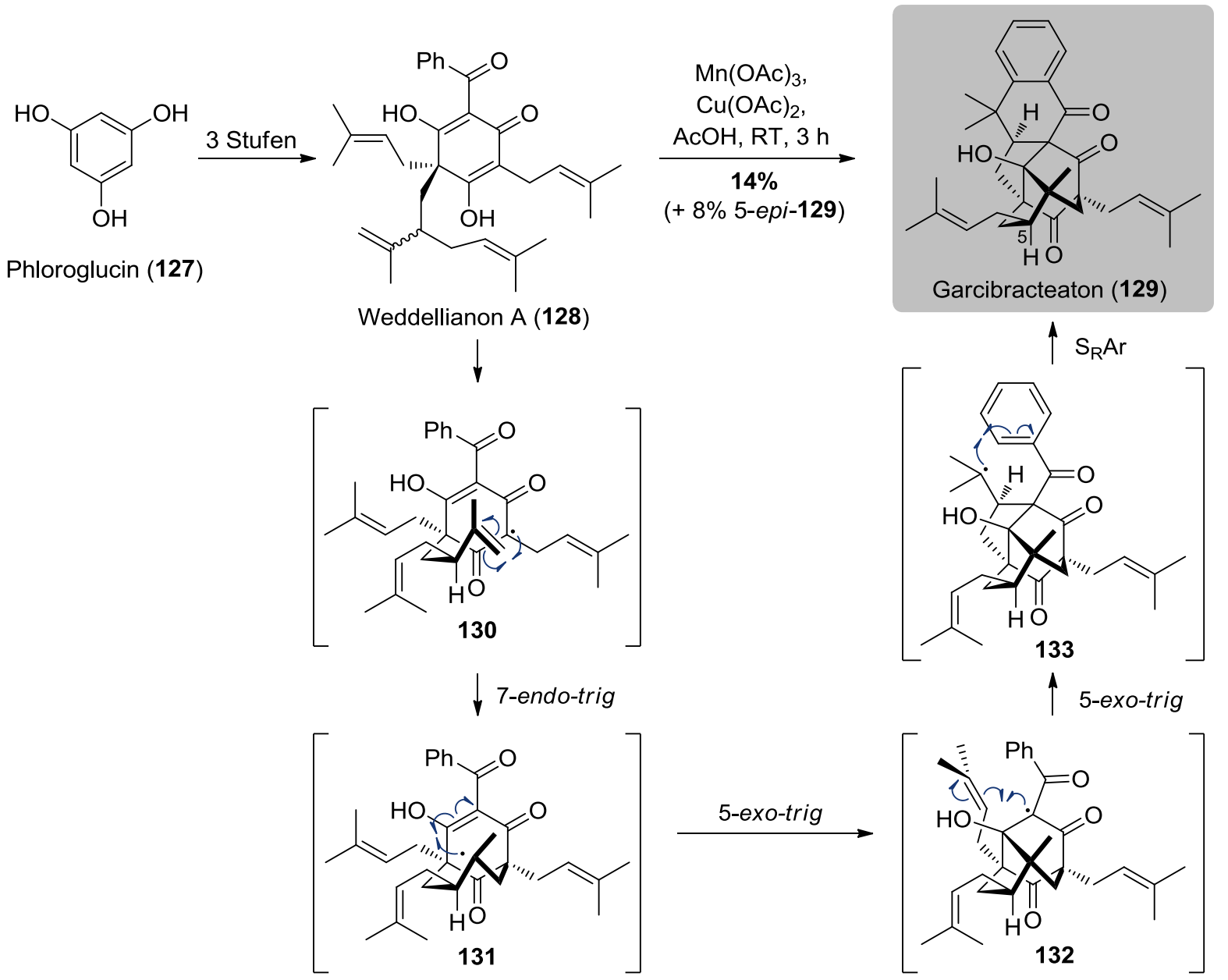

Abbildung 39: Radikalische Domino-Reaktion in der Synthese von Garcibracteaton (129) nach George et al.

Ein weiteres Beispiel für die moderne, elegante Totalsynthese von Naturstoffen über eine Domino-Reaktion ist die 2014 von Tietze et al. veröffentlichte Totalsynthese von (-)-Blennolid C (140) und (-)-Gonytolid C (141). ${ }^{104}$ Zunächst wurde der Domino-Vorläufer 135 in sechs Stufen ausgehend von kommerziell erhältlichem Orcinol (134) hergestellt (Abbildung 40). Eine Palladium-katalysierte Domino-Reaktion mit dem chiralen $(S, S)-i \operatorname{Pr}$ BOXAX-Liganden (136) in Methanol lieferte unter CO-Atmosphäre das Chroman 139 in 62\% Ausbeute und einer exzellenten Enantioselektivität von über 99\%. Mechanistisch betrachtet erfolgt zunächst eine Wacker-Oxidation, in der das Chroman-System 137 gebildet wird. In diesem System gibt es kein $\beta$-ständiges H-Atom, daher kann selektiv eine COInsertion und Methoxylierung der resultierenden Palladium-Acyl-Spezies 138 erfolgen. Der reduzierte Palladiumkomplex wird nach der Domino-Reaktion mit Hilfe von paraBenzochinon reoxidiert. Ausgehend von Methylester 139 wurden (-)-Blennolid C (140) und (-)-Gonytolid C (141) in 15 bzw. 14 weiteren Synthesestufen erhalten. 

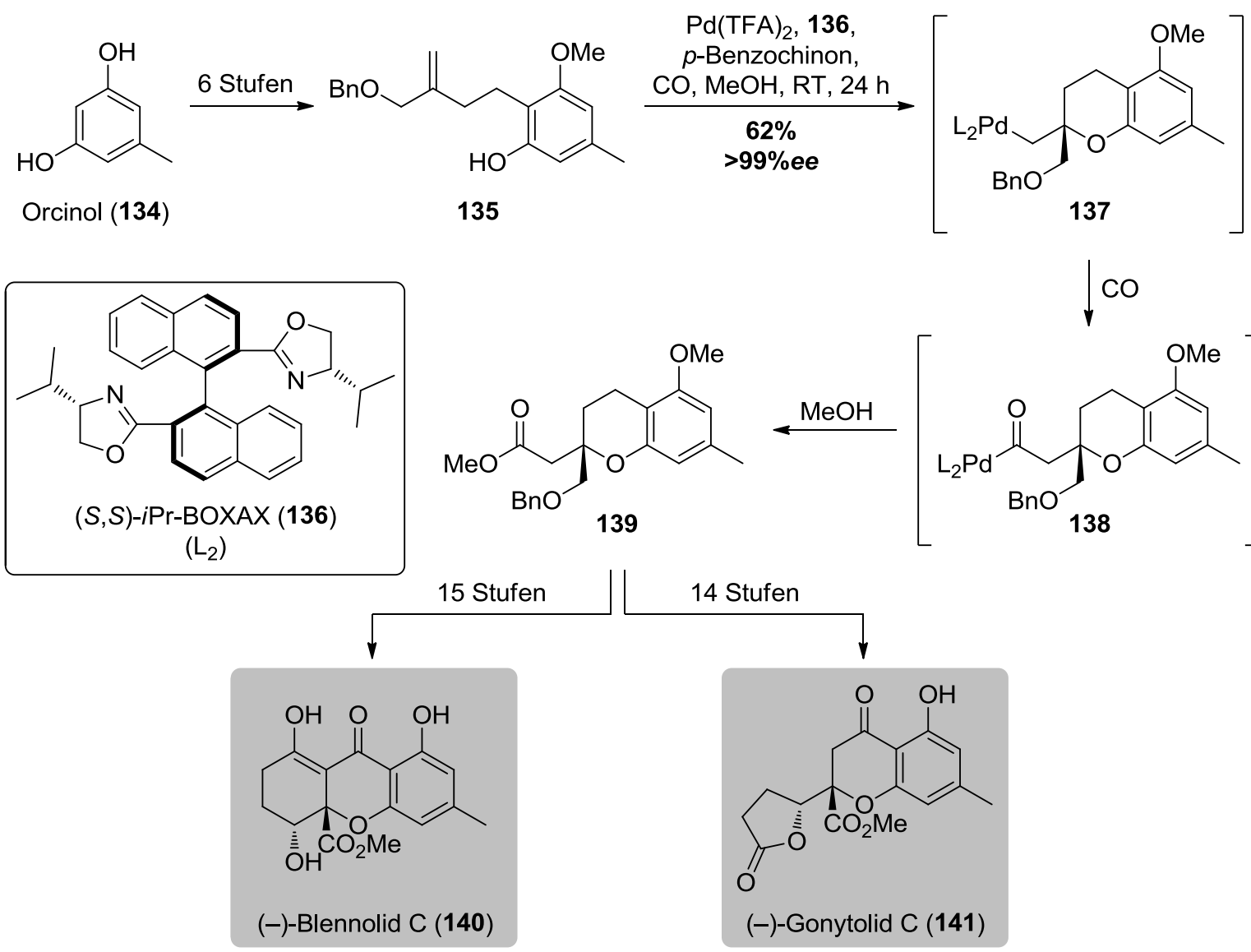

Abbildung 40: Synthese von (-)-Blennolid C (140) und (-)-Gonytolid C (141) über eine Domino-Wacker/ Carbonylierung/Methoxylierung nach Tietze et al.

Eine weitere interessante Anwendung fand das Prinzip der Domino-Reaktionen in der 2012 von Werz et al. veröffentlichten Synthese von chiralen Biphenylen. ${ }^{105}$ In diesem TandemDomino-Prozess finden zeitgleich zwei Domino-Reaktionen an beiden Seiten des $C_{2^{-}}$ symmetrischen Domino-Vorläufers 142 statt (Abbildung 41). Zunächst werden durch zwei aufeinanderfolgende Carbopalladierungen sukzessive der Tetrahydrofuranring (vgl. 145) und ein weiterer Cyclopentan- oder -hexanring (vgl. 146) aufgebaut. Ausgehend von Intermediat 146 erfolgt im nächsten Teilschritt ein elektrocyclischer Ringschluss, bevor durch eine abschließende syn- $\beta$-Hydrid-Eliminierung das hochsubstituierte Biphenylsystem 143 entsteht. Während dieses Prozesses werden sechs neue $\mathrm{C}-\mathrm{C}$-Bindungen, sechs neue Ringsysteme und eine chirale Achse aufgebaut. Da die Stereoinformation der chiralen Achse des Moleküls durch die Sterik des Moleküls selbst induziert wird, wurde die selektive Bildung eines einzelnen Diastereomers beobachtet. 


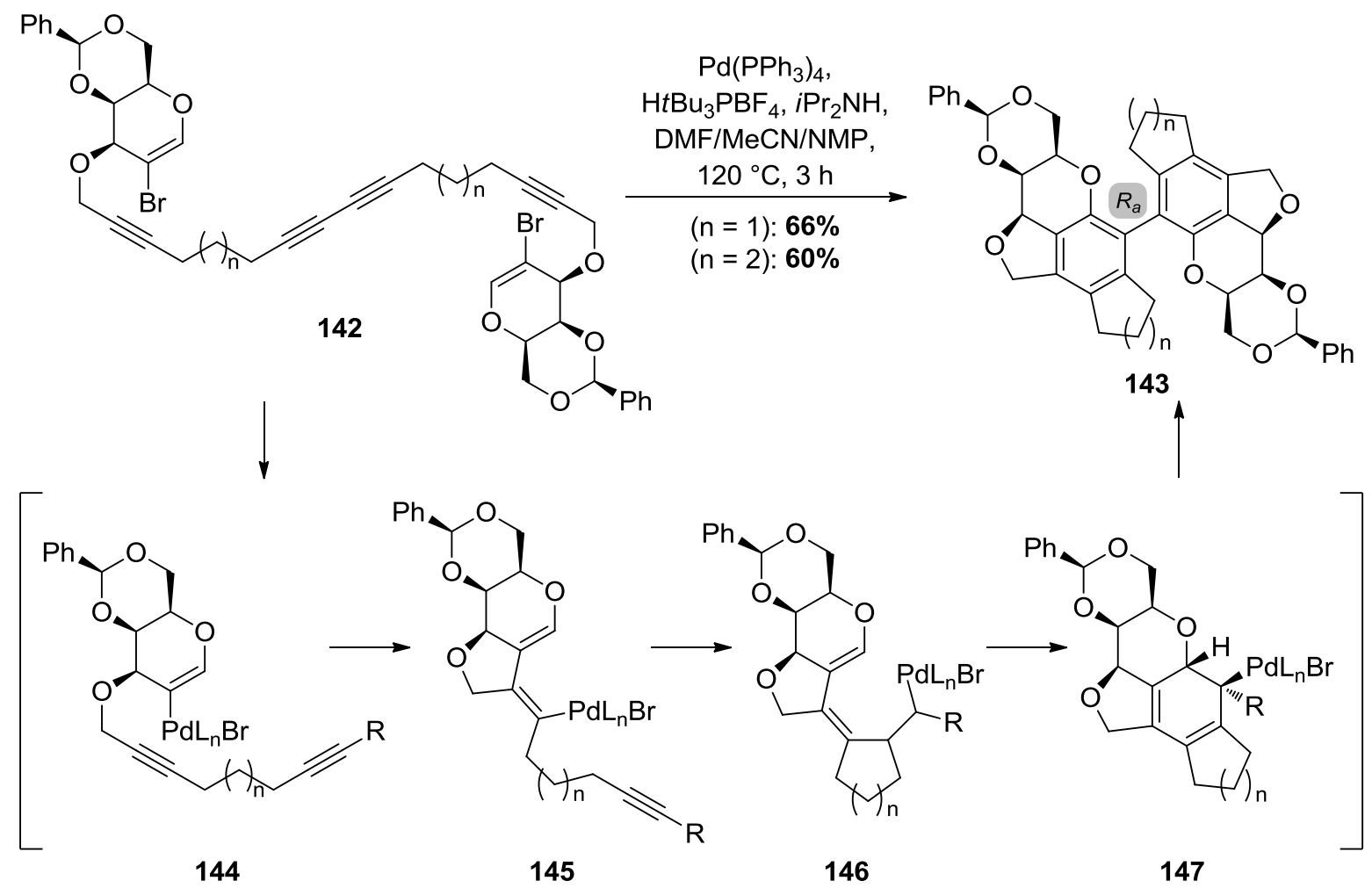

Abbildung 41: Tandem-Domino-Reaktion zur Synthese von chiralen Biphenylen nach Werz et al. 


\section{Aufgabenstellung und Planung der Arbeit}

Naturstoffe dienen aufgrund ihrer strukturellen Vielfalt seit jeher als Leitmotive in der Wirkstoffsynthese. Auch wenn moderne Verfahren wie das High-Throughput-Screening die Tür $\mathrm{zu}$ wesentlich einfacher $\mathrm{zu}$ synthetisierenden niedermolekularen Verbindungen aufgestoßen haben, sind doch immer noch ein Großteil der Wirkstoffe auf zum Teil komplex aufgebaute Naturstoffe und deren semisynthetische Analoga zurückzuführen. Da sich die Gewinnung von biologisch aktiven Naturstoffen aus natürlichen Quellen oftmals als schwierig darstellt und das Auftreten von Resistenzen teilweise Modifikationen des Grundgerüsts erfordert, ist ein synthetischer Zugang zu diesen Verbindungen erstrebenswert.

Ziel dieser Arbeit ist die Entwicklung von Synthesemethoden zu neuartigen Analoga des hochpotenten Pflanzenschutzmittels Spinosyn A (19), ein enantioselektiver Zugang zu dem Naturstoff (+)-Linoxepin (69) sowie die Bereitstellung von niedermolekularen Verbindungen mit potentiell inhibitorischer Wirkung gegenüber dem Hitzeschockprotein 47.

Das Pflanzenschutzmittel Spinosad, das aus den beiden natürlichen Spinosynen A (19) und D (20) besteht, findet auf dem amerikanischen Kontinent in der Bekämpfung von Insekten seit fast 20 Jahren eine breite Anwendung. Mit dem Auftreten der ersten Resistenzen gewinnt ein synthetischer Zugang zu dem bisher aus Fermentation gewonnenen Insektizid an Bedeutung.

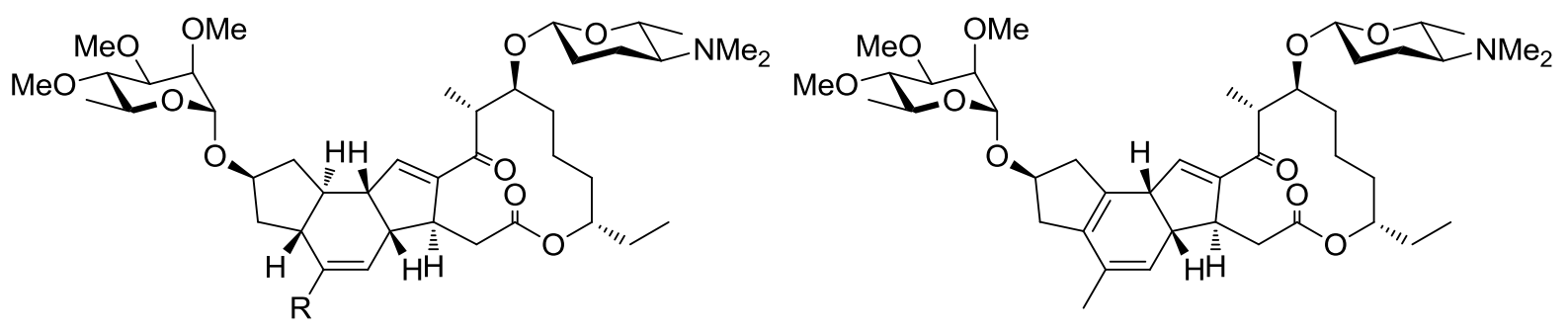

$\mathrm{R}=\mathrm{H}: \quad$ (-)-Spinosyn A (19)

7,11-Dehydro-Spinosyn D (22)

$\mathrm{R}=\mathrm{Me}:(-)-S p i n o s y n \mathrm{D}(\mathbf{2 0})$

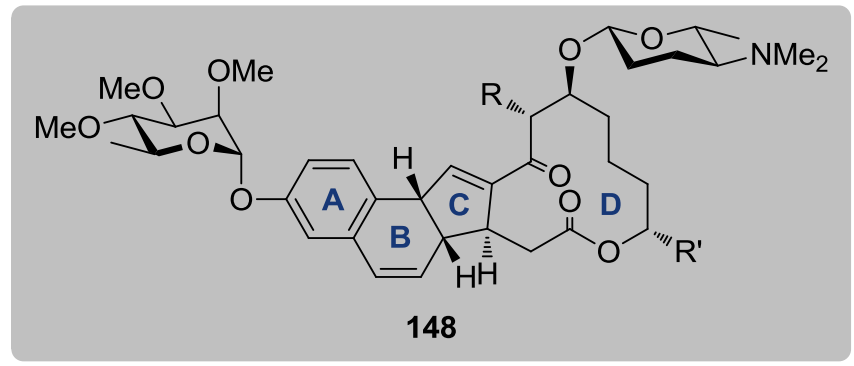

Abbildung 42: Natürlich vorkommende Spinosyne 19 und 20, Spinosynderivat 22 sowie das auf StrukturAktivitäts-Beziehungen zurückzuführende neuartige Spinosyn-Analogon 148.

Da Struktur-Aktivitäts-Beziehungen gezeigt haben, dass die Eliminierung von zwei stereogenen Zentren zum 7,11-Dehydro-Spinosyn D (22) zu keinerlei Wirkverlust führt, 
sollen im Rahmen dieser Arbeit neuartige Spinosyn-Analoga des Typs 148 synthetisiert werden (Abbildung 42). In diesem Analogon-Typ wurde der Rhamnose-tragende Cyclopentanring durch ein phenylisches System ersetzt. Die Verbindungen zeichnen sich durch eine deutlich geringere Anzahl von stereogenen Zentren aus, deren synthetischer Zugang sich häufig als schwierig erweist und in der industriellen Herstellung folglich mit hohen Kosten verbunden ist. Die synthetischen Herausforderungen des neuartigen AnalogonTyps 148 bestehen in der cis-Verknüpfung der Ringe B und C und der damit einhergehenden Anordnung der Doppelbindungen sowie in der selektiven Einführung der beiden Zuckereinheiten. In vorangegangenen Arbeiten der Arbeitsgruppe Tietze konnten bereits mehrfach Ringsysteme der Art A-B-C über zwei aufeinanderfolgende Heck-Reaktionen hochselektiv aufgebaut werden (vgl. Kapitel A. 5.2). ${ }^{106}$ Überdies konnten in jüngsten Arbeiten neue Erkenntnisse über die $\beta$-selektive Einführung des Aminozuckers gewonnen werden, die aufgrund der fehlenden Substituenten an 2-Position des Forosamins ein großes Problem in den bisherigen Synthesen darstellte. ${ }^{107}$

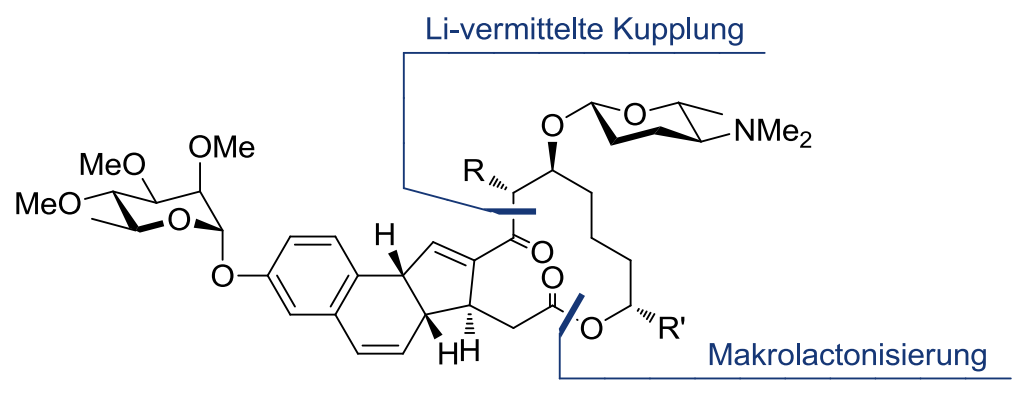

148

$\int \mathrm{R}=\mathrm{R}^{\prime}=\mathrm{H}$

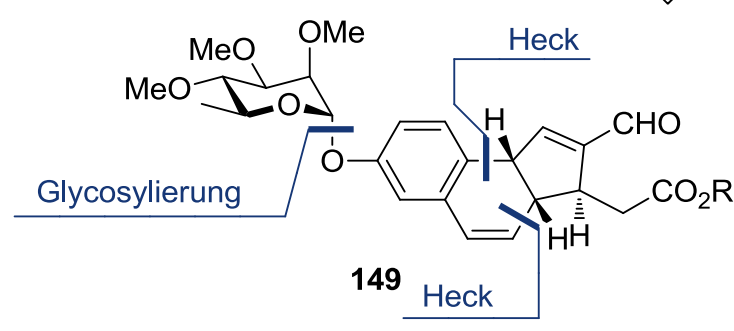

$\sqrt{1}$

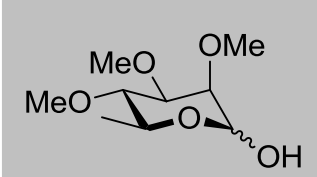

151

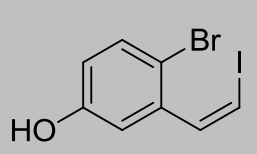

152

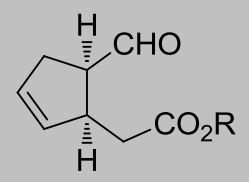

153

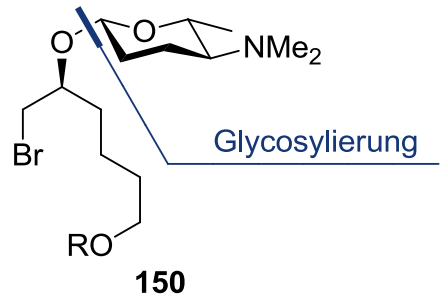

$\prod$

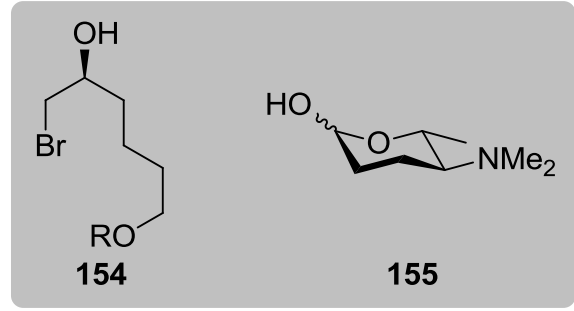

Abbildung 43: Retrosynthetischer Zugang zu Spinosyn-Analoga des Typs 148. 
Ein Ziel dieses Teilgebietes der Arbeit ist die Synthese der Bausteine 149 und 150, die über eine Lithium-vermittelte 1,2-Addition mit anschließender Makrolactonisierung in das Spinosyn-Analogon 148 überführt werden können (Abbildung 43). Da den beiden alkylischen Substituenten am Makrolacton ( $\mathrm{R}$ und $\mathrm{R}^{\prime}$ ) bislang keine aktivitätsrelevante Wirkung nachgesagt werden konnte, wurden diese zunächst in den Syntheseplanungen durch Wasserstoffatome ausgetauscht.

Der Aufbau des zuckertragenden Tricyclus 149 sieht hierbei eine Glycosylierung des aromatischen Bausteins 152 mit 2,3,4-Tri- $O$-methyl-L-rhamnose (151) sowie zwei aufeinanderfolgende Heck-Reaktionen mit dem Cyclopentenderivat 153 vor. Die Seitenkette 150 sollte über eine $\beta$-selektive Glycosylierung des C-6-Fragments 154 mit dem Aminozucker D-Forosamin (155) zugänglich sein.

In vorangegangenen Arbeiten hat sich herausgestellt, dass die Kupplung mit der Forosamineinheit möglichst früh in der Synthese erfolgen sollte, da eine spätere Glycosylierung an komplexeren Verbindungen entweder gar nicht oder nur in geringen Ausbeuten zu realisieren war. Des Weiteren konnten durch einen Austausch der AminFunktionalität gegen eine unreaktivere Azid-Einheit höhere Selektivitäten und Ausbeuten erzielt werden. Um die neu entwickelte Methode der selektiven Glycosylierung auch bei komplexeren Molekülen $\mathrm{zu}$ etablieren, sollte in Kooperation mit der Bayer AG das Spinosyn A-Aglycon $157 \beta$-selektiv mit der Azidverbindung 158 gekuppelt werden (Abbildung 44).
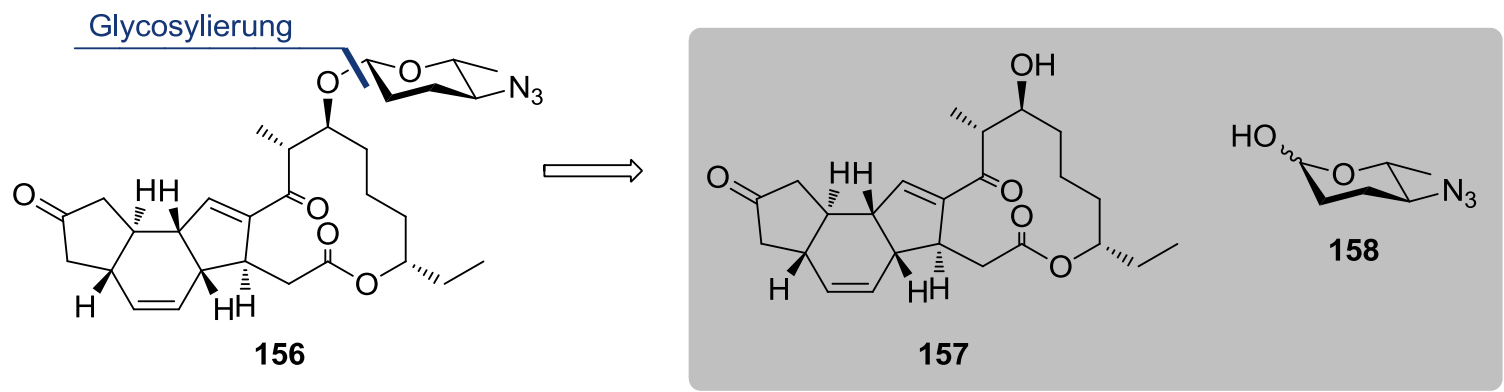

Abbildung 44: Glycosylierung des Spinosyn A-Grundgerüsts 157.

Da in neueren Studien gezeigt wurde, dass die Forosamineinheit ohne signifikanten Wirkverlust gegen andere stickstoffhaltige Kohlenhydrate und Nicht-Kohlenhydrate ausgetauscht werden kann, ${ }^{42}$ ist auch die Herstellung von leicht zugänglichen ForosaminAnaloga Teil dieser Arbeit (Abbildung 45). Um eine größtmögliche Ähnlichkeit zur bioidentischen Substanz zu erhalten, sollen sich weiterhin vier Kohlenstoffatome zwischen dem veretherten Sauerstoff und dem Stickstoff der Aminofunktion befinden. 


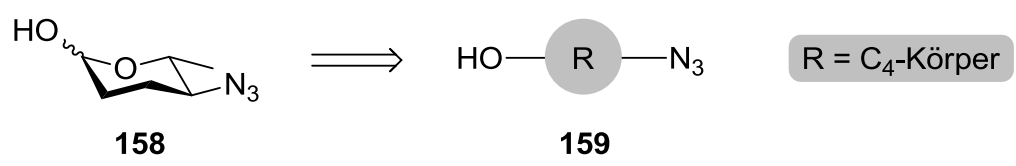

Abbildung 45: Geplante Synthese von Forosamin-Analoga.

Zusammenfassend ergeben sich für diesen Teil der Arbeit folgende Aufgabenfelder:

- Aufbau des tricyclischen Grundgerüsts 149 mit cis-Verknüpfung der B-C-Einheit durch zwei aufeinanderfolgende Heck-Reaktionen

- Synthese der Seitenkette $\mathbf{1 5 0}$ mit selektiv $\beta$-gebundener Forosamineinheit

- Untersuchungen zur Kupplung der Bausteine 149 und 150 zum Aufbau von Spinosynanaloga des Typs 148

- Etablierung der Glycosylierungsmethode an dem Spinosyngrundgerüst 157 im Rahmen einer Kooperation mit der Bayer AG

- Aufbau von chemisch leicht zugänglichen Forosamin-Analoga des Typs 159.

Der zweite Teil der Arbeit befasst sich mit einer enantioselektiven Totalsynthese des Naturstoffes (+)-Linoxepin (69) über eine Palladium-katalysierte Domino-Reaktion. Da der in der Arbeitsgruppe Tietze zuvor gezeigte synthetische Ansatz racemischer Natur war und eine aufwendige Trennung der Enantiomere über HPLC erforderte, ist die Entwicklung eines asymmetrischen Ansatzes mit höherer Gesamtausbeute von Interesse.

Die Retrosynthese sieht zunächst die Kupplung des Vinylsilans 164 mit dem Benzylbromid 165 zu Benzylarylether 162 vor (Abbildung 46). Eine anschließende Sonogashira-Reaktion mit dem geschützten Propargylalkohol (163) und eine nachfolgende Palladium-katalysierte Domino-Carbopalladierung-Heck-Reaktion sollten den Aufbau des Ringsystems 161 ermöglichen. Da in vorangegangenen Arbeiten häufig eine Isomerisierung der exoDoppelbindung zum aromatischen Produkt auftrat, wird in dieser Arbeit ein Vinylsilan in der Heck-Reaktion verwendet, da dieses im Idealfall eine hohe Regio- und Chemoselektivität in Bezug auf die Terminierung der Palladium-katalysierten Domino-Reaktion aufweist. ${ }^{108}$ Die Stereoinformation an C-9a soll in dieser Synthese durch eine asymmetrische Hydroborierung zu Diol 160 induziert werden. Da in der Vergangenheit bereits eine asymmetrische Hydrierung an ähnlichen Substraten erprobt wurde und nicht zu positiven Ergebnissen geführt hat, ${ }^{109}$ soll auf eine weitere Anwendung dieser Reaktion verzichtet werden. Eine Oxidation des Allylalkohols mit anschließender Lactonisierung sollte abschließend den Naturstoff (+)-Linoxepin (69) liefern. 


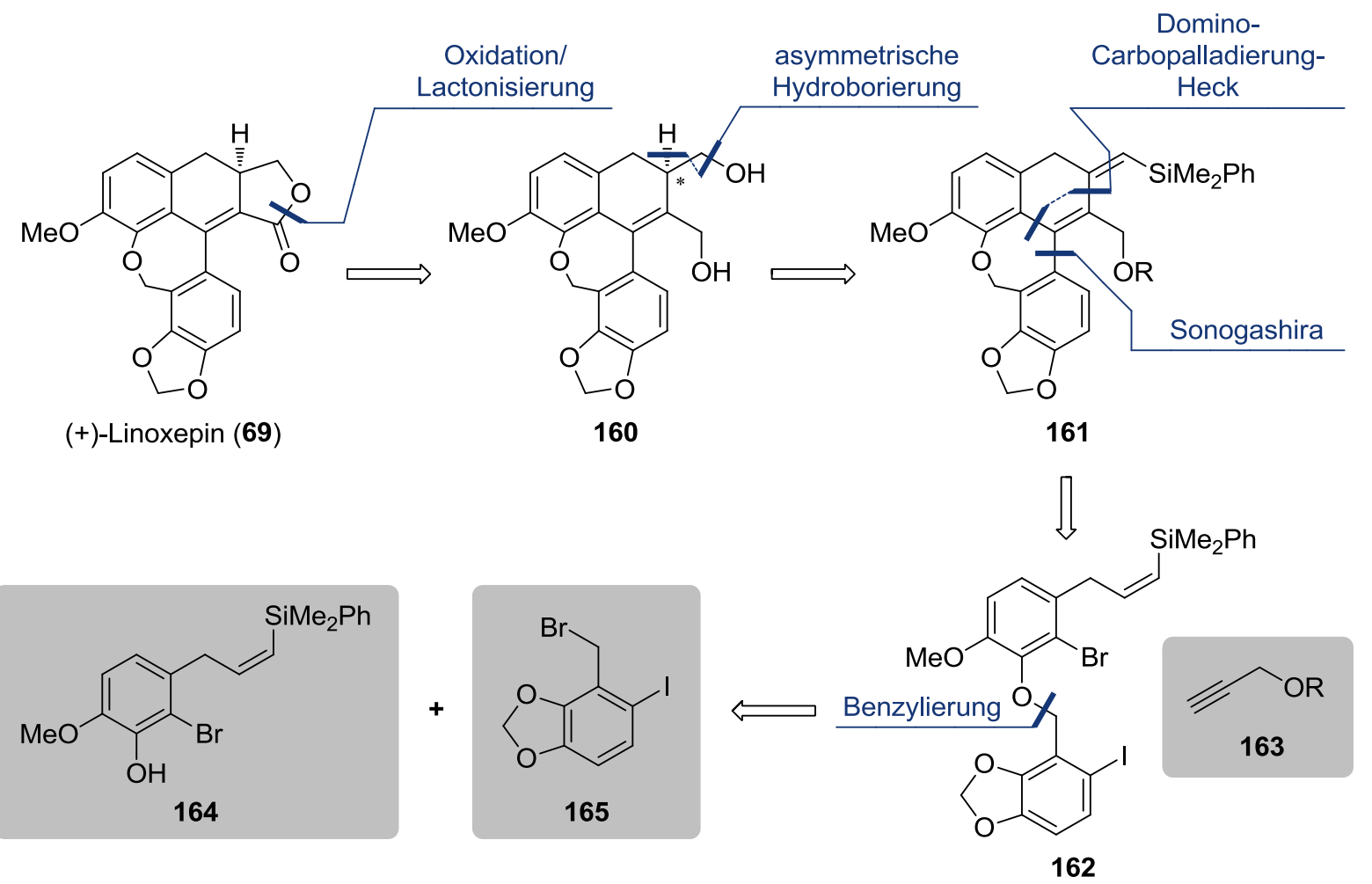

Abbildung 46: Retrosynthetischer Zugang zu (+)-Linoxepin (69).

Die Aufgaben des zweiten Teils der Arbeit sind folglich:

- Aufbau des Dominovorläufers 162

- Durchführung der Palladium-katalysierten Domino-Carbopalladierung-Heck-Reaktion unter Unterbindung der Ausbildung des aromatischen Produktes

- Realisierung eines enantioselektiven Zugangs zu (+)-Linoxepin unter Verwendung einer asymmetrischen Hydroborierung

- Fertigstellung der Totalsynthese von (+)-Linoxepin (69).

Der letzte Teil dieser Doktorarbeit befasst sich mit der Synthese von niedermolekularen Verbindungen des Typs 84 mit potentieller Hsp47-Inhibitionsaktivität. Dabei gilt es, in Kooperation mit der Arbeitsgruppe Zimmermann des Universitätsklinikums in Göttingen die Auswirkungen von Änderungen des Substitutionsmusters auf die biologische Aktivität der Verbindung zu untersuchen.

Retrosynthetisch betrachtet sind die potentiellen Hsp47-Inhibitoren des Typs 84 durch eine Kupplung des Dihydropyrimidons 166 mit den $\alpha$-Bromacetophenonen 167 zugänglich (Abbildung 47). Während das Dihydropyrimidon 166 über eine Mehrkomponenten-Reaktion auf die drei kommerziell erhältlichen Bausteine 168, 169 und 170 zurückzuführen ist, sollte 
eine Methylierung und anschließende Bromierung der Benzoesäurederivate $171 \mathrm{zu}$ den $\alpha$-Bromacetophenonen 167 führen.

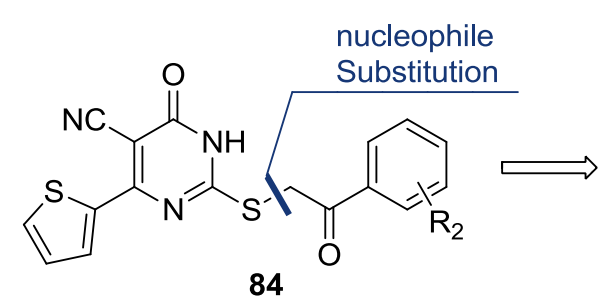

84

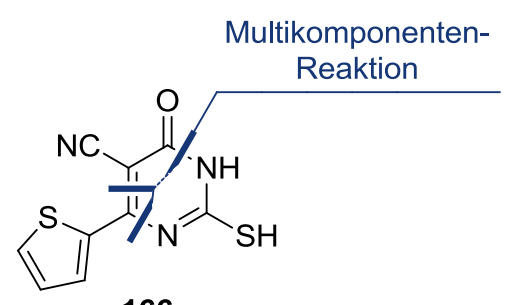

166
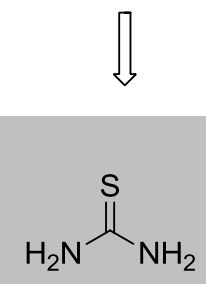

169<smiles>CCOC(=O)CC#N</smiles>

170

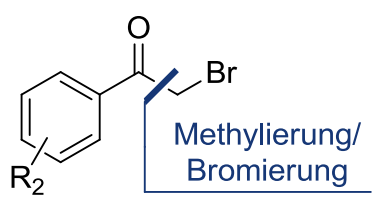

167<smiles>C=C</smiles>

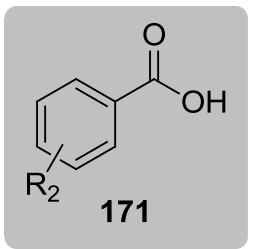

Abbildung 47: Retrosynthese für potentielle Hsp47-Inhibitoren des Typs 84.

Da in vorangegangenen Arbeiten das Augenmerk auf eine Substitution des Thiophenrings gerichtet wurde, ${ }^{110}$ besteht in dieser Arbeit das Ziel in einer Modulation des aromatischen Systems am Acetophenon. Hierbei sollen sowohl die Art der Substituenten als auch das Substitutionsmusters untersucht werden.

Für den letzten Teilabschnitt dieser Arbeit ergeben sich dementsprechend folgende Aufgabenfelder:

- Synthese des Thiophen-substituierten Dihydropyrimidons 166 über eine Mehrkomponenten-Reaktion

- Aufbau von verschiedenen $\alpha$-Bromacetophenonen 167 mit unterschiedlichem Substitutionsmuster und Varianz der Substituenten

- Bereitstellung von potentiellen Hsp47-Inhibitoren für die Evaluation der biologischen Aktivität in der Arbeitsgruppe Zimmermann. 


\section{B. ERGEBNISSE \& DISKUSSION}

\section{Synthese von Spinosyn-Analoga}

\subsection{Synthese des Tricyclus 149}

Bei der Synthese von neuartigen Spinosyn-Analoga konnte auf ausführliche Vorarbeiten aus dem Arbeitskreis Tietze zurückgegriffen werden. ${ }^{111}$ Besonders bei dem Aufbau des tricyclischen Grundgerüsts und den Glycosylierungsmethoden wurde von den zahlreichen Studien und Optimierungen profitiert.

\subsubsection{Synthese des Glycosyldonors 175}

Als Glycosyldonor für die Einführung der Rhamnoseeinheit wurde das Trichloracetimidat 175 gewählt. Nach Schmidt et al. zeichnen sich diese Verbindungen durch ihre hohe Stabilität sowie Reaktivität aus. Als Ausgangsstoff für die Synthese diente das kommerziell erhältliche L-Rhamnose Monohydrat 172. Um einen Syntheseschritt einzusparen, wurde direkt eine Permethylierung der Zuckereinheit durchgeführt und nicht, wie sonst üblich, zunächst das anomere Zentrum und anschließend die übrigen Hydroxyfunktionen methyliert. Die Umsetzung zur permethylierten L-Rhamnose wurde mittels Natriumhydrid und Methyliodid in Dimethylsulfoxid in einer Ausbeute von $64 \%$ und einer $\alpha / \beta$-Selektivität von $1: 1$ bewerkstelligt (Abbildung 48).
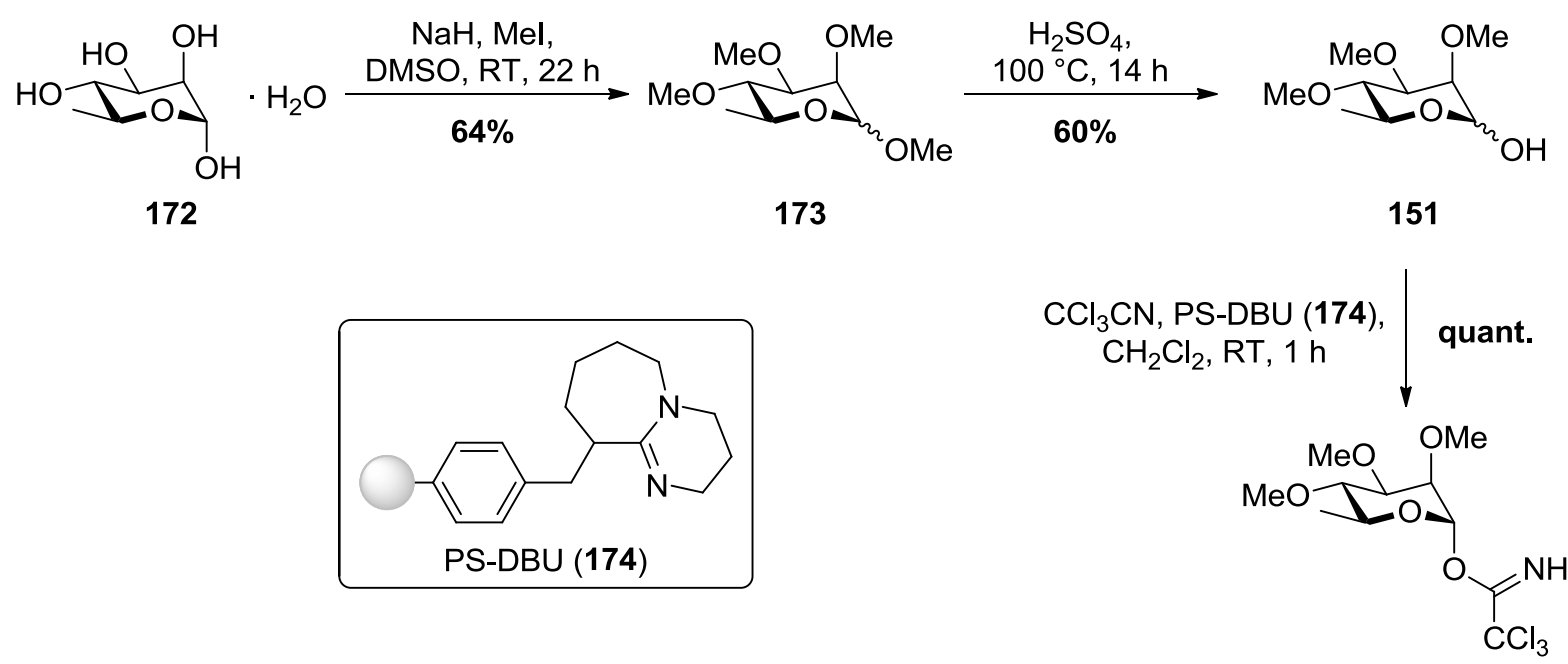

Abbildung 48: Synthese des Trichloracetimidats 175. 
Anschließende Entschützung des anomeren Zentrums mit verdünnter Schwefelsäure bei $100{ }^{\circ} \mathrm{C}$ lieferte den Zucker 151 in $60 \%$ Ausbeute mit einer $\alpha / \beta$-Selektivität von 6:1. Die Überführung in den Glycosyldonor 175 gelang unter basenvermittelter Zugabe von Trichloracetonitril in Dichlormethan. Durch die Verwendung von polymergebundenem Diazabicycloundecen (174) als Base konnte auf eine säulenchromatographische Reinigung verzichtet und das Rohprodukt nach Filtration des Polymers direkt in der nachfolgenden Glycosylierung verwendet werden.

\subsubsection{Synthese des aromatischen Glycosylakzeptors 178}

Die Synthese des aromatischen Bausteins 178 wurde nach einem leicht modifizierten literaturbekannten Verfahren ausgehend von kommerziell erhältlichem 3-Methoxybenzaldehyd (176) durchgeführt. ${ }^{112}$ Im ersten Schritt der Synthese wurde Aldehyd 176 unter Verwendung von elementarem Brom in Dichlormethan bei Raumtemperatur regioselektiv in para-Position zur Methoxygruppe bromiert (Abbildung 49). Nach Umkristallisation aus n-Pentan konnte die gewünschte bromierte Spezies 177 in $82 \%$ Ausbeute erhalten werden.

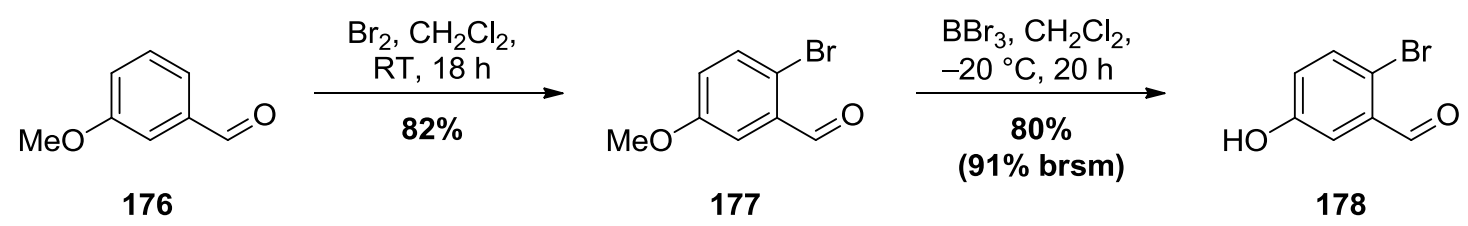

Abbildung 49: Synthese des aromatischen Glycosylakzeptors 178.

Eine anschließende Spaltung des aromatischen Methylethers nach einem Standardprotokoll mit der starken Lewis-Säure Bortribromid in Dichlormethan bei $-20{ }^{\circ} \mathrm{C}$ führte in $80 \%$ Ausbeute zu dem gewünschten Glycosylakzeptor 178. Des Weiteren wurden in dieser Reaktion $11 \%$ des nicht umgesetzten Startmaterials reisoliert.

\subsubsection{Synthese des Vinyliodids 180}

Die Lewis-Säure-katalysierte Kupplung der beiden Bausteine erfolgte dank der hohen Reaktivität des Trichloracetimidats in einer exzellenten Ausbeute von 85\% über zwei Stufen bezogen auf das Rhamnopyranosid 175 (Abbildung 50). ${ }^{113}$ Eine Überführung des Aldehyds 178 in das Vinyliodid 180 wurde durch eine Wittig-Reaktion mit einem Phosphoniumsalz und Kaliumhexamethyldisilazid als Base in THF bei $-78^{\circ} \mathrm{C}$ realisiert. ${ }^{114}$ Die moderate Ausbeute von 55\% ist dabei auf die Bildung und mühsame Abtrennung des ungewünschten $(E)$ konfigurierten Vinyliodids zurückzuführen. 


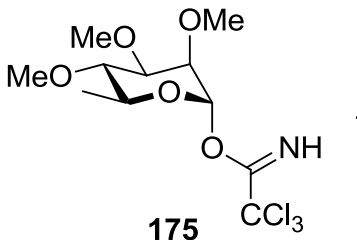

175<smiles>O=Cc1cc(O)ccc1Br</smiles>

178

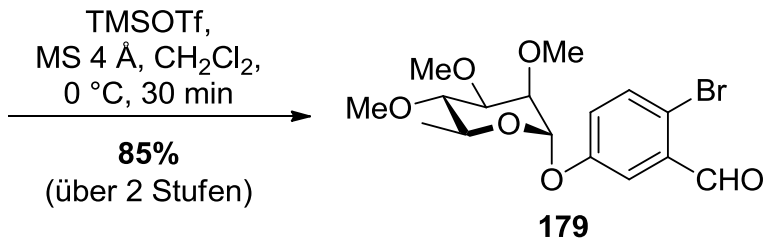

$\left[\mathrm{Ph}_{3} \mathrm{PCH}_{2} \mathrm{I}^{+} \mathrm{I}^{-}\right.$, KHMDS, THF, $-78^{\circ} \mathrm{C}, 90 \mathrm{~min}$ $55 \%$

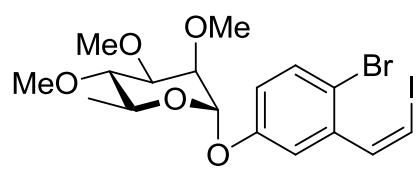

180

Abbildung 50: Synthese des Vinyliodids 180.

\subsubsection{Mechanistische Aspekte der zweifachen Heck-Reaktion}

Eine besondere chemische Herausforderung bei der Synthese von Spinosyn-Analoga des Typs 148 ist die cis-Verknüpfung zwischen dem B- und C-Ring des tricyclischen Grundgerüsts. Zur Bewerkstelligung dieses Aufbaus war eine Synthesesequenz aus zwei aufeinanderfolgenden Heck-Reaktionen vorgesehen. Eine wesentliche Voraussetzung für die Ausbildung dieser $c i s$-Verknüpfung ist die Verwendung eines 1,2-cis-disubstituierten Cyclopentenderivats 182. Zur Verdeutlichung dient der in Abbildung 51 gezeigte Reaktionsmechanismus.

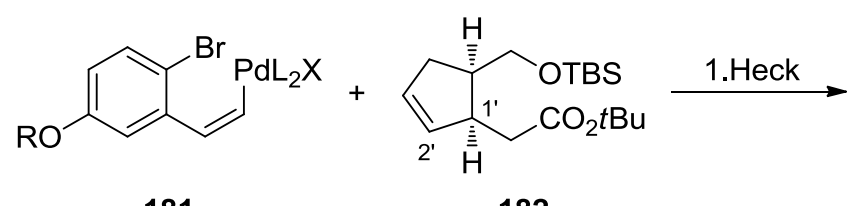

181

182

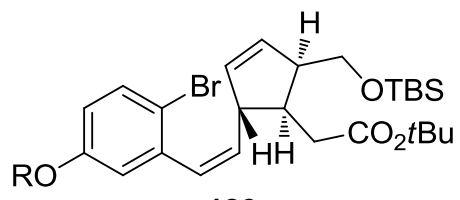

183

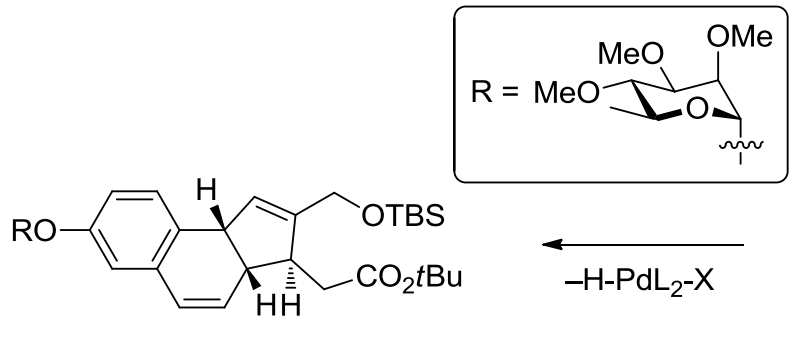

185

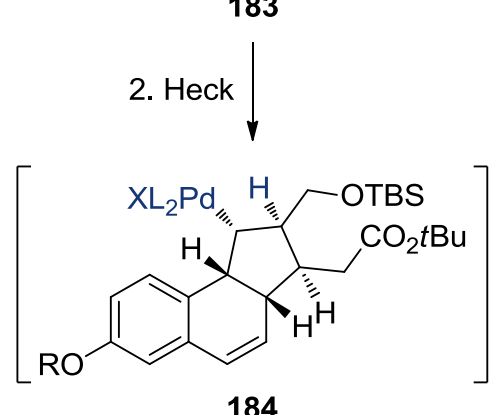

Abbildung 51: Stereochemische Aspekte der zweifachen Heck-Reaktion.

Unter Annahme eines regioselektiven Angriffs der Palladiumspezies 181 an C-2' des Cyclopentenderivats $\mathbf{1 8 2}$ erfolgt eine diastereoselektive Reaktionssteuerung durch das benachbarte stereogene Zentrum C-1' (vgl. Kapitel A. 5.2). Dieses begünstigt einen Angriff des Palladium von der $\alpha$-Seite und somit den selektiven Aufbau des Heck- 
Kupplungsproduktes 183. In der zweiten, intramolekular verlaufenen Heck-Reaktion findet zunächst eine oxidative Addition des Palladium-Katalysators in die Aryl-Br-Bindung statt. Der Insertionsprozess des neu gebildeten Palladium-Komplexes in die olefinische Doppelbindung erfolgt hierbei aufgrund der geringen Flexibilität der Verbindung wiederum von der $\alpha$-Seite und resultiert in der Bildung der intermediären Spezies 184, die bereits die gewünschte cis-Verknüpfung aufweist. Das für die $\beta$-Hydrid-Eliminierung benötigte syn-ständige Wasserstoffatom befindet sich an C-5' und erlaubt eine selektive Eliminierung in diese Richtung. Diese mechanistischen Überlegungen bestätigen die Verwendung eines 1,2-cis-disubstituierten Cyclopentenderivats, da das korrespondierende trans-Derivat neben einer fraglichen Selektivität in der ersten Heck-Reaktion auch nicht die syn- $\beta$-HydridEliminierung in der intramolekular verlaufenden Reaktion eingehen könnte.

\subsubsection{Synthese des tricyclischen Bausteins 185}

Die intermolekulare Heck-Reaktion wurde bei Raumtemperatur mit Palladium(II)acetat als Katalysator und Tetrabutylammoniumchlorid sowie Natriumacetat als Additive durchgeführt. Hierbei konnte auf das in vorangegangenen Arbeiten synthetisierte racemische Cyclopentenderivat $r a c-\mathbf{1 8 2}$ zurückgegriffen werden. ${ }^{115}$

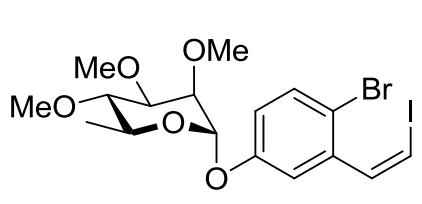

180

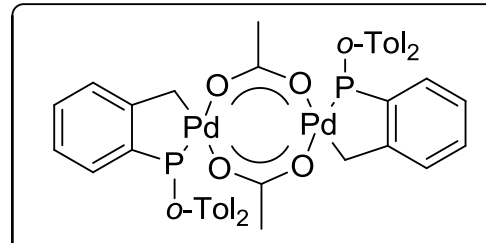

Herrmann-Beller-Katalysator (122)

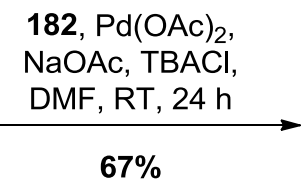

$67 \%$

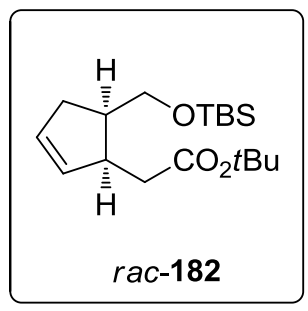

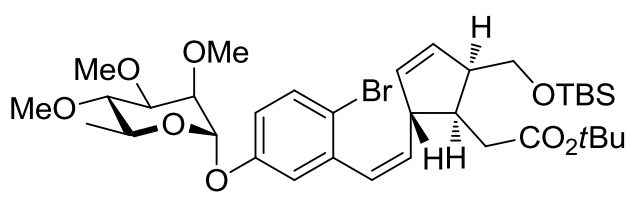

183

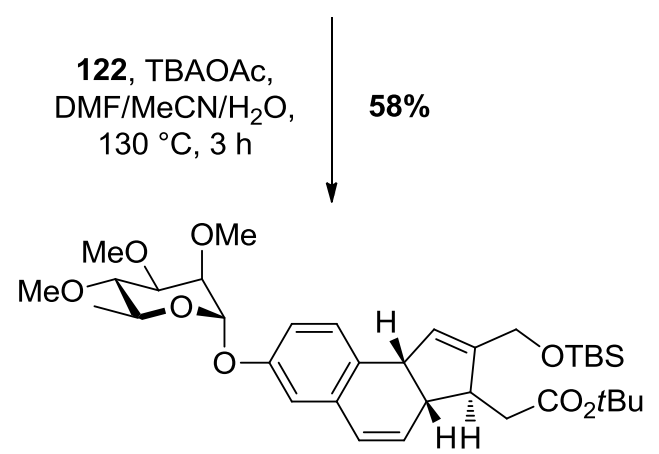

185

Abbildung 52: Zweifache Heck-Reaktion zum Aufbau des Tricyclus 185.

Das gewünschte Kupplungsprodukt wurde dabei in einer hervorragenden Ausbeute von 67\% als 2:1-Gemisch beider Diastereomere erhalten, von denen aus Gründen der Übersicht im weiteren Verlauf des Kapitels nur das Hauptdiastereomer abgebildet ist (Abbildung 52). Als 
zusätzliche Nebenprodukte dieser Heck-Reaktion wurden das an C-3' funktionalisierte Regioisomer sowie einige (E)-konfigurierte Kupplungsprodukte isoliert.

Die zweite, intramolekular verlaufende Heck-Reaktion erfolgte mit katalytischen Mengen des Herrmann-Beller-Katalysators (122) unter Zugabe des Additivs Tetrabutylammoniumacetat in einem Lösungsmittelgemisch aus Dimethylformamid, Acetonitril und Wasser bei $130{ }^{\circ} \mathrm{C}$. Die mäßige Ausbeute von 58\% ist dabei höchstwahrscheinlich auf eine alte, inaktive Charge des Palladacyclus 122 zurückzuführen. Bei früheren Arbeiten wurden für diesen Schritt Ausbeuten von bis zu $99 \%$ erzielt. $^{111}$

\subsubsection{Synthese des Aldehyds 149}

Um ein geeignetes Elektrophil für die geplante Lithium-vermittelte 1,2-Addition zu schaffen, galt es im Folgenden, die allylische Hydroxyfunktion des Tricyclus 185 zu entschützen und zu dem korrespondierenden Aldehyd 149 zu oxidieren. Zunächst wurde der primäre Alkohol 186 unter Verwendung von katalytischen Mengen $p$-Toluolsulfonsäure in Methanol bei $0{ }^{\circ} \mathrm{C}$ in einer ausgezeichneten Ausbeute von 96\% freigesetzt (Abbildung 53).
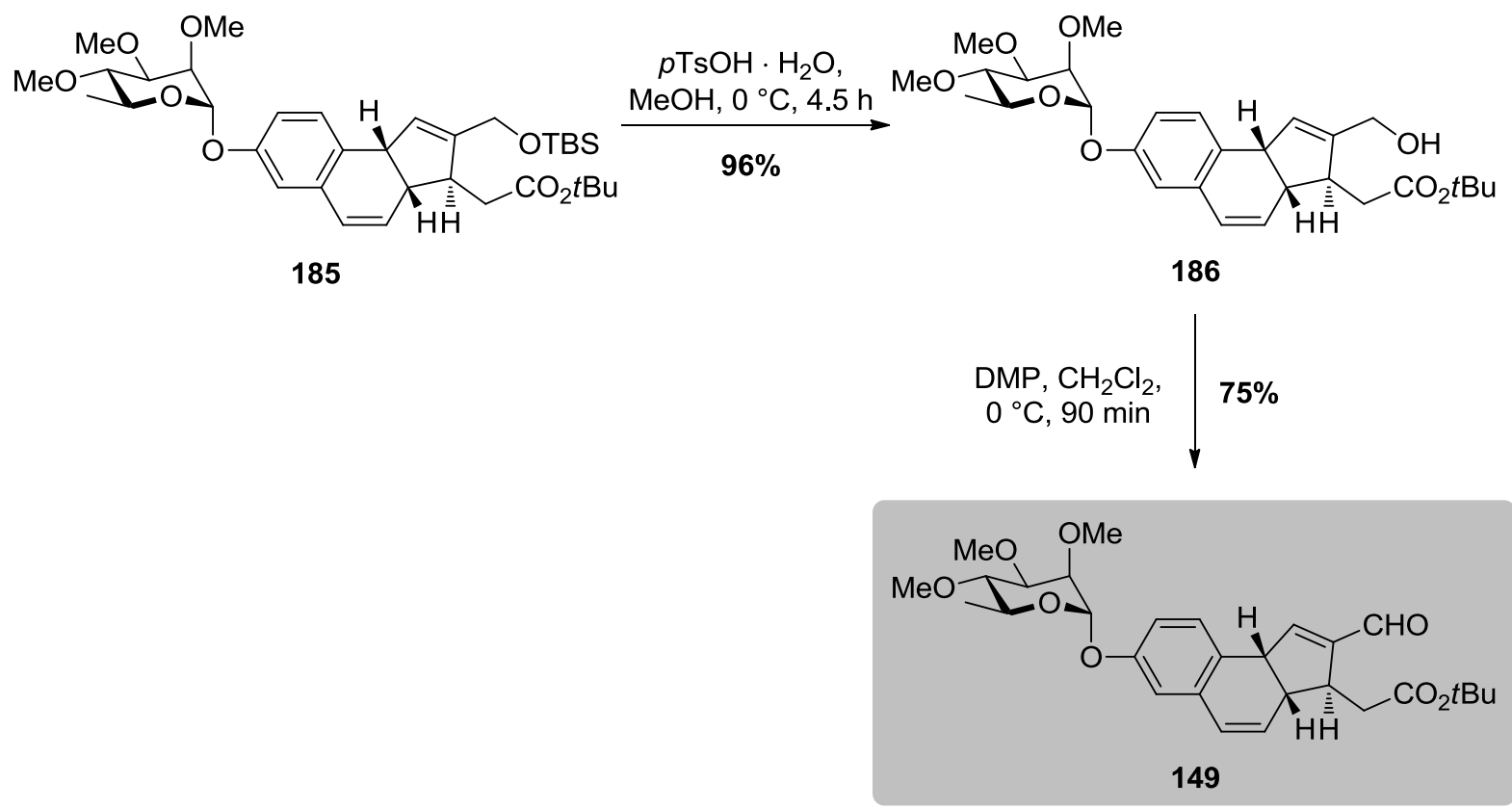

Abbildung 53: Synthese des tricyclischen Bausteins 149.

Auf dieser Synthesestufe gelang außerdem die Trennung der beiden Diastereomere durch präparative HPLC. Nach der Isolierung des enantio- und diastereomerenreinen Alkohols 186 konnte die cis-Verknüpfung des B- und C-Rings und die trans-Stellung der Protonen 3'-H und 3a'-H durch NOESY-Experimente zweifelsfrei nachgewiesen werden. 
Der freie Allylalkohol wurde im nächsten Schritt unter Verwendung von Dess-MartinPeriodinan und Dichlormethan als Lösungsmittel in 75\% Ausbeute zum korrespondierenden Aldehyd 149 oxidiert und die Synthese des enantiomerenreinen cis-verknüpften Tricyclus somit fertiggestellt.

Da die Trennung der beiden Diastereomere des Alkohols 186 lediglich in semi-präparativem Maßstab bewerkstelligt werden konnte und sich außerdem als äußerst zeitintensiv erwies, wurde für weitere Studien zur Synthese von Spinosyn-Analoga das Diastereomerengemisch des Alkohols 186 verwendet und eine säulenchromatographische Trennung zu einem späteren Zeitpunkt der Synthese angestrebt.

\subsubsection{Diskussion der spektroskopischen Daten von Verbindung 186}

Im Folgenden sollen einige ausgewählte spektroskopische Daten des enantiomerenreinen Alkohols 186 näher diskutiert werden. Die genaue Zuordnung der Signale erfolgte über entsprechende zweidimensionale NMR-Spektren (COSY, HSQC und HMBC) sowie über die unterschiedlichen Intensitäten der Signale im ${ }^{13} \mathrm{C}$-NMR. Das ${ }^{1} \mathrm{H}-\mathrm{NMR}$-Spektrum wurde bei Raumtemperatur in deuteriertem Chloroform bei einer Frequenz von $600 \mathrm{MHz}$ aufgenommen und ist in Abbildung 54 dargestellt.
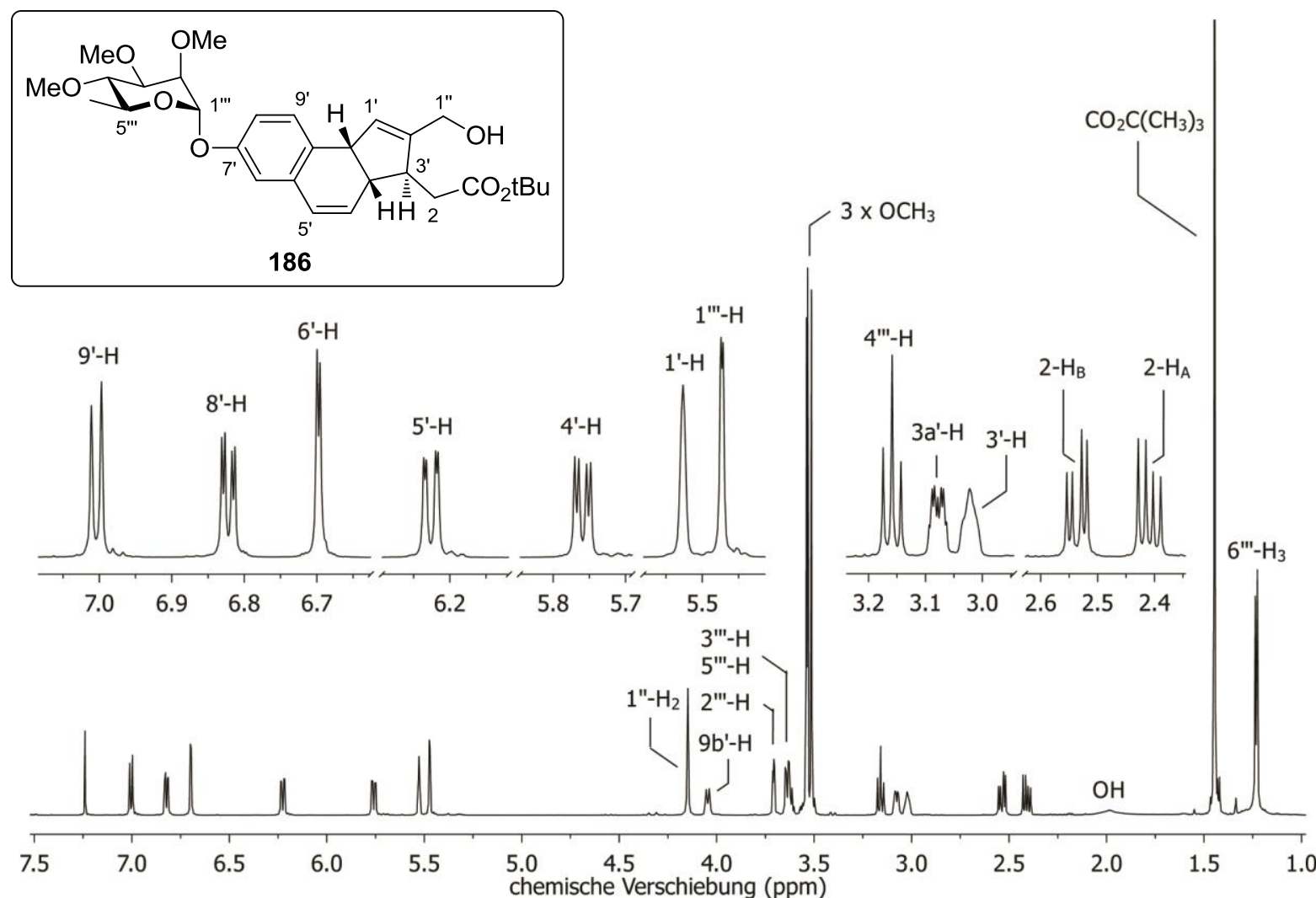

Abbildung 54: ${ }^{1} \mathrm{H}-\mathrm{NMR}-S p e k t r u m$ von Verbindung 186. 
Im Hochfeldbereich des Spektrums resonieren bei $\delta=1.23 \mathrm{ppm}$ erwartungsgemäß die Wasserstoffatome der Methylgruppe an der Rhamnoseeinheit $\left(6 " '-\mathrm{H}_{3}\right)$ in Form eines Dubletts mit der Kopplungskonstante $J=6.2 \mathrm{~Hz}$. Das Singulett mit einer Intensität von neun bei $\delta=1.45$ ppm ist auf die Resonanz der Wasserstoffatome des tert-Butylesters zurückzuführen. Aufgrund von Austauschprozessen resoniert das Wasserstoffatom der Hydroxyfunktion an C-1" in Form eines breiten Singuletts und ist bei einer chemischen Verschiebung von $\delta=1.99 \mathrm{ppm} \mathrm{zu}$ finden. Die beiden diastereotopen Wasserstoffatome $2^{\prime}-\mathrm{H}_{\mathrm{A}}$ und $2^{\prime}-\mathrm{H}_{\mathrm{B}}$ resonieren bei $\delta=2.41 \mathrm{ppm}$ und $\delta=2.54 \mathrm{ppm}$ jeweils als Dublett vom Dublett. Neben der gemeinsamen geminalen Kopplungskonstante von $J=15.6 \mathrm{~Hz}$ sind auch die vicinalen Kopplungskonstanten von $J=8.0 \mathrm{~Hz}\left(2^{\prime}-\mathrm{H}_{\mathrm{A}}\right)$ und $J=5.8 \mathrm{~Hz}\left(2^{\prime}-\mathrm{H}_{\mathrm{B}}\right)$ zu dem Wasserstoffatom 3'-H am stereogenen Zentrum zu finden. Das Signal dieses Wasserstoffatoms befindet sich bei einer chemischen Verschiebung von $\delta=3.02 \mathrm{ppm}$ und erscheint ebenso wie das Signal des benachbarten Wasserstoffatoms $3 \mathrm{a}^{\prime}-\mathrm{H}$ in Form eines zentrierten Multipletts. Die transVerknüpfung dieser beiden Wasserstoffatome sowie die cis-Ständigkeit von 3a'-H und 9b'-H konnte durch NOE-Experimente bestätigt werden. Etwas weiter tieffeldverschoben bei $\delta=3.16 \mathrm{ppm}$ befindet sich das Signal des 4"'-H in Form eines Pseudotripletts mit der Kopplungskonstante von $J=9.4 \mathrm{~Hz}$. Dieser Wert ist charakteristisch für die beiden, in diesem Fall identischen, axial-axial-Kopplungen zu den benachbarten axialen Wasserstoffatomen.

Die drei Methoxygruppen der Rhamnoseeinheit zeigen eine charakteristische chemische Verschiebung von $\delta=3.51,3.53$ und $3.54 \mathrm{ppm}$ und ergeben je ein Signal in Form eines Singuletts mit der Intensität von drei. In dem Bereich von $\delta=3.60-3.66 \mathrm{ppm}$ erscheint das Signal des 5"'-H ein Form eines schlecht aufgelösten Multipletts. Innerhalb dieses Multipletts befindet sich mit einer chemischen Verschiebung von $\delta=3.64 \mathrm{ppm}$ das Signal des 3 "'-H in Form eines Dubletts vom Dublett mit den charakteristischen Kopplungskonstanten von $J=9.4 \mathrm{~Hz}$ (axial-axial) und $J=3.3 \mathrm{~Hz}$ (axial-äquatorial). Das Signal bei $\delta=3.71 \mathrm{ppm}$ erscheint ebenfalls in Form eines Dubletts vom Dublett mit den Kopplungskonstanten $J=3.3$ (axial-äquatorial) und $1.9 \mathrm{~Hz}$ (äquatorial-äquatorial) und ist dem Wasserstoffatom 2"'-H zuzuordnen. Bei einer Resonanzfrequenz von $\delta=4.05 \mathrm{ppm}$ ist ein zentriertes Multiplett zu finden, dass dem in benzylischer Position vorliegenden Wasserstoffatom 9b'-H zuzuordnen ist. Neben weiteren schlecht aufgelösten Kopplungen ist in diesem Multiplett auch ein Dublett mit der Kopplungskonstante von $J=9.5 \mathrm{~Hz}$ zu finden, das höchstwahrscheinlich auf die Kopplung mit dem cis-ständigen 3a'-H zurückzuführen ist. Die beiden Wasserstoffatome $1 "-\mathrm{H}_{2}$ des Allylalkohols resonieren bei $\delta=4.15 \mathrm{ppm}$ als Singulett. 
Weiter ins Tieffeld verschoben bei $\delta=5.47 \mathrm{ppm}$ befindet sich das Signal des Wasserstoffatoms 1"'-H am anomeren Zentrum der Rhamnoseeinheit. Die starke Tieffeldverschiebung und die Kopplungskonstante von $J=1.9 \mathrm{~Hz}$ (äquatorial-äquatorial) bestätigen die $\alpha$ Verknüpfung des Glycosids. Bei einer chemischen Verschiebung von $\delta=5.52 \mathrm{ppm}$ befindet sich das Signal des $\mathrm{sp}^{2}$-hybridisierten Wasserstoffatoms 1'-H in Form eines zentrierten Multipletts. Die beiden anderen olefinischen Wasserstoffatome resonieren bei $\delta=5.76 \mathrm{ppm}$ (4'-H) und $\delta=6.23$ ppm (5'-H) als Dublett vom Dublett mit einer gemeinsamen Kopplungskonstante von $J=9.8 \mathrm{~Hz}$ sowie der ${ }^{3} J$-Kopplungskonstante von $J=3.4 \mathrm{~Hz}\left(4^{\prime}-\mathrm{H}\right)$ und der ${ }^{4} J$-Kopplungskonstante von $J=2.0 \mathrm{~Hz}\left(5^{\prime}-\mathrm{H}\right)$. Aufgrund des Einflusses des benachbarten aromatischen $\pi$-Systems ist das Signal für das 5 '-H weiter ins Tieffeld verschoben.

Im aromatischen Bereich des Spektrums resonieren die Wasserstoffatome 6'-H ( $\delta=6.89 \mathrm{ppm})$ und 9'-H $(\delta=7.00 \mathrm{ppm})$ als zwei Dubletts mit den Kopplungskonstanten von $J=2.6 \mathrm{~Hz}$ (6'-H) sowie $J=8.3 \mathrm{~Hz}\left(9^{\prime}-\mathrm{H}\right)$. Das Wasserstoffatom 8'-H zeigt bei $\delta=6.83 \mathrm{ppm}$ ein Signal in Form eines Dubletts vom Dublett mit den beiden oben genannten Kopplungskonstanten.

Das ${ }^{13} \mathrm{C}$-NMR-Spektrum wurde bei Raumtemperatur in deuteriertem Chloroform bei einer Frequenz von $126 \mathrm{MHz}$ aufgenommen und ist in Abbildung 55 dargestellt.

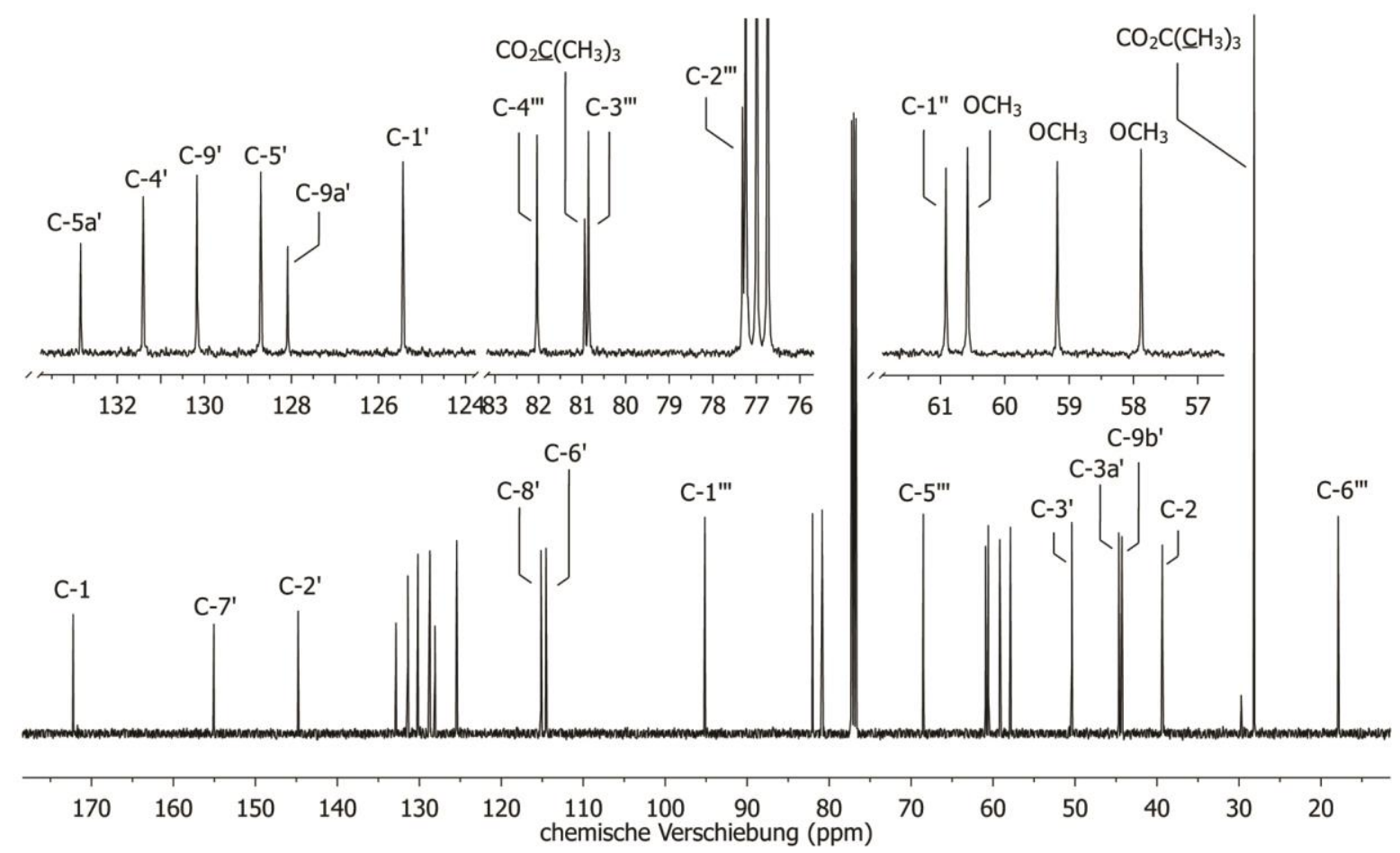

Abbildung 55: ${ }^{13} \mathrm{C}-\mathrm{NMR}$-Spektrum von Verbindung 186. 
Im Hochfeldbereich des Spektrums sind erwartungsgemäß das Signal der Methylgruppe C-6"' bei $\delta=17.9 \mathrm{ppm}$ und das Signal der $\mathrm{CH}_{3}$-Einheiten des tert-Butylesters bei $\delta=28.1 \mathrm{ppm} \mathrm{zu}$ finden. Darüber hinaus resoniert das Kohlenstoffatom C-2 ebenfalls im Hochfeld bei $\delta=39.3$ ppm. Die Signale der tertiären Kohlenstoffatome, die die Verknüpfung des B- und C-Ringes bilden, weisen eine sehr ähnliche chemische Verschiebung mit $\delta=44.3 \mathrm{ppm}$ (C-9b') und $\delta=44.7$ ppm (C-3a') auf, während das Kohlenstoffatom C-3', welches das dritte stereogene Zentrum im tricyclischen Grundgerüst bildet, eine chemische Verschiebung von $\delta=50.4 \mathrm{ppm}$ zeigt. Neben den drei Methoxygruppen bei $\delta=57.9,59.2$ und $60.9 \mathrm{ppm}$ resoniert auch das Kohlenstoffatom an C-1" bei $\delta=60.6 \mathrm{ppm}$. Die Signale der Ringkohlenstoffatome der Rhamnoseeinheit sind bei $\delta=68.5$ (C-5'"'), 77.3 (C-2'"), 80.9 (C-3"'), 82.0 (C-4"') und $95.1 \mathrm{ppm}\left(\mathrm{C}-1^{\prime \prime \prime}\right)$ zu finden, wobei das Kohlenstoffatom des anomeren Zentrum erwartungsgemäß am weitesten tieffeldverschoben ist. In diesem Bereich des Spektrums ist ebenfalls das charakteristische Signal des quartären Kohlenstoffatoms des tert-Butylesters bei $\delta=80.9$ ppm zu beobachten.

Im aromatischen und olefinischen Bereich des Spektrums resonieren die Kohlenstoffatome in ortho-Stellung $\mathrm{zu}$ der glycosidischen Bindung am weitesten hochfeldverschoben bei $\delta=114.6 \mathrm{ppm}\left(\mathrm{C}-6^{\prime}\right)$ und $\delta=115.1 \mathrm{ppm}\left(\mathrm{C}-8^{\prime}\right)$. Darüber hinaus sind die Signale der tertiären Kohlenstoffatome C-1' $(\delta=125.4$ ppm $), C^{\prime}-5^{\prime}(\delta=128.7$ ppm $), C^{\prime}-9^{\prime}(\delta=130.2$ ppm) und C-4' $(\delta=131.4 \mathrm{ppm})$ sowie die quartären Kohlenstoffatome C-9a' $(\delta=128.1 \mathrm{ppm})$, und C-5a' $(\delta=132.9 \mathrm{ppm})$ in diesem Bereich zu finden. Etwas weiter tieffeldverschoben resonieren die Kohlenstoffatome C-2' bei $\delta=144.8 \mathrm{ppm}$ und C-7' bei $\delta=155.0 \mathrm{ppm}$. Die größte Entschirmung zeigt erwartungsgemäß das Carbonyl-Kohlenstoffatom C-1 bei einer chemischen Verschiebung von $\delta=172.2 \mathrm{ppm}$.

Das ESI-Spektrum der Verbindung weist im positiven Modus neben dem Basispeak bei dem Masse-zu-Ladungs-Verhältnis $m / z=1055.6$ für das $[2 \mathrm{M}+\mathrm{Na}]^{+}$-Addukt einen Peak für das erwartungsgemäße Konjugat $[\mathrm{M}+\mathrm{Na}]^{+}(\mathrm{m} / z=539.3)$ auf. Die Summenformel $\mathrm{C}_{29} \mathrm{H}_{40} \mathrm{O}_{8}$ mit der berechneten exakten Masse von $m / z=539.2615$ für das $[\mathrm{M}+\mathrm{Na}]^{+}$-Addukt wurde überdies durch ESI-HRMS mit einer gefundenen Masse von $\mathrm{m} / \mathrm{z}=539.2618$ bestätigt.

\subsection{Synthese von Seitenketten des Typs 150}

Als besondere synthetische Herausforderungen in der Synthese der Seitenkette gelten die selektive Einführung des stereogenen Zentrums und die $\beta$-selektive Glycosylierung. Während bei der Kupplung mit der Forosamineinheit von vorangegangenen Arbeiten zu 
Glycosylierungsmethoden ${ }^{107}$ profitiert werden konnte, sollte die Einführung des stereogenen Zentrums über eine selektive $\alpha$-Oxidation nach MacMillan erfolgen. ${ }^{116}$

\subsubsection{Untersuchungen zur $\alpha$-Oxidation nach MacMillan}

Der Vorläufer für die $\alpha$-Oxidation wurde ausgehend von kommerziell erhältlichem 1,6-Hexandiol (187) hergestellt. Im ersten Schritt erfolgte die selektive mono-Schützung einer Hydroxyfunktion mit MEM-Chlorid und Hünig-Base in Dichlormethan bei Raumtemperatur in 51\% Ausbeute (Abbildung 56). Neben dem gewünschten Produkt konnten auch nicht umgesetztes Startmaterial und zweifach geschütztes Diol isoliert werden, welches durch Entschützung in nahezu quantitativer Ausbeute wieder in das Startmaterial überführt wurde.

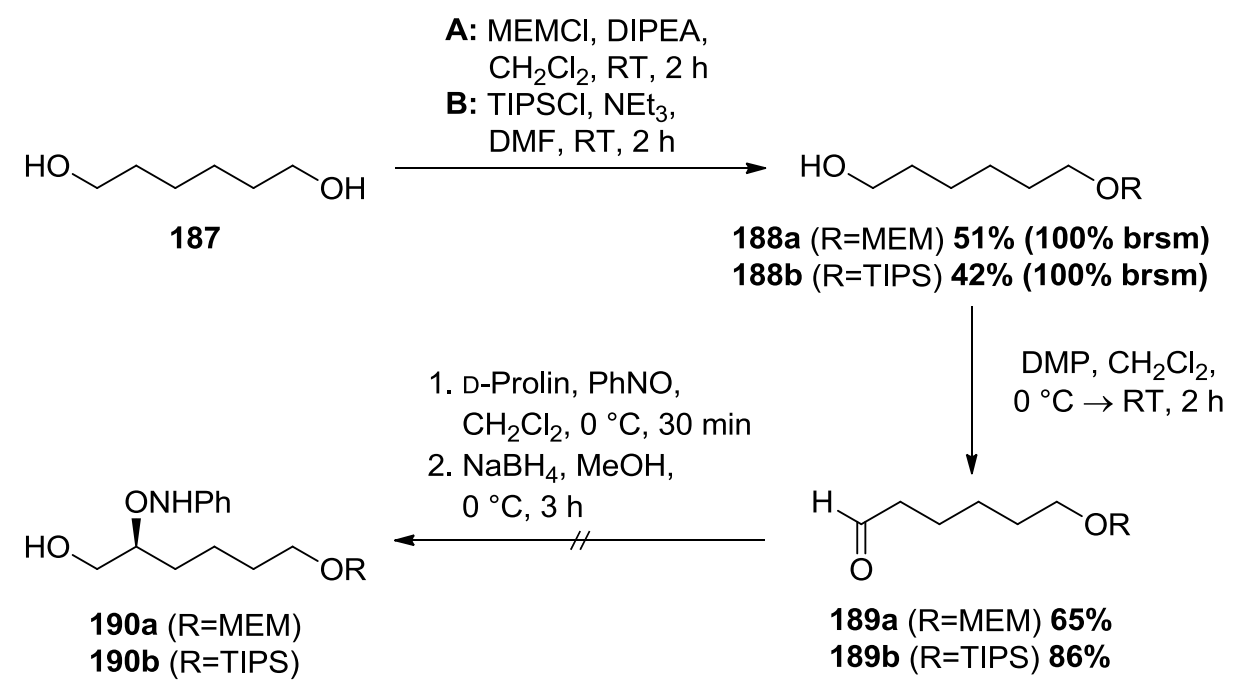

Abbildung 56: Untersuchungen zur $\alpha$-Oxidation nach MacMillan.

Im zweiten Schritt wurde die freie Alkoholfunktionalität unter Verwendung von Dess-MartinPeriodinan in Dichlormethan zu dem korrespondierenden Aldehyd oxidiert. Obwohl die $\alpha$-Oxidation in der Literatur bei ähnlichen Substraten hervorragende Ausbeuten und Enantioselektivitäten von bis zu 99\% lieferte, ${ }^{117}$ führte die organokatalysierte Umsetzung des Aldehyds 189a lediglich zu einer Zersetzung des Startmaterials und nicht trennbaren Produktgemischen. Auch die Verwendung des weniger koordinativen TIPS-geschützten Aldehyds 189b, der analog zu 189a in zwei Stufen ausgehend von Diol 187 in 36\% Ausbeute synthetisiert wurde, führte nicht zum gewünschten Produkt. Die Einführung des stereogenen Zentrums über eine enantioselektive Oxidation wurde daher verworfen.

\subsubsection{Synthese des Benzoyl-geschützten Glycosylakzeptors 194}

Als alternative Syntheseroute für die Einführung des stereogenen Zentrums wurde die asymmetrische Sharpless-Dihydroxylierung gewählt. Um die Enantioselektivität der Reaktion 
mit Hilfe analytischer HPLC an chiraler Phase zu überprüfen und gegebenenfalls eine präparative Trennung der Enantiomere zu ermöglichen, wurde zunächst die chromophore Benzoyl-Schutzgruppe eingeführt.

Ausgehend von dem kommerziell erhältlichem Hexenol 191 wurde unter Zugabe von Benzoesäureanhydrid, katalytischen Mengen an 4-Dimethylaminopyridin und Kaliumcarbonat als Base der Benzoyl-geschützte Alkohol 192 in quantitativer Ausbeute erhalten (Abbildung 57).
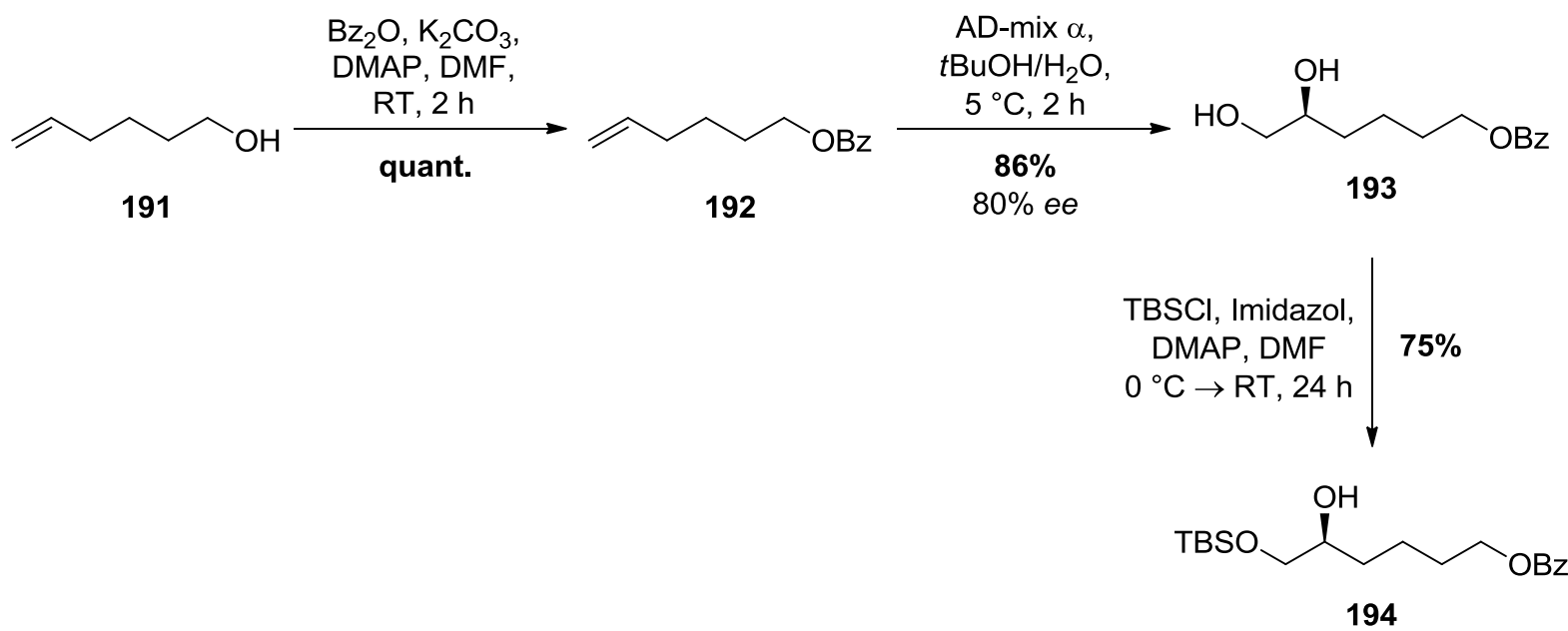

Abbildung 57: Synthese des Benzoyl-geschützten Glycosylakzeptors 194.

Anschließende Sharpless-Dihydroxylierung mit AD-mix $\alpha$ in einem tert-Butanol-WasserGemisch lieferte bei $5{ }^{\circ} \mathrm{C}$ das Diol 193 in einer Ausbeute von 86\%. ${ }^{118}$ Die Enantioselektivität der Reaktion wurde anhand von Verbindung 193 über analytische HPLC an chiraler Phase bestimmt. Obwohl die Trennleistung für eine Bestimmung des $e e$-Wertes $(80 \%$ ee $)$ ausreichend war, zeigte sich eine präparative Trennung der Enantiomere auf dieser Stufe als nicht umsetzbar.

Um eine selektive Glycosylierung an der sekundären Hydroxyfunktion des Diols 193 zu verwirklichen, wurde zunächst die primäre Hydroxyfunktion mit TBS-Chlorid, katalytischen Mengen an 4-Dimethylaminopyridin und Imidazol als Base in $75 \%$ Ausbeute in den resultierenden Silylether 194 überführt. Da auch auf dieser Stufe eine präparative Anreicherung des benötigten Enantiomers über HPLC nicht möglich war, wurden im Folgenden Versuche unternommen, um die Bereitstellung der enantiomerenreinen Verbindung mittels Kristallisation zu erreichen. 


\subsubsection{Untersuchungen zur Enantiomerenanreicherung über Kristallisation}

Da sämtliche Kristallisationsversuche des Diols 193 sowie Verbindung 194 erfolglos blieben, wurden zur Erleichterung der Kristallisation Nitrophenolsubstituenten eingeführt. Ausgehend von Diol 193 erfolgte die Synthese der Nitroverbindung 195 durch die Zugabe von p-Nitrobenzaldehyd und katalytischen Mengen an para-Toluolsulfonsäure in $87 \%$ Ausbeute (Abbildung 58). Die doppelte Veresterung der Hydroxyfunktionen mit DNB-Chlorid, Triethylamin und katalytischen Mengen an 4-Dimethylaminopyridin in Dichlormethan lieferte hingegen Verbindung 196 in 64\% Ausbeute.

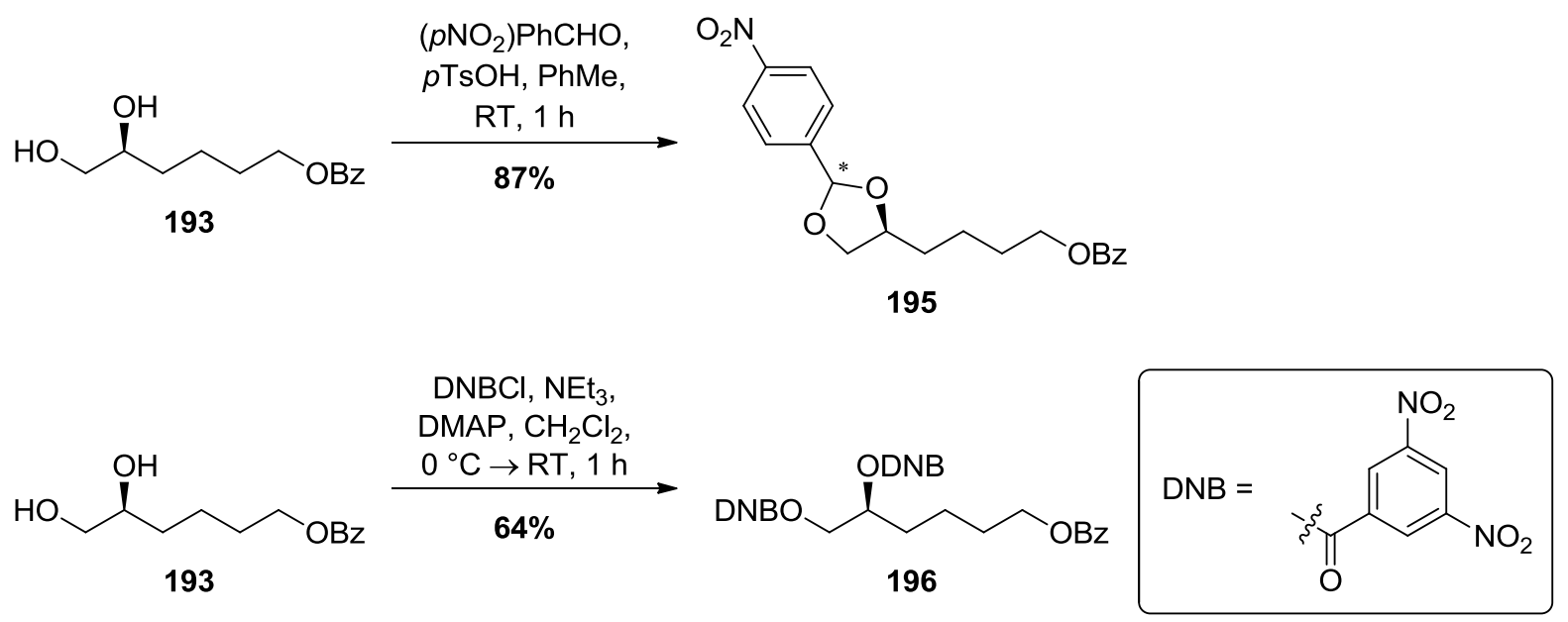

Abbildung 58: Synthese der Nitrophenolverbindungen 195 und 196.

Entgegen der Erwartungen konnten allerdings weder das Acetal 195 noch das Bisdinitrobenzoat 196 in eine kristalline Form überführt werden, so dass eine Trennung der Enantiomere über Kristallisation nicht verwirklicht werden konnte.

\subsubsection{Glycosylierung von Verbindung 194}

Da sowohl die Enantiomerenanreicherung der ungeschützten sowie geschützten Diole 193, 194, 195 und 196 fehlschlug, wurde die Glycosylierung zunächst an der Verbindung 194 mit einem Enantiomerenüberschuss von $86 \%$ getestet. Zur Vorbereitung der Glycosylierungsreaktion wurde die Azidoverbindung 158 mit einem Überschuss an Trichloracetonitril und Caesiumcarbonat in Dichlormethan umgesetzt (Abbildung 59). Das entstandene Trichloracetimidat 197 wurde nach Filtration der Base in quantitativer Ausbeute als Gemisch der Anomere $(\alpha / \beta \approx 1: 9)$ isoliert und ohne weitere Aufarbeitung in der nachfolgenden Glycosylierung verwendet.

In vorangegangenen Arbeiten zur Untersuchung der Glycosylierungsreaktion hatte sich die Azidoverbindung 158 als am besten geeigneter Glycosyldonor herausgestellt. ${ }^{107}$ Über die 
Azid-Funktion an C-4 besteht überdies die Möglichkeit zu einem beliebigen späteren Zeitpunkt eine Vielzahl an Derivaten bereitzustellen oder auf kurzem Weg die für das Spinosyn-Analogon 148 benötigte Dimethylaminofunktion der D-Forosamineinheit zu installieren.

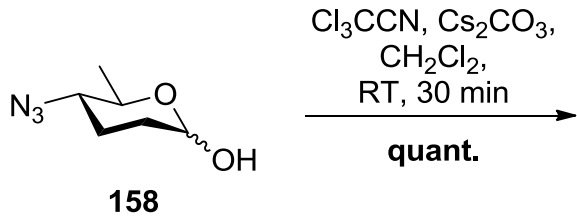

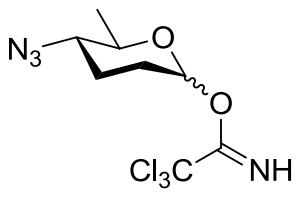
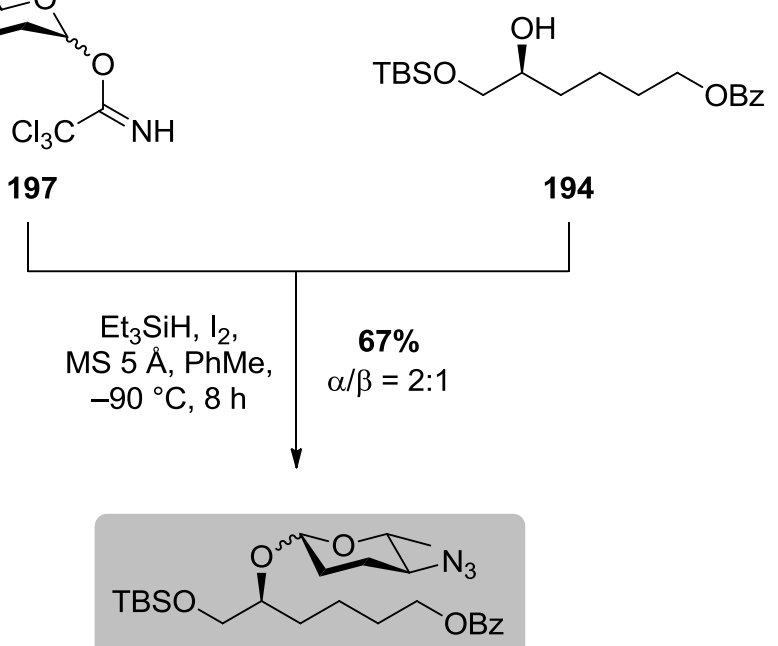

$(\alpha / \beta)-198$

Abbildung 59: Glycosylierung von Verbindung 194.

Die Glycosylierung erfolgte unter Verwendung von Triethylsilan und elementarem Iod bei einer Temperatur von $-90{ }^{\circ} \mathrm{C}$. Es wurde ein 2:1-Gemisch der Anomere $\alpha-198$ und $\beta-198$ in 67\% Ausbeute erhalten. Da sämtliche Versuche zur Trennung der Anomere fehlschlugen, wurde die Synthese einer Seitenkette mit alternativer Schutzgruppe mit Kristallisationspotential angestrebt.

\subsubsection{Synthese des DNB-geschützten Glycosylakzeptors 201}

Um trotz der bisherigen Probleme den Zugang $\mathrm{zu}$ enantiomerenreinem Alkohol zu verwirklichen, wurde im Folgenden die chromophore Benzoyl-Schutzgruppe durch die zur Kristallisation neigende Dinitrobenzoyl-Schutzgruppe ersetzt. Eine Einbringung der Schutzgruppe an dem Hexenol 191 konnte durch Einsatz von DNB-Chlorid, katalytischen Mengen an 4-Dimethylaminopyridin und Triethylamin als Base in Dichlormethan realisiert werden. Die weitere Oxidation der in quantitativer Ausbeute erhaltenen Verbindung 199 wurde analog zur Sharpless-Dihydroxylierung von Alken 192 durchgeführt und lieferte unter vollständigem Umsatz das Diol 200 (Abbildung 60). Da auch diese Verbindung nicht in ihre kristalline Form überführt werden konnte, erfolgte im Anschluss die selektive TBS-Schützung der primären Hydroxyfunktion entsprechend der Benzoyl-geschützen Verbindung. 
Erfreulicherweise war der TBS-Ether 201 kristallin und wurde in der Folge bis zur Einstellung eines konstanten Drehwertes umkristallisiert. Das reine $(S)$-Enantiomer konnte so in einer Ausbeute von 65\% erhalten werden. Der exzellente Enantiomerenüberschuss von $>99 \%$ wurde nach zweistufiger Überführung der Verbindung in das Benzoyl-geschützte Diol 193 mittels analytischer HPLC ermittelt.
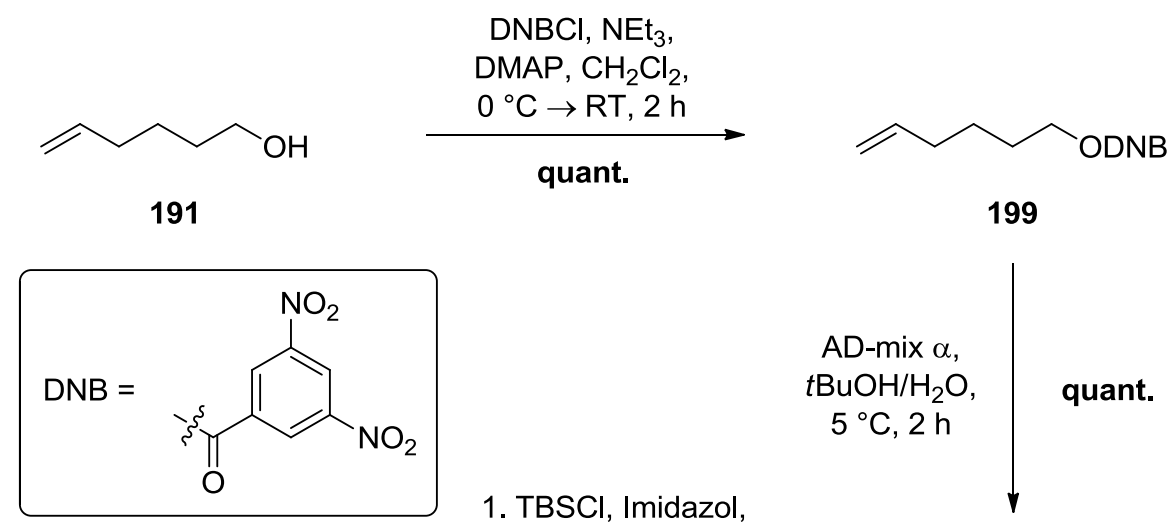

1. TBSCI, Imidazol,
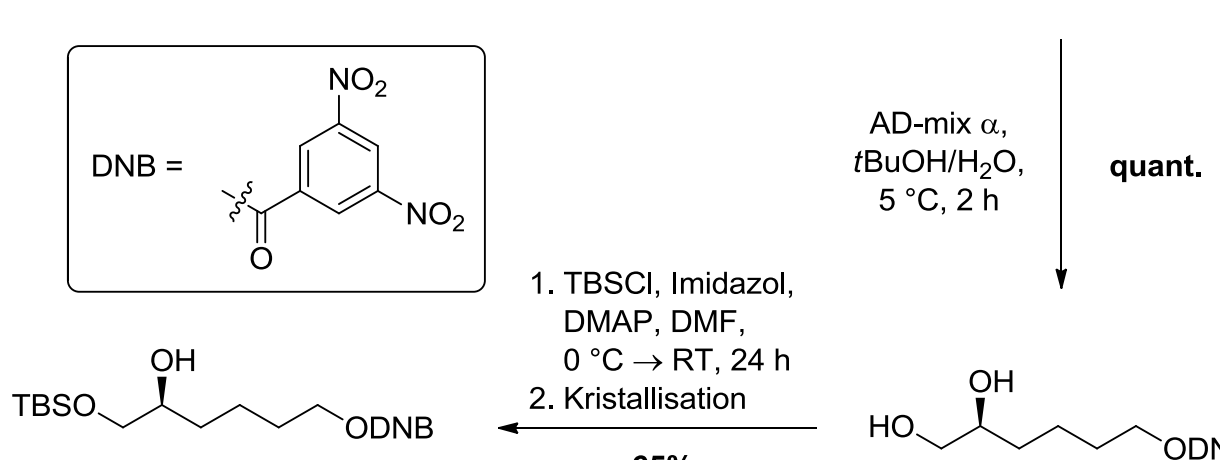

DMAP, DMF,

$0^{\circ} \mathrm{C} \rightarrow \mathrm{RT}, 24 \mathrm{~h}$

201

2. Kristallisation

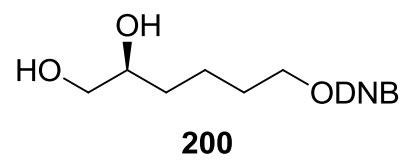

$>99 \%$ ee

$65 \%$

200

Abbildung 60: Synthese des enantiomerenreinen DNB-geschützten Glycosylakzeptors 201.

\subsubsection{Glycosylierung von Verbindung 201}

Die Glycosylierung der enantiomerenreinen Verbindung 201 mit dem Azid 197 erfolgte unter den gleichen Bedingungen wie bei dem Benzoyl-geschützten Glycosylakzeptor 194 (Abbildung 61).

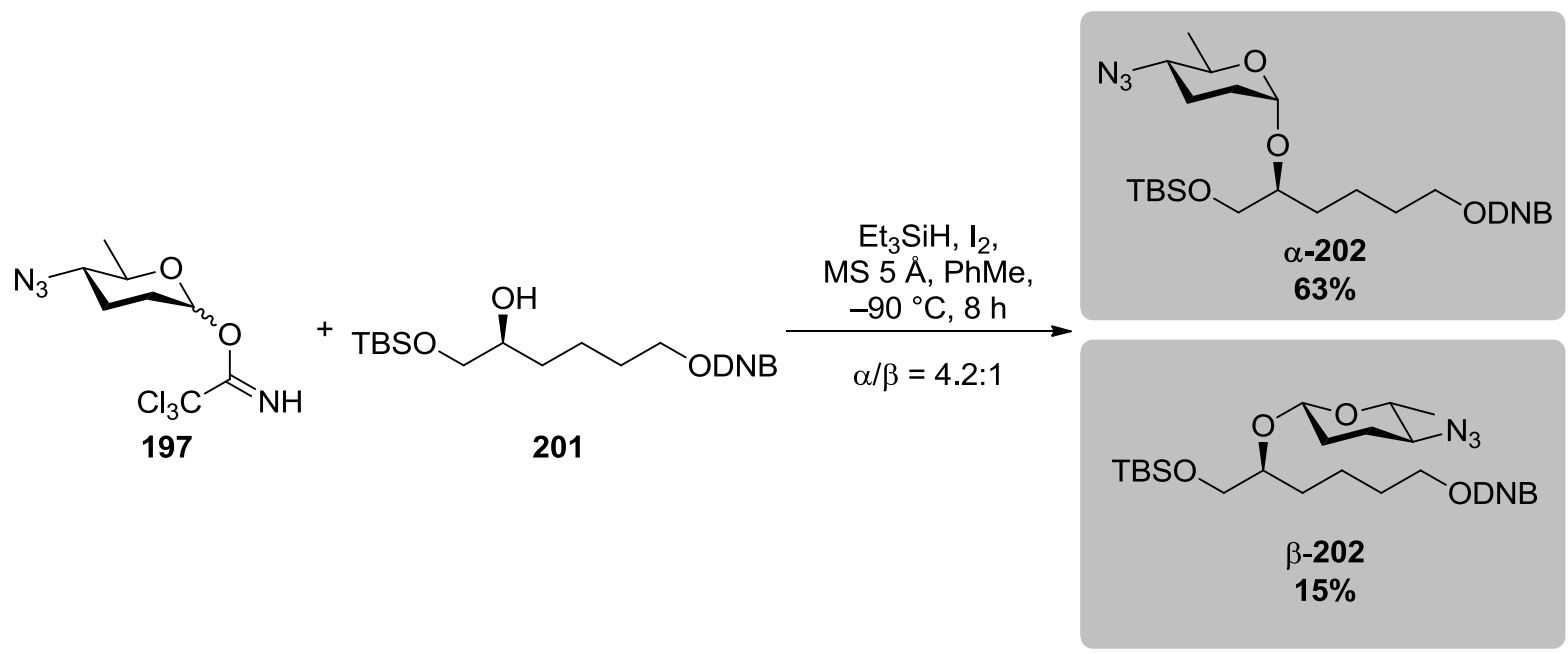

Abbildung 61: Glycosylierung von Verbindung 201. 
Während bei der vorangegangenen Kupplung der Forosamineinheit ein untrennbares Produktgemisch der beiden Anomere entstand, wurden in diesem Fall sowohl $\alpha$-Anomer als auch $\beta$-Anomer in ihrer reinen Form isoliert. Die Gesamtausbeute konnte sogar auf sehr gute $78 \%$ gesteigert werden, allerdings erfolgte die Reaktion unter einer wesentlich schlechteren $\alpha / \beta$-Selektivität. So wurden lediglich $15 \%$ des gewünschten $\beta$-Anomers erhalten. Unter Berücksichtigung der Tatsache, dass diese Reaktion nur in kleinem Maßstab $(<500 \mu \mathrm{mol})$ akzeptable Ausbeuten und Selektivitäten liefert, ist eine isolierte Ausbeute des gewünschten Produktes von $15 \%$ für die weitere Synthese von Spinosyn-Analoga nicht zufriedenstellend.

Da der Schutzgruppenwechsel zu einer signifikanten Änderung der $\alpha / \beta$-Selektivität während der Glycosylierung geführt hat, wurde ausgehend von dem enantiomerenreinen $\mathrm{C}_{6}$-Baustein 201 die Synthese eines MEM-geschützten Glycosylakzeptors durchgeführt.

\subsubsection{Synthese des MEM-geschützten Glycosyldonors 204}

Das enantiomerenreine Dinitrobenzoat 201 wurde zunächst bei Raumtemperatur mit Lithiumhydroxid in einem Dichlormethan-Methanol-Wasser-Gemisch in quantitativer Ausbeute hydrolysiert (Abbildung 62). Eine anschließende selektive Schützung der primären Hydroxyfunktion mit MEM-Chlorid und Hünig-Base in Dichlormethan führte in 65\% Ausbeute zu dem Glycosylakzeptor 204.

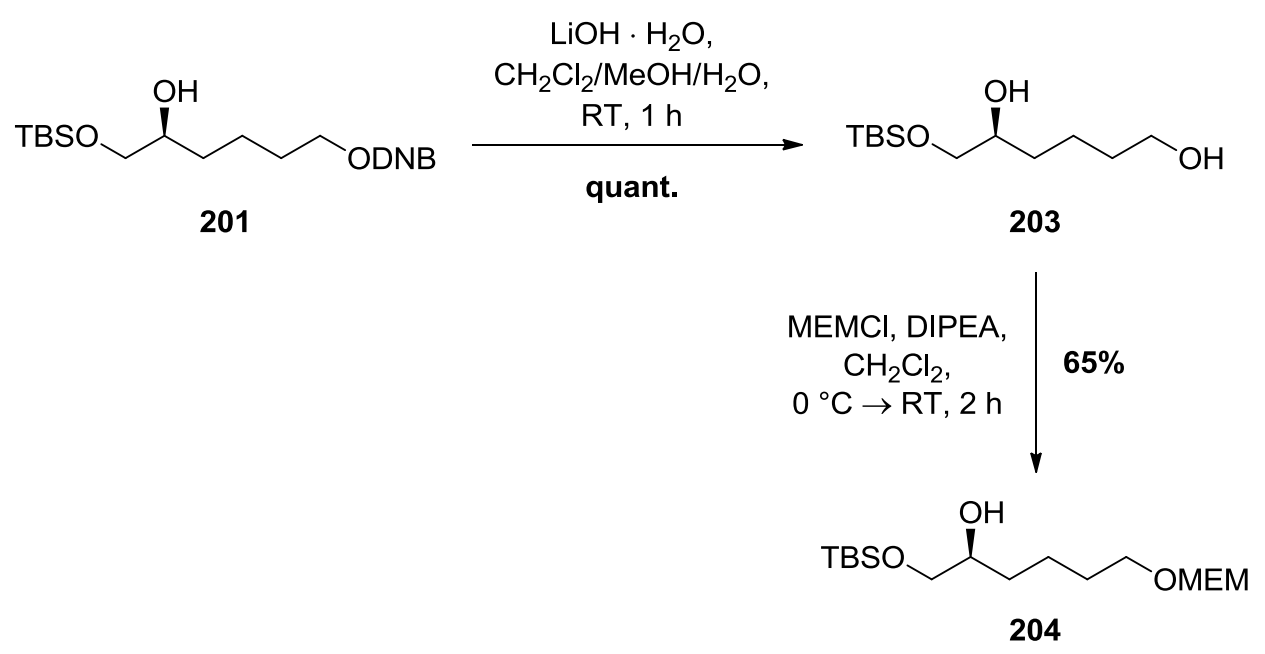

Abbildung 62: Synthese des MEM-geschützten Glycosyldonors 204.

\subsubsection{Glycosylierung von Verbindung 204}

Die Glycosylierung der enantiomerenreinen Verbindung 204 wurde analog der bisherigen Transformationen durchgeführt. Wie bei dem DNB-geschützten Glycosylakzeptor war auch in diesem Fall die separate Isolierung beider gebildeter Anomere möglich. Entgegen der bisher durchgeführten Glycosylierungen wurde in dieser Reaktion jedoch bevorzugt das 
gewünschte $\beta$-Anomer in $41 \%$ Ausbeute gebildet (Abbildung 63). Für die weitere Synthese von Spinosyn-Analoga des Typs 148 scheint das MEM-geschützte Kupplungsprodukt 205 folglich die am besten geeignete Verbindung zu sein.

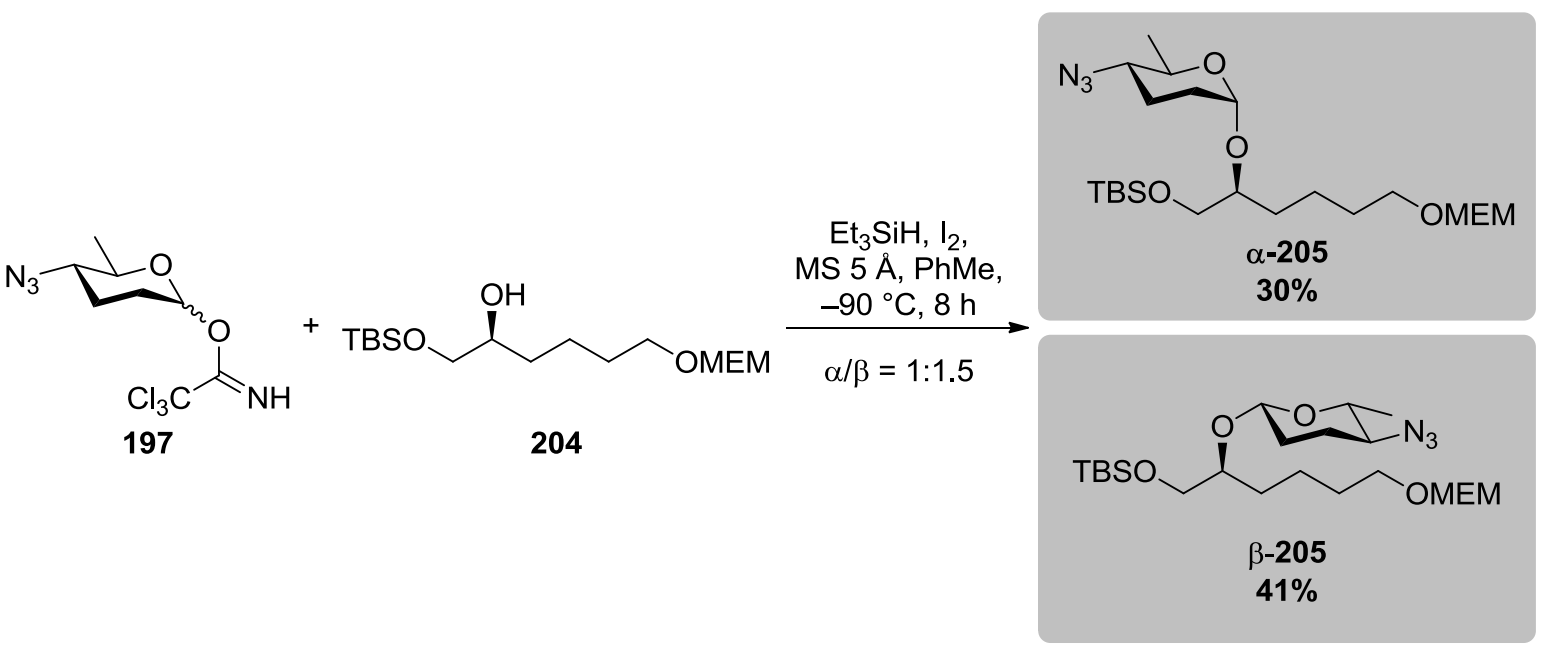

Abbildung 63: Glycosylierung von Verbindung 205.

\subsubsection{Optimierung des Synthesewegs zum Glycosylakzeptor 204}

Um einen besseren synthetischen Zugang zu dem Glycosylakzeptor 204 zu erlangen, wurde im Folgenden eine Optimierung des Synthesewegs durchgeführt. Zunächst wurde ausgehend von dem kommerziell erhältlichen Hexenol 191 mit MEM-Chlorid und Hünig-Base in Dichlormethan der MEM-Ether 206 hergestellt (Abbildung 64).

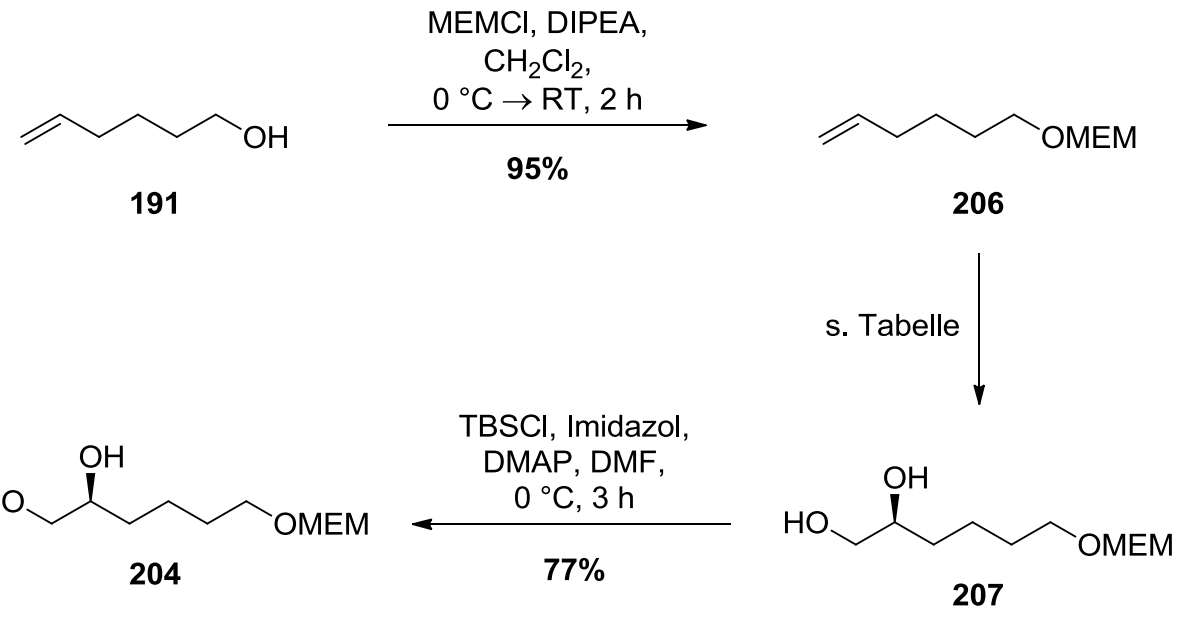

Abbildung 64: Verbesserter Syntheseweg zum enantiomerenreinen Glycosylakzeptor 204.

Zur Vermeidung des Umwegs über die zu Substanzverlust führende Kristallisation und den anschließenden Schutzgruppenwechsel, war eine Optimierung der Sharpless-Dihydroxylierung unabdingbar. Die Reaktion wurde ursprünglich mit kommerziellem AD-mix $\alpha$, einem 
Gemisch aus Kaliumcarbonat, Kaliumosmat, rotem Blutlaugensalz und dem chiralen Liganden 209, durchgeführt. In Studien zu dieser asymmetrisch verlaufenden Reaktion wurde gezeigt, dass der Substituent an 9-O des Dihydrochinins einen erheblichen Einfluss auf die Stereoselektivität hat (vgl. Abbildung 65).

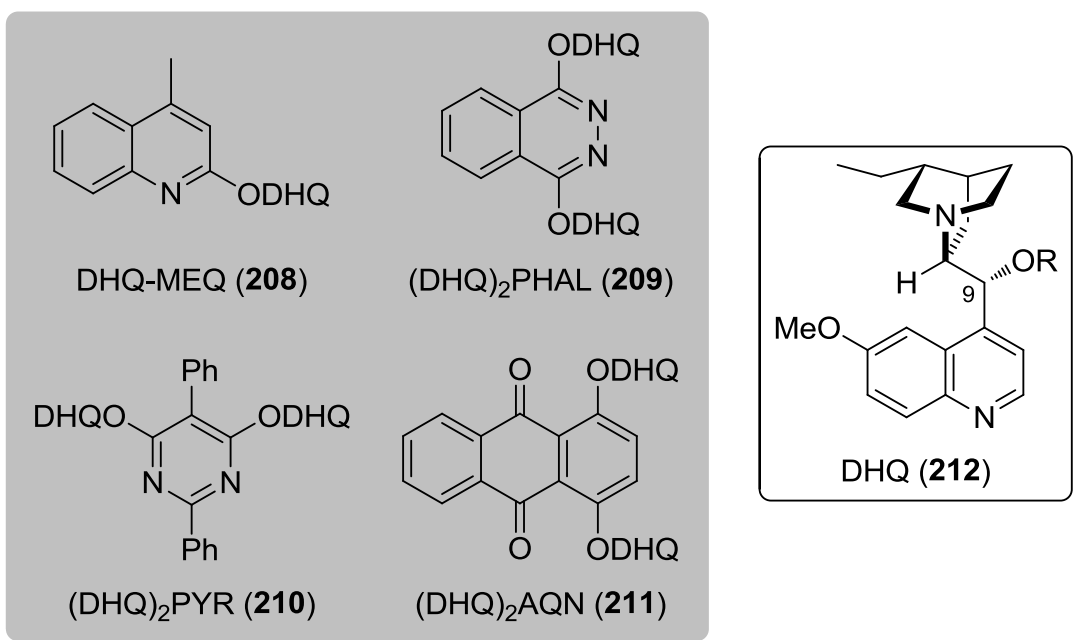

Abbildung 65: Liganden für die asymmetrische Sharpless-Dihydroxylierung.

Die erste Umsetzung mit kommerziell erhältlichem AD-mix $\alpha$ lieferte bei $5{ }^{\circ} \mathrm{C}$ das Diol 207 in quantitativer Ausbeute und einer Selektivität von 81:19 (Tabelle 4, Eintrag 1).

Tabelle 4: Sharpless Dihydroxylierung des MEM-Ethers 206.

\begin{tabular}{|c|c|c|c|}
\hline Eintrag & Reaktionsbedingungen & Ausbeute & $S / R$ \\
\hline 1 & AD-mix $\alpha, t \mathrm{BuOH} / \mathrm{H}_{2} \mathrm{O} 1: 1,5^{\circ} \mathrm{C}, 3 \mathrm{~h}$ & quant. & $81: 19$ \\
\hline 2 & AD-mix $\alpha, t \mathrm{BuOH} / \mathrm{H}_{2} \mathrm{O} 1: 1,0{ }^{\circ} \mathrm{C}, 3 \mathrm{~h}$ & quant. & $85: 15$ \\
\hline 3 & $\begin{array}{l}\mathrm{K}_{2} \mathrm{OsO}_{4} \cdot 2 \mathrm{H}_{2} \mathrm{O}, \text { DHQ-MEQ }(\mathbf{2 0 8}), \mathrm{K}_{3} \mathrm{Fe}(\mathrm{CN})_{6} \\
\mathrm{~K}_{2} \mathrm{CO}_{3}, t \mathrm{BuOH} / \mathrm{H}_{2} \mathrm{O} 1: 1,5{ }^{\circ} \mathrm{C}, 3 \mathrm{~h}\end{array}$ & quant. & $72: 28$ \\
\hline 4 & $\begin{array}{l}\mathrm{K}_{2} \mathrm{OsO}_{4} \cdot 2 \mathrm{H}_{2} \mathrm{O},(\mathrm{DHQ})_{2} \mathrm{PYR}(\mathbf{2 1 0}), \mathrm{K}_{3} \mathrm{Fe}(\mathrm{CN})_{6} \\
\mathrm{~K}_{2} \mathrm{CO}_{3}, t \mathrm{BuOH} / \mathrm{H}_{2} \mathrm{O} 1: 1,5{ }^{\circ} \mathrm{C}, 3 \mathrm{~h}\end{array}$ & quant. & $85: 15$ \\
\hline 5 & $\begin{array}{l}\mathrm{K}_{2} \mathrm{OsO}_{4} \cdot 2 \mathrm{H}_{2} \mathrm{O},(\mathrm{DHQ})_{2} \mathrm{AQN}(\mathbf{2 1 1}), \mathrm{K}_{3} \mathrm{Fe}(\mathrm{CN})_{6}, \\
\mathrm{~K}_{2} \mathrm{CO}_{3}, t \mathrm{BuOH} / \mathrm{H}_{2} \mathrm{O} 1: 1,5^{\circ} \mathrm{C}, 3 \mathrm{~h}\end{array}$ & quant. & $83: 17$ \\
\hline 6 & $\mathrm{AD}-\operatorname{mix} \alpha, t \mathrm{BuOH} / \mathrm{H}_{2} \mathrm{O} 1: 1,-10^{\circ} \mathrm{C}, 3 \mathrm{~h}$ & quant. & $>99: 1$ \\
\hline 7 & AD-mix $\alpha, t \mathrm{BuOH} / \mathrm{H}_{2} \mathrm{O} 1: 1,-10^{\circ} \mathrm{C}, 3 \mathrm{~h}$ & $84 \%{ }^{\mathrm{a}}$ & $>99: 1$ \\
\hline
\end{tabular}


Bei einer um $5^{\circ} \mathrm{C}$ erniedrigten Temperatur wurde eine verbesserte Selektivität von 85:15 bei gleichbleibender Ausbeute beobachtet (Tabelle 4, Eintrag 2). Um eine höhere Selektivität der asymmetrischen Umsetzung zu erlangen, wurden nachfolgend die Substituenten an 9-O des Dihydrochinins 212 verändert.

Für das Liganden-Screening wurden 5 mol\% des Osmium(VI)-Katalysators, 10 mol\% des chiralen Dihydrochinin-Liganden und stöchiometrische Mengen an Kaliumhexacyanoferrat(III) und Kaliumcarbonat (jeweils 6 Äq.) eingesetzt. Die Umsetzung mit einem Monomer-Liganden der ersten Generation, DHQ-MEQ (208), führte bei quantitativer Ausbeute zu einer reduzierten Selektivität von 72:28 für das gewünschte $(S)$-Enantiomer (Tabelle 4, Eintrag 3). Der dimere (DHQ) 2 PYR-Ligand (210), der bei monosubstituierten terminalen Olefinen oft der Ligand der Wahl ist, zeigte bei der Reaktion mit 85:15 die höchste Selektivität bei $5^{\circ} \mathrm{C}$ (Tabelle 4, Eintrag 4). Die Verwendung des (DHQ) 2 AQN-Liganden (211) führte lediglich $\mathrm{zu}$ einer leichten Verbesserung der Selektivität gegenüber dem kommerziell erhältlichen AD-mix $\alpha$ (Tabelle 4, Eintrag 5).

Da mit den verwendeten Ligandensystemen das gewünschte $(S)$-Diol 207 bei $0{ }^{\circ} \mathrm{C}$ und höheren Temperaturen nur in nicht zufriedenstellenden Selektivitäten gebildet wurde, wurde mit dem kommerziell erhältlichen AD-mix $\alpha$ eine Sharpless-Dihydroxylierung bei möglichst tiefer Temperatur durchgeführt. Durch die Verwendung einer hochkonzentrierten Reaktionslösung konnte die Umsetzung in dem Zwei-Phasen-Gemisch tert-Butanol/Wasser bei $-10{ }^{\circ} \mathrm{C}$ verwirklicht werden. Bei dieser Temperatur wurde ausschließlich die Bildung des gewünschten $(S)$-Enantiomers beobachtet (Tabelle 4, Eintrag 6).

Die Sharpless-Dihydroxylierung mit AD-mix $\alpha$ ließ sich zudem hochkonzentriert bei $-10{ }^{\circ} \mathrm{C}$ im Multigramm-Maßstab mit guten Ausbeuten von $84 \%$ bei gleichbleibenden Selektivitäten durchführen (Tabelle 4, Einträge 7).

Ausgehend von enantiomerenreinem Diol 207 erfolgte im Anschluss die selektive Schützung der primären Hydroxyfunktion mit TBS-Chlorid, Imidazol, katalytischen Mengen an 4-Dimethylaminopyridin und Dimethylformamid als Lösungsmittel in 77\% Ausbeute (vgl. Abbildung 64).

\subsubsection{Synthese der Seitenketten $\alpha-214$ und $\beta-214$}

Da die Kupplung der Seitenkette mit dem Tricyclus 149 über eine Lithium-vermittelte 1,2-Addition erfolgen sollte, galt es, die TBS-Ether $\alpha-205$ und $\beta$-205 im weiteren Verlauf der Synthese in ein Halogenid zu überführen. Um die Auswirkung der glycosidischen Bindung 
auf die biologische Aktivität von Spinosyn-Analoga zu beurteilen, wurden die nachfolgenden Reaktionen sowohl mit dem $\alpha$-als auch mit dem $\beta$-Anomer durchgeführt.

Zunächst erfolgte die Spaltung des Silylethers mit Tetrabutylammoniumfluorid in Tetrahydrofuran bei Raumtemperatur (Abbildung 66).

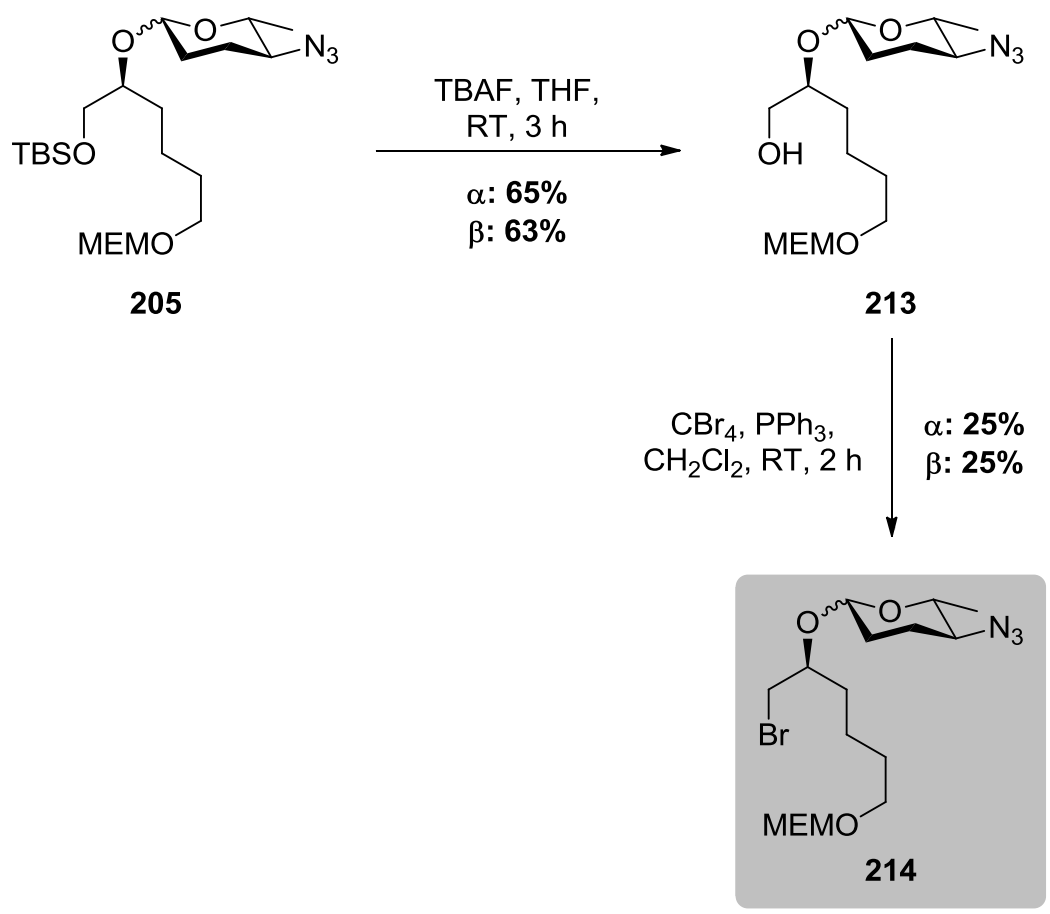

Abbildung 66: Synthese der Seitenketten $\alpha-214$ und $\beta-215$.

Die resultierenden primären Alkohole $\alpha-213$ und $\beta$-213 wurden in moderaten Ausbeuten von 65\% und 63\% isoliert und anschließend einer Appel-Reaktion unterzogen. Unter Verwendung der klassischen Bedingungen mit Tetrabrommethan und Triphenylphosphin in Dichlormethan konnten die beiden Bromide $\alpha-214$ und $\beta-214$ nur in geringer Ausbeute von 25\% erhalten werden.

Mit dem Abschluss der Synthese von Tricyclus 149 und Seitenkette 214 sah die weitere Synthesestrategie eine Lithium-vermittelte 1,2-Addition vor. Die Kupplung der beiden Bausteine wurde in einer separaten Arbeit untersucht und führte nicht zu dem gewünschten Kupplungsprodukt 215 (Abbildung 67). ${ }^{119}$ Statt des erhofften nucleophilen Angriffs der Seitenkette an die Aldehydfunktion wurde nur die intramolekulare Eliminierung des Substituenten am stereogenen Zentrum beobachtet. Da die geplante 1,2-Addition keine erfolgversprechenden Ergebnisse lieferte, wurden auch keine Optimierungen der letzten Synthesestufen zum Aufbau der Seitenkette 214 durchgeführt. 


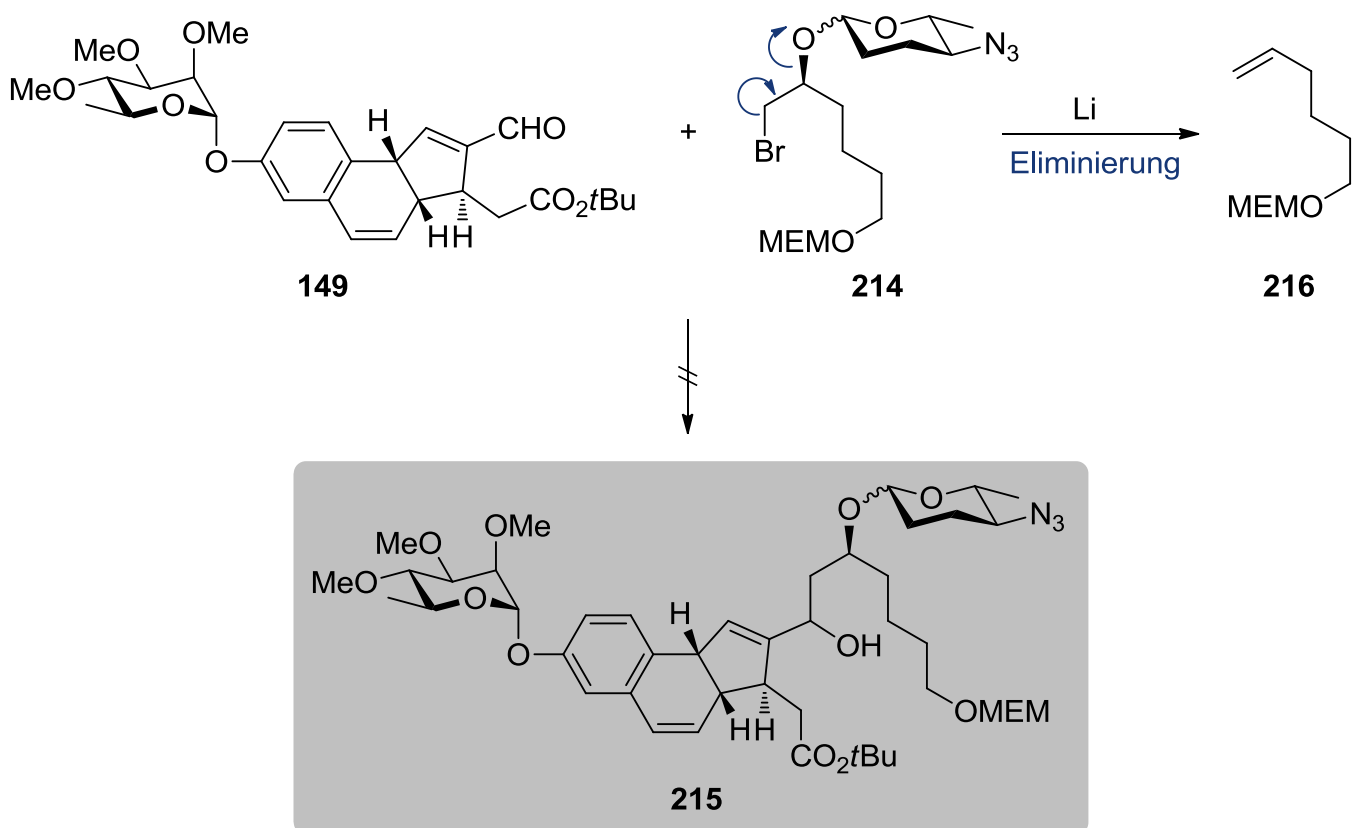

Abbildung 67: Kupplung von Tricyclus 149 und Seitenkette 214.

\subsubsection{Diskussion der spektroskopischen Daten von Verbindung 214}

Im Folgenden sollen einige ausgewählte spektroskopische Daten der Verbindung 214 näher diskutiert werden. Die genaue Zuordnung der Signale erfolgte über entsprechende zweidimensionale NMR-Spektren (COSY, HSQC und HMBC) sowie über die unterschiedlichen Intensitäten der Signale im ${ }^{13} \mathrm{C}-\mathrm{NMR}$. Das ${ }^{1} \mathrm{H}-\mathrm{NMR}$-Spektrum wurde bei Raumtemperatur in deuteriertem Chloroform bei einer Frequenz von $600 \mathrm{MHz}$ aufgenommen und ist in Abbildung 68 dargestellt.

Im aliphatischen Bereich des Spektrums resonieren die Wasserstoffatome der Methylgruppe an der Forosamineinheit erwartungsgemäß bei $\delta=1.28 \mathrm{ppm}$ in Form eines Dubletts mit der Kopplungskonstante von $J=6.2 \mathrm{~Hz}$. Die Wasserstoffatome $3-\mathrm{H}_{2}$ zeigen bei $\delta=1.38 \mathrm{ppm}$ ein Signal in Form eines zentrierten Multipletts, während die benachbarten Methylengruppen bei $\delta=1.58 \mathrm{ppm}\left(2-\mathrm{H}_{2}\right)$ und $\delta=1.63 \mathrm{ppm}\left(4-\mathrm{H}_{2}\right)$ jeweils als Dublett vom Triplett mit den Kopplungskonstanten $J=14.3$ und $7.0 \mathrm{~Hz}\left(2-\mathrm{H}_{2}\right)$ und $J=12.2$ und $6.1 \mathrm{~Hz}\left(4-\mathrm{H}_{2}\right)$ Resonanz zeigen. Die Signale der diastereotopen Wasserstoffatome der Forosamineinheit sind bei $\delta=1.69-1.77 \mathrm{ppm}\left(2^{\prime}-\mathrm{H}_{\mathrm{A}}\right), \delta=1.82-1.88 \mathrm{ppm}\left(2^{\prime}-\mathrm{H}_{\mathrm{B}}\right)$ und $\delta=1.87-1.93 \mathrm{ppm}\left(3^{\prime}-\mathrm{H}_{2}\right)$ als Multipletts zu finden. Einzig das Wasserstoffatom 4'- $\mathrm{H}_{2}$ zeigt ein als Dublett vom Triplett aufgelöstes Signal bei $\delta=2.97 \mathrm{ppm}$ mit den Kopplungskonstanten $J=9.8$ und $6.2 \mathrm{~Hz}$. Das Singulett bei der charakteristischen chemischen Verschiebung von $\delta=3.37 \mathrm{ppm}$ mit einem Integral von drei ist auf die Methoxygruppe der MEM-Schutzgruppe zurückzuführen. Die beiden Wasserstoffatome $6-\mathrm{H}_{2}$ zeigen bei $\delta=3.44-3.50 \mathrm{ppm}$ ein Signal in Form eines 
Multipletts, während die beiden Wasserstoffatome $1-\mathrm{H}_{2}$ in Form eines Tripletts mit einer Kopplungskonstante von $J=6.7 \mathrm{~Hz}$ resonieren. In diesem Bereich des Spektrums sind überdies die beiden Signale der Methylengruppen der MEM-Schutzgruppe mit charakteristischen chemischen Verschiebungen von $\delta=3.54 \mathrm{ppm}$ und $\delta=3.66 \mathrm{ppm} \mathrm{zu}$ finden. Beide Signale spalten in ein Triplett mit der Kopplungskonstante $J=4.8 \mathrm{~Hz}$ auf. Das Wasserstoffatom 5-H am stereogenen Zentrum resoniert bei $\delta=3.71 \mathrm{ppm}$ in Form eines Quintetts mit der Kopplungskonstante $J=5.5 \mathrm{~Hz}$, während das Signal des 5'-H bei $\delta=3.85 \mathrm{ppm}$ in ein Dublett vom Quartett mit den Kopplungskonstanten $J=9.9$ und $6.2 \mathrm{~Hz}$ aufspaltet.

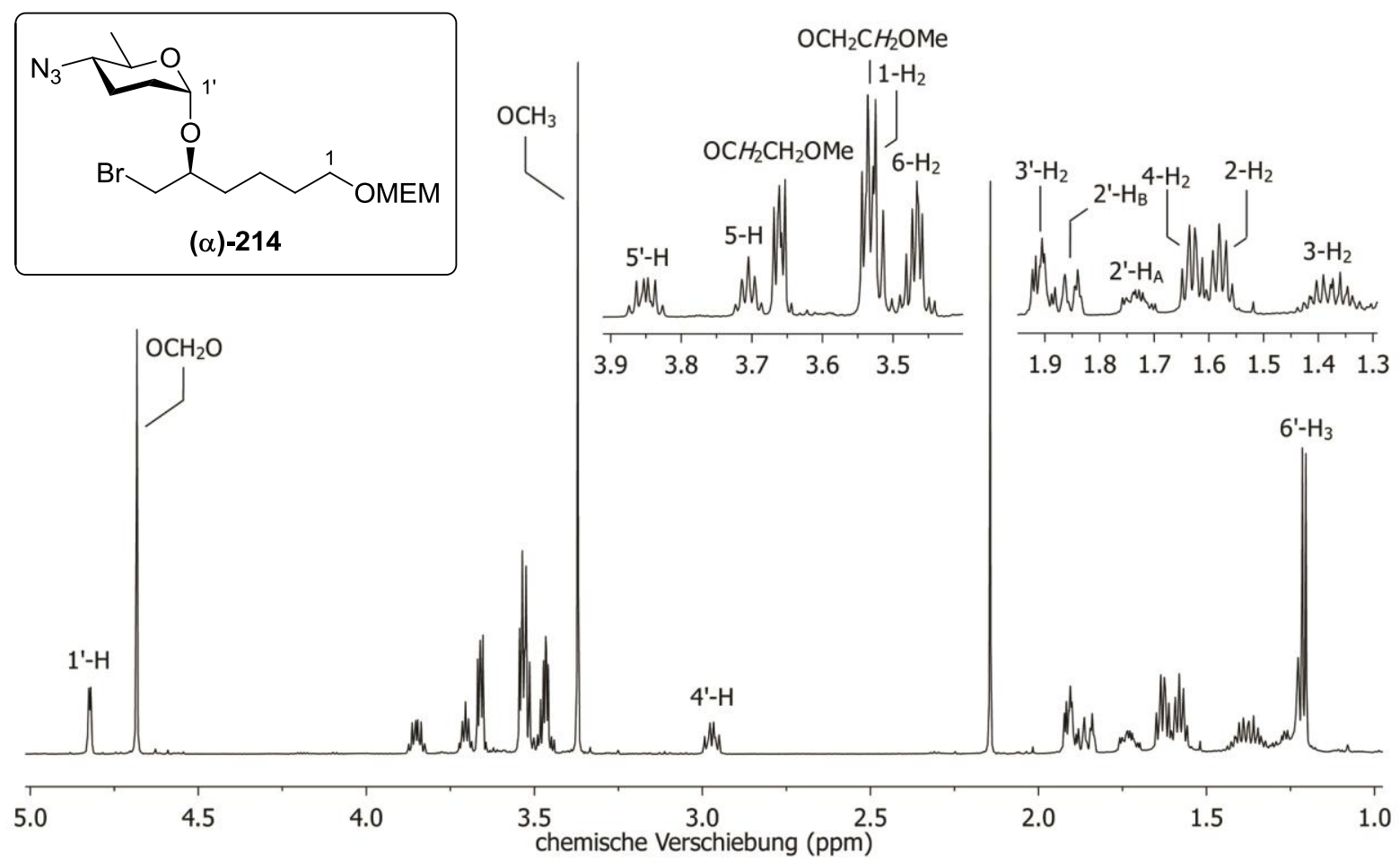

Abbildung 68: ${ }^{1} \mathrm{H}-\mathrm{NMR}-$ Spektrum von Verbindung 214.

Die acetalischen Wasserstoffatome der MEM-Schutzgruppe zeigen bei einer charakteristischen chemischen Verschiebung von $\delta=4.68 \mathrm{ppm}$ ein Signal in Form eines Singuletts. Am weitesten ins Tieffeld verschoben ist das Signal des Wasserstoffatoms 1'-H am anomeren Zentrum bei $\delta=4.82 \mathrm{ppm}$. Die Kopplungskonstante von $J=3.2 \mathrm{~Hz}$ ist ein zweifelfreies Zeichen für das Vorhandensein eines $\alpha$-Glycosids. Zum Vergleich weist das anomere Wasserstoffatom des $\beta$-Glycosids eine für axial-axial-Kopplungen übliche Kopplungskonstante von $J=9.2 \mathrm{~Hz}$ auf und resoniert bei $4.76 \mathrm{ppm}$.

Das ${ }^{13}$ C-NMR-Spektrum der Verbindung wurde ebenfalls in deuteriertem Chloroform und bei einer Frequenz von $126 \mathrm{~Hz}$ aufgenommen und ist in Abbildung 69 abgebildet. 


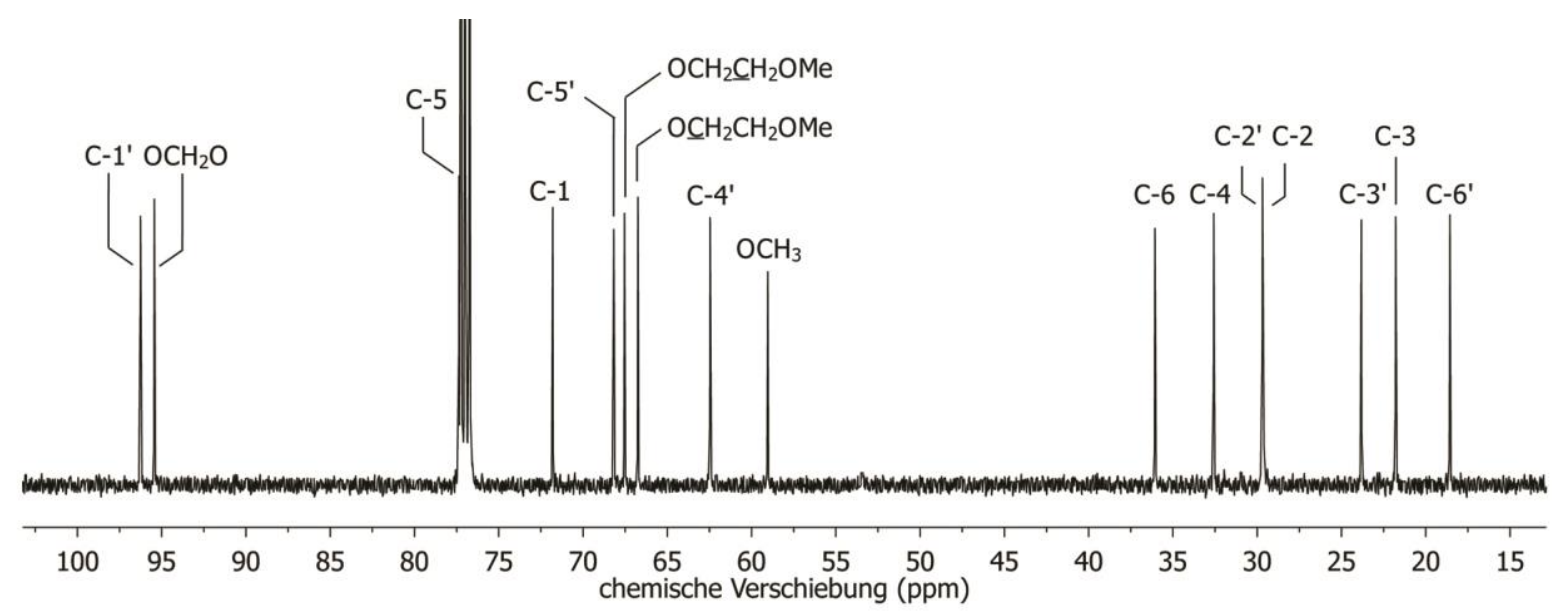

Abbildung 69: ${ }^{13} \mathrm{C}-\mathrm{NMR}-$ Spektrum von Verbindung 214.

Im aliphatischen Bereich des Spektrums resonieren erwartungsgemäß die abgeschirmten Wasserstoffatome der Methylgruppe bei $\delta=18.6 \mathrm{ppm}$. Etwas weiter ins Tieffeld verschoben befinden sich die Signale für das C-3 bei $\delta=21.8 \mathrm{ppm}$, das C-3' bei $\delta=23.8 \mathrm{ppm}$, das C-2 bei $\delta=29.6 \mathrm{ppm}$, das C-2' bei $\delta=29.7 \mathrm{ppm}$, das C-4 bei $\delta=31.0 \mathrm{ppm}$ und das C-6 bei $\delta=32.6 \mathrm{ppm}$. Die Methoxygruppe der MEM-Schutzgruppe resoniert wie erwartet bei $\delta=59.0$ ppm, während das C-4' durch die benachbarte Azidfunktion entschirmt bei $\delta=62.5 \mathrm{ppm}$ ein Signal zeigt. Durch den entschirmenden Effekt eines benachbarten Sauerstoffatoms weisen die Kohlenstoffatome der Methylengruppen der MEM-Schutzgruppe (bei $\delta=66.8$ und $71.8 \mathrm{ppm}$ ), das C-1 (bei $\delta=67.6 \mathrm{ppm}$ ) und das C-5' (bei $\delta=68.2 \mathrm{ppm}$ ) eine sehr ähnliche chemische Verschiebung auf. Das Kohlenstoffatom an der glycosidischen Bindung (C-5) resoniert indes bei $\delta=77.4 \mathrm{ppm}$. Durch die direkte Nachbarschaft von zwei Sauerstoffatomen entschirmt sind die Signale der acetalischen Kohlenstoffatome am stärksten tieffeldverschoben bei $\delta=95.5 \mathrm{ppm}\left(\mathrm{OCH}_{2} \mathrm{O}\right)$ und $\delta=96.3 \mathrm{ppm}\left(\mathrm{C}-1^{\prime}\right)$ zu finden.

Im ESI-Spektrum sind neben den Basispeaks bei einem Masse-zu-Ladungs-Verhältnis von $m / z=446.1$ und 448.1 für das $[\mathrm{M}+\mathrm{Na}]^{+}$-Addukt auch Signale bei $m / z=441.2$ und 443.2, die auf das $\left[\mathrm{M}+\mathrm{NH}_{4}\right]^{+}-$Konjugat zurückzuführen sind, vorhanden. Das Isotopenmuster von ungefähr 1:1 deutet dabei auf eine einfach bromierte Verbindung hin. Die Summenformel von $\mathrm{C}_{16} \mathrm{H}_{31} \mathrm{~N}_{3} \mathrm{O}_{6}$ mit einer berechneten exakten Masse von $m / z=446.1261$ bzw. 448.1241 für das $[\mathrm{M}+\mathrm{Na}]^{+}$-Addukt wurde überdies durch ESI-HRMS mit gefundenen Massen von $m / z=446.1256$ und 448.1236 bestätigt. 


\subsection{Synthese von Forosamin-Analoga}

Ein grundlegendes Problem der Synthese von Spinosyn-Analoga ist die selektive Einführung der Forosamineinheit am Makrolacton. Vorangegangene Arbeiten zeigten, dass bei einer Glycosylierung von relativ kleinen Molekülen zu Beginn der Synthese die besten Ergebnisse erzielt werden konnten. ${ }^{107}$ In neueren Studien wurde überdies dargelegt, dass die Forosamineinheit ohne signifikanten Wirkverlust gegen andere stickstoffhaltige Kohlenhydrate und Nicht-Kohlenhydrate ausgetauscht werden kann. ${ }^{42}$ Um einen hohen Substanzverlust über die Glycosylierungsreaktion zu verhindern, wurden einige Forosamin-Analoga hergestellt, die über eine nucleophile Substitution mit der Seitenkette gekuppelt werden können.

Zunächst erfolgte die Bromierung der kommerziell erhältlichen Diole 217 und 219 mit Phosphortribromid und Pyridin in Diethylether (Abbildung 70).

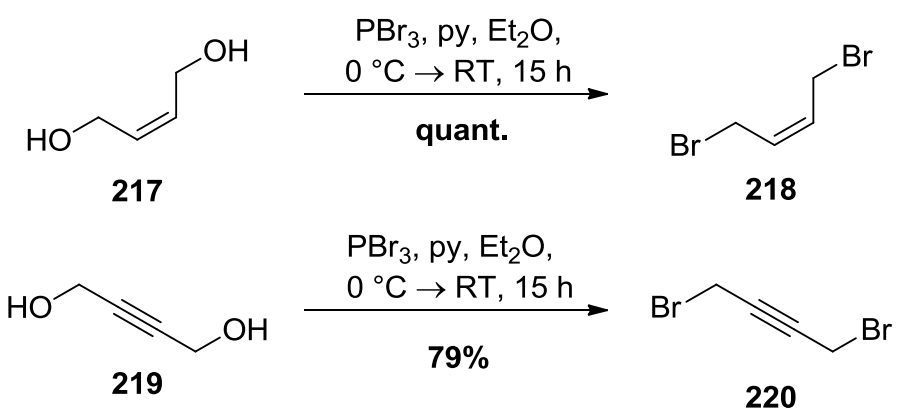

Abbildung 70: Bromierung der Verbindungen 217 und 219.

Während bei der Umsetzung des (Z)-Alkens 217 zu 218 eine quantitative Ausbeute erreicht wurde, führte die Bromierung des Alkins 219 in 79\% Ausbeute zum gewünschten Dibromid 220.

Zusammen mit kommerziell erhältlichen Dibromiden wurden diese Verbindungen anschließend unter Verwendung von Natriumazid in Dimethylformamid bei $50{ }^{\circ} \mathrm{C}$ in die resultierenden Azide 222a-f überführt (Abbildung 71).

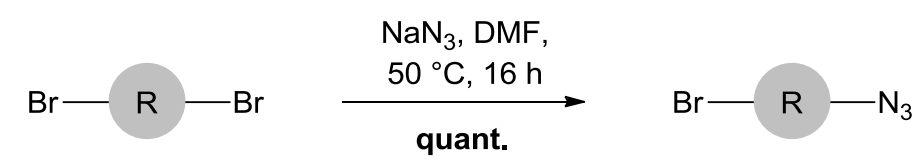

221

222

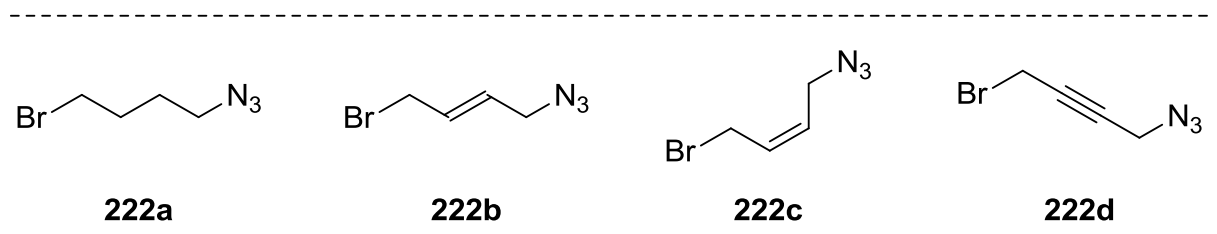

Abbildung 71: Synthese der Forosamin-Analoga 222a-f. 
Aufgrund der geringen chromophoren Eigenschaften der Verbindungen war eine Aufreinigung der Verbindungen über traditionelle Säulenchromatographie sehr mühsam. Glücklicherweise waren die Rohprodukte laut NMR und IR-Spektroskopie nahezu rein, weshalb auf eine chromatographische Reinigung der Verbindungen verzichtet werden konnte. Während sich die Alkan- und Alkenspezies 222a-c als stabile Verbindungen herausstellten, neigte die Alkinverbindung 222d jedoch zur Polymerisation und sollte in einer potentiellen Analogasynthese unverzüglich eingesetzt werden.

Neben den gezeigten elektrophilen Forosamin-Analoga wurde zusätzlich das nucleophile Cyclohexanolderivat 225 hergestellt. Durch Zugabe von Kupfer(II)sulfat, Kaliumcarbonat und der Imidazolverbindung 224 zu 223 in Methanol bei Raumtemperatur konnte das Azid 225 in einer hervorragenden Ausbeute von 93\% erhalten werden (Abbildung 72).

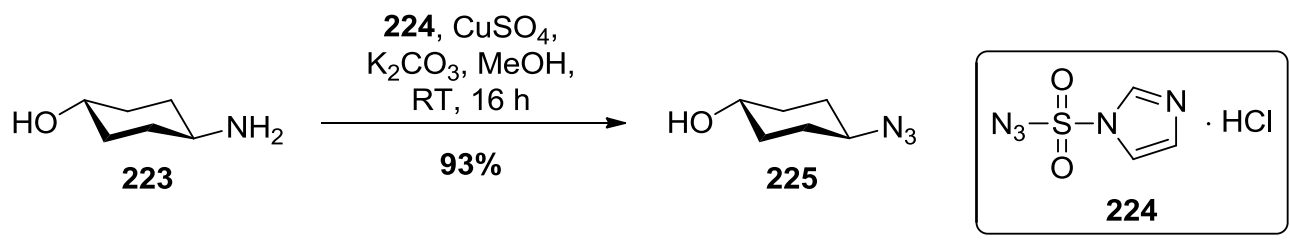

Abbildung 72: Synthese des Forosamin-Analogons 225.

Die hergestellten Forosamin-Analoga erlauben eine größere Freiheit bei der Gestaltung der Seitenkette und können ohne die problematische Glycosylierungsreaktion auch zu einem späteren Zeitpunkt der Synthese eingeführt werden. Erste Untersuchungen zur Einbringung der Forosamin-Analoga an dem vorliegenden Glycosyldonor 204 führten jedoch unter verschiedenen Reaktionsbedingungen nicht zu zufriedenstellenden Ergebnissen. So konnte im Falle einer Kupplung mit dem Alkylderivat 222a das gewünschte Produkt 226 nur in Ausbeuten von bis zu 10\% isoliert werden (Abbildung 73).

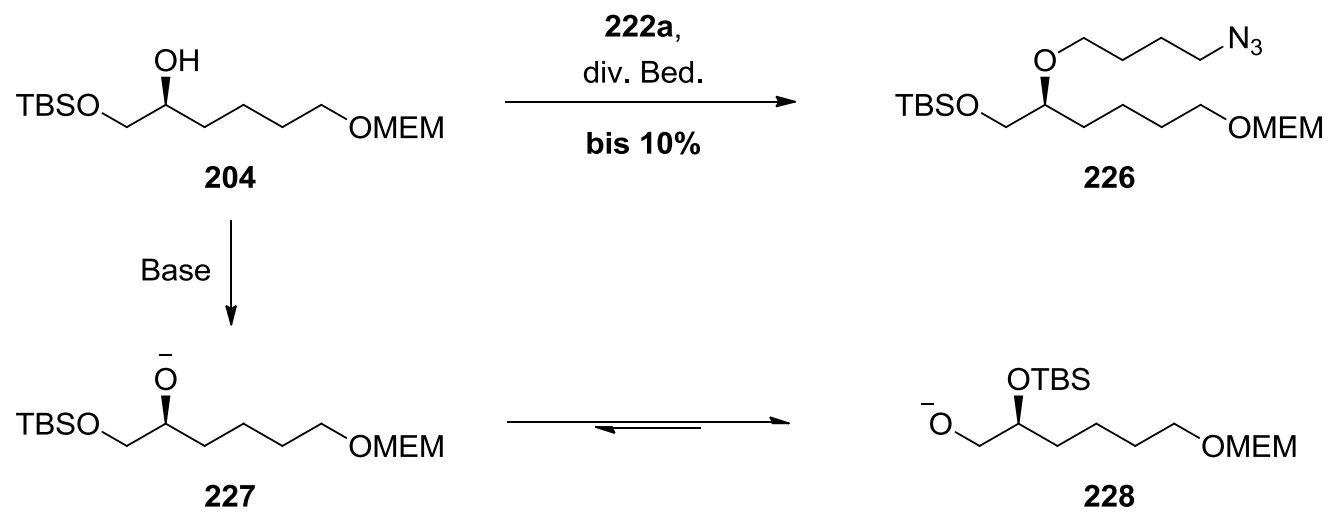

Abbildung 73: Silylshift bei der Deprotonierung von Verbindung 204. 
Die Analyse von Nebenprodukten führte zu dem Schluss, dass Verbindung 204 nach Deprotonierung der freien Hydroxyfunktion über einen 1,3-Silylshift zu dem primären Alkoholat 228 umgelagert wird. Infolge dieser Umlagerung kommt es zu schwer trennbaren Produktgemischen. Um die Isomerisierung zu vermeiden, wäre eine Überführung der sekundären Hydroxyfunktion in 204 unter Appel-Bedingungen in das resultierende Bromid und eine anschließende Kupplung mit dem entsprechenden hydroxyfunktionalisierten Forosamin-Analogon 225 denkbar.

Diese Ergebnisse unterstützen die Strategie, die Einführung der Forosamin-Analoga zu einem späteren Zeitpunkt der Synthese durchzuführen.

\subsection{Alternative Synthesestrategien zur Kupplung der Spinosyn-Bausteine}

Einem neuen Ansatz folgend sollte die Kupplung von Tricyclus und Seitenkette über eine nucleophile Substitution erfolgen. Diese alternative Synthesestrategie sah eine Verwendung des bereits synthetisierten Allylalkohols 186 vor. Die Seitenkette sollte analog zu Verbindung 229 eine gute Abgangsgruppe für die nucleophile Substitution beinhalten (Abbildung 74).

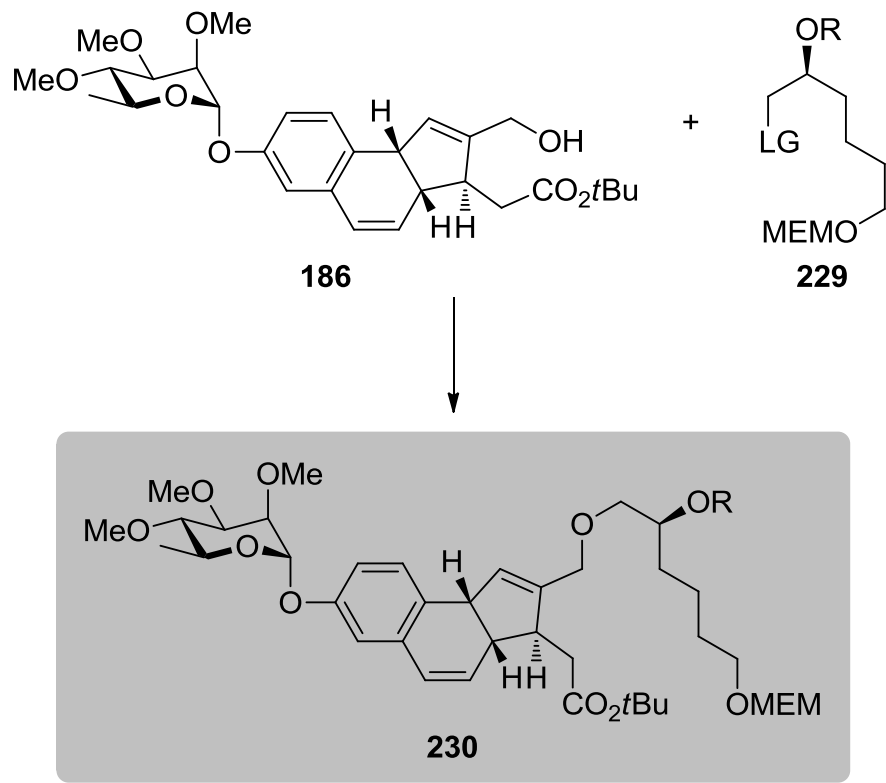

Abbildung 74: Alternative Syntheseroute zur Bildung des Makrolactons.

Bei der Synthese der neu gestalteten Seitenkette konnte auf das bereits synthetisierte enantiomerenreine Diol 207 zurückgegriffen werden. Im ersten Schritt wurden beide Hydroxyfunktionen mit TBS-Triflat und 2,6-Lutidin in hervorragender Ausbeute von 99\% in die TBS-Ether 231 überführt (Abbildung 75). 


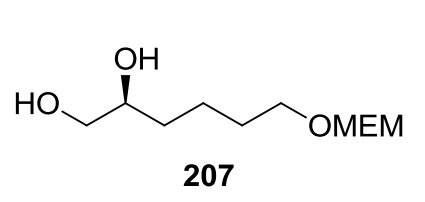

207
TBSOTf, 2,6-Lutidin,

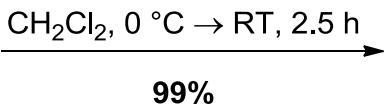

$99 \%$

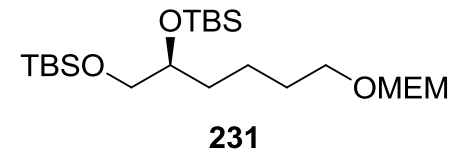

231

s. Tabelle<smiles>COCCCC[C@@H](CO)O[SbH2]</smiles>

232

Abbildung 75: Synthese des primären Alkohols 232.

Anschließend sollte eine selektive Spaltung des primären TBS-Ethers erfolgen. Die Verwendung von Pyridin-Hydrofluorid in einem Tetrahydrofuran-Pyridin-Gemisch lieferte den primären Alkohol 232 in einer moderaten Ausbeute von 66\% (Tabelle 5, Eintrag 1). Auch die Anwendung von alternativen Entschützungsbedingungen führte nicht $\mathrm{zu}$ einer Verbesserung der Ausbeute. Während die Verwendung von Tetrabutylammoniumfluorid in Essigsäure und Tetrahydrofuran das gewünschte Produkt in $47 \%$ Ausbeute lieferte, führte eine Umsetzung mit Camphersulfonsäure in einem Chloroform-Methanol-Gemisch lediglich in 43\% Ausbeute zum primären Alkohol 232 (Tabelle 5, Einträge 2 \& 3).

Tabelle 5: Selektive Entschützung des primären TBS-Ethers in 231.

\begin{tabular}{clc}
\hline Eintrag & Reaktionsbedingungen & Ausbeute \\
\hline 1 & $\mathrm{HF} \cdot \mathrm{py}, \mathrm{THF}, \mathrm{py}, 0^{\circ} \mathrm{C} \rightarrow \mathrm{RT}, 24 \mathrm{~h}$ & $66 \%$ \\
2 & $\mathrm{TBAF}, \mathrm{AcOH}, \mathrm{THF}, \mathrm{RT}, 12 \mathrm{~h}$ & $47 \%$ \\
3 & $\mathrm{CSA}, \mathrm{CHCl}_{3}, \mathrm{MeOH},-10{ }^{\circ} \mathrm{C}, 8 \mathrm{~h}$ & $43 \%$ \\
\hline
\end{tabular}

Ausgehend von Verbindung $\mathbf{2 3 2}$ sollte im nächsten Schritt die Einführung einer Abgangsgruppe erfolgen (Abbildung 76).

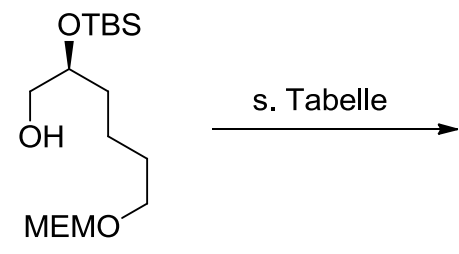

232

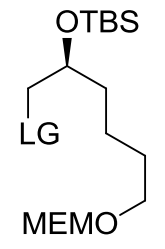

233
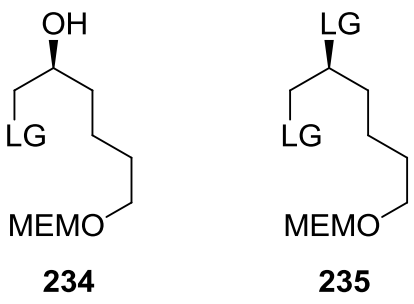

$\mathrm{LG}=\mathrm{Br}$, OTs, OMs, OTf

Abbildung 76: Einführung der Abgangsgruppe. 
Zunächst wurde unter Appel-Bedingungen mit Tetrabromkohlenstoff und Triphenylphosphin in Dichlormethan das Bromid 233a synthetisiert (Tabelle 6, Eintrag 1). Analog zur Synthese der Seitenkette 214 konnte bei der Bromierung nur eine geringe Ausbeute von 14\% erzielt werden. Aufgrund der Präsenz der primären Hydroxyfunktion bot sich eine Überführung in die guten Abgangsgruppen Mesylat, Tosylat und Triflat an.

Die Synthese des Tosylats unter Standardbedingungen mit Tosylchlorid, Triethylamin und katalytischen Mengen an 4-Dimethylaminopyridin in Dichlormethan führte in 85\% Ausbeute zu dem TBS-entschützten Tosylat 234 (Tabelle 6, Eintrag 2). Des Weiteren wurden 8\% des doppelt substituierten Produktes 235 erhalten. Um eine TBS-Entschützung zu vermeiden, wurde die Reaktion bei $10{ }^{\circ} \mathrm{C}$ durchgeführt, was allerdings zu keinem Umsatz führte (Tabelle 6, Eintrag 3). Nach Anwendung von alternativen Reaktionsbedingungen mit Tosylchlorid in Pyridin bei Raumtemperatur konnte das gewünschte Tosylat 233b in $87 \%$ Ausbeute erhalten werden (Tabelle 6, Eintrag 4). Darüber hinaus wurden 7\% des eingesetzten Alkohols 232 reisoliert.

Tabelle 6: Reaktionsbedingungen für die Einführung einer Abgangsgruppe an Molekül 232.

\begin{tabular}{cclc}
\hline Eintrag & LG & Reaktionsbedingungen & Ergebnis \\
\hline 1 & $\mathrm{Br}$ & $\mathrm{CBr}_{4}, \mathrm{PPh}_{3}, \mathrm{CH}_{2} \mathrm{Cl}_{2}, 0{ }^{\circ} \mathrm{C} \rightarrow \mathrm{RT}, 18 \mathrm{~h}$ & $14 \%$ \\
2 & $\mathrm{OTs}$ & $\mathrm{TsCl}, \mathrm{NEt}_{3}, \mathrm{DMAP}, \mathrm{CH}_{2} \mathrm{Cl}_{2}, 0{ }^{\circ} \mathrm{C} \rightarrow \mathrm{RT}, 16 \mathrm{~h}$ & $85 \%(\mathbf{2 3 4}), 8 \%(\mathbf{2 3 5})$ \\
3 & $\mathrm{OTs}$ & $\mathrm{TsCl}, \mathrm{NEt}_{3}, \mathrm{DMAP}, \mathrm{CH}_{2} \mathrm{Cl}_{2}, 0{ }^{\circ} \mathrm{C} \rightarrow 10{ }^{\circ} \mathrm{C}, 16 \mathrm{~h}$ & kein Umsatz \\
4 & $\mathrm{OTs}$ & $\mathrm{TsCl}, \mathrm{py}, \mathrm{RT}, 16 \mathrm{~h}$ & $87 \%$ (94\% brsm) \\
5 & $\mathrm{OMs}$ & $\mathrm{MsCl}_{2} \mathrm{NEt}_{3}, \mathrm{CH}_{2} \mathrm{Cl}_{2}, 0{ }^{\circ} \mathrm{C}, 30 \mathrm{~min}$ & quant. \\
6 & $\mathrm{OTf}$ & $\mathrm{Tf}_{2} \mathrm{O}, \mathrm{NEt}_{3}, \mathrm{CH}_{2} \mathrm{Cl}_{2}, 0{ }^{\circ} \mathrm{C}, 2 \mathrm{~h}$ & $70 \%$ \\
\hline
\end{tabular}

Die Synthese des Mesylats 233c erfolgte unter Verwendung von Mesylchlorid und Triethylamin in Dichlormethan bei $0{ }^{\circ} \mathrm{C}$ und erbrachte die gewünschte Verbindung in quantitativer Ausbeute (Tabelle 6, Eintrag 5). Unter analogen Bedingungen wurde mit Trifluormethansulfonsäureanhydrid das zu Zersetzung neigende Triflat 233d in 70\% Ausbeute hergestellt (Tabelle 6, Eintrag 6).

Die synthetisierten $\mathrm{C}_{6}$-Ketten sollten im weiteren Verlauf der Synthese nach Deprotonierung des Allylalkohols in Tricyclus 186 mit dem Grundgerüst gekuppelt werden (Abbildung 77). Die Verwendung von Natriumhydrid als Base in Dimethylformamid (Tabelle 7, Eintrag 1) erwies sich als zu harsch. So konnte unter diesen Reaktionsbedingungen und der Verwendung 
des Tosylats 233b nur eine komplette Zersetzung des tricyclischen Grundgerüsts beobachtet werden.<smiles>[R6]c1ccc2c(c1)C=C[C@@H]1[C@H](CCOC(C)(C)C)CC(CO)=C[C@H]21</smiles><smiles>CCC(CCCOC)C(CC)OC</smiles>
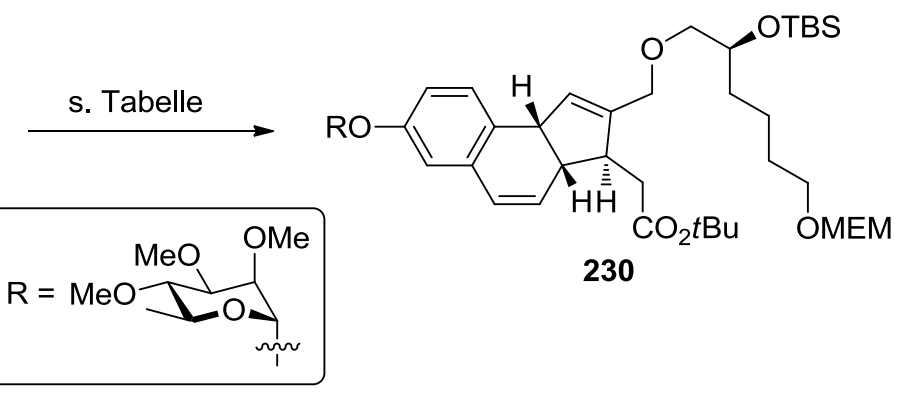

233

Abbildung 77: Kupplung der $\mathrm{C}_{6}$-Kette mit dem tricyclischen Grundgerüst.

Mit Kaliumcarbonat in Aceton wurde bei allen drei Seitenketten auch nach zwei Tagen unter Rückfluss nur ein geringer Umsatz beobachtet (Tabelle 7, Eintrag 2). Zwar zeigte die Reaktionskontrolle einen neu gebildeten, UV-aktiven Spot mit erwarteter Polarität, die Bildung eines Kupplungsproduktes konnte nach NMR-spektroskopischen und massenspektrometrischen Untersuchungen jedoch sicher ausgeschlossen werden. Bei dem isolierten Spot handelte es sich höchstwahrscheinlich um ein Zersetzungsprodukt des tricyclischen Grundgerüsts. Die Verwendung von Kalium-tert-butanolat als Base in Tetrahydrofuran führte wie auch die Behandlung mit Natriumhydrid zu einer Zersetzung des Alkohols 186 (Tabelle 7, Eintrag 3).

Tabelle 7: Bedingungen zur Kupplung der Bausteine 186 und 233.

\begin{tabular}{|c|c|c|c|}
\hline Eintrag & LG & Reaktionsbedingungen & Ergebnis \\
\hline 1 & OTs & $\mathrm{NaH}, \mathrm{DMF}, \mathrm{RT} \rightarrow 0{ }^{\circ} \mathrm{C} \rightarrow \mathrm{RT}, 12 \mathrm{~h}$ & Zersetzung \\
\hline 2 & OMs/OTs/OTf & $\mathrm{K}_{2} \mathrm{CO}_{3}$, Aceton, $56{ }^{\circ} \mathrm{C}, 2 \mathrm{~d}$ & kaum Umsatz \\
\hline 3 & OTs & $\mathrm{KO} t \mathrm{Bu}, \mathrm{THF}, 0^{\circ} \mathrm{C}$ & Zersetzung \\
\hline 4 & OTs & $\mathrm{NEt}_{3}, \mathrm{CH}_{2} \mathrm{Cl}_{2}, \mathrm{RT}, 2 \mathrm{~d}$ & kein Umsatz \\
\hline 5 & OMs/OTs/OTf & $\mathrm{NEt}_{3}, \mathrm{EDC}, 80^{\circ} \mathrm{C}, 2 \mathrm{~d}$ & kein Umsatz \\
\hline 6 & OMs/OTs/OTf & 2,6-Lutidin, EDC, $80{ }^{\circ} \mathrm{C}, 2 \mathrm{~d}$ & kein Umsatz \\
\hline 7 & OMs/OTs/OTf & DIPEA, EDC, $80^{\circ} \mathrm{C}, 2 \mathrm{~d}$ & kein Umsatz \\
\hline
\end{tabular}


Da die oben genannten anorganischen Basen für eine Kupplung der Bausteine nicht geeignet waren, wurden in der Folge organische Aminbasen zur Deprotonierung der primären Hydroxyfunktion in 186 eingesetzt. Mit der Standardbase Triethylamin konnte sowohl bei Raumtemperatur in Dichlormethan (Tabelle 7, Eintrag 4) als auch bei $80{ }^{\circ} \mathrm{C}$ in 1,2-Dichlorethan (Tabelle 7, Eintrag 5) kein Umsatz erzielt werden. Auch der Einsatz von 2,6-Lutidin (Tabelle 7, Eintrag 6) und Hünig-Base (Tabelle 7, Eintrag 7) führte unter erhöhter Temperatur nicht zu dem gewünschten Kupplungsprodukt.

Die überarbeitete Synthesestrategie sah nun eine Kupplung der hydroxy-tragenden Seitenkette 232 und der aktivierten Carbonsäure des Tricyclus, die durch Oxidation des Aldehyds 149 zugänglich ist, vor. Um eine Trennung des Diastereomerengemisches von 186 über Säulenchromatographie an Kieselgel zu ermöglichen, wurde der Allylalkohol 186 nicht direkt zur polaren Carbonsäure oxidiert, sondern zunächst in den Methylester 236 überführt. Unter Verwendung von Mangan(IV)oxid und Natriumcyanid in einem Methanol- $n$-Hexan-Gemisch konnte Methylester 236 als Rohprodukt isoliert werden. (Abbildung 78).

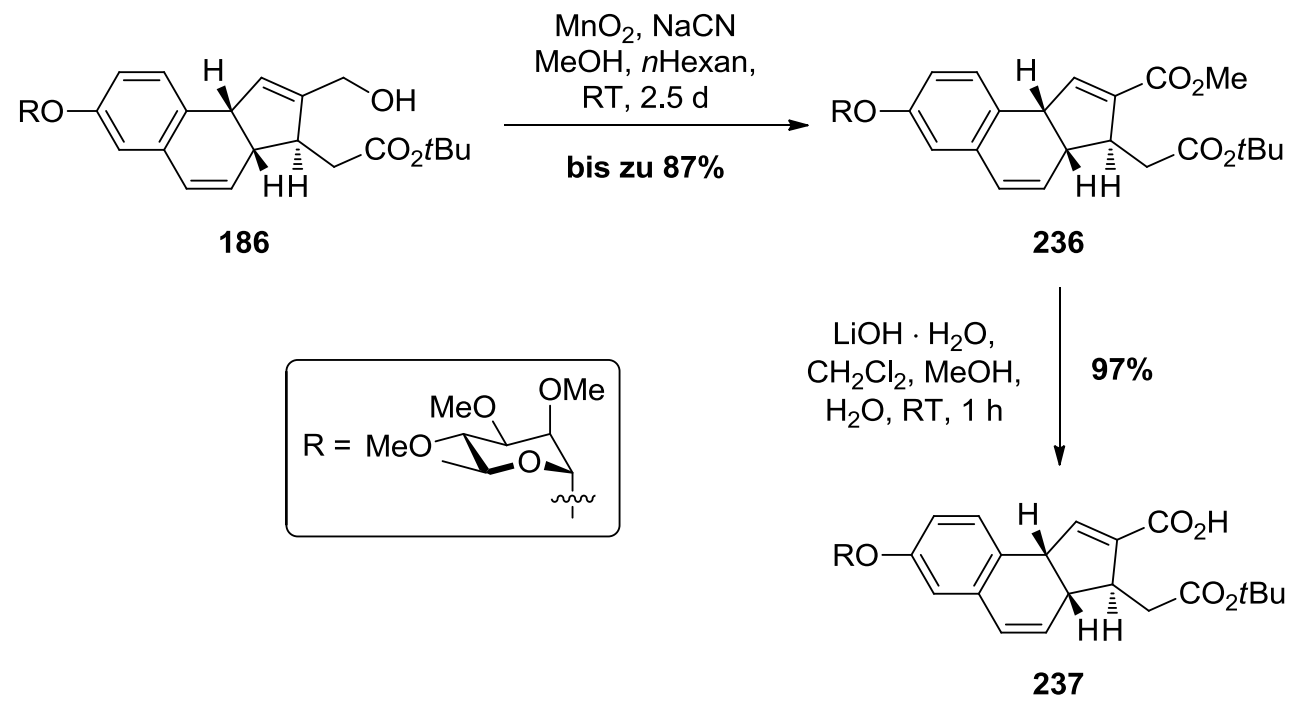

Abbildung 78: Synthese des Carbonsäure 237.

Eine anschließende säulenchromatographische Reinigung des Rohproduktes an Kieselgel lieferte den diastereomerenreinen Methylester 236 in 61\% Ausbeute. Die vergleichsweise leichte präparative Trennung der Diastereomere auf dieser Stufe der Synthese erspart die äußerst zeitaufwendige Aufreinigung der Alkohole 186 über präparative HPLC und ermöglicht so einen leichteren Zugang $\mathrm{zu}$ einem diastereomerenreinen tricyclischen Grundgerüst. Überdies konnte bei der Oxidation von enantiomerenreinem Alkohol 186 eine Ausbeute von $87 \%$ erreicht werden. 
Ausgehend von dem diastereomerenreinen Tricyclus 236 konnte unter Verwendung von Lithiumhydroxid in einem Dichlormethan-Methanol-Wasser-Gemisch die resultierende Carbonsäure 237 in einer exzellenten Ausbeute von 97\% synthetisiert werden.

Für die nachfolgende Kupplungsreaktion wurde Verbindung 236 unter Verwendung von Dicyclohexylcarbodiimid und 4-Dimethylaminopyridin in einem Diethylether-ChloroformGemisch in situ aktiviert und mit einem Überschuss von Verbindung 232 versetzt (Abbildung 79).

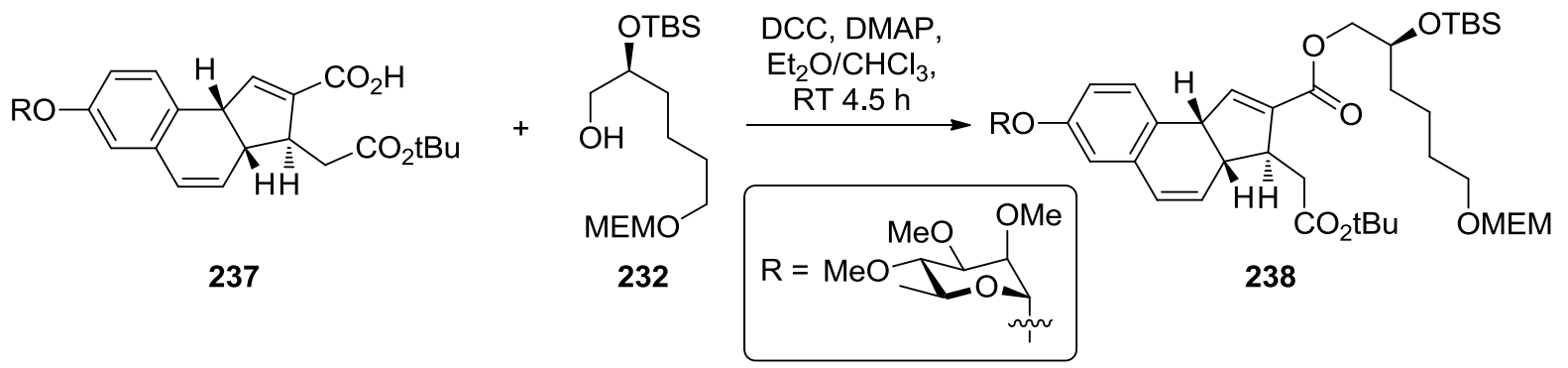

Abbildung 79: Aktivierung der Carbonsäure 237 und Kupplung mit der $\mathrm{C}_{6}$-Kette 232.

Leider konnte das entstandene Produkt 238 nicht durch chromatographische Methoden von dem überschüssigen Alkohol 232 abgetrennt werden. So zeigten massenspektrometrische Untersuchungen der isolierten Fraktion das Vorhandensein von 238 und 232. Die Summenformel des Kupplungsproduktes $\mathbf{2 3 8}$ wurde durch hochauflösende Massenspektrometrie bestätigt. Ein weiterer Kupplungsversuch unter Einsatz äquimolarer Mengen an Carbonsäure und Seitenkette konnte nicht mehr untersucht werden, da die vorhandenen Substanzmengen von 237 dafür nicht ausreichten. Eine erneute Synthese der Carbonsäure 237 konnte aus Zeitgründen nicht mehr durchgeführt werden.

\subsection{Glycosylierung von Verbindung 156}

Ein weiteres Ziel dieser Arbeit war eine Überprüfung des Anwendungsgebiets der im Arbeitskreis Tietze entwickelten $\beta$-selektiven Glycosylierungsmethode. Traditionell wurden hierbei sekundäre Alkohole unter Verwendung von Triethylsilan und Iod in Toluol mit dem Trichloracetimidat 197 umgesetzt. Da sich in vorangegangenen Arbeiten die Einführung der Forosamineinheit bei komplexeren Molekülen aufgrund von geringen Ausbeuten und schlechten Selektivitäten als schwierig darstellte, ${ }^{107}$ eine Einführung des Zuckersubstituenten zu einem späten Zeitpunkt der Synthese jedoch von Vorteil ist, wurde die Glycosylierung des Akzeptors 157 genauer untersucht (Abbildung 80). Das Aglycon 157 ${ }^{120}$ wurde dabei von der Bayer AG bereitgestellt und beinhaltet das bioidentische tricyclische Grundgerüst von Spinosyn A. 


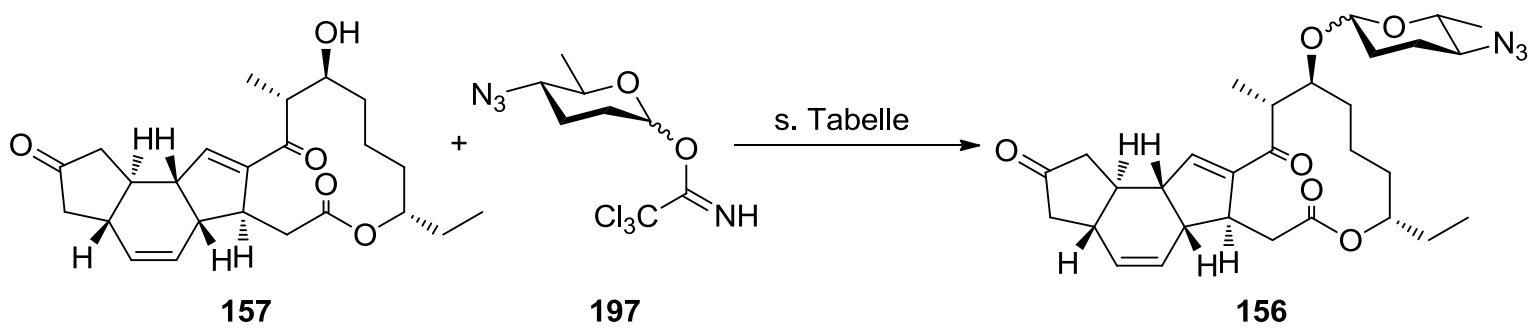

Abbildung 80: Glycosylierung des Aglycons 157.

Bei der Umsetzung des in situ hergestellten Trichloracetimidats 197 mit dem Glycosylakzeptor 157 unter den etablierten Reaktionsbedingungen mit Triethylsilan und Iod in Toluol über Molsieb bei einer Temperatur von $-87^{\circ} \mathrm{C}$ konnte analog $\mathrm{zu}$ anderen komplexen Aglycons kein Umsatz beobachtet werden (Tabelle 8, Eintrag 1). Eine Erhöhung der Temperatur auf $-60{ }^{\circ} \mathrm{C}$ führte lediglich zu einem geringen Umsatz. So konnten bei dieser Temperatur 3\% des $\alpha$-Anomers und $4 \%$ des gewünschten $\beta$-Anomers von 156 isoliert werden (Tabelle 8, Eintrag 2).

Tabelle 8: Reaktionsbedingungen zur Glycosylierung von Verbindung 156.

Eintrag Reaktionsbedingungen

1

2

3

$$
\begin{aligned}
& \mathrm{Et}_{3} \mathrm{SiH}, \mathrm{I}_{2}, \mathrm{MS} 3 \AA, \mathrm{PhMe},-87{ }^{\circ} \mathrm{C}, 4 \mathrm{~d} \\
& \mathrm{Et}_{3} \mathrm{SiH}, \mathrm{I}_{2}, \mathrm{MS} 3 \AA, \mathrm{PhMe},-60{ }^{\circ} \mathrm{C} \\
& \mathrm{Et}_{3} \mathrm{SiH}, \mathrm{I}_{2}, \mathrm{MS} 3 \AA, \mathrm{CH}_{2} \mathrm{Cl}_{2},-87^{\circ} \mathrm{C}
\end{aligned}
$$

\section{Ergebnis}

kein Umsatz

$\alpha-156: 3 \%, \beta-156: 4 \%$

$\alpha-156: 21 \%, \beta-156: 30 \%$

Neben Toluol als Lösungsmittel lieferte bei Testreaktionen in vorangegangenen Arbeiten Dichlormethan die besten Selektivitäten bezüglich eines gewünschten $\beta$-Anomers, zeichnete sich allerdings auch durch eine geringere Schutzgruppentoleranz aus. ${ }^{107}$ Da Aglycon 157 keine Schutzgruppen beinhaltet, konnte in Dichlormethan bei tiefen Temperaturen eine hervorragende Gesamtausbeute von $51 \%$ bei sehr guter $\alpha / \beta$-Selektivität von 1:1.5 erzielt werden (Tabelle 8, Eintrag 3).

Die Ergebnisse dieser Experimente zeigen, dass eine Einführung der Forosamineinheit gegen Ende der Syntheseroute möglich ist. Dies ermöglicht eine variablere Strategie in der Synthese von Spinosyn-Analoga. 


\section{Enantioselektive Synthese von (+)-Linoxepin}

Die enantioselektive Synthese von (+)-Linoxepin basiert auf der im Arbeitskreis Tietze durchgeführten und bereits veröffentlichten ersten Totalsynthese des Naturstoffes. Bei der Synthese einiger Bausteine und Bedingungen für die Domino-Carbopalladierung-HeckReaktion konnte auf vorangegangene Arbeiten zurückgegriffen werden. ${ }^{109,121}$

\subsection{Synthese des Benzylbromids 165}

Bei der Synthese des Benzylbromid-Bausteins 165 wurde von kommerziell erhältlichem 5-Brom-1,3-benzodioxol (239) ausgegangen. Im ersten Schritt wurde die Ausgangsverbindung mit $n$ Butyllithium in Tetrahydrofuran bei $-78^{\circ} \mathrm{C}$ zunächst lithiiert und das gebildete Phenylanion anschließend durch die Addition von Iod in einer guten Ausbeute von $84 \%$ in das Aryliodid 240 überführt (Abbildung 81). ${ }^{122}$

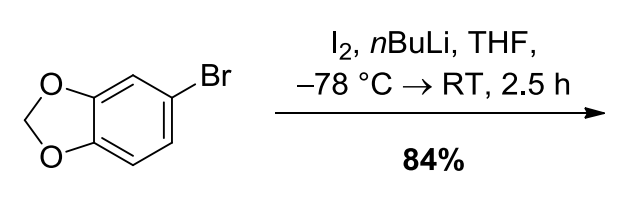

239
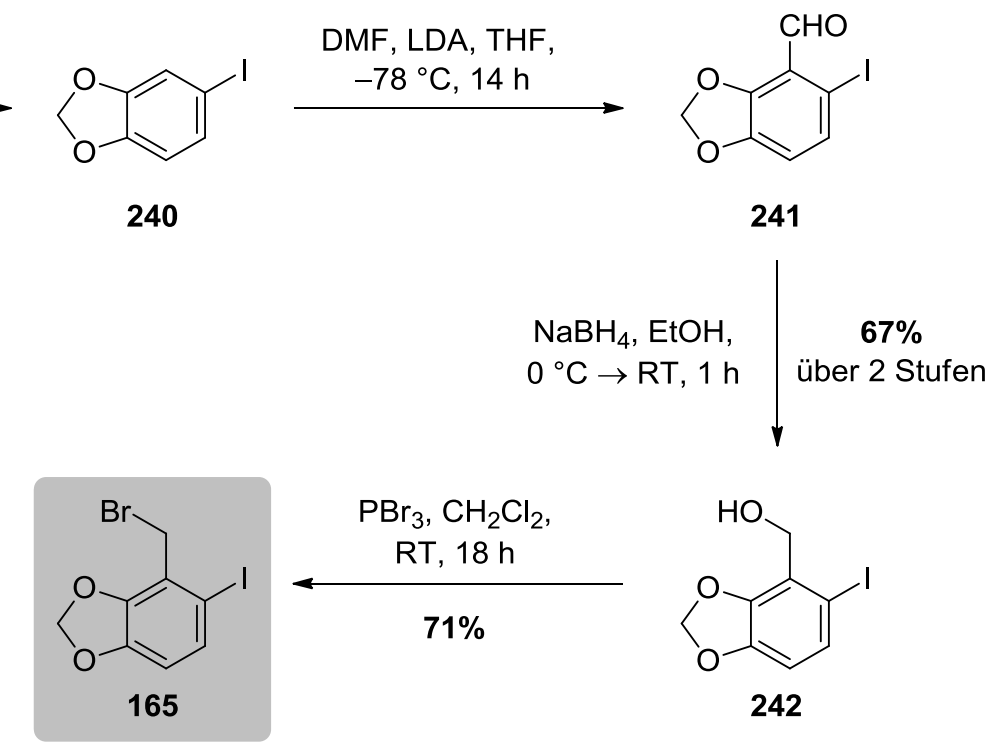

Abbildung 81: Synthese des Benzylbromids 165.

Eine Deprotonierung in 4-Position durch Lithiumdiisopropylamid in Tetrahydrofuran bei $-78{ }^{\circ} \mathrm{C}$ führte durch anschließende Formylierung mit Dimethylformamid zum Aldehyd 241, der nach wässriger Aufarbeitung mit Natriumborhydrid in Ethanol in 67\% Ausbeute über zwei Stufen zum resultierenden Benzylalkohol 242 reduziert wurde. Von dieser Verbindung ausgehend wurde unter Verwendung von Phosphortribromid in Dichlormethan das gewünschte Benzylbromid 165 in 71\% Ausbeute erhalten. 


\subsection{Synthese des Vinylsilans 164}

Für die Synthese des Vinylsilans 164 wurde zunächst kommerziell erhältliches Bromovanin (243) mit Essigsäureanhydrid und Kaliumcarbonat in Acetonitril in quantitativer Ausbeute zu Verbindung 244 acetyliert (Abbildung 82). Im nächsten Schritt erfolgte eine Lithiumvermittelte Addition des Alkins 245 an die Aldehydfunktion. Unter Verwendung von $n$ Butyllithium in Tetrahydrofuran bei $-78^{\circ} \mathrm{C}$ führte die Kupplung in $53 \%$ Ausbeute zum Alkin 246. Unter diesen Reaktionsbedingungen wurde eine in situ Umesterung beobachtet. Die Migration der Acetyl-Schutzgruppe des Phenols zu der propargylischen Hydroxyfunktion ist dabei höchstwahrscheinlich auf die Stabilität der jeweiligen Alkoholationen zurückzuführen. In einer weiteren im Arbeitskreis Tietze durchgeführten Arbeit konnte die Ausbeute dieser Reaktion auf $81 \%$ verbessert werden. ${ }^{109}$
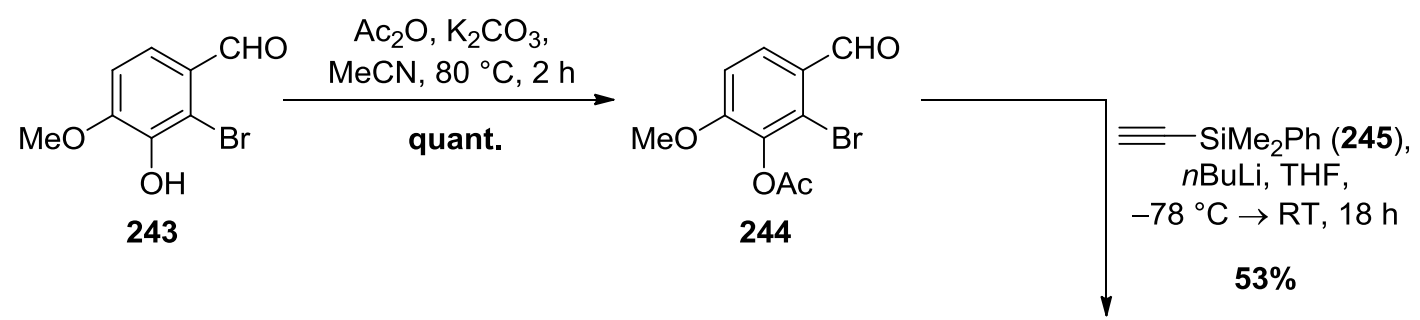

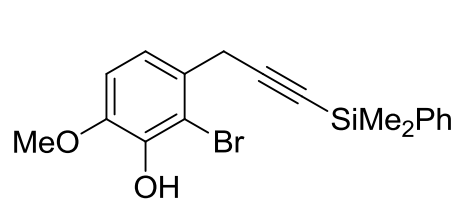

247
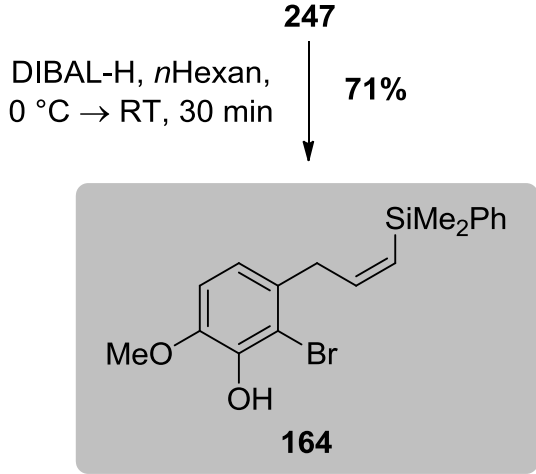

Abbildung 82: Synthese des Vinylsilans 164.

Die weitere Synthese sah eine reduktive Eliminierung der Acetat-Gruppe vor. Nach Umsetzung mit Dimethylphenylsilan als Wasserstoffquelle und Trifluoressigsäure in Toluol bei $0{ }^{\circ} \mathrm{C}$ konnte das Alkin 247 in $80 \%$ Ausbeute isoliert werden. Die abschließende Reduktion der Dreifachbindung mit Diisobutylaluminiumhydrid in $n$-Hexan führte in $71 \%$ Ausbeute zum gewünschten Vinylsilan 164. 


\subsection{Synthese des Domino-Vorläufers 249}

Für die Synthese des Domino-Vorläufers 249 sollten die beiden Bausteine 164 und 165 unter basischen Bedingungen gekuppelt und anschließend der TIPS-geschützte Propargylalkohol 163 über eine Sonogashira-Reaktion eingeführt werden. Die Alkylierung des Phenols 164 mit Kaliumcarbonat in Acetonitril führte bei $80{ }^{\circ} \mathrm{C}$ in quantitativer Ausbeute zu dem gewünschten Benzylarylether 162 (Abbildung 83).

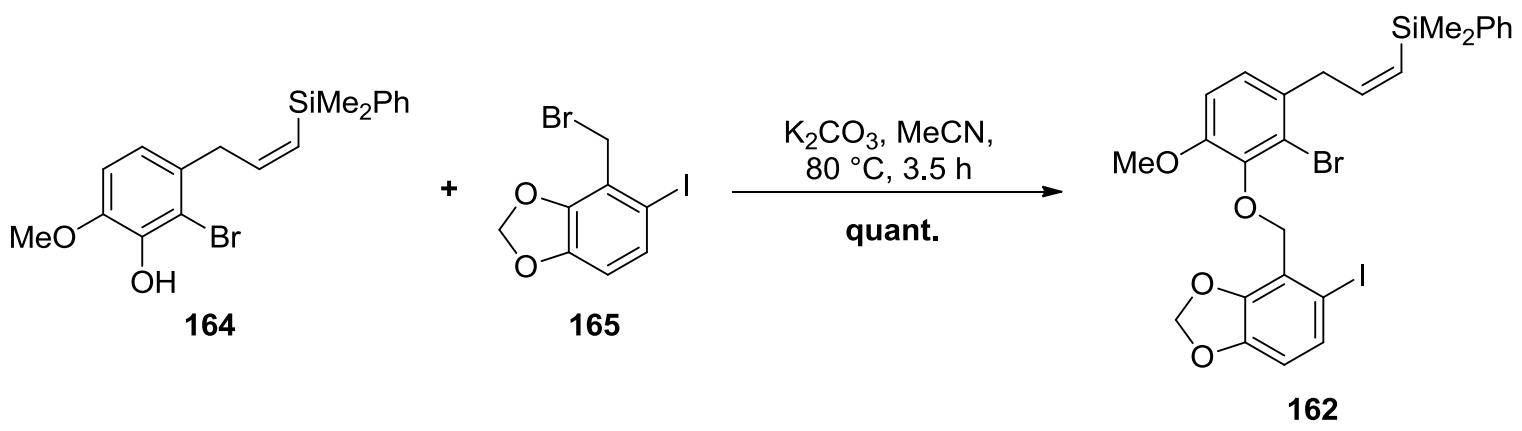

Abbildung 83: Alkylierung des Phenols 164 mit Benzylbromid 165 zum Benzylarylether 162.

Als letzter Baustein für die Synthese von (+)-Linoxepin (69) wurde der Silylether 249 für die bevorstehende Sonogashira-Reaktion hergestellt. Kommerziell erhältlicher Propargylalkohol (248) konnte unter Verwendung von TIPS-Chlorid, Triethylamin und katalytischen Mengen an 4-Dimethylaminopyridin in Dichlormethan in 83\% Ausbeute in das gewünschte Alkin 163 überführt werden (Abbildung 84).

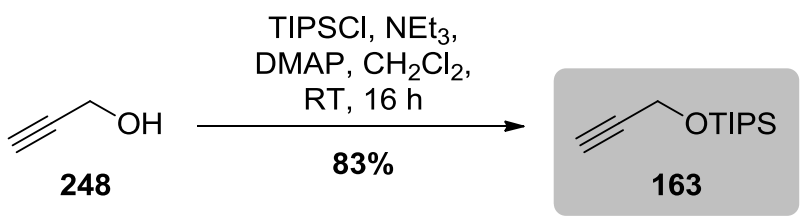

Abbildung 84: Synthese des Silylethers 163.

Da in vorangegangenen Arbeiten eine Einbindung der Sonogashira-Reaktion in die DominoSequenz zu untrennbaren Produktgemischen und verringerten Ausbeuten des gewünschten Dominoproduktes geführt hatte, sollte zunächst die Kupplung des Alkins 163 mit dem Benzylarylether 162 in einer separaten Reaktion erfolgen und das entstandene SonogashiraProdukt anschließend in einer Domino-Carbopalladierung-Heck-Reaktion in das gewünschte annellierte Oxepin 249 überführt werden.

Da die übliche Amin-basierte Variante der Sonogashira-Reaktion sich in dieser Substratklasse als nicht zielführend erwies, ${ }^{109}$ wurde für die Palladium-katalysierte Kupplung Tetrabutylammoniumacetat eingesetzt. ${ }^{123}$ Die Transformation lieferte mit katalytischen Mengen an 
Tetrakis(triphenylphosphin)palladium(0) und Kupfer(I)iodid das gewünschte SonogashiraProdukt 249 in einer sehr guten Ausbeute von 93\% (Abbildung 85).<smiles>COc1ccc(C/C=C\CSc2ccccc2)c(Br)c1OCc1c(I)ccc2c1OCO2</smiles>

162

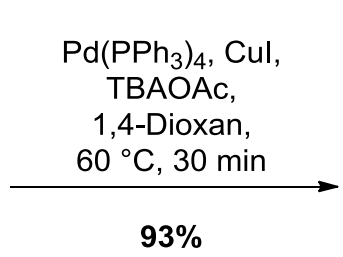<smiles>COc1ccc(C/C=C\CSc2ccccc2)c(Br)c1OCc1c(C#CCO[Na])ccc2c1OCO2</smiles>

Abbildung 85: Synthese des Domino-Vorläufers 249.

\subsection{Domino-Carbopalladierung-Heck-Reaktion zu Oxepin 161}

Bei der Durchführung der Domino-Carbopalladierung-Heck-Reaktion wurde auf Reaktionsbedingungen zurückgegriffen, die sich bei strukturell ähnlichen Substraten in vorangegangenen Arbeiten bewährt hatten. ${ }^{109}$ Mit Palladium(II)acetat, DavePhos (250), äquimolaren Mengen Silber(I)carbonat und katalytischen Mengen an 4-Dimethylaminopyridin in Toluol wurde das gewünschte Domino-Produkt 161 in exzellenten Ausbeuten von bis zu $99 \%$ erhalten (Abbildung 86).

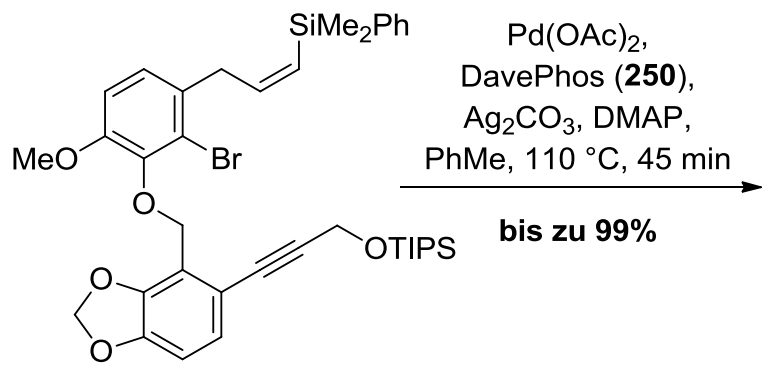

249

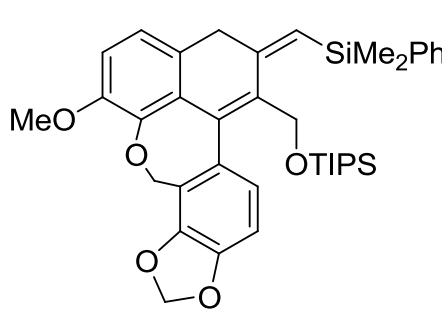

161

Abbildung 86: Synthese des Oxepins 161.

Durch den Einsatz des sterisch sehr anspruchsvollen und gleichzeitig sehr elektronenreichen Biarylsystems 250 als Ligand kann eine Reaktivitätssteigerung in der oxidativen Addition sowie der reduktiven Eliminierung herbeigeführt werden. Dies ist darauf zurückzuführen, dass durch den hohen sterischen Anspruch der Liganden hochreaktive monosubstituierte Palladiumkomplexe des Typs $\left[\mathrm{L}_{1} \mathrm{Pd}^{0}\right]$ gebildet werden können (vgl. Kapitel A. 5.1.1). ${ }^{124}$

Bei längeren Reaktionszeiten wurde vermehrt die Bildung des aromatischen Produktes 251 beobachtet (Abbildung 87). Die Isomerisierung der Doppelbindung ist höchstwahrscheinlich 
auf eine Readdition des Palladium-Katalysators mit anschließender $\beta$-Hydrid-Eliminierung zurückzuführen.

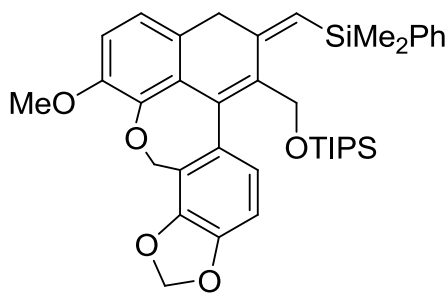

161

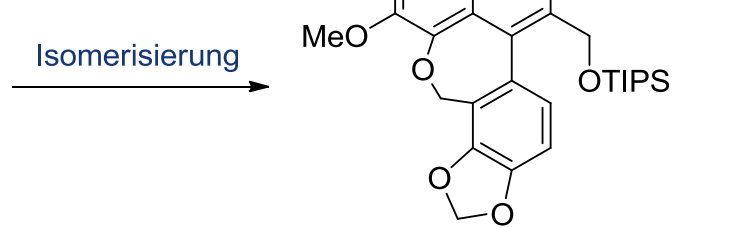

251

Abbildung 87: Isomerisierung des Domino-Produktes 161 zu dem aromatischen Oxepin 251.

Um diese ungewünschte Nebenreaktion $\mathrm{zu}$ verhindern, wurden äquimolare Mengen Silber(I)carbonat zugegeben, die über eine Aktivierung des Alkins die syn-Addition des Palladiums an die Dreifachbindung gegenüber einer Readdition an bereits gebildetes Produkt bevorzugen sollten. Zur Veranschaulichung dieser Tatsache ist in Abbildung 88 der postulierte Mechanismus der Domino-Carbopalladierung-Heck-Reaktion gezeigt.

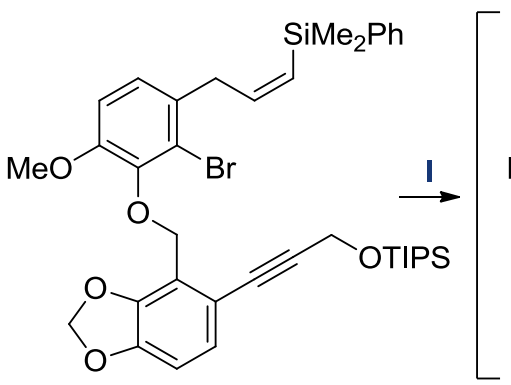

249<smiles>COc1ccc(C/C=C\[SH2+]c2ccccc2)c(Br)c1OCc1c(C#CC[OH+])ccc2c1OCO2</smiles>

252

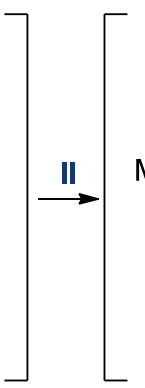

$\rightarrow$<smiles>CCCc1c(C#CCO[P-])ccc2c1OCO2</smiles>

253

III

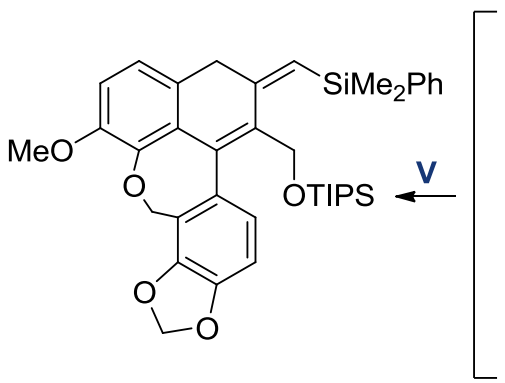

161

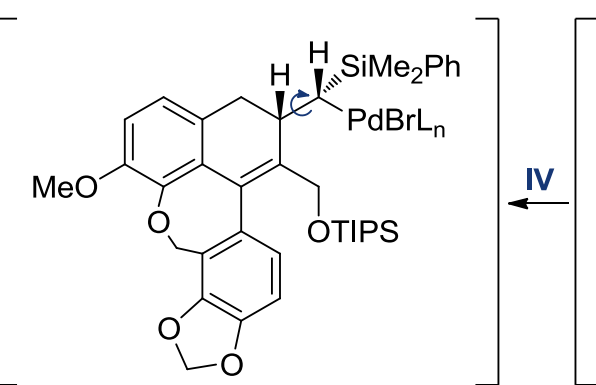

255

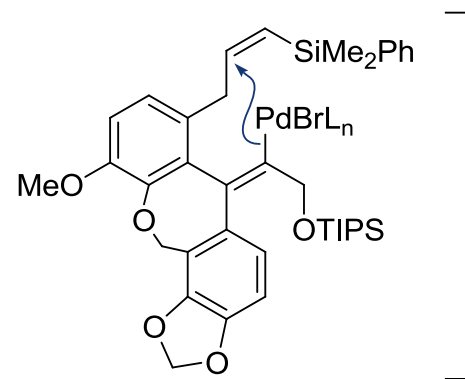

254

Abbildung 88: Postulierter Mechanismus der Domino-Carbopalladierung-Heck-Reaktion.

Zunächst erfolgt eine Aktivierung der Dreifachbindung über eine Koordination des als LewisSäure fungierenden Silberions (Schritt I). Nach der oxidativen Addition der PalladiumSpezies in die Aryl-Bromid-Bindung (Schritt II) kommt es zu einer intramolekularen Carbopalladierung der Dreifachbindung unter Ausbildung der Vinyl-Palladium-Spezies 254 
(Schritt III). Die Bildung der exocyclischen Doppelbindung $\mathrm{zu}$ dem favorisierten siebengliedrigen Ring ist dabei üblicherweise gegenüber der Bildung des achtgliedrigen Ringes bevorzugt. Im nächsten Schritt erfolgt eine 6-exo-trig-Insertion des Palladiums in die Vinylsilan-Doppelbindung, die zu der Akyl-Palladium-Spezies 255 mit neu gebildetem sechsgliedrigen Ring führt (Schritt IV). Nach Rotation der Einfachbindung wird im letzten Teil der Sequenz unter $\beta$-Hydrid-Eliminierung das gewünschte Domino-Produkt 161 gebildet (Schritt V).

\subsection{Asymmetrische Synthese des Diols 160}

Da die Versuche zur Einführung des stereogenen Zentrums über eine asymmetrische Hydrierung mit anschließender Tamao-Fleming-Oxidation in vorangegangenen Arbeiten nicht zielführend waren, ${ }^{109}$ sollte das Domino-Produkt 161 über eine asymmetrische Hydroborierung in den sekundären Alkohol 258 überführt werden. Unter Verwendung des in situ aus $\alpha$-Pinen generierten Borans 257 in Tetrahydrofuran konnte nach oxidativer Aufarbeitung mit Natriumhydroxid und Wasserstoffperoxid die gewünschte Verbindung 258 in einer guten Ausbeute von $77 \%$ erhalten werden (Abbildung 89). ${ }^{125}$

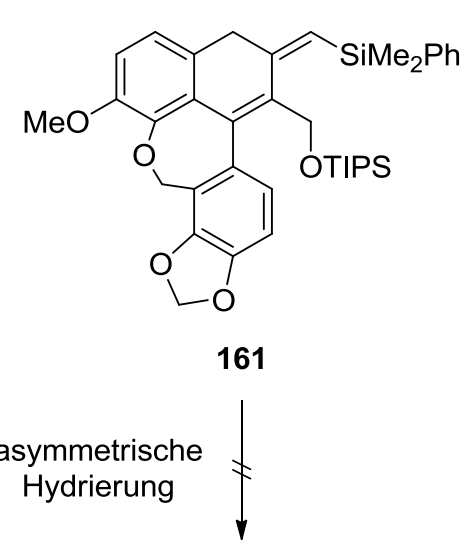

1. (-)-(ipc) $\mathrm{BH}_{2}(257)$, THF,

$0^{\circ} \mathrm{C} \rightarrow \mathrm{RT}, 17 \mathrm{~h}$

2. $\mathrm{NaOH}, \mathrm{H}_{2} \mathrm{O}_{2}$,
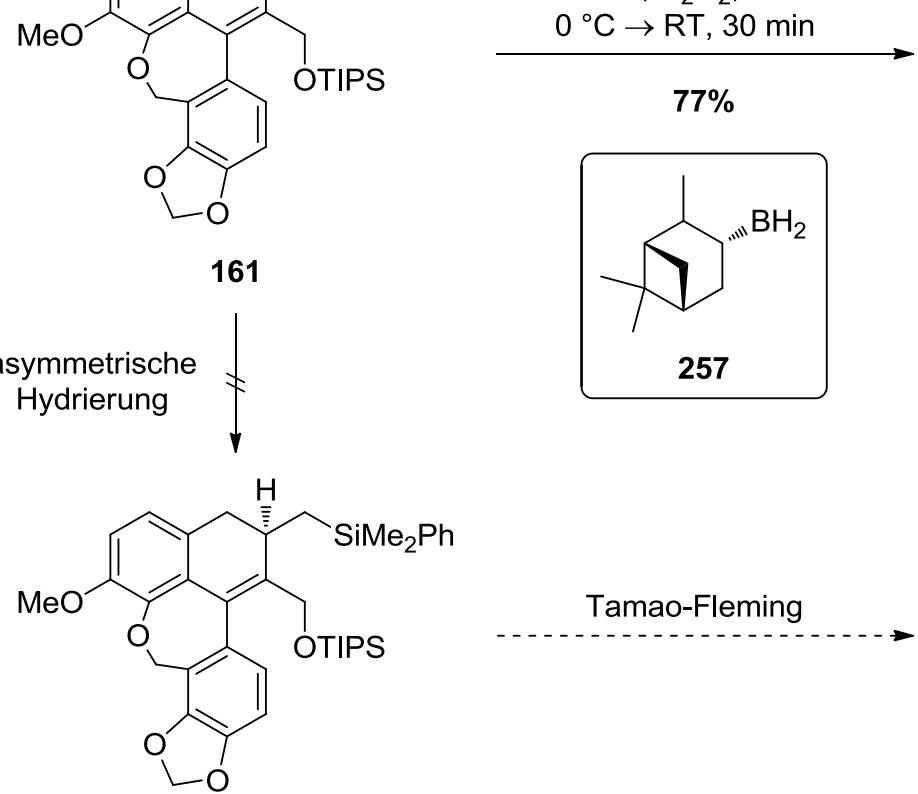

256<smiles>COc1ccc2c3c1OCc1c(ccc4c1OCO4)C3=C(CO[SnH3])[C@H]([C@H](O)[SiH2]c1ccccc1)C2</smiles>

\begin{tabular}{c|} 
TBAF, \\
THF, $0{ }^{\circ} \mathrm{C}, 1 \mathrm{~h}$
\end{tabular}<smiles>COc1ccc2c3c1CC[C@H](CO)C(CO)=C3c1ccc3c(c1CO2)OCO3</smiles>

160

$52 \%$ ee

Abbildung 89: Asymmetrische Hydroborierung des Domino-Produktes 161 und anschließende Desilylierung.

Das erhaltene Produkt wurde anschließend mit Tetrabutylammoniumfluorid in Tetrahydrofuran bei $0{ }^{\circ} \mathrm{C}$ in $89 \%$ Ausbeute in das Diol 160 überführt. An dieser Verbindung konnte 
mithilfe von analytischer HPLC an chiraler stationärer Phase ein Enantiomerenüberschuss von $52 \%$ ermittelt werden. In detaillierten Untersuchungen zur asymmetrischen Hydroborierung des Domino-Produktes 161, die auch eine Verwendung des Masamune-Borans ${ }^{126}$ beinhalten und nicht Teil dieser Arbeit sind, konnte der Enantiomerenüberschuss nicht weiter verbessert werden. ${ }^{127}$

Für den synthetischen Zugang zu dem enantiomerenreinen Naturstoff (+)-Linoxepin (69) wurden die beiden Enantiomere des Diols 160 über präparative HPLC an chiraler Phase ohne weiteren Substanzverlust getrennt. So konnten $76 \%$ des gewünschten $(R)$-Enantiomers von Verbindung 160 und 24\% des $(S)$-Diols 160 erhalten werden.

\subsection{Untersuchungen zur Stabilität der chiralen Achse des Diols 160}

Neben dem durch die asymmetrische Hydroborierung induzierten stereogenen Zentrum enthält das Diol 160 ebenso wie der Naturstoff (+)-Linoxepin (69) bei genauerer Betrachtung ein weiteres stereogenes Element in Form einer chiralen Achse. Die Verbindung kann nach der präparativen Abtrennung des ungewollten $R$-Enantiomers folglich in der $\left(S, S_{a}\right)$-Form oder der diastereomeren $\left(S, R_{a}\right)$-Form vorliegen (Abbildung 90).

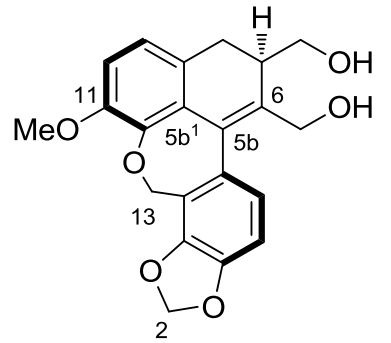

$\left(S, S_{a}\right)-160$
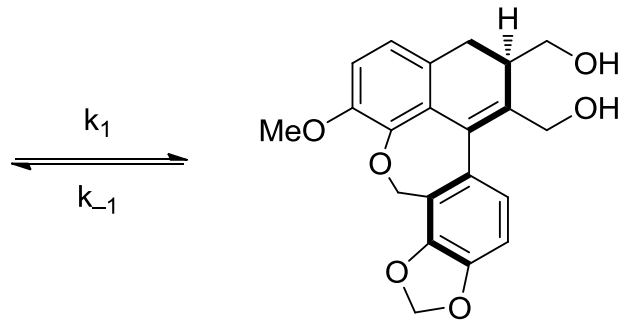

$\left(S, R_{a}\right)-160$

Abbildung 90: Beide möglichen Diastereomere des Diols 160.

Bei genauerer Untersuchung der analytischen Daten wurde ein zweiter Signalsatz in den NMR-Spektren der Verbindung entdeckt. Da die anderen Daten keine Hinweise auf Zersetzungsprodukte lieferten, muss davon ausgegangen werden, dass es sich um zwei bei Raumtemperatur interkonvertierbare Diastereomere handelt. Die unterschiedlichen Intensitäten der beiden Signalsätze deuten dabei auf unterschiedliche Grundzustandsenergien der möglichen Diastereomere $\left(S, S_{a}\right)$-160 und $\left(S, R_{a}\right)$-160 hin. Überraschenderweise zeigte sich, dass die Lage des thermodynamischen Gleichgewichts stark von dem Lösungsmittel beeinflusst wird. Während in deuteriertem Chloroform ein zweiter Signalsatz deutlich zu erkennen ist, fällt dieser in deuteriertem Acetonitril erst bei genauerer Betrachtung auf (Abbildung 91). Besonders deutlich ist der zweite Signalsatz bei den Methylengruppen des 
Oxepinrings $\left(4\right.$ '- $\left.\mathrm{H}_{2}\right)$ und des Allylalkohols $\left(1-\mathrm{H}_{2}\right)$ sowie den aromatischen Wasserstoffatomen zu beobachten (Abbildung 91).

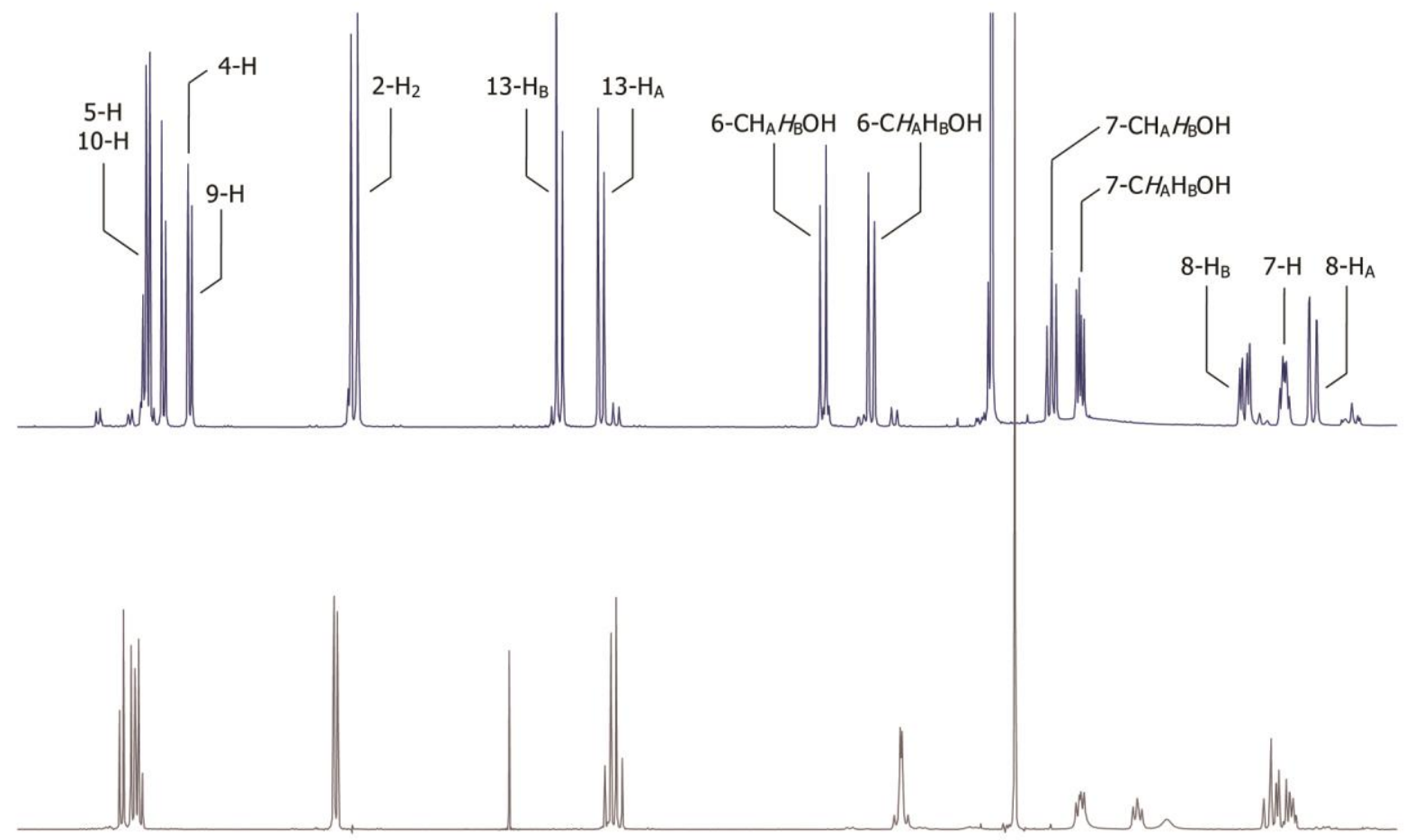

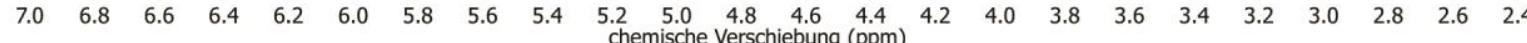

Abbildung 91: ${ }^{1}$ H-NMR-Spektren von Verbindung 160 in Acetonitril (grau) und Chloroform (blau).

Zur Untersuchung der Stabilität der chiralen Achse eignet sich die EXSY-Methode (EXSY = exchange spectroscopy). ${ }^{128}$ Diese Methode ist eine Form des zweidimensionalen NOEExperiments, bei der die Probe bei unterschiedlichen Mischzeiten und/oder Temperaturen gemessen wird. Bei dem Vorhandensein von zwei ineinander umwandelbaren Verbindungen sind sogenannte Crosspeaks zu beobachten, mit deren Intensität sich die Geschwindigkeitskonstante der jeweiligen Umwandlung bestimmen lässt. Mit Hilfe der Eyring-Gleichung kann aus diesen Werten anschließend die Höhe der Energiebarriere $\Delta \mathrm{G}^{\ddagger}$ bestimmt werden.

Die Aufnahme der NOESY-Spektren erfolgte in Acetonitril und Chloroform jeweils bei 273, 298 und $323 \mathrm{~K}$. Während bei niedrigen Temperaturen bereits ein deutlicher Austausch zwischen den Wasserstoffatomen der Haupt- und der Nebenkomponente zu verzeichnen ist, ist die Bestimmung der Geschwindigkeitskonstanten von Hin- und Rückreaktion bei $323 \mathrm{~K}$ aufgrund der starken Linienverbreiterung nicht mehr genau durchführbar. Der deutlich 
schnellere Austausch der Wasserstoffatome lässt dabei auf eine erleichterte Umwandlung der beiden Spezies schließen (Abbildung 92).

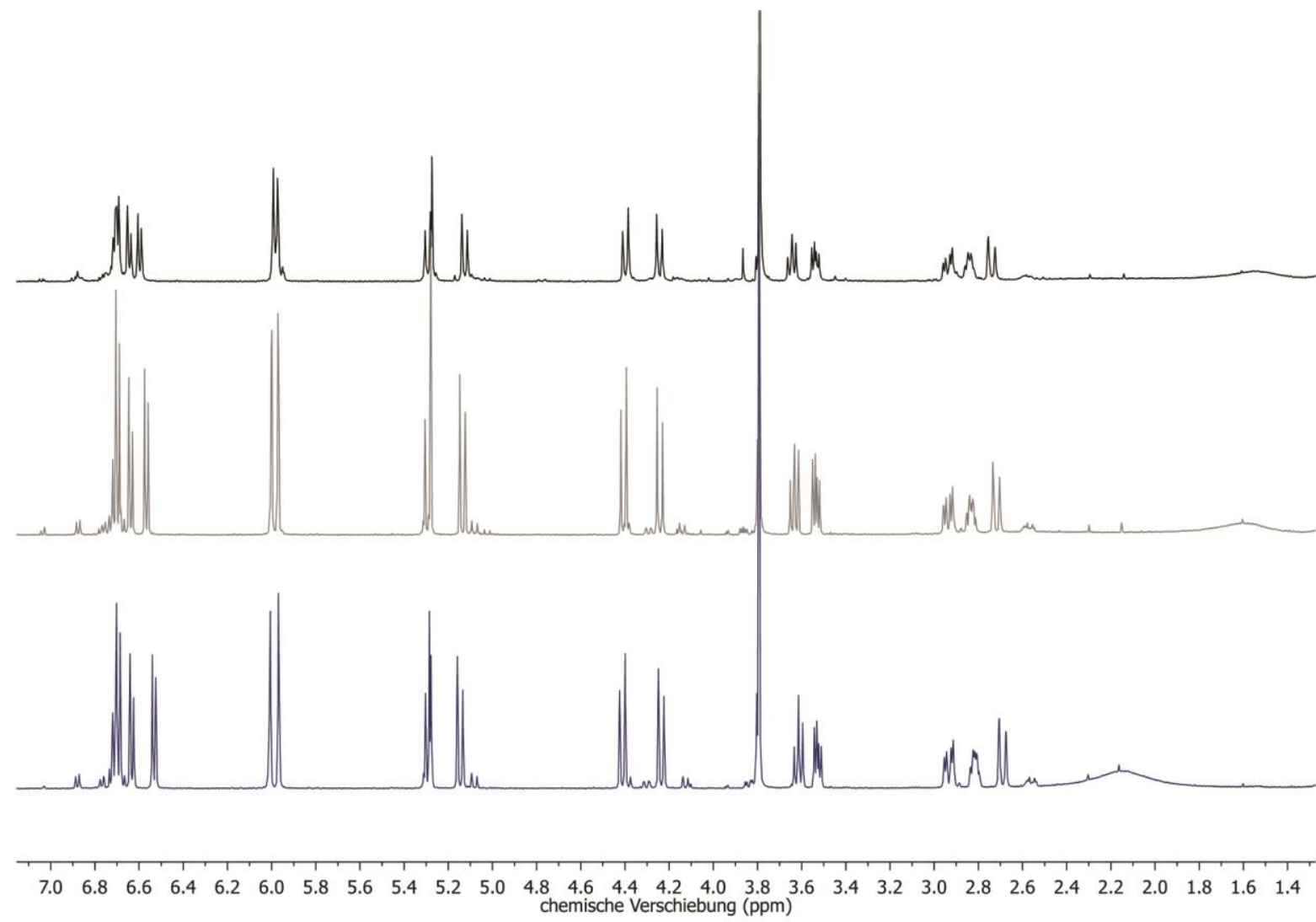

Abbildung 92: Temperaturabhängigkeit des ${ }^{1} \mathrm{H}$-NMR-Spektrums von Verbindung 160 in $\mathrm{CDCl}_{3}$ : $273 \mathrm{~K}$ (blau), $298 \mathrm{~K}$ (grau) und $323 \mathrm{~K}$ (schwarz).

Die quantitative Auswertung der NOESY-Spektren wurde mithilfe des Programms EXSYCalc der Firma MestreLab Research durchgeführt. Durch Matrixanalyse der Amplituden liefert dieses Programm die Geschwindigkeitskonstanten von Hin- und Rückreaktion. Über die Eyring-Gleichung wurden letztlich die Aktivierungsenthalpien $\Delta \mathrm{G}^{\ddagger}$ des Übergangs bestimmt (Tabelle 9).

$$
k=x \frac{k_{B} T}{h} e^{\frac{-\Delta G^{\ddagger}}{R T}}
$$

$k=$ Geschwindigkeitskonstante

$k_{B}=$ Boltzmann-Konstante, $1.381 \cdot 10^{-23} \mathrm{~J} \cdot \mathrm{K}^{-1}$

$T=$ Temperatur in Kelvin

$R=$ universelle Gaskonstante, $8.314 \mathrm{~J} \cdot \mathrm{mol}^{-1} \cdot \mathrm{K}^{-1}$

$h=$ Plancksches Wirkungsquantum, $6.626 \cdot 10^{-34} \mathrm{~J} \cdot \mathrm{s}$

$x=$ Transmissionskoeffizient (wird in der Regel $=1$ gesetzt). 
Bei der Bestimmung der Aktivierungsenthalpien wurde ein Mittelwert aus den Amplituden einiger signifikanter Crosspeaks gebildet. Die charakteristischen Signale sind in Abbildung 93 dargestellt.

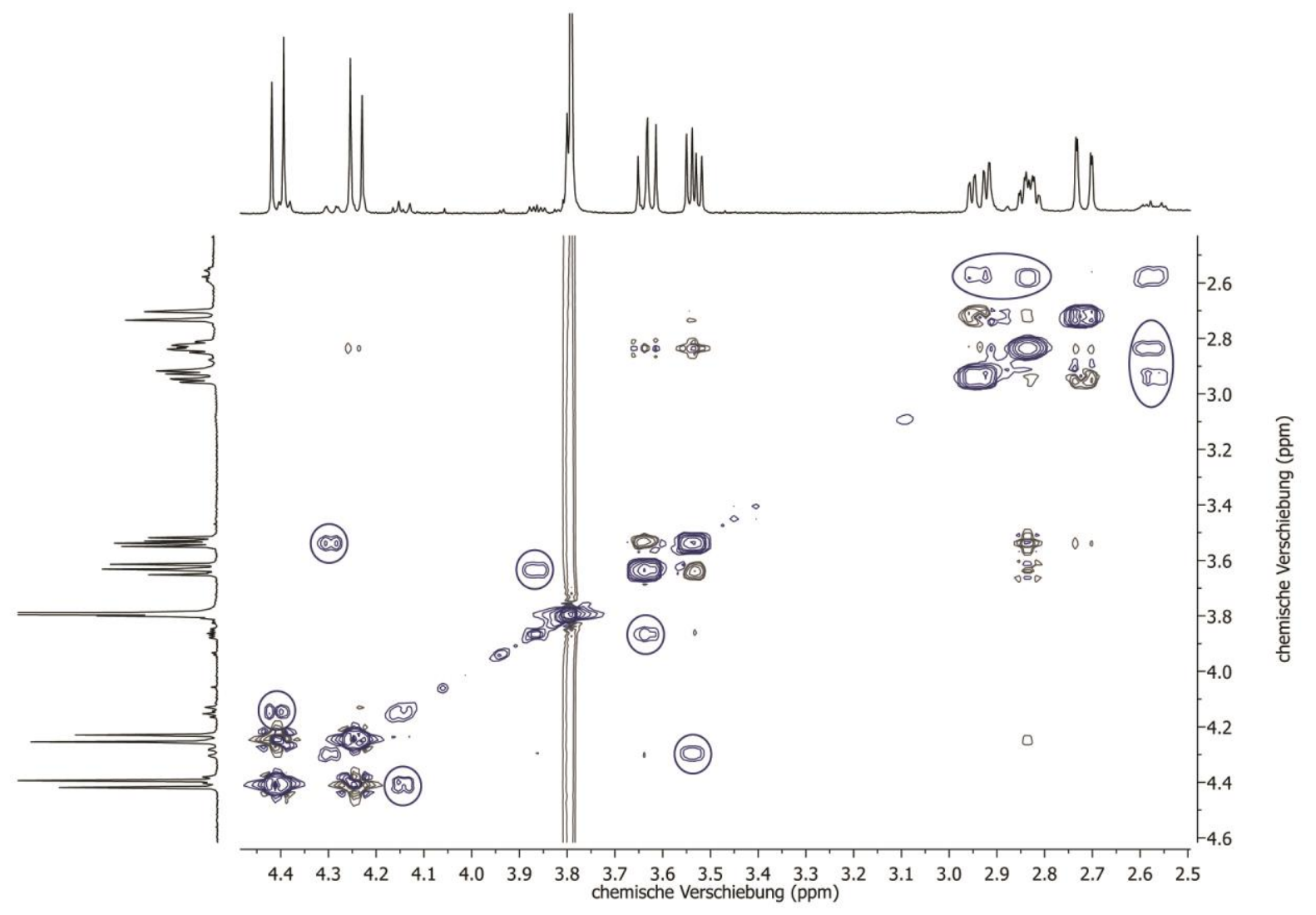

Abbildung 93: NOE-Spektrum von Verbindung 160 mit signifikanten Crosspeaks (Kreise).

Bei genauerer Betrachtung der Werte fällt auf, dass die berechneten Energiebarrieren bei hohen Temperaturen entgegen der Erwartungen sinken. Dies ist besonders in Acetonitril auf die durch die starke Linienverbreiterung ausgelöste hohe Fehlerhaftigkeit der berechneten Geschwindigkeitskonstanten zurückzuführen.

Damit zwei Verbindungen eine Lebensdauer von über $24 \mathrm{~h}$ aufweisen und somit physikalisch trennbar sind, müssen die Inversionsbarrieren der Verbindungen bei Raumtemperatur mindestens $100 \mathrm{~kJ} / \mathrm{mol}$ betragen. ${ }^{129}$ Die ermittelten Werte bestätigen somit die Vermutung, dass es sich um zwei Atropisomere handelt, die bei Raumtemperatur nicht stabil sind. Außerdem ist aus der Differenz der in Tabelle 9 aufgezeigten Aktivierungsenergien für die Hin- und Rückreaktion der Isomerisierung der Unterschied der freien Bildungsenthalpien der beiden Diastereomere zu entnehmen. Unter Nichtberücksichtigung der ungenauen Werte bei $323 \mathrm{~K}$ ergibt sich in Chloroform ein Wert von ca. $4.6 \mathrm{~kJ} / \mathrm{mol}$, während in dem polareren Acetonitril der Unterschied der Energiebarrieren und somit auch der Grundzustandsenergien der Isomere ca. $6.4 \mathrm{~kJ} / \mathrm{mol}$ beträgt. Da die Aktivierungsenthalpie der Rückreaktion in 
Acetonitril auch ca. $3.4 \mathrm{~kJ} / \mathrm{mol}$ niedriger als in Chloroform ist, liegt in Acetonitril hauptsächlich das Hauptdiastereomer vor, während in Chloroform deutlich ein zweiter Signalsatz zu erkennen ist.

Tabelle 9: Geschwindigkeitskonstanten und Aktivierungsenthalpien des Übergangs.

\begin{tabular}{ccccccc}
\hline $\boldsymbol{T}[\mathbf{K}]$ & Solvens & $\boldsymbol{t}_{\boldsymbol{m i x}}[\mathbf{m s}]$ & $\boldsymbol{k}_{\boldsymbol{l}}\left[\mathbf{s}^{-\mathbf{1}}\right]$ & $\boldsymbol{k} \boldsymbol{- I}_{\boldsymbol{I}}\left[\mathbf{s}^{\mathbf{- 1}}\right]$ & $\boldsymbol{\Delta} \boldsymbol{G}_{\mathbf{1}}^{\ddagger}[\mathbf{k J} / \mathbf{m o l}]$ & $\boldsymbol{\Delta} \boldsymbol{G}_{-\mathbf{1}}^{\ddagger}[\mathbf{k J} / \mathbf{m o l}]$ \\
\hline 273 & $\mathrm{CDCl}_{3}$ & 100 & $0.017 \pm 0.001$ & $0.13 \pm 0.01$ & 75.5 & 70.9 \\
298 & $\mathrm{CDCl}_{3}$ & 100 & $0.23 \pm 0.02$ & $1.6 \pm 0.2$ & 76.1 & 71.3 \\
323 & $\mathrm{CDCl}_{3}$ & 500 & $3 \pm 0.5$ & $20 \pm 3$ & 75.9 & 70.8 \\
273 & $\mathrm{CD}_{3} \mathrm{CN}$ & 100 & $0.032 \pm 0.002$ & $0.53 \pm 0.02$ & 74.0 & 67.7 \\
298 & $\mathrm{CD}_{3} \mathrm{CN}$ & 100 & $0.51 \pm 0.03$ & $6.6 \pm 0.3$ & 74.2 & 67.8 \\
323 & $\mathrm{CD}_{3} \mathrm{CN}$ & 500 & $8 \pm 2$ & $80 \pm 20$ & 70.8 & 67.0 \\
\hline
\end{tabular}

Die Ergebnisse der temperaturabhängigen EXSY-Experimente führen außerdem zu der Erkenntnis, dass eine selektive Bildung des stereogenen Zentrums während einer Dominoreaktion bei $110{ }^{\circ} \mathrm{C}$ in Abhängigkeit der chiralen Achse nicht möglich ist, da die berechneten Energiebarrieren zu gering sind, um bei dieser Temperatur eine Isomerisierung der Achse zu verhindern.

\subsection{Synthese von (+)-Linoxepin}

Ausgehend von dem enantiomerenreinen $(R)$-Diol 160 sollte die Synthese von (+)-Linoxepin (69) über eine selektive Oxidation des Allylalkohols und eine anschließende Lactonisierung erfolgen. Die Oxidation des Allylalkohols in Gegenwart einer aliphatischen Hydroxyfunktion wurde mit Mangan(IV)oxid in Dichlormethan bei Raumtemperatur realisiert (Abbildung 94).

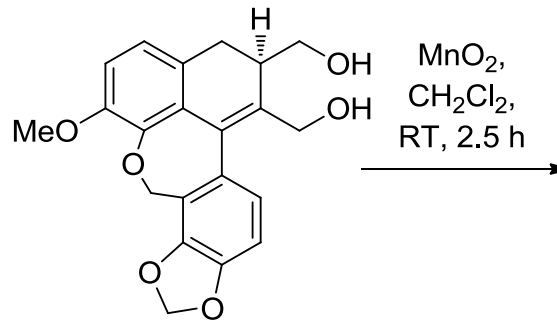

160

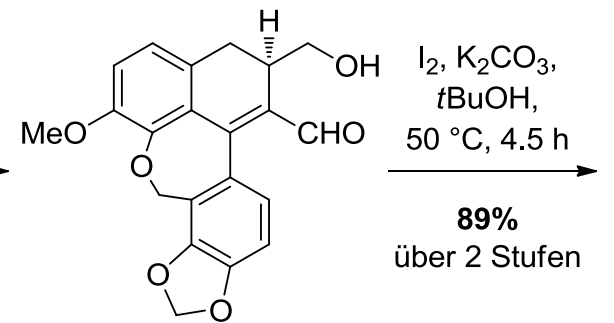

259

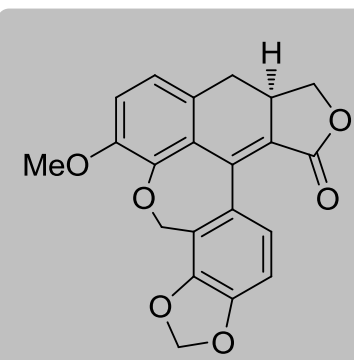

$(+)$-Linoxepin (69)

Abbildung 94: Synthese von (+)-Linoxepin (69). 
Der entstandene Aldehyd wurde ohne weitere Aufarbeitung unter Verwendung von Iod und Kaliumcarbonat in tert-Butanol bei $50{ }^{\circ} \mathrm{C}$ in die korrespondierende Carbonsäure überführt, die während der Aufarbeitung unter Bildung des Lactons in hervorragender Ausbeute von $89 \%$ über zwei Stufen zum gewünschten Naturstoff 69 führte. Analog zu der Synthese des bioidentischen (+)-Linoxepins (69) wurde aus dem $(S)$-Diol 160 auch das Enantiomer des Naturstoffes (ent-69) hergestellt. Bei dieser Synthesesequenz konnte ebenfalls eine Ausbeute von $89 \%$ über zwei Stufen erreicht werden.

Die enantioselektive Totalsynthese von (+)-Linoxepin (69) umfasst von Bromovanin (243) ausgehend elf Stufen und wurde mit einer Gesamtausbeute von 32\% abgeschlossen. Aufgrund des geringeren Substanzverlustes bei der Enantiomerentrennung ist sie somit die bis heute effizienteste Totalsynthese dieses Naturstoffes.

\subsection{Diskussion der spektroskopischen Daten von (+)-Linoxepin}

Im Folgenden sollen einige ausgewählte spektroskopische Daten des Naturstoffes (+)-Linoxepin (69) näher diskutiert werden. Die genaue Zuordnung der Signale in den NMRSpektren erfolgte unter Berücksichtigung der entsprechenden zweidimensionalen Spektren (COSY, HSQC und HMBC) sowie über die unterschiedlichen Intensitäten der Signale im ${ }^{13} \mathrm{C}-\mathrm{NMR}$. Außerdem war ein Vergleich mit den spektroskopischen Daten des bereits in der ersten Totalsynthese hergestellten (+)-Linoxepins möglich. ${ }^{54}$ Das ${ }^{1} \mathrm{H}-\mathrm{NMR}-$ Spektrum wurde bei Raumtemperatur in deuteriertem Chloroform bei einer Frequenz von $600 \mathrm{MHz}$ aufgenommen und ist in Abbildung 95 dargestellt.

Die beiden diastereotopen Wasserstoffatome an C-9 sind am stärksten hochfeldverschoben und resonieren getrennt bei $\delta=2.64 \mathrm{ppm}$ und $\delta=2.98 \mathrm{ppm}$. Das Signal bei $\delta=2.64 \mathrm{ppm}$ spaltet dabei in ein Dublett vom Triplett mit den Kopplungskonstanten von $J=14.7$ und $1.0 \mathrm{~Hz}$ auf, während das Signal bei $\delta=2.98 \mathrm{ppm}$ als Dublett vom Dublett mit den Kopplungskonstanten $J=14.7$ und $5.7 \mathrm{~Hz}$ erscheint. Die größere der beiden Kopplungskonstanten ist dabei auf die geminale Kopplung der beiden Wasserstoffatome sowie bei dem Dublett vom Triplett auf die vicinale Kopplung mit 9a-H zurückzuführen. Bei dem tieffeldverschobenerem Signal bei $\delta=2.98 \mathrm{ppm}$ beträgt die Kopplung zu dem am stereogenen Zentrum befindlichen 9a-H 5.7 Hz. Die Kopplungskonstante von $J=1.0 \mathrm{~Hz}$ von $9-\mathrm{H}_{\mathrm{A}}$ ist auf eine ${ }^{4} J$-Kopplung mit dem aromatischen Wasserstoffatom an C-8 zurückzuführen und bei $9-\mathrm{H}_{\mathrm{B}}$ nicht zu beobachten.

Das Wasserstoffatom 9a-H am stereogenen Zentrum zeigt bei $\delta=3.26 \mathrm{ppm}$ als Dublett vom Dublett vom Triplett mit den Kopplungskonstanten $J=14.7$, 8.8 und 5.7 Hz Resonanz und 
koppelt erwartungsgemäß mit den Methylengruppen an 9- und 10-Position. Die Wasserstoffatome der an C-6 substituierten Methoxygruppe zeigen ein Signal in Form eines Singuletts mit der typischen chemischen Verschiebung von $\delta=3.83 \mathrm{ppm}$. Etwas weiter tieffeldverschoben sind die Signale der beiden diastereotopen Wasserstoffatome $10-\mathrm{H}_{2}$ bei $\delta=4.01 \mathrm{ppm}$ und $\delta=4.66 \mathrm{ppm}$ zu finden. Beide Signale erscheinen als Triplett mit einer Kopplungskonstante von $J=8.8 \mathrm{~Hz}$, die in dem Signal des 9a-H wiederzufinden ist.

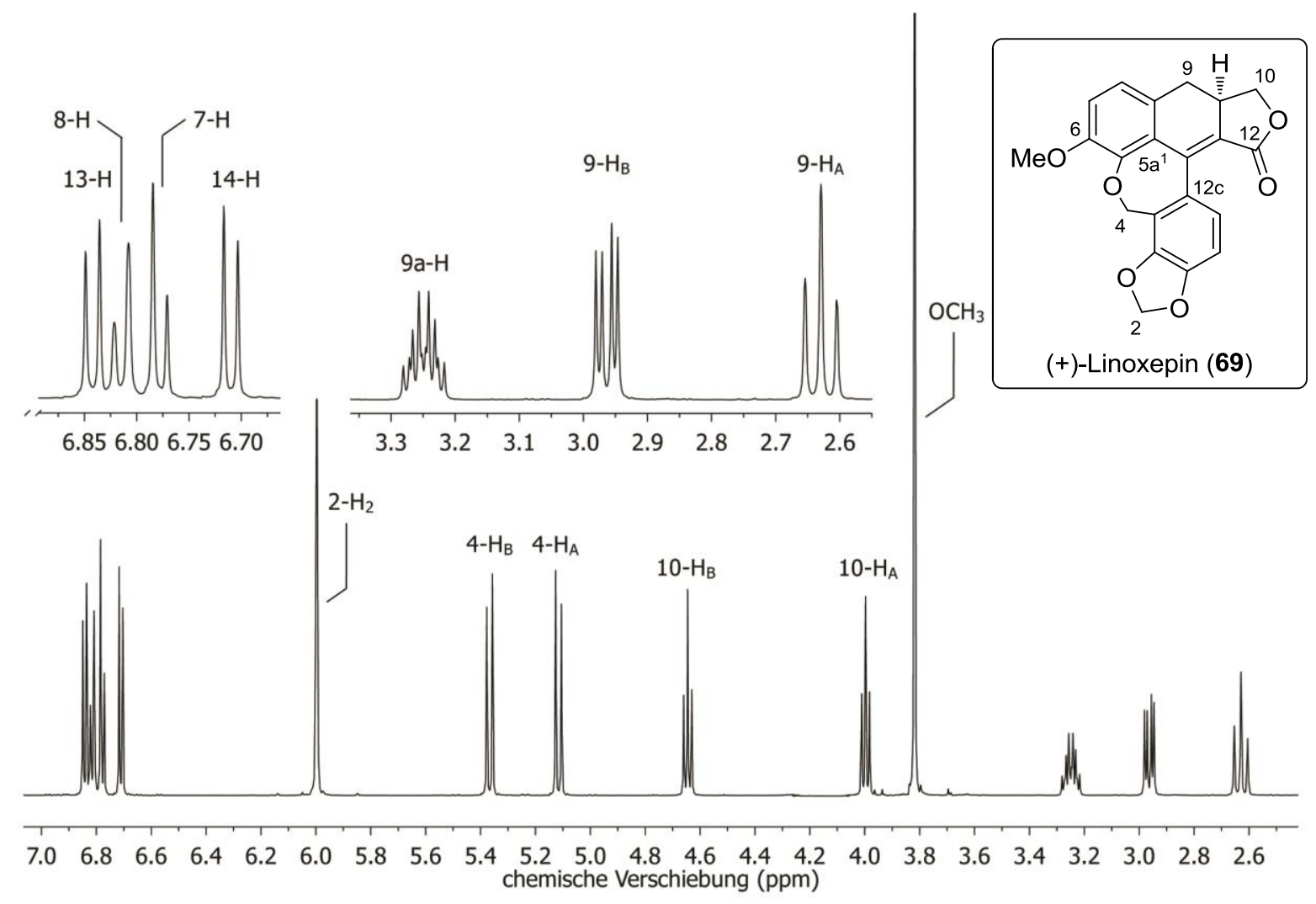

Abbildung 95: ${ }^{1} \mathrm{H}-\mathrm{NMR}-$ Spektrum von (+)-Linoxepin (69).

Die ebenfalls diastereotopen Wasserstoffatome 4- $\mathrm{H}_{2}$ des Oxepinrings zeigen bei $\delta=5.12 \mathrm{ppm}$ und $\delta=5.37 \mathrm{ppm}$ in Form eines Dubletts mit einer geminalen Kopplung von $J=12.5 \mathrm{~Hz}$ Resonanz. Durch einen weiteren Sauerstoff-Substituenten entschirmt erscheinen die Signale der Methylendioxy-Einheit 2- $\mathrm{H}_{2}$ weiter tieffeldverschoben bei $\delta=6.01 \mathrm{~Hz}$ in Form zweier Dubletts mit einer Kopplungskonstante von $J=1.9 \mathrm{~Hz}$ für die geminale Kopplung.

In dem für aromatische Wasserstoffatome charakteristischen Bereich sind von $\delta=6.70-6.90 \mathrm{ppm}$ vier Signale zu finden. Die Signale bei $\delta=6.78 \mathrm{ppm}$ und $\delta=6.83 \mathrm{ppm}$ zeigen beide eine für Aromaten typische ortho-Kopplung von $J=8.2 \mathrm{~Hz}$, wobei bei dem stark tieffeldverschobenem Dublett vom Dublett zusätzlich die ${ }^{4} J$-Kopplung zu dem 9- $\mathrm{H}_{\mathrm{A}}$ von $J=1.0 \mathrm{~Hz}$ wiederzufinden ist. Da die ortho-Kopplung der beiden Wasserstoffatome über einen Crosspeak im COSY-Spektrum bestätigt wurde, wurde das Signal bei $\delta=6.78$ ppm 7-H 
zugeordnet und das Signal bei $\delta=6.83 \mathrm{ppm}$ dem Wasserstoffatom am C-8. Das Dublett bei $\delta=6.72 \mathrm{ppm}$ weist eine für Aromaten typische ortho-Kopplung von $J=8.0 \mathrm{~Hz}$ auf, die in dem Dublett bei $\delta=6.85 \mathrm{ppm}$ wiederzufinden ist. Mit Hilfe des HMBC-Spektrums konnte das Signal bei $\delta=6.72 \mathrm{ppm}$ eindeutig als $14-\mathrm{H}$ identifiziert werden. Dem über das COSYSpektrum bestätigten Kopplungspartner 13-H wurde folglich das Signal bei $\delta=6.85 \mathrm{ppm}$ zugeordnet.

Das ${ }^{13} \mathrm{C}-\mathrm{NMR}-$ Spektrum wurde bei einer Frequenz von $126 \mathrm{MHz}$ und ebenfalls in deuteriertem Chloroform bei Raumtemperatur aufgenommen (Abbildung 96). Die Zuordnung der Wasserstoff-tragenden Kohlenstoffatome erfolgt größtenteils über das HSQC-Spektrum, während die quartären Kohlenstoffatome mithilfe des HMBC-Spektrums identifiziert wurden.

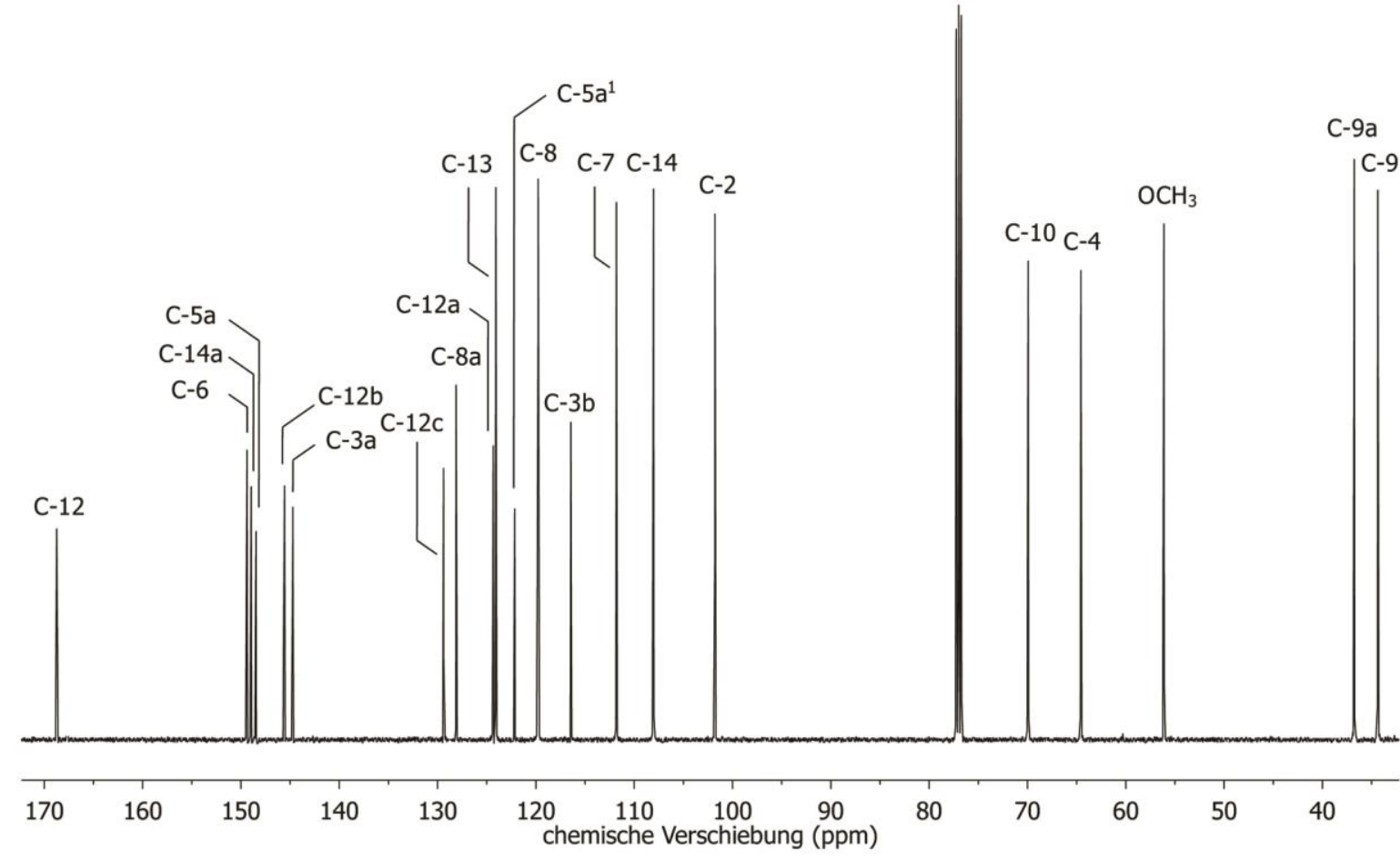

Abbildung 96: ${ }^{13}$ C-NMR-Spektrum von (+)-Linoxepin (69).

Analog zu dem ${ }^{1} \mathrm{H}-\mathrm{NMR}-$ Spektrum resonieren die Methylengruppe an C-9 und das Kohlenstoffatom am stereogenen Zentrum C-9a mit $\delta=34.5 \mathrm{ppm}$ und $\delta=36.9 \mathrm{ppm}$ bei hohem Feld. Das Signal der aromatensubstituierenden Methoxygruppe an C-6 zeigt indes eine erwartungsgemäße chemische Verschiebung von $\delta=56.2 \mathrm{ppm}$, während die Signale bei $\delta=64.7 \mathrm{ppm}$ und $\delta=70.0 \mathrm{ppm}$ der Oxepin-Methylengruppe C-4 und der lactonischen Methylengruppe C-10 zugeordnet wurden. Aufgrund der zwei Sauerstoff-Substituenten ist das Signal der acetalischen Methylengruppe C-2 am stärksten entschirmt und resoniert tieffeldverschoben bei $\delta=101.8 \mathrm{ppm}$. Im aromatischen Bereich des ${ }^{13} \mathrm{C}-\mathrm{NMR}$-Spektrums 
konnten die Signale bei $\delta=108.1,111.8,119.8$ und 124.1 ppm mit Hilfe des HSQCSpektrums eindeutig den Wasserstoff-tragenden Kohlenstoffatomen C-14, C-7, C-8 und C-13 zugeteilt werden.

Die Signale mit geringerer Intensität wurden mithilfe des HMBC-Spektrums analysiert. So konnten die Resonanzen bei $\delta=116.5,122.2,124.3,128.1$ und 129.4 ppm den quartären Kohlenstoffatomen C-3b, C-5 $\mathrm{a}^{1}, \mathrm{C}-12 \mathrm{a}, \mathrm{C}-8 \mathrm{a}$ und C-12c zugeordnet werden. Die Kohlenstoffatome C-3a, C-5a, C-14a und C-6 werden durch das benachbarte Sauerstoffatom stark entschirmt und resonieren so bei $\delta=144.7,148.5,149.0$ und $149.4 \mathrm{ppm}$. Aufgrund der Ladungsalternanz der benachbarten $\pi$-Systeme wird das C-12b gegenüber den anderen quartären, nicht Heteroatom-substituierten Kohlenstoffatomen sehr stakt entschirmt und zeigt ein Signal bei $\delta=145.6$ ppm. Das Kohlenstoffatom der Carbonyleinheit des Lactons zeigt erwartungsgemäß stark tieffeldverschoben bei $\delta=168.7$ ppm Resonanz.

Neben der NMR-Spektroskopie wurde der Naturstoff 69 auch über massenspektrometrische Analysen eindeutig identifiziert. Das ESI-Spektrum weist einen Basispeak bei dem Masse-zuLadungs-Verhältnis $m / z=741.2$ für das $[2 \mathrm{M}+\mathrm{Na}]^{+}$-Addukt auf. Neben den erwartungsgemäßen Konjugaten $[\mathrm{M}+\mathrm{H}]^{+}(\mathrm{m} / \mathrm{z}=365.1,46 \%)$ und $[\mathrm{M}+\mathrm{Na}]^{+}(\mathrm{m} / \mathrm{z}=387.1)$ lag auch das $[3 \mathrm{M}+\mathrm{Na}]^{+}$-Addukt bei $\mathrm{m} / z=1115.3$ mit $17 \%$ Intensität vor. Die Summenformel $\mathrm{C}_{21} \mathrm{H}_{16} \mathrm{O}_{6}$ von (+)-Linoxepin (69) mit der berechneten exakten Masse von $\mathrm{m} / \mathrm{z}=365.1020$ für das $[\mathrm{M}+\mathrm{H}]^{+}$-Addukt wurde überdies durch ESI-HRMS mit einer gefundenen Masse von $m / z=365.1019$ bestätigt. Die analytischen Daten stimmen somit mit denen des in vorangegangenen Arbeiten auf racemischem Wege synthetisierten (+)-Linoxepin überein. ${ }^{54}$ Da der erhaltene Naturstoff auffällige Fluoreszenzeigenschaften aufwies, wurden außerdem ein Anregungsspektrum und ein Emissionsspektrum aufgenommen (Abbildung 97).

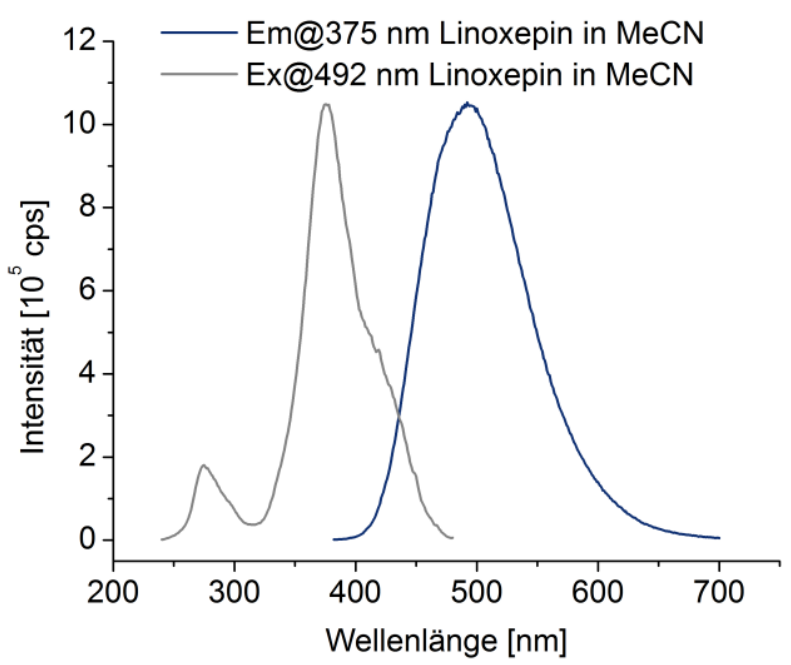

Abbildung 97: Anregungsspektrum (grau) und Emissionsspektrum (blau) von (+)-Linoxepin (69). 
Die kleine Bande bei einer Wellenlänge von $275 \mathrm{~nm}$ im Anregungsspektrum ist auf den für aromatische Systeme typischen $\pi-\pi *$-Übergang zwischen dem elektronischen Grundzustand $\mathrm{S}_{0}$ und dem dritten elektronisch angeregten Zustand $\mathrm{S}_{3}$ zurückzuführen. ${ }^{130}$ (+)-Linoxepin (69) absorbiert im nahen UV-Bereich Licht einer Wellenlänge von $375 \mathrm{~nm}$ und emittiert türkises Licht bei 492 nm. Der Stokes-Shift, also die Differenz der höchsten Absorptionsbande des Anregungsspektrums und des Emissionsmaximums beträgt ca. $117 \mathrm{~nm}$. 


\section{Synthese von potentiellen Hsp47-Inhibitoren}

Der dritte Teil dieser Arbeit beschäftigt sich mit der Synthese von potentiellen Hsp47Inhibitoren des Typs 84. Da in vorangegangenen Untersuchungen bereits Variationen am Thiophen-Substituenten der Verbindung durchgeführt wurden, ${ }^{110}$ war eine Modifikation des Phenylrings Hauptaugenmerk dieses Projekts.

\subsection{Synthese des Dihydropyrimidons 166}

Das Dihydropyrimidon 166 wurde über eine Multikomponenten-Eintopf-Reaktion aus den kommerziell erhältlichen Substanzen Thiophen-2-carbaldehyd (168), Thioharnstoff (169) und Ethylcyanoacetat (170) hergestellt (Abbildung 98). Die Zugabe von Kaliumcarbonat in Ethanol lieferte bei $78{ }^{\circ} \mathrm{C}$ das gewünschte Produkt nach Umkristallisation aus Wasser in 54\% Ausbeute.

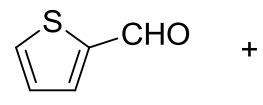

168<smiles>NC(N)=S</smiles>

169

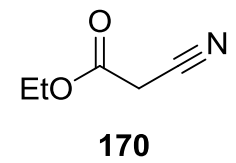

170
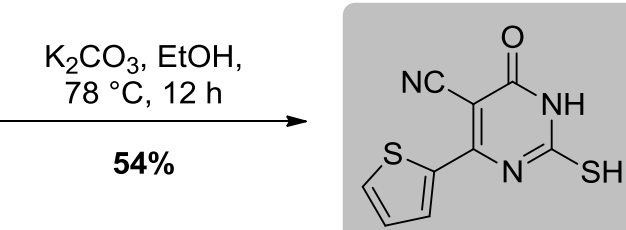

166

Abbildung 98: Synthese des Dihydropyrimidons 166 über eine Multikomponenten-Reaktion.

\subsection{Synthese der $\alpha$-Bromacetophenone 167a-g}

Die bisherige Synthese des Methylketons 261a verlief über zwei Stufen und sah zunächst eine Bildung des Weinreb-Amids 260a vor. Eine anschließende Methylierung führte zu dem gewünschten Acetophenon 261a in 70\% Gesamtausbeute (Abbildung 99).<smiles>Cc1cc(C(=O)O)ccc1Cl</smiles>

$171 \mathrm{a}$

A: $M e L i, \mathrm{Et}_{2} \mathrm{O}, \mathrm{THF}$,

$0{ }^{\circ} \mathrm{C} \rightarrow \mathrm{RT}, 2.5 \mathrm{~h}, 65 \%$

B: CuCN, MeLi, THF, $0^{\circ} \mathrm{C} \rightarrow \mathrm{RT}, 14 \mathrm{~h}, 73 \%$

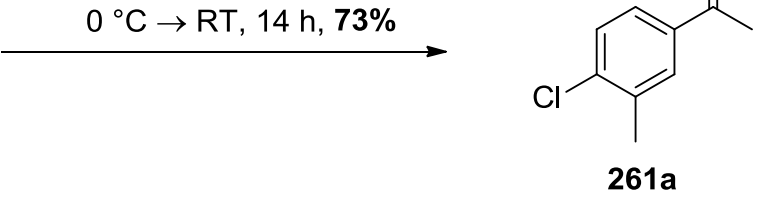

$N, O$-Dimethylhydroxylamin, HOBt, EDCI, DIPEA, DMF, RT, 22 h
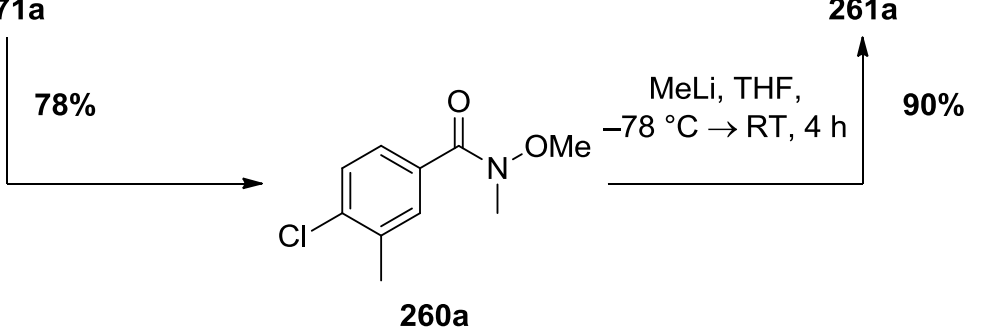

Abbildung 99: Unterschiedliche Reaktionspfade zu Methylketon 261a. 
Um einen kürzeren Zugang zu den Methylketonen zu ermöglichen und die Syntheseroute somit zu verkürzen, wurde die direkte Methylierung der Benzoesäure 171a untersucht. Die Umsetzung mit Methyllithium in einem Diethylether-Tetrahydrofuran-Gemisch analog zu der Gilman-van Ess-Methode lieferte das gewünschte Acetophenon 261a in einer Ausbeute von $65 \% .{ }^{131}$ Als unerwünschtes Nebenprodukt wurde in dieser Reaktion der durch zweifache Methylierung entstandene tertiäre Alkohol isoliert.

Eine weitere Möglichkeit der direkten Ketonsynthese bildet der Einsatz von in situ generierten Organocupraten. ${ }^{132}$ Unter Verwendung von Kupfer(I)cyanid und Methyllithium in Tetrahydrofuran konnte die Ausbeute auf $73 \%$ gesteigert werden. Analog zu dieser Methode wurden sechs weitere, unterschiedlich substituierte Acetophenone 261b-g mit Ausbeuten von bis zu 89\% hergestellt (Abbildung 100).<smiles>[R][R]1ccc(C(=O)O)cc1</smiles>
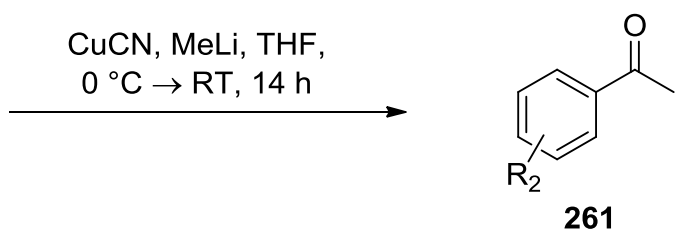<smiles>CC(=O)c1ccc(Cl)c(C(C)=O)c1</smiles>

$73 \%$<smiles>CC(=O)c1ccc(Cl)cc1C</smiles>

261b

$71 \%$<smiles>CCCCCC(=O)c1ccccc1C</smiles>

$261 \mathrm{c}$ $52 \%$<smiles>CC(=O)c1ccc(C)c(Cl)c1</smiles>

261d $87 \%$<smiles>CC(=O)c1ccc(Cl)c(C(C)(F)F)c1</smiles>

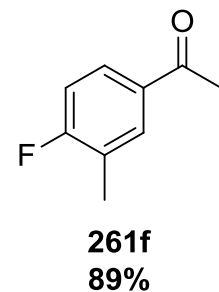

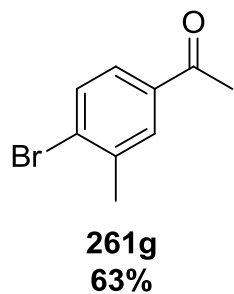

Abbildung 100: Synthese der Methylketone 261a-g.

Neben den Konstitutionsisomeren 261b-d, die sich durch die Position des Methyl- oder Chlor-Substituenten von 261a unterscheiden, wurde auch die Methylgruppe gegen einen Trifluormethyl-Substituenten ausgetauscht (261e) sowie das Halogen in para-Position variiert (261f-g). Der Einsatz von elektronegativeren Substituenten bringt durch eine geringere Elektronendichte im Ring eine Verbesserung der Ausbeute mit sich $(\mathrm{f}(\mathrm{F})>\mathrm{a}(\mathrm{Cl})>\mathrm{g}(\mathrm{Br})$; e $\left(\mathrm{CF}_{3}\right)>$ a $\left.\left(\mathrm{CH}_{3}\right)\right)$. Bei der Änderung des Substitutionsmusters fiel auf, dass die orthosubstituierten Acetophenone 261b und 261c $\mathrm{zu}$ einer Deaktivierung führen, die höchstwahrscheinlich auf sterische Aspekte zurückzuführen ist. Besonders Verbindung 261c 
mit einem Chlor-Substituenten in ortho-Position konnte nur in einer geringen Ausbeute von lediglich 52\% erhalten werden. Durch den Austausch der beiden Substituenten in 3- und 4-Position (261d) wurde jedoch eine sehr gute Ausbeute von $87 \%$ erreicht.

Die Bromierung der resultierenden Methylketone 261a-g in $\alpha$-Position erfolgte unter Verwendung von Phenyltrimethylammoniumtribromid (PTAT) in Tetrahydrofuran und führte in guten Ausbeuten von bis zu 87\% zu den gewünschten $\alpha$-Bromacetophenonen 167a-g (Abbildung 101). ${ }^{133}$ Die elektronischen und sterischen Faktoren scheinen in dieser Reaktion eher eine untergeordnete Rolle zu spielen, da die geringe Varianz in der Ausbeute keinem eindeutigen Muster zu folgen scheint. Eine nennenswerte Ausnahme bildet das $\alpha$-Bromacetophenon 167d, das als einzige Verbindung einen Halogen-Substituenten in meta-Stellung aufweist und die mit Abstand beste Ausbeute von 87\% erbringt.

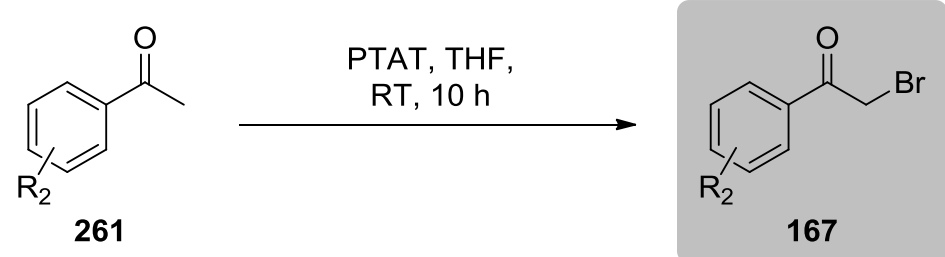<smiles>Cc1cc(C(=O)CBr)ccc1Cl</smiles><smiles>Cc1cc(Cl)ccc1C(=O)CBr</smiles>

$70 \%$<smiles>Cc1cccc(C(=O)CBr)c1Cl</smiles>

$167 \mathrm{c}$ $63 \%$<smiles>Cc1ccc(C(=O)CBr)cc1Cl</smiles><smiles>O=C(CBr)c1ccc(Cl)c(C(F)(F)F)c1</smiles>

$167 e$

$66 \%$<smiles>[B]CC(=O)c1ccc(F)c(C)c1</smiles>

$167 f$

$77 \%$<smiles>Cc1cc(C(=O)CBr)ccc1Br</smiles>

Abbildung 101: Synthese der $\alpha$-Bromacetophenone 167a-g.

\subsection{Synthese der potentiellen Hsp47-Inhibitoren 84a-g}

Die Kupplung der beiden Bausteine erfolgte über eine nucleophile Substitution unter Zusatz von Kaliumcarbonat in Dimethylformamid. Um die zweifache Substitution des $\alpha$-Bromacetophenons 167 am Dihydropyrimidonring zu vermeiden, wurden die beiden Komponenten im Verhältnis 1:1 eingesetzt. Eine Umkristallisation aus Methanol oder Ethanol führte zu den gewünschten potentiellen Hsp47-Inhibitoren 84a-g in bis zu 54\% Ausbeute (Abbildung 102). Ein Rückschluss vom Substitutionsmuster auf die Ausbeute ist schwierig, da die sehr 
unterschiedlichen aber durchweg problematischen Lösungs- und Kristallisationseigenschaften der Verbindungen zu hohen Ausbeuteverlusten führten. Als auffällig erwiesen sich aber die besonders guten Ausbeuten für die Fluor-substituierte Verbindung 84f (53\%) und den potentiellen Inhibitor mit Chlor-Substituenten in meta-Position 84d (54\%) sowie die mäßige Ausbeute bei der Verwendung eines ortho-Chlor-Substituenten 84c (20\%). Bereits in den vorangegangenen Syntheseschritten wurden diese Trends in der Ausbeute beobachtet. Da die geplanten medizinischen Tests zur Bestimmung der Aktivität besonders reine Verbindungen erforderten und aufgrund der geringen Löslichkeit der Substanzen eine präparative chromatographische Trennung nicht möglich war, war eine Reinigung über Kristallisation unabdingbar.

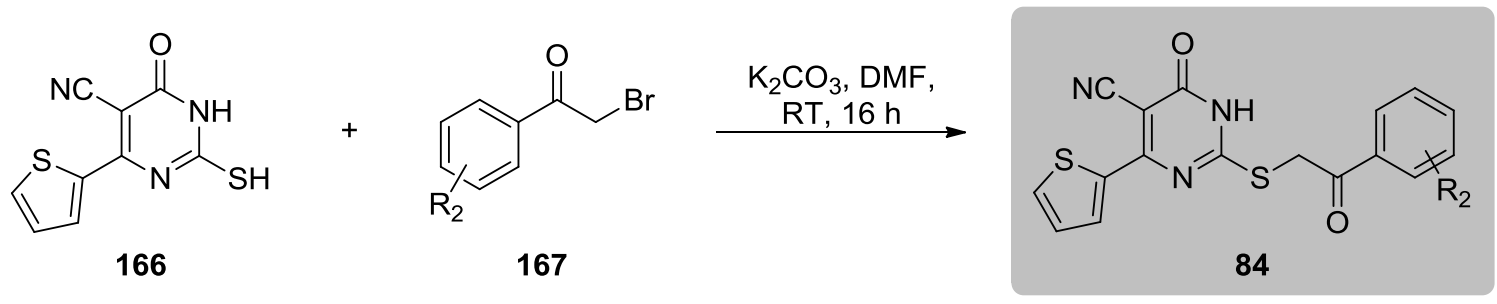<smiles>Cc1cc(C(=O)CSc2nc(-c3cccs3)c(C#N)c(=O)[nH]2)ccc1Cl</smiles><smiles>Cc1cc(Cl)ccc1C(=O)CSc1nc(-c2cccs2)c(C#N)c(=O)[nH]1</smiles><smiles>Cc1cccc(C(=O)CSc2nc(-c3cccs3)c(C#N)c(=O)[nH]2)c1Cl</smiles><smiles>Cc1ccc(C(=O)CSc2nc(-c3cccs3)c(C#N)c(=O)[nH]2)cc1Cl</smiles><smiles>N#Cc1c(-c2cccs2)nc(SCC(=O)c2ccc(Cl)c(C(F)(F)F)c2)[nH]c1=O</smiles><smiles>Cc1cc(C(=O)CSc2nc(-c3cccs3)c(C#N)c(=O)[nH]2)ccc1F</smiles><smiles>Cc1cc(C(=O)CSc2nc(-c3cccs3)c(C#N)c(=O)[nH]2)ccc1Br</smiles>

Abbildung 102: Synthese der potentiellen Hsp47-Inhibitoren 84a-g.

Zusammengefasst wurden neben der bereits bekannten Verbindung 84a sechs weitere potentielle Hsp47-Inhibitoren mit unterschiedlichem Substitutionsmuster am aromatischen 
Benzolsystem hergestellt. Die ursprüngliche Syntheseroute konnte bei leicht verbesserten Ausbeuten um eine Stufe verkürzt werden und führte in drei linearen Stufen ausgehend von den kommerziell erhältlichen Benzoesäuren 171a-g in bis zu $41 \%$ Gesamtausbeute zu den gewünschten Zielverbindungen. Nach dem in Kapitel A. 4.4 beschriebenen Verfahren ${ }^{71}$ erfolgt die Bestimmung der biologischen Aktivität im Rahmen einer Kooperation mit dem Arbeitskreis Zimmermann. Bei der Verfassung dieser Dissertation lagen noch keine Ergebnisse zur inhibitorischen Wirkung der synthetisierten Verbindungen vor.

\subsection{Diskussion der spektroskopischen Daten von Verbindung 84f}

Im Folgenden sollen einige ausgewählte spektroskopische Daten eines potentiellen Hsp47Inhibitors näher diskutiert werden. Aufgrund der pharmakologischen Bedeutung von FluorSubstituenten und der resultierenden interessanten Aufspaltung der Signale wurde Verbindung 84f für die Diskussion ausgewählt. Die genaue Zuordnung der Signale erfolgte über entsprechende zweidimensionale NMR-Spektren (COSY, HSQC und HMBC) sowie über die unterschiedlichen Intensitäten der Signale im ${ }^{13} \mathrm{C}-\mathrm{NMR}$. Das ${ }^{1} \mathrm{H}-\mathrm{NMR}$-Spektrum wurde bei $35^{\circ} \mathrm{C}$ in deuteriertem Dimethylsulfoxid bei einer Frequenz von $600 \mathrm{MHz}$ aufgenommen und ist in Abbildung 103 dargestellt.

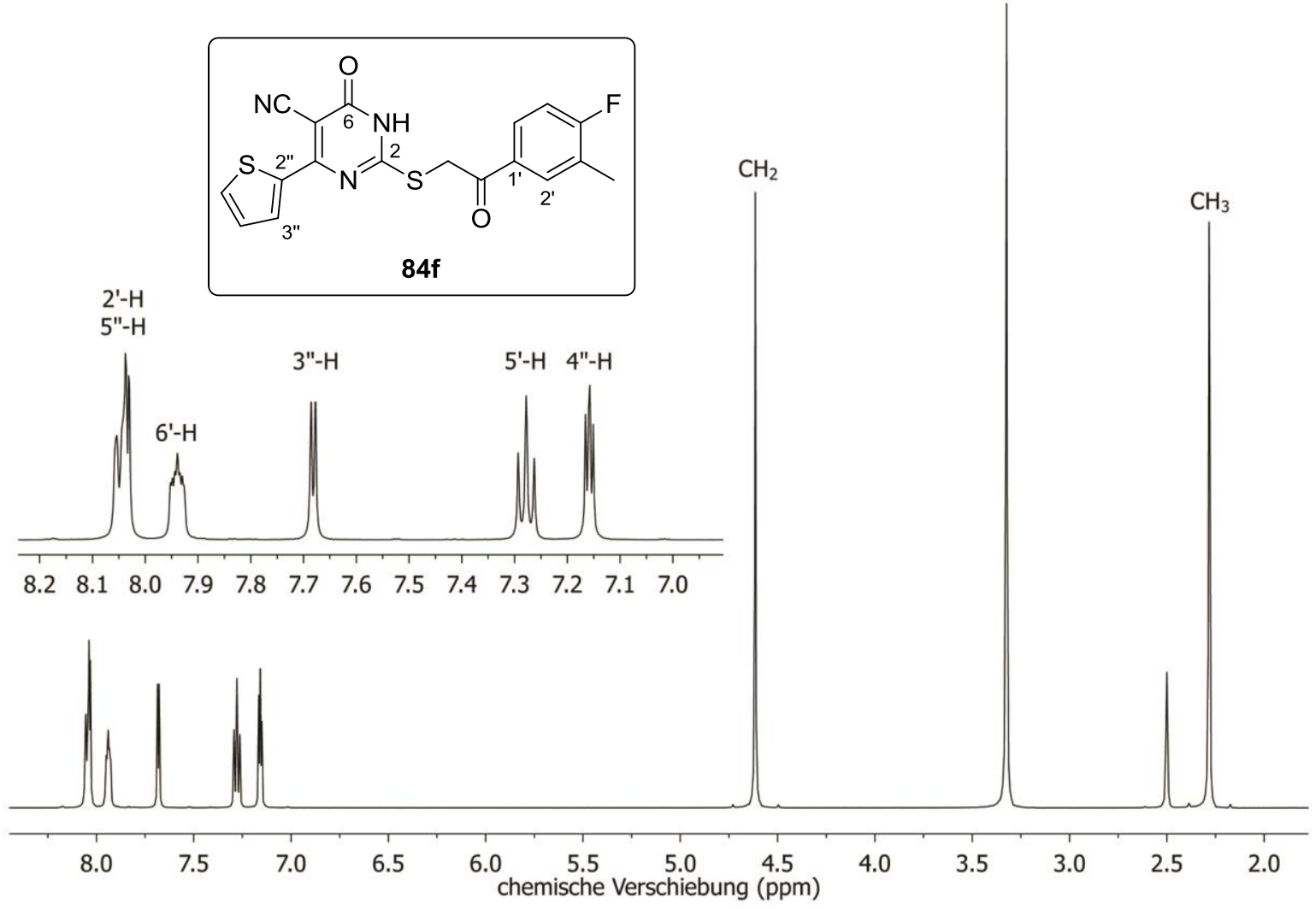

Abbildung 103: ${ }^{1} \mathrm{H}-\mathrm{NMR}$ Spektrum von Verbindung $84 f$. 
Am stärksten ins Hochfeld verschoben resonieren erwartungsgemäß die Wasserstoffatome der aromatischen Methylgruppe bei $\delta=2.32 \mathrm{ppm}$ in Form eines Singuletts. Ebenfalls als Singulett und durch den benachbarten Thioether und die Carbonylgruppe entschirmt erscheint das Signal der verknüpfenden Methylengruppe bei $\delta=4.65 \mathrm{ppm}$.

Im aromatischen Bereich des Spektrums wurden die Signale über das Kopplungsmuster und die Crosspeaks im COSY-Spektrum zugeteilt. Das Dublett vom Dublett bei $\delta=7.20$ mit den Kopplungskonstanten $J=4.9 \mathrm{~Hz}$ und $4.0 \mathrm{~Hz}$ konnte so mit eindeutig dem 4"-H am ThiophenRing zugeordnet werden. Das Signal des 5'-H erscheint als Pseudotriplett mit einer Kopplungskonstante von $J=9.1 \mathrm{~Hz}$ bei $\delta=7.32 \mathrm{ppm}$. Neben der Interaktion mit dem benachbarten aromatischen Wasserstoffatom 6'-H liegt auch eine Kopplung mit dem FluorSubstituenten an 4'-Position vor. Das Dublett vom Dublett bei $\delta=7.72 \mathrm{ppm}$ ist dem 3"-H zuzuordnen und zeigt neben der ortho-Kopplung von $J=4.9 \mathrm{~Hz}$ auch eine meta-Kopplung von $J=0.7 \mathrm{~Hz}$ zu dem 5"-H. Bei $\delta=7.98 \mathrm{ppm}$ zeigt das 6'-H ein Signal in Form eines zentrierten Multipletts. Die erwartete ortho-Kopplung zu 5'-H sowie die meta-Kopplung zu 2'-H und die Kopplung zu dem Fluor-Substituenten konnten nicht eindeutig aufgeschlüsselt werden. Bei $\delta=8.07$ ppm resoniert das 5"-H in Form eines Dubletts vom Dublett mit den erwarteten Kopplungskonstanten von $J=4.0$ und $0.7 \mathrm{~Hz}$. Das Signal überlagert sich mit dem Dublett vom Dublett des $2 '-\mathrm{H}$ bei $\delta=8.09 \mathrm{ppm}$. Die Kopplungskonstante von $J=7.8 \mathrm{~Hz}$ ist dabei auf die Kopplung mit dem Fluor-Substituenten zurückzuführen während die kleinere Kopplungskonstante von $J=2.0 \mathrm{~Hz}$ über eine meta-Kopplung mit dem Wasserstoffatom an C-6' zu erklären ist. Aufgrund von schnellen Austauschprozessen ist in dem Spektrum kein Signal für das Wasserstoffatom an N-1 bzw. N-3 zu finden.

Das ${ }^{13} \mathrm{C}$-NMR-Spektrum wurde bei einer Frequenz von $126 \mathrm{MHz}$ und in deuteriertem Dimethylsulfoxid bei $35^{\circ} \mathrm{C}$ aufgenommen (Abbildung 104). Die Zuordnung der Wasserstofftragenden Kohlenstoffatome erfolgte größtenteils über das HSQC-Spektrum, während die quartären Kohlenstoffatome mithilfe des HMBC-Spektrums identifiziert wurden.

Im Hochfeldbereich des Spektrums sind erwartungsgemäß das Signal für die aromatische Methylgruppe bei $\delta=14.9 \mathrm{ppm}$ sowie das Signal der verknüpfenden Methylengruppe bei $\delta=38.2 \mathrm{ppm} z u$ finden. Das $\mathrm{sp}^{2}$-hybridisierte C-5 des Dihydropyrimidonsystems zeigt eine typische Verschiebung von $\delta=86.2 \mathrm{ppm}$ während die substituierte Cyano-Gruppe wie für aromatische Nitrile üblich bei $\delta=121.2 \mathrm{ppm}$ resoniert. Das Dublett bei $\delta=116.2 \mathrm{ppm}$ konnte dem C-5' zugeordnet werden, wobei die Kopplungskonstante von $J=22.9 \mathrm{~Hz}$ auf den benachbarten Fluor-Substituenten zurückzuführen ist. Ebenfalls als Dublett mit einer 
Kopplungskonstante von $J=17.8 \mathrm{~Hz}$ erscheint das Signal des quartären Kohlenstoffatoms in 3'-Position.

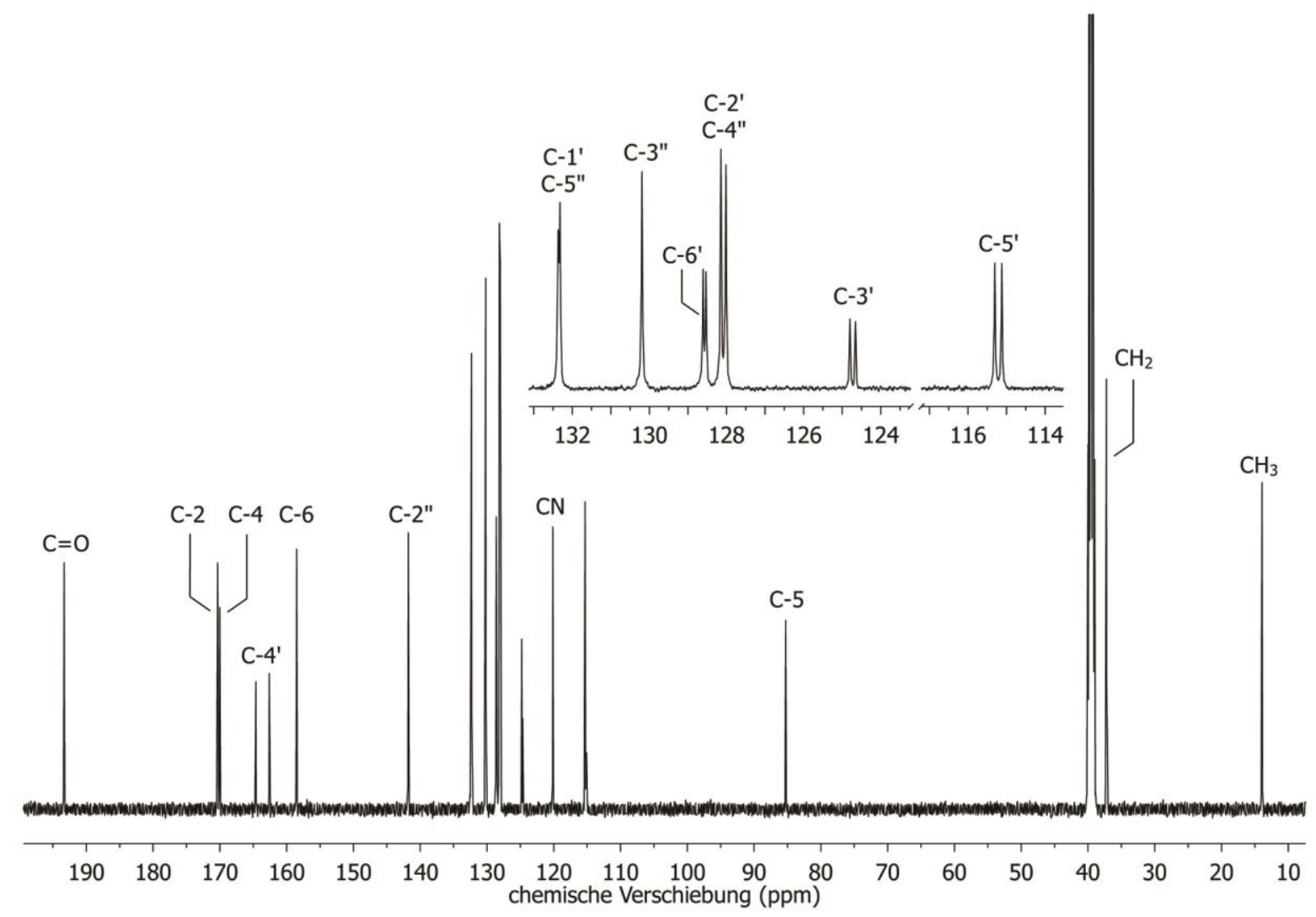

Abbildung 104: ${ }^{13} \mathrm{C}-\mathrm{NMR}-$ Spektrum von Verbindung $84 \mathrm{f}$.

In dem Bereich von $\delta=128.9-129.1 \mathrm{ppm}$ befinden sich die Signale von C-4" und C-2'. Aufgrund der durch den Fluor-Substituenten hervorgerufenen Aufspaltung des C-2'-Signals ist eine genauere Zuordnung jedoch nicht möglich. Das Signal für das aromatische C-6' bei $\delta=129.5 \mathrm{ppm}$ zeigt ebenfalls eine Aufspaltung von $J=9.4 \mathrm{~Hz}$, während das Wasserstoffatom-tragende Kohlenstoffatom C-3" des Thiophens bei $\delta=131.2$ ppm resoniert. Bei $\delta=133.3 \mathrm{ppm}$ sind zwei Signale zu erkennen, die mithilfe des HSQC- und HMBCSpektrums dem C-5" und dem quartären C-1' zugeordnet werden konnten.

Durch das benachbarte Schwefelatom stark entschirmt, ist das Signal für das C-2" bei $\delta=142.8$ ppm zu finden. Das Signal des C-6 des Dihydropyrimidons zeigt eine für AmidKohlenstoffatome typische chemische Verschiebung von $\delta=159.5 \mathrm{ppm}$. Die direkte Bindung des C-4' zu dem Fluoratom in ipso-Position führt bei dem Signal bei $\delta=164.6 \mathrm{ppm}$ zu einer sehr starken Aufspaltung von $J=251.3 \mathrm{~Hz}$. Mit einer chemischen Verschiebung von $\delta=194.3 \mathrm{ppm}$ am stärksten ins Tieffeld verschoben ist das Signal der verbrückenden Carbonyleinheit zu finden. 
In dem ${ }^{19} \mathrm{~F}-\mathrm{NMR}$-Spektrum, das ebenfalls bei $35^{\circ} \mathrm{C}$ in deuteriertem Dimethylsulfoxid aufgenommen wurde, resoniert das Fluoratom bei $\delta=105.3 \mathrm{ppm}$ in Form eines zentrierten Multipletts.

Neben der NMR-Spektroskopie wurde der potentielle Hsp47-Inhibitor auch über massenspektrometrische Analysen identifiziert. Das ESI-Spektrum weist im positiven Modus einen Basispeak bei dem Masse-zu-Ladungs-Verhältnis $m / z=424.0$ für das $[\mathrm{M}+\mathrm{K}]^{+}$-Addukt auf. Des Weiteren sind auch die erwartungsmäßen Konjugate $[\mathrm{M}+\mathrm{H}]^{+}(\mathrm{m} / \mathrm{z}=386.0)$ und $[\mathrm{M}+\mathrm{Na}]^{+}(\mathrm{m} / \mathrm{z}=408.0)$ vorhanden. Im negativen Modus ist der Basispeak bei $\mathrm{m} / \mathrm{z}=384.0$ dem $[\mathrm{M}-\mathrm{H}]^{-}$-Konjugat sowie der Peak bei $\mathrm{m} / \mathrm{z}=769.1 \mathrm{dem}[2 \mathrm{M}-\mathrm{H}]^{-}$-Addukt zuzuordnen. Die Summenformel $\mathrm{C}_{18} \mathrm{H}_{11} \mathrm{FN}_{3} \mathrm{O}_{2} \mathrm{~S}_{2}$ mit der berechneten exakten Masse von $\mathrm{m} / \mathrm{z}=384.0282$ für das $[\mathrm{M}-\mathrm{H}]^{-}$-Addukt wurde überdies durch ESI-HRMS mit einer gefundenen Masse von $m / z=384.0285$ bestätigt. 


\section{ZUSAMMENFASSUNG \& AUSBLICK}

Das Ziel dieser Arbeit war der Zugang zu Agrar- und Pharmawirkstoffen durch organische Synthese. Aufgrund der teils schwierigen und kostspieligen Gewinnung von biologisch aktiven Naturstoffen aus natürlichen Quellen nimmt die technische Herstellung von neuen Wirkstoffen immer mehr an Bedeutung zu. Im ersten Teil wurde ein neuer Zugang zu Spinosyn-Analoga untersucht, in dem mit dem Ziel der Syntheseeffizienz und Wirtschaftlichkeitssteigerung strukturelle Veränderungen eingebaut wurden, welche die Komplexität der natürlichen Struktur verringern.

Zunächst wurde das tricyclische Grundgerüst 149 ausgehend von den kommerziell erhältlichen Bausteinen 3-Methoxybenzaldehyd (176) und L-Rhamnose sowie dem bereits synthetisierten Cyclopentenderivat rac-182 hergestellt. Ausgehend von Benzaldehyd 176 gelang die Synthese des Tricyclus 149 in acht Stufen mit einer Gesamtausbeute von 10\% (Abbildung 105).
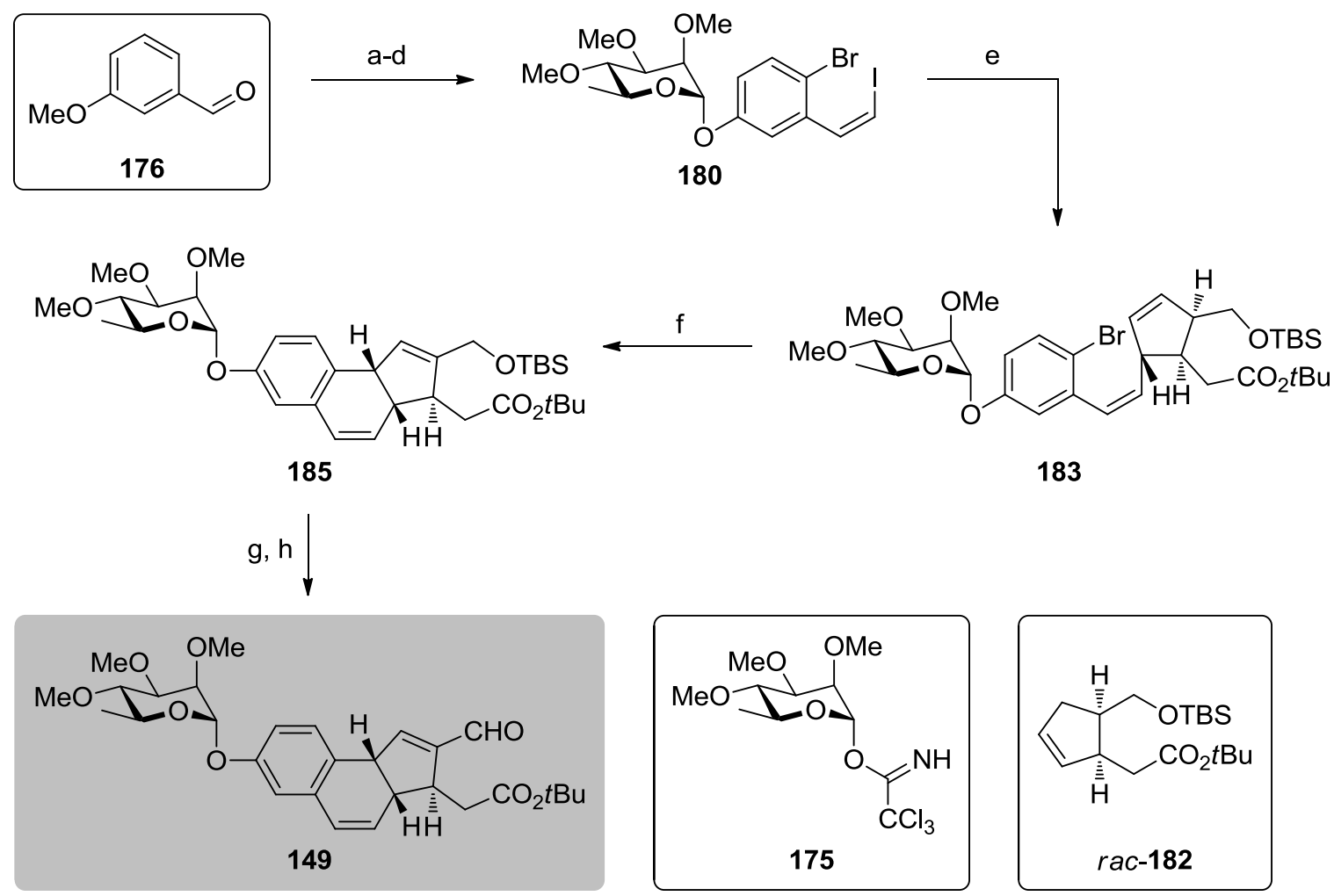

Abbildung 105: Synthese des tricyclischen Aldehyds 149: a) $\mathrm{Br}_{2}, \mathrm{CH}_{2} \mathrm{Cl}_{2}, \mathrm{RT}, 18 \mathrm{~h}, 82 \%$; b) $\mathrm{BBr}_{3}, \mathrm{CH}_{2} \mathrm{Cl}_{2}$, $-20{ }^{\circ} \mathrm{C}, 20 \mathrm{~h}, 80 \%$ (91\% brsm); c) 175, TMSOTf, MS $4 \AA \mathrm{A}^{2} \mathrm{CH}_{2} \mathrm{Cl}_{2}, 0{ }^{\circ} \mathrm{C}, 30 \mathrm{~min}, 85 \%$; d) $\left[\mathrm{Ph}_{3} \mathrm{PCH}_{2} \mathrm{I}\right]^{+} \mathrm{I}^{-}$, KHMDS, THF, $-78{ }^{\circ} \mathrm{C}, 90 \mathrm{~min}, 55 \%$; e) rac-182, Pd(OAc) $)_{2}, \mathrm{NaOAc}, \mathrm{TBACl}, \mathrm{DMF}, \mathrm{RT}, 24 \mathrm{~h}, 67 \%$; f) Herrmann-Beller-Katalysator, TBAOAc, DMF, MeCN, $\mathrm{H}_{2} \mathrm{O}, 130{ }^{\circ} \mathrm{C}, 3 \mathrm{~h}, 58 \%$; g) $p \mathrm{TsOH} \cdot \mathrm{H}_{2} \mathrm{O}, \mathrm{MeOH}$, $0{ }^{\circ} \mathrm{C}, 4.5 \mathrm{~h}, 96 \%$; h) DMP, $\mathrm{CH}_{2} \mathrm{Cl}_{2}, 0{ }^{\circ} \mathrm{C}, 90 \mathrm{~min}, 75 \%$. 
Die Schlüsselschritte dieser Synthesesequenz bestehen in zwei aufeinanderfolgenden Palladium-katalysierten Heck-Reaktionen, über die die beiden Ringsysteme 180 und 182 miteinander verknüpft werden.

Bei dem Aufbau der Seitenkette 214, die im Verlauf der Synthese den Makrocyclus D stellen soll, bestand die Hauptproblematik in dem enantioselektiven Aufbau des sekundären Alkohols und der Vermeidung von Schutzgruppenscrambling. Der MEM-Ether 204 stellte sich schließlich als das beste Substrat für die Glycosylierungsreaktion heraus. So konnte bei der Verknüpfung des sekundären Alkohols 204 mit dem Trichloracetimidat 197 eine Gesamtausbeute von $71 \%$ und eine $\alpha / \beta$-Selektivität von 1:1.5 erzielt werden (Abbildung 106). Die $\alpha / \beta$-Selektivität ist aufgrund eines fehlenden Substituenten an C-2 der Zuckereinheit erwartungsgemäß nicht besonders hoch.
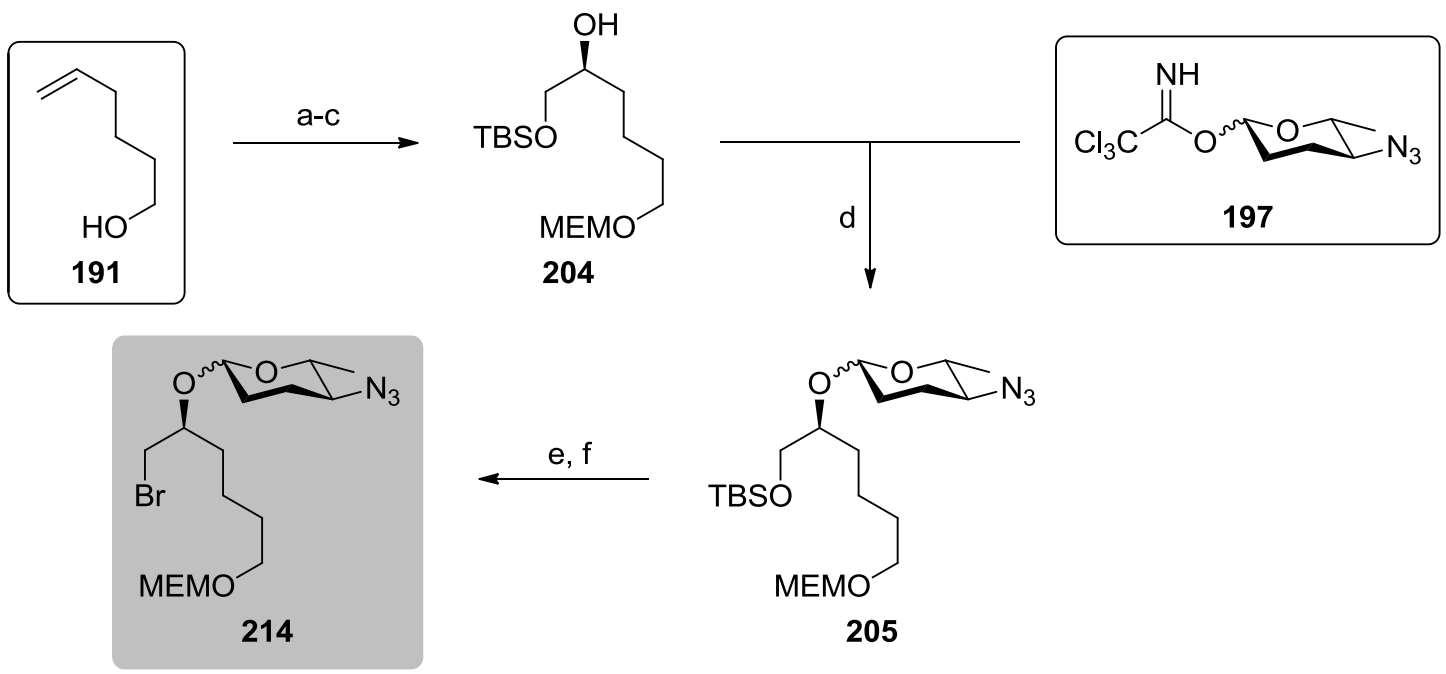

Abbildung 106: Synthese der Seitenkette 214: a) MEMCl, DIPEA, $\mathrm{CH}_{2} \mathrm{Cl}_{2}, 0{ }^{\circ} \mathrm{C} \rightarrow \mathrm{RT}, 2 \mathrm{~h}, 95 \%$; b) AD-mix $\alpha, t \mathrm{BuOH}, \mathrm{H}_{2} \mathrm{O},-10{ }^{\circ} \mathrm{C}, 3 \mathrm{~h}$, quant. (>99\% ee); c) TBSCl, Imidazol, DMAP, DMF, $0{ }^{\circ} \mathrm{C}, 3 \mathrm{~h}, 77 \%$; d) $\mathrm{Et}_{3} \mathrm{SiH}_{\text {, }}$ $\mathrm{I}_{2}$, MS $5 \AA$ A $\mathrm{PhMe},-90{ }^{\circ} \mathrm{C}, 8 \mathrm{~h}, \alpha: 30 \%, \beta: 41 \%$; e) TBAF, THF, RT, 3 h, $\alpha: 65 \%, \beta: 63 \%$; $\mathrm{CBr}_{4}, \mathrm{PPh}_{3}$, $\mathrm{CH}_{2} \mathrm{Cl}_{2}, \mathrm{RT}, 2 \mathrm{~h}, \alpha: 25 \%, \beta: 25 \%$.

Ein weiterer Schlüsselschritt dieser Synthese ist die stereoselektive Einführung der Hydroxygruppe ausgehend von dem kommerziell erhältlichen Hexenol 191 über eine Sharpless Dihydroxylierung. In einer hochkonzentrierten Lösung konnte bei sehr tiefen Temperaturen selektiv der $(S)$-Alkohol gebildet werden. Über den gesamten Syntheseabschnitt konnten aus 191 in sechs linearen Schritten 5\% der gewünschten $\beta$-verknüpften Seitenkette 214 und $4 \%$ des $\alpha$-Glycosids 214 erhalten.

Da in einer parallel durchgeführten Untersuchung eine Kupplung der beiden Bausteine 149 und 214 über eine Lithium-vermittelte 1,2-Addition nicht bewerkstelligt werden konnte, wurden die letzten beiden Stufen dieser Synthese nicht weiter optimiert. 
Als aussichtsreichstes alternatives Derivat stellte sich das über eine Ester-Brücke verknüpfte Analogon 262 heraus, bei dem die Kupplung der Bausteine 237 und 232 nach in situ Aktivierung der Carbonsäure erfolgt (Abbildung 107). Beide Kupplungspartner wurden in jeweils zwei Schritten aus Zwischenstufen der ursprünglichen Synthesesequenz aufgebaut. Die massenspektrometrische Reaktionskontrolle der finalen Kupplungsreaktion zeigte einen kompletten Umsatz der Carbonsäure 237 mit dem Alkohol 232, wobei ausschließlich das gewünschte Kupplungsprodukt 238 gebildet wurde, das allerdings nicht von dem im Überschuss eingesetzten Alkohol abgetrennt werden konnte. Eine erneute Reaktionsführung unter dem Einsatz von äquimolaren Mengen von 232 war aufgrund von fehlenden Substanzmengen an $\mathbf{2 3 7}$ nicht mehr möglich.
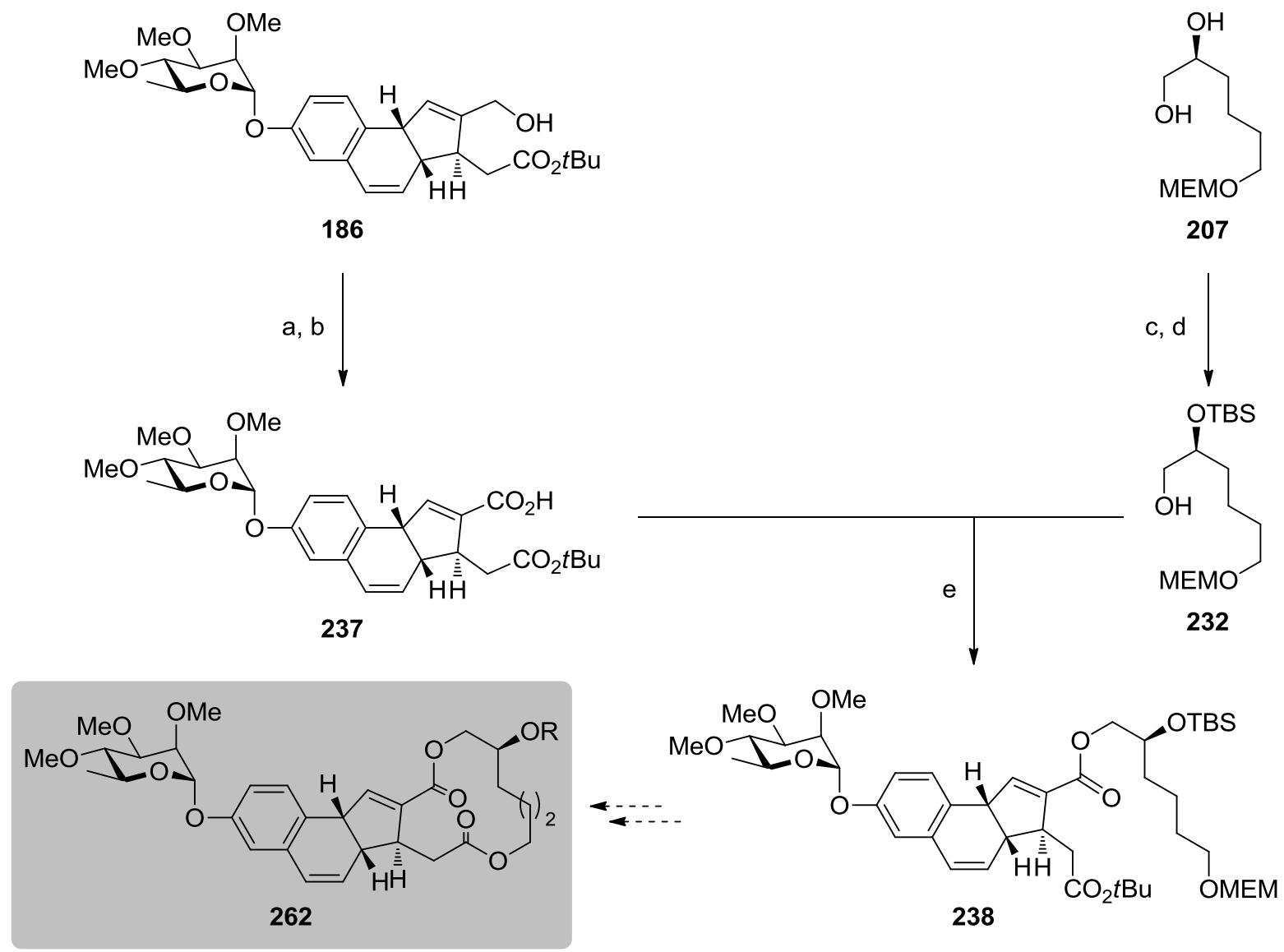

$\mathrm{R}=\mathrm{D}-$ Forosamin oder Analoga

Abbildung 107: Syntheseweg zu alternativen Spinosyn-Analoga des Typs 262: a) $\mathrm{MnO}_{2}, \mathrm{NaCN}, \mathrm{MeOH}$, $n$ Hexan, RT, 2.5 d, 87\%; b) LiOH $\cdot \mathrm{H}_{2} \mathrm{O}, \mathrm{CH}_{2} \mathrm{Cl}_{2}, \mathrm{MeOH}, \mathrm{H}_{2} \mathrm{O}$, RT, 1 h, 97\%; c) TBSOTf, 2,6-Lutidin, $\mathrm{CH}_{2} \mathrm{Cl}_{2}$, $0{ }^{\circ} \mathrm{C} \rightarrow \mathrm{RT}, 2.5$ h, 99\%; d) HF $\cdot$ py, THF, py, $0{ }^{\circ} \mathrm{C} \rightarrow \mathrm{RT}, 24 \mathrm{~h}, 66 \%$; e) DCC, DMAP, Et $2 \mathrm{O}, \mathrm{CHCl}_{3}, \mathrm{RT}, 4.5 \mathrm{~h}$.

Ausgehend von dem Kupplungsprodukt 238 ist nach Entschützung der Carbonsäure und des MEM-geschützen Alkohols eine Makrolactonisierung nach Yamaguchi denkbar. In vorangegangenen Arbeiten wurde diese Methode bei ähnlichen Systemen bereits erfolgreich 
angewendet. ${ }^{134}$ Im Anschluss an den Aufbau des Makrolactons kann nach TBS-Entschützung entweder der Aminozucker Forosamin oder ein beliebiger Aminoalkohol eingeführt werden, um zu Spinosyn-Analoga des Typs 262 zu gelangen.

Hierzu wurden fünf Forosamin-Analoga in ein bis zwei Stufen mit hervorragenden Ausbeuten synthetisiert (Abbildung 108). Neben der Synthese der vier elektrophilen Verbindungen 222a-d gelang auch die Herstellung eines nucleophilen Analogons (225), das in alternativen Synthesestrategien für die Fertigung neuer Spinosyn-Analoga eingesetzt werden kann.<smiles></smiles>

222a<smiles>N#C/C=C/CBr</smiles>

$222 b$<smiles>N#C/C=C\CBr</smiles>

222c<smiles>NCC#CCBr</smiles>

222d

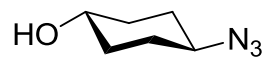

225

Abbildung 108: Synthetisierte Forosamin-Analoga.

Um die im Arbeitskreis Tietze entwickelte Glycosylierungsmethode zur selektiven Einführung des Aminozuckers Forosamin weiter $\mathrm{zu}$ etablieren, wurde in einer Kooperationsarbeit mit der Bayer AG das Spinosyn-Aglycon 156 mit dem Trichloracetimidat 197 umgesetzt (Abbildung 109). Hierbei ist die Wahl des Lösungsmittels von großer Bedeutung. So wurden in Toluol nur 7\%, in Dichlormethan dagegen 51\% des gewünschten Glycosids 156 bei einer verbesserten $\alpha / \beta$-Selektivität von 1:1.5 erhalten. Dies ist beachtlich, da diese Glycosylierung üblicherweise nur an sehr kleinen Molekülen in akzeptablen Ausbeuten durchführbar ist.

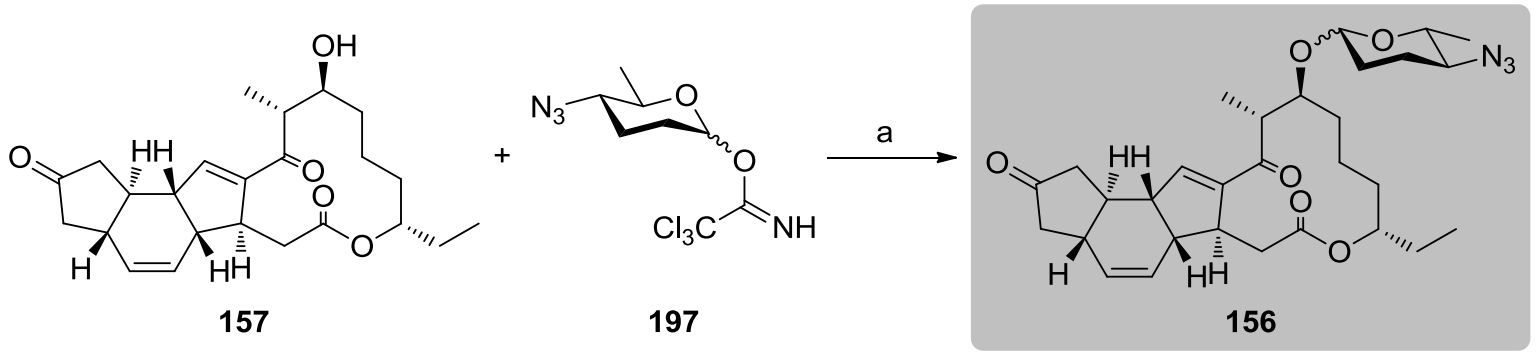

Abbildung 109: Glycosylierung des Spinosyn-Analogons 156: a) $\mathrm{Et}_{3} \mathrm{SiH}, \mathrm{I}_{2}, \mathrm{MS} 3 \AA \mathrm{A}_{2} \mathrm{Cl}_{2},-87^{\circ} \mathrm{C}, 8 \mathrm{~h}$, $\alpha: 21 \%, \beta: 30 \%$.

Neben einem synthetischen Zugang zu Analoga des Agrarwirkstoffes Spinosyn A beschäftigte sich diese Arbeit außerdem mit einer enantioselektiven Synthese des Naturstoffes (+)-Linoxepin.

Zunächst wurden das Vinylsilan 164 und das Benzylbromid 165 in jeweils vier Stufen aus den kommerziell erhältlichen Verbindungen 243 und 239 hergestellt und anschließend über eine Benzylierung miteinander verbunden (Abbildung 110). Die Synthese des Benzylbromids 165 
erfolgte ausgehend von dem Dioxol 239 in einer Gesamtausbeute von 40\%, während das Vinylsilan 164 in 30\% Gesamtausbeute erhalten wurde. Eine basenvermittelte Kupplung der beiden Bausteine konnte mit quantitativer Ausbeute realisiert werden.<smiles>COc1ccc(C=O)c(Br)c1O</smiles>

243

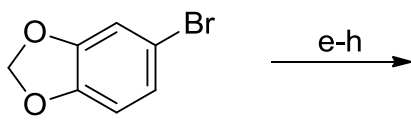

239

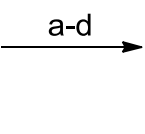

$\mathrm{MeO}$<smiles>C=C(O)CCC</smiles>

164

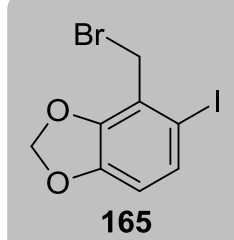

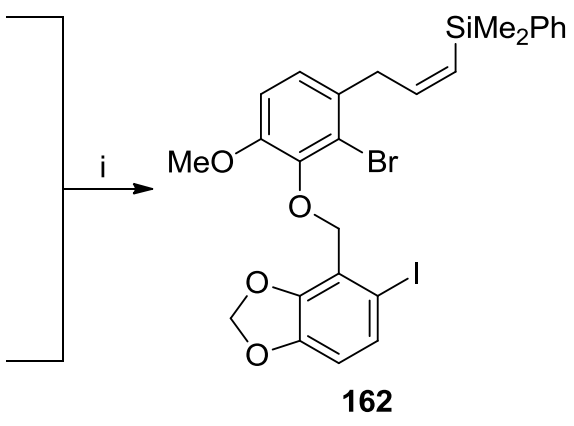

162

Abbildung 110: Synthese und Kupplung der Bausteine 164 und 165: a) $\mathrm{I}_{2}, n$ Buli, THF $-78{ }^{\circ} \mathrm{C} \rightarrow \mathrm{RT}, 90 \mathrm{~min}$, $84 \%$; b) DMF, LDA, THF, $-78^{\circ} \mathrm{C}, 14 \mathrm{~h}$; c) $\mathrm{NaBH}_{4}$, EtOH, $0{ }^{\circ} \mathrm{C} \rightarrow \mathrm{RT}, 1 \mathrm{~h}, 67 \%$ über 2 Stufen; d) $\mathrm{PBr}_{3}$, $\mathrm{CH}_{2} \mathrm{Cl}_{2}, \mathrm{RT}, 18 \mathrm{~h}, 71 \%$; e) $\mathrm{Ac}_{2} \mathrm{O}, \mathrm{K}_{2} \mathrm{CO}_{3}, \mathrm{MeCN}, 80^{\circ} \mathrm{C}, 2 \mathrm{~h}$, quant.; f) Ethinyldimethylphenylsilan, $n \mathrm{BuLi}$, THF, $-78{ }^{\circ} \mathrm{C} \rightarrow \mathrm{RT}, 18 \mathrm{~h}, 53 \%$; g) $\mathrm{HSiMe}_{2} \mathrm{Ph}$, TFA, PhMe, $0{ }^{\circ} \mathrm{C}, 30 \mathrm{~min}, 80 \%$; h) DIBAL-H, $n \mathrm{Hexan}$, $0{ }^{\circ} \mathrm{C} \rightarrow \mathrm{RT}, 30 \mathrm{~min}, 71 \%$; i) $\mathrm{K}_{2} \mathrm{CO}_{3}, \mathrm{MeCN}, 80{ }^{\circ} \mathrm{C}, 3.5 \mathrm{~h}$, quant..

Ausgehend von Verbindung 162 wurde der Naturstoffe (+)-Linoxepin (69) in sechs weiteren Schritten mit einer Gesamtausbeute von 56\% aufgebaut (Abbildung 111). Nach einer Sonogashira-Reaktion mit anschließender Domino-Carbopalladierung-Heck-Reaktion wurde das stereogene Zentrum über eine asymmetrische Hydroborierung eingeführt.

Drei weitere Stufen führten nach Enantiomerenanreicherung über HPLC an chiraler stationärer Phase zu dem gewünschten Naturstoff in $>99 \%$ ee.

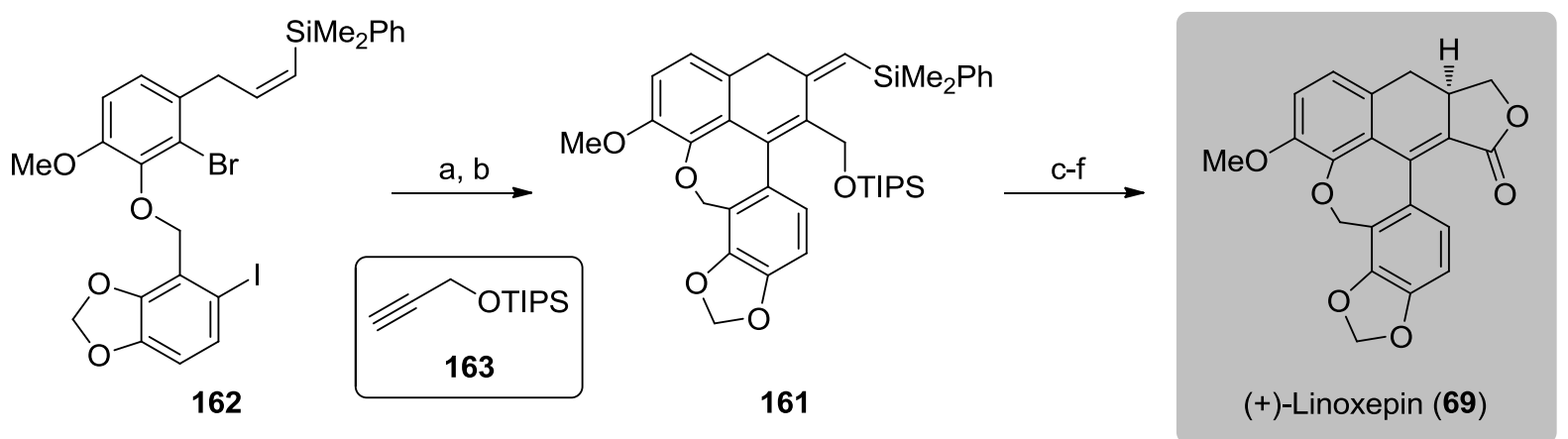

Abbildung 111: Synthese von (+)-Linoxepin (69): a) 163, $\mathrm{Pd}\left(\mathrm{PPh}_{3}\right)_{4}, \mathrm{CuI}, \mathrm{TBAOAc}, 1,4-\mathrm{Dioxan}, 60{ }^{\circ} \mathrm{C}$, 30 min, 93\%; b) $\mathrm{Pd}(\mathrm{OAc})_{2}$, DavePhos, $\mathrm{Ag}_{2} \mathrm{CO}_{3}$, DMAP, PhMe, $110{ }^{\circ} \mathrm{C}, 45 \mathrm{~min}$, bis zu 99\%; c) 1. (-)-(ipc) $\mathrm{BH}_{2}$, $\left.\mathrm{THF}, 0{ }^{\circ} \mathrm{C} \rightarrow \mathrm{RT}, 2 \mathrm{NaOH}, \mathrm{H}_{2} \mathrm{O}_{2}, 0{ }^{\circ} \mathrm{C} \rightarrow \mathrm{RT}, 30 \mathrm{~min}, 77 \%, 52 \% e e ; \mathrm{d}\right) \mathrm{TBAF}, \mathrm{THF}, 0{ }^{\circ} \mathrm{C}, 1 \mathrm{~h}, 89 \%$; e) $\mathrm{MnO}_{2}$, $\mathrm{CH}_{2} \mathrm{Cl}_{2}$, RT, $2.5 \mathrm{~h}$; f) $\mathrm{I}_{2}, \mathrm{~K}_{2} \mathrm{CO}_{3}, t \mathrm{BuOH}, 50{ }^{\circ} \mathrm{C}, 4.5 \mathrm{~h}, 89 \%$ über 2 Stufen. 
Durch den im Rahmen dieser Arbeit optimierten enantioselektiven Aufbau des Stereozentrums konnte die Gesamtausbeute von 30\% auf hervorragende 32\% über elf Stufen ausgehend von kommerziell erhältlichem Bromovanin (243) verbessert werden. Die Naturstoffsynthese von (+)-Linoxepin mit einer Domino-Carbopalladierung-Heck-Reaktion und einer asymmetrischen Hydroborierung als Schlüsselschritte ist somit die bis zu diesem Zeitpunkt effektivste Syntheseroute zu dem enantiomerenreinen Naturstoff.

Darüber hinaus konnte durch EXSY-Experimente die Stabilität der chiralen Achse der Atropisomere $\left(S, S_{a}\right)$-160 und $\left(S, R_{a}\right)-\mathbf{1 6 0}$ bestimmt werden (Abbildung 112). Hierbei fiel besonders die Lösungsmittelabhängigkeit der Aktivierungsenthalpien auf.<smiles>COc1ccc2c(c1)C(c1ccc3c(c1)OCO3)=C(CO)CC2CO</smiles>

$\left(S, S_{a}\right)-160$
$\Delta \mathrm{G}^{\ddagger}\left(\mathrm{CDCl}_{3}\right)=71.3 \mathrm{~kJ} \cdot \mathrm{mol}^{-1}$
$\Delta \mathrm{G}^{\ddagger}\left(\mathrm{CD}_{3} \mathrm{CN}\right)=67.8 \mathrm{~kJ} \cdot \mathrm{mol}^{-1}$

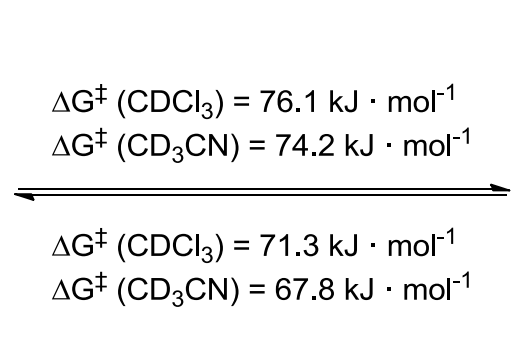

\begin{abstract}
Abbildung 112: Aktivierungsenthalpien des Isomerisierung bei $298 \mathrm{~K}$.
\end{abstract}
Der letzte Teil dieser Arbeit befasste sich mit einem schnellen synthetischen Zugang zu potentiellen Hsp47-Inhibitoren. Der Grundgedanke war es, in dem aromatischen Phenylring verschiedene Substituenten und Substitutionsmuster einzuführen.

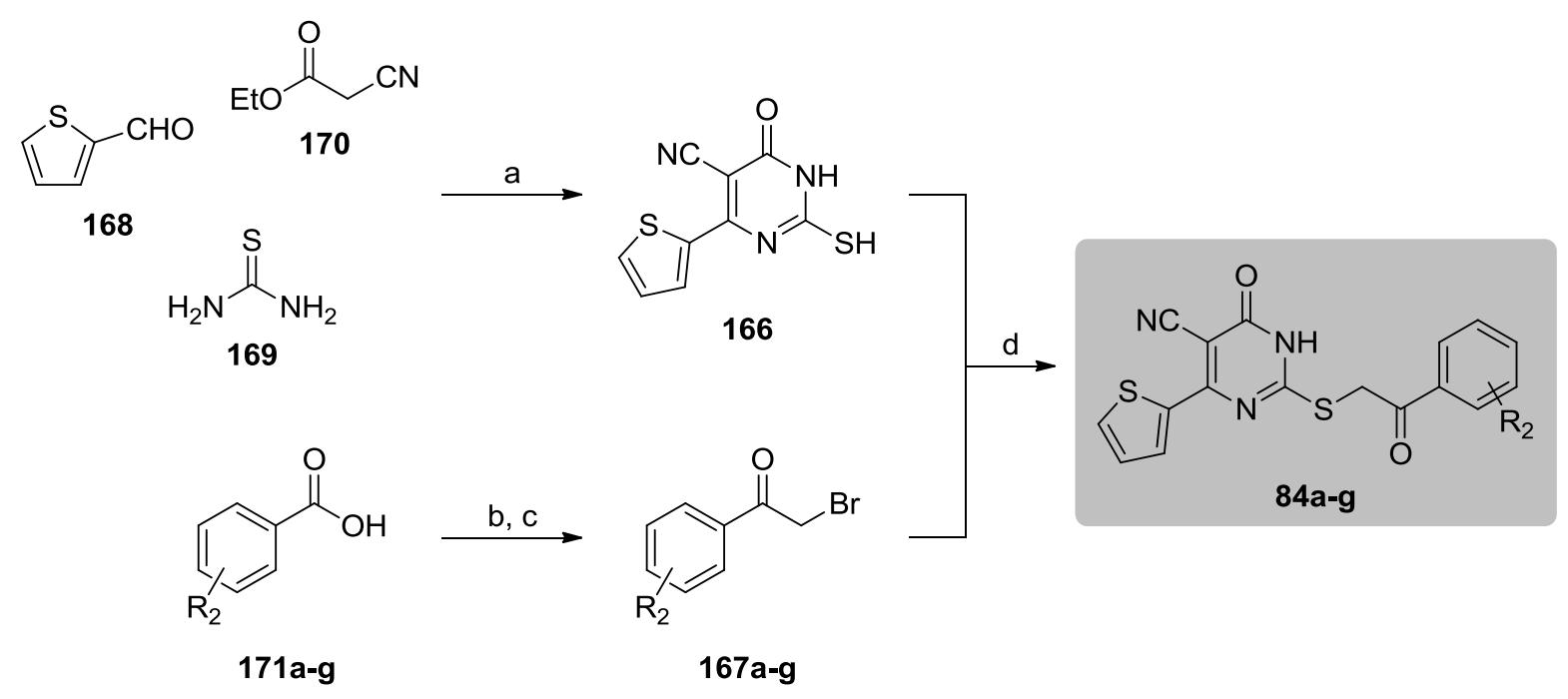

Abbildung 113: Synthese von potentiellen Hsp47-Inhibitoren 84a-g: a) $\mathrm{K}_{2} \mathrm{CO}_{3}$, EtOH, $78{ }^{\circ} \mathrm{C}, 12 \mathrm{~h}, 54 \%$; b) MeLi, CuCN, THF, $0{ }^{\circ} \mathrm{C} \rightarrow \mathrm{RT}, 16 \mathrm{~h}$, bis zu 89\%; c) PTAT, THF, RT, $14 \mathrm{~h}$, bis zu 87\%; d) $\mathrm{K}_{2} \mathrm{CO}_{3}$, DMF, $\mathrm{RT}, 16 \mathrm{~h}$, bis zu $54 \%$ nach Kristallisation. 
Ausgehend von den Benzoesäuren 171a-g gelang die Synthese der sieben potentiellen Inhibitoren 84a-g in einer Gesamtausbeute von bis $\mathrm{zu} 41 \%$ nach Kristallisation über drei Stufen (Abbildung 113). Das Dihydropyrimidongrundgerüst 166 wurde dabei über eine Multikomponenten-Eintopf-Reaktion in 54\% Ausbeute aufgebaut.

Die ursprüngliche Syntheseroute, die bei der Synthese von Hsp47-Inhibitoren mit Modifikationen des Thiophenrings angewandt wurde, konnte dabei unter verbesserten Ausbeuten um eine Stufe verkürzt werden. Die Ergebnisse der biologischen Aktivitäten der Inhibitoren 84a-g, die in einer Kooperationsarbeit mit dem Arbeitskreis von Prof. Zimmermann analysiert werden, lagen zum Zeitpunkt der Fertigstellung dieser Arbeit noch nicht vor. 


\section{EXPERIMENTELLER TEIL}

\section{Allgemeine Methoden}

\subsection{Allgemeine Arbeitstechniken}

Sämtliche Umsetzungen wurden, soweit wasserfreies Arbeiten unter Ausschluss von Luft erforderlich war, in ausgeheizten Glasapparaturen unter Argonatmosphäre durchgeführt.

\subsection{Lösungsmittel und Reagenzien}

Alle verwendeten Lösungsmittel wurden über 3-Ångström-Molekularsieb getrocknet, welches zuvor in einem Vakuumtrockenschrank Vacutherm 6025 der Firma Heraeus Instruments bei $100{ }^{\circ} \mathrm{C}$ in vacuo aktiviert wurde. Kommerziell erhältliche Reagenzien wurden entweder in p.a.-Qualität oder nach vorheriger Reinigung durch Destillation bzw. Umkristallisation eingesetzt. Zur Gewährleistung der Trockenheit von wasser- oder sauerstoffempfindlichen Reagenzien wurden diese in einer Trockenbox des Modells LABmaster sp der Firma MBraun gelagert und abgewogen.

\subsection{Verwendete Geräte}

\subsubsection{Drehwerte}

Drehwerte wurden mit einem Polarimeter P-2000 der Firma Jasco gemessen.

\subsubsection{Schmelzpunkte}

Die Bestimmung der Schmelzpunkte erfolgte mit Hilfe eines vollautomatischen Schmelzpunktbestimmungsgeräts Modell MPA120 (EZ-Melt) der Firma Stanford Research Systems.

\subsubsection{Infrarotspektren}

Infrarotspektren wurden mit dem Modell FT/IR-4100 der Firma Jasco aufgenommen. Alle Substanzen wurden direkt auf eine ATR-Einheit aufgetragen (neat).

\subsubsection{UV/Vis-Spektren}

Die Aufnahme der UV-Spektren erfolgte mit dem Modell V-630 der Firma Jasco. Die Messung erfolgte im Wellenlängenbereich von 190-600 nm. 


\subsection{5 ${ }^{1}$ H-NMR-Spektren}

${ }^{1}$ H-NMR-Spektren wurden mit dem Mercury 300 (300 MHz), Unity 300 (300 MHz) und Inova $600(600 \mathrm{MHz})$ der Firma Varian sowie dem Gerät Avance 300 der Firma Bruker aufgenommen. Die chemischen Verschiebungen sind in Einheiten der $\delta$-Skala angegeben. Referenziert wurde auf das jeweilige Lösungsmittel. Die Multiplizitäten von Signalen erster Ordnung wurden wie folgt bezeichnet: s (Singulett), $\mathrm{s}_{\mathrm{br}}$ (breites Singulett), d (Dublett), $\mathrm{t}$ (Triplett), q (Quartett), quint. (Quintett), dd (Dublett von Dubletts) usw. Signale, die durch Überlagerung oder Anteile höherer Ordnung nicht interpretierbar waren, wurden mit m (Multiplett) bzw. $\mathrm{m}_{\mathrm{c}}$ (symmetrisches, zentriertes Multiplett) bezeichnet. Zur Bezeichnung aromatischer Protonen oder Kohlenstoffatome werden folgende Abkürzungen verwendet: $i$ (ipso), $o$ (ortho), $m$ (meta), $p$ (para). Kopplungskonstanten $J$ sind in Hertz (Hz) aufgeführt und beziehen sich, sofern nicht anders angegeben, auf ${ }^{1} \mathrm{H}-{ }^{1} \mathrm{H}-$ Kopplungen.

\subsection{6 ${ }^{13}$ C-NMR-Spektren}

${ }^{13}$ C-NMR-Spektren wurden mit dem Modell Inova $500(125.7 \mathrm{MHz})$ der Firma Varian aufgenommen. Die chemischen Verschiebungen sind den ${ }^{1} \mathrm{H}$-breitbandentkoppelten Spektren entnommen und in Einheiten der $\delta$-Skala angegeben. Die Signalmultiplizitäten wurden durch die APT-Pulsfolge ermittelt. Referenziert wurde auf das jeweilige Lösungsmittel. Die Zuordnung der Signale erfolgte mit Hilfe eines Korrelationsspektrums zwischen ${ }^{1} \mathrm{H}$ - und ${ }^{13} \mathrm{C}$ Kernen.

\subsection{7 $\quad{ }^{19}$ F-NMR-Spektren}

${ }^{19}$ F-NMR-Spektren wurden mit dem Modell Mercury 300 (282 Mhz) der Firma Varian aufgenommen. Die chemischen Verschiebungen sind in Einheiten der $\delta$-Skala angegeben.

\subsection{8 $\quad{ }^{31}$ P-NMR-Spektren}

${ }^{31}$ P-NMR-Spektren wurden mit dem Modell Mercury 300 (122 Mhz) der Firma Varian aufgenommen. Die chemischen Verschiebungen sind in Einheiten der $\delta$-Skala angegeben.

\subsubsection{Massenspektren}

Zur Aufnahme der EI-Spektren diente ein doppelfokussierendes Sektorfeld-Massenspektrometer MAT 95 der Firma Finnigan. Als Ionisierungsmethode kam dabei die Elektronenstoß-Ionisation (EI, $70 \mathrm{eV}$ ) zum Einsatz. ESI-MS-Spektren wurden mit dem Timeof-Flight Massenspektrometer micrOTOF der Firma Bruker aufgenommen. Die Messung der ESI-HRMS-Spektren erfolgte an dem 7-Tesla-Fourier-Transform-Ion-Cyclotron-Resonance 
(FTICR)-Massenspektrometer Apex IV der Firma Bruker sowie am micrOTOF. Angegeben werden die Quotienten aus Masse zu Ladung, sowie in Klammern die relativen Intensitäten bezogen auf den Basispeak $(\mathrm{I}=100)$. Zur Aufnahme und Auswertung der Spektren diente das Programm XMASS.

\subsubsection{Kältetechnik}

Die Reaktionsführung bei tiefen Temperaturen erfolgte unter der Verwendung von Kryostaten (Haake EK90, Haake EK101 \& Julabo FT902 mit Ethanol/isoPropanol als Kühlmittel) und geeigneten Kühlmischungen (Trockeneis/Aceton; Natriumchlorid/Eis).

\subsection{Chromatographische Methoden}

\subsubsection{Dünnschichtchromatographie (DC)}

Es wurden DC-Folien Kieselgel $60 \mathrm{~F}_{254}$ der Firma Merck verwendet. Angegeben sind $\mathrm{R}_{f}$-Werte (Laufhöhe relativ zur Laufmittelfront). Neben der UV-Detektion (254 nm, $365 \mathrm{~nm}$ ) dienten Vanillin-Schwefelsäure-Lösung (0.75 g Vanillin, $5 \mathrm{~mL}$ konz. $\mathrm{H}_{2} \mathrm{SO}_{4}, 130 \mathrm{~mL}$ Methanol und $15 \mathrm{~mL}$ Essigsäure), Molybdatophosphorsäure-Lösung (5 g Molybdatophosphorsäure, $100 \mathrm{~mL}$ EtOH) und Hanessian's Stain (5 g Ammoniummolybdat, $1 \mathrm{~g}$ Cersulfat, $10 \mathrm{ml} \mathrm{konz.} \mathrm{H}_{2} \mathrm{SO}_{4}, 90 \mathrm{~mL} \mathrm{H}_{2} \mathrm{O}$ ) als Anfärbereagenzien.

\subsubsection{Säulenchromatographie (SC)}

Säulenchromatographische Reinigungen erfolgten mit Kieselgel der Firma Merck (Geduran ${ }^{\circledR}$ Si60, $\varnothing=40-63 \mu \mathrm{m}$ ) unter erhöhtem Druck (Flashchromatographie, typ. 0.1-0.8 bar). Die analog der DC verwendeten Eluentien wurden vor Verwendung nach den üblichen Labormethoden getrocknet und destilliert.

\subsubsection{Analytische HPLC}

Analytische Trennungen wurden auf einer HPLC-Anlage der Firma Jasco, ausgestattet mit einer Lösungsmittelpumpe PU-2080, einer Mischkammer LG-1590-04, einem Multiwellenlängendetektor MD-2010 Plus und der Steuerung LC-Net II/ADC vorgenommen. Für die Injektion wurde ein automatischer Probenwechsler (Autosampler AS-2055) derselben Firma verwendet. Zur Bedienung, Datenerfassung und Datenauswertung wurden die Computerprogramme Borwin PDA, HSS 2000 und Borwin Chromatography der Firma Jasco eingesetzt. Für die analytischen Messungen wurden die Säulen Chiralpak ${ }^{\circledR}$ IA $(250 \times 4.6$ mm, $5 \mu \mathrm{m}$, Daicel Chemical Industries Ltd.) sowie Chiralpak ${ }^{\circledR} \mathrm{IB}(250 \times 4.6 \mathrm{~mm}, 5 \mu \mathrm{m}$, Daicel Chemical Industries Ltd.) mit chiraler stationärer Phase verwendet. Die verwendeten 
Lösungsmittel waren von HPLC-Qualität. Alle Proben wurden membranfiltriert mit Polytetrafluorethylen-(PTFE)-Filtern der Firma Roth $(\varnothing 25 \mathrm{~mm}, 0.2 \mu \mathrm{m})$ bzw. VWR (Ø $13 \mathrm{~mm}, 0.2 \mu \mathrm{m})$.

\subsubsection{Präparative HPLC}

Präparative Trennungen wurden auf einem HPLC-System der Firma Jasco, ausgestattet mit zwei Lösungsmittelpumpen Modell PU-2087 PLUS und einem UV-Detektor Modell UV2075 PLUS, vorgenommen. Verwendet wurden die Säulen Chiralpak ${ }^{\circledR}$ IA $(250 \times 20$ mm, $5 \mu \mathrm{m}$, Daicel Chemical Industries Ltd.) sowie Chiralpak ${ }^{\circledR}$ IB $(250 \times 10 \mathrm{~mm}, 5 \mu \mathrm{m}$, Daicel Chemical Industries Ltd.) mit chiraler stationärer Phase. Die verwendeten Lösungsmittel waren von HPLC-Qualität. Alle Proben wurden vor der Trennung membranfiltriert (siehe analytische HPLC). 


\section{Allgemeine Arbeitsvorschriften}

\subsection{AAV1: Synthese der Acetophenone 261a-g}

$\mathrm{Zu}$ einer gerührten Suspension von $\mathrm{CuCN}$ (5.0 Äq.) in THF $(\sim 1.7 \mathrm{~mL} / \mathrm{mmol})$ wurde bei $0{ }^{\circ} \mathrm{C}$ $\mathrm{MeLi}$ (1.6 $\mathrm{M}$ in $\mathrm{Et}_{2} \mathrm{O}, 10.0$ Äq.) getropft. Anschließend wurde bei $0{ }^{\circ} \mathrm{C}$ zu der klaren Lösung langsam die Benzoesäure 171 (1.0 Äq.) in THF ( 5 mL/mmol) getropft und nach beendeter Zugabe $14 \mathrm{~h}$ bei Raumtemperatur gerührt. Nach Neutralisation mit gesättigter wässriger $\mathrm{NH}_{4} \mathrm{Cl}$-Lösung $(50 \mathrm{~mL})$ wurde das Reaktionsgemisch filtriert, die wässrige Phase mit DCM $(3 \times 20 \mathrm{~mL})$ extrahiert und die vereinigten organischen Phasen über $\mathrm{Na}_{2} \mathrm{SO}_{4}$ getrocknet. Das Lösungsmittel wurde unter vermindertem Druck entfernt und der Rückstand säulenchromatographisch an Kieselgel gereinigt.

\subsection{AAV2: Synthese der $\alpha$-Bromacetophenone 167a-g}

Zu einer Lösung des Acetophenons 261 (1.0 Äq.) in THF ( 10 mL/mmol) wurde bei Raumtemperatur PTAT (1.0 Äq.) gegeben und die entstandene Suspension $10 \mathrm{~h}$ gerührt. Anschließend wurde der entstandene Feststoff abfiltriert, das Lösungsmittel unter vermindertem Druck entfernt und der Rückstand säulenchromatographisch an Kieselgel gereinigt.

\subsection{AAV3: Synthese der potentiellen Hsp47-Inhibitoren 84a-g}

Dihydropyrimidon 166 (1.0 Äq.) wurde in DMF ( 1 mL/mmol) gelöst und mit dem $\alpha$-Bromacetophenon (1.0 Äq.) versetzt. $\mathrm{Zu}$ der Suspension wurde bei Raumtemperatur $\mathrm{K}_{2} \mathrm{CO}_{3}$ (1.0 Äq.) gegeben und die Reaktionsmischung $16 \mathrm{~h}$ gerührt. Anschließend wurde das Lösungsmittel unter vermindertem Druck entfernt, der resultierende Rückstand mit demineralisiertem $\mathrm{H}_{2} \mathrm{O}(15 \mathrm{~mL})$ und $1 \mathrm{M} \mathrm{HCl}(3 \mathrm{~mL})$ versetzt, filtriert und aus $\mathrm{MeOH}$ umkristallisiert. 


\section{Synthese von verwendeten Reagenzien}

\subsection{Polymer-gebundenes 1,8-Diazabicyclo[5.4.0]undec-7-en (174)}

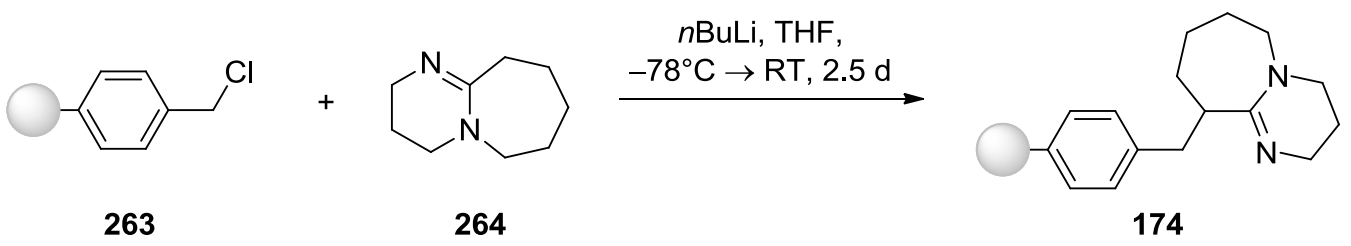

$\mathrm{Zu}$ einer Lösung aus 1,8-Diazabicyclo[5.4.0]undec-7-en (264) (14.9 mL, 15.0 g, 100 mmol, 1.0 Äq.) in frisch destilliertem THF $(500 \mathrm{~mL})$ wurde bei $-78{ }^{\circ} \mathrm{C}$ langsam $n \operatorname{BuLi}(2.5 \mathrm{M}$ in $n$ Hexan, $38.4 \mathrm{~mL}, 96.0 \mathrm{mmol}, 1.0$ Äq.) getropft. Die Reaktionslösung wurde $2 \mathrm{~h}$ bei $-78{ }^{\circ} \mathrm{C}$ gerührt und anschließend portionsweise Merrifield-Harz (263) (31.8 g, 2-2.5 MEQ Cl/g, 200-400 mesh, 2\% crosslinked with DVB) zugegeben. Nach der Zugabe wurde langsam auf Raumtemperatur erwärmt und $2.5 \mathrm{~d}$ gerührt. Die Reaktion wurde durch Zugabe von absolutem $\mathrm{MeOH}(10 \mathrm{~mL})$ beendet, der Feststoff abfiltriert und mit absolutem THF/MeOH (1:1), $\mathrm{MeOH}, \mathrm{MeOH} / \mathrm{H}_{2} \mathrm{O}$ (1:1), Aceton und THF (jeweils $300 \mathrm{~mL}$ ) gewaschen. Nach $4 \mathrm{~h}$ Trocknen im Hochvakuum bei $70{ }^{\circ} \mathrm{C}$ wurde die Titelverbindung 174 (36.5 g) als hellgelber Feststoff erhalten.

Zur Bestimmung der Beladung wurden $200 \mathrm{mg}$ des Produktes in einem Gemisch aus absolutem MeOH/1,4-Dioxan (1:2, $60 \mathrm{~mL})$ suspendiert und $2 \mathrm{~h}$ bei RT gerührt. Anschließende Titration mit $0.1 \mathrm{M} \mathrm{HCl}$ unter Verwendung von Methylrot als Indikator ergab eine Beladung von $1.13 \mathrm{mmol} / \mathrm{g}$. 


\subsection{Iodmethyl-triphenylphosphoniumiodid (265)}

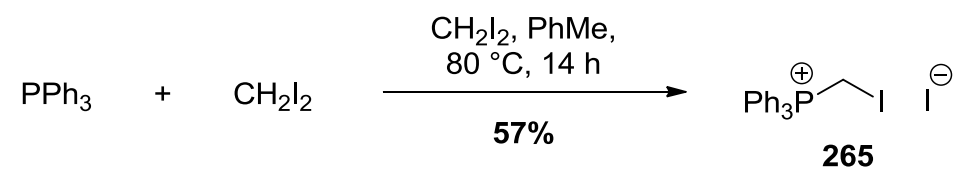

$\mathrm{Zu}$ einer Lösung aus $\mathrm{PPh}_{3}(5.32 \mathrm{~g}, 20.3 \mathrm{mmol}, 1.0$ Äq.) in Toluol (15 mL) wurde bei Raumtemperatur Diiodmethan (1.72 mL, $5.71 \mathrm{~g}, 21.3 \mathrm{mmol}, 1.1$ Äq.) gegeben und anschließend $14 \mathrm{~h}$ bei $80^{\circ} \mathrm{C}$ gerührt. Der ausgefallene Feststoff wurde abfiltriert, mit $n$ Pentan $(8 \times 40 \mathrm{~mL})$ gewaschen und im Hochvakuum getrocknet. Das Wittig-Salz 265 (6.50 g, $12.3 \mathrm{mmol}, 57 \%$ ) wurde in Form eines farblosen Pulvers analysenrein erhalten.

UV $\left(\mathrm{CH}_{3} \mathrm{CN}\right): \lambda_{\max }(\lg \varepsilon)=202 \mathrm{~nm}$ (4.8376), 221 (4.5971), 268 (3.5931), 275 (3.5093).

IR: $\tilde{v}\left(\mathrm{~cm}^{-1}\right)=3041,2987,2916,2848,2742,2147,1586,1482,1438,1335,1317$.

${ }^{1}$ H-NMR $\left(300 \mathrm{MHz}, \mathrm{DMSO}-\mathrm{d}_{6}\right): \delta(\mathrm{ppm})=5.03\left(\mathrm{~d}, J=8.6 \mathrm{~Hz}, 2 \mathrm{H}, \mathrm{CH}_{2}\right), 7.74-7.96(\mathrm{~m}$, $\left.15 \mathrm{H}, 3 \times \mathrm{Ph}-\mathrm{H}_{5}\right)$.

${ }^{13}$ C-NMR $\left(126 \mathrm{MHz}, \mathrm{DMSO}-\mathrm{d}_{6}\right): \delta(\mathrm{ppm})=118.3\left(\mathrm{~d}, J_{\mathrm{C}, \mathrm{P}}=88.7 \mathrm{~Hz}, 3 \times \mathrm{Ph}-\mathrm{C}_{i}\right), 130.0(\mathrm{~d}$, $\left.J_{\mathrm{C}, \mathrm{P}}=12.7 \mathrm{~Hz}, 6 \times \mathrm{Ph}-\mathrm{C}_{o}\right), 133.7\left(\mathrm{~d}, J_{\mathrm{C}, \mathrm{P}}=10.1 \mathrm{~Hz}, 6 \times \mathrm{Ph}-\mathrm{C}_{m}\right), 135.0\left(\mathrm{~d}, J_{\mathrm{C}, \mathrm{P}}=2.9 \mathrm{~Hz}\right.$, $\left.3 \times \mathrm{Ph}-\mathrm{C}_{p}\right), 223.5\left(\mathrm{~d}, J_{\mathrm{C}, \mathrm{P}}=51.8 \mathrm{~Hz}, \mathrm{CH}_{2}\right)$.

${ }^{31}$ P-NMR $\left(122 \mathrm{MHz}, \mathrm{DMSO}-\mathrm{d}_{6}\right): \delta(\mathrm{ppm})=24.7$.

MS (EI, $70 \mathrm{eV}): m / z(\%)=275.4(100)[\mathrm{M}-\mathrm{I}-\mathrm{HI}]^{+}, 403.0(56)[\mathrm{M}-\mathrm{I}]^{+}, 932.4(15)[2 \mathrm{M}-\mathrm{I}]^{+}$.

HRMS von $\mathrm{C}_{19} \mathrm{H}_{17} \mathrm{I}_{2} \mathrm{P}$ ber.: 403.0107 gef.: $\quad 403.0105[\mathrm{M}-\mathrm{I}]^{+}(\mathrm{ESI}-\mathrm{HRMS})$.

$\mathbf{C}_{19} \mathrm{H}_{17} \mathbf{I}_{2} \mathbf{P}$ (530.12). 


\section{3 $1 H$-Imidazol-1-sulfonylazid Hydrochlorid (224)}

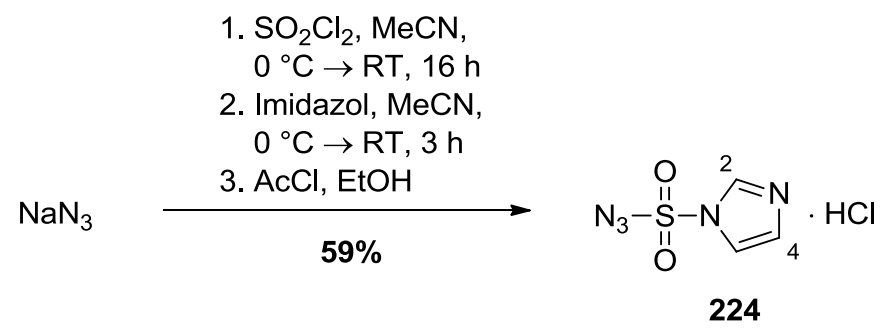

$\mathrm{Zu}$ einer Lösung aus $\mathrm{NaN}_{3}\left(2.60 \mathrm{~g}, 40.0 \mathrm{mmol}, 1.0\right.$ Äq.) in $\mathrm{MeCN}(50 \mathrm{~mL})$ wurde bei $0{ }^{\circ} \mathrm{C}$ langsam $\mathrm{SO}_{2} \mathrm{Cl}_{2}(3.23 \mathrm{~mL}, 5.40 \mathrm{~g}, 40.0 \mathrm{mmol}, 1.0 \mathrm{Äq}$.) gegeben, auf Raumtemperatur erwärmt und $16 \mathrm{~h}$ gerührt. Anschließend wurde bei $0{ }^{\circ} \mathrm{C}$ portionsweise Imidazol $(5.17 \mathrm{~g}$, 76.0 mmol, 1.9 Äq.) zugegeben, auf Raumtemperatur erwärmt und $3 \mathrm{~h}$ gerührt. Die Reaktionsmischung wurde mit EtOAc $(100 \mathrm{~mL})$ versetzt, mit $\mathrm{H}_{2} \mathrm{O}(2 \times 100 \mathrm{~mL})$, und ges. $\mathrm{NaHCO}_{3}$-Lösung $(2 \times 100 \mathrm{~mL})$ gewaschen, über $\mathrm{Na}_{2} \mathrm{SO}_{4}$ getrocknet und filtriert. $\mathrm{Zu}$ dem Filtrat wurde tropfenweise eine Lösung aus $\mathrm{HCl}$ in $\mathrm{EtOH}$, welche bei $0{ }^{\circ} \mathrm{C}$ durch langsame Zugabe von $\mathrm{AcCl}$ (4.26 mL, $4.69 \mathrm{~g}, 60.0 \mathrm{mmol}, 1.5$ Äq.) in EtOH (15 mL) erhalten wurde, gegeben. Die Lösung wurde 15 min bei $0{ }^{\circ} \mathrm{C}$ gelagert, filtriert und der Rückstand mit EtOAc (3×100 mL) gewaschen. Das Hydrochlorid 224 (4.92 g, $23.5 \mathrm{mmol}, 59 \%)$ wurde in Form eines farblosen Feststoffes erhalten.

UV $\left(\mathrm{CH}_{3} \mathrm{CN}\right)$ : keine Absorption.

IR: $\tilde{v}\left(\mathrm{~cm}^{-1}\right)=2169,1913,1581,1508,1425,1298,1229,1158,1067,774,582$.

${ }^{1} \mathbf{H}-\mathbf{N M R}\left(300 \mathrm{MHz}, \mathrm{D}_{2} \mathrm{O}\right): \delta(\mathrm{ppm})=7.70(\mathrm{~s}, 1 \mathrm{H}, 4-\mathrm{H}), 8.09(\mathrm{~s}, 1 \mathrm{H}, 5-\mathrm{H}), 9.52(\mathrm{~s}, 1 \mathrm{H}$, 2-H).

${ }^{13}$ C-NMR (126 MHz, D 20$): \delta(p p m)=122.7$ (C-5), $125.8(\mathrm{C}-4), 140.3(\mathrm{C}-2)$.

MS (ESI): $\mathrm{m} / \mathrm{z}(\%)=174.0(100)[\mathrm{M}-\mathrm{HCl}+\mathrm{H}]^{+}$.

HRMS von $\mathrm{C}_{3} \mathrm{H}_{3} \mathrm{~N}_{5} \mathrm{O}_{2} \mathrm{~S}$

ber.: $\quad 174.0080$

gef.: $\quad 174.0075[\mathrm{M}+\mathrm{H}]^{+}(\mathrm{ESI}-\mathrm{HRMS})$.

$\mathbf{C}_{3} \mathrm{H}_{4} \mathrm{CIN}_{5} \mathrm{O}_{2} \mathrm{~S}(209.61)$. 


\subsection{Lithiumdi-iso-propylamid (266)}

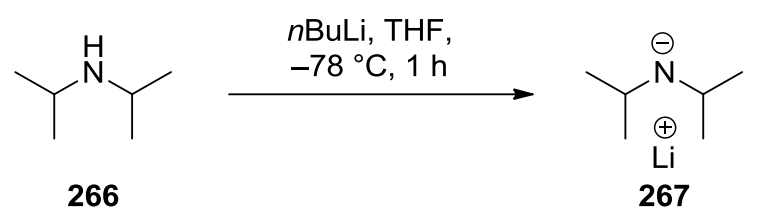

$\mathrm{Zu}$ einer Lösung aus DIPA (266) (8.69 mL, $6.25 \mathrm{~g}, 61.8 \mathrm{mmol}, 1.0$ Äq) in THF (60 mL) wurde bei $-78^{\circ} \mathrm{C} n$-BuLi (1.6 M in $n$ Hexan, $38.6 \mathrm{~mL}, 61.8 \mathrm{mmol}, 1.0$ Äq) gegeben und $1 \mathrm{~h}$ gerührt. Die entstandene Lösung von 267 wurde direkt für die Umsetzung verwendet. 


\subsection{Tri-iso-propyl(prop-2-in-1-yloxy)silan (163)}

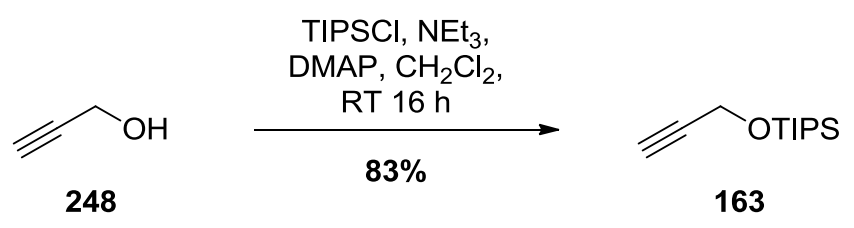

$\mathrm{Zu}$ einer Lösung des Propargylalkohols (248) (2.00 mL, $2.06 \mathrm{~g}, 36.8 \mathrm{mmol}, 1.0$ Äq.) in $\mathrm{CH}_{2} \mathrm{Cl}_{2}(80 \mathrm{~mL})$ wurde bei Raumtemperatur $\mathrm{NEt}_{3}(8.15 \mathrm{~mL}, 11.2 \mathrm{~g}, 110 \mathrm{mmol}, 3.0$ Äq.) gegeben und $30 \mathrm{~min}$ gerührt. Anschließend wurden DMAP (270 mg, $2.21 \mathrm{mmol}, 6 \mathrm{~mol} \%$ ) und TIPSCl (7.73 mL, 8.51 g, $44.1 \mathrm{mmol}, 1.2$ Äq.) zugegeben und weitere 16 h gerührt. Nach Zugabe von $2 \mathrm{M} \mathrm{HCl}(100 \mathrm{~mL})$ wurden die Phasen getrennt, die wässrige Phase mit $\mathrm{CH}_{2} \mathrm{Cl}_{2}$ $(3 \times 100 \mathrm{~mL})$ extrahiert und die vereinten organischen Phasen über $\mathrm{Na}_{2} \mathrm{SO}_{4}$ getrocknet. Entfernung des Lösungsmittels unter vermindertem Druck und säulenchromatographische Reinigung an Kieselgel (nPentan/EtOAc 20:1) lieferte das Produkt 163 (6.74 g, 30.5 mmol, $83 \%$ ) als farblose Flüssigkeit.

DC: $\mathrm{R}_{f}=0.35(n$ Pentan/EtOAc 20:1).

UV $\left(\mathrm{CH}_{3} \mathrm{CN}\right)$ : keine Absorption.

IR: $\tilde{v}\left(\mathrm{~cm}^{-1}\right)=2942,2865,1463,1369,1262,1096,999,881,770,658$.

${ }^{1} \mathbf{H}-\mathbf{N M R}\left(300 \mathrm{MHz}, \mathrm{CDCl}_{3}\right): \delta(\mathrm{ppm})=1.04-1.09\left(\mathrm{~m}, 21 \mathrm{H}, \mathrm{Si}\left(\mathrm{CH}\left(\mathrm{CH}_{3}\right)_{2}\right)_{3}\right), 2.37(\mathrm{t}, J=$ $2.4 \mathrm{~Hz}, 1 \mathrm{H}, 3-\mathrm{H}), 4.36$ (d, $\left.J=2.4 \mathrm{~Hz}, 2 \mathrm{H}, 1-\mathrm{H}_{2}\right)$.

${ }^{13}$ C-NMR $\left(126 \mathrm{MHz}, \mathrm{CDCl}_{3}\right): \delta(\mathrm{ppm})=12.1\left(\mathrm{Si}\left(\underline{\mathrm{CH}}\left(\mathrm{CH}_{3}\right)_{2}\right)_{3}\right), 18.0\left(\mathrm{Si}\left(\mathrm{CH}\left(\underline{\mathrm{CH}_{3}}\right)_{2}\right)_{3}\right), 51.8$ (C-1), $72.6(\mathrm{C}-3), 82.5$ (C-2).

MS (ESI): $\mathrm{m} / \mathrm{z}(\%)=213.2(100)[\mathrm{M}+\mathrm{Na}]^{+}, 235.2(75)[2 \mathrm{M}+\mathrm{Na}]^{+}$.

HRMS von $\mathrm{C}_{12} \mathrm{H}_{24} \mathrm{OSi}$ ber.: 213.1669 235.1489 gef.: $\quad 213.1668[\mathrm{M}+\mathrm{H}]^{+}$ $235.1486[\mathrm{M}+\mathrm{Na}]^{+}(\mathrm{ESI}-\mathrm{HRMS})$.

$\mathbf{C}_{12} \mathbf{H}_{24} \mathbf{O S i}(212.41)$. 


\subsection{Bis-(((1S,3R,5S)-2,6,6-trimethylbicyclo[3.1.1]heptan-3-yl)boran)- TMEDA-Komplex (269)}

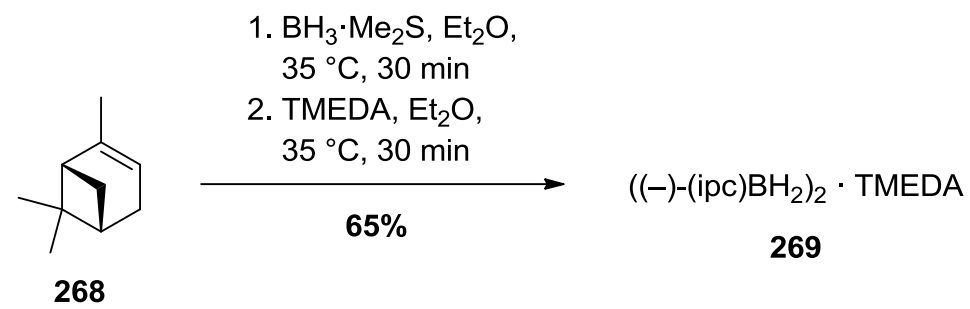

a-Pinen (268) $(7.36 \mathrm{~mL}, 6.27 \mathrm{~g}, 46.0 \mathrm{mmol}, 4.6$ Äq.) wurde bei Raumtemperatur zu $\mathrm{BH}_{3} \cdot \mathrm{Me}_{2} \mathrm{~S}\left(2 \mathrm{M}\right.$ in $\mathrm{Et}_{2} \mathrm{O}, 10.0 \mathrm{~mL}, 20.0 \mathrm{mmol}, 2.0$ Äq.) getropft und die Reaktionslösung $30 \mathrm{~min}$ bei $35^{\circ} \mathrm{C}$ gerührt. Dann wurde frisch destilliertes TMEDA (1.51 mL, $1.16 \mathrm{~g}$, $10.0 \mathrm{mmol}, 1.0$ Äq.) zugefügt und weitere $30 \mathrm{~min}$ bei $35^{\circ} \mathrm{C}$ gerührt. Anschließend wurde die Mischung langsam auf $0{ }^{\circ} \mathrm{C}$ gekühlt, der entstandene Feststoff abfiltriert und mit auf $0{ }^{\circ} \mathrm{C}$ gekühltem $n$ Pentan $(3 \times 5 \mathrm{~mL})$ gewaschen. Die Zielverbindung 269 (2.73 g, 6.54 mmol, 65\%) wurde in Form von farblosen Kristallen erhalten.

Smp.: $140{ }^{\circ} \mathrm{C}$.

Drehwert: $[\alpha]_{\mathrm{D}}^{20}=+48.3\left(\mathrm{c}=1.3, \mathrm{CHCl}_{3}\right)$.

UV $\left(\mathrm{CH}_{3} \mathrm{CN}\right)$ : keine Absorption.

IR: $\tilde{v}\left(\mathrm{~cm}^{-1}\right)=2889,2843,2321,1458,1364,1214,1141,903,835,662$.

${ }^{1} \mathbf{H}-\mathbf{N M R}\left(300 \mathrm{MHz}, \mathrm{CDCl}_{3}\right): \delta(\mathrm{ppm})=0.98\left(\mathrm{~d}, J=7.0 \mathrm{~Hz}, 6 \mathrm{H}, 2 \times \mathrm{CH}_{3}\right), 1.07(\mathrm{~s}, 6 \mathrm{H}$, $\left.2 \times \mathrm{CH}_{3}\right), 1.14\left(\mathrm{~s}, 6 \mathrm{H}, 2 \times \mathrm{CH}_{3}\right), 1.47-2.38(\mathrm{~m}, 16 \mathrm{H}, 16 \times \mathrm{ipc}-\mathrm{H}), 2.57\left(\mathrm{~s}, 6 \mathrm{H}, 2 \times \mathrm{NCH}_{3}\right), 2.61$ (s, $\left.6 \mathrm{H}, 2 \times \mathrm{NCH}_{3}\right), 3.19\left(\mathrm{~m}_{\mathrm{c}}, 4 \mathrm{H}, \mathrm{NCH}_{2} \mathrm{CH}_{2} \mathrm{~N}\right)$.

$\mathbf{C}_{19} \mathbf{H}_{17} \mathbf{I}_{2} \mathbf{P}$ (530.12). 


\section{7 ((1S,3R,5S)-2,6,6-Trimethylbicyclo[3.1.1]heptan-3-yl)boran (257)}

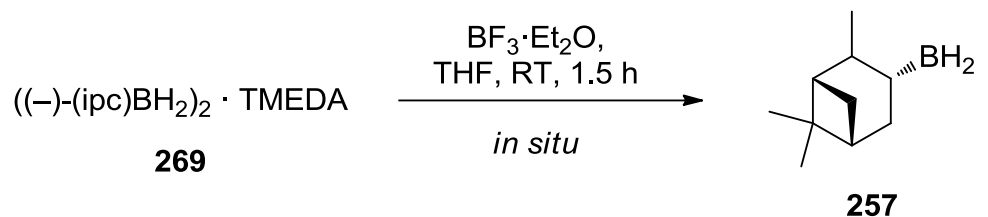

Der TMEDA-Komplex 269 (130 mg, $312 \mu \mathrm{mol}, 1.0$ Äq.) wurde mit $\mathrm{BF}_{3} \cdot \mathrm{OEt}_{2}(77.0 \mu \mathrm{L}$, $88.6 \mathrm{mg}, 624 \mu \mathrm{mol}, 2.0$ Äq.) und THF ( $3 \mathrm{~mL}$ ) versetzt und $1 \mathrm{~h}$ bei Raumtemperatur gerührt. Die Reaktionsmischung wurde anschließend mit einem PTFE-Filter ( $\varnothing 25 \mathrm{~mm}, 0.2 \mu \mathrm{m}$ ) membranfiltriert und das Filtrat ohne weitere Aufarbeitung direkt für die Umsetzung benutzt. 


\section{Synthese von Spinosynanaloga}

\subsection{Synthese des Glykosyldonors 175}

\subsubsection{1,2,3,4-Tetra- $O$-methyl- $\alpha / \beta$-L-rhamnopyranosid (173)}

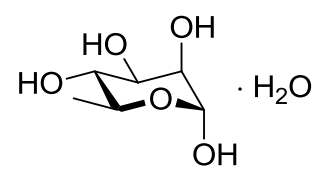

172

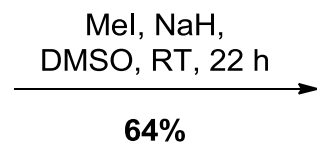

$64 \%$

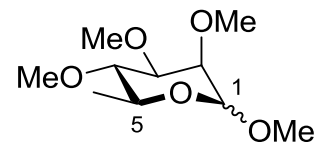

173

Zu einer Lösung des L-Rhamnose-Monohydrats 172 (500 mg, 2.74 mmol, 1.0 Äq.) in DMSO $(20 \mathrm{~mL})$ wurde bei $0{ }^{\circ} \mathrm{C}$ portionsweise $\mathrm{NaH}$ (60\%ig in Mineralöl, $770 \mathrm{mg}, 32.1 \mathrm{mmol}$, 12.0 Äq.) gegeben und 30 min gerührt. Anschließend wurde über 4 h MeI (6.64 mL, $2.14 \mathrm{~g}$, $15.1 \mathrm{mmol}, 5.5$ Äq.) zugetropft und nach erfolgter Zugabe $12 \mathrm{~h}$ bei Raumtemperatur gerührt. Nach Zugabe von $\mathrm{MeOH}(10 \mathrm{~mL})$ wurde $1 \mathrm{~h}$ bei Raumtemperatur gerührt, die Reaktionsmischung auf $\mathrm{H}_{2} \mathrm{O}(100 \mathrm{~mL})$ gegeben und mit $\mathrm{Et}_{2} \mathrm{O}(3 \times 150 \mathrm{~mL})$ extrahiert. Die vereinigten organischen Phasen wurden über $\mathrm{Na}_{2} \mathrm{SO}_{4}$ getrocknet, filtriert und das Lösungsmittel unter vermindertem Druck entfernt. Nach säulenchromatographischer Reinigung an Kieselgel (Petrolether/EtOAc 9:1 $\rightarrow 1: 1$ ) wurde die Zielverbindung 173 (494 mg, $2.24 \mathrm{mmol}, 64 \%, \alpha: \beta=1: 1)$ als farbloses Öl erhalten.

DC: $\mathbf{R}_{f}=0.13$ (Petrolether/EtOAc 5:1).

UV $\left(\mathrm{CH}_{3} \mathrm{CN}\right)$ : keine Absorption.

IR: $\tilde{v}\left(\mathrm{~cm}^{-1}\right)=2935,2834,1749,1447,1372,1274,1110,1012,861,778$.

${ }^{1} \mathbf{H}$-NMR (300 MHz, $\mathrm{CDCl}_{3}, \alpha$-Anomer beschrieben): $\delta(\mathrm{ppm})=1.26(\mathrm{~d}, J=6.3 \mathrm{~Hz}, 3 \mathrm{H}$, 6-CH3), 3.07 (t, $J=9.3 \mathrm{~Hz}, 1 \mathrm{H}, 4-\mathrm{H}), 3.31$ (s, $3 \mathrm{H}, 1-\mathrm{OCH}_{3}$ ), 3.39-3.52 (m, $3 \mathrm{H}, 2-\mathrm{H}, 3-\mathrm{H}$, 5-H), 3.44, 3.45, $3.50\left(3 \mathrm{~s}, 9 \mathrm{H}, 3 \times \mathrm{OCH}_{3}\right), 4.67(\mathrm{~d}, J=1.8 \mathrm{~Hz}, 1 \mathrm{H}, 1-\mathrm{H})$.

${ }^{13}$ C-NMR $\left(126 \mathrm{MHz}, \mathrm{CDCl}_{3}\right): \delta(\mathrm{ppm})=17.8(\mathrm{C}-6), 54.7\left(1-\mathrm{OCH}_{3}\right), 57.7,59.0,60.9$ $\left(3 \times \mathrm{OCH}_{3}\right), 67.6(\mathrm{C}-5), 77.3,81.1,82.1(\mathrm{C}-2, \mathrm{C}-3, \mathrm{C}-4), 97.8(\mathrm{C}-1)$.

MS (ESI): m/z (\%) = $243.1(100)[\mathrm{M}+\mathrm{Na}]^{+}, 463.3(36)[2 \mathrm{M}+\mathrm{Na}]^{+}$.

HRMS von $\mathrm{C}_{10} \mathrm{H}_{20} \mathrm{O}_{5}$ ber.: $\quad 243.1203$ gef.: $243.1204[\mathrm{M}+\mathrm{Na}]^{+}(\mathrm{ESI}-\mathrm{HRMS})$.

$\mathbf{C}_{10} \mathbf{H}_{20} \mathbf{O}_{5}$ (220.26). 


\subsubsection{2,3,4-Tri- $O$-methyl- $\alpha / \beta$-L-rhamnopyranosid (151)}

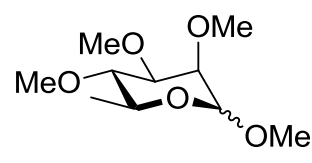

173

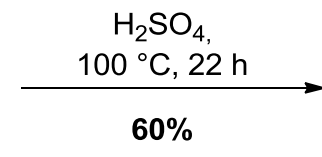

$60 \%$

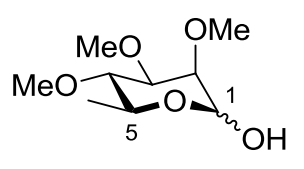

151

Die permethylierte L-Rhamnose $173\left(2.25 \mathrm{~g}, 11.8 \mathrm{mmol}, 1.0\right.$ Äq.) wurde in $0.5 \mathrm{M} \mathrm{H}_{2} \mathrm{SO}_{4}$ $(150 \mathrm{~mL})$ gelöst, $14 \mathrm{~h}$ bei $100{ }^{\circ} \mathrm{C}$ gerührt und anschließend die Reaktion durch Zugabe von $\mathrm{NaHCO}_{3}$ beendet. Es wurde ges. NaCl-Lösung $(50 \mathrm{~mL})$ zugegeben und mit EtOAc $(3 \times 50 \mathrm{~mL})$ extrahiert. Die vereinigten organischen Phasen wurden über $\mathrm{Na}_{2} \mathrm{SO}_{4}$ getrocknet, filtriert und das Lösungsmittel unter vermindertem Druck entfernt. Nach säulenchromatographischer Reinigung an Kieselgel (Petrolether/EtOAc $2: 1 \rightarrow 1: 2$ ) wurde die Titelverbindung 151 ( $2.00 \mathrm{~g}, 9.70 \mathrm{mmol}, 60 \%, \alpha: \beta=6: 1)$ in Form eines gelben Öls erhalten.

DC: $\mathrm{R}_{f}=0.29$ (Petrolether/EtOAc 1:1).

UV $\left(\mathrm{CH}_{3} \mathrm{CN}\right): \lambda_{\max }(\lg \varepsilon)=($ keine Absorption $)$.

IR: $\tilde{v}\left(\mathrm{~cm}^{-1}\right)=3388,2932,1137,1096,1086,1060,1024,987,788,586$.

${ }^{1} \mathbf{H}-\mathbf{N M R}\left(300 \mathrm{MHz}, \mathrm{CDCl}_{3}\right): \delta(\mathrm{ppm})=1.24\left(\mathrm{~d}, J=6.3 \mathrm{~Hz}, 3 \mathrm{H}, 6-\mathrm{H}_{3}\right), 2.46(\mathrm{~d}, J=3.3 \mathrm{~Hz}$, $1 \mathrm{H}, \mathrm{OH}), 3.09$ (t, $J=9.5 \mathrm{~Hz}, 1 \mathrm{H}, 4-\mathrm{H}), 3.46-3.54\left(\mathrm{~m}, 10 \mathrm{H}, 3 \times \mathrm{OCH}_{3}, 3-\mathrm{H}\right), 3.54-3.58$ (m, $1 \mathrm{H}, 2-\mathrm{H}), 3.77$ (dd, $J=9.5,6.3 \mathrm{~Hz}, 1 \mathrm{H}, 5-\mathrm{H}), 5.21$ (s, $1 \mathrm{H}, 1-\mathrm{H})$.

${ }^{13}$ C-NMR $\left(126 \mathrm{MHz}, \mathrm{CDCl}_{3}\right): \delta(\mathrm{ppm})=17.7(\mathrm{C}-6), 57.7,59.1,60.8\left(3 \times \mathrm{OCH}_{3}\right), 67.8(\mathrm{C}-5)$, 77.6, 80.6, 82.1 (C-2, C-3, C-4), 91.6 (C-1).

MS (ESI): $\mathrm{m} / \mathrm{z}(\%)=229.1(100)[\mathrm{M}+\mathrm{Na}]^{+}, 435.2(36)[2 \mathrm{M}+\mathrm{Na}]^{+}$.

HRMS von $\mathrm{C}_{9} \mathrm{H}_{18} \mathrm{O}_{5}$ ber.: $\quad 229.1046$ gef.: $229.1056[\mathrm{M}+\mathrm{Na}]^{+}(\mathrm{ESI}-\mathrm{HRMS})$.

$\mathbf{C}_{9} \mathbf{H}_{18} \mathbf{O}_{5}$ (206.24). 


\subsection{3 $O$-(2,3,4-Tri- $O$-methyl- $\alpha$-L-rhamnopyranosyl)trichloracetimidat (175)}

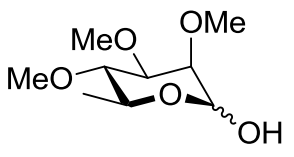

151

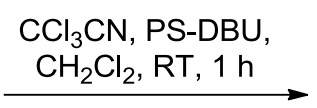

$\mathrm{CH}_{2} \mathrm{Cl}_{2}, \mathrm{RT}, 1 \mathrm{~h}$

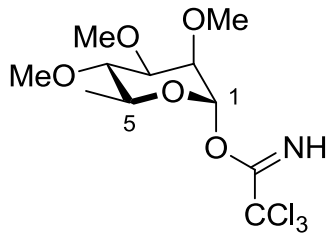

175

$\mathrm{Zu}$ einer Lösung des Pyranosids 151 (3.09 g, $15.0 \mathrm{mmol}, 1.0$ Äq.) in $\mathrm{CH}_{2} \mathrm{Cl}_{2}$ (280 mL) wurde bei $0{ }^{\circ} \mathrm{C} \mathrm{CCl}_{3} \mathrm{CN}(30.0 \mathrm{~mL}, 43.3 \mathrm{~g}, 300 \mathrm{mmol}, 20.0$ Äq.) und PS-DBU (174) $(6.56 \mathrm{mmol} / \mathrm{g}$, 3.43 g, 22.5 mmol, 1.5 Äq.) gegeben, auf Raumtemperatur erwärmt und $1 \mathrm{~h}$ gerührt. Die Reaktionslösung wurde durch Celite ${ }^{\circledR}$ filtriert, der Rückstand mit $\mathrm{CH}_{2} \mathrm{Cl}_{2}$ (400 mL) gewaschen und das Lösungsmittel des Filtrats unter vermindertem Druck entfernt. Das Trichloracetimidat 175 (5.01 g) wurde in Form eines gelben Öls analysenrein erhalten und ohne weitere Reinigung in der nächsten Reaktion verwendet.

DC: $\mathrm{R}_{f}=0.31(\mathrm{PE} / \mathrm{EE} 4: 1)$.

${ }^{1} \mathbf{H}-\mathbf{N M R}\left(300 \mathrm{MHz}, \mathrm{CDCl}_{3}\right): \delta(\mathrm{ppm})=1.31(\mathrm{~d}, J=6.2 \mathrm{~Hz}, 3 \mathrm{H}, 6-\mathrm{H} 3), 3.21(\mathrm{t}, J=9.5 \mathrm{~Hz}$, $1 \mathrm{H}, 4-\mathrm{H}), 3.50,3.54,3.56\left(3 \mathrm{~s}, 9 \mathrm{H}, 3 \times \mathrm{OCH}_{3}\right), 3.50-3.53(\mathrm{~m}, 1 \mathrm{H}, 3-\mathrm{H}), 3.71-3.80(\mathrm{~m}$, $2 \mathrm{H}, 2-\mathrm{H}, 5-\mathrm{H}), 6.29$ (d, $J=1.9 \mathrm{~Hz}, 1 \mathrm{H}, 1-\mathrm{H}), 8.57$ (s, $1 \mathrm{H}, \mathrm{NH})$.

MS (ESI): m/z (\%) = $374.0(42)[\mathrm{M}+\mathrm{Na}]^{+}, 723.1(100)[2 \mathrm{M}+\mathrm{Na}]^{+}$.

HRMS von $\mathrm{C}_{11} \mathrm{H}_{18} \mathrm{Cl}_{3} \mathrm{NO}_{5}$ ber.: $\quad 374.0106$ gef.: $374.0114[\mathrm{M}+\mathrm{Na}]^{+}(\mathrm{ESI}-\mathrm{HRMS})$.

$\mathrm{C}_{11} \mathrm{H}_{18} \mathrm{Cl}_{3} \mathrm{NO}_{5}$ (350.62). 


\subsection{Synthese des tricyclischen Aldehyds 149}

\subsubsection{2-Brom-5-methoxybenzaldehyd (177)}

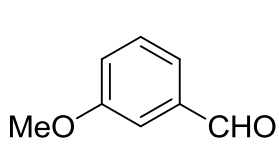

176

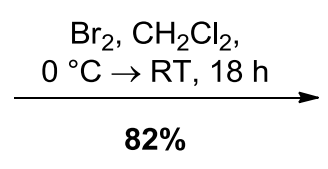

$82 \%$<smiles>COc1ccc(Br)c(C=O)c1</smiles>

177

$\mathrm{Zu}$ einer Lösung aus 3-Methoxybenzaldehyd (176) (5.00 g, $36.7 \mathrm{mmol}, 1.0$ Äq.) in $\mathrm{CH}_{2} \mathrm{Cl}_{2}$ $(700 \mathrm{~mL})$ wurde bei $0{ }^{\circ} \mathrm{C}$ langsam Brom $(1.88 \mathrm{~mL}, 5.87 \mathrm{~g}, 36.7 \mathrm{mmol}, 1.0$ Äq.) gegeben, anschließend auf Raumtemperatur erwärmt und $18 \mathrm{~h}$ unter Lichtausschluss gerührt. Das Reaktionsgemisch wurde bis zur vollständigen Entfärbung mit 5\%iger $\mathrm{Na}_{2} \mathrm{~S}_{2} \mathrm{O}_{3}$-Lösung und danach bis zur Beendigung der Gasentwicklung mit ges. $\mathrm{NaHCO}_{3}$-Lösung versetzt. Die organische Phase wurde abgetrennt, über $\mathrm{Na}_{2} \mathrm{SO}_{4}$ getrocknet und das Lösungsmittel unter vermindertem Druck entfernt. Umkristallisation des Rohprodukts aus $n$ Pentan lieferte die Zielverbindung 177 (6.48 g, 30.1 mmol, 82\%) als hellgelbe Nadeln.

DC: $\mathrm{R}_{f}=0.50$ (Petrolether/Et $\left.2 \mathrm{O} 4: 1\right)$.

UV $\left(\mathrm{CH}_{3} \mathrm{CN}\right): \lambda_{\max }(\lg \varepsilon)=225 \mathrm{~nm}$ (4.3388), 253 (3.8498), 329 (3.4456).

IR: $\tilde{v}\left(\mathrm{~cm}^{-1}\right)=2873,2363,1978,1895,1673,1469,1277,1197,931,864,819,647,597$.

${ }^{1} \mathbf{H}-\mathbf{N M R}\left(300 \mathrm{MHz}, \mathrm{CDCl}_{3}\right): \delta(\mathrm{ppm})=3.81\left(\mathrm{~s}, 3 \mathrm{H}, \mathrm{OCH}_{3}\right), 7.00(\mathrm{dd}, J=8.8,3.2 \mathrm{~Hz}, 1 \mathrm{H}$, 4-H), 7.38 (d, J=3.2 Hz, $1 \mathrm{H}, 6-\mathrm{H}), 7.49$ (d, $J=8.8 \mathrm{~Hz}, 1 \mathrm{H}, 3-\mathrm{H}), 10.28$ (s, $1 \mathrm{H}, \mathrm{CHO}$ ).

${ }^{13}$ C-NMR $\left(126 \mathrm{MHz}, \mathrm{CDCl}_{3}\right): \delta(\mathrm{ppm})=55.7\left(\mathrm{OCH}_{3}\right), 112.6(\mathrm{C}-6), 117.8(\mathrm{C}-2), 123.0(\mathrm{C} 4)$, 133.8 (C-1), 134.4 (C-3), 159.1 (C-5), 191.5 (CHO).

MS (ESI): $\mathrm{m} / \mathrm{z}(\%)=269.0(63)[\mathrm{M}+\mathrm{MeOH}+\mathrm{Na}]^{+}, 516.9(100)[2 \mathrm{M}+2 \mathrm{MeOH}+\mathrm{Na}]^{+}$.

HRMS von $\mathrm{C}_{8} \mathrm{H}_{7} \mathrm{BrO}_{2}$ ber.: 236.9522 gef.: $236.9519[\mathrm{M}+\mathrm{Na}]^{+}(\mathrm{ESI}-\mathrm{HRMS})$.

$\mathrm{C}_{8} \mathrm{H}_{7} \mathrm{BrO}_{2}$ (215.04). 


\subsubsection{2-Brom-5-hydroxybenzaldehyd (178)}<smiles>COc1ccc(Br)c(C=O)c1</smiles>

177

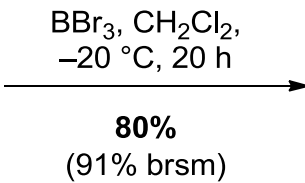

$(91 \%$ brsm)

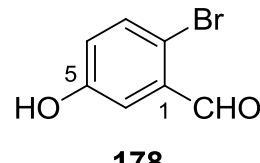

178

$\mathrm{Zu}$ einer Lösung des Aromaten 177 (10.3 g, 47.9 mmol, 1.00 Äq.) in absolutem $\mathrm{CH}_{2} \mathrm{Cl}_{2}$ (200 mL) wurde bei $-20{ }^{\circ} \mathrm{C}$ langsam $\mathrm{BBr}_{3}\left(1 \mathrm{M}\right.$ in $\mathrm{CH}_{2} \mathrm{Cl}_{2}, 90.0 \mathrm{~mL}, 90.0 \mathrm{mmol}, 1.9$ Äq.) getropft. Die Reaktionsmischung wurde $22 \mathrm{~h}$ bei dieser Temperatur gerührt, anschließend auf Raumtemperatur erwärmt und die Reaktion durch Zugabe von ges. $\mathrm{NH}_{4} \mathrm{Cl}$-Lösung (500 mL) beendet. Nach Zugabe von $\mathrm{Et}_{2} \mathrm{O}(500 \mathrm{~mL})$ wurde die organische Phase abgetrennt und die wässrige Phase mit $\mathrm{Et}_{2} \mathrm{O}(4 \times 150 \mathrm{~mL})$ extrahiert. Die vereinigten organischen Phasen wurden mit ges. NaCl-Lösung $(150 \mathrm{~mL})$ gewaschen, über $\mathrm{Na}_{2} \mathrm{SO}_{4}$ getrocknet und das Lösungsmittel unter vermindertem Druck entfernt. Nach säulenchromatographischer Reinigung des Rückstands an Kieselgel ( $n$ Pentan/EtOAc 7:1 $\rightarrow 5: 1$ ) wurde die Titelverbindung 178 (7.68 g, $38.2 \mathrm{mmol}, 80 \%, 91 \%$ brsm) in Form eines hellbraunen Feststoffes erhalten.

DC: $\mathbf{R}_{f}=0.15(n$ Pentan/EtOAc 6:1).

UV $\left(\mathrm{CH}_{3} \mathrm{CN}\right): \lambda_{\max }(\lg \varepsilon)=223 \mathrm{~nm}(4.3220), 253$ (3.8522), 330 (3.4494).

IR: $\tilde{v}\left(\mathrm{~cm}^{-1}\right)=3314,1921,1733,1672,1590,1438,1303,1233,1169,864,831,761,656$, 584.

${ }^{1} \mathrm{H}-\mathrm{NMR}\left(300 \mathrm{MHz}, \mathrm{CDCl}_{3}\right): \delta(\mathrm{ppm})=5.72\left(\mathrm{~s}_{\mathrm{br}}, 1 \mathrm{H}, \mathrm{OH}\right), 7.00(\mathrm{dd}, J=8.6,3.2 \mathrm{~Hz}, 1 \mathrm{H}$ 4-H), 7.42 (d, $J=3.2 \mathrm{~Hz}, 1 \mathrm{H}, 6-\mathrm{H}), 7.50$ (d, $J=8.6 \mathrm{~Hz}, 1 \mathrm{H}, 3-\mathrm{H}), 10.27$ (s, $1 \mathrm{H}, \mathrm{CHO}$ ).

${ }^{13}$ C-NMR $\left(126 \mathrm{MHz}, \mathrm{CDCl}_{3}\right): \delta(\mathrm{ppm})=115.7(\mathrm{C}-6), 117.8(\mathrm{C}-2), 123.5(\mathrm{C}-4), 134.0(\mathrm{C}-1)$, 135.0 (C-3), 155.6 (C-5), 192.3 (CHO).

MS (ESI): $\mathrm{m} / \mathrm{z}(\%)=224.9(29)[\mathrm{M}+\mathrm{Na}]^{+}, 255.0(100)[\mathrm{M}+\mathrm{MeOH}+\mathrm{Na}]^{+}$.

HRMS von $\mathrm{C}_{7} \mathrm{H}_{5} \mathrm{BrO}_{2}$

ber.: 222.9365

gef.: $222.9365[\mathrm{M}+\mathrm{Na}]^{+}(\mathrm{ESI}-\mathrm{HRMS})$.

$\mathbf{C}_{7} \mathrm{H}_{5} \mathrm{BrO}_{2}(201.02)$. 


\subsubsection{2-Brom-5-(2,3,4-tri- $O$-methyl- $\alpha$-L-rhamnopyranosyl)benzaldehyd (179)}<smiles>COC1C(OC)C(OC)C(OC(=N)C(F)(F)F)C1OC</smiles>

175<smiles>O=Cc1cc(O)ccc1Br</smiles>

178

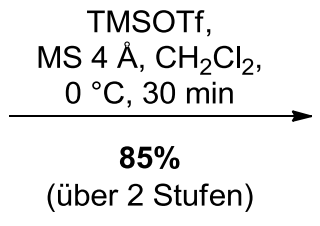

(über 2 Stufen)

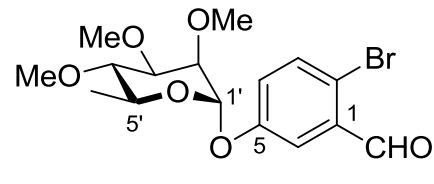

179

Eine Lösung des Aldehyds 178 (4.22 g, 21.0 mmol, 1.9 Äq.) in $\mathrm{CH}_{2} \mathrm{Cl}_{2}$ (500 mL) wurde bei Raumtemperatur 90 min über aktiviertem $4 \AA$ Molekularsieb (30 g) gerührt. Die Reaktionsmischung wurde auf $0{ }^{\circ} \mathrm{C}$ gekühlt und eine Lösung des Trichloracetimidats 175 (4.45 g, $12.7 \mathrm{mmol}, \quad 1.0$ Äq.) in $\mathrm{CH}_{2} \mathrm{Cl}_{2}$ (20 mL) sowie TMSOTf (190 $\mu \mathrm{L}, 233 \mathrm{mg}$, $1.05 \mathrm{mmol}, 10 \mathrm{~mol} \%)$ gelöst in $\mathrm{CH}_{2} \mathrm{Cl}_{2}(20 \mathrm{~mL})$ zugetropft. Es wurde $75 \mathrm{~min}$ bei $0{ }^{\circ} \mathrm{C}$ gerührt und anschließend $\mathrm{NEt}_{3}$ (684 $\mu \mathrm{L}, 499 \mathrm{mg}, 4.99 \mathrm{mmol}, 0.5$ Äq.) zugegeben. Nach Erwärmen auf Raumtemperatur wurde durch Celite ${ }^{\circledR}$ filtriert und mit $\mathrm{CH}_{2} \mathrm{Cl}_{2}(750 \mathrm{~mL})$ gewaschen. Das Filtrat wurde unter vermindertem Druck eingeengt und der Rückstand durch Säulenchromatographie an Kieselgel ( $n$ Pentan/EtOAc 7:1) gereinigt. Die Titelverbindung 179 (4.22 g, $10.8 \mathrm{mmol}, 85 \%$ ) wurde als farbloses Öl erhalten.

DC: $\mathrm{R}_{f}=0.24(n$ Pentan/EtOAc $3: 1)$.

Drehwert: $[\alpha]_{\mathrm{D}}^{20}=-66.5^{\circ}\left(c=1.3, \mathrm{CHCl}_{3}\right)$.

UV $\left(\mathrm{CH}_{3} \mathrm{CN}\right): \lambda_{\max }(\lg \varepsilon)=223 \mathrm{~nm}$ (4.3412), 251 (3.8337), 322 (3.3332).

IR: $\tilde{v}\left(\mathrm{~cm}^{-1}\right)=1692,1469,1389,1300,1271,1228,1169,1133,1120,1101,1005,824$.

${ }^{1} \mathbf{H}$-NMR $\left(300 \mathrm{MHz}, \mathrm{CDCl}_{3}\right): \delta(\mathrm{ppm})=1.22\left(\mathrm{~d}, J=6.3 \mathrm{~Hz}, 3 \mathrm{H}, 6^{\prime}-\mathrm{H}_{3}\right), 3.18(\mathrm{t}, J=9.3 \mathrm{~Hz}$, $\left.1 \mathrm{H}, 4^{\prime}-\mathrm{H}\right), 3.54$ (s, $\left.9 \mathrm{H}, 3 \times \mathrm{OCH}_{3}\right), 3.56-3.64$ (m, $\left.2 \mathrm{H}, 3^{\prime}-\mathrm{H}, 5^{\prime}-\mathrm{H}\right), 3.74$ (dd, J = 3.3, $2.0 \mathrm{~Hz}$, $\left.1 \mathrm{H}, 2^{\prime}-\mathrm{H}\right), 5.54$ (d, $\left.J=2.0 \mathrm{~Hz}, 1 \mathrm{H}, 1^{\prime}-\mathrm{H}\right), 7.16(\mathrm{dd}, J=8.8,3.1 \mathrm{~Hz}, 1 \mathrm{H}, 4-\mathrm{H}), 7.53(\mathrm{~d}, J=$ $8.8 \mathrm{~Hz}, 1 \mathrm{H}, 3-\mathrm{H}), 7.58$ (d, J=3.1 Hz, $1 \mathrm{H}, 6-\mathrm{H}), 10.27$ (s, $1 \mathrm{H}, \mathrm{CHO}$ ).

${ }^{13}$ C-NMR $\left(126 \mathrm{MHz}, \mathrm{CDCl}_{3}\right): \delta(\mathrm{ppm})=17.9\left(\mathrm{C}^{\prime} 6^{\prime}\right), 58.0,59.3,60.9\left(3 \times \mathrm{OCH}_{3}\right), 69.0\left(\mathrm{C}^{\prime} 5^{\prime}\right)$, 77.0, 80.7, 81.8 (C-2', C-3', C-4'), 95.4 (C-1'), 116.9 (C-6), 119.1 (C-2), 123.7 (C-4), 134.1 (C-1), 134.7 (C-3), 155.8 (C-5), 191.2 (CHO).

MS (ESI): $\mathrm{m} / \mathrm{z}(\%)=405.1(100)\left[\mathrm{M}+\mathrm{NH}_{4}\right]^{+}, 443.1(50)[\mathrm{M}+\mathrm{MeOH}+\mathrm{Na}]^{+}$.

HRMS von $\mathrm{C}_{16} \mathrm{H}_{21} \mathrm{BrO}_{6}$ ber.: 411.0414 gef.: $411.0413[\mathrm{M}+\mathrm{Na}]^{+}(\mathrm{ESI}-\mathrm{HRMS})$.

$\mathrm{C}_{16} \mathrm{H}_{21} \mathrm{BrO}_{6}$ (389.24). 


\subsection{4 (Z)-2-(2-Iodethenyl)-4-(2,3,4-tri- $O$-methyl- $\alpha$-L-rhamnopyranosyl)-brombenzol} (180)

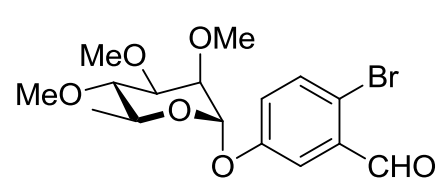

179

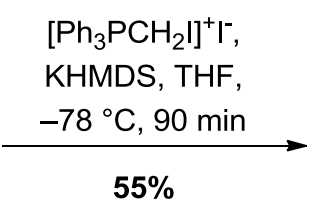

$55 \%$

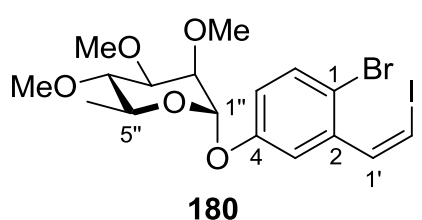

180

Zu einer Suspension des Wittig-Salzes 265 (8.42 g, 15.9 mmol, 1.5 Äq.) in THF (150 mL) wurde bei Raumtemperatur langsam KHMDS (0.5 M in Toluol, $36.9 \mathrm{~mL}, 18.4 \mathrm{mmol}, 1.8$ Äq.) getropft und 5 min gerührt. Das Reaktionsgemisch wurde auf $-78^{\circ} \mathrm{C}$ gekühlt und langsam eine Lösung des Aldehyds 179 (4.10 g, 10.5 mmol, 1.0 Äq.) in THF (60 mL) zugefügt. Es wurde $1 \mathrm{~h}$ bei $-78{ }^{\circ} \mathrm{C}$ und $1.5 \mathrm{~h}$ bei Raumtemperatur gerührt. Die Reaktion wurde durch Zugabe von ges. $\mathrm{NH}_{4} \mathrm{Cl}$-Lösung $(200 \mathrm{~mL}$ ) abgebrochen, die Phasen getrennt und die wässrige Phase mit $\mathrm{Et}_{2} \mathrm{O}(4 \times 100 \mathrm{~mL})$ extrahiert. Die organischen Extrakte wurden vereint, mit ges. $\mathrm{NaCl}$-Lösung $(150 \mathrm{~mL})$ gewaschen, über $\mathrm{Na}_{2} \mathrm{SO}_{4}$ getrocknet und das Lösungsmittel unter vermindertem Druck entfernt. Säulenchromatographie an Kieselgel (nPentan/MTBE 7:1) lieferte die Titelverbindung 180 (2.96 g, $5.77 \mathrm{mmol}$, 55\%) in Form eines hellbraunen Feststoffes.

DC: $\mathbf{R}_{f}=0.34$ (Petrolether/MTBE 1:1).

Drehwert: $[\alpha]_{\mathrm{D}}^{20}=-58.7^{\circ}\left(c=1.1, \mathrm{CHCl}_{3}\right)$.

UV $\left(\mathrm{CH}_{3} \mathrm{CN}\right): \lambda_{\max }(\lg \varepsilon)=200.0 \mathrm{~nm}(4.380)$.

IR: $\tilde{v}\left(\mathrm{~cm}^{-1}\right)=1458,1197,1148,1119,1102,1090,1046,1037,1008,991,931,873,799$, $692,657$.

${ }^{1} \mathbf{H}-\mathbf{N M R}\left(300 \mathrm{MHz}, \mathrm{CDCl}_{3}\right): \delta(\mathrm{ppm})=1.25\left(\mathrm{~d}, J=6.3 \mathrm{~Hz}, 3 \mathrm{H}, 6^{\prime \prime}-\mathrm{H}_{3}\right), 3.18(\mathrm{t}, J=9.6 \mathrm{~Hz}$, $1 \mathrm{H}, 4$ "-H), 3.54, 3.54, 3.55 (3 s, 9 H, 3× $\mathrm{OCH}_{3}$ ), 3.57-3.67 (m, 2 H, 3"-H, 5"-H), 3.75 (dd, $J=$ 3.3, 2.0 Hz, $\left.1 \mathrm{H}, 2^{2}-\mathrm{H}\right), 5.52$ (d, $\left.J=2.0 \mathrm{~Hz}, 1 \mathrm{H}, 1^{\prime \prime}-\mathrm{H}\right), 6.72$ (d, J = 8.7 Hz, $\left.1 \mathrm{H}, 2^{\prime}-\mathrm{H}\right), 6.91$ $(\mathrm{dd}, J=8.7,2.9 \mathrm{~Hz}, 1 \mathrm{H}, 5-\mathrm{H}), 7.27\left(\mathrm{~d}, J=8.4 \mathrm{~Hz}, 1 \mathrm{H}, 1^{\prime}-\mathrm{H}\right), 7.36(\mathrm{~d}, J=2.9 \mathrm{~Hz}, 1 \mathrm{H}, 3-\mathrm{H})$, $7.46(\mathrm{~d}, J=8.7 \mathrm{~Hz}, 1 \mathrm{H}, 6-\mathrm{H})$.

${ }^{13}$ C-NMR $\left(126 \mathrm{MHz}, \mathrm{CDCl}_{3}\right): \delta(\mathrm{ppm})=17.9(\mathrm{C}-6 ")$, 57.9, 59.3, $61.0\left(3 \times \mathrm{OCH}_{3}\right), 68.8(\mathrm{C}-5 ")$, 77.2, 80.8, 81.9 (C-2", C-3", C-4"), 83.9 (C-2'), 95.5 (C-1"), 115.5 (C-1), 117.9, 118.0 (C-3, C-5), 133.4 (C-6), 138.4 (C-2), 138.7 (C-1'), 155.1 (C-4).

MS (ESI): $\mathrm{m} / \mathrm{z}(\%)=535.0(59)[\mathrm{M}+\mathrm{Na}]^{+}, 1049.0(100)[2 \mathrm{M}+\mathrm{Na}]^{+}$. 
HRMS von $\mathrm{C}_{17} \mathrm{H}_{22} \mathrm{BrIO}_{5}$

ber.: $\quad 534.9588$

gef.: $\quad 534.9580[\mathrm{M}+\mathrm{Na}]^{+}(\mathrm{ESI}-\mathrm{HRMS})$.

$\mathrm{C}_{17} \mathrm{H}_{22} \mathrm{BrIO}_{5}$ (513.16). 
4.2.5 2-\{2-[(Z)-2-(2-Brom-5-(2,3,4-tri- $O$-methyl- $\alpha$-L-rhamnopyranosyl)phenyl) vinyl]-5tert-butyldimethylsilyloxymethylcyclopent-3-enyl\}essigsäure-tert-butylester (183)
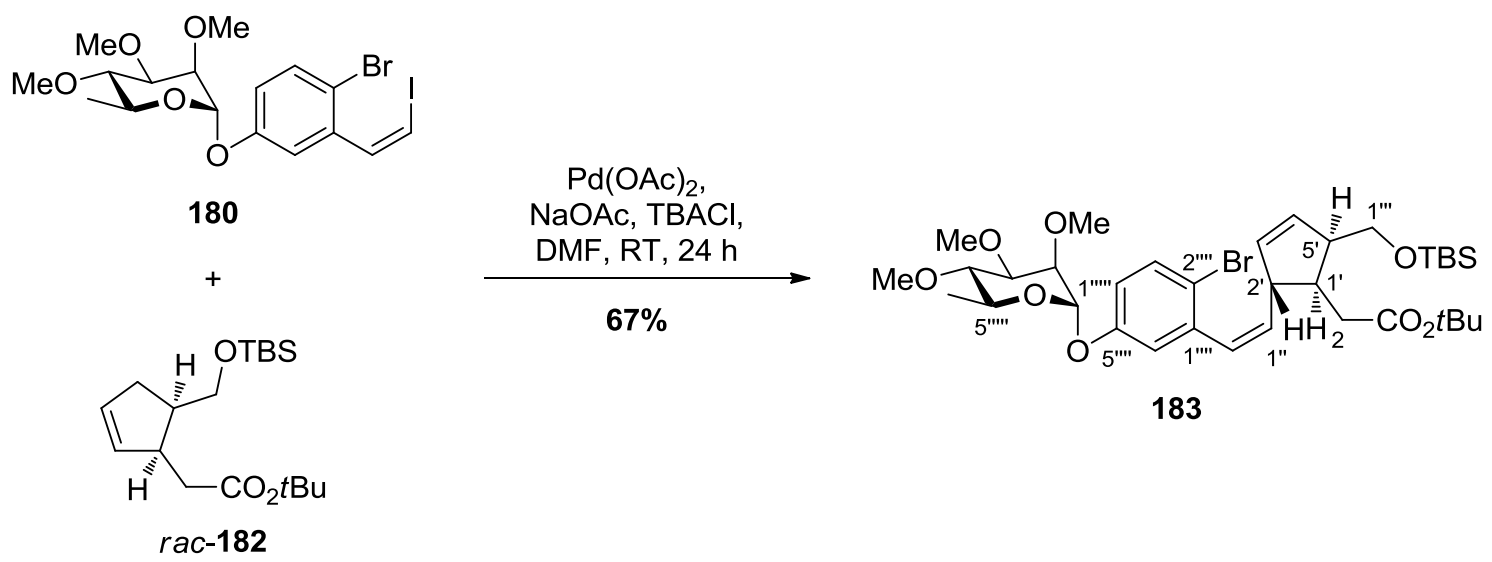

183

Eine Lösung aus Vinyliodid $\mathbf{1 8 0}(2.09 \mathrm{~g}, 4.07 \mathrm{mmol}, \quad 1.0$ Äq.) und racemischem Cyclopentenderivat 182 (2.00 mg, $6.14 \mathrm{mmol}, 1.5$ Äq.) in DMF (100 mL) wurde $15 \mathrm{~min}$ entgast anschließend $\mathrm{Pd}(\mathrm{OAc})_{2}(92.0 \mathrm{mg}, 410 \mu \mathrm{mol}, 10 \mathrm{~mol} \%)$, NaOAc (1.00 g, $12.3 \mathrm{mmol}$, 3.0 Äq.) und TBACl (1.36 g, 4.91 mmol, 1.2 Äq.) zugefügt und die Reaktionsmischung 24 h bei Raumtemperatur unter Lichtausschluss gerührt. Das Reaktionsgemisch wurde anschließend mit $\mathrm{Et}_{2} \mathrm{O}(200 \mathrm{~mL})$ verdünnt und mit $\mathrm{H}_{2} \mathrm{O}(100 \mathrm{~mL})$ gewaschen. Die wässrige Phase wurde mit $\mathrm{Et}_{2} \mathrm{O}(3 \times 150 \mathrm{~mL})$ extrahiert. Die vereinigten organischen Extrakte wurden mit ges. NaCl-Lösung. (300 mL) gewaschen, über $\mathrm{Na}_{2} \mathrm{SO}_{4}$ getrocknet und das Lösungsmittel unter vermindertem Druck entfernt. Nach säulenchromatographischer Reinigung an Kieselgel ( $n$ Pentan/MTBE 7:1 $\rightarrow 4: 1$ ) wurde die Zielverbindung 183 (1.95 mg, $2.74 \mathrm{mmol}, 67 \%$ ) als 2:1Gemisch der Diastereomere in Form eines farblosen Öls erhalten.

DC: $\mathrm{R}_{f}=0.70$ (Petrolether/MTBE 1:1).

${ }^{1} \mathbf{H}-\mathbf{N M R}\left(300 \mathrm{MHz}, \mathrm{CDCl}_{3}, 2: 1-G e m i s c h\right.$ der Diastereomere): $\delta(\mathrm{ppm})=-0.09,-0.07(2 \mathrm{~s}$, $\left.6 \mathrm{H}, \mathrm{Si}\left(\mathrm{CH}_{3}\right)_{2}\right), 0.74\left(\mathrm{~s}, 9 \mathrm{H}, \mathrm{SiC}\left(\mathrm{CH}_{3}\right)_{3}\right), 1.21$ (d, J = 6.0 Hz, $3 \mathrm{H}, 6$ 6"'"-H $\left.\mathrm{H}_{3}\right), 1.39$ (s, $9 \mathrm{H}$, $\left.\mathrm{CO}_{2} \mathrm{C}\left(\mathrm{CH}_{3}\right)_{3}\right), 2.26-2.43$ (m, 3 H, 2-H $\left.{ }_{2}, 1^{\prime}-\mathrm{H}\right), 2.83$ (m, 1 H, 5'-H), 3.14-3.19 (m, 2 H, 2'-H,

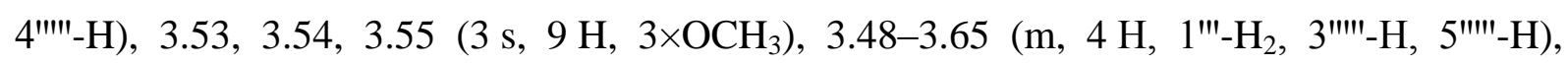
3.72-3.75 (m, 1 H, 2"'"'-H), 5.48 (d, J = 1.8 Hz, 1 H, 1"'"'-H), 5.44-5.53 (m, 1 H, 1"-H), 5.645.77 (m, 2 H, 3'-H, 4'-H), 6.40 (d, J = 11.4 Hz, 1 H, 2"-H), 6.82-6.89 (m, 2 H, 4"'--H, 6'"'-H), $7.42\left(\mathrm{~d}, J=8.7 \mathrm{~Hz}, 1 \mathrm{H}, 3^{\prime \prime \prime}-\mathrm{H}\right)$.

${ }^{13}$ C-NMR (126 MHz, $\mathrm{CDCl}_{3}, 2: 1-$ Gemisch der Diastereomere): $\delta(\mathrm{ppm})=-5.7,-5.5$ $\left(\mathrm{Si}\left(\mathrm{CH}_{3}\right)_{2}\right), 17.8\left(\mathrm{C}-6\right.$ '"''), $18.0\left(\mathrm{SiC}\left(\mathrm{CH}_{3}\right)_{3}\right), 25.8\left(\mathrm{SiC}\left(\underline{\mathrm{CH}_{3}}\right)_{3}\right), 28.1\left(\mathrm{CO}_{2} \mathrm{C}\left(\underline{\mathrm{C}} \mathrm{H}_{3}\right)_{3}\right), 34.6(\mathrm{C}-2)$,

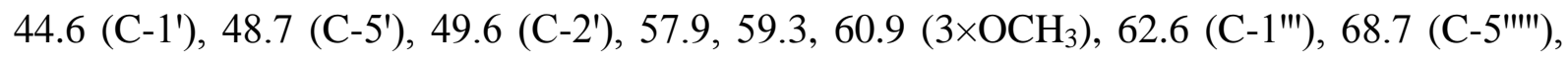
77.3 (C-2'"'"), $80.0\left(\mathrm{CO}_{2} \underline{\mathrm{C}}\left(\mathrm{CH}_{3}\right)_{3}\right), 80.8$ (C-3'"'"), 81.9 (C-4"'"'), 95.3 (C-1"'"'), 116.0 (C-4'"'), 
116.1 (C-2"'"), 118.8 (C-6"''), 129.7 (C-2"), 133.1 (C-3"'"), 133.8, 134.4 (C-3', C-4'), 136.4 (C-1"), 138.8 (C-1"'), 155.2 (C-5"''), 172.8 (C-1).

MS (ESI): $\mathrm{m} / \mathrm{z}(\%)=735.3(100)[\mathrm{M}+\mathrm{Na}]^{+}, 1445.6(30)[2 \mathrm{M}+\mathrm{Na}]^{+}$.

HRMS von $\mathrm{C}_{35} \mathrm{H}_{55} \mathrm{BrO}_{8} \mathrm{Si}$ ber.: 733.2742

gef.: $733.2742[\mathrm{M}+\mathrm{Na}]^{+}(\mathrm{ESI}-\mathrm{HRMS})$.

$\mathrm{C}_{35} \mathrm{H}_{55} \mathrm{BrO} \mathrm{O}_{8} \mathrm{Si}(711.80)$. 


\subsubsection{2-[2-(tert-Butyldimethylsilyloxymethyl)-7-(2,3,4-tri- $O$-methyl- $\alpha$-L-rhamno- pyranosyl)-3a,9b-dihydro-3H-cyclopenta[a]naphthalin-3-yl]essigsäure-tert- butylester (185)}
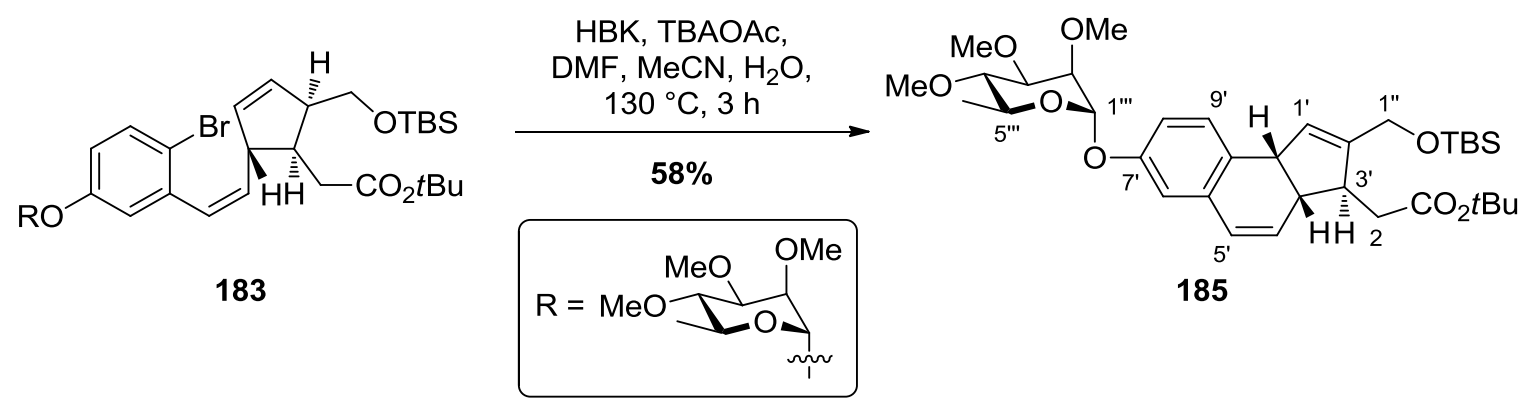

Eine Lösung aus Verbindung 183 (1.95 g, $2.74 \mathrm{mmol}, 1.0$ Äq.) und $n \mathrm{Bu}_{4} \mathrm{NOAc}$ (1.65 g, $5.48 \mathrm{mmol}, 2.0$ Äq.) in einem Lösungsmittelgemisch aus DMF/ $\mathrm{CH}_{3} \mathrm{CN} / \mathrm{H}_{2} \mathrm{O}(5: 5: 1,100 \mathrm{~mL})$ wurde 15 min entgast, bei Raumtemperatur mit dem Herrmann-Beller-Katalysator (122) (181 mg, $192 \mu \mathrm{mol}, 7 \mathrm{~mol} \%$ ) versetzt und anschließend $3 \mathrm{~h}$ bei $130{ }^{\circ} \mathrm{C}$ gerührt. Nach Abkühlen auf Raumtemperatur wurde die Reaktionslösung mit MTBE (100 mL) verdünnt, mit $\mathrm{H}_{2} \mathrm{O}(100 \mathrm{~mL})$ gewaschen und die wässrige Phase mit MTBE $(3 \times 100 \mathrm{~mL})$ extrahiert. Die vereinigten organischen Extrakte wurden über $\mathrm{Na}_{2} \mathrm{SO}_{4}$ getrocknet und das Lösungsmittel unter vermindertem Druck entfernt. Säulenchromatographische Reinigung an Kieselgel ( $n$ Pentan/EtOAc 5:1) lieferte die Zielverbindung 185 (988 mg, $1.57 \mathrm{mmol}, 58 \%$ ) als 2:1Gemisch der Diastereomere in Form eines farblosen Schaums.

DC: $\mathrm{R}_{f}=0.52($ Petrolether/EtOAc 3:1).

UV (MeOH): $\lambda_{\max }(\lg \varepsilon)=228 \mathrm{~nm}$ (4.4565), 265 (3.7604), 274 (3.6873), 298 (3.4165), 309 (3.3836).

IR: $\tilde{v}\left(\mathrm{~cm}^{-1}\right)=2929,1990,1726,1602,1366,1255,1137,1100,1088,1008,992,835776$.

${ }^{1} \mathbf{H}$-NMR $\left(600 \mathrm{MHz}, \mathrm{CDCl}_{3}, 2: 1\right.$-Gemisch der Diastereomere): $\delta(\mathrm{ppm})=0.00(\mathrm{~s}, 6 \mathrm{H}$, $\left.\mathrm{Si}\left(\mathrm{CH}_{3}\right)_{2}\right), 0.86\left(\mathrm{~s}, 9 \mathrm{H}, \mathrm{SiC}\left(\mathrm{CH}_{3}\right)_{3}\right), 1.23\left(\mathrm{~d}, J=6.0 \mathrm{~Hz}, 3 \mathrm{H}, 66^{\prime \prime}-\mathrm{H}_{3}\right), 1.44(\mathrm{~s}, 9 \mathrm{H}$, $\left.\mathrm{CO}_{2} \mathrm{C}\left(\mathrm{CH}_{3}\right)_{3}\right), 2.27\left(\mathrm{dd}, J=15.6,6.0 \mathrm{~Hz}, 1 \mathrm{H}, 2-\mathrm{H}_{\mathrm{A}}\right), 2.58\left(\mathrm{dd}, J=15.6,4.3 \mathrm{~Hz}, 1 \mathrm{H}, 2-\mathrm{H}_{\mathrm{B}}\right)$, 2.94-3.11 ( $\left.\mathrm{m}_{\mathrm{c}}, 1 \mathrm{H}, 3^{\prime}-\mathrm{H}\right), 3.11-3.14\left(\mathrm{~m}_{\mathrm{c}}, 1 \mathrm{H}, 3 \mathrm{a}^{\prime}-\mathrm{H}\right), 3.16$ (t, $\left.J=9.6 \mathrm{~Hz}, 1 \mathrm{H}, 4^{\prime \prime \prime}-\mathrm{H}\right), 3.52$, 3.54, $3.55\left(3 \mathrm{~s}, 9 \mathrm{H}, 3 \times \mathrm{OCH}_{3}\right), 3.62-3.74\left(\mathrm{~m}, 1 \mathrm{H}, 5^{\prime \prime}-\mathrm{H}\right), 3.64(\mathrm{dd}, J=9.6,3.3 \mathrm{~Hz}, 1 \mathrm{H}$, 3'"-H), 3.71 (dd, $\left.J=3.3,1.7 \mathrm{~Hz}, 1 \mathrm{H}, 2^{\prime \prime \prime}-\mathrm{H}\right), 4.04$ (m $\left., 1 \mathrm{H}, 9 \mathrm{~b}^{\prime}-\mathrm{H}\right), 4.17$ (d, $J=1.8 \mathrm{~Hz}, 2 \mathrm{H}$, 1"- $\left.\mathrm{H}_{2}\right), 5.41\left(\mathrm{~m}_{\mathrm{c}}, 1 \mathrm{H}, 1^{\prime}-\mathrm{H}\right), 5.47(\mathrm{~d}, J=1.8 \mathrm{~Hz}, 1 \mathrm{H}, 1$ '"-H), $5.72(\mathrm{dd}, J=9.7,3.0 \mathrm{~Hz}, 1 \mathrm{H}$, 4'-H), 6.20 (dd, $J=9.7,1.8 \mathrm{~Hz}, 1 \mathrm{H}, 5$ '-H), 6.68 (dd, $J=8.1,2.4 \mathrm{~Hz}, 1 \mathrm{H}, 6$ '-H), 6.82 (m $\mathrm{m}_{\mathrm{c}}, 1 \mathrm{H}$, 8'-H), $7.01\left(\mathrm{~d}, J=8.1 \mathrm{~Hz}, 1 \mathrm{H}, 9^{\prime}-\mathrm{H}\right)$. 
${ }^{13}$ C-NMR (126 MHz, $\mathrm{CDCl}_{3}, 2: 1-$ Gemisch der Diastereomere): $\delta(\mathrm{ppm})=-5.4,-5.4$ $\left(\mathrm{Si}\left(\mathrm{CH}_{3}\right)_{2}\right), 17.8\left(\mathrm{C}-6{ }^{\prime \prime \prime}\right), 18.3\left(\mathrm{Si} \underline{\mathrm{C}}\left(\mathrm{CH}_{3}\right)_{3}\right), 25.9\left(\mathrm{SiC}\left(\underline{\mathrm{CH}}_{3}\right)_{3}\right), 28.1\left(\mathrm{CO}_{2} \mathrm{C}\left(\underline{\mathrm{CH}}_{3}\right)_{3}\right), 39.3(\mathrm{C}-2)$, $44.3\left(\mathrm{C}-9 \mathrm{~b}^{\prime}\right), 44.6\left(\mathrm{C}-3 \mathrm{a}^{\prime}\right), 50.6\left(\mathrm{C}-3^{\prime}\right), 57.9,59.2,60.9\left(3 \times \mathrm{OCH}_{3}\right), 61.1\left(\mathrm{C}-1^{\prime \prime}\right), 68.5\left(\mathrm{C}-5^{\prime \prime \prime}\right)$, 77.3 (C-2"'), $80.5\left(\mathrm{CO}_{2} \underline{\mathrm{C}}\left(\mathrm{CH}_{3}\right)_{3}\right), 80.9$ (C-3"'), 82.1 (C-4"'), 95.2 (C-1'"'), 114.6 (C-6'), 114.9 (C-8'), 125.3 (C-5'), 128.5 (C-9a'), 128.7 (C-1'), 128.8 (C-9'), 132.1 (C-4'), 133.1 (C-5a'), 144.6 (C-2'), 155.1 (C-7'), 172.0 (C-1).

MS (ESI): $\mathrm{m} / \mathrm{z}(\%)=653.3(100)[\mathrm{M}+\mathrm{Na}]^{+}, 1283.7(44)[2 \mathrm{M}+\mathrm{Na}]^{+}$.

HRMS von $\mathrm{C}_{35} \mathrm{H}_{54} \mathrm{O}_{8} \mathrm{Si}$

ber.: $\quad 653.3480$

gef.: $653.3482[\mathrm{M}+\mathrm{Na}]^{+}(\mathrm{ESI}-\mathrm{HRMS})$.

$\mathrm{C}_{35} \mathrm{H}_{54} \mathrm{O}_{8} \mathrm{Si}$ (630.88). 


\subsubsection{2-[2-Hydroxymethyl-7-(2,3,4-tri- $O$-methyl- $\alpha$-L-rhamnopyranosyl)-3a,9b-dihydro-} $3 H$-cyclopenta $[a]$ naphthalin-3-yl]essigsäure-tert-butylester (186)

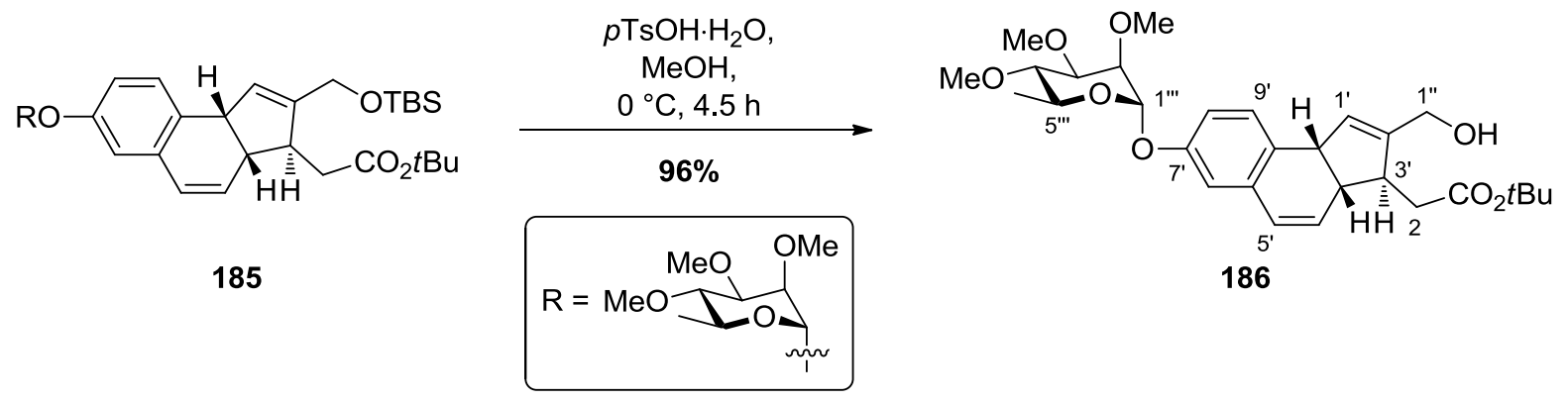

Zu einer Lösung des TBS-geschützten Alkohols 185 (600 mg, $951 \mu \mathrm{mol}, 1.0$ Äq.) in MeOH (25 mL) wurde bei $0{ }^{\circ} \mathrm{C} p \mathrm{TsOH} \cdot \mathrm{H}_{2} \mathrm{O}(27.1 \mathrm{mg}, 143 \mu \mathrm{mol}, 15 \mathrm{~mol} \%)$ und $4.5 \mathrm{~h}$ bei dieser Temperatur gerührt. Das Reaktionsgemisch wurde mit $\mathrm{CH}_{2} \mathrm{Cl}_{2}(15 \mathrm{~mL})$ verdünnt, mit $\mathrm{H}_{2} \mathrm{O}$ (20 mL) gewaschen und die wässrige Phase mit $\mathrm{CH}_{2} \mathrm{Cl}_{2}(3 \times 15 \mathrm{~mL})$ extrahiert. Die vereinigten organischen Phasen wurden über $\mathrm{Na}_{2} \mathrm{SO}_{4}$ getrocknet und das Lösungsmittel unter vermindertem Druck entfernt. Nach säulenchromatographischer Reinigung des Rückstands an Kieselgel ( $n$ Pentan/MTBE 1:1) wurde Die Zielverbindung 186 (472 mg, $913 \mu \mathrm{mol}, 96 \%$ ) als farbloser Schaum erhalten. Eine semipräparative Trennung der Diastereomere konnte für analytische Zwecke an dieser Stelle bewerkstelligt werden, allerdings wurden nachfolgende Reaktionen zu Optimierungszwecken mit dem Gemisch der Diastereomere durchgeführt.

DC: $\mathrm{R}_{f}=0.35$ (Petrolether/EtOAc 1:1).

Drehwert: $\quad(-)-(S, S, S)-\mathbf{1 8 6}: \quad[\alpha]_{\mathrm{D}}^{20}=-204.9^{\circ} \quad\left(c=0.37, \mathrm{CHCl}_{3}\right)$,

$$
(+)-(R, R, R)-186: \quad[\alpha]_{\mathrm{D}}^{20}=+88.2^{\circ} \quad\left(c=0.34, \mathrm{CHCl}_{3}\right) .
$$

UV $\left(\mathrm{CH}_{3} \mathrm{CN}\right): \lambda_{\max }(\lg \varepsilon)=227 \mathrm{~nm}$ (4.4583), 256 (3.7375), 265 (3.7754), 274 (3.7037), 299 (4.3051), 309 (3.2400).

IR: $\tilde{v}\left(\mathrm{~cm}^{-1}\right)=3447,2923,2852,2362,1722,1601,1574,1498,1365,1258,1137,1118$, 1099, 1046, 1012, 989, 810.

${ }^{1} \mathbf{H}-\mathbf{N M R}\left(600 \mathrm{MHz}, \mathrm{CDCl}_{3}\right): \delta(\mathrm{ppm})=1.23\left(\mathrm{~d}, J=6.2 \mathrm{~Hz}, 3 \mathrm{H}, 66^{\prime \prime}-\mathrm{H}_{3}\right), 1.45(\mathrm{~s}, 9 \mathrm{H}$, $\left.\mathrm{CO}_{2} \mathrm{C}\left(\mathrm{CH}_{3}\right)_{3}\right), 1.99\left(\mathrm{~s}_{\mathrm{br}}, 1 \mathrm{H}, \mathrm{OH}\right), 2.41\left(\mathrm{dd}, J=15.6,8.0 \mathrm{~Hz}, 1 \mathrm{H}, 2-\mathrm{H}_{\mathrm{A}}\right), 2.54(\mathrm{dd}, J=15.6$, $\left.5.8 \mathrm{~Hz}, 1 \mathrm{H}, 2-\mathrm{H}_{\mathrm{B}}\right), 3.02\left(\mathrm{~m}_{\mathrm{c}}, 1 \mathrm{H}, 3\right.$ '-H), $3.08\left(\mathrm{~m}_{\mathrm{c}}, 1 \mathrm{H}, 3 \mathrm{a}^{\prime}-\mathrm{H}\right), 3.16$ (t, J = 9.4 Hz, $1 \mathrm{H}, 4$ '"'-H), 3.51, 3.53, 3.54 (3 s, 9 H, 3× $\left.\mathrm{OCH}_{3}\right), 3.60-3.66(\mathrm{~m}, 1 \mathrm{H}, 5$ "'-H), 3.64 (dd, $J=9.4,3.3 \mathrm{~Hz}, 1 \mathrm{H}$, 3"'-H), 3.71 (dd, J = 3.3, 1.9 Hz, 1 H, 2'"-H), 4.04 (m, 1 H, 9b'-H), 4.15 (s, 2 H, 1"-H $), 5.47$ (d, $\left.J=1.9 \mathrm{~Hz}, 1 \mathrm{H}, 1^{\prime \prime}-\mathrm{H}\right), 5.52\left(\mathrm{~m}_{\mathrm{c}}, 1 \mathrm{H}, 1^{\prime}-\mathrm{H}\right), 5.76$ (dd, $\left.J=9.8,3.4 \mathrm{~Hz}, 1 \mathrm{H}, 4^{\prime}-\mathrm{H}\right), 6.23$ (dd, $\left.J=9.8,2.0 \mathrm{~Hz}, 1 \mathrm{H}, 5^{\prime}-\mathrm{H}\right), 6.69\left(\mathrm{~d}, J=2.6 \mathrm{~Hz}, 1 \mathrm{H}, 6{ }^{\prime}-\mathrm{H}\right), 6.83(\mathrm{dd}, J=8.3,2.6 \mathrm{~Hz}, 1 \mathrm{H}$, 8'-H), $7.00\left(\mathrm{~d}, J=8.3 \mathrm{~Hz}, 1 \mathrm{H}, 9^{\prime}-\mathrm{H}\right)$. 
${ }^{13} \mathbf{C}-\mathbf{N M R}\left(126 \mathrm{MHz}, \mathrm{CDCl}_{3}\right): \delta(\mathrm{ppm})=17.9\left(\mathrm{C}-6\right.$ '"'), $28.1\left(\mathrm{CO}_{2} \mathrm{C}\left(\underline{\mathrm{CH}}_{3}\right)_{3}\right), 39.3(\mathrm{C}-2), 44.3$ (C-9b'), 44.7 (C-3a'), 50.4 (C-3'), 57.9, 59.2, 60.9 (3×OCH 3$), 60.6$ (C-1"), 68.5 (C-5"'), 77.3 (C-2"'), 80.9 (C-3"'), 80.9 ( $\left.\mathrm{CO}_{2} \underline{\mathrm{C}}\left(\mathrm{CH}_{3}\right)_{3}\right), 82.0$ (C-4"'), 95.1 (C-1'"'), 114.6 (C-6'), 115.1 (C-8'), 125.4 (C-5'), 128.1 (C-9a'), 128.7 (C-9'), 130.2 (C-1'), 131.4 (C-4'), 132.9 (C-5a'), 144.8 (C-2'), 155.0 (C-7'), $172.2(\mathrm{C}-1)$.

MS (ESI): m/z (\%) = $539.3(43)[\mathrm{M}+\mathrm{Na}]^{+}, 1055.6(100)[2 \mathrm{M}+\mathrm{Na}]^{+}$.

HRMS von $\mathrm{C}_{29} \mathrm{H}_{40} \mathrm{O}_{8}$ ber.: $\quad 539.2615$ gef.: $\quad 539.2618[\mathrm{M}+\mathrm{Na}]^{+}(\mathrm{ESI}-\mathrm{HRMS})$.

$\mathrm{C}_{29} \mathrm{H}_{40} \mathrm{O}_{8}(516.62)$.

HPLC (analytisch): Säule: $\quad$ Chiralpak $^{\circledR} \mathrm{IB}, 250 \times 4.6 \mathrm{~mm}, 5 \mu \mathrm{m}$

Eluens: $\quad n \mathrm{Hexan} / \mathrm{iPrOH}$ 93:7

Fluß: $\quad 0.8 \mathrm{~mL} / \mathrm{min}$

$\lambda: \quad 230 \mathrm{~nm}$

$t_{\mathrm{R}}: \quad(-)-(S, S, S)-186: 23.7 \mathrm{~min},(+)-(R, R . R)-186: 13.7 \mathrm{~min}$.

HPLC (präparativ): Säule: $\quad$ Chiralpak $^{\circledR} \mathrm{IB}, 250 \times 10 \mathrm{~mm}, 5 \mu \mathrm{m}$

Eluens: $\quad n \mathrm{Hexan} / \mathrm{iPrOH}$ 95:5

Fluß: $\quad 6 \mathrm{~mL} / \mathrm{min}$

$\lambda: \quad 230 \mathrm{~nm}$

$t_{\mathrm{R}}: \quad(-)-(S, S, S)-\mathbf{1 8 6}: 12.6 \mathrm{~min},(+)-(R, R \cdot R)-\mathbf{1 8 6}: 3.5 \mathrm{~min}$. 
4.2.8 2-(2-Formyl-7-(2,3,4-tri- $O$-methyl- $\alpha$-L-rhamnopyranosyl)-3a,9b-dihydro-3Hcyclopenta[a]naphthalin-3-yl)essigsäure-tert-butylester (149)

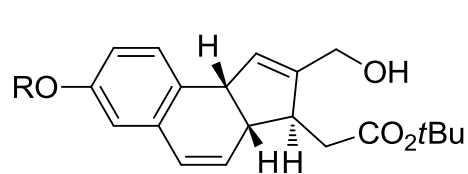

186

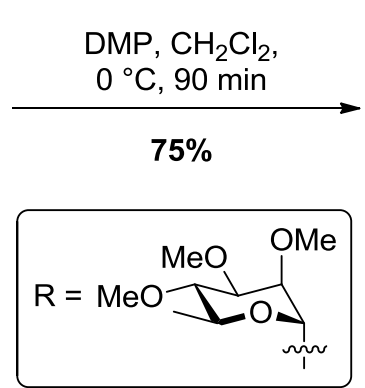

Zu einer Lösung des Alkohols 186 (20.0 mg, $38.7 \mu$ mol, 1.0 Äq.) in $\mathrm{CH}_{2} \mathrm{Cl}_{2}$ (7 mL) wurde bei $0{ }^{\circ} \mathrm{C}$ DMP (28.7 mg, $67.7 \mu \mathrm{mol}, 1.8$ Äq.) gegeben und $90 \mathrm{~min}$ bei dieser Temperatur gerührt. Die Reaktion wurde durch Zugabe von ges. $\mathrm{NaHCO}_{3}$-Lösung $(5 \mathrm{~mL})$ und ges. $\mathrm{Na}_{2} \mathrm{~S}_{2} \mathrm{O}_{3^{-}}$ Lösung $(5 \mathrm{~mL})$ beendet und das Reaktionsgemisch mit $\mathrm{CH}_{2} \mathrm{Cl}_{2}(3 \times 5 \mathrm{~mL})$ extrahiert. Die vereinigten organischen Phasen wurden über $\mathrm{Na}_{2} \mathrm{SO}_{4}$ getrocknet und das Lösungsmittel unter vermindertem Druck entfernt. Nach säulenchromatographischer Reinigung des Rückstands an Kieselgel (Petrolether/MTBE 1:1) wurde die Zielverbindung 149 (14:9 mg, 29.0 mmol, 75\%) als farbloser Schaum erhalten.

DC: $\mathbf{R}_{f}=0.28$ (Petrolether/EtOAc 2:1).

Drehwert: $[\alpha]_{\mathrm{D}}^{20}=-158.0^{\circ}\left(c=0.87, \mathrm{CHCl}_{3}\right)$.

UV $\left(\mathrm{CH}_{3} \mathrm{CN}\right): \lambda_{\max }(\lg \varepsilon)=232 \mathrm{~nm}$ (4.5319), 309 (3.3689), 335 (3.2892), 354 (3.2729).

IR: $\tilde{v}\left(\mathrm{~cm}^{-1}\right)=2976,2930,2825,1724,1677,1498,1366,1259,1138,1119,1100,1046$, 1010, 988, 841, 812, 793.

${ }^{1} \mathbf{H}-\mathbf{N M R}\left(600 \mathrm{MHz}, \mathrm{CDCl}_{3}\right): \delta(\mathrm{ppm})=1.23\left(\mathrm{~d}, J=6.2 \mathrm{~Hz}, 3 \mathrm{H}, 6 "-\mathrm{H}_{3}\right), 1.44(\mathrm{~s}, 9 \mathrm{H}$, $\left.\mathrm{CO}_{2} \mathrm{C}\left(\mathrm{CH}_{3}\right)_{3}\right), 2.27\left(\mathrm{dd}, J=15.9,10.7 \mathrm{~Hz}, 1 \mathrm{H}, 2-\mathrm{H}_{\mathrm{A}}\right), 2.77\left(\mathrm{dd}, J=15.9,3.6 \mathrm{~Hz}, 1 \mathrm{H}, 2-\mathrm{H}_{\mathrm{B}}\right)$, 3.17 (t, $\left.J=9.4 \mathrm{~Hz}, 1 \mathrm{H}, 4^{\prime \prime}-\mathrm{H}\right), 3.24$ ( $\left.\mathrm{m}_{\mathrm{c}}, 1 \mathrm{H}, 3 \mathrm{a}^{\prime}-\mathrm{H}\right), 3.33$ ( $\left.\mathrm{m}_{\mathrm{c}}, 1 \mathrm{H}, 3^{\prime}-\mathrm{H}\right), 3.52,3.54,3.54$ (3 s, 9 H, 3× $\mathrm{OCH}_{3}$ ), 3.59-3.64 (m, 1 H, 5"-H), 3.64 (dd, $\left.J=9.4,3.3 \mathrm{~Hz}, 1 \mathrm{H}, 3 "-\mathrm{H}\right), 3.72$ (dd, $\left.J=3.1,2.1 \mathrm{~Hz}, 1 \mathrm{H}, 2^{\prime \prime}-\mathrm{H}\right), 4.29$ (m $\left.\mathrm{c}, 1 \mathrm{H}, 9 \mathrm{~b}^{\prime}-\mathrm{H}\right), 5.49$ (d, $\left.J=1.8 \mathrm{~Hz}, 1 \mathrm{H}, 1 "-\mathrm{H}\right), 5.74$ (dd, $\left.J=9.8,3.0 \mathrm{~Hz}, 1 \mathrm{H}, 4^{\prime}-\mathrm{H}\right), 6.23\left(\mathrm{dd}, J=9.8,2.4 \mathrm{~Hz}, 1 \mathrm{H}, 5^{\prime}-\mathrm{H}\right), 6.59\left(\mathrm{~m}_{\mathrm{c}}, 1 \mathrm{H}, 1^{\prime}-\mathrm{H}\right), 6.72(\mathrm{~d}$, $\left.J=2.6 \mathrm{~Hz}, 1 \mathrm{H}, 66^{\prime}-\mathrm{H}\right), 6.87\left(\mathrm{dd}, J=8.3,2.6 \mathrm{~Hz}, 1 \mathrm{H}, 8^{\prime}-\mathrm{H}\right), 7.08\left(\mathrm{~d}, J=8.3 \mathrm{~Hz}, 1 \mathrm{H}, 9^{\prime}-\mathrm{H}\right)$, $9.72(\mathrm{~s}, 1 \mathrm{H}, \mathrm{CHO})$.

${ }^{13} \mathbf{C}-\mathbf{N M R}\left(126 \mathrm{MHz}, \mathrm{CDCl}_{3}\right): \delta(\mathrm{ppm})=17.9(\mathrm{C}-6 "), 28.2\left(\mathrm{CO}_{2} \mathrm{C}\left(\underline{\mathrm{CH}}_{3}\right)_{3}\right), 38.4(\mathrm{C}-2), 44.1$ (C-3a'), 45.9 (C-9b'), 47.4 (C-3'), 57.9, 59.2, 60.9 (3×OCH 3$), 68.6$ (C-5"), 77.3 (C-2"), 80.7 $\left(\mathrm{CO}_{2} \underline{\mathrm{C}}\left(\mathrm{CH}_{3}\right)_{3}\right), 80.8$ (C-3"), 82.0 (C-4"), 95.2 (C-1"), 114.8 (C-6'), 115.3 (C-8'), 125.1 (C-9a'), 
$125.4\left(\mathrm{C}-5^{\prime}\right), 128.8$ (C-9'), $131.3\left(\mathrm{C}-4^{\prime}\right), 133.4\left(\mathrm{C}-5 \mathrm{a}^{\prime}\right), 146.4\left(\mathrm{C}-2^{\prime}\right), 155.2\left(\mathrm{C}-1^{\prime}\right), 155.6\left(\mathrm{C}-7^{\prime}\right)$, 171.1 (C-1), 189.1 (CHO).

MS (ESI): $m / z(\%)=537.2(57)[\mathrm{M}+\mathrm{Na}]^{+}, 1051.5(100)[2 \mathrm{M}+\mathrm{Na}]^{+}$.

HRMS von $\mathrm{C}_{29} \mathrm{H}_{38} \mathrm{O}_{8}$ ber.: $\quad 537.2459$

gef.: $\quad 537.2458[\mathrm{M}+\mathrm{Na}]^{+}(\mathrm{ESI}-\mathrm{HRMS})$.

$\mathrm{C}_{29} \mathrm{H}_{38} \mathrm{O}_{8}(514.61)$. 


\subsubsection{1-tert-Butyl-2-[2-methyl-7-(2,3,4-tri- $O$-methyl- $\alpha$-L-rhamnopyranosyl)-3a,9b-}

\section{dihydro-3H-cyclopenta[a]naphthalin-3-yl]methandicarboxylat (236)}
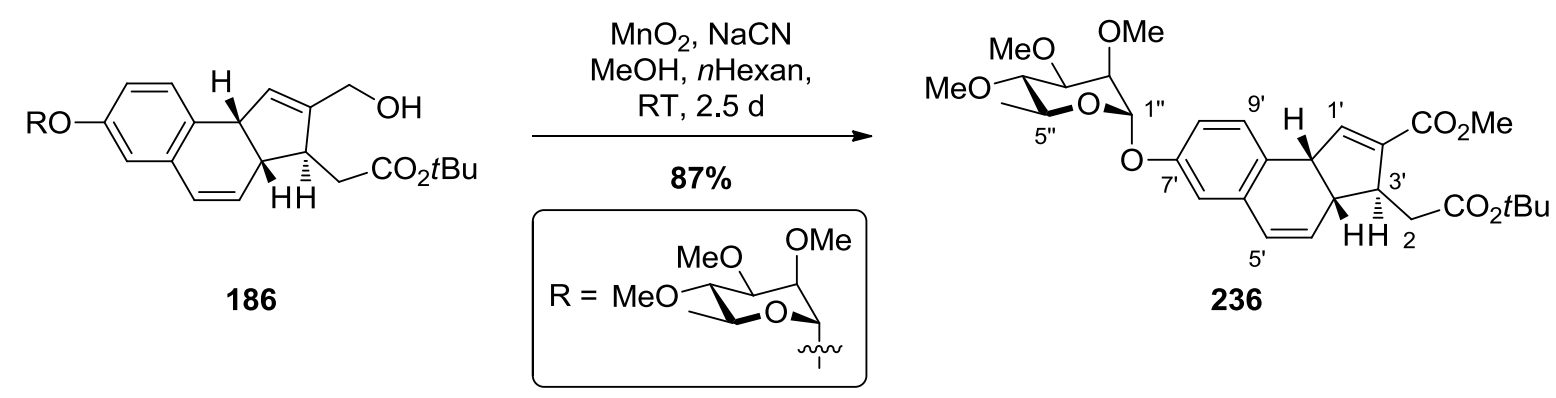

Zu einer Lösung des Alkohols 186 (10.0 mg, $19.4 \mu \mathrm{mol}, 1.0$ Äq.) in $n$ Hexan (3 mL) und $\mathrm{MeOH}(0.5 \mathrm{~mL})$ wurde bei Raumtemperatur $\mathrm{NaCN}(95.0 \mu \mathrm{g}, 1.94 \mu \mathrm{mol}, 10 \mathrm{~mol} \%)$ und portionsweise $\mathrm{MnO}_{2}$ (101 mg, $1.16 \mathrm{mmol}, 60.0$ Äq.) gegeben und $2.5 \mathrm{~d}$ bei dieser Temperatur gerührt. Das Reaktionsgemisch wurde mit $\mathrm{CH}_{2} \mathrm{Cl}_{2}(15 \mathrm{~mL})$ verdünnt, durch Celite ${ }^{\circledR}$ filtriert und das Lösungsmittel unter vermindertem Druck entfernt. Säulenchromatographische Reinigung des Rückstands an Kieselgel (Petrolether/EtOAc 5:1 $\rightarrow 2: 1$ ) lieferte die Zielverbindung 236 (9.21 mg, $16.9 \mu \mathrm{mol}, 87 \%)$ in Form eines farblosen Öls.

DC: $\mathbf{R}_{f}=0.34$ (Petrolether/EtOAc 3:1).

${ }^{1} \mathbf{H}-\mathbf{N M R}\left(600 \mathrm{MHz}, \mathrm{CDCl}_{3}\right): \delta(\mathrm{ppm})=1.23\left(\mathrm{~d}, J=6.3 \mathrm{~Hz}, 3 \mathrm{H}, 6 "-\mathrm{H}_{3}\right), 1.44(\mathrm{~s}, 9 \mathrm{H}$, $\left.\mathrm{CO}_{2} \mathrm{C}\left(\mathrm{CH}_{3}\right)_{3}\right), 2.31\left(\mathrm{dd}, J=15.7,10.7 \mathrm{~Hz}, 1 \mathrm{H}, 2-\mathrm{H}_{\mathrm{A}}\right), 2.77\left(\mathrm{dd}, J=15.7,3.5 \mathrm{~Hz}, 1 \mathrm{H}, 2-\mathrm{H}_{\mathrm{B}}\right)$, 3.16 (t, $J=9.4$ Hz, 1 H, 4"-H), 3.21 (dd, $\left.J=9.0,1.6 \mathrm{~Hz}, 1 \mathrm{H}, 3 \mathrm{a}^{\prime}-\mathrm{H}\right), 3.29$ (d, $J=8.8 \mathrm{~Hz}, 1 \mathrm{H}$, 3'-H), 3.52, 3.53, $3.54\left(3 \mathrm{~s}, 9 \mathrm{H}, 3 \times \mathrm{OCH}_{3}\right), 3.59-3.65(\mathrm{~m}, 1 \mathrm{H}, 5 "-\mathrm{H}), 3.64(\mathrm{dd}, J=9.3$, $3.3 \mathrm{~Hz}, 1 \mathrm{H}, 3 "-\mathrm{H}), 3.68$ (s, $\left.3 \mathrm{H}, \mathrm{CO}_{2} \mathrm{CH}_{3}\right), 3.71\left(\mathrm{~m}_{\mathrm{c}}, 1 \mathrm{H}, 2^{\prime \prime}-\mathrm{H}\right), 4.19$ (d, J = 8.5 Hz, $1 \mathrm{H}$, $\left.9 \mathrm{~b}^{\prime}-\mathrm{H}\right), 5.48$ (d, $\left.J=2.2 \mathrm{~Hz}, 1 \mathrm{H}, 1^{\prime \prime}-\mathrm{H}\right), 5.72$ (dd, $\left.J=9.8,3.0 \mathrm{~Hz}, 1 \mathrm{H}, 4^{\prime}-\mathrm{H}\right), 6.22$ (dd, $J=9.8$, $\left.2.6 \mathrm{~Hz}, 1 \mathrm{H}, 5^{\prime}-\mathrm{H}\right), 6.51$ (d, J = 2.4 Hz, $\left.1 \mathrm{H}, 1^{\prime}-\mathrm{H}\right), 6.69$ (d, J = 2.6 Hz, $1 \mathrm{H}, 6$ '-H), 6.84 (d, $J=$ $\left.8.1 \mathrm{~Hz}, 1 \mathrm{H}, 8^{\prime}-\mathrm{H}\right), 7.03$ (d, $\left.J=8.2 \mathrm{~Hz}, 1 \mathrm{H}, 9^{\prime}-\mathrm{H}\right)$.

${ }^{13}$ C-NMR $\left(126 \mathrm{MHz}, \mathrm{CDCl}_{3}\right): \delta(\mathrm{ppm})=17.8(\mathrm{C}-6 "), 28.1\left(\mathrm{CO}_{2} \mathrm{C}\left(\underline{\mathrm{CH}}_{3}\right)_{3}\right), 38.9(\mathrm{C}-2), 43.9$

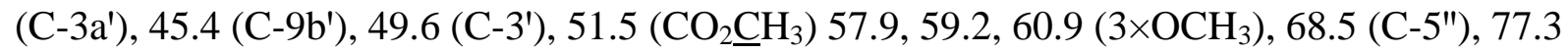
(C-2"), $80.7\left(\mathrm{CO}_{2} \underline{\mathrm{C}}\left(\mathrm{CH}_{3}\right)_{3}\right), 80.9$ (C-3"), 82.0 (C-4"), 95.2 (C-1"), 114.7 (C-6'), 115.2 (C-8'), 125.5 (C-5'), 125.8 (C-9a'),128.9 (C-9'), 131.6 (C-4'), 133.4 (C-5a'), 136.4 (C-2'), 147.1 (C-1'), 155.5 (C-7'), $164.9\left(\mathrm{CO}_{2} \mathrm{CH}_{3}\right), 171.6(\mathrm{C}-1)$.

MS (ESI): $\mathrm{m} / \mathrm{z}(\%)=567.3(17)[\mathrm{M}+\mathrm{Na}]^{+}, 583.2(100)[\mathrm{M}+\mathrm{K}]^{+}$.

HRMS von $\mathrm{C}_{30} \mathrm{H}_{40} \mathrm{O}_{9}$ ber.: 583.2304 gef.: $583.2287[\mathrm{M}+\mathrm{Na}]^{+}(\mathrm{ESI}-\mathrm{HRMS})$.

$\mathbf{C}_{30} \mathbf{H}_{40} \mathbf{O}_{9}$ (544.63). 
4.2.10 2-[2-Carboxy-7-(2,3,4-tri- $O$-methyl- $\alpha$-L-rhamnopyranosyl)-3a,9b-dihydro-3Hcyclopenta[a]naphthalin-3-yl]essigsäure-tert-butylester (237)

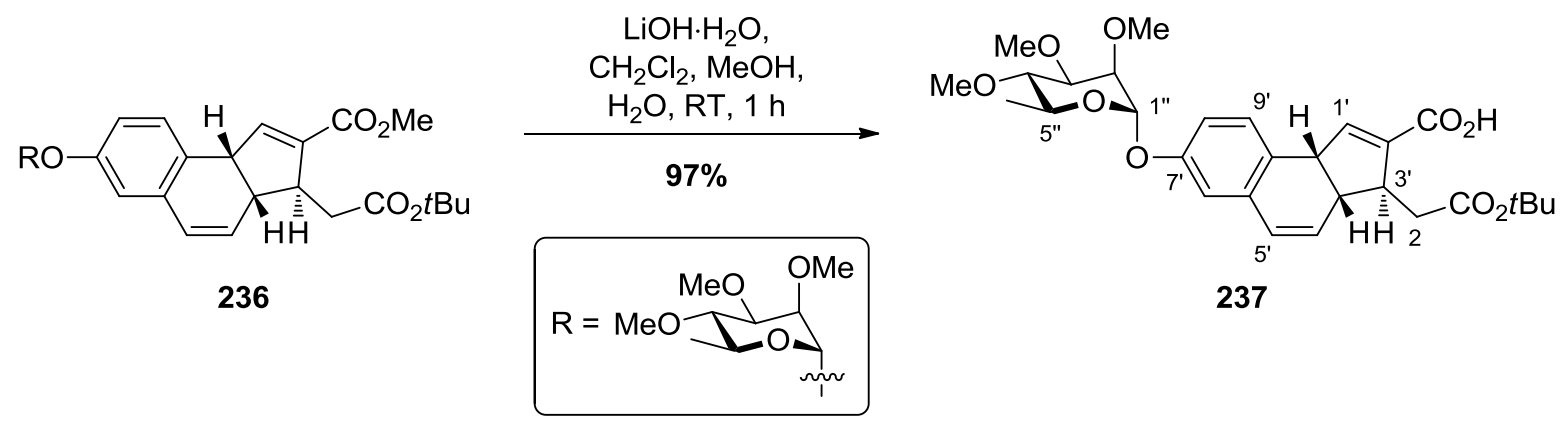

$\mathrm{Zu}$ einer Lösung des Methylesters 236 (2.30 mg, $4.22 \mu \mathrm{mol}, 1.0$ Äq.) in $\mathrm{CH}_{2} \mathrm{Cl}_{2} / \mathrm{MeOH} / \mathrm{H}_{2} \mathrm{O}$ (1:1:0.01, $1 \mathrm{~mL})$ wurde bei Raumtemperatur $\mathrm{LiOH} \cdot \mathrm{H}_{2} \mathrm{O} \quad(270 \mu \mathrm{g}, 6.33 \mu \mathrm{mol}, 1.5$ Äq. $)$ gegeben und $1 \mathrm{~d}$ bei dieser Temperatur gerührt. Die Reaktion wurde durch Zugabe von $\mathrm{HCl}$ $(2 \mathrm{M}, 500 \mu \mathrm{L})$ beendet und die Reaktionsmischung mit $\mathrm{CH}_{2} \mathrm{Cl}_{2}(3 \times 2 \mathrm{~mL})$ extrahiert. Die vereinigten organischen Phasen wurden mit ges. NaCl-Lösung $(5 \mathrm{~mL})$ gewaschen, über $\mathrm{Na}_{2} \mathrm{SO}_{4}$ getrocknet und das Lösungsmittel unter vermindertem Druck entfernt. Die Zielverbindung 237 (2.15 mg, $4.09 \mu \mathrm{mol}$, 97\%) wurde in Form eines farblosen Feststoffes isoliert und direkt in der folgenden Reaktion eingesetzt.

MS (ESI): m/z (\%) = $553.2(75)[\mathrm{M}+\mathrm{Na}]^{+}, 569.2(100)[\mathrm{M}+\mathrm{K}]^{+} ; 529.3(100)[\mathrm{M}-\mathrm{H}]^{-}$.

HRMS von $\mathrm{C}_{29} \mathrm{H}_{38} \mathrm{O}_{9}$

ber.: $\quad 529.2443$

553.2408

gef.: $\quad 529.2431[\mathrm{M}-\mathrm{H}]^{-}$

$553.2406[\mathrm{M}+\mathrm{Na}]^{+}(\mathrm{ESI}-\mathrm{HRMS})$.

$\mathrm{C}_{29} \mathrm{H}_{38} \mathrm{O}_{9}$ (530.61). 
4.2.11 2-((tert-Butyldimethylsilyl)oxy)-6-((2-methoxyethoxy)methoxy)hexyl 3-(2-(tertbutoxy)-2-oxoethyl)-7-((3,4,5-trimethoxy-6-methyltetrahydro-2H-pyran-2yl)oxy)-3a,9b-dihydro-3H-cyclopenta[ $[a]$ naphthalin-2-carboxylat (238)<smiles>[R6]c1ccc2c(c1)C=C[C@@H]1[C@H](CCOC)C(C(=O)O)=C[C@H]21</smiles>

237<smiles>C#[M]OCCCC[C@@H]([OH2+])CO</smiles>
232

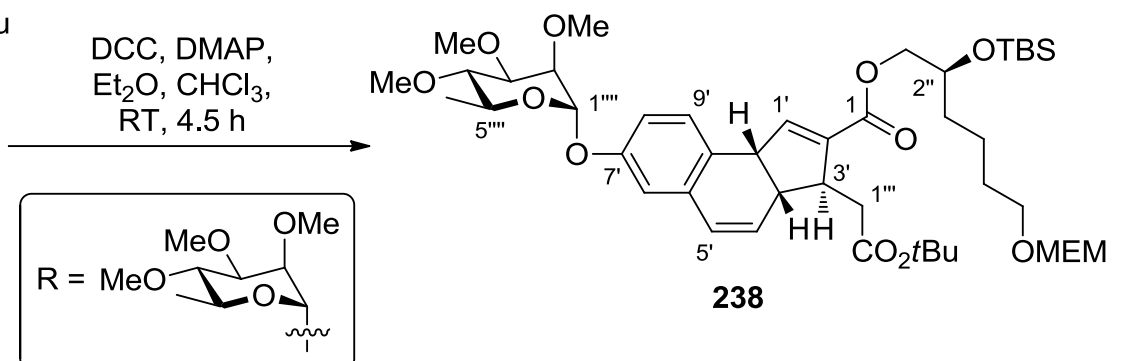

$\mathrm{Zu}$ einer Lösung aus Carbonsäure 237 (2.00 mg, $3.77 \mu \mathrm{mol}, 1.0$ Äq.) und Alkohol 232 (5.55 mg, $11.3 \mu \mathrm{mol}, 3.0$ Äq.) in $\mathrm{Et}_{2} \mathrm{O}$ (1 mL) wurden bei Raumtemperatur eine Lösung aus DMAP (2.76 mg, $22.6 \mu \mathrm{mol}, 6.0$ Äq.) in $\mathrm{Et}_{2} \mathrm{O} / \mathrm{CHCl}_{3}(1: 1,1 \mathrm{~mL})$ und eine Lösung aus DCC (3.11 mg, $15.1 \mu \mathrm{mol}, 4.0$ Äq.) in $\mathrm{Et}_{2} \mathrm{O} / \mathrm{CHCl}_{3}(1: 1,1 \mathrm{~mL})$ gegeben und $4.5 \mathrm{~d}$ bei dieser Temperatur gerührt. Das Lösungsmittel wurde unter vermindertem Druck entfernt und der Rückstand über Säulenchromatographie an Kieselgel (Petrolether/EtOAc 3:1 $\rightarrow 1: 1$ ) gereinigt. Die Zielverbindung 238 konnte nicht vom überstöchiometrisch eingesetzten Alkohol abgetrennt werden, aber eindeutig massenspektroskopisch nachgewiesen werden. Der Umsatz der Reaktion betrug $100 \%$

DC: $\mathbf{R}_{f}=0.38$ (Petrolether/EtOAc 2:1).

MS (ESI): $\mathrm{m} / \mathrm{z}(\%)=871.5(100)[\mathrm{M}+\mathrm{Na}]^{+}$.

HRMS von $\mathrm{C}_{45} \mathrm{H}_{72} \mathrm{O}_{13} \mathrm{Si}$

ber.: 871.4634

gef.: $871.4641[\mathrm{M}+\mathrm{Na}]^{+}$(ESI-HRMS).

$\mathrm{C}_{45} \mathrm{H}_{72} \mathrm{O}_{13} \mathbf{S i}$ (530.61). 


\subsection{Untersuchungen zur $\alpha$-Oxidation nach MacMillan}

\subsubsection{6-((2-Methoxyethoxy)methoxy)hexan-1-ol (188a)}

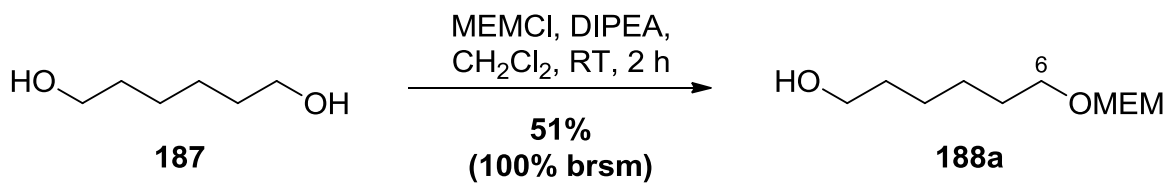

Zu einer Lösung des Diols 187 (10.0 g, 84.6 mmol, 1.0 Äq.) in $\mathrm{CH}_{2} \mathrm{Cl}_{2}$ (100 mL) wurde bei Raumtemperatur DIPEA (17.7 mL, $13.1 \mathrm{~g}, 102 \mathrm{mmol}, 1.2$ Äq.) gegeben und 5 min gerührt. Anschließend wurde langsam MEMCl (9.67 mL, 10.5 g, 84.6 mmol, 1.0 Äq.) zugegeben, und weitere $2 \mathrm{~h}$ gerührt. Die Reaktion wurde durch Zugabe von ges. $\mathrm{NaHCO}_{3}$-Lösung $(100 \mathrm{~mL})$ beendet und die Reaktionsmischung mit $\mathrm{CH}_{2} \mathrm{Cl}_{2}(3 \times 50 \mathrm{~mL})$ extrahiert. Die vereinigten organischen Phasen wurden mit ges. NaCl-Lösung $(100 \mathrm{~mL})$ gewaschen, über $\mathrm{Na}_{2} \mathrm{SO}_{4}$ getrocknet und das Lösungsmittel unter vermindertem Druck entfernt. Säulenchromatographische Reinigung des Rückstandes an Kieselgel ( $n$ Pentan/EtOAc 2:1 $\rightarrow 0: 1)$ lieferte die Zielverbindung 188a (8.87 g, $43.0 \mathrm{mmol}, 51 \%)$ in Form einer farblosen Flüssigkeit. Doppelt geschütztes Nebenprodukt konnte in quantitativer Ausbeute wieder in das Substrat 187 umgewandelt werden.

DC: $\mathrm{R}_{f}=0.18(n$ Pentan/EtOAc 1:1).

${ }^{1} \mathbf{H}-\mathbf{N M R}\left(300 \mathrm{MHz}, \mathrm{CDCl}_{3}\right): \delta(\mathrm{ppm})=1.33-1.44\left(\mathrm{~m}, 4 \mathrm{H}, 3-\mathrm{H}_{2}, 4-\mathrm{H}_{2}\right), 1.50-1.63(\mathrm{~m}, 4 \mathrm{H}$, 2- $\left.\mathrm{H}_{2}, 5-\mathrm{H}_{2}\right), 3.37\left(\mathrm{~s}, 3 \mathrm{H}, \mathrm{OCH}_{3}\right), 3.52\left(\mathrm{t}, J=6.5 \mathrm{~Hz}, 2 \mathrm{H}, 6-\mathrm{H}_{2}\right), 3.53-3.56(\mathrm{~m}, 2 \mathrm{H}$, $\mathrm{OCH}_{2} \mathrm{CH}_{2} \mathrm{OMe}$ ), 3.62 (t, $\left.J=6.5 \mathrm{~Hz}, 2 \mathrm{H}, 1-\mathrm{H}_{2}\right), 3.64-3.69\left(\mathrm{~m}, 2 \mathrm{H}, \mathrm{OCH}_{2} \mathrm{CH}_{2} \mathrm{OMe}\right), 4.69$ (s, $\left.2 \mathrm{H}, \mathrm{OCH}_{2} \mathrm{O}\right)$.

$\mathrm{C}_{10} \mathrm{H}_{22} \mathrm{O}_{4}$ (206.28). 


\subsubsection{6-((2-Methoxyethoxy)methoxy)hexanal (189a)}

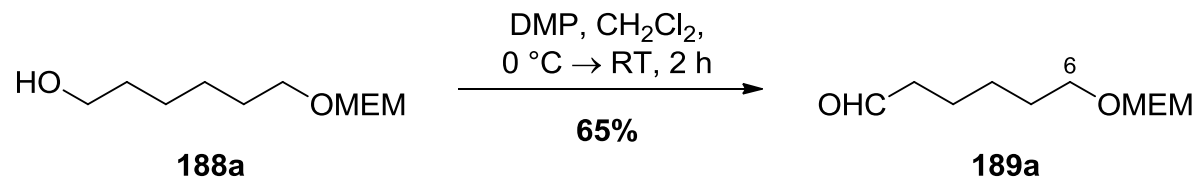

Zu einer Lösung des Alkohols 188a (750 mg, 3.64 mmol, 1.0 Äq.) in $\mathrm{CH}_{2} \mathrm{Cl}_{2}$ (15 mL) wurde bei $0{ }^{\circ} \mathrm{C}$ DMP (3.08 g, $7.27 \mathrm{mmol}, 2.0$ Äq.) gegeben und 5 min gerührt. Anschließend wurde die Lösung auf Raumtemperatur erwärmt und weitere $2 \mathrm{~h}$ gerührt. Die Reaktion wurde durch Zugabe von ges. $\mathrm{NaHCO}_{3}$-Lösung $(10 \mathrm{~mL})$ und ges. $\mathrm{Na}_{2} \mathrm{~S}_{2} \mathrm{O}_{3}$-Lösung $(10 \mathrm{~mL})$ beendet und die Reaktionsmischung mit $\mathrm{CH}_{2} \mathrm{Cl}_{2}(3 \times 10 \mathrm{~mL})$ extrahiert. Die vereinigten organischen Phasen wurden mit ges. NaCl-Lösung $(30 \mathrm{~mL})$ gewaschen, über $\mathrm{Na}_{2} \mathrm{SO}_{4}$ getrocknet und das Lösungsmittel unter vermindertem Druck entfernt. Säulenchromatographische Reinigung des Rückstandes an Kieselgel ( $n$ Pentan/EtOAc 4:1) lieferte die Zielverbindung 189a (481 mg, $2.36 \mathrm{mmol}, 65 \%$ ) in Form einer farblosen Flüssigkeit.

DC: $\mathbf{R}_{f}=0.48(n$ Pentan/EtOAc $1: 1)$.

${ }^{1} \mathbf{H}-\mathbf{N M R}\left(300 \mathrm{MHz}, \mathrm{CDCl}_{3}\right): \delta(\mathrm{ppm})=1.31-1.44\left(\mathrm{~m}, 2 \mathrm{H}, 4-\mathrm{H}_{2}\right), 1.52-1.69\left(\mathrm{~m}_{\mathrm{c}}, 4 \mathrm{H}, 3-\mathrm{H}_{2}\right.$, 5- $\left.\mathrm{H}_{2}\right), 2.42\left(\mathrm{dt}, J=7.3,1.8 \mathrm{~Hz}, 2 \mathrm{H}, 2-\mathrm{H}_{2}\right), 3.36\left(\mathrm{~s}, 3 \mathrm{H}, \mathrm{OCH}_{3}\right), 3.51(\mathrm{t}, J=6.4 \mathrm{~Hz}, 2 \mathrm{H}$, 6- $\mathrm{H}_{2}$ ), 3.51-3.54 (m, $2 \mathrm{H}, \mathrm{OCH}_{2} \mathrm{CH}_{2} \mathrm{OMe}$ ), 3.62-3.67 (m, $\left.2 \mathrm{H}, \mathrm{OCH}_{2} \mathrm{CH}_{2} \mathrm{OMe}\right), 4.67$ (s, $2 \mathrm{H}$, $\left.\mathrm{OCH}_{2} \mathrm{O}\right), 9.73(\mathrm{t}, J=1.8 \mathrm{~Hz}, 1 \mathrm{H}, \mathrm{CHO})$.

${ }^{13}$ C-NMR $\left(126 \mathrm{MHz}, \mathrm{CDCl}_{3}\right): \delta(\mathrm{ppm})=21.8(\mathrm{C}-4), 25.8$ (C-3), 29.4 (C-5), 43.8 (C-2), 59.0 $\left(\mathrm{OCH}_{3}\right), 66.7\left(\mathrm{OCH}_{2} \mathrm{CH}_{2} \mathrm{OMe}\right), 67.5(\mathrm{C}-6), 71.8\left(\mathrm{OCH}_{2} \underline{\mathrm{CH}}_{2} \mathrm{OMe}\right), 95.4\left(\mathrm{OCH}_{2} \mathrm{O}\right), 202.5$ (CHO).

MS (ESI): $\mathrm{m} / \mathrm{z} \quad(\%)=222.2 \quad(6) \quad\left[\mathrm{M}+\mathrm{NH}_{4}\right]^{+}, \quad 227.1 \quad(18) \quad[\mathrm{M}+\mathrm{Na}]^{+}, \quad 259.2$ (100) $[\mathrm{M}+\mathrm{MeOH}+\mathrm{Na}]^{+} ; 203.1(66)[\mathrm{M}-\mathrm{H}]^{-}$.

HRMS von $\mathrm{C}_{10} \mathrm{H}_{20} \mathrm{O}_{4}$

ber.: $\quad 203.1289$

227.1254

gef.: $203.1285[\mathrm{M}-\mathrm{H}]$

$227.1255[\mathrm{M}+\mathrm{Na}]^{+}(\mathrm{ESI}-\mathrm{HRMS})$.

$\mathbf{C}_{10} \mathbf{H}_{20} \mathbf{O}_{4}$ (204.26). 


\subsubsection{6-((Triisopropylsilyl)oxy)hexan-1-ol (188b)}

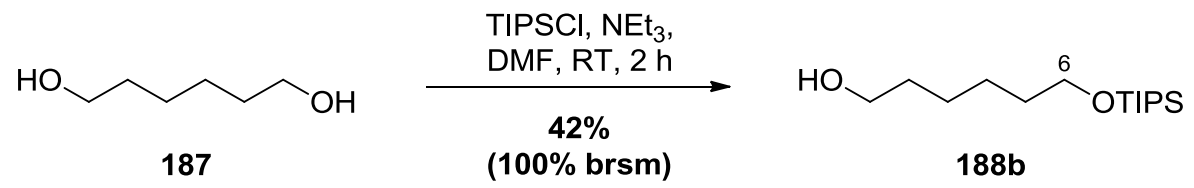

Zu einer Lösung des Diols 187 (1.00 g, 8.46 mmol, 1.0 Äq.) in DMF (5 mL) wurde bei Raumtemperatur $\mathrm{NEt}_{3}(1.29 \mathrm{~mL}, 942 \mathrm{mg}, 9.31 \mathrm{mmol}, 1.1$ Äq.) gegeben und $5 \mathrm{~min}$ gerührt. Anschließend wurden langsam DMAP (103 mg, $846 \mu \mathrm{mol}, 10 \mathrm{~mol} \%)$ und TIPSCl (1.80 mL, $1.63 \mathrm{~g}, 8.46 \mathrm{mmol}, 1.0$ Äq.) zugegeben und weitere $2 \mathrm{~h}$ gerührt. Die Reaktion wurde durch Zugabe von ges. $\mathrm{NaHCO}_{3}$-Lösung $(5 \mathrm{~mL})$ beendet und die Reaktionsmischung mit $\mathrm{Et}_{2} \mathrm{O}$ $(3 \times 10 \mathrm{~mL})$ extrahiert. Die vereinigten organischen Phasen wurden mit ges. NaCl-Lösung $(20 \mathrm{~mL})$ gewaschen, über $\mathrm{Na}_{2} \mathrm{SO}_{4}$ getrocknet und das Lösungsmittel unter vermindertem Druck entfernt. Säulenchromatographische Reinigung des Rückstandes an Kieselgel ( $n$ Pentan/EtOAc 15:1 $\rightarrow 5: 1$ ) lieferte die Zielverbindung 188b (976 mg, $3.56 \mathrm{mmol}, 42 \%$ ) in Form einer farblosen Flüssigkeit. Doppelt geschütztes Nebenprodukt konnte in quantitativer Ausbeute wieder in das Substrat 187 umgewandelt werden.

DC: $\mathrm{R}_{f}=0.40(n$ Pentan/EtOAc 10:1).

${ }^{1} \mathbf{H}-\mathbf{N M R}\left(300 \mathrm{MHz}, \mathrm{CDCl}_{3}\right): \delta(\mathrm{ppm})=1.02-1.05\left(\mathrm{~m}, 21 \mathrm{H}, \mathrm{Si}\left(\mathrm{CH}\left(\mathrm{CH}_{3}\right)_{2}\right)_{3}\right), 1.32-1.41(\mathrm{~m}$, $\left.4 \mathrm{H}, 3-\mathrm{H}_{2}, 4-\mathrm{H}_{2}\right), 1.48-1.60\left(\mathrm{~m}, 4 \mathrm{H}, 2-\mathrm{H}_{2}, 5-\mathrm{H}_{2}\right), 3.62\left(\mathrm{t}, J=6.6 \mathrm{~Hz}, 2 \mathrm{H}, 1-\mathrm{H}_{2}\right), 3.65(\mathrm{t}, J=$ $\left.6.5 \mathrm{~Hz}, 2 \mathrm{H}, 6-\mathrm{H}_{2}\right)$.

${ }^{13}$ C-NMR $\left(126 \mathrm{MHz}, \mathrm{CDCl}_{3}\right): \delta(\mathrm{ppm})=12.0\left(\mathrm{Si}\left(\underline{\mathrm{CH}}\left(\mathrm{CH}_{3}\right)_{2}\right)_{3}\right), 18.0\left(\mathrm{Si}\left(\mathrm{CH}\left(\mathrm{CH}_{3}\right)_{2}\right)_{3}\right), 25.6$, 25.6 (C-3, C-4), 32.8 (C-2), 32.9 (C-5), 63.0 (C-6), 63.3 (C-1).

MS (ESI): $\mathrm{m} / \mathrm{z}(\%)=275.2(100)[\mathrm{M}+\mathrm{H}]^{+}, 297.2(65)[\mathrm{M}+\mathrm{Na}]^{+}$.

HRMS von $\mathrm{C}_{15} \mathrm{H}_{34} \mathrm{O}_{2} \mathrm{Si}$ ber.: $\quad 275.2401$ gef.: $\quad 275.2402[\mathrm{M}+\mathrm{H}]^{+}($ESI-HRMS).

$\mathrm{C}_{15} \mathrm{H}_{34} \mathrm{O}_{2} \mathrm{Si}$ (274.51). 


\subsubsection{6-((Triisopropylsilyl)oxy)hexanal (189b)}

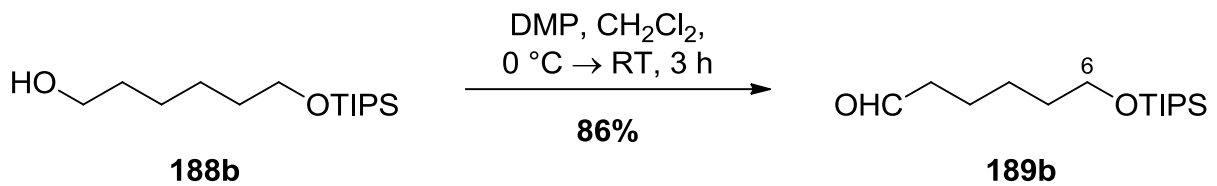

Zu einer Lösung des Alkohols $\mathbf{1 8 8 b}$ (950 mg, 3.46 mmol, 1.0 Äq.) in $\mathrm{CH}_{2} \mathrm{Cl}_{2}$ (15 mL) wurde bei $0{ }^{\circ} \mathrm{C}$ DMP (2.94 g, $6.92 \mathrm{mmol}, 2.0$ Äq.) gegeben und 5 min gerührt. Anschließend wurde die Lösung auf Raumtemperatur erwärmt und weitere $3 \mathrm{~h}$ gerührt. Die Reaktion wurde durch Zugabe von ges. $\mathrm{NaHCO}_{3}$-Lösung $(10 \mathrm{~mL})$ und ges. $\mathrm{Na}_{2} \mathrm{~S}_{2} \mathrm{O}_{3}$-Lösung $(10 \mathrm{~mL})$ beendet und die Reaktionsmischung mit $\mathrm{CH}_{2} \mathrm{Cl}_{2}(3 \times 10 \mathrm{~mL})$ extrahiert. Die vereinigten organischen Phasen wurden mit ges. NaCl-Lösung $(30 \mathrm{~mL})$ gewaschen, über $\mathrm{Na}_{2} \mathrm{SO}_{4}$ getrocknet und das Lösungsmittel unter vermindertem Druck entfernt. Säulenchromatographische Reinigung des Rückstandes an Kieselgel ( $n$ Pentan/EtOAc 100:1 $\rightarrow 20: 1)$ lieferte die Zielverbindung 189b (812 mg, $2.98 \mathrm{mmol}, 86 \%$ ) in Form einer farblosen Flüssigkeit.

DC: $\mathbf{R}_{f}=0.46(n$ Pentan/EtOAc 20:1).

${ }^{\mathbf{1}} \mathbf{H}$-NMR $\left(300 \mathrm{MHz}, \mathrm{CDCl}_{3}\right): \delta(\mathrm{ppm})=0.98-1.10\left(\mathrm{~m}, 21 \mathrm{H}, \mathrm{Si}\left(\mathrm{CH}\left(\mathrm{CH}_{3}\right)_{2}\right)_{3}\right), 1.32-1.45(\mathrm{~m}$, $\left.2 \mathrm{H}, 4-\mathrm{H}_{2}\right), 1.47-1.58\left(\mathrm{~m}_{\mathrm{c}}, 2 \mathrm{H}, 5-\mathrm{H}_{2}\right), 1.64$ (quint., $\left.J=7.6 \mathrm{~Hz}, 2 \mathrm{H}, 3-\mathrm{H}_{2}\right), 2.41$ (dt, $J=7.4$, $\left.1.9 \mathrm{~Hz}, 2 \mathrm{H}, 2-\mathrm{H}_{2}\right), 3.65$ (t, $\left.J=6.3 \mathrm{~Hz}, 2 \mathrm{H}, 6-\mathrm{H}_{2}\right), 9.74$ (t, $\left.J=1.9 \mathrm{~Hz}, 1 \mathrm{H}, \mathrm{CHO}\right)$.

${ }^{13} \mathrm{C}-\mathrm{NMR}\left(126 \mathrm{MHz}, \mathrm{CDCl}_{3}\right): \delta(\mathrm{ppm})=12.0\left(\mathrm{Si}\left(\underline{\mathrm{CH}}\left(\mathrm{CH}_{3}\right)_{2}\right)_{3}\right), 18.0\left(\mathrm{Si}\left(\mathrm{CH}\left(\underline{\mathrm{CH}_{3}}\right)_{2}\right)_{3}\right), 21.9$ (C-5), 25.5 (C-3), 32.7 (C-4), 43.9 (C-2), 63.1 (C-6), 202.8 (CHO).

MS (ESI): $\mathrm{m} / \mathrm{z}(\%)=273.2(48)[\mathrm{M}+\mathrm{H}]^{+}, 311.2(57)[\mathrm{M}+\mathrm{K}]^{+}, 327.2(100)[\mathrm{M}+\mathrm{MeOH}+\mathrm{Na}]^{+}$; $271.2(100)[\mathrm{M}-\mathrm{H}]^{-}$.

HRMS von $\mathrm{C}_{15} \mathrm{H}_{32} \mathrm{O}_{2} \mathrm{Si}$

ber.: $\quad 271.2099$

273.2244

gef.: $271.2095[\mathrm{M}-\mathrm{H}]^{-}$

273.2239 $[\mathrm{M}+\mathrm{H}]^{+}(\mathrm{ESI}-\mathrm{HRMS})$.

$\mathbf{C}_{15} \mathbf{H}_{32} \mathrm{O}_{2} \mathrm{Si}$ (272.50). 


\subsection{Synthese der Seitenkette 214}

\subsubsection{Hex-5-en-1-ylbenzoat (192)}

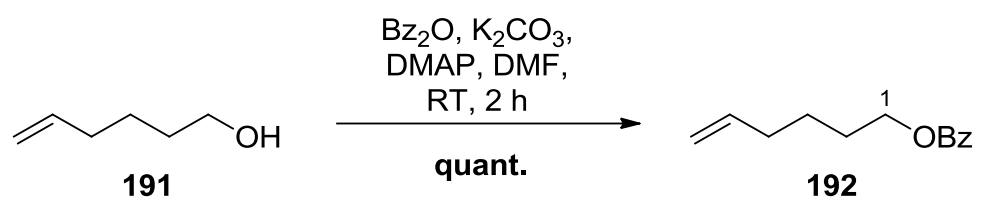

Zu einer Lösung des Alkohols 191 (5.40 mL, 4.50 g, 45.0 mmol, 1.0 Äq.) in DMF (50 mL) wurde bei Raumtemperatur $\mathrm{K}_{2} \mathrm{CO}_{3}(6.84 \mathrm{~g}, 49.5 \mathrm{mmol}, 1.1$ Äq.) gegeben und $5 \mathrm{~min}$ gerührt. Anschließend wurden DMAP (550 mg, $4.50 \mathrm{mmol}, 10 \mathrm{~mol} \%$ ) und $\mathrm{Bz}_{2} \mathrm{O}$ (11.2 g, 49.5 mmol, 1.1 Äq.) zugegeben und weitere $2 \mathrm{~h}$ bei dieser Temperatur gerührt. Die Reaktion wurde durch Zugabe von ges. $\mathrm{NaHCO}_{3}$-Lösung $(20 \mathrm{~mL})$ beendet und das Reaktionsgemisch mit $\mathrm{Et}_{2} \mathrm{O}$ $(4 \times 30 \mathrm{~mL})$ extrahiert. Die vereinigten organischen Phasen wurden mit ges. NaCl-Lösung $(50 \mathrm{~mL})$ gewaschen, über $\mathrm{Na}_{2} \mathrm{SO}_{4}$ getrocknet und das Lösungsmittel unter vermindertem Druck entfernt. Säulenchromatographische Reinigung des Rückstandes an Kieselgel (Petrolether/EtOAc 10:1) lieferte die Zielverbindung 192 (9.19 g, $45.0 \mathrm{mmol}$, quant.) in Form einer farblosen Flüssigkeit.

DC: $\mathrm{R}_{f}=0.61$ (Petrolether/EtOAc 10:1).

UV $\left(\mathrm{CH}_{3} \mathrm{CN}\right): \lambda_{\max }(\lg \varepsilon)=195 \mathrm{~nm}$ (4.5810), 228 (4.0960), 272 (2.9575), 279 (2.8649).

IR (ATR): $\tilde{v}\left(\mathrm{~cm}^{-1}\right)=2935,1715,1451,1268,1110,1069,1026,910,708$.

${ }^{1} \mathbf{H}-\mathbf{N M R}\left(300 \mathrm{MHz}, \mathrm{CDCl}_{3}\right): \delta(\mathrm{ppm})=1.47-1.60\left(\mathrm{~m}_{\mathrm{c}}, 2 \mathrm{H}, 3-\mathrm{H}_{2}\right), 1.71-1.83\left(\mathrm{~m}_{\mathrm{c}}, 2 \mathrm{H}\right.$, 2- $\left.\mathrm{H}_{2}\right), 2.06-2.17\left(\mathrm{~m}_{\mathrm{c}}, 2 \mathrm{H}, 4-\mathrm{H}_{2}\right), 4.31\left(\mathrm{t}, J=6.6 \mathrm{~Hz}, 2 \mathrm{H}, 1-\mathrm{H}_{2}\right), 4.93-4.95\left(\mathrm{~m}_{\mathrm{c}}, 0.5 \mathrm{H}\right.$, 6- $\left.\mathrm{H}_{\mathrm{A} 1}\right), 4.96-5.00\left(\mathrm{~m}_{\mathrm{c}}, 1 \mathrm{H}, 6-\mathrm{H}_{\mathrm{A} 2}, 6-\mathrm{H}_{\mathrm{B} 1}\right), 5.03-5.06\left(\mathrm{~m}_{\mathrm{c}}, 0.5 \mathrm{H}, 6-\mathrm{H}_{\mathrm{B} 2}\right), 5.80(\mathrm{ddt}, J=16.9$, 10.2, 6.6 Hz, $1 \mathrm{H}, 5-\mathrm{H}), 7.38-7.46\left(\mathrm{~m}_{\mathrm{c}}, 2 \mathrm{H}, 2 \times \mathrm{Ph}-\mathrm{H}_{m}\right), 7.50-7.58\left(\mathrm{~m}_{\mathrm{c}}, 1 \mathrm{H}, \mathrm{Ph}-\mathrm{H}_{p}\right)$, 8.00-8.06 ( $\left.\mathrm{m}_{\mathrm{c}}, 2 \mathrm{H}, 2 \times \mathrm{Ph}-\mathrm{H}_{o}\right)$.

${ }^{13}$ C-NMR (126 MHz, $\left.\mathrm{CDCl}_{3}\right): \delta(\mathrm{ppm})=25.4$ (C-3), 28.2 (C-2), 33.4 (C-4), 64.9 (C-1), 114.8 (C-6), $128.2\left(2 \times \mathrm{Ph}-\mathrm{C}_{m}\right), 129.4\left(2 \times \mathrm{Ph}-\mathrm{C}_{o}\right), 130.4\left(\mathrm{Ph}-\mathrm{C}_{i}\right), 132.7\left(\mathrm{Ph}-\mathrm{C}_{p}\right), 138.2(\mathrm{C}-5), 166.5$ $(\mathrm{C}=\mathrm{O})$.

MS (ESI): m/z $(\%)=227.1(60)[\mathrm{M}+\mathrm{Na}]^{+}$.

HRMS von $\mathrm{C}_{13} \mathrm{H}_{16} \mathrm{O}_{2} \quad$ ber.: 227.1043 gef.: $227.1040[\mathrm{M}+\mathrm{Na}]^{+}(\mathrm{ESI}-\mathrm{HRMS})$.

$\mathbf{C}_{13} \mathbf{H}_{16} \mathbf{O}_{2}$ (204.26). 


\subsection{2 (S)-5,6-Dihydroxyhexylbenzoat (193)}

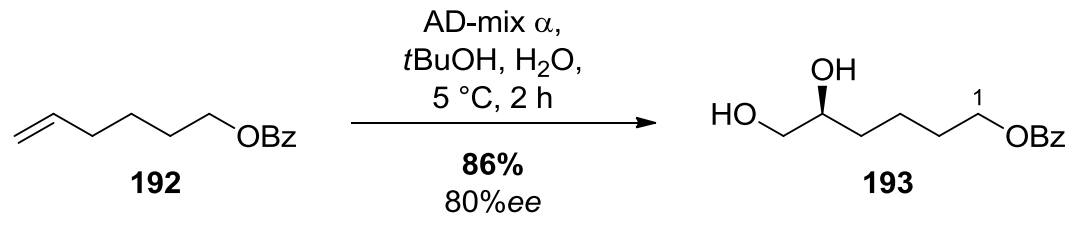

Zu einer Lösung des Alkens 192 (500 mg, $2.45 \mathrm{mmol}, 1.0$ Äq.) in $t \mathrm{BuOH} / \mathrm{H}_{2} \mathrm{O}$ (1:1,30 mL) wurde bei $5{ }^{\circ} \mathrm{C}$ portionsweise AD-mix $\alpha(3.43 \mathrm{~g}, 1.4 \mathrm{~g} / \mathrm{mmol})$ gegeben und $2 \mathrm{~h}$ bei dieser Temperatur gerührt. Die Reaktion wurde durch Zugabe von ges. $\mathrm{NaHCO}_{3}$-Lösung $(10 \mathrm{~mL})$ und ges. $\mathrm{Na}_{2} \mathrm{~S}_{2} \mathrm{O}_{3}$-Lösung $(10 \mathrm{~mL})$ beendet und die Reaktionsmischung mit $\mathrm{Et}_{2} \mathrm{O}(3 \times 25 \mathrm{~mL})$ extrahiert. Die vereinigten organischen Phasen wurden mit ges. NaCl-Lösung $(50 \mathrm{~mL})$ gewaschen, über $\mathrm{Na}_{2} \mathrm{SO}_{4}$ getrocknet und das Lösungsmittel unter vermindertem Druck entfernt. Säulenchromatographische Reinigung des Rückstandes an Kieselgel ( $n$ Pentan/EtOAc 1:3 $\rightarrow 1: 5$ ) lieferte die Zielverbindung 193 (503 mg, $2.11 \mathrm{mmol}, 86 \%$, 80\%ee) in Form einer farblosen Flüssigkeit.

DC: $\mathbf{R}_{f}=0.23$ (Petrolether/EtOAc 1:3).

${ }^{1} \mathbf{H}-\mathbf{N M R}\left(300 \mathrm{MHz}, \mathrm{CDCl}_{3}\right): \delta(\mathrm{ppm})=1.41-1.67\left(\mathrm{~m}_{\mathrm{c}}, 4 \mathrm{H}, 3-\mathrm{H}_{2}, 4-\mathrm{H}_{2}\right), 1.70-1.85\left(\mathrm{~m}_{\mathrm{c}}, 2 \mathrm{H}\right.$, 2- $\left.\mathrm{H}_{2}\right), 2.64,2.71\left(2 \mathrm{~s}_{\mathrm{br}}, 2 \mathrm{H}, 2 \times \mathrm{OH}\right), 3.42\left(\mathrm{dd}, J=11.1,7.6 \mathrm{~Hz}, 1 \mathrm{H}, 6-\mathrm{H}_{\mathrm{A}}\right), 3.63(\mathrm{dd}, J=11.1$, $\left.3.0 \mathrm{~Hz}, 1 \mathrm{H}, 6-\mathrm{H}_{\mathrm{B}}\right), 3.66-3.75\left(\mathrm{~m}_{\mathrm{c}}, 1 \mathrm{H}, 5-\mathrm{H}\right), 4.30\left(\mathrm{t}, J=6.5 \mathrm{~Hz}, 2 \mathrm{H}, 1-\mathrm{H}_{2}\right), 7.41(\mathrm{t}, J=$ $\left.7.5 \mathrm{~Hz}, 2 \mathrm{H}, 2 \times \mathrm{Ph}-\mathrm{H}_{m}\right), 7.53\left(\mathrm{tt}, J=7.4,1.2 \mathrm{~Hz}, 1 \mathrm{H}, \mathrm{Ph}-\mathrm{H}_{p}\right), 8.00(\mathrm{~d}, J=7.1 \mathrm{~Hz}, 2 \mathrm{H}$, $\left.2 \times \mathrm{Ph}-\mathrm{H}_{o}\right)$.

${ }^{13}$ C-NMR $\left(126 \mathrm{MHz}, \mathrm{CDCl}_{3}\right): \delta(\mathrm{ppm})=22.2(\mathrm{C}-3), 28.8$ (C-2), 32.7 (C-4), 64.8 (C-1), 66.7 (C-6), $72.0(\mathrm{C}-5), 128.3\left(2 \times \mathrm{Ph}-\mathrm{C}_{m}\right), 129.4\left(2 \times \mathrm{Ph}-\mathrm{C}_{o}\right), 130.2\left(\mathrm{Ph}-\mathrm{C}_{i}\right), 132.8\left(\mathrm{Ph}-\mathrm{C}_{p}\right), 166.6$ $(\mathrm{C}=\mathrm{O})$.

MS (ESI): m/z (\%) = $239.1(6)[\mathrm{M}+\mathrm{H}]^{+}, 261.1(100)[\mathrm{M}+\mathrm{Na}]^{+}$.

HRMS von $\mathrm{C}_{13} \mathrm{H}_{18} \mathrm{O}_{4}$ ber.: 261.1097 gef.: $261.1102[\mathrm{M}+\mathrm{Na}]^{+}(\mathrm{ESI}-\mathrm{HRMS})$.

$\mathbf{C}_{13} \mathbf{H}_{18} \mathbf{O}_{4}$ (238.28).

HPLC (analytisch): Säule: $\quad$ Chiralpak $^{\circledR}$ IC, $250 \times 4.6 \mathrm{~mm}, 5 \mu \mathrm{m}$

Eluens: $\quad n \mathrm{Hexan} / \mathrm{iPrOH}$ 95:5

Fluß: $\quad 0.8 \mathrm{~mL} / \mathrm{min}$

$\lambda: \quad 236 \mathrm{~nm}$

$t_{\mathrm{R}}: \quad(S)$-193: $85.7 \mathrm{~min},(R)-193: 91.7 \mathrm{~min}$. 


\subsection{3 (R)-5,6-Dihydroxyhexylbenzoat (272)}

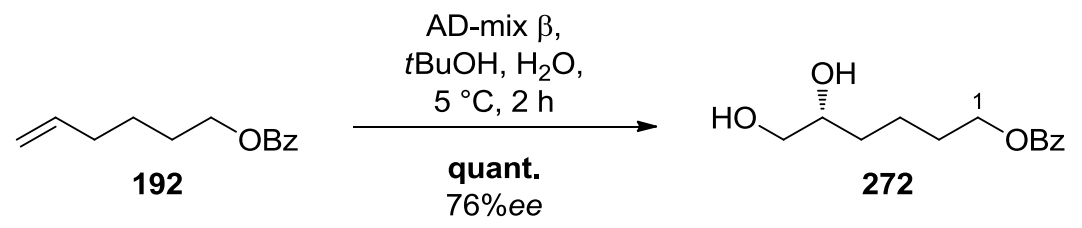

Zu einer Lösung des Alkens $192\left(10.0 \mathrm{mg}, 49.0 \mu \mathrm{mol}, 1.0\right.$ Äq.) in $t \mathrm{BuOH} / \mathrm{H}_{2} \mathrm{O}(1: 1,1 \mathrm{~mL})$ wurde bei $5{ }^{\circ} \mathrm{C}$ portionsweise AD-mix $\beta(67.0 \mathrm{mg}, 1.4 \mathrm{~g} / \mathrm{mmol})$ gegeben und $2 \mathrm{~h}$ bei dieser Temperatur gerührt. Die Reaktion wurde durch Zugabe von ges. $\mathrm{NaHCO}_{3}$-Lösung (2 mL) und ges. $\mathrm{Na}_{2} \mathrm{~S}_{2} \mathrm{O}_{3}$-Lösung $(2 \mathrm{~mL})$ beendet und die Reaktionsmischung mit $\mathrm{Et}_{2} \mathrm{O}(3 \times 5 \mathrm{~mL})$ extrahiert. Die vereinigten organischen Phasen wurden mit ges. NaCl-Lösung (15 mL) gewaschen, über $\mathrm{Na}_{2} \mathrm{SO}_{4}$ getrocknet und das Lösungsmittel unter vermindertem Druck entfernt. Säulenchromatographische Reinigung des Rückstandes an Kieselgel ( $n$ Pentan/EtOAc 1:3 $\rightarrow 1: 5$ ) lieferte die Zielverbindung 272 (11.7 mg, $49.0 \mu$ mol, quant., $76 \% e e$ ) in Form einer farblosen Flüssigkeit.

DC: $\mathrm{R}_{f}=0.23$ (Petrolether/EtOAc 1:3).

${ }^{1} \mathbf{H}-\mathbf{N M R}\left(300 \mathrm{MHz}, \mathrm{CDCl}_{3}\right): \delta(\mathrm{ppm})=1.41-1.67\left(\mathrm{~m}_{\mathrm{c}}, 4 \mathrm{H}, 3-\mathrm{H}_{2}, 4-\mathrm{H}_{2}\right), 1.70-1.85\left(\mathrm{~m}_{\mathrm{c}}, 2 \mathrm{H}\right.$, 2- $\left.\mathrm{H}_{2}\right), 2.64,2.71\left(2 \mathrm{~s}_{\mathrm{br}}, 2 \mathrm{H}, 2 \times \mathrm{OH}\right), 3.42\left(\mathrm{dd}, J=11.1,7.6 \mathrm{~Hz}, 1 \mathrm{H}, 6-\mathrm{H}_{\mathrm{A}}\right), 3.63(\mathrm{dd}, J=11.1$, $\left.3.0 \mathrm{~Hz}, 1 \mathrm{H}, 6-\mathrm{H}_{\mathrm{B}}\right), 3.66-3.75\left(\mathrm{~m}_{\mathrm{c}}, 1 \mathrm{H}, 5-\mathrm{H}\right), 4.30\left(\mathrm{t}, J=6.5 \mathrm{~Hz}, 2 \mathrm{H}, 1-\mathrm{H}_{2}\right), 7.41(\mathrm{t}, J=$ $\left.7.5 \mathrm{~Hz}, 2 \mathrm{H}, 2 \times \mathrm{Ph}_{-} \mathrm{H}_{m}\right), 7.53\left(\mathrm{tt}, J=7.4,1.2 \mathrm{~Hz}, 1 \mathrm{H}, \mathrm{Ph}-\mathrm{H}_{p}\right), 8.00(\mathrm{~d}, J=7.1 \mathrm{~Hz}, 2 \mathrm{H}$, $\left.2 \times \mathrm{Ph}-\mathrm{H}_{o}\right)$.

${ }^{13}$ C-NMR $\left(126 \mathrm{MHz}, \mathrm{CDCl}_{3}\right): \delta(\mathrm{ppm})=22.2(\mathrm{C}-3), 28.8(\mathrm{C}-2), 32.7(\mathrm{C}-4), 64.8(\mathrm{C}-1), 66.7$ (C-6), $72.0(\mathrm{C}-5), 128.3\left(2 \times \mathrm{Ph}-\mathrm{C}_{m}\right), 129.4\left(2 \times \mathrm{Ph}-\mathrm{C}_{o}\right), 130.2\left(\mathrm{Ph}-\mathrm{C}_{i}\right), 132.8\left(\mathrm{Ph}-\mathrm{C}_{p}\right), 166.6$ $(\mathrm{C}=\mathrm{O})$.

MS (ESI): $\mathrm{m} / \mathrm{z}(\%)=239.1(6)[\mathrm{M}+\mathrm{H}]^{+}, 261.1(100)[\mathrm{M}+\mathrm{Na}]^{+}$.

HRMS von $\mathrm{C}_{13} \mathrm{H}_{18} \mathrm{O}_{4}$ ber.: 261.1097 gef.: $261.1102[\mathrm{M}+\mathrm{Na}]^{+}(\mathrm{ESI}-\mathrm{HRMS})$.

$\mathrm{C}_{13} \mathrm{H}_{18} \mathrm{O}_{4}$ (238.28).

HPLC (analytisch): Säule: $\quad$ Chiralpak $^{\circledR}$ IC, $250 \times 4.6 \mathrm{~mm}, 5 \mu \mathrm{m}$

Eluens: $\quad n$ Hexan/iPrOH 95:5

Fluß: $\quad 0.8 \mathrm{~mL} / \mathrm{min}$

$\lambda: \quad 236 \mathrm{~nm}$

$t_{\mathrm{R}}: \quad(R)-272: 91.7 \mathrm{~min},(S)-272: 85.7 \mathrm{~min}$. 


\subsection{4 (S)-6-Benzoyloxyhexan-1,2-diylbis(3,5-dinitrobenzoat) (196)}
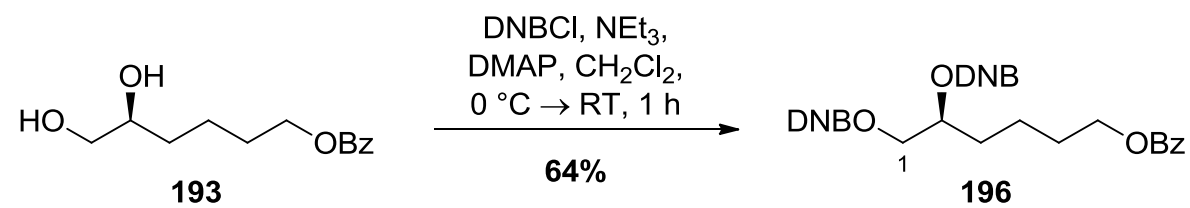

$\mathrm{Zu}$ einer Lösung des Diols 193 (25.7 mg, $115 \mu \mathrm{mol}, 1.0$ Äq.) in $\mathrm{CH}_{2} \mathrm{Cl}_{2}$ (8 mL) wurde bei $0{ }^{\circ} \mathrm{C} \mathrm{NEt}_{3}(40.0 \mu \mathrm{L}, 29.2 \mathrm{mg}, 289 \mu \mathrm{mol}, 2.5$ Äq.) gegeben und $5 \mathrm{~min}$ gerührt. Anschließend wurden DMAP (1.40 mg, $11.5 \mu \mathrm{mol}, 10 \mathrm{~mol} \%)$ und DNBCl (66.5 mg, $289 \mu \mathrm{mol}, 2.5$ Äq.) zugegeben, die Lösung auf Raumtemperatur erwärmt und weitere 60 min bei dieser Temperatur gerührt. Die Reaktion wurde durch Zugabe von $\mathrm{HCl}(2 \mathrm{M}, 5 \mathrm{~mL})$ beendet und das Reaktionsgemisch mit $\mathrm{CH}_{2} \mathrm{Cl}_{2}(3 \times 5 \mathrm{~mL})$ extrahiert. Die vereinigten organischen Phasen wurden mit ges. NaCl-Lösung $(10 \mathrm{~mL})$ gewaschen, über $\mathrm{Na}_{2} \mathrm{SO}_{4}$ getrocknet und das Lösungsmittel unter vermindertem Druck entfernt. Säulenchromatographische Reinigung des Rückstandes an Kieselgel (Petrolether/EtOAc 5:1 $\rightarrow 2: 1$ ) lieferte die Zielverbindung 196 (46.1 mg, $73.6 \mu \mathrm{mol}, 64 \%$ ) in Form eines gelben Schaums.

DC: $\mathbf{R}_{f}=0.36$ (Petrolether/EtOAc 2:1).

${ }^{1} \mathbf{H}-\mathbf{N M R}\left(300 \mathrm{MHz}, \mathrm{CDCl}_{3}\right): \delta(\mathrm{ppm})=1.57-1.73\left(\mathrm{~m}_{\mathrm{c}}, 2 \mathrm{H}, 4-\mathrm{H}_{2}\right), 1.80-2.08\left(\mathrm{~m}_{\mathrm{c}}, 4 \mathrm{H}, 3-\mathrm{H}_{2}\right.$, $\left.5-\mathrm{H}_{2}\right), 4.34\left(\mathrm{td}, J=6.2,1.6 \mathrm{~Hz}, 2 \mathrm{H}, 6-\mathrm{H}_{2}\right), 4.55\left(\mathrm{dd}, J=12.2,7.3 \mathrm{~Hz}, 1 \mathrm{H}, 1-\mathrm{H}_{\mathrm{A}}\right), 4.86(\mathrm{dd}$, $\left.J=12.2,2.8 \mathrm{~Hz}, 1 \mathrm{H}, 1-\mathrm{H}_{\mathrm{B}}\right), 5.60-5.70\left(\mathrm{~m}_{\mathrm{c}}, 1 \mathrm{H}, 2-\mathrm{H}\right), 7.35(\mathrm{tt}, J=7.8,1.2 \mathrm{~Hz}, 2 \mathrm{H}$, $\left.2 \times \mathrm{Ph}-\mathrm{H}_{m}\right), 7.50\left(\mathrm{tt}, J=7.4,1.3 \mathrm{~Hz}, 1 \mathrm{H}, \mathrm{Ph}-\mathrm{H}_{p}\right), 7.89-7.94\left(\mathrm{~m}, 2 \mathrm{H}, 2 \times \mathrm{Ph}-\mathrm{H}_{o}\right), 9.03(\mathrm{~d}, J=$ $\left.2.2 \mathrm{~Hz}, 2 \mathrm{H}, 2 \times \mathrm{DNB}_{-} \mathrm{H}_{o}\right), 9.08\left(\mathrm{~d}, J=2.1 \mathrm{~Hz}, 2 \mathrm{H}, 2 \times \mathrm{DNB}^{-\mathrm{H}_{o}}\right) 9.15-9.18(\mathrm{~m}, 2 \mathrm{H}$, $2 \times$ DNB-H ${ }_{p}$.

${ }^{13}$ C-NMR $\left(126 \mathrm{MHz}, \mathrm{CDCl}_{3}\right): \delta(\mathrm{ppm})=21.9(\mathrm{C}-4), 28.4$ (C-5), 30.2 (C-3), 64.1 (C-6), 67.0 (C-1), $74.1(\mathrm{C}-2), 122.64\left(2 \times \mathrm{DNB}^{-\mathrm{C}_{p}}\right), 128.2\left(2 \times \mathrm{Ph}-\mathrm{C}_{m}\right), 129.3\left(2 \times \mathrm{Ph}-\mathrm{C}_{o}\right), 129.3\left(\mathrm{DNB}-\mathrm{C}_{o}\right)$, $129.3\left(\right.$ DNB-C $\left._{o}\right), 129.9\left(\mathrm{Ph}-\mathrm{C}_{i}\right), 132.9\left(\mathrm{Ph}-\mathrm{C}_{p}\right), 132.9\left(\mathrm{DNB}-\mathrm{C}_{i}\right), 133.0\left(\mathrm{DNB}-\mathrm{C}_{i}\right), 148.6$ $\left(4 \times\right.$ DNB-C $\left._{m}\right), 162.1(\mathrm{DNB}-\mathrm{C}=\mathrm{O}), 162.2(\mathrm{DNB}-\mathrm{C}=\mathrm{O}), 166.6(\mathrm{C}=\mathrm{O})$.

MS (ESI): $\mathrm{m} / \mathrm{z}(\%)=239.1(5)[\mathrm{M}-2 \mathrm{DNB}+\mathrm{H}]^{+}, 261.1(100)[\mathrm{M}-2 \mathrm{DNB}+\mathrm{Na}]^{+}, 455.1(10)$ $[\mathrm{M}-\mathrm{DNB}+\mathrm{Na}]^{+}$.

$\mathbf{C}_{27} \mathbf{H}_{22} \mathbf{N}_{4} \mathbf{O}_{14}$ (626.48). 


\subsection{5 (S)-6-((tert-Butyldimethylsilyl)oxy)-5-hydroxyhexylbenzoat (194)}

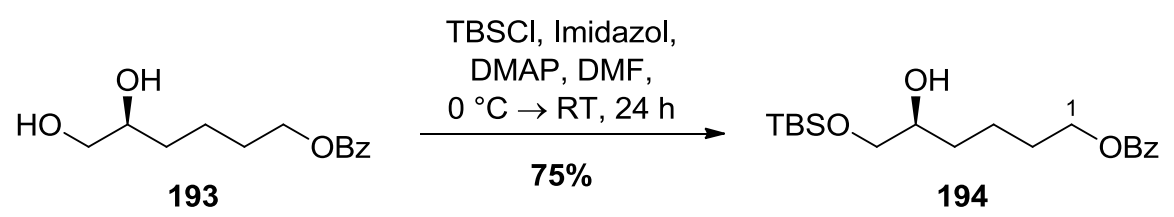

Zu einer Lösung des Diols 193 (385 mg, 1.62 mmol, 1.0 Äq.), in DMF (20 mL) wurde bei $0{ }^{\circ} \mathrm{C}$ Imidazol (157 mg, $2.42 \mathrm{mmol}, 1.5$ Äq.) gegeben und $5 \mathrm{~min}$ gerührt. Anschließend wurden DMAP (8.00 mg, $64.6 \mu \mathrm{mol}, 4 \mathrm{~mol} \%)$ und TBSCl (232 mg, $1.62 \mathrm{mmol}, 1.0$ Äq.) zugegeben, die Lösung auf Raumtemperatur erwärmt und weitere $24 \mathrm{~h}$ bei dieser Temperatur gerührt. Die Reaktion wurde durch Zugabe von ges. NaCl-Lösung (15 mL) beendet und das Reaktionsgemisch mit $\mathrm{Et}_{2} \mathrm{O}(3 \times 15 \mathrm{~mL})$ extrahiert. Die vereinigten organischen Phasen wurden mit ges. NaCl-Lösung $(30 \mathrm{~mL})$ gewaschen, über $\mathrm{Na}_{2} \mathrm{SO}_{4}$ getrocknet und das Lösungsmittel unter vermindertem Druck entfernt. Säulenchromatographische Reinigung des Rückstandes an Kieselgel (Petrolether/EtOAc 5:1 $\rightarrow 2: 1$ ) lieferte die Zielverbindung 194 (438 mg, $1.22 \mathrm{mmol}, 75 \%$ ) in Form einer farblosen Flüssigkeit.

DC: $\mathrm{R}_{f}=0.24$ (Petrolether/EtOAc 5:1).

${ }^{1} \mathbf{H}$-NMR $\left(300 \mathrm{MHz}, \mathrm{CDCl}_{3}\right): \delta(\mathrm{ppm})=0.05\left(\mathrm{~s}, 6 \mathrm{H}, \mathrm{Si}\left(\mathrm{CH}_{3}\right)_{2}\right), 0.88\left(\mathrm{~s}, 9 \mathrm{H}, \mathrm{SiC}\left(\mathrm{CH}_{3}\right)_{3}\right)$, 1.39-1.71 (m, $\left.4 \mathrm{H}, 3-\mathrm{H}_{2}, 4-\mathrm{H}_{2}\right), 1.74-1.85\left(\mathrm{~m}_{\mathrm{c}}, 2 \mathrm{H}, 2-\mathrm{H}_{2}\right), 2.43(\mathrm{~s}, 1 \mathrm{H}, \mathrm{OH}), 3.42(\mathrm{dd}, J=$ 9.8, 7.4 Hz, $\left.1 \mathrm{H}, 6-\mathrm{H}_{\mathrm{A}}\right), 3.61\left(\mathrm{dd}, J=10.1,2.7 \mathrm{~Hz}, 1 \mathrm{H}, 6-\mathrm{H}_{\mathrm{B}}\right), 3.61-3.69\left(\mathrm{~m}_{\mathrm{c}}, 1 \mathrm{H}, 5-\mathrm{H}\right)$, $4.31\left(\mathrm{t}, J=6.5 \mathrm{~Hz}, 2 \mathrm{H}, 1-\mathrm{H}_{2}\right), 7.41$ (tt, $\left.J=6.6,1.4 \mathrm{~Hz}, 2 \mathrm{H}, 2 \times \mathrm{Ph}-\mathrm{H}_{m}\right), 7.53$ (tt, $J=7.4$, $\left.1.4 \mathrm{~Hz}, 1 \mathrm{H}, \mathrm{Ph}-\mathrm{H}_{p}\right), 8.00-8.04\left(\mathrm{~m}_{\mathrm{c}}, 2 \mathrm{H}, 2 \times \mathrm{Ph}-\mathrm{H}_{o}\right)$.

${ }^{13}$ C-NMR $\left(126 \mathrm{MHz}, \mathrm{CDCl}_{3}\right): \delta(\mathrm{ppm})=-5.3,-5.2\left(\mathrm{Si}\left(\mathrm{CH}_{3}\right)_{2}\right), 18.4\left(\mathrm{SiC}\left(\mathrm{CH}_{3}\right)_{3}\right), 22.3(\mathrm{C}-3)$, $26.0\left(\mathrm{SiC}\left(\underline{\mathrm{CH}}_{3}\right)_{3}\right), 28.9$ (C-2), 32.5 (C-4), 64.9 (C-1), 67.2 (C-6), 71.6 (C-5), $128.2\left(2 \times \mathrm{Ph}-\mathrm{C}_{m}\right)$, $129.5\left(2 \times \mathrm{Ph}-\mathrm{C}_{o}\right), 130.4\left(\mathrm{Ph}-\mathrm{C}_{i}\right), 132.7\left(\mathrm{Ph}-\mathrm{C}_{p}\right), 166.5(\mathrm{C}=\mathrm{O})$.

MS (ESI): $\mathrm{m} / \mathrm{z}(\%)=239.1(5)[\mathrm{M}-\mathrm{TBS}+\mathrm{H}]^{+}, 261.1(100)[\mathrm{M}-\mathrm{TBS}+\mathrm{Na}]^{+}, 375.2(2)[\mathrm{M}+\mathrm{Na}]^{+}$. HRMS von $\mathrm{C}_{19} \mathrm{H}_{32} \mathrm{O}_{4} \mathrm{Si}$ ber.: 375.1962 gef.: $\quad 375.1956[\mathrm{M}+\mathrm{Na}]^{+}$(ESI-HRMS).

$\mathrm{C}_{19} \mathrm{H}_{32} \mathrm{O}_{4} \mathrm{Si}$ (352.54). 


\subsubsection{2,3,4,6-Tetradesoxy-4-azido- $\alpha / \beta$-D-glucopyranosyltrichloracetimidat (197)}
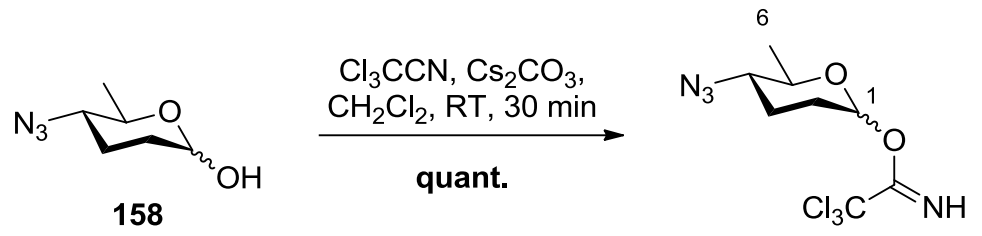

197

Zu einer Lösung des Azids 158 (22.0 mg, $142 \mu \mathrm{mol}, 1.0$ Äq.) in $\mathrm{CH}_{2} \mathrm{Cl}_{2}$ (8 mL) wurde bei Raumtemperatur $\mathrm{Cl}_{3} \mathrm{CCN}$ (152 $\mu \mathrm{L}, 205 \mathrm{mg}, 1.42 \mathrm{mmol}, 10.0$ Äq.) und $\mathrm{Cs}_{2} \mathrm{CO}_{3}$ (4.63 mg, $14.2 \mu \mathrm{mol}, 0.1$ Äq.) gegeben und $2 \mathrm{~h}$ bei dieser Temperatur gerührt. Anschließend wurde das $\mathrm{Cs}_{2} \mathrm{CO}_{3}$ mit Hilfe eines PTFE-Filters ( $\varnothing 25 \mathrm{~mm}, 0.2 \mu \mathrm{m}$ ) abfiltriert und dieser mit $\mathrm{CH}_{2} \mathrm{Cl}_{2}$ $(10 \mathrm{~mL})$ gespült. Entfernen des Lösungsmittels unter vermindertem Druck lieferte die Zielverbindung 197 (42.8 mg, $142 \mu \mathrm{mol}$, quant.) in Form eines Farblosen Öls. Die Verbindung wurde ohne weitere Aufreinigung direkt für die Umsetzung benutzt.

${ }^{1} \mathbf{H}$-NMR (300 MHz, $\mathrm{C}_{6} \mathrm{D}_{6}, \alpha$-Anomer beschrieben): $\delta(\mathrm{ppm})=0.81-1.62\left(\mathrm{~m}, 7 \mathrm{H}, 2-\mathrm{H}_{2}\right.$, 3- $\left.\mathrm{H}_{2}, 6-\mathrm{H}_{3}\right), 2.31$ (ddd, $\left.J=10.0,4.6,1.5 \mathrm{~Hz}, 1 \mathrm{H}, 4-\mathrm{H}\right), 3.84(\mathrm{dq}, J=10.0,6.6 \mathrm{~Hz}, 1 \mathrm{H}, 5-\mathrm{H})$, $6.20(\mathrm{~d}, J=3.7 \mathrm{~Hz}, 1 \mathrm{H}, 1-\mathrm{H}), 8.36(\mathrm{~s}, 1 \mathrm{H}, \mathrm{NH})$.

${ }^{13}$ C-NMR (126 MHz, $\mathrm{C}_{6} \mathrm{D}_{6}, \alpha$-Anomer beschrieben): $\delta(\mathrm{ppm})=18.8$ (C-6), 23.6 (C-3), 27.9 (C-2), 62.1 (C-4), 70.5 (C-5), $90.5\left(\mathrm{CCl}_{3}\right), 95.4(\mathrm{C}-1), 160.6(\mathrm{C}=\mathrm{N})$.

$\mathrm{C}_{8} \mathrm{H}_{11} \mathrm{Cl}_{3} \mathrm{~N}_{4} \mathrm{O}_{2}$ (301.56). 
4.4.7 (S)-5-(2,3,4,6-Tetradesoxy-4-azido- $\alpha / \beta$-D-glucopyranosyl)-6-((tert-butyldimethylsilyl)oxy)hexylbenzoat $((\alpha / \beta)-198)$

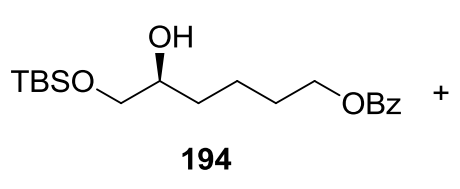

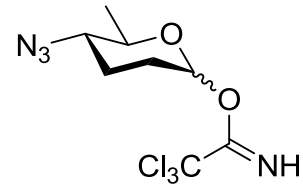

197

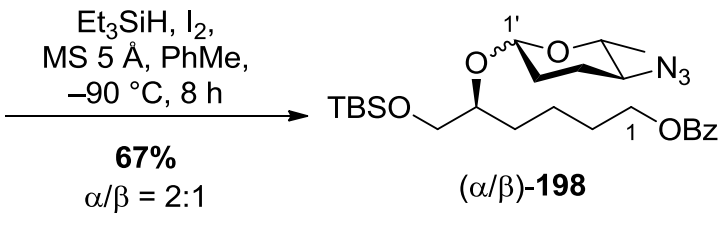

$(\alpha / \beta)-198$

Eine Lösung aus Alkohol 194 (50.0 mg, $142 \mu \mathrm{mol}, 1.0$ Äq.) und frisch hergestelltem Azid 197 (64.2 mg, $213 \mu \mathrm{mol}, 1.5$ Äq.) in Toluol ( $8 \mathrm{~mL}$ ) wurde bei Raumtemperatur 30 min über fein gemörsertem, aktiviertem $5 \AA$ Molekularsieb gerührt. Anschließend wurde die Reaktionslösung auf $-90{ }^{\circ} \mathrm{C}$ gekühlt, langsam $\mathrm{Et}_{3} \mathrm{SiH}(42.4 \mu \mathrm{L}, 31.0 \mathrm{mg}, 267 \mu \mathrm{mol}, 1.9$ Äq.) und eine Lösung aus $\mathrm{I}_{2}(54.1 \mathrm{mg}, 213 \mu \mathrm{mol}, 1.5$ Äq.) in Toluol $(10 \mathrm{~mL})$ zugegeben und $8 \mathrm{~h}$ bei dieser Temperatur gerührt. Die Reaktion wurde durch Zugabe von $\mathrm{NEt}_{3}(1 \mathrm{~mL})$ beendet und ges. $\mathrm{NaHCO}_{3}$-Lösung ( $5 \mathrm{~mL}$ ) sowie ges. $\mathrm{Na}_{2} \mathrm{~S}_{2} \mathrm{O}_{3}$-Lösung $(5 \mathrm{~mL})$ zugegeben. Es wurde mit $\mathrm{CH}_{2} \mathrm{Cl}_{2}(3 \times 5 \mathrm{~mL})$ extrahiert, die vereinigten organischen Phasen mit ges. NaCl-Lösung $(10 \mathrm{~mL})$ gewaschen und über $\mathrm{Na}_{2} \mathrm{SO}_{4}$ getrocknet. Säulenchromatographische Reinigung an Kieselgel (Petrolether/EtOAc 40:1) lieferte die Zielverbindung ( $\alpha / \beta)-(S)-198$ (46.8 mg, $95.2 \mu \mathrm{mol}, 67 \%, \alpha / \beta \sim 2: 1)$ als Gemisch beider Anomere in Form eines farblosen Öls.

DC: $\mathrm{R}_{f}=0.40$ (Petrolether/EtOAc 10:1).

${ }^{1} \mathbf{H}$-NMR $\left(300 \mathrm{MHz}, \mathrm{CDCl}_{3}\right): \delta(\mathrm{ppm})=0.03\left(\mathrm{~s}, 12 \mathrm{H}, \quad \mathrm{Si}\left(\mathrm{CH}_{3}\right)_{2 \alpha \beta}\right), 0.87(\mathrm{~s}, 18 \mathrm{H}$, $\left.\mathrm{SiC}\left(\mathrm{CH}_{3}\right)_{3 \alpha \beta}\right), 1.19\left(\mathrm{~d}, J=6.1 \mathrm{~Hz}, 3 \mathrm{H}, 6{ }^{\prime}-\mathrm{H}_{3 \alpha}\right), 1.19-1.28\left(\mathrm{~m}, 4 \mathrm{H}, 4-\mathrm{H}_{2 \alpha \beta}\right), 1.29(\mathrm{~d}, J=$ $\left.6.1 \mathrm{~Hz}, 3 \mathrm{H}, 6{ }^{\prime}-\mathrm{H}_{3 \beta}\right), 1.37-1.69\left(\mathrm{~m}, 8 \mathrm{H}, 3-\mathrm{H}_{2 \alpha \beta}, 2^{\prime}-\mathrm{H}_{2 \alpha \beta}\right), 1.68-1.81\left(\mathrm{~m}, 4 \mathrm{H}, 2-\mathrm{H}_{2 \alpha \beta}\right)$, 1.81-1.98 (m, 2 H, 3'- $\mathrm{H}_{2 \alpha}$ ), 2.09-2.21 (m, 2 H, 3'- $\left.\mathrm{H}_{2 \beta}\right), 2.89-3.04$ (m, 2 H, 4'- $\left.\mathrm{H}_{\alpha \beta}\right), 3.32$ (dq, $\left.J=9.6,6.1 \mathrm{~Hz}, 1 \mathrm{H}, 5^{\prime}-\mathrm{H}_{\beta}\right), 3.53\left(\mathrm{dd}, J=9.1,5.3 \mathrm{~Hz}, 2 \mathrm{H}, 6-\mathrm{H}_{\mathrm{A} \alpha \beta}\right.$ ), 3.60 (quint., $J=5.4 \mathrm{~Hz}$, $\left.2 \mathrm{H}, 5-\mathrm{H}_{\alpha \beta}\right), 3.67\left(\mathrm{dd}, J=9.1,4.9 \mathrm{~Hz}, 2 \mathrm{H}, 6-\mathrm{H}_{\mathrm{B} \alpha \beta}\right), 3.77\left(\mathrm{dq}, J=9.8,6.4 \mathrm{~Hz}, 1 \mathrm{H}, 5 '-\mathrm{H}_{\alpha}\right)$, $4.30\left(\mathrm{t}, J=6.5 \mathrm{~Hz}, 4 \mathrm{H}, 1-\mathrm{H}_{2 \alpha \beta}\right), 4.60\left(\mathrm{dd}, J=9.1,2.0 \mathrm{~Hz}, 1 \mathrm{H}, 1^{\prime}-\mathrm{H}_{\beta}\right), 4.82(\mathrm{~d}, J=1.6 \mathrm{~Hz}$, $\left.1 \mathrm{H}, 1^{\prime}-\mathrm{H}_{\alpha}\right), 7.42\left(\mathrm{t}, J=7.7 \mathrm{~Hz}, 4 \mathrm{H}, 2 \times \mathrm{Ph}-\mathrm{H}_{m \alpha \beta}\right), 7.53\left(\mathrm{t}, J=7.4 \mathrm{~Hz}, 2 \mathrm{H}, \mathrm{Ph}-\mathrm{H}_{p \alpha \beta}\right), 8.01$ (d, $J$ $\left.=7.1 \mathrm{~Hz}, 4 \mathrm{H}, 2 \times \mathrm{Ph}-\mathrm{H}_{o \alpha \beta}\right)$.

${ }^{13}$ C-NMR $\left(126 \mathrm{MHz}, \mathrm{CDCl}_{3}\right): \delta(\mathrm{ppm})=-5.3,-5.3\left(\mathrm{Si}\left(\mathrm{CH}_{3}\right)_{2 \alpha \beta}\right), 18.4\left(\mathrm{Si} \underline{\mathrm{C}}\left(\mathrm{CH}_{3}\right)_{3 \alpha \beta}\right), 18.6$ $\left(\mathrm{C}-6^{\prime}{ }_{\alpha}\right), 18.8\left(\mathrm{C}-6^{\prime}{ }_{\beta}\right), 21.7\left(\mathrm{C}-3_{\alpha \beta}\right), 23.8\left(\mathrm{C}-3^{\prime}{ }_{\alpha}\right), 26.0\left(\mathrm{SiC}\left(\underline{\mathrm{CH}}_{3}\right)_{3 \alpha \beta}\right), 27.6\left(\mathrm{C}-3^{\prime}{ }_{\beta}\right), 29.0\left(\mathrm{C}-2_{\alpha \beta}\right)$, $29.6\left(\mathrm{C}-4_{\alpha \beta}\right), 30.2\left(\mathrm{C}-2^{\prime}{ }_{\beta}\right), 30.6\left(\mathrm{C}-2^{\prime}{ }_{\alpha}\right), 61.9\left(\mathrm{C}-4_{\beta}^{\prime}\right), 62.7\left(\mathrm{C}-4_{\alpha}^{\prime}\right), 64.8\left(\mathrm{C}-1_{\alpha \beta}\right), 65.2\left(\mathrm{C}-6_{\alpha \beta}\right)$, 
$67.4\left(\mathrm{C}-5_{\alpha}^{\prime}\right), 74.4\left(\mathrm{C}-5^{\prime}{ }_{\beta}\right), 77.6\left(\mathrm{C}-5_{\alpha \beta}\right), 95.2\left(\mathrm{C}-1_{\alpha}^{\prime}\right), 100.6\left(\mathrm{C}-1_{\beta}^{\prime}\right), 128.2\left(2 \times \mathrm{Ph}-\mathrm{C}_{m \alpha \beta}\right), 129.4$ $\left(2 \times \mathrm{Ph}-\mathrm{C}_{o \alpha \beta}\right), 130.3\left(\mathrm{Ph}-\mathrm{C}_{i \alpha \beta}\right), 132.7\left(\mathrm{Ph}-\mathrm{C}_{p \alpha \beta}\right), 166.4\left(\mathrm{C}=\mathrm{O}_{\alpha \beta}\right)$.

MS (ESI): $\mathrm{m} / \mathrm{z}(\%)=514.3(100)[\mathrm{M}+\mathrm{Na}]^{+}$.

HRMS von $\mathrm{C}_{25} \mathrm{H}_{41} \mathrm{~N}_{3} \mathrm{O}_{5} \mathrm{Si}$

ber.: $\quad 514.2708$

gef.: $\quad 514.2705[\mathrm{M}+\mathrm{Na}]^{+}(\mathrm{ESI}-\mathrm{HRMS})$.

$\mathbf{C}_{25} \mathbf{H}_{41} \mathbf{N}_{3} \mathbf{O}_{5} \mathrm{Si}$ (491.70). 


\subsubsection{Hex-5-en-1-yl-3,5-dinitrobenzoat (199)}

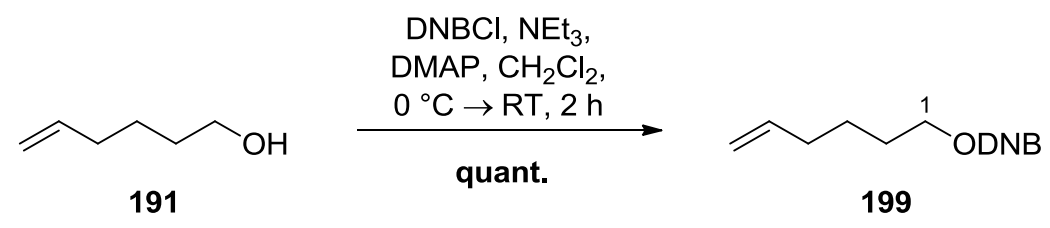

Zu einer Lösung des Alkohols 191 (844 mg, 8.43 mmol, 1.0 Äq.) in $\mathrm{CH}_{2} \mathrm{Cl}_{2}$ (50 mL) wurde bei $0{ }^{\circ} \mathrm{C} \mathrm{NEt}_{3}(1.87 \mathrm{~mL}, 1.36 \mathrm{~g}, 13.5 \mathrm{mmol}, 1.6$ Äq.) gegeben und $5 \mathrm{~min}$ gerührt. Anschließend wurden DMAP (103 mg, $843 \mu \mathrm{mol}, 10 \mathrm{~mol} \%)$ und $\mathrm{DNBCl}, \quad(2.33 \mathrm{~g}$, $10.1 \mathrm{mmol}, 1.2$ Äq.) zugegeben, langsam auf Raumtemperatur erwärmt und weitere $2 \mathrm{~h}$ gerührt. Die Reaktion wurde durch Zugabe von $\mathrm{HCl}(2 \mathrm{M}, 30 \mathrm{~mL})$ beendet und die Reaktionsmischung mit $\mathrm{CH}_{2} \mathrm{Cl}_{2}(3 \times 15 \mathrm{~mL})$ extrahiert. Die vereinigten organischen Phasen wurden mit ges. NaCl-Lösung $(50 \mathrm{~mL})$ gewaschen, über $\mathrm{Na}_{2} \mathrm{SO}_{4}$ getrocknet und das Lösungsmittel unter vermindertem Druck entfernt. Säulenchromatographische Reinigung des Rückstandes an Kieselgel (Petrolether/EtOAc 20:1) lieferte die Zielverbindung 199 (2.51 g, 8.43 mmol, quant.) in Form eines farblosen kristallinen Feststoffes.

DC: $\mathrm{R}_{f}=0.51$ (Petrolether/EtOAc 10:1).

${ }^{1} \mathbf{H}-\mathbf{N M R}\left(300 \mathrm{MHz}, \mathrm{CDCl}_{3}\right): \delta(\mathrm{ppm})=1.48-1.64\left(\mathrm{~m}_{\mathrm{c}}, 2 \mathrm{H}, 3-\mathrm{H}_{2}\right), 1.77-1.90\left(\mathrm{~m}_{\mathrm{c}}, 2 \mathrm{H}\right.$, 2- $\mathrm{H}_{2}$ ), 2.13 (q, $\left.J=7.3 \mathrm{~Hz}, 2 \mathrm{H}, 4-\mathrm{H}_{2}\right), 4.43\left(\mathrm{t}, J=6.7 \mathrm{~Hz}, 2 \mathrm{H}, 1-\mathrm{H}_{2}\right), 4.94(\mathrm{dt}, J=2.1,1.2 \mathrm{~Hz}$, $\left.0.5 \mathrm{H}, 6-\mathrm{H}_{\mathrm{A} 1}\right), 4.96-5.01\left(\mathrm{~m}_{\mathrm{c}}, 1 \mathrm{H}, 6-\mathrm{H}_{\mathrm{A} 2}, 6-\mathrm{H}_{\mathrm{B} 1}\right), 5.05\left(\mathrm{dt}, J=10.3,1.6 \mathrm{~Hz}, 0.5 \mathrm{H}, 6-\mathrm{H}_{\mathrm{B} 2}\right)$, 5.79 (ddt, $J=16.9,10.2,6.6 \mathrm{~Hz}, 1 \mathrm{H}, 5-\mathrm{H}), 9.12\left(\mathrm{~d}, J=2.2 \mathrm{~Hz}, 2 \mathrm{H}, 2 \times \mathrm{Ph}_{-} \mathrm{H}_{o}\right), 9.18(\mathrm{t}, J=$ $\left.2.2 \mathrm{~Hz}, 1 \mathrm{H}, \mathrm{Ph}-\mathrm{H}_{p}\right)$.

${ }^{13}$ C-NMR $\left(126 \mathrm{MHz}, \mathrm{CDCl}_{3}\right): \delta(\mathrm{ppm})=25.1(\mathrm{C}-3), 27.9(\mathrm{C}-2), 33.1(\mathrm{C}-4), 66.8(\mathrm{C}-1), 115.2$ (C-6), $122.3\left(\mathrm{Ph}-\mathrm{C}_{p}\right), 129.3\left(2 \times \mathrm{Ph}^{-\mathrm{C}_{o}}\right), 134.1\left(\mathrm{Ph}-\mathrm{C}_{i}\right), 137.9(\mathrm{C}-5), 148.6\left(2 \times \mathrm{Ph}-\mathrm{C}_{m}\right), 162.5$ $(\mathrm{C}=\mathrm{O})$.

MS (ESI): $\mathrm{m} / \mathrm{z}(\%)=317.2(13)[\mathrm{M}+\mathrm{Na}]^{+}$.

HRMS von $\mathrm{C}_{13} \mathrm{H}_{14} \mathrm{~N}_{2} \mathrm{O}_{6}$ ber.: 317.0744 gef.: $317.0735[\mathrm{M}+\mathrm{Na}]^{+}$(ESI-HRMS).

$\mathbf{C}_{13} \mathbf{H}_{14} \mathbf{N}_{2} \mathbf{O}_{6}$ (294.26). 


\subsection{9 (S)-5,6-Dihydroxyhexyl-3,5-dinitrobenzoat (200)}

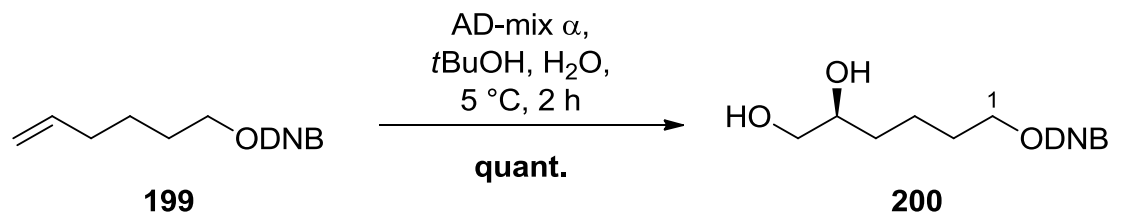

Zu einer Lösung des Alkens 199 (2.51 g, $8.53 \mathrm{mmol}, 1.0$ Äq.) in $t \mathrm{BuOH} / \mathrm{H}_{2} \mathrm{O}$ (1:1, $\left.30 \mathrm{~mL}\right)$ wurde bei $5{ }^{\circ} \mathrm{C}$ portionsweise AD-mix $\alpha(11.9 \mathrm{~g}, 1.4 \mathrm{~g} / \mathrm{mmol})$ gegeben und $2 \mathrm{~h}$ bei dieser Temperatur gerührt. Die Reaktion wurde durch Zugabe von ges. $\mathrm{NaHCO}_{3}$-Lösung $(10 \mathrm{~mL})$ und ges. $\mathrm{Na}_{2} \mathrm{~S}_{2} \mathrm{O}_{3}$-Lösung $(10 \mathrm{~mL})$ beendet und die Reaktionsmischung mit $\mathrm{Et}_{2} \mathrm{O}(3 \times 30 \mathrm{~mL})$ extrahiert. Die vereinigten organischen Phasen wurden mit ges. NaCl-Lösung $(50 \mathrm{~mL})$ gewaschen, über $\mathrm{Na}_{2} \mathrm{SO}_{4}$ getrocknet und das Lösungsmittel unter vermindertem Druck entfernt. Säulenchromatographische Reinigung des Rückstandes an Kieselgel (Petrolether/EtOAc 1:2 $\rightarrow 1: 5$ ) lieferte die Zielverbindung 200 (2.80 g, 8.53 mmol, quant.) in Form eines farblosen Öls, welches nicht weiter kristallisiert werden konnte. Eine Bestimmung des Enantiomerenüberschusses über chirale GC-MS war auf dieser Stufe nicht möglich.

DC: $\mathrm{R}_{f}=0.31$ (Petrolether/EtOAc 1:3).

UV $\left(\mathrm{CH}_{3} \mathrm{CN}\right): \lambda_{\max }(\lg \varepsilon)=208 \mathrm{~nm}$ (4.4178), 228 (4.2867).

IR (ATR): $\tilde{v}\left(\mathrm{~cm}^{-1}\right)=3286,1725,1538,1342,1276,1164,1079,1057,999,920,719$.

${ }^{1} \mathbf{H}-\mathbf{N M R}\left(300 \mathrm{MHz}, \mathrm{CDCl}_{3}\right): \delta(\mathrm{ppm})=1.45-1.56\left(\mathrm{~m}_{\mathrm{c}}, 2 \mathrm{H}, 3-\mathrm{H}_{2}\right), 1.56-1.72\left(\mathrm{~m}_{\mathrm{c}}, 2 \mathrm{H}\right.$, 4- $\left.\mathrm{H}_{2}\right), 1.79-1.95\left(\mathrm{~m}_{\mathrm{c}}, 2 \mathrm{H}, 2-\mathrm{H}_{2}\right), 2.47,2.63(2 \mathrm{sbr}, 2 \mathrm{H}, 2 \times \mathrm{OH}), 3.43(\mathrm{dd}, J=11.0,7.5 \mathrm{~Hz}$, $\left.1 \mathrm{H}, 6-\mathrm{H}_{\mathrm{A}}\right), 3.64\left(\mathrm{dd}, J=11.0,3.0 \mathrm{~Hz}, 1 \mathrm{H}, 6-\mathrm{H}_{\mathrm{B}}\right), 3.66-3.76\left(\mathrm{~m}_{\mathrm{c}}, 1 \mathrm{H}, 5-\mathrm{H}\right), 4.44(\mathrm{t}, J=$ $\left.6.6 \mathrm{~Hz}, 2 \mathrm{H}, 1-\mathrm{H}_{2}\right), 9.11\left(\mathrm{~d}, J=2.1 \mathrm{~Hz}, 2 \mathrm{H}, 2 \times \mathrm{Ph}-\mathrm{H}_{o}\right), 9.19\left(\mathrm{t}, J=2.1 \mathrm{~Hz}, 1 \mathrm{H}, \mathrm{Ph}-\mathrm{H}_{p}\right)$.

${ }^{13}$ C-NMR $\left(126 \mathrm{MHz}, \mathrm{CDCl}_{3}\right): \delta(\mathrm{ppm})=22.0(\mathrm{C}-3), 28.5(\mathrm{C}-2), 32.5(\mathrm{C}-4), 66.7,66.8(\mathrm{C}-1$, C-6), 71.9 (C-5), $122.3\left(\mathrm{Ph}-\mathrm{C}_{p}\right), 129.4\left(2 \times \mathrm{Ph}-\mathrm{C}_{o}\right), 134.0\left(\mathrm{Ph}-\mathrm{C}_{i}\right), 148.6\left(2 \times \mathrm{Ph}-\mathrm{C}_{m}\right), 162.6$ $(\mathrm{C}=\mathrm{O})$.

MS (ESI): m/z (\%) = $351.1(100)[\mathrm{M}+\mathrm{Na}]^{+}, 679.2(70)[2 \mathrm{M}+\mathrm{Na}]^{+} ; 327.1(100)[\mathrm{M}-\mathrm{H}]^{-}$.

HRMS von $\mathrm{C}_{13} \mathrm{H}_{16} \mathrm{~N}_{2} \mathrm{O}_{6}$ ber.: 327.0834 351.0799 gef.: $\quad 327.0826[\mathrm{M}-\mathrm{H}]^{-}$ $351.0797[\mathrm{M}+\mathrm{Na}]^{+}(\mathrm{ESI}-\mathrm{HRMS})$.

$\mathbf{C}_{13} \mathbf{H}_{16} \mathbf{N}_{2} \mathbf{O}_{8}(328.27)$. 


\subsubsection{0 (S)-6-((tert-Butyldimethylsilyl)oxy)-5-hydroxyhexyl-3,5-dinitrobenzoat (201)}

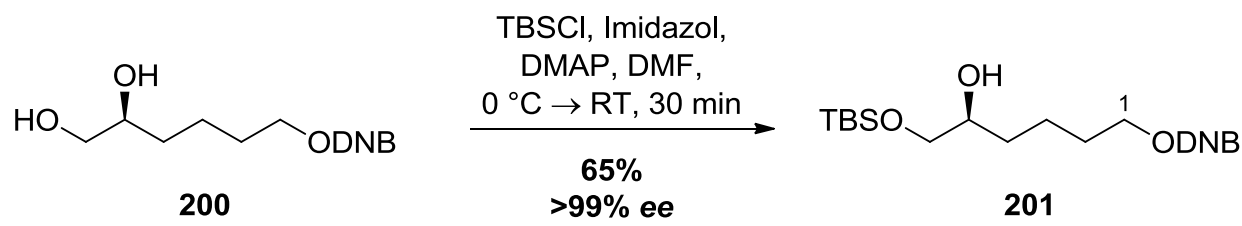

$\mathrm{Zu}$ einer Lösung des Diols 200 (465 mg, $1.42 \mathrm{mmol}, 1.0$ Äq.) in DMF (15 mL) wurde bei $0{ }^{\circ} \mathrm{C}$ Imidazol (145 mg, $2.12 \mathrm{mmol}, 1.5$ Äq.) gegeben und $5 \mathrm{~min}$ gerührt. Anschließend wurden DMAP (17.3 mg, $142 \mu \mathrm{mol}, 10 \mathrm{~mol} \%)$ und TBSCl (213 mg, $1.42 \mathrm{mmol}, 1.0$ Äq.) zugegeben, die Lösung auf Raumtemperatur erwärmt und weitere $30 \mathrm{~min}$ bei dieser Temperatur gerührt. Die Reaktion wurde durch Zugabe von ges. $\mathrm{NaHCO}_{3}$-Lösung $(10 \mathrm{~mL})$ beendet und das Reaktionsgemisch mit $\mathrm{Et}_{2} \mathrm{O}(3 \times 10 \mathrm{~mL})$ extrahiert. Die vereinigten organischen Phasen wurden mit ges. NaCl-Lösung $(20 \mathrm{~mL})$ gewaschen, über $\mathrm{Na}_{2} \mathrm{SO}_{4}$ getrocknet und das Lösungsmittel unter vermindertem Druck entfernt. Säulenchromatographische Reinigung des Rückstandes an Kieselgel (Petrolether/EtOAc 7:1) und anschließende Kristallisation aus $n$ Heptan/EtOAc lieferte die Zielverbindung 201 (411 mg, $929 \mu \mathrm{mol}, 65 \%$ ) in Form von farblosen Kristallen.

DC: $\mathrm{R}_{f}=0.21$ (Petrolether/EtOAc 5:1).

${ }^{1} \mathbf{H}-\mathbf{N M R}\left(300 \mathrm{MHz}, \mathrm{CDCl}_{3}\right): \delta(\mathrm{ppm})=0.04\left(\mathrm{~s}, 6 \mathrm{H}, \mathrm{Si}\left(\mathrm{CH}_{3}\right)_{2}\right), 0.87\left(\mathrm{~s}, 9 \mathrm{H}, \mathrm{SiC}\left(\mathrm{CH}_{3}\right)_{3}\right)$, 1.40-1.55 (m, 3 H, 3- $\left.\mathrm{H}_{2}, 4-\mathrm{H}_{\mathrm{A}}\right), 1.56-1.74\left(\mathrm{~m}, 1 \mathrm{H}, 4-\mathrm{H}_{\mathrm{B}}\right), 1.78-1.92\left(\mathrm{~m}_{\mathrm{c}}, 2 \mathrm{H}, 2-\mathrm{H}_{2}\right), 2.45(\mathrm{~s}$, $1 \mathrm{H}, \mathrm{OH}), 3.38\left(\mathrm{dd}, J=9.8,7.4 \mathrm{~Hz}, 1 \mathrm{H}, 6-\mathrm{H}_{\mathrm{A}}\right), 3.61\left(\mathrm{dd}, J=10.1,2.7 \mathrm{~Hz}, 1 \mathrm{H}, 6-\mathrm{H}_{\mathrm{B}}\right)$, 3.60-3.69 ( $\left.\mathrm{m}_{\mathrm{c}}, 1 \mathrm{H}, 5-\mathrm{H}\right), 4.44\left(\mathrm{t}, J=6.7 \mathrm{~Hz}, 2 \mathrm{H}, 1-\mathrm{H}_{2}\right), 9.12\left(\mathrm{~d}, J=2.1 \mathrm{~Hz}, 2 \mathrm{H}, 2 \times \mathrm{Ph}^{-\mathrm{H}_{o}}\right)$, $9.19\left(\mathrm{t}, J=2.1 \mathrm{~Hz}, 1 \mathrm{H}, \mathrm{Ph}-\mathrm{H}_{p}\right)$.

${ }^{13}$ C-NMR $\left(126 \mathrm{MHz}, \mathrm{CDCl}_{3}\right): \delta(\mathrm{ppm})=-5.5,-5.4\left(\mathrm{Si}\left(\mathrm{CH}_{3}\right)_{2}\right), 18.2\left(\mathrm{SiC}\left(\mathrm{CH}_{3}\right)_{3}\right), 22.1(\mathrm{C}-3)$, $25.8\left(\mathrm{SiC}\left(\underline{\mathrm{CH}}_{3}\right)_{3}\right), 28.6$ (C-2), 32.2 (C-4), 66.9 (C-1), 67.2 (C-6), 71.5 (C-5), $122.3\left(\mathrm{Ph}-\mathrm{C}_{p}\right)$, $129.4\left(2 \times \mathrm{Ph}^{-\mathrm{C}_{o}}\right), 134.1\left(\mathrm{Ph}-\mathrm{C}_{i}\right), 148.6\left(2 \times \mathrm{Ph}-\mathrm{C}_{m}\right), 162.5(\mathrm{C}=\mathrm{O})$.

MS (ESI): m/z (\%) = $465.2(100)[\mathrm{M}+\mathrm{Na}]^{+}, 907.4(93)[2 \mathrm{M}+\mathrm{Na}]^{+} ; 441.4(100)[\mathrm{M}-\mathrm{H}]^{-}$.

HRMS von $\mathrm{C}_{19} \mathrm{H}_{30} \mathrm{~N}_{2} \mathrm{O}_{8} \mathrm{Si}$ ber.: 465.1664 gef.: $465.1662[\mathrm{M}+\mathrm{Na}]^{+}(\mathrm{ESI}-\mathrm{HRMS})$.

$\mathrm{C}_{19} \mathrm{H}_{30} \mathrm{~N}_{2} \mathrm{O}_{8} \mathrm{Si}$ (442.54). 


\subsubsection{1 (S)-6-((tert-Butyldimethylsilyl)oxy)-5-(2,3,4,6-tetradesoxy-4-azido- $\alpha / \beta$-D-gluco-} pyranosyl)hexyl-3,5-dinitrobenzoat $((\alpha / \beta)-202)$

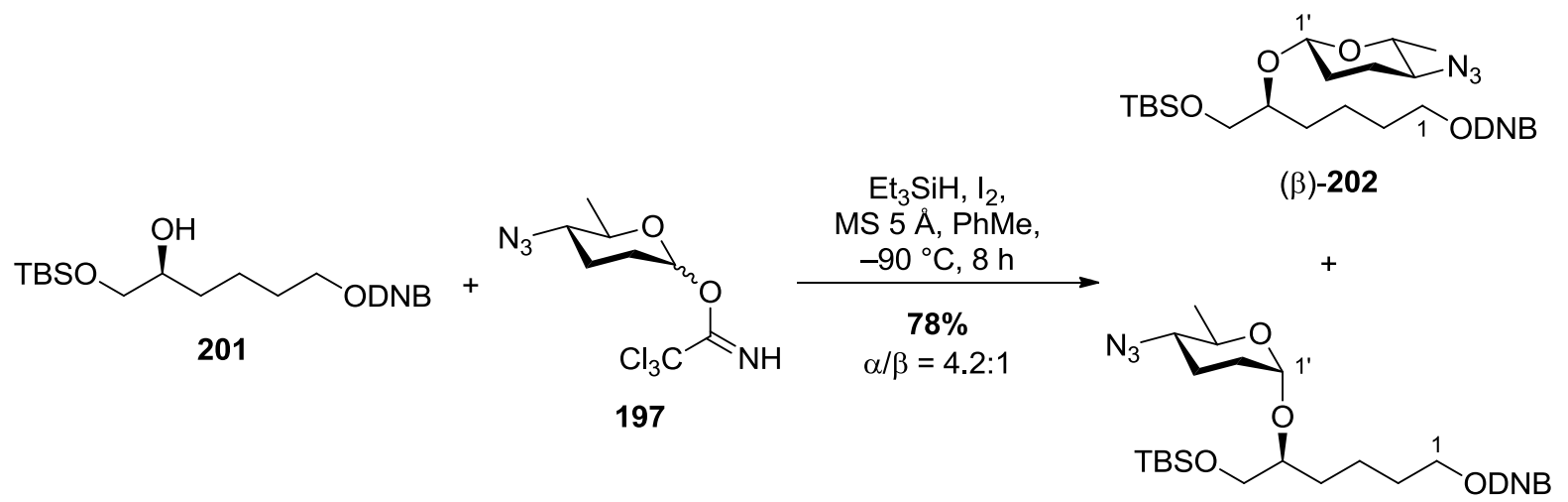

$(\alpha)-202$

Eine Lösung aus Alkohol 201 (225 mg, 508 mol, 1.0 Äq.) und frisch hergestelltem Azid 197 (230 mg, $763 \mu \mathrm{mol}, 1.5$ Äq.) in Toluol (40 mL) wurde bei Raumtemperatur 30 min über fein gemörsertem, aktiviertem $5 \AA$ Molekularsieb gerührt. Anschließend wurde die Reaktionslösung auf $-90{ }^{\circ} \mathrm{C}$ gekühlt, langsam $\mathrm{Et}_{3} \mathrm{SiH}(152 \mu \mathrm{L}, 111 \mathrm{mg}, 956 \mu \mathrm{mol}, 1.9$ Äq.) und eine Lösung aus $\mathrm{I}_{2}$ (194 mg, $763 \mu \mathrm{mol}, 1.5$ Äq.) in Toluol (35 mL) zugegeben und $8 \mathrm{~h}$ bei dieser Temperatur gerührt. Die Reaktion wurde durch Zugabe von $\mathrm{NEt}_{3}$ (3 mL) beendet und ges. $\mathrm{NaHCO}_{3}$-Lösung (15 mL) sowie ges. $\mathrm{Na}_{2} \mathrm{~S}_{2} \mathrm{O}_{3}$-Lösung $(15 \mathrm{~mL})$ zugegeben. Es wurde mit $\mathrm{CH}_{2} \mathrm{Cl}_{2}(3 \times 15 \mathrm{~mL})$ extrahiert, die vereinigten organischen Phasen mit ges. NaCl-Lösung (30 mL) gewaschen und über $\mathrm{Na}_{2} \mathrm{SO}_{4}$ getrocknet. Säulenchromatographische Reinigung an Kieselgel (Petrolether/EtOAc 10:1 $\rightarrow 1: 5$ ) lieferte die Zielverbindung ( $\beta$ )-202 (44.9 mg, $77.2 \mu \mathrm{mol}, 15 \%)$ und das Anomer $(\alpha)$-202 (186 mg, $320 \mu \mathrm{mol}, 63 \%)$ jeweils in Form eines farblosen Öls.

Analytische Daten für $(\alpha)-202$ :

DC: $\mathrm{R}_{f}=0.18$ (Petrolether/EtOAc 7:1).

${ }^{1} \mathbf{H}-\mathbf{N M R}\left(600 \mathrm{MHz}, \mathrm{CDCl}_{3}\right): \delta(\mathrm{ppm})=0.05\left(\mathrm{~s}, 6 \mathrm{H}, \mathrm{Si}\left(\mathrm{CH}_{3}\right)_{2}\right), 0.87\left(\mathrm{~s}, 9 \mathrm{H}, \mathrm{SiC}\left(\mathrm{CH}_{3}\right)_{3}\right)$, $1.21\left(\mathrm{~d}, J=6.3 \mathrm{~Hz}, 3 \mathrm{H}, 6{ }^{\prime}-\mathrm{H}_{3}\right), 1.39-1.57$ (m, 4 H, 3- $\left.\mathrm{H}_{2}, 4-\mathrm{H}_{2}\right), 1.58-1.80$ (m, $\left.2 \mathrm{H}, 2^{\prime}-\mathrm{H}_{2}\right)$, 1.79-1.95 (m, 2 H, 2-H $), 1.94-2.02$ (m, 2 H, 3'- H $\left._{2}\right), 2.91-3.04$ (m, 1 H, 4'-H), 3.39 (dd, $J=$ 9.7, $\left.2.3 \mathrm{~Hz}, 1 \mathrm{H}, 6-\mathrm{H}_{\mathrm{A}}\right), 3.61\left(\mathrm{dd}, J=9.7,3.4 \mathrm{~Hz}, 1 \mathrm{H}, 6-\mathrm{H}_{\mathrm{B}}\right), 3.61-3.69$ (m, $\left.1 \mathrm{H}, 5-\mathrm{H}\right), 3.86$ (dq, $\left.J=9.8,6.2 \mathrm{~Hz}, 1 \mathrm{H}, 5^{\prime}-\mathrm{H}\right), 4.44\left(\mathrm{t}, J=6.7 \mathrm{~Hz}, 2 \mathrm{H}, 1-\mathrm{H}_{2}\right), 5.21\left(\mathrm{~d}, J=3.1 \mathrm{~Hz}, 1 \mathrm{H}, 1^{\prime}-\mathrm{H}\right)$, $9.13\left(\mathrm{~d}, J=2.2 \mathrm{~Hz}, 2 \mathrm{H}, 2 \times \mathrm{Ph}-\mathrm{H}_{o}\right), 9.20\left(\mathrm{t}, J=2.2 \mathrm{~Hz}, 1 \mathrm{H}, \mathrm{Ph}-\mathrm{H}_{p}\right)$.

${ }^{13}$ C-NMR $\left.\left(126 \mathrm{MHz}, \mathrm{CDCl}_{3}\right): \delta(\mathrm{ppm})=-5.4,-5.4\left(\mathrm{Si}_{(\mathrm{CH}}\right)_{2}\right), 18.3\left(\mathrm{SiC}\left(\mathrm{CH}_{3}\right)_{3}\right), 18.7(\mathrm{C}-6$ ' $)$, 22.1 (C-3), $23.0\left(\mathrm{C}-3^{\prime}\right), 25.8\left(\mathrm{SiC}\left(\mathrm{CH}_{3}\right)_{3}\right), 28.6$ (C-2), 29.2 (C-4), $32.2\left(\mathrm{C}-2^{\prime}\right), 62.6\left(\mathrm{C}-4^{\prime}\right), 66.9$ 
(C-1), 67.2 (C-6), 67.4 (C-5'), $71.5(\mathrm{C}-5), 90.7\left(\mathrm{C}-1^{\prime}\right), 122.3\left(\mathrm{Ph}^{-\mathrm{C}_{p}}\right), 129.4\left(2 \times \mathrm{Ph}-\mathrm{C}_{o}\right), 134.1$ $\left(\mathrm{Ph}-\mathrm{C}_{i}\right), 148.6\left(2 \times \mathrm{Ph}-\mathrm{C}_{m}\right), 162.5(\mathrm{C}=\mathrm{O})$.

MS (ESI): $\mathrm{m} / \mathrm{z}(\%)=604.2(100)[\mathrm{M}+\mathrm{Na}]^{+}$.

HRMS von $\mathrm{C}_{25} \mathrm{H}_{39} \mathrm{~N}_{5} \mathrm{O}_{9} \mathrm{Si}$ ber.: $\quad 604.2409$ gef.: $\quad 604.2411[\mathrm{M}+\mathrm{Na}]^{+}(\mathrm{ESI}-\mathrm{HRMS})$.

$\mathrm{C}_{25} \mathrm{H}_{39} \mathrm{~N}_{5} \mathrm{O}_{9} \mathrm{Si}$ (581.69).

Analytische Daten für $(\beta)-202$ :

DC: $\mathrm{R}_{f}=0.33$ (Petrolether/EtOAc 7:1).

${ }^{1} \mathbf{H}-\mathbf{N M R}\left(300 \mathrm{MHz}, \mathrm{CDCl}_{3}\right): \delta(\mathrm{ppm})=0.05\left(\mathrm{~s}, 6 \mathrm{H}, \mathrm{Si}\left(\mathrm{CH}_{3}\right)_{2}\right), 0.87\left(\mathrm{~s}, 9 \mathrm{H}, \mathrm{SiC}\left(\mathrm{CH}_{3}\right)_{3}\right)$, $1.29\left(\mathrm{~d}, J=6.2 \mathrm{~Hz}, 3 \mathrm{H}, 6^{\prime}-\mathrm{H}_{3}\right), 1.39-1.57$ (m, $\left.4 \mathrm{H}, 3-\mathrm{H}_{2}, 4-\mathrm{H}_{2}\right), 1.58-1.80$ (m, $\left.2 \mathrm{H}, 2^{\prime}-\mathrm{H}_{2}\right)$, 1.79-1.95 (m, 2 H, 2-H $)$, 2.11-2.21 (m, 2 H, 3'- $\left.\mathrm{H}_{2}\right), 2.91-3.04$ (m, $1 \mathrm{H}, 4$ '-H), 3.35 (dq, $J=$ 9.6, 6.2 Hz, $1 \mathrm{H}, 5 '-\mathrm{H}), 3.39\left(\mathrm{dd}, J=9.7,2.3 \mathrm{~Hz}, 1 \mathrm{H}, 6-\mathrm{H}_{\mathrm{A}}\right), 3.61(\mathrm{dd}, J=9.7,3.4 \mathrm{~Hz}, 1 \mathrm{H}$, 6- $\left.\mathrm{H}_{\mathrm{B}}\right), 3.61-3.69(\mathrm{~m}, 1 \mathrm{H}, 5-\mathrm{H}), 4.44\left(\mathrm{t}, J=6.7 \mathrm{~Hz}, 2 \mathrm{H}, 1-\mathrm{H}_{2}\right), 4.75(\mathrm{~d}, J=8.9 \mathrm{~Hz}, 1 \mathrm{H}$, 1'-H), 9.13 (d, $\left.J=2.2 \mathrm{~Hz}, 2 \mathrm{H}, 2 \times \mathrm{Ph}-\mathrm{H}_{o}\right), 9.20\left(\mathrm{t}, J=2.2 \mathrm{~Hz}, 1 \mathrm{H}, \mathrm{Ph}-\mathrm{H}_{p}\right)$.

${ }^{13}$ C-NMR $\left(126 \mathrm{MHz}, \mathrm{CDCl}_{3}\right): \delta(\mathrm{ppm})=-5.4,-5.4\left(\mathrm{Si}\left(\mathrm{CH}_{3}\right)_{2}\right), 18.3\left(\mathrm{SiC}\left(\mathrm{CH}_{3}\right)_{3}\right), 18.7(\mathrm{C}-6$ '), 22.1 (C-3), $25.8\left(\mathrm{SiC}\left(\underline{\mathrm{CH}}_{3}\right)_{3}\right), 27.6$ (C-3'), 28.6 (C-2), 29.2 (C-4), 31.7 (C-2'), $61.8\left(\mathrm{C}-4^{\prime}\right), 66.9$ (C-1), 67.2 (C-6), 71.5 (C-5), 74.5 (C-5'), $95.6\left(\mathrm{C}-1^{\prime}\right), 122.3\left(\mathrm{Ph}^{-\mathrm{C}_{p}}\right), 129.4\left(2 \times \mathrm{Ph}-\mathrm{C}_{o}\right), 134.1$ $\left(\mathrm{Ph}-\mathrm{C}_{i}\right), 148.6\left(2 \times \mathrm{Ph}-\mathrm{C}_{m}\right), 162.5(\mathrm{C}=\mathrm{O})$.

MS (ESI): $\mathrm{m} / \mathrm{z}(\%)=604.2(100)[\mathrm{M}+\mathrm{Na}]^{+}$.

HRMS von $\mathrm{C}_{25} \mathrm{H}_{39} \mathrm{~N}_{5} \mathrm{O}_{9} \mathrm{Si}$ ber.: 604.2409 gef.: $604.2407[\mathrm{M}+\mathrm{Na}]^{+}(\mathrm{ESI}-\mathrm{HRMS})$.

$\mathrm{C}_{25} \mathrm{H}_{39} \mathrm{~N}_{5} \mathrm{O}_{9} \mathrm{Si}$ (581.69). 


\subsubsection{6-((2-Methoxyethoxy)methoxy)hex-1-en (206)}

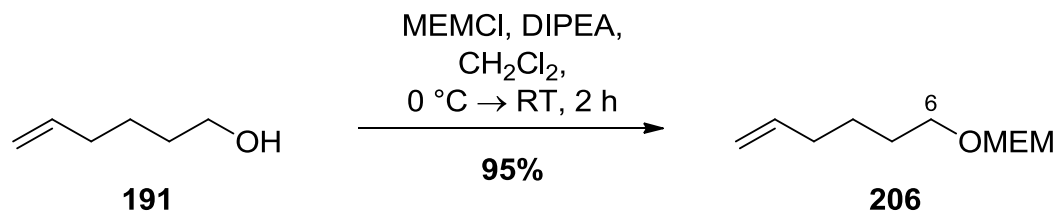

$\mathrm{Zu}$ einer Lösung des Alkohols 191 (20.0 g, 200 mmol, 1.0 Äq.) in $\mathrm{CH}_{2} \mathrm{Cl}_{2}$ (100 mL) wurde bei $0{ }^{\circ} \mathrm{C}$ DIPEA $(52.1 \mathrm{~mL}, 38.7 \mathrm{~g}, 300 \mathrm{mmol}, 1.5$ Äq.) gegeben und $5 \mathrm{~min}$ gerührt. Anschließend wurde langsam MEMCl (34.2 mL, 37.3 g, 300 mmol, 1.5 Äq.) zugegeben, langsam auf Raumtemperatur erwärmt und weitere $2 \mathrm{~h}$ gerührt. Die Reaktion wurde durch Zugabe von ges. $\mathrm{NaHCO}_{3}$-Lösung $(100 \mathrm{~mL})$ beendet und die Reaktionsmischung mit $\mathrm{CH}_{2} \mathrm{Cl}_{2}$ $(3 \times 100 \mathrm{~mL})$ extrahiert. Die vereinigten organischen Phasen wurden mit ges. NaCl-Lösung $(150 \mathrm{~mL})$ gewaschen, über $\mathrm{Na}_{2} \mathrm{SO}_{4}$ getrocknet und das Lösungsmittel unter vermindertem Druck entfernt. Säulenchromatographische Reinigung des Rückstandes an Kieselgel ( $n$ Pentan/EtOAc 20:1 $\rightarrow$ 10:1) lieferte die Zielverbindung 206 (35.9 g, $191 \mathrm{mmol}, 95 \%)$ in Form einer farblosen Flüssigkeit.

DC: $\mathrm{R}_{f}=0.52($ Petrolether/EtOAc 10:1).

UV $\left(\mathrm{CH}_{3} \mathrm{CN}\right)$ : keine Absorption.

IR (ATR): $\tilde{v}\left(\mathrm{~cm}^{-1}\right)=2928,2873,1640,1456,1114,1094,1042,908,850,637$.

${ }^{1} \mathbf{H}-\mathbf{N M R}\left(300 \mathrm{MHz}, \mathrm{CDCl}_{3}\right): \delta(\mathrm{ppm})=1.35-1.49\left(\mathrm{~m}_{\mathrm{c}}, 2 \mathrm{H}, 4-\mathrm{H}_{2}\right), 1.50-1.63\left(\mathrm{~m}_{\mathrm{c}}, 2 \mathrm{H}\right.$, 5- $\left.\mathrm{H}_{2}\right), 2.04\left(\mathrm{q}, J=7.1 \mathrm{~Hz}, 2 \mathrm{H}, 3-\mathrm{H}_{2}\right), 3.35\left(\mathrm{~s}, 3 \mathrm{H}, \mathrm{OCH}_{3}\right), 3.51\left(\mathrm{t}, J=6.7 \mathrm{~Hz}, 2 \mathrm{H}, 6-\mathrm{H}_{2}\right)$, 3.50-3.55 (m, $\left.2 \mathrm{H}, \mathrm{OCH}_{2} \mathrm{CH}_{2} \mathrm{OMe}\right), 3.62-3.67\left(\mathrm{~m}, 2 \mathrm{H}, \mathrm{OCH}_{2} \mathrm{CH}_{2} \mathrm{OMe}\right), 4.67(\mathrm{~s}, 2 \mathrm{H}$, $\left.\mathrm{OCH}_{2} \mathrm{O}\right), 4.89$ (dt, $\left.J=1.9,1.2 \mathrm{~Hz}, 0.5 \mathrm{H}, 1-\mathrm{H}_{\mathrm{A} 1}\right), 4.90-4.95\left(\mathrm{~m}, 1 \mathrm{H}, 1-\mathrm{H}_{\mathrm{A} 2}, 1-\mathrm{H}_{\mathrm{B} 1}\right), 4.99$ (dt, $\left.J=10.3,2.0 \mathrm{~Hz}, 0.5 \mathrm{H}, 1-\mathrm{H}_{\mathrm{B} 2}\right), 5.76(\mathrm{ddt}, J=16.9,10.3,6.7 \mathrm{~Hz}, 1 \mathrm{H}, 2-\mathrm{H})$.

${ }^{13}$ C-NMR $\left(126 \mathrm{MHz}, \mathrm{CDCl}_{3}\right): \delta(\mathrm{ppm})=25.4(\mathrm{C}-4), 29.1(\mathrm{C}-5), 33.4(\mathrm{C}-3), 58.9\left(\mathrm{OCH}_{3}\right)$, $66.6\left(\mathrm{O}_{\mathrm{CH}} \mathrm{CH}_{2} \mathrm{OMe}\right), 67.6(\mathrm{C}-6), 71.8\left(\mathrm{OCH}_{2} \underline{\mathrm{CH}}_{2} \mathrm{OMe}\right), 95.4\left(\mathrm{OCH}_{2} \mathrm{O}\right), 114.5(\mathrm{C}-1), 138.6$ (C-2).

MS (ESI): $\mathrm{m} / \mathrm{z}(\%)=211.1(100)[\mathrm{M}+\mathrm{Na}]^{+}, 399.3(10)[2 \mathrm{M}+\mathrm{Na}]^{+}$.

HRMS von $\mathrm{C}_{10} \mathrm{H}_{20} \mathrm{O}_{3}$ ber.: 211.1305 gef.: $\quad 211.1307[\mathrm{M}+\mathrm{Na}]^{+}(\mathrm{ESI}-\mathrm{HRMS})$.

$\mathbf{C}_{10} \mathbf{H}_{20} \mathbf{O}_{3}$ (188.26). 


\subsubsection{3 (S)-6-((2-Methoxyethoxy)methoxy)hexan-1,2-diol (207)}

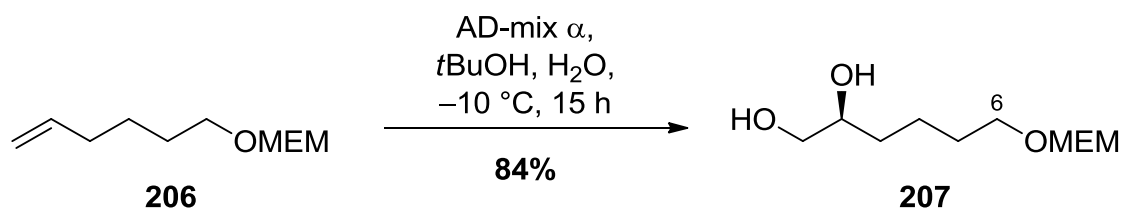

$\mathrm{Zu}$ einer Lösung des Alkens 206 (10.0 g, $53.1 \mathrm{mmol}, 1.0$ Äq.) in $t \mathrm{BuOH} / \mathrm{H}_{2} \mathrm{O}(1: 1,30 \mathrm{~mL})$ wurde bei $-10{ }^{\circ} \mathrm{C}$ portionsweise AD-mix $\alpha(74.4 \mathrm{~g}, 1.4 \mathrm{~g} / \mathrm{mmol})$ gegeben und $15 \mathrm{~h}$ bei dieser Temperatur gerührt. Die Reaktion wurde durch Zugabe von ges. $\mathrm{NaHCO}_{3}$-Lösung $(10 \mathrm{~mL})$ und ges. $\mathrm{Na}_{2} \mathrm{~S}_{2} \mathrm{O}_{3}$-Lösung $(10 \mathrm{~mL})$ beendet und die Reaktionsmischung mit $\mathrm{Et}_{2} \mathrm{O}(3 \times 30 \mathrm{~mL})$ extrahiert. Die vereinigten organischen Phasen wurden mit ges. NaCl-Lösung $(50 \mathrm{~mL})$ gewaschen, über $\mathrm{Na}_{2} \mathrm{SO}_{4}$ getrocknet und das Lösungsmittel unter vermindertem Druck entfernt. Säulenchromatographische Reinigung des Rückstandes an Kieselgel (Petrolether/EtOAc 1:2 $\rightarrow$ 1:5) lieferte die Zielverbindung 207 (9.95 g, 44.8 mmol, 84\%, $>99 \% e e$ ) in Form eines farblosen Öls.

DC: $\mathrm{R}_{f}=0.38$ (EtOAc).

Drehwert: $[\alpha]_{\mathrm{D}}^{20}=-2.2^{\circ}\left(\mathrm{c}=1.1, \mathrm{CHCl}_{3}\right)$.

UV $\left(\mathrm{CH}_{3} \mathrm{CN}\right)$ : keine Absorption.

IR (ATR): $\tilde{v}\left(\mathrm{~cm}^{-1}\right)=2931,2870,1651,1456,1111,1093,1039,1021,931,848$.

${ }^{1} \mathbf{H}-\mathbf{N M R}\left(300 \mathrm{MHz}, \mathrm{CDCl}_{3}\right): \delta(\mathrm{ppm})=1.33-1.47\left(\mathrm{~m}_{\mathrm{c}}, 4 \mathrm{H}, 3-\mathrm{H}_{2}, 4-\mathrm{H}_{2}\right), 1.48-1.62(\mathrm{~m}, 2 \mathrm{H}$, 5- $\left.\mathrm{H}_{2}\right), 2.86(\mathrm{~s}, 2 \mathrm{H}, 2 \times \mathrm{OH}), 3.34\left(\mathrm{~s}, 3 \mathrm{H}, \mathrm{OCH}_{3}\right), 3.36\left(\mathrm{dd}, J=11.1,7.6 \mathrm{~Hz}, 1 \mathrm{H}, 1-\mathrm{H}_{\mathrm{A}}\right), 3.51$ (t, $\left.J=6.1 \mathrm{~Hz}, 2 \mathrm{H}, 6-\mathrm{H}_{2}\right), 3.48-3.54\left(\mathrm{~m}, 2 \mathrm{H}, \mathrm{OCH}_{2} \mathrm{CH}_{2} \mathrm{OMe}\right), 3.57(\mathrm{dd}, J=11.1,3.1 \mathrm{~Hz}$, $\left.1 \mathrm{H}, 1-\mathrm{H}_{\mathrm{B}}\right), 3.61-3.66\left(\mathrm{~m}, 3 \mathrm{H}, 2-\mathrm{H}, \mathrm{OCH}_{2} \mathrm{CH}_{2} \mathrm{OMe}\right), 4.65\left(\mathrm{~s}, 2 \mathrm{H}, \mathrm{OCH}_{2} \mathrm{O}\right)$.

${ }^{13}$ C-NMR $\left(126 \mathrm{MHz}, \mathrm{CDCl}_{3}\right): \delta(\mathrm{ppm})=22.2(\mathrm{C}-4), 29.5(\mathrm{C}-5), 32.7(\mathrm{C}-3), 58.9\left(\mathrm{OCH}_{3}\right)$, 66.6 (C-1), $66.7\left(\mathrm{OCH}_{2} \mathrm{CH}_{2} \mathrm{OMe}\right), 67.7$ (C-6), $71.7\left(\mathrm{OCH}_{2} \underline{\mathrm{CH}}_{2} \mathrm{OMe}\right), 72.0$ (C-2), 95.4 $\left(\mathrm{OCH}_{2} \mathrm{O}\right)$.

MS (ESI): m/z (\%) = $245.1(100)[\mathrm{M}+\mathrm{Na}]^{+} ; 221.1(77)[\mathrm{M}-\mathrm{H}]^{-}$.

HRMS von $\mathrm{C}_{10} \mathrm{H}_{22} \mathrm{O}_{5} \quad$ ber.: 221.1394 245.1359

gef.: $221.1396[\mathrm{M}-\mathrm{H}]^{-}$

$245.1363[\mathrm{M}+\mathrm{Na}]^{+}(\mathrm{ESI}-\mathrm{HRMS})$.

$\mathrm{C}_{10} \mathrm{H}_{22} \mathrm{O}_{5}$ (222.28). 


\subsubsection{4 (S)-6-((tert-Butyldimethylsilyl)oxy)hexan-1,5-diol (203)}

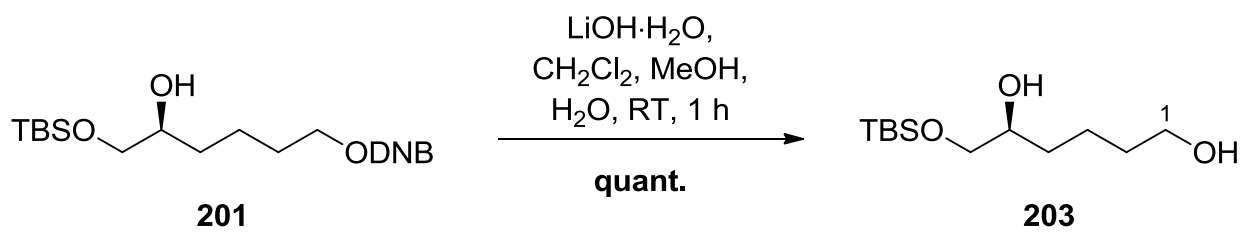

$\mathrm{Zu}$ einer Lösung des Alkohols 201 (100 mg, $226 \mu \mathrm{mol}$, 1.0 Äq.) in $\mathrm{CH}_{2} \mathrm{Cl}_{2} / \mathrm{MeOH} / \mathrm{H}_{2} \mathrm{O}$ (1:1:0.01, $10 \mathrm{~mL})$ wurde bei Raumtemperatur $\mathrm{LiOH} \cdot \mathrm{H}_{2} \mathrm{O}$ (14.2 mg, $339 \mu \mathrm{mol}, 1.5$ Äq.) gegeben und $1 \mathrm{~h}$ bei dieser Temperatur gerührt. Die Reaktion wurde durch Zugabe von $\mathrm{HCl}$ $(2 \mathrm{M}, 5 \mathrm{~mL})$ beendet und die Reaktionsmischung mit $\mathrm{CH}_{2} \mathrm{Cl}_{2}(3 \times 5 \mathrm{~mL})$ extrahiert. Die vereinigten organischen Phasen wurden mit ges. NaCl-Lösung $(10 \mathrm{~mL})$ gewaschen, über $\mathrm{Na}_{2} \mathrm{SO}_{4}$ getrocknet und das Lösungsmittel unter vermindertem Druck entfernt. Säulenchromatographische Reinigung des Rückstandes an Kieselgel (Petrolether/EtOAc 1.5:1) lieferte die Zielverbindung 203 (56.1 mg, $226 \mu \mathrm{mol}$, quant.) in Form einer farblosen Flüssigkeit.

DC: $\mathbf{R}_{f}=0.36$ (Petrolether/EtOAc 1:1).

${ }^{1} \mathbf{H}-\mathbf{N M R}\left(300 \mathrm{MHz}, \mathrm{CDCl}_{3}\right): \delta(\mathrm{ppm})=0.05\left(\mathrm{~s}, 6 \mathrm{H}, \mathrm{Si}\left(\mathrm{CH}_{3}\right)_{2}\right), 0.88\left(\mathrm{~s}, 9 \mathrm{H}, \mathrm{SiC}\left(\mathrm{CH}_{3}\right)_{3}\right)$, 1.20-1.66 (m, $\left.6 \mathrm{H}, 2-\mathrm{H}_{2}, 3-\mathrm{H}_{2}, 4-\mathrm{H}_{2}\right), 3.33-3.41\left(\mathrm{~m}_{\mathrm{c}}, 1 \mathrm{H}, 5-\mathrm{H}\right), 3.52(\mathrm{dd}, J=13.7,4.4 \mathrm{~Hz}$, $\left.1 \mathrm{H}, 6-\mathrm{H}_{\mathrm{A}}\right), 3.56-3.68\left(\mathrm{~m}_{\mathrm{c}}, 3 \mathrm{H}, 1-\mathrm{H}_{2}, 6-\mathrm{H}_{\mathrm{B}}\right)$.

MS (ESI): $\mathrm{m} / \mathrm{z}(\%)=271.2(100)[\mathrm{M}+\mathrm{Na}]^{+}, 519.4(24)[2 \mathrm{M}+\mathrm{Na}]^{+} ; 247.2(100)[\mathrm{M}-\mathrm{H}]^{-}$.

HRMS von $\mathrm{C}_{12} \mathrm{H}_{28} \mathrm{O}_{3} \mathrm{Si}$ ber.: $\quad 247.1735$

271.1700

gef.: $247.1735[\mathrm{M}-\mathrm{H}]^{-}$

$271.1703[\mathrm{M}+\mathrm{Na}]^{+}(\mathrm{ESI}-\mathrm{HRMS})$.

$\mathbf{C}_{12} \mathbf{H}_{28} \mathbf{O}_{3} \mathbf{S i}(248.43)$. 


\subsubsection{5 (S)-6-((tert-Butyldimethylsilyl)oxy)-1-((2-methoxyethoxy)methoxy)hexan-5-ol} (204)

\section{Methode A:}

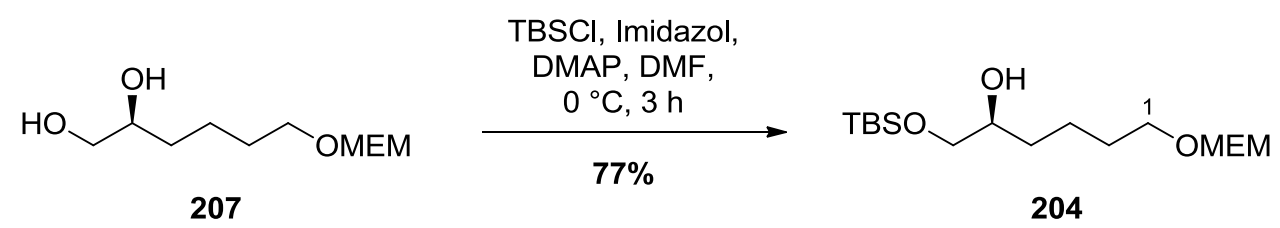

Zu einer Lösung des Diols 207 (9.92 g, 44.6 mmol, 1.0 Äq.) in DMF (150 mL) wurde bei $0{ }^{\circ} \mathrm{C}$ Imidazol (3.19 g, 46:8 mmol, 1.1 Äq.) gegeben und 5 min gerührt. Anschließend wurden DMAP (545 mg, 4.46 mmol, 10 mol\%) und TBSCl (6.73 g, 44.6 mmol, 1.0 Äq.) zugegeben und weitere $3 \mathrm{~h}$ bei dieser Temperatur gerührt. Die Reaktion wurde durch Zugabe von ges. $\mathrm{NaHCO}_{3}$-Lösung $(100 \mathrm{~mL})$ beendet und das Reaktionsgemisch mit $\mathrm{Et}_{2} \mathrm{O}(3 \times 100 \mathrm{~mL})$ extrahiert. Die vereinigten organischen Phasen wurden mit ges. NaCl-Lösung $(200 \mathrm{~mL})$ gewaschen, über $\mathrm{Na}_{2} \mathrm{SO}_{4}$ getrocknet und das Lösungsmittel unter vermindertem Druck entfernt. Säulenchromatographische Reinigung des Rückstandes an Kieselgel (Petrolether/EtOAc 5:1 $\rightarrow 3: 1$ ) lieferte die Zielverbindung 204 (11.6 g, $34.5 \mathrm{mmol}, 77 \%$ ) in Form eines farblosen Öls.

\section{Methode B:}

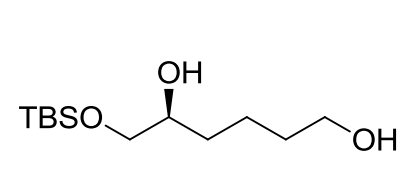

203

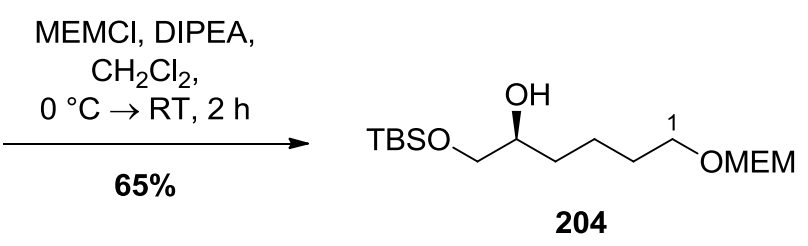

204

Zu einer Lösung des Diols 203 (18.0 mg, $72.5 \mu \mathrm{mol}, 1.0$ Äq.) in $\mathrm{CH}_{2} \mathrm{Cl}_{2}$ (5 mL) wurde bei $0{ }^{\circ} \mathrm{C}$ DIPEA $(15.1 \mu \mathrm{L}, 11.2 \mathrm{mg}, \quad 86.9 \mu \mathrm{mol}, \quad 1.2$ Äq. $)$ gegeben und $5 \mathrm{~min}$ gerührt. Anschließend wurde MEMCl (8.7 $\mu \mathrm{L}, 9.50 \mathrm{mg}, 72.5 \mu \mathrm{mol}, 1.0$ Äq.) zugegeben, die Lösung auf Raumtemperatur erwärmt und weitere $2 \mathrm{~h}$ bei dieser Temperatur gerührt. Die Reaktion wurde durch Zugabe von ges. $\mathrm{NaHCO}_{3}$-Lösung $(5 \mathrm{~mL})$ beendet und das Reaktionsgemisch mit $\mathrm{CH}_{2} \mathrm{Cl}_{2}(3 \times 3 \mathrm{~mL})$ extrahiert. Die vereinigten organischen Phasen wurden mit ges. $\mathrm{NaCl}-$ Lösung $(10 \mathrm{~mL})$ gewaschen, über $\mathrm{Na}_{2} \mathrm{SO}_{4}$ getrocknet und das Lösungsmittel unter vermindertem Druck entfernt. Säulenchromatographische Reinigung des Rückstandes an Kieselgel (Petrolether/EtOAc 3:1) lieferte die Zielverbindung 204 (15.4 mg, 45.8 mol, 65\%) in Form einer farblosen Flüssigkeit. 
DC: $\mathbf{R}_{f}=0.27$ (Petrolether/EtOAc 5:2).

UV $\left(\mathrm{CH}_{3} \mathrm{CN}\right)$ : keine Absorption.

IR: $\tilde{v}\left(\mathrm{~cm}^{-1}\right)=2928,2858,1461,1251,1093,1043,835,776,668$.

${ }^{1} \mathbf{H}-\mathbf{N M R}\left(300 \mathrm{MHz}, \mathrm{CDCl}_{3}\right): \delta(\mathrm{ppm})=0.04\left(\mathrm{~s}, 6 \mathrm{H}, \mathrm{Si}\left(\mathrm{CH}_{3}\right)_{2}\right), 0.87\left(\mathrm{~s}, 9 \mathrm{H}, \mathrm{SiC}\left(\mathrm{CH}_{3}\right)_{3}\right)$, 1.31-1.47 (m $\left.\mathrm{c}, 4 \mathrm{H}, 3-\mathrm{H}_{2}, 4-\mathrm{H}_{2}\right), 1.49-1.65$ (m, $\left.2 \mathrm{H}, 2-\mathrm{H}_{2}\right), 2.34$ (s, $\left.1 \mathrm{H}, \mathrm{OH}\right), 3.36$ (s, $3 \mathrm{H}$, $\left.\mathrm{OCH}_{3}\right), 3.36\left(\mathrm{dd}, J=10.6,8.1 \mathrm{~Hz}, 1 \mathrm{H}, 6-\mathrm{H}_{\mathrm{A}}\right), 3.52\left(\mathrm{t}, J=6.1 \mathrm{~Hz}, 2 \mathrm{H}, 1-\mathrm{H}_{2}\right), 3.50-3.54(\mathrm{~m}$, $\left.2 \mathrm{H}, \mathrm{OCH}_{2} \mathrm{CH}_{2} \mathrm{OMe}\right), 3.58\left(\mathrm{dd}, J=10.6,3.4 \mathrm{~Hz}, 1 \mathrm{H}, 6-\mathrm{H}_{\mathrm{B}}\right), 3.57-3.63(\mathrm{~m}, 1 \mathrm{H}, 5-\mathrm{H})$, 3.63-3.67 (m, $\left.2 \mathrm{H}, \mathrm{OCH}_{2} \mathrm{CH}_{2} \mathrm{OMe}\right), 4.67$ (s, $\left.2 \mathrm{H}, \mathrm{OCH}_{2} \mathrm{O}\right)$.

${ }^{13}$ C-NMR $\left(126 \mathrm{MHz}, \mathrm{CDCl}_{3}\right): \delta(\mathrm{ppm})=-5.4,-5.4\left(\mathrm{Si}\left(\mathrm{CH}_{3}\right)_{2}\right), 18.3\left(\mathrm{Si} \underline{\mathrm{C}}\left(\mathrm{CH}_{3}\right)_{3}\right), 22.2(\mathrm{C}-3)$, $25.8\left(\mathrm{SiC}\left(\mathrm{CH}_{3}\right)_{3}\right), 29.7(\mathrm{C}-2), 32.5(\mathrm{C}-4), 59.0\left(\mathrm{OCH}_{3}\right), 66.7\left(\mathrm{OCH}_{2} \mathrm{CH}_{2} \mathrm{OMe}\right), 67.2(\mathrm{C}-6)$, 67.7 (C-1), $71.7(\mathrm{C}-5), 71.8\left(\mathrm{OCH}_{2} \underline{\mathrm{CH}}_{2} \mathrm{OMe}\right), 95.4\left(\mathrm{OCH}_{2} \mathrm{O}\right)$.

MS (ESI): m/z (\%) = $337.2(21)[\mathrm{M}+\mathrm{H}]^{+}, 359.2(100)[\mathrm{M}+\mathrm{Na}]^{+} ; 335.2(100)[\mathrm{M}-\mathrm{H}]^{-}$.

HRMS von $\mathrm{C}_{16} \mathrm{H}_{36} \mathrm{O}_{5} \mathrm{Si}$ ber.: $\quad 335.2259$ 359.2224

gef.: $\quad 335.2255[\mathrm{M}-\mathrm{H}]^{-}$ $359.2226[\mathrm{M}+\mathrm{Na}]^{+}(\mathrm{ESI}-\mathrm{HRMS})$.

$\mathrm{C}_{16} \mathrm{H}_{36} \mathrm{O}_{5} \mathrm{Si}$ (336.54). 
4.4.16 (S)-6-((tert-Butyldimethylsilyl)oxy)-5-(2,3,4,6-tetradesoxy-4-azido- $\alpha / \beta$-D-glucopyranosyl)-1-((2-methoxyethoxy)methoxy)hexan $((\alpha / \beta)-205)$

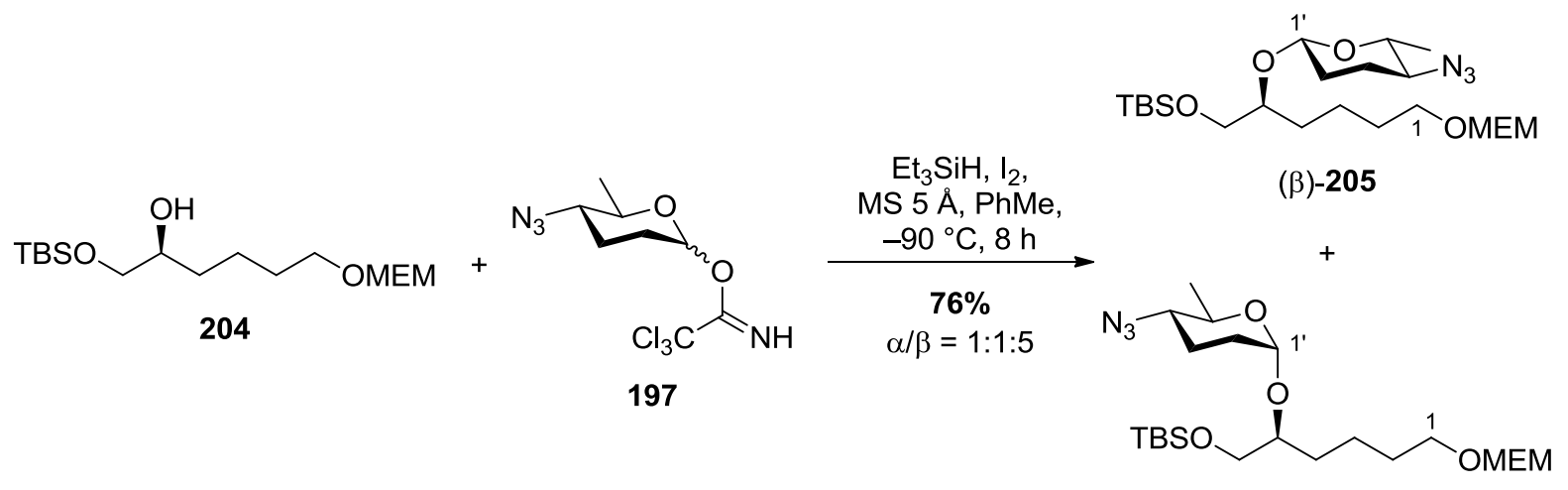

$(\alpha)-205$

Eine Lösung aus Alkohol 204 (15.0 mg, $44.6 \mu \mathrm{mol}, 1.0$ Äq.) und frisch hergestelltem Azid 197 (20.2 mg, 66.9 mol, 1.5 Äq.) in Toluol ( $5 \mathrm{~mL}$ ) wurde bei Raumtemperatur 30 min über fein gemörsertem, aktiviertem $5 \AA$ Molekularsieb gerührt. Anschließend wurde die Reaktionslösung auf $-90^{\circ} \mathrm{C}$ gekühlt, langsam $\mathrm{Et}_{3} \mathrm{SiH}(13.3 \mu \mathrm{L}, 9.72 \mathrm{mg}, 83.8 \mu \mathrm{mol}, 1.9$ Äq.) und eine Lösung aus $\mathrm{I}_{2}(17.0 \mathrm{mg}, 66.9 \mu \mathrm{mol}, 1.5 \mathrm{Äq}$.) in Toluol ( $3 \mathrm{~mL})$ zugegeben und $8 \mathrm{~h}$ bei dieser Temperatur gerührt. Die Reaktion wurde durch Zugabe von $\mathrm{NEt}_{3}(1 \mathrm{~mL})$ beendet und ges. $\mathrm{NaHCO}_{3}$-Lösung $(5 \mathrm{~mL})$ sowie ges. $\mathrm{Na}_{2} \mathrm{~S}_{2} \mathrm{O}_{3}$-Lösung $(5 \mathrm{~mL})$ zugegeben. Es wurde mit $\mathrm{CH}_{2} \mathrm{Cl}_{2}(3 \times 5 \mathrm{~mL})$ extrahiert, die vereinigten organischen Phasen mit ges. NaCl-Lösung $(10 \mathrm{~mL})$ gewaschen und über $\mathrm{Na}_{2} \mathrm{SO}_{4}$ getrocknet. Säulenchromatographische Reinigung an Kieselgel (Petrolether/EtOAc 7:1) lieferte die Zielverbindung ( $\beta$ )-205 (8.70 mg, $18.3 \mu \mathrm{mol}$, 41\%) und das Anomer $(\alpha)$-205 (6.36 mg, $13.4 \mu \mathrm{mol}, 30 \%)$ jeweils in Form eines farblosen Öls.

Analytische Daten für $(\alpha)-\mathbf{2 0 5}$ :

DC: $\mathrm{R}_{f}=0.26$ (Petrolether/EtOAc 5:1).

${ }^{1} \mathbf{H}-\mathbf{N M R}\left(600 \mathrm{MHz}, \mathrm{CDCl}_{3}\right): \delta(\mathrm{ppm})=0.03\left(\mathrm{~s}, 6 \mathrm{H}, \mathrm{Si}\left(\mathrm{CH}_{3}\right)_{2}\right), 0.86\left(\mathrm{~s}, 9 \mathrm{H}, \mathrm{SiC}\left(\mathrm{CH}_{3}\right)_{3}\right)$, $1.18\left(\mathrm{~d}, J=6.2 \mathrm{~Hz}, 3 \mathrm{H}, 6{ }^{\prime}-\mathrm{H}_{3}\right), 1.30-1.40\left(\mathrm{~m}_{\mathrm{c}}, 2 \mathrm{H}, 3-\mathrm{H}_{2}\right), 1.39-1.48\left(\mathrm{~m}_{\mathrm{c}}, 1 \mathrm{H}, 2^{\prime}-\mathrm{H}_{\mathrm{A}}\right)$, 1.51-1.60 (m, $\left.3 \mathrm{H}, 4-\mathrm{H}_{2}, 2^{\prime}-\mathrm{H}_{\mathrm{B}}\right), 1.67-1.75\left(\mathrm{~m}, 1 \mathrm{H}, 2-\mathrm{H}_{\mathrm{A}}\right), 1.81(\mathrm{dt}, J=13.7,2.8 \mathrm{~Hz}, 1 \mathrm{H}$, 2- $\left.\mathrm{H}_{\mathrm{B}}\right), 1.87\left(\mathrm{ddd}, J=11.0,9.8,3.8 \mathrm{~Hz}, 2 \mathrm{H}, 3^{\prime}-\mathrm{H}_{2}\right), 2.87-3.00\left(\mathrm{~m}, 1 \mathrm{H}, 4^{\prime}-\mathrm{H}_{2}\right), 3.36(\mathrm{~s}, 3 \mathrm{H}$, $\left.\mathrm{OCH}_{3}\right), 3.51\left(\mathrm{t}, J=6.4 \mathrm{~Hz}, 2 \mathrm{H}, 1-\mathrm{H}_{2}\right), 3.49-3.54\left(\mathrm{~m}, 3 \mathrm{H}, 6-\mathrm{H}_{\mathrm{A}}, \mathrm{OCH}_{2} \mathrm{CH}_{2} \mathrm{OMe}\right), 3.57$ (quint., $J=5.8 \mathrm{~Hz}, 1 \mathrm{H}, 5-\mathrm{H}), 3.49-3.54\left(\mathrm{~m}, 3 \mathrm{H}, 6-\mathrm{H}_{\mathrm{B}}, \mathrm{OCH}_{2} \mathrm{CH}_{2} \mathrm{OMe}\right), 3.74-3.80\left(\mathrm{~m}_{\mathrm{c}}, 1 \mathrm{H}\right.$, 5'-H), 4.67 (s, $\left.2 \mathrm{H}, \mathrm{OCH}_{2} \mathrm{O}\right), 4.81\left(\mathrm{~d}, J=3.3 \mathrm{~Hz}, 1 \mathrm{H}, 1^{\prime}-\mathrm{H}\right)$. 
${ }^{13}$ C-NMR $\left(126 \mathrm{MHz}, \mathrm{CDCl}_{3}\right): \delta(\mathrm{ppm})=-5.4,-5.4\left(\mathrm{Si}\left(\mathrm{CH}_{3}\right)_{2}\right), 18.3\left(\mathrm{SiC}\left(\mathrm{CH}_{3}\right)_{3}\right), 18.6\left(\mathrm{C}-6{ }^{\prime}\right)$, 21.8 (C-3), 23.7 (C-3'), $25.9\left(\mathrm{SiC}\left(\underline{\mathrm{CH}}_{3}\right)_{3}\right), 29.6$ (C-2), 29.9 (C-4), $30.7\left(\mathrm{C}-2^{\prime}\right), 59.0\left(\mathrm{OCH}_{3}\right)$, 62.7 (C-4'), 65.3 (C-6), $66.7\left(\mathrm{OCH}_{2} \mathrm{CH}_{2} \mathrm{OMe}\right), 67.3$ (C-5'), 67.7 (C-1), $71.8\left(\mathrm{OCH}_{2} \mathrm{C}_{2} \mathrm{OMe}\right)$, $77.6(\mathrm{C}-5), 95.0\left(\mathrm{C}-1^{\prime}\right), 95.4\left(\mathrm{OCH}_{2} \mathrm{O}\right)$.

MS (ESI): m/z (\%) = $493.4(69)\left[\mathrm{M}+\mathrm{NH}_{4}\right]^{+}, 498.3(100)[\mathrm{M}+\mathrm{Na}]^{+}, 514.3(9)[\mathrm{M}+\mathrm{K}]^{+}$.

HRMS von $\mathrm{C}_{22} \mathrm{H}_{45} \mathrm{~N}_{3} \mathrm{O}_{6} \mathrm{Si}$

ber.: $\quad 498.2970$

gef.: $498.2969[\mathrm{M}+\mathrm{Na}]^{+}(\mathrm{ESI}-\mathrm{HRMS})$.

$\mathrm{C}_{22} \mathbf{H}_{45} \mathbf{N}_{3} \mathbf{O}_{6} \mathrm{Si}$ (475.69).

Analytische Daten für $(\beta)-\mathbf{2 0 5}$ :

DC: $\mathbf{R}_{f}=0.36$ (Petrolether/EtOAc 5:1).

${ }^{1} \mathbf{H}-\mathbf{N M R}\left(600 \mathrm{MHz}, \mathrm{CDCl}_{3}\right): \delta(\mathrm{ppm})=0.03\left(\mathrm{~s}, 6 \mathrm{H}, \mathrm{Si}\left(\mathrm{CH}_{3}\right)_{2}\right), 0.86\left(\mathrm{~s}, 9 \mathrm{H}, \mathrm{SiC}\left(\mathrm{CH}_{3}\right)_{3}\right)$, $1.29\left(\mathrm{~d}, J=6.1 \mathrm{~Hz}, 3 \mathrm{H}, 6{ }^{\prime}-\mathrm{H}_{3}\right), 1.30-1.40\left(\mathrm{~m}_{\mathrm{c}}, 2 \mathrm{H}, 3-\mathrm{H}_{2}\right), 1.39-1.48\left(\mathrm{~m}_{\mathrm{c}}, 1 \mathrm{H}, 2^{\prime}-\mathrm{H}_{\mathrm{A}}\right)$, 1.51-1.60 (m, $\left.3 \mathrm{H}, 4-\mathrm{H}_{2}, 2^{\prime}-\mathrm{H}_{\mathrm{B}}\right), 1.67-1.75\left(\mathrm{~m}, 1 \mathrm{H}, 2-\mathrm{H}_{\mathrm{A}}\right), 1.81(\mathrm{dt}, J=13.7,2.8 \mathrm{~Hz}, 1 \mathrm{H}$, 2- $\left.\mathrm{H}_{\mathrm{B}}\right), 2.11-2.20\left(\mathrm{~m}, 2 \mathrm{H}, 3^{\prime}-\mathrm{H}_{2}\right), 2.87-3.00$ (m, $\left.1 \mathrm{H}, 4^{\prime}-\mathrm{H}\right), 3.35$ (dq, J = 9.4, 6.2 Hz, $1 \mathrm{H}, 5^{\prime}-$ $\mathrm{H}), 3.36\left(\mathrm{~s}, 3 \mathrm{H}, \mathrm{OCH}_{3}\right), 3.51\left(\mathrm{t}, J=6.4 \mathrm{~Hz}, 2 \mathrm{H}, 1-\mathrm{H}_{2}\right), 3.49-3.54\left(\mathrm{~m}, 3 \mathrm{H}, 6-\mathrm{H}_{\mathrm{A}}\right.$, $\mathrm{OCH}_{2} \mathrm{CH}_{2} \mathrm{OMe}$ ), 3.57 (quint., $\left.J=5.8 \mathrm{~Hz}, 1 \mathrm{H}, 5-\mathrm{H}\right), 3.49-3.54\left(\mathrm{~m}, 3 \mathrm{H}, 6-\mathrm{H}_{\mathrm{B}}\right.$, $\mathrm{OCH}_{2} \mathrm{CH}_{2} \mathrm{OMe}$ ), 4.67 (s, $\left.2 \mathrm{H}, \mathrm{OCH}_{2} \mathrm{O}\right), 4.75$ (d, $\left.J=8.1 \mathrm{~Hz}, 1 \mathrm{H}, 1^{\prime}-\mathrm{H}\right)$.

${ }^{13} \mathrm{C}-\mathrm{NMR}\left(126 \mathrm{MHz}, \mathrm{CDCl}_{3}\right): \delta(\mathrm{ppm})=-5.4,-5.4\left(\mathrm{Si}\left(\mathrm{CH}_{3}\right)_{2}\right), 18.3\left(\mathrm{SiC}\left(\mathrm{CH}_{3}\right)_{3}\right), 18.6\left(\mathrm{C}-6{ }^{\prime}\right)$, $21.8\left(\mathrm{C}-3{ }^{\prime}\right), 23.0(\mathrm{C}-3), 25.9\left(\mathrm{SiC}\left(\underline{\mathrm{CH}}_{3}\right)_{3}\right), 29.6(\mathrm{C}-2), 29.9(\mathrm{C}-4), 31.7\left(\mathrm{C}-2^{\prime}\right), 59.0\left(\mathrm{OCH}_{3}\right)$,

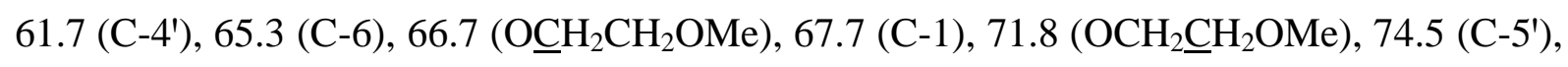
$77.6(\mathrm{C}-5), 95.4\left(\mathrm{OCH}_{2} \mathrm{O}\right), 95.7\left(\mathrm{C}-1^{\prime}\right)$.

MS (ESI): $\mathrm{m} / \mathrm{z}(\%)=493.4(23)\left[\mathrm{M}+\mathrm{NH}_{4}\right]^{+}, 498.3(100)[\mathrm{M}+\mathrm{Na}]^{+}, 514.3(7)[\mathrm{M}+\mathrm{K}]^{+}$.

HRMS von $\mathrm{C}_{22} \mathrm{H}_{45} \mathrm{~N}_{3} \mathrm{O}_{6} \mathrm{Si}$ ber.: $\quad 498.2970$ gef.: $\quad 498.2959[\mathrm{M}+\mathrm{Na}]^{+}(\mathrm{ESI}-\mathrm{HRMS})$.

$\mathrm{C}_{22} \mathrm{H}_{45} \mathbf{N}_{3} \mathrm{O}_{6} \mathrm{Si}$ (475.69). 


\subsubsection{7 (S)-2-(2,3,4,6-Tetradesoxy-4-azido- $\alpha$-D-glucopyranosyl)-6-((2-methoxyethoxy)-} methoxy)hexan-1-ol $((\alpha)-213)$

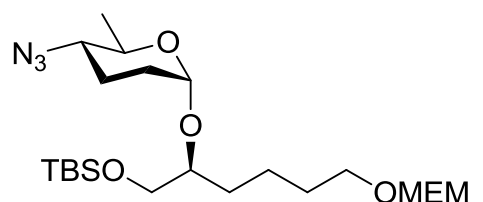

$(\alpha)-205$

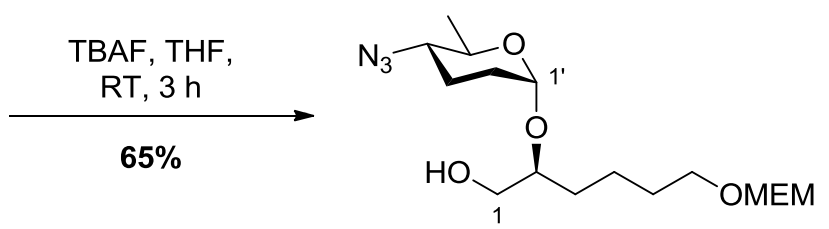

$(\alpha)-213$

$\mathrm{Zu}$ einer Lösung des TBS-Ethers $(\alpha)$-205 (89.0 mg, $187 \mu \mathrm{mol}, 1.0$ Äq.) in THF (15 mL) wurde bei Raumtemperatur TBAF (1 M in THF, $281 \mu \mathrm{L}, 281 \mu \mathrm{mol}, 1.5$ Äq.) gegeben und die resultierende Lösung $3 \mathrm{~h}$ bei dieser Temperatur gerührt. Die Reaktion wurde durch Zugabe von ges. $\mathrm{NaHCO}_{3}$-Lösung $(10 \mathrm{~mL})$ beendet und die Reaktionsmischung mit $\mathrm{Et}_{2} \mathrm{O}(3 \times 10 \mathrm{~mL})$ extrahiert. Die vereinigten organischen Phasen wurden mit ges. NaCl-Lösung $(20 \mathrm{~mL})$ gewaschen, über $\mathrm{Na}_{2} \mathrm{SO}_{4}$ getrocknet und das Lösungsmittel unter vermindertem Druck entfernt. Säulenchromatographische Reinigung an Kieselgel $(n$ Pentan/EtOAc $2: 1 \rightarrow 1: 1)$ lieferte die Zielverbindung ( $\alpha$ )-213 (44.1 mg, $122 \mu \mathrm{mol}, 65 \%)$ in Form einer farblosen Flüssigkeit.

DC: $\mathbf{R}_{f}=0.24(n$ Pentan/EtOAc $1: 1)$.

UV $\left(\mathrm{CH}_{3} \mathrm{CN}\right)$ : keine Absorption.

IR (ATR): $\tilde{v}\left(\mathrm{~cm}^{-1}\right)=2933,2873,2095,1454,1259,1116,1042,990,849$.

${ }^{1} \mathbf{H}-\mathbf{N M R}\left(600 \mathrm{MHz}, \mathrm{CDCl}_{3}\right): \delta(\mathrm{ppm})=1.23\left(\mathrm{~d}, J=6.2 \mathrm{~Hz}, 3 \mathrm{H}, 66^{\prime}-\mathrm{H}_{3}\right), 1.30-1.62(\mathrm{~m}, 6 \mathrm{H}$, 4- $\left.\mathrm{H}_{2}, 3-\mathrm{H}_{2}, 5-\mathrm{H}_{2}\right), 1.65-1.99$ (m, $4 \mathrm{H}, 2^{\prime}-\mathrm{H}_{2}, 3$ '- $\left.\mathrm{H}_{2}\right), 2.96-3.06$ (m, $1 \mathrm{H}, 4$ '-H), 3.36 (s, $3 \mathrm{H}$, $\left.\mathrm{OCH}_{3}\right), 3.51\left(\mathrm{t}, J=6.4 \mathrm{~Hz}, 2 \mathrm{H}, 6-\mathrm{H}_{2}\right), 3.48-3.55\left(\mathrm{~m}, 5 \mathrm{H}, 2-\mathrm{H}, 1-\mathrm{H}_{2}, \mathrm{OCH}_{2} \mathrm{CH}_{2} \mathrm{OMe}\right)$, 3.62-3.68 (m, $\left.2 \mathrm{H}, \mathrm{OCH}_{2} \mathrm{CH}_{2} \mathrm{OMe}\right), 3.76(\mathrm{dq}, J=9.7,6.2 \mathrm{~Hz}, 1 \mathrm{H}, 5 '-\mathrm{H}), 4.67$ (s, $2 \mathrm{H}$, $\left.\mathrm{OCH}_{2} \mathrm{O}\right), 4.83\left(\mathrm{~d}, J=3.3 \mathrm{~Hz}, 1 \mathrm{H}, 1^{\prime}-\mathrm{H}\right)$.

${ }^{13}$ C-NMR $\left(126 \mathrm{MHz}, \mathrm{CDCl}_{3}\right): \delta(\mathrm{ppm})=18.6\left(\mathrm{C}-6\right.$ '), $22.3(\mathrm{C}-4), 23.7\left(\mathrm{C}-3\right.$ '), $29.6\left(\mathrm{C}-2^{\prime}\right), 29.6$ (C-5), 31.1 (C-3), $59.0\left(\mathrm{OCH}_{3}\right), 62.4(\mathrm{C}-4)$ ') 66.3 (C-1), $66.7\left(\mathrm{OCH}_{2} \mathrm{CH}_{2} \mathrm{OMe}\right), 67.6(\mathrm{C}-6)$, 68.5 (C-5'), $71.8\left(\mathrm{OCH}_{2} \underline{\mathrm{CH}}_{2} \mathrm{OMe}\right), 82.1(\mathrm{C}-2), 95.4\left(\mathrm{OCH}_{2} \mathrm{O}\right), 96.7\left(\mathrm{C}-1^{\prime}\right)$.

MS (ESI): $\mathrm{m} / \mathrm{z}(\%)=362.2(5)[\mathrm{M}+\mathrm{H}]^{+}, 379.3(59)\left[\mathrm{M}+\mathrm{NH}_{4}\right]^{+}, 384.2(100)[\mathrm{M}+\mathrm{Na}]^{+} ; 360.2$ (9) $[\mathrm{M}-\mathrm{H}]^{-}$.

HRMS von $\mathrm{C}_{16} \mathrm{H}_{31} \mathrm{~N}_{3} \mathrm{O}_{6}$

$\mathbf{C}_{16} \mathrm{H}_{31} \mathbf{N}_{3} \mathbf{O}_{6}(361.43)$. ber.: $\quad 384.2105$

gef.: $384.2106[\mathrm{M}+\mathrm{Na}]^{+}$(ESI-HRMS). 


\subsubsection{8 (S)-2-(2,3,4,6-Tetradesoxy-4-azido- $\beta$-D-glucopyranosyl)-6-((2-methoxyethoxy)-} methoxy)hexan-1-ol $((\beta)-213)$

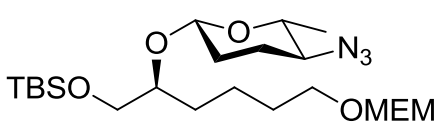

$(\beta)-205$

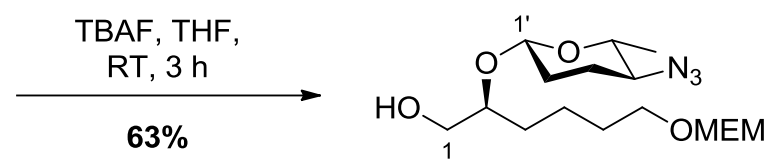

$(\beta)-213$

Zu einer Lösung des TBS-Ethers ( $\beta$ )-205 (91.0 mg, $191 \mu \mathrm{mol}, 1.0$ Äq.) in THF (15 mL) wurde bei Raumtemperatur TBAF (1 M in THF, $287 \mu \mathrm{L}, 287 \mu \mathrm{mol}, 1.5$ Äq.) gegeben und die resultierende Lösung $3 \mathrm{~h}$ bei dieser Temperatur gerührt. Die Reaktion wurde durch Zugabe von ges. $\mathrm{NaHCO}_{3}$-Lösung $(10 \mathrm{~mL})$ beendet und die Reaktionsmischung mit $\mathrm{Et}_{2} \mathrm{O}(3 \times 10 \mathrm{~mL})$ extrahiert. Die vereinigten organischen Phasen wurden mit ges. NaCl-Lösung (20 mL) gewaschen, über $\mathrm{Na}_{2} \mathrm{SO}_{4}$ getrocknet und das Lösungsmittel unter vermindertem Druck entfernt. Säulenchromatographische Reinigung an Kieselgel ( $n$ Pentan/EtOAc $2: 1 \rightarrow 1: 1)$ lieferte die Zielverbindung ( $\beta$ )-213 (43.5 mg, $120 \mu \mathrm{mol}, 63 \%)$ in Form einer farblosen Flüssigkeit.

DC: $\mathbf{R}_{f}=0.28(n$ Pentan/EtOAc 1:1).

UV $\left(\mathrm{CH}_{3} \mathrm{CN}\right)$ : keine Absorption.

IR (ATR): $\tilde{v}\left(\mathrm{~cm}^{-1}\right)=2933,2873,2095,1454,1259,1116,1042,990,849$.

${ }^{1} \mathbf{H}-\mathbf{N M R}\left(600 \mathrm{MHz}, \mathrm{CDCl}_{3}\right): \delta(\mathrm{ppm})=1.28\left(\mathrm{~d}, J=6.2 \mathrm{~Hz}, 3 \mathrm{H}, 6{ }^{\prime}-\mathrm{H}_{3}\right), 1.30-1.62(\mathrm{~m}, 6 \mathrm{H}$, 4- $\left.\mathrm{H}_{2}, 3-\mathrm{H}_{2}, 5-\mathrm{H}_{2}\right), 1.65-1.99\left(\mathrm{~m}, 2 \mathrm{H}, 2^{\prime}-\mathrm{H}_{2}\right), 2.08-2.18$ (m, $\left.2 \mathrm{H}, 3^{\prime}-\mathrm{H}_{2}\right), 2.96-3.06$ (m, $1 \mathrm{H}$, 4'-H), $3.36\left(\mathrm{~s}, 3 \mathrm{H}, \mathrm{OCH}_{3}\right), 3.51\left(\mathrm{t}, J=6.4 \mathrm{~Hz}, 2 \mathrm{H}, 6-\mathrm{H}_{2}\right), 3.42-3.56\left(\mathrm{~m}, 5 \mathrm{H}, 2-\mathrm{H}, 1-\mathrm{H}_{2}\right.$, $\mathrm{OCH}_{2} \mathrm{CH}_{2} \mathrm{OMe}$ ), 3.62-3.68 (m, $2 \mathrm{H}, \mathrm{OCH}_{2} \mathrm{CH}_{2} \mathrm{OMe}$ ), 3.76 (dq, $J=9.7,6.2 \mathrm{~Hz}, 1 \mathrm{H}, 5^{\prime}-\mathrm{H}$ ), 4.67 (s, $\left.2 \mathrm{H}, \mathrm{OCH}_{2} \mathrm{O}\right), 4.77$ (d, $\left.J=9.2 \mathrm{~Hz}, 1 \mathrm{H}, 1^{\prime}-\mathrm{H}\right)$.

${ }^{13}$ C-NMR $\left(126 \mathrm{MHz}, \mathrm{CDCl}_{3}\right): \delta(\mathrm{ppm})=18.6\left(\mathrm{C}^{\prime} 6^{\prime}\right), 21.9\left(\mathrm{C}-3^{\prime}\right), 22.3(\mathrm{C}-4), 29.6\left(\mathrm{C}-2^{\prime}\right), 29.6$ (C-5), 31.9 (C-3), $59.0\left(\mathrm{OCH}_{3}\right), 61.9(\mathrm{C}-4)$ ') 66.3 (C-1), $66.7\left(\mathrm{OCH}_{2} \mathrm{CH}_{2} \mathrm{OMe}\right), 67.6(\mathrm{C}-6)$, $71.8\left(\mathrm{OCH}_{2} \underline{\mathrm{CH}}_{2} \mathrm{OMe}\right), 74.8\left(\mathrm{C}-5{ }^{\prime}\right), 82.1(\mathrm{C}-2), 95.4\left(\mathrm{OCH}_{2} \mathrm{O}\right), 97.3\left(\mathrm{C}-1^{\prime}\right)$.

MS (ESI): $\mathrm{m} / \mathrm{z}(\%)=362.2(7)[\mathrm{M}+\mathrm{H}]^{+}, 379.3(53)\left[\mathrm{M}+\mathrm{NH}_{4}\right]^{+}, 384.2(100)[\mathrm{M}+\mathrm{Na}]^{+} ; 360.2$ (8) $[\mathrm{M}-\mathrm{H}]^{-}$.

HRMS von $\mathrm{C}_{16} \mathrm{H}_{31} \mathrm{~N}_{3} \mathrm{O}_{6}$

$\mathbf{C}_{16} \mathbf{H}_{31} \mathbf{N}_{3} \mathbf{O}_{6}$ (361.43). ber.: $\quad 384.2105$

gef.: $384.2103[\mathrm{M}+\mathrm{Na}]^{+}$(ESI-HRMS). 


\subsubsection{9 (S)-6-Brom-5-(2,3,4,6-tetradesoxy-4-azido- $\alpha$-D-glucopyranosyl)-1-((2-methoxy-} ethoxy)methoxy)hexan $((\alpha)-214)$

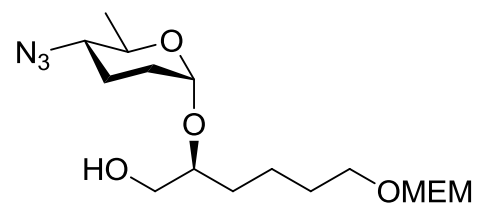

$(\alpha)-213$

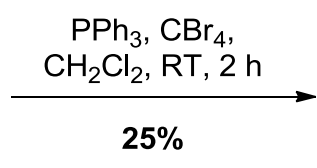

$25 \%$

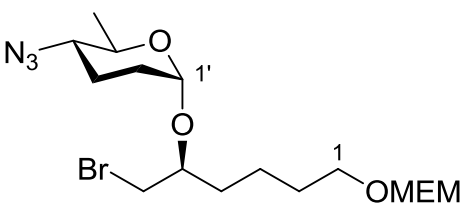

$(\alpha)-214$

$\mathrm{Zu}$ einer Lösung des Alkohols $(\alpha)-213\left(12.0 \mathrm{mg}, 33.2 \mu \mathrm{mol}, 1.0\right.$ Äq.) in $\mathrm{CH}_{2} \mathrm{Cl}_{2}(5 \mathrm{~mL})$ wurde bei Raumtemperatur $\mathrm{PPh}_{3}$ (27.9 mg, $106 \mu \mathrm{mol}, 3.2$ Äq.) und $\mathrm{CBr}_{4}$ (33.0 mg, $99.6 \mu \mathrm{mol}, 3.0$ Äq.) gegeben und die resultierende Lösung $2 \mathrm{~h}$ bei dieser Temperatur gerührt. Die Reaktion wurde durch Zugabe von ges. $\mathrm{NaHCO}_{3}$-Lösung $(5 \mathrm{~mL})$ beendet und die Reaktionsmischung mit $\mathrm{CH}_{2} \mathrm{Cl}_{2}(3 \times 5 \mathrm{~mL})$ extrahiert. Die vereinigten organischen Phasen wurden mit ges. NaCl-Lösung $(10 \mathrm{~mL})$ gewaschen, über $\mathrm{Na}_{2} \mathrm{SO}_{4}$ getrocknet und das Lösungsmittel unter vermindertem Druck entfernt. Säulenchromatographische Reinigung an Kieselgel ( $n$ Pentan/EtOAc $3: 1 \rightarrow 1: 1)$ lieferte die Zielverbindung $(\alpha)-214 \quad(3.50 \mathrm{mg}$, $8.25 \mu \mathrm{mol}, 25 \%)$ in Form eines farblosen Feststoffes.

DC: $\mathbf{R}_{f}=0.49(n$ Pentan/EtOAc 2:1).

UV $\left(\mathrm{CH}_{3} \mathrm{CN}\right)$ : keine Absorption.

IR (ATR): $\tilde{v}\left(\mathrm{~cm}^{-1}\right)=2930,2870,2091,1452,1259,1111,1040,991,851$.

${ }^{1} \mathbf{H}-\mathbf{N M R}\left(600 \mathrm{MHz}, \mathrm{CDCl}_{3}\right): \delta(\mathrm{ppm})=1.21\left(\mathrm{~d}, J=6.2 \mathrm{~Hz}, 3 \mathrm{H}, 6{ }^{\prime}-\mathrm{H}_{3}\right), 1.31-1.44\left(\mathrm{~m}_{\mathrm{c}}, 2 \mathrm{H}\right.$, $\left.3-\mathrm{H}_{2}\right), 1.58\left(\mathrm{dt}, J=14.3,7.0 \mathrm{~Hz}, 2 \mathrm{H}, 2-\mathrm{H}_{2}\right), 1.63\left(\mathrm{dt}, J=12.2,6.1 \mathrm{~Hz}, 2 \mathrm{H}, 4-\mathrm{H}_{2}\right), 1.69-1.77$ $\left(\mathrm{m}, 1 \mathrm{H}, 2^{\prime}-\mathrm{H}_{\mathrm{A}}\right), 1.82-1.88\left(\mathrm{~m}, 1 \mathrm{H}, 2^{\prime}-\mathrm{H}_{\mathrm{B}}\right), 1.87-1.93\left(\mathrm{~m}, 2 \mathrm{H}, 3^{\prime}-\mathrm{H}_{2}\right), 2.97(\mathrm{dt}, J=9.8$, $\left.6.2 \mathrm{~Hz}, 1 \mathrm{H}, 4^{\prime}-\mathrm{H}\right), 3.37$ (s, $\left.3 \mathrm{H}, \mathrm{OCH}_{3}\right), 3.44-3.50\left(\mathrm{~m}, 2 \mathrm{H}, 6-\mathrm{H}_{2}\right), 3.52(\mathrm{t}, J=6.7 \mathrm{~Hz}, 2 \mathrm{H}$, 1- $\mathrm{H}_{2}$ ), 3.54 (t, $J=4.8 \mathrm{~Hz}, 2 \mathrm{H}, \mathrm{OCH}_{2} \mathrm{CH}_{2} \mathrm{OMe}$ ), 3.66 (t, $J=4.8 \mathrm{~Hz}, 2 \mathrm{H}, \mathrm{OCH}_{2} \mathrm{CH}_{2} \mathrm{OMe}$ ), 3.71 (quint., $J=5.5 \mathrm{~Hz}, 1 \mathrm{H}, 5-\mathrm{H}), 3.85$ (dq, $J=9.9,6.2 \mathrm{~Hz}, 1 \mathrm{H}, 5 '-\mathrm{H}), 4.68$ (s, $2 \mathrm{H}$, $\left.\mathrm{OCH}_{2} \mathrm{O}\right), 4.82\left(\mathrm{~d}, J=3.2 \mathrm{~Hz}, 1 \mathrm{H}, 1^{\prime}-\mathrm{H}\right)$.

${ }^{13}$ C-NMR $\left(126 \mathrm{MHz}, \mathrm{CDCl}_{3}\right): \delta(\mathrm{ppm})=18.6\left(\mathrm{C}-6{ }^{\prime}\right), 21.8(\mathrm{C}-3), 23.8(\mathrm{C}-3$ '), $29.6(\mathrm{C}-2), 29.7$ (C-2'), 31.0 (C-4), 32.6 (C-6), $59.0\left(\mathrm{OCH}_{3}\right), 62.5\left(\mathrm{C}-4\right.$ '), $66.8\left(\mathrm{OCH}_{2} \mathrm{CH}_{2} \mathrm{OMe}\right), 67.6(\mathrm{C}-1)$, $68.2\left(\mathrm{C}^{-5}\right), 71.8\left(\mathrm{OCH}_{2} \mathrm{CH}_{2} \mathrm{OMe}\right), 77.4(\mathrm{C}-5), 95.5\left(\mathrm{OCH}_{2} \mathrm{O}\right), 96.3\left(\mathrm{C}-1^{\prime}\right)$.

MS (ESI): $\mathrm{m} / \mathrm{z}(\%)=441.2(68), 443.2(72)\left[\mathrm{M}+\mathrm{NH}_{4}\right]^{+}, 446.1(98), 448.1(100)[\mathrm{M}+\mathrm{Na}]^{+}$.

HRMS von $\mathrm{C}_{16} \mathrm{H}_{31} \mathrm{~N}_{3} \mathrm{O}_{6}$ ber.: $\quad 446.1261,448.1241$

gef.: $\quad 446.1256,448.1236[\mathrm{M}+\mathrm{Na}]^{+}$(ESI-HRMS).

$\mathbf{C}_{16} \mathbf{H}_{31} \mathbf{N}_{3} \mathbf{O}_{6}(361.43)$. 


\subsubsection{0 (S)-6-Brom-5-(2,3,4,6-tetradesoxy-4-azido- $\alpha$-D-glucopyranosyl)-1-((2-methoxy-} ethoxy)methoxy)hexan $((\beta)-214)$

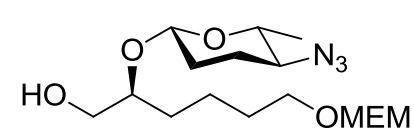

$(\beta)-213$

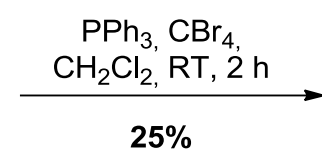

$25 \%$

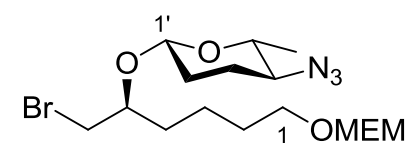

( $\beta)-214$

Zu einer Lösung des Alkohols ( $\beta$ )-213 (12.0 mg, $33.2 \mu$ mol, 1.0 Äq.) in $\mathrm{CH}_{2} \mathrm{Cl}_{2}$ (5 mL) wurde bei Raumtemperatur $\mathrm{PPh}_{3}\left(27.9 \mathrm{mg}, 106 \mu \mathrm{mol}, 3.2\right.$ Äq.) und $\mathrm{CBr}_{4}(33.0 \mathrm{mg}, 99.6 \mu \mathrm{mol}$, 3.0 Äq.) gegeben und die resultierende Lösung $2 \mathrm{~h}$ bei dieser Temperatur gerührt. Die Reaktion wurde durch Zugabe von ges. $\mathrm{NaHCO}_{3}$-Lösung $(5 \mathrm{~mL})$ beendet und die Reaktionsmischung mit $\mathrm{CH}_{2} \mathrm{Cl}_{2}(3 \times 5 \mathrm{~mL})$ extrahiert. Die vereinigten organischen Phasen wurden mit ges. NaCl-Lösung $(10 \mathrm{~mL})$ gewaschen, über $\mathrm{Na}_{2} \mathrm{SO}_{4}$ getrocknet und das Lösungsmittel unter vermindertem Druck entfernt. Säulenchromatographische Reinigung an Kieselgel ( $n$ Pentan/EtOAc 3:1 $\rightarrow 1: 1)$ lieferte die Zielverbindung ( $\beta$ )-213 (3.50 mg, $8.25 \mu \mathrm{mol}$, $25 \%$ ) in Form eines farblosen Feststoffes.

DC: $\mathbf{R}_{f}=0.52(n$ Pentan/EtOAc 2:1).

UV $\left(\mathrm{CH}_{3} \mathrm{CN}\right)$ : keine Absorption.

IR (ATR): $\tilde{v}\left(\mathrm{~cm}^{-1}\right)=2935,2871,2096,1454,1261,1118,1042,987,850$.

${ }^{1} \mathbf{H}-\mathbf{N M R}\left(600 \mathrm{MHz}, \mathrm{CDCl}_{3}\right): \delta(\mathrm{ppm})=1.27\left(\mathrm{~d}, J=6.2 \mathrm{~Hz}, 3 \mathrm{H}, 6{ }^{\prime}-\mathrm{H}_{3}\right), 1.30-1.45\left(\mathrm{~m}_{\mathrm{c}}, 2 \mathrm{H}\right.$, $\left.3-\mathrm{H}_{2}\right), 1.57\left(\mathrm{dt}, J=14.2,7.0 \mathrm{~Hz}, 2 \mathrm{H}, 2-\mathrm{H}_{2}\right), 1.63\left(\mathrm{dt}, J=12.2,6.2 \mathrm{~Hz}, 2 \mathrm{H}, 4-\mathrm{H}_{2}\right), 1.67-1.80$ $\left(\mathrm{m}, 1 \mathrm{H}, 2^{\prime}-\mathrm{H}_{\mathrm{A}}\right), 1.82-1.89\left(\mathrm{~m}, 1 \mathrm{H}, 2^{\prime}-\mathrm{H}_{\mathrm{B}}\right), 2.09-2.18\left(\mathrm{~m}, 2 \mathrm{H}, 3^{\prime}-\mathrm{H}_{2}\right), 2.96(\mathrm{dt}, J=9.8$, $\left.6.1 \mathrm{~Hz}, 1 \mathrm{H}, 4^{\prime}-\mathrm{H}\right), 3.38$ (s, $3 \mathrm{H}, \mathrm{OCH}_{3}$ ), 3.41 (dq, $J=9.9,6.2 \mathrm{~Hz}, 1 \mathrm{H}, 5$ '-H), 3.43-3.50 (m, $\left.2 \mathrm{H}, 6-\mathrm{H}_{2}\right), 3.51\left(\mathrm{t}, J=6.7 \mathrm{~Hz}, 2 \mathrm{H}, 1-\mathrm{H}_{2}\right), 3.53\left(\mathrm{t}, J=4.8 \mathrm{~Hz}, 2 \mathrm{H}, \mathrm{OCH}_{2} \mathrm{CH}_{2} \mathrm{OMe}\right), 3.66(\mathrm{t}$, $J=4.8 \mathrm{~Hz}, 2 \mathrm{H}, \mathrm{OCH}_{2} \mathrm{CH}_{2} \mathrm{OMe}$ ), 3.70 (quint., $\left.J=5.4 \mathrm{~Hz}, 1 \mathrm{H}, 5-\mathrm{H}\right), 4.68\left(\mathrm{~s}, 2 \mathrm{H}, \mathrm{OCH}_{2} \mathrm{O}\right.$ ), $4.76\left(\mathrm{~d}, J=9.2 \mathrm{~Hz}, 1 \mathrm{H}, 1^{\prime}-\mathrm{H}\right)$.

${ }^{13}$ C-NMR $\left(126 \mathrm{MHz}, \mathrm{CDCl}_{3}\right): \delta(\mathrm{ppm})=18.6\left(\mathrm{C}-6^{\prime}\right), 21.8(\mathrm{C}-3), 21.9(\mathrm{C}-3$ '), $29.6(\mathrm{C}-2), 30.5$ (C-2'), 31.0 (C-4), 32.6 (C-6), $59.0\left(\mathrm{OCH}_{3}\right), 61.7\left(\mathrm{C}-4\right.$ '), $66.8\left(\mathrm{OCH}_{2} \mathrm{CH}_{2} \mathrm{OMe}\right), 67.6(\mathrm{C}-1)$, $71.8\left(\mathrm{OCH}_{2} \underline{\mathrm{CH}}_{2} \mathrm{OMe}\right), 75.3\left(\mathrm{C}-5{ }^{\prime}\right), 77.4(\mathrm{C}-5), 95.5\left(\mathrm{OCH}_{2} \mathrm{O}\right), 97.0\left(\mathrm{C}-1^{\prime}\right)$.

MS (ESI): m/z (\%) = $441.2(70), 443.2(72)\left[\mathrm{M}+\mathrm{NH}_{4}\right]^{+}, 446.1(97), 448.1(100)[\mathrm{M}+\mathrm{Na}]^{+}$.

HRMS von $\mathrm{C}_{16} \mathrm{H}_{31} \mathrm{~N}_{3} \mathrm{O}_{6}$ ber.: $\quad 446.1261,448.1241$ gef.: $\quad 446.1257,448.1238[\mathrm{M}+\mathrm{Na}]^{+}(\mathrm{ESI}-\mathrm{HRMS})$.

$\mathrm{C}_{16} \mathbf{H}_{31} \mathbf{N}_{3} \mathbf{O}_{6}$ (361.43). 


\subsubsection{1 (S)-5,6-((Bis-tert-butyldimethylsilyl)oxy)-1-((2-methoxyethoxy)methoxy)hexan} (231)

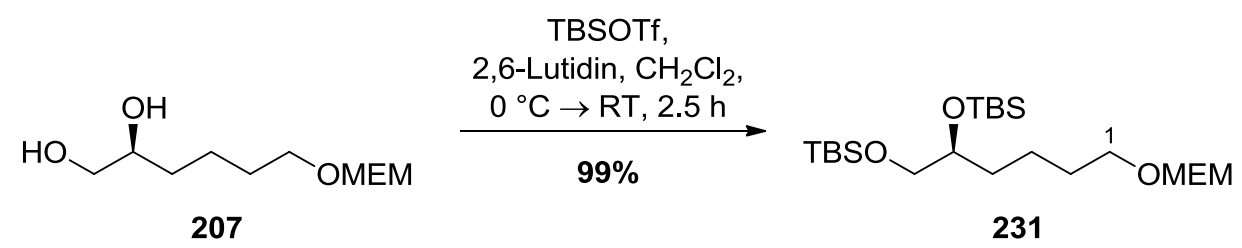

Zu einer Lösung aus Diol 207 (3.00 g, 13.5 mmol, 1.0 Äq.) in $\mathrm{CH}_{2} \mathrm{Cl}_{2}$ (100 mL) wurde bei $0{ }^{\circ} \mathrm{C}$ 2,6-Lutidin $(6.27 \mathrm{~mL}, 5.79 \mathrm{~g}, 54.0 \mathrm{mmol}, 4.0$ Äq.) gegeben und $5 \mathrm{~min}$ bei dieser Temperatur gerührt. Anschließend wurde langsam TBSOTf (10.8 mL, $12.5 \mathrm{~g}, 47.2 \mathrm{mmol}$, 3.5 Äq.) zugegeben, auf Raumtemperatur erwärmt und weitere $2.5 \mathrm{~h}$ gerührt. Die Reaktion wurde durch Zugabe von ges. $\mathrm{NaHCO}_{3}$-Lösung $(100 \mathrm{~mL})$ beendet und mit $\mathrm{CH}_{2} \mathrm{Cl}_{2}$ $(3 \times 100 \mathrm{~mL})$ extrahiert. Die vereinigten organischen Phasen wurden mit ges. NaCl-Lösung (200 mL) gewaschen, über $\mathrm{Na}_{2} \mathrm{SO}_{4}$ getrocknet und das Lösungsmittel unter vermindertem Druck entfernt. Säulenchromatographische Reinigung an Kieselgel ( $n$ Pentan/EtOAc 50:1 $\rightarrow 20: 1)$ lieferte die Zielverbindung 231 (5.98 g, $13.3 \mathrm{mmol}$, 99\%) in Form eines farblosen Öls.

DC: $\mathbf{R}_{f}=0.34(n$ Pentan/EtOAc 25:1).

UV $\left(\mathrm{CH}_{3} \mathrm{CN}\right)$ : keine Absorption.

IR (ATR): $\tilde{v}\left(\mathrm{~cm}^{-1}\right)=2928,2857,1471,1462,1252,1098,1044,831,773,667$.

${ }^{1} \mathbf{H}-\mathbf{N M R}\left(300 \mathrm{MHz}, \mathrm{CDCl}_{3}\right): \delta(\mathrm{ppm})=0.01\left(\mathrm{~s}, 6 \mathrm{H}, \mathrm{Si}\left(\mathrm{CH}_{3}\right)_{2}\right), 0.03\left(\mathrm{~s}, 6 \mathrm{H}, \mathrm{Si}\left(\mathrm{CH}_{3}\right)_{2}\right), 0.85$ (s, $\left.9 \mathrm{H}, \mathrm{SiC}\left(\mathrm{CH}_{3}\right)_{3}\right), 0.86$ (s, $\left.9 \mathrm{H}, \mathrm{SiC}\left(\mathrm{CH}_{3}\right)_{3}\right), 1.28-1.50$ (m, $\left.4 \mathrm{H}, 3-\mathrm{H}_{2}, 4-\mathrm{H}_{2}\right), 1.50-1.62\left(\mathrm{~m}_{\mathrm{c}}\right.$, $\left.2 \mathrm{H}, 2-\mathrm{H}_{2}\right), 3.37\left(\mathrm{~s}, 3 \mathrm{H}, \mathrm{OCH}_{3}\right), 3.37\left(\mathrm{dd}, J=10.0,6.3 \mathrm{~Hz}, 1 \mathrm{H}, 6-\mathrm{H}_{\mathrm{A}}\right), 3.49(\mathrm{dd}, J=10.0$, $\left.5.5 \mathrm{~Hz}, 1 \mathrm{H}, 6-\mathrm{H}_{\mathrm{B}}\right), 3.52\left(\mathrm{t}, J=6.7 \mathrm{~Hz}, 2 \mathrm{H}, 1-\mathrm{H}_{2}\right), 3.51-3.56\left(\mathrm{~m}, 2 \mathrm{H}, \mathrm{OCH}_{2} \mathrm{CH}_{2} \mathrm{OMe}\right)$, 3.57-3.67 (m $\mathrm{c}, 1 \mathrm{H}, 5-\mathrm{H}), 3.63-3.69$ (m, $\left.2 \mathrm{H}, \mathrm{OCH}_{2} \mathrm{CH}_{2} \mathrm{OMe}\right), 4.68$ (s, $2 \mathrm{H}, \mathrm{OCH}_{2} \mathrm{O}$ ).

${ }^{13} \mathrm{C}-\mathrm{NMR}\left(126 \mathrm{MHz}, \mathrm{CDCl}_{3}\right): \delta(\mathrm{ppm})=-5.4,-5.3,-4.8,-4.3\left(2 \times \mathrm{Si}\left(\mathrm{CH}_{3}\right)_{2}\right), 18.1,18.3$ $\left(2 \times \mathrm{SiC}\left(\mathrm{CH}_{3}\right)_{3}\right), 21.8(\mathrm{C}-3), 25.9,26.0\left(2 \times \mathrm{SiC}\left(\mathrm{CH}_{3}\right)_{3}\right), 29.9(\mathrm{C}-2), 34.2(\mathrm{C}-4), 59.0\left(\mathrm{OCH}_{3}\right)$,

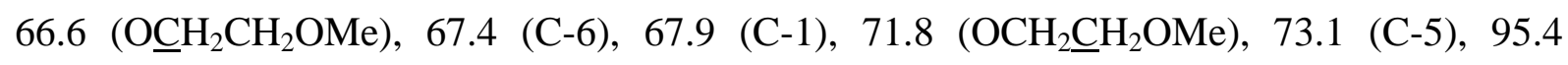
$\left(\mathrm{OCH}_{2} \mathrm{O}\right)$.

MS (ESI): $\mathrm{m} / \mathrm{z}(\%)=451.3(12)[\mathrm{M}+\mathrm{H}]^{+}, 473.3(100)[\mathrm{M}+\mathrm{Na}]^{+}$.

HRMS von $\mathrm{C}_{22} \mathrm{H}_{50} \mathrm{O}_{5} \mathrm{Si}_{2}$ ber.: 473.3089 gef.: $473.3092[\mathrm{M}+\mathrm{Na}]^{+}(\mathrm{ESI}-\mathrm{HRMS})$.

$\mathbf{C}_{22} \mathbf{H}_{50} \mathrm{O}_{5} \mathrm{Si}_{2}$ (450.80). 


\subsubsection{2 (S)-2-((tert-Butyldimethylsilyl)oxy)-6-((2-methoxyethoxy)methoxy)hexan-1-ol} (232)

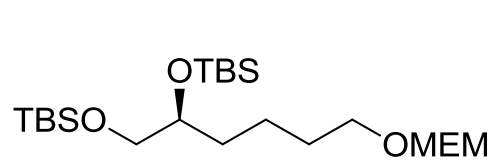

231

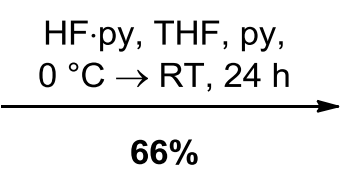

$66 \%$

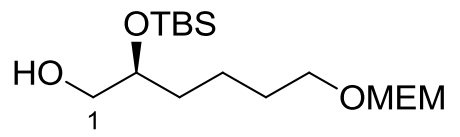

232

Zu einer Lösung aus 231 (1.00 g, 2.22 mmol, 1.0 Äq.) in THF (180 mL) und Pyridin (25 mL) wurde bei $0{ }^{\circ} \mathrm{C}$ HF.py $(2.31 \mathrm{~mL}, 2.54 \mathrm{~g}, 88.7 \mathrm{mmol}, 40.0$ Äq.) gegeben, langsam auf Raumtemperatur erwärmt und $24 \mathrm{~h}$ gerührt. Die Reaktion wurde durch Zugabe von ges. $\mathrm{NaHCO}_{3}$-Lösung $(200 \mathrm{~mL})$ beendet und mit EtOAc $(3 \times 200 \mathrm{~mL})$ extrahiert. Die vereinigten organischen Phasen wurden mit ges. NaCl-Lösung $(400 \mathrm{~mL})$ gewaschen, über $\mathrm{Na}_{2} \mathrm{SO}_{4}$ getrocknet und das Lösungsmittel unter vermindertem Druck entfernt. Säulenchromatographische Reinigung an Kieselgel ( $n$ Pentan/EtOAc 20:1 $\rightarrow 0: 1$ ) lieferte die Zielverbindung 232 (495 mg, 1.47 mmol, 66\%) in Form eines farblosen Öls.

DC: $\mathrm{R}_{f}=0.22(n$ Pentan/EtOAc 5:1).

UV $\left(\mathrm{CH}_{3} \mathrm{CN}\right)$ : keine Absorption.

IR (ATR): $\tilde{v}\left(\mathrm{~cm}^{-1}\right)=2928,2857,1471,1462,1252,1114,1093,1043,833,774,665$.

${ }^{1} \mathbf{H}-\mathbf{N M R}\left(300 \mathrm{MHz}, \mathrm{CDCl}_{3}\right): \delta(\mathrm{ppm})=0.04\left(\mathrm{~s}, 6 \mathrm{H}, \mathrm{Si}\left(\mathrm{CH}_{3}\right)_{2}\right), 0.86\left(\mathrm{~s}, 9 \mathrm{H}, \mathrm{SiC}\left(\mathrm{CH}_{3}\right)_{3}\right)$, 1.34 (quint., $\left.J=7.7 \mathrm{~Hz} .2 \mathrm{H}, 3-\mathrm{H}_{2}\right), 1.42-1.53\left(\mathrm{~m}_{\mathrm{c}}, 2 \mathrm{H}, 4-\mathrm{H}_{2}\right), 1.55$ (quint., $J=7.7 \mathrm{~Hz}, 2 \mathrm{H}$, 2- $\left.\mathrm{H}_{2}\right), 1.94\left(\mathrm{~s}_{\mathrm{br}}, 1 \mathrm{H}, \mathrm{OH}\right), 3.35\left(\mathrm{~s}, 3 \mathrm{H}, \mathrm{OCH}_{3}\right), 3.40\left(\mathrm{dd}, J=11.0,5.2 \mathrm{~Hz}, 1 \mathrm{H}, 6-\mathrm{H}_{\mathrm{A}}\right), 3.50$ (t, $\left.J=6.7 \mathrm{~Hz}, 2 \mathrm{H}, 1-\mathrm{H}_{2}\right), 3.48-3.55\left(\mathrm{~m}, 3 \mathrm{H}, 6-\mathrm{H}_{\mathrm{B}}, \mathrm{OCH}_{2} \mathrm{CH}_{2} \mathrm{OMe}\right), 3.62-3.67(\mathrm{~m}, 2 \mathrm{H}$, $\mathrm{OCH}_{2} \mathrm{CH}_{2} \mathrm{OMe}$ ), 3.62-3.67 (m $\left.\mathrm{c}, 1 \mathrm{H}, 5-\mathrm{H}\right), 4.66\left(\mathrm{~s}, 2 \mathrm{H}, \mathrm{OCH}_{2} \mathrm{O}\right)$.

${ }^{13} \mathrm{C}-\mathrm{NMR}\left(126 \mathrm{MHz}, \mathrm{CDCl}_{3}\right): \delta(\mathrm{ppm})=-4.6,-4.6\left(\mathrm{Si}\left(\mathrm{CH}_{3}{ }_{2}\right), 18.0\left(\mathrm{SiC}\left(\mathrm{CH}_{3}\right)_{3}\right), 22.0(\mathrm{C}-3)\right.$, $25.8\left(\mathrm{SiC}\left(\underline{\mathrm{CH}}_{3}\right)_{3}\right), 29.8(\mathrm{C}-2), 33.7(\mathrm{C}-4), 58.9\left(\mathrm{OCH}_{3}\right), 66.1(\mathrm{C}-6), 66.6\left(\mathrm{OC}_{2} \mathrm{CH}_{2} \mathrm{OMe}\right)$, $67.6(\mathrm{C}-1), 71.8\left(\mathrm{OCH}_{2} \mathrm{CH}_{2} \mathrm{OMe}\right), 72.7$ (C-5), $95.4\left(\mathrm{OCH}_{2} \mathrm{O}\right)$.

MS (ESI): m/z (\%) = $359.2(100)[\mathrm{M}+\mathrm{Na}]^{+}, 375.2(14)[\mathrm{M}+\mathrm{K}]^{+} ; 335.2(83)[\mathrm{M}-\mathrm{H}]^{-}$.

HRMS von $\mathrm{C}_{16} \mathrm{H}_{36} \mathrm{O}_{5} \mathrm{Si}$ ber.: $\quad 335.2259$ 359.2224 gef.: $\quad 335.2247[\mathrm{M}-\mathrm{H}]^{-}$ $359.2226[\mathrm{M}+\mathrm{Na}]^{+}(\mathrm{ESI}-\mathrm{HRMS})$.

$\mathrm{C}_{16} \mathrm{H}_{36} \mathrm{O}_{5} \mathrm{Si}$ (336.54). 


\subsubsection{3 (S)-6-Brom-5-((tert-butyldimethylsilyl)oxy)-1-((2-methoxyethoxy)methoxy)-} hexan (233a)
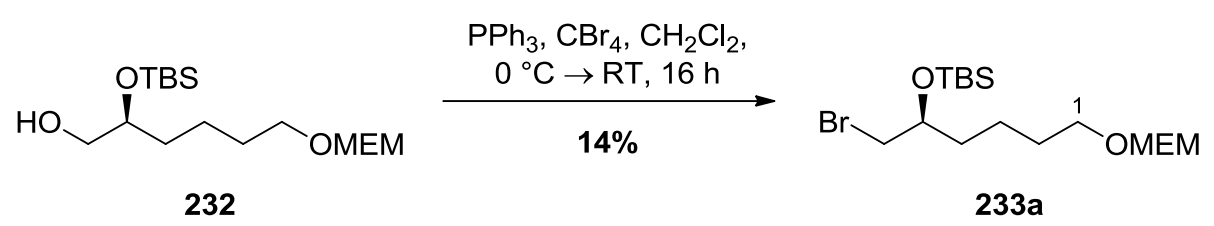

$\mathrm{Zu}$ einer Lösung aus $\mathrm{PPh}_{3}\left(234 \mathrm{mg}, 891 \mu \mathrm{mol}, 3.0\right.$ Äq.) und $\mathrm{CBr}_{4}(296 \mathrm{mg}, 891 \mu \mathrm{mol}$, 3.0 Äq.) in $\mathrm{CH}_{2} \mathrm{Cl}_{2}$ (10 mL) wurde bei $0{ }^{\circ} \mathrm{C}$ Alkohol 232 (100 mg, $297 \mu \mathrm{mol}, 1.0$ Äq.) gegeben und $30 \mathrm{~min}$ bei dieser Temperatur gerührt. Anschließend wurde langsam auf Raumtemperatur erwärmt und weitere $3.5 \mathrm{~h}$ gerührt. Die Reaktion wurde durch Zugabe von ges. $\mathrm{NaHCO}_{3}$-Lösung $(10 \mathrm{~mL})$ beendet und mit $\mathrm{CH}_{2} \mathrm{Cl}_{2}(3 \times 10 \mathrm{~mL})$ extrahiert. Die vereinigten organischen Phasen wurden mit ges. NaCl-Lösung $(20 \mathrm{~mL})$ gewaschen, über $\mathrm{Na}_{2} \mathrm{SO}_{4}$ getrocknet und das Lösungsmittel unter vermindertem Druck entfernt. Säulenchromatographische Reinigung an Kieselgel ( $n$ Pentan/EtOAc 20:1 $\rightarrow 5: 1$ ) lieferte die Zielverbindung 233a (16.5 mg, $41.3 \mu \mathrm{mol}, 14 \%)$ in Form eines farblosen Öls.

DC: $\mathrm{R}_{f}=0.54(n$ Pentan/EtOAc 5:1).

${ }^{1} \mathbf{H}-\mathbf{N M R}\left(300 \mathrm{MHz}, \mathrm{CDCl}_{3}\right): \delta(\mathrm{ppm})=0.03,0.06\left(2 \mathrm{~s}, 6 \mathrm{H}, \mathrm{Si}\left(\mathrm{CH}_{3}\right)_{2}\right), 0.86(\mathrm{~s}, 9 \mathrm{H}$, $\left.\mathrm{SiC}\left(\mathrm{CH}_{3}\right)_{3}\right), 1.27-1.47\left(\mathrm{~m}_{\mathrm{c}}, 2 \mathrm{H}, 3-\mathrm{H}_{2}\right), 1.46-1.70\left(\mathrm{~m}, 4 \mathrm{H}, 2-\mathrm{H}_{2}, 4-\mathrm{H}_{2}\right), 3.28(\mathrm{~d}, J=6.1 \mathrm{~Hz}$, $\left.1 \mathrm{H}, 6-\mathrm{H}_{\mathrm{A}}\right), 3.28\left(\mathrm{~d}, J=4.8 \mathrm{~Hz}, 1 \mathrm{H}, 6-\mathrm{H}_{\mathrm{B}}\right), 3.36\left(\mathrm{~s}, 3 \mathrm{H}, \mathrm{OCH}_{3}\right), 3.51(\mathrm{t}, J=6.5 \mathrm{~Hz}, 2 \mathrm{H}$, 1- $\mathrm{H}_{2}$ ), 3.50-3.55 (m, $2 \mathrm{H}, \mathrm{OCH}_{2} \mathrm{CH}_{2} \mathrm{OMe}$ ), 3.63-3.68 (m, $2 \mathrm{H}, \mathrm{OCH}_{2} \mathrm{CH}_{2} \mathrm{OMe}$ ), 3.78 (quint., $J=5.6 \mathrm{~Hz}, 1 \mathrm{H}, 5-\mathrm{H}), 4.67\left(\mathrm{~s}, 2 \mathrm{H}, \mathrm{OCH}_{2} \mathrm{O}\right)$.

${ }^{13} \mathrm{C}-\mathrm{NMR}\left(126 \mathrm{MHz}, \mathrm{CDCl}_{3}\right): \delta(\mathrm{ppm})=-4.7,-4.5\left(\mathrm{Si}\left(\mathrm{CH}_{3}\right)_{2}\right), 18.0\left(\mathrm{SiC}\left(\mathrm{CH}_{3}\right)_{3}\right), 21.5(\mathrm{C}-3)$, $25.7\left(\mathrm{SiC}\left(\underline{\mathrm{CH}}_{3}\right)_{3}\right), 29.6(\mathrm{C}-2), 35.3(\mathrm{C}-4), 37.6(\mathrm{C}-6), 59.0\left(\mathrm{OCH}_{3}\right), 66.6\left(\mathrm{OC}_{2} \mathrm{CH}_{2} \mathrm{OMe}\right)$, $67.6(\mathrm{C}-1), 71.8,71.8\left(\mathrm{OCH}_{2} \mathrm{CH}_{2} \mathrm{OMe}, \mathrm{C}-5\right), 95.4\left(\mathrm{OCH}_{2} \mathrm{O}\right)$.

MS (ESI): $\mathrm{m} / \mathrm{z}(\%)=416.2(24), 418.2(25)\left[\mathrm{M}+\mathrm{NH}_{4}\right]^{+}, 421.2(95), 423.2(100)[\mathrm{M}+\mathrm{Na}]^{+}$, $437.1(20), 439.1(21)[\mathrm{M}+\mathrm{K}]^{+}$.

HRMS von $\mathrm{C}_{16} \mathrm{H}_{35} \mathrm{BrO}_{4} \mathrm{Si}$ ber.: $\quad 421.1380,423.1360$ gef.: $\quad 421.1378,423.1359[\mathrm{M}+\mathrm{Na}]^{+}$(ESI-HRMS).

$\mathbf{C}_{\mathbf{1 6}} \mathrm{H}_{35} \mathrm{BrO}_{4} \mathrm{Si}$ (399.44). 


\subsubsection{4 (S)-2-((tert-Butyldimethylsilyl)oxy)-6-((2-methoxyethoxy)methoxy)hexyl-4-} methylphenylsulfonat (233b)
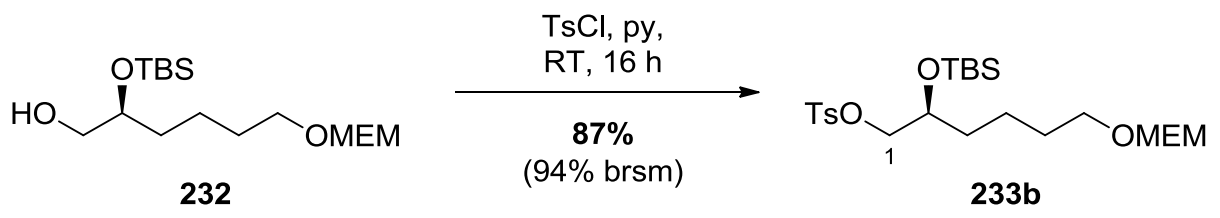

Zu einer Lösung aus Alkohol 232 (270 mg, $802 \mu \mathrm{mol}, 1.0$ Äq.) in Pyridin (10 mL) wurde bei $0{ }^{\circ} \mathrm{C} \mathrm{TsCl}$ (306 mg, $1.61 \mathrm{mmol}, 2.0$ Äq.) gegeben, langsam auf Raumtemperatur erwärmt und $16 \mathrm{~h}$ bei dieser Temperatur gerührt. Die Reaktion wurde durch Zugabe von ges. $\mathrm{NH}_{4} \mathrm{Cl}-$ Lösung $(10 \mathrm{~mL})$ beendet und mit $\mathrm{CH}_{2} \mathrm{Cl}_{2}(3 \times 20 \mathrm{~mL})$ extrahiert. Die vereinigten organischen Phasen wurden mit ges. NaCl-Lösung $(50 \mathrm{~mL})$ gewaschen, über $\mathrm{Na}_{2} \mathrm{SO}_{4}$ getrocknet und das Lösungsmittel unter vermindertem Druck entfernt. Säulenchromatographische Reinigung an Kieselgel ( $n$ Pentan/EtOAc 5:1 $\rightarrow 2: 1$ ) lieferte die Zielverbindung 233b (344 mg, $701 \mu \mathrm{mol}$, $87 \%, 94 \%$ brsm) in Form eines farblosen Öls.

DC: $\mathrm{R}_{f}=0.50(n$ Pentan/EtOAc 3:1).

UV $\left(\mathrm{CH}_{3} \mathrm{CN}\right): \lambda_{\max }(\lg \varepsilon)=225 \mathrm{~nm}$ (4.2138), 262 (3.0766).

IR (ATR): $\tilde{v}\left(\mathrm{~cm}^{-1}\right)=2934,2873,1357,1174,1095,1042,1020,965,814,666,554$.

${ }^{1} \mathbf{H}-\mathbf{N M R}\left(300 \mathrm{MHz}, \mathrm{CDCl}_{3}\right): \delta(\mathrm{ppm})=-0.04,-0.02\left(2 \mathrm{~s}, 6 \mathrm{H}, \mathrm{Si}\left(\mathrm{CH}_{3}\right)_{2}\right), 0.79(\mathrm{~s}, 9 \mathrm{H}$, $\left.\mathrm{SiC}\left(\mathrm{CH}_{3}\right)_{3}\right), 1.19-1.45\left(\mathrm{~m}, 4 \mathrm{H}, 3-\mathrm{H}_{2}, 4-\mathrm{H}_{2}\right), 1.50$ (quint., $\left.J=7.4 \mathrm{~Hz}, 2 \mathrm{H}, 5-\mathrm{H}_{2}\right), 2.40$ (s, $3 \mathrm{H}$, Ts- $\left.\mathrm{CH}_{3}\right), 3.35\left(\mathrm{~s}, 3 \mathrm{H}, \mathrm{OCH}_{3}\right), 3.46\left(\mathrm{t}, J=6.4 \mathrm{~Hz}, 2 \mathrm{H}, 6-\mathrm{H}_{2}\right), 3.49-3.54(\mathrm{~m}, 2 \mathrm{H}$, $\mathrm{OCH}_{2} \mathrm{CH}_{2} \mathrm{OMe}$ ), 3.61-3.66 (m, $2 \mathrm{H}, \mathrm{OCH}_{2} \mathrm{CH}_{2} \mathrm{OMe}$ ), 3.73-3.86 (m, $3 \mathrm{H}, 1-\mathrm{H}_{2}, 2-\mathrm{H}$ ), 4.65 (s, $\left.2 \mathrm{H}, \mathrm{OCH}_{2} \mathrm{O}\right), 7.30\left(\mathrm{~d}, J=8.0 \mathrm{~Hz}, 2 \mathrm{H}, 2 \times \mathrm{Ts}-\mathrm{H}_{m}\right), 7.73\left(\mathrm{~d}, J=8.3 \mathrm{~Hz}, 2 \mathrm{H}, 2 \times \mathrm{Ts}_{\mathrm{H}}-\mathrm{H}_{o}\right)$.

${ }^{13} \mathrm{C}-\mathrm{NMR}\left(126 \mathrm{MHz}, \mathrm{CDCl}_{3}\right): \delta(\mathrm{ppm})=-4.9,-4.7\left(\mathrm{Si}\left(\mathrm{CH}_{3}\right)_{2}\right), 17.9\left(\mathrm{SiC}\left(\mathrm{CH}_{3}\right)_{3}\right), 21.5(\mathrm{C}-4)$, $21.5\left(\mathrm{Ts}-\mathrm{CH}_{3}\right), 25.7\left(\mathrm{SiC}\left(\underline{\mathrm{CH}}_{3}\right)_{3}\right), 29.6(\mathrm{C}-5), 33.8(\mathrm{C}-3), 58.9\left(\mathrm{OCH}_{3}\right), 66.6\left(\mathrm{OCL}_{2} \mathrm{CH}_{2} \mathrm{OMe}\right)$, 67.5 (C-6), 69.8 (C-2), $71.7\left(\mathrm{OCH}_{2} \underline{\mathrm{CH}}_{2} \mathrm{OMe}\right), 73.0(\mathrm{C}-1), 95.4\left(\mathrm{OCH}_{2} \mathrm{O}\right), 127.9$ (2×Ts- $\left.\mathrm{C}_{o}\right)$, $129.7\left(2 \times \mathrm{Ts}^{-\mathrm{C}_{m}}\right), 132.9\left(\mathrm{Ts}_{\mathrm{p}} \mathrm{C}_{\mathrm{p}}\right), 144.7\left(\mathrm{Ts}_{\mathrm{C}} \mathrm{C}_{i}\right)$.

MS (ESI): m/z (\%) = $508.3(24)\left[\mathrm{M}+\mathrm{NH}_{4}\right]^{+}, 513.3(86)[\mathrm{M}+\mathrm{Na}]^{+}, 529.2(100)[\mathrm{M}+\mathrm{K}]^{+}$.

HRMS von $\mathrm{C}_{23} \mathrm{H}_{42} \mathrm{O}_{7} \mathrm{SSi}$ ber.: 529.2052 gef.: $\quad 529.2054[\mathrm{M}+\mathrm{K}]^{+}(\mathrm{ESI}-\mathrm{HRMS})$.

$\mathbf{C}_{23} \mathbf{H}_{42} \mathbf{O}_{7} \mathbf{S S i}$ (490.73). 


\subsubsection{5 (S)-2-((tert-Butyldimethylsilyl)oxy)-6-((2-methoxyethoxy)methoxy)hexyl-} methansulfonat $(\mathbf{2 3 3 c})$

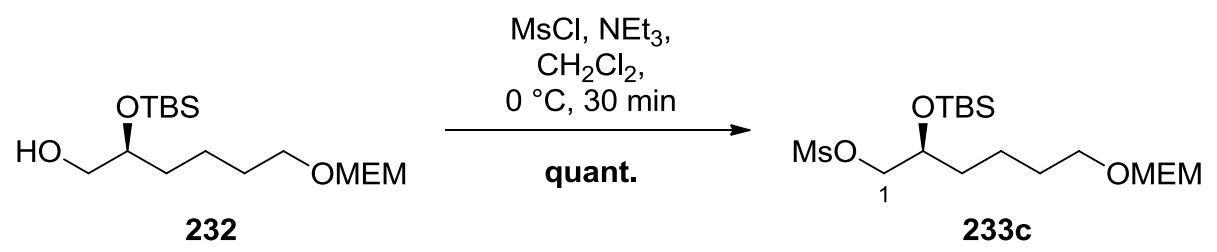

Zu einer Lösung aus Alkohol 232 (170 mg, $505 \mu \mathrm{mol}, 1.0$ Äq.) und $\mathrm{MsCl}$ (86.0 $\mu \mathrm{L}, 127$ mg, $1.11 \mathrm{mmol}, 2.2 \mathrm{Äq}$.) in $\mathrm{CH}_{2} \mathrm{Cl}_{2}(25 \mathrm{~mL})$ wurde bei $0{ }^{\circ} \mathrm{C} \mathrm{NEt}_{3}(77.0 \mu \mathrm{L}, 56.2 \mathrm{mg}, 556 \mu \mathrm{mol}$, 1.1 Äq.) gegeben und $1 \mathrm{~h}$ bei dieser Temperatur gerührt. Die Reaktion wurde durch Zugabe von ges. $\mathrm{NH}_{4} \mathrm{Cl}$-Lösung $(25 \mathrm{~mL})$ beendet und mit $\mathrm{CH}_{2} \mathrm{Cl}_{2}(3 \times 25 \mathrm{~mL})$ extrahiert. Die vereinigten organischen Phasen wurden mit ges. NaCl-Lösung $(50 \mathrm{~mL})$ gewaschen, über $\mathrm{Na}_{2} \mathrm{SO}_{4}$ getrocknet und das Lösungsmittel unter vermindertem Druck entfernt. Die Zielverbindung 233c (209 mg, $505 \mu \mathrm{mol}$, quant.) wurde analysenrein in Form einer farblosen Flüssigkeit isoliert und ohne weitere Aufreinigung eingesetzt.

DC: $\mathbf{R}_{f}=0.59(n$ Pentan/EtOAc 1:1).

UV $\left(\mathrm{CH}_{3} \mathrm{CN}\right)$ : keine Absorption.

IR (ATR): $\tilde{v}\left(\mathrm{~cm}^{-1}\right)=2929,2857,1463,1357,1254,1174,1114,1044,958,835,776,527$.

${ }^{1} \mathbf{H}$-NMR $\left(300 \mathrm{MHz}, \mathrm{CDCl}_{3}\right): \delta(\mathrm{ppm})=0.04,0.05\left(2 \mathrm{~s}, 6 \mathrm{H}, \mathrm{Si}\left(\mathrm{CH}_{3}\right)_{2}\right), 0.85(\mathrm{~s}, 9 \mathrm{H}$, $\left.\mathrm{SiC}\left(\mathrm{CH}_{3}\right)_{3}\right), 1.33-1.44\left(\mathrm{~m}_{\mathrm{c}}, 2 \mathrm{H}, 4-\mathrm{H}_{2}\right), 1.43-1.55\left(\mathrm{~m}_{\mathrm{c}}, 2 \mathrm{H}, 3-\mathrm{H}_{2}\right), 1.56$ (quint., $J=7.0 \mathrm{~Hz}$, $\left.2 \mathrm{H}, 5-\mathrm{H}_{2}\right), 2.98\left(\mathrm{~s}, 3 \mathrm{H}, \mathrm{Ms}_{-} \mathrm{CH}_{3}\right), 3.36\left(\mathrm{~s}, 3 \mathrm{H}, \mathrm{OCH}_{3}\right), 3.51\left(\mathrm{t}, J=6.5 \mathrm{~Hz}, 2 \mathrm{H}, 6-\mathrm{H}_{2}\right)$, 3.50-3.55 (m, $2 \mathrm{H}, \mathrm{OCH}_{2} \mathrm{CH}_{2} \mathrm{OMe}$ ), 3.62-3.68 (m, $2 \mathrm{H}, \mathrm{OCH}_{2} \mathrm{CH}_{2} \mathrm{OMe}$ ), 3.87 (quint., $J=$ $5.5 \mathrm{~Hz}, 1 \mathrm{H}, 2-\mathrm{H}), 4.00\left(\mathrm{dd}, J=10.1,6.0 \mathrm{~Hz}, 1 \mathrm{H}, 1-\mathrm{H}_{\mathrm{A}}\right), 4.09(\mathrm{dd}, J=10.1,4.3 \mathrm{~Hz}, 1 \mathrm{H}$, $\left.1-\mathrm{H}_{\mathrm{B}}\right), 4.67\left(\mathrm{~s}, 2 \mathrm{H}, \mathrm{OCH}_{2} \mathrm{O}\right)$.

${ }^{13} \mathrm{C}-\mathrm{NMR}\left(126 \mathrm{MHz}, \mathrm{CDCl}_{3}\right): \delta(\mathrm{ppm})=-4.8,-4.5\left(\mathrm{Si}\left(\mathrm{CH}_{3}\right)_{2}\right), 18.0\left(\mathrm{SiC}\left(\mathrm{CH}_{3}\right)_{3}\right), 21.6(\mathrm{C}-4)$, $25.7 \quad\left(\mathrm{SiC}\left(\underline{\mathrm{CH}}_{3}\right)_{3}\right), \quad 29.6 \quad(\mathrm{C}-5), \quad 33.8 \quad(\mathrm{C}-3), \quad 37.3 \quad\left(\mathrm{Ms}-\mathrm{CH}_{3}\right), \quad 59.0 \quad\left(\mathrm{OCH}_{3}\right), \quad 66.7$ $\left(\mathrm{O}_{\underline{C}} \mathrm{CH}_{2} \mathrm{OMe}\right), 67.5$ (C-6), 70.1 (C-2), $71.8\left(\mathrm{OCH}_{2} \underline{\mathrm{CH}}_{2} \mathrm{OMe}\right), 72.7$ (C-1), $95.4\left(\mathrm{OCH}_{2} \mathrm{O}\right)$. MS (ESI): $\mathrm{m} / \mathrm{z}(\%)=437.2(28)[\mathrm{M}+\mathrm{Na}]^{+}, 453.2(100)[\mathrm{M}+\mathrm{K}]^{+}$.

HRMS von $\mathrm{C}_{17} \mathrm{H}_{38} \mathrm{O}_{7} \mathrm{SSi}$ ber.: 453.1739 gef.: $\quad 453.1742[\mathrm{M}+\mathrm{K}]^{+}(\mathrm{ESI}-\mathrm{HRMS})$.

$\mathrm{C}_{17} \mathrm{H}_{38} \mathrm{O}_{7} \mathrm{SSi}$ (414.63). 


\subsubsection{6 (S)-2-((tert-Butyldimethylsilyl)oxy)-6-((2-methoxyethoxy)methoxy)hexyltri-}

fluormethanesulfonat (233d)
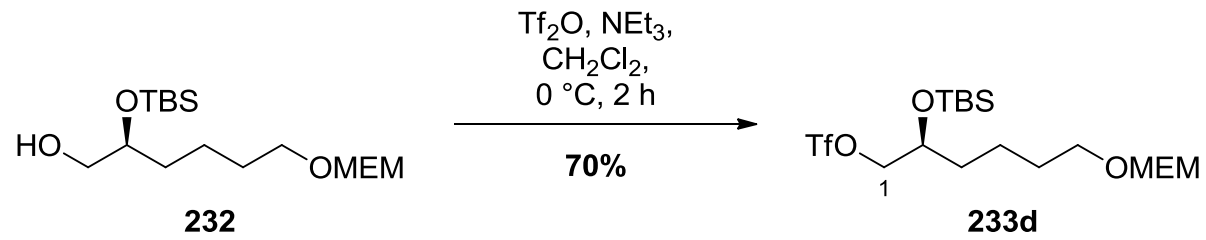

Zu einer Lösung aus Alkohol 232 (50.0 mg, $149 \mu$ mol, 1.0 Äq.) und $\mathrm{Tf}_{2} \mathrm{O}\left(1 \mathrm{M}\right.$ in $\mathrm{CH}_{2} \mathrm{Cl}_{2}$, $178 \mu \mathrm{L}, 178 \mu \mathrm{mol}, 1.2$ Äq.) in $\mathrm{CH}_{2} \mathrm{Cl}_{2}(3 \mathrm{~mL})$ wurde bei $0{ }^{\circ} \mathrm{C} \mathrm{NEt}_{3}(24.7 \mu \mathrm{L}, 18.0 \mathrm{mg}$, $178 \mu \mathrm{mol}, 1.2$ Äq.) gegeben und $2 \mathrm{~h}$ bei dieser Temperatur gerührt. Die Reaktion wurde durch Zugabe von ges. $\mathrm{NaHCO}_{3}$-Lösung $(3 \mathrm{~mL})$ beendet und mit $\mathrm{CH}_{2} \mathrm{Cl}_{2}(3 \times 3 \mathrm{~mL})$ extrahiert. Die vereinigten organischen Phasen wurden mit ges. NaCl-Lösung $(10 \mathrm{~mL})$ gewaschen, über $\mathrm{Na}_{2} \mathrm{SO}_{4}$ getrocknet und das Lösungsmittel unter vermindertem Druck entfernt. Säulenchromatographische Reinigung an Kieselgel ( $n$ Pentan/EtOAc $5: 1 \rightarrow 1: 1$ ) lieferte die Zielverbindung 233d (49.2 mg, $105 \mu \mathrm{mol}, 70 \%$ ) in Form eines farblosen Öls. Aufgrund der geringen Stabilität des Produktes wurde es direkt umgesetzt.

DC: $\mathbf{R}_{f}=0.70(n$ Pentan/EtOAc $1: 1)$

UV $\left(\mathrm{CH}_{3} \mathrm{CN}\right)$ : keine Absorption.

IR (ATR): $\tilde{v}\left(\mathrm{~cm}^{-1}\right)=2930,2860,1414,1246,1201,1133,1044,946,836,776.611$.

${ }^{1} \mathbf{H}-\mathbf{N M R}\left(300 \mathrm{MHz}, \mathrm{CDCl}_{3}\right): \delta(\mathrm{ppm})=0.06,0.07\left(2 \mathrm{~s}, 6 \mathrm{H}, \mathrm{Si}\left(\mathrm{CH}_{3}\right)_{2}\right), 0.87(\mathrm{~s}, 9 \mathrm{H}$, $\left.\mathrm{SiC}\left(\mathrm{CH}_{3}\right)_{3}\right), 1.33-1.47\left(\mathrm{~m}_{\mathrm{c}}, 2 \mathrm{H}, 4-\mathrm{H}_{2}\right), 1.47-1.65\left(\mathrm{~m}, 4 \mathrm{H}, 3-\mathrm{H}_{2}, 5-\mathrm{H}_{2}\right), 3.38\left(\mathrm{~s}, 3 \mathrm{H}, \mathrm{OCH}_{3}\right)$, $3.53\left(\mathrm{t}, J=6.3 \mathrm{~Hz}, 2 \mathrm{H}, 6-\mathrm{H}_{2}\right), 3.52-3.57\left(\mathrm{~m}, 2 \mathrm{H}, \mathrm{OCH}_{2} \mathrm{CH}_{2} \mathrm{OMe}\right), 3.63-3.69(\mathrm{~m}, 2 \mathrm{H}$, $\mathrm{OCH}_{2} \mathrm{CH}_{2} \mathrm{OMe}$ ), 3.88-3.97 (m $\left., 1 \mathrm{H}, 2-\mathrm{H}\right), 4.30\left(\mathrm{dd}, J=10.0,6.2 \mathrm{~Hz}, 1 \mathrm{H}, 1-\mathrm{H}_{\mathrm{A}}\right), 4.36(\mathrm{dd}$, $\left.J=10.0,4.1 \mathrm{~Hz}, 1 \mathrm{H}, 1-\mathrm{H}_{\mathrm{B}}\right), 4.69\left(\mathrm{~s}, 2 \mathrm{H}, \mathrm{OCH}_{2} \mathrm{O}\right)$.

${ }^{13}$ C-NMR $\left(126 \mathrm{MHz}, \mathrm{CDCl}_{3}\right): \delta(\mathrm{ppm})=-4.8,-4.7\left(\mathrm{Si}\left(\mathrm{CH}_{3}\right)_{2}\right), 18.0\left(\mathrm{SiC}\left(\mathrm{CH}_{3}\right)_{3}\right), 21.6(\mathrm{C}-4)$, $25.7\left(\mathrm{SiC}\left(\mathrm{CH}_{3}\right)_{3}\right), 29.6(\mathrm{C}-5), 33.6(\mathrm{C}-3), 59.0\left(\mathrm{OCH}_{3}\right), 66.8\left(\mathrm{OCH}_{2} \mathrm{CH}_{2} \mathrm{OMe}\right), 67.4(\mathrm{C}-6)$, 69.7 (C-2), $71.8\left(\mathrm{OCH}_{2} \mathrm{CH}_{2} \mathrm{OMe}\right), 79.3(\mathrm{C}-1), 95.5\left(\mathrm{OCH}_{2} \mathrm{O}\right)$.

MS (ESI): $\mathrm{m} / \mathrm{z}(\%)=491.2(83)[\mathrm{M}+\mathrm{Na}]^{+}, 507.1(57)[\mathrm{M}+\mathrm{K}]^{+}$.

HRMS von $\mathrm{C}_{17} \mathrm{H}_{35} \mathrm{~F}_{3} \mathrm{O}_{7} \mathrm{SSi}$

ber.: $\quad 507.1456$

gef.: $507.1457[\mathrm{M}+\mathrm{K}]^{+}(\mathrm{ESI}-\mathrm{HRMS})$.

\section{$\mathrm{C}_{17} \mathrm{H}_{35} \mathrm{~F}_{3} \mathbf{O}_{7} \mathrm{SSi}(468.60)$.}




\subsection{Synthese der Forosamin-Analoga 222a-d und 225}

\subsubsection{1-Azido-4-brombutan (222a)}
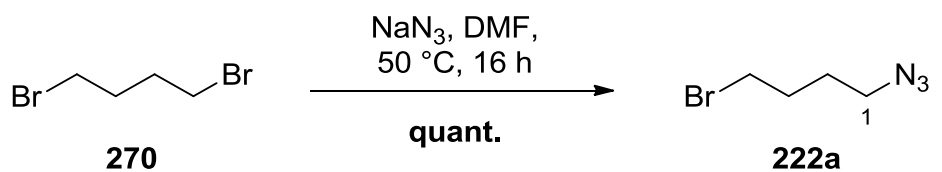

Zu einer Lösung des Dibromids 270 (553 $\mu \mathrm{L}, 1.00 \mathrm{~g}, 4.63 \mathrm{mmol}, 1.0$ Äq.) in DMF (10 mL) wurde bei Raumtemperatur $\mathrm{NaN}_{3}$ (301 mg, $4.63 \mathrm{mmol}, 1.0$ Äq.) gegeben, auf $50{ }^{\circ} \mathrm{C}$ erhitzt und $16 \mathrm{~h}$ gerührt. Anschließend wurde die Lösung auf Raumtemperatur abgekühlt und mit ges. $\mathrm{NaHCO}_{3}$-Lösung $(10 \mathrm{~mL})$ und ges. $\mathrm{Na}_{2} \mathrm{~S}_{2} \mathrm{O}_{3}$-Lösung $(10 \mathrm{~mL})$ versetzt. Es wurde mit $\mathrm{Et}_{2} \mathrm{O}(3 \times 10 \mathrm{~mL})$ extrahiert, die vereinigten organischen Phasen mit ges. NaCl-Lösung (20 mL) gewaschen, über $\mathrm{Na}_{2} \mathrm{SO}_{4}$ getrocknet und das Lösungsmittel unter vermindertem Druck entfernt. Da das Zielprodukt 222a ( $825 \mathrm{mg}$, $4.63 \mathrm{mmol}$, quant.) weder UV-aktiv noch durch gängige Anfärbereagenzien nachweisbar ist, wurde es ohne weitere Aufarbeitung verwendet.

${ }^{1} \mathbf{H}-\mathbf{N M R}\left(300 \mathrm{MHz}, \mathrm{CDCl}_{3}\right): \delta(\mathrm{ppm})=1.62-1.72\left(\mathrm{~m}_{\mathrm{c}}, 4 \mathrm{H}, 2-\mathrm{H}_{2}, 3-\mathrm{H}_{2}\right), 3.25-3.37\left(\mathrm{~m}_{\mathrm{c}}, 4 \mathrm{H}\right.$, $\left.1-\mathrm{H}_{2}, 4-\mathrm{H}_{2}\right)$.

$\mathbf{C}_{4} \mathbf{H}_{8} \mathrm{BrN}_{3}$ (178.03). 


\subsection{2 (E)-1-Azido-4-brombut-2-en (222b)}

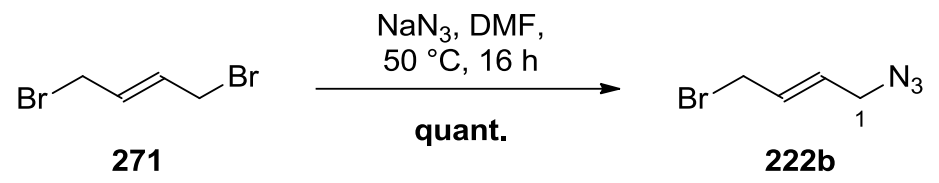

Zu einer Lösung des Dibromids 271 (1.00 g, 4.68 mmol, 1.0 Äq.) in DMF (10 mL) wurde bei Raumtemperatur $\mathrm{NaN}_{3}$ (304 mg, $4.68 \mathrm{mmol}, 1.0$ Äq.) gegeben, auf $50{ }^{\circ} \mathrm{C}$ erhitzt und $16 \mathrm{~h}$ gerührt. Anschließend wurde die Lösung auf Raumtemperatur abgekühlt und mit ges. $\mathrm{NaHCO}_{3}$-Lösung $(10 \mathrm{~mL})$ und ges. $\mathrm{Na}_{2} \mathrm{~S}_{2} \mathrm{O}_{3}$-Lösung $(10 \mathrm{~mL})$ versetzt. Es wurde mit $\mathrm{Et}_{2} \mathrm{O}$ $(3 \times 10 \mathrm{~mL})$ extrahiert, die vereinigten organischen Phasen mit ges. NaCl-Lösung $(20 \mathrm{~mL})$ gewaschen, über $\mathrm{Na}_{2} \mathrm{SO}_{4}$ getrocknet und das Lösungsmittel unter vermindertem Druck entfernt. Da das Zielprodukt weder UV-aktiv noch durch gängige Anfärbereagenzien nachweisbar ist, wurden die einzelnen Fraktionen nach säulenchromatographischer Reinigung an Kieselgel ( $n$ Pentan) eingedampft und der entstandene Feststoff isoliert. Die Zielverbindung 222b (823 mg, 4.68 mmol, quant.) wurde in Form eines farblosen Feststoffes erhalten.

${ }^{\mathbf{1}} \mathbf{H}-\mathbf{N M R}\left(300 \mathrm{MHz}, \mathrm{CDCl}_{3}\right): \delta(\mathrm{ppm})=3.87-3.98\left(\mathrm{~m}_{\mathrm{c}}, 4 \mathrm{H}, 1-\mathrm{H}_{2}, 4-\mathrm{H}_{2}\right), 5.93-5.99\left(\mathrm{~m}_{\mathrm{c}}, 2 \mathrm{H}\right.$, 2-H, 3-H).

$\mathbf{C}_{4} \mathbf{H}_{6} \mathbf{B r N}_{3}(176.01)$. 


\subsection{3 (Z)-1,4-Dibrombut-2-en (218)}

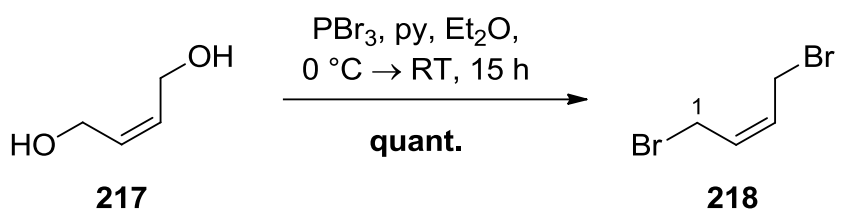

Zu einer Lösung des Diols 217 (5.00 mL, 5.36 g, 60.8 mmol, 1.0 Äq.) in $\mathrm{Et}_{2} \mathrm{O}$ (40 mL) wurde bei $0{ }^{\circ} \mathrm{C}$ Pyridin $(982 \mu \mathrm{L}, 962 \mathrm{mg}, 12.2 \mathrm{mmol}, 0.2 \mathrm{Äq}$.) gegeben und $5 \mathrm{~min}$ gerührt. Anschließend wurde $\mathrm{PBr}_{3}$ (5.15 mL, $14.8 \mathrm{~g}, 54.7 \mathrm{mmol}, 0.9$ Äq.) zugegeben, weitere $30 \mathrm{~min}$ bei $0{ }^{\circ} \mathrm{C}$ gerührt und auf Raumtemperatur erwärmt. Nach $16 \mathrm{~h}$ wurde die Reaktion durch Zugabe von ges. $\mathrm{NaHCO}_{3}$-Lösung $(25 \mathrm{~mL})$ und ges. $\mathrm{Na}_{2} \mathrm{~S}_{2} \mathrm{O}_{3}$-Lösung $(25 \mathrm{~mL}$ ) beendet und mit $\mathrm{Et}_{2} \mathrm{O}(3 \times 50 \mathrm{~mL})$ extrahiert. Die vereinigten organischen Phasen wurden mit ges. $\mathrm{NaCl}-$ Lösung (100 mL) gewaschen, über $\mathrm{Na}_{2} \mathrm{SO}_{4}$ getrocknet und das Lösungsmittel unter vermindertem Druck entfernt. Die Zielverbindung 218 (13.2 g, $60.8 \mathrm{mmol}$, quant.) wurde in Form einer farblosen Flüssigkeit isoliert.

${ }^{\mathbf{1}} \mathbf{H}$-NMR $\left(300 \mathrm{MHz}, \mathrm{CDCl}_{3}\right): \delta(\mathrm{ppm})=3.91-3.94\left(\mathrm{~m}, 4 \mathrm{H}, 1-\mathrm{H}_{2}, 4-\mathrm{H}_{2}\right), 5.94(\mathrm{ddd}, J=6.4$ 4.4, $2.1 \mathrm{~Hz}, 2 \mathrm{H}, 2-\mathrm{H}, 3-\mathrm{H})$.

${ }^{13}$ C-NMR (126 MHz, $\left.\mathrm{CDCl}_{3}\right): \delta(\mathrm{ppm})=30.9(\mathrm{C}-1, \mathrm{C}-4), 130.7$ (C-2, C-3).

$\mathbf{C}_{4} \mathbf{H}_{6} \mathbf{B r}_{2}(213.90)$. 


\subsection{4 (Z)-1-Azido-4-brombut-2-en (222c)}
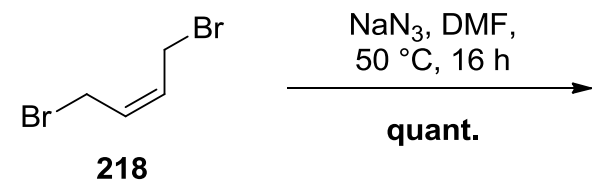

quant.<smiles>N[C@H](C=CCBr)C(=O)O</smiles>

222c

Zu einer Lösung des Dibromids 218 (1.00 g, 4.68 mmol, 1.0 Äq.) in DMF (10 mL) wurde bei Raumtemperatur $\mathrm{NaN}_{3}$ (304 mg, $4.68 \mathrm{mmol}, 1.0$ Äq.) gegeben, auf $50{ }^{\circ} \mathrm{C}$ erhitzt und $16 \mathrm{~h}$ gerührt. Anschließend wurde die Lösung auf Raumtemperatur abgekühlt und mit ges. $\mathrm{NaHCO}_{3}$-Lösung $(10 \mathrm{~mL})$ und ges. $\mathrm{Na}_{2} \mathrm{~S}_{2} \mathrm{O}_{3}$-Lösung $(10 \mathrm{~mL})$ versetzt. Es wurde mit $\mathrm{Et}_{2} \mathrm{O}$ $(3 \times 10 \mathrm{~mL})$ extrahiert, die vereinigten organischen Phasen mit ges. NaCl-Lösung $(20 \mathrm{~mL})$ gewaschen, über $\mathrm{Na}_{2} \mathrm{SO}_{4}$ getrocknet und das Lösungsmittel unter vermindertem Druck entfernt. Die Zielverbindung 222c (824 mg, $4.68 \mathrm{mmol}$, quant.) wurde in Form einer farblosen Flüssigkeit erhalten.

${ }^{\mathbf{1}} \mathbf{H}$-NMR $\left(300 \mathrm{MHz}, \mathrm{CDCl}_{3}\right): \delta(\mathrm{ppm})=3.75-3.83\left(\mathrm{~m}, 2 \mathrm{H}, 1-\mathrm{H}_{2}\right), 3.88-3.96\left(\mathrm{~m}, 2 \mathrm{H}, 4-\mathrm{H}_{2}\right)$, 5.74-5.84 (m, 1 H. 2-H), 5.89-5.98 (m, 1 H, 3-H).

$\mathbf{C}_{4} \mathbf{H}_{6} \mathbf{B r N}_{3}(176.01)$. 


\subsubsection{1,4-Dibrombut-2-in (220)}

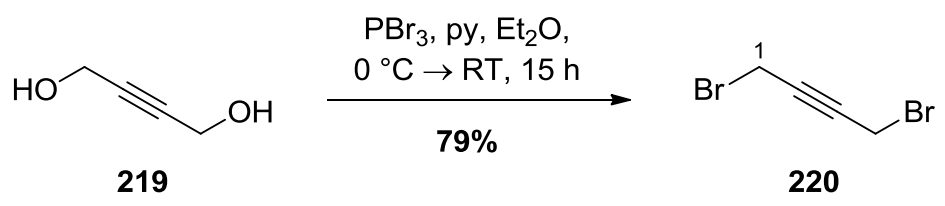

Zu einer Lösung des Diols 219 (5.00 g, $58.1 \mathrm{mmol}, 1.0$ Äq.) in $\mathrm{Et}_{2} \mathrm{O}$ (75 mL) wurde bei $0{ }^{\circ} \mathrm{C}$ Pyridin (937 $\mu \mathrm{L}, 919 \mathrm{mg}, 11.6 \mathrm{mmol}, 0.2$ Äq.) gegeben und $5 \mathrm{~min}$ gerührt. Anschließend wurde $\mathrm{PBr}_{3}$ (4.91 mL, $14.1 \mathrm{~g}, 52.3 \mathrm{mmol}, 0.9$ Äq.) zugegeben, weitere $30 \mathrm{~min}$ bei $0{ }^{\circ} \mathrm{C}$ gerührt und auf Raumtemperatur erwärmt. Nach 15 h wurde die Reaktion durch Zugabe von ges. $\mathrm{NaHCO}_{3}$-Lösung $(25 \mathrm{~mL})$ und ges. $\mathrm{Na}_{2} \mathrm{~S}_{2} \mathrm{O}_{3}$-Lösung $(25 \mathrm{~mL})$ beendet und mit $\mathrm{Et}_{2} \mathrm{O}$ $(3 \times 50 \mathrm{~mL})$ extrahiert. Die vereinigten organischen Phasen wurden mit ges. NaCl-Lösung (100 mL) gewaschen, über $\mathrm{Na}_{2} \mathrm{SO}_{4}$ getrocknet und das Lösungsmittel unter vermindertem Druck entfernt. Die Zielverbindung 220 (9.74 g, 46.0 mmol, 79\%) wurde in Form einer farblosen Flüssigkeit isoliert.

${ }^{\mathbf{1}} \mathbf{H}-\mathbf{N M R}\left(300 \mathrm{MHz}, \mathrm{CDCl}_{3}\right): \delta(\mathrm{ppm})=3.93\left(\mathrm{~s}, 4 \mathrm{H}, 1-\mathrm{H}_{2}, 4-\mathrm{H}_{2}\right)$.

MS (EI, $70 \mathrm{eV}): \mathrm{m} / \mathrm{z}(\%)=131.0$ (99), 132.9 (100) [M-Br] $]^{+}, 209.9$ (9), 211.9 (16), 213.9 (9) $[\mathrm{M}]^{+*}$.

HRMS von $\mathrm{C}_{4} \mathrm{H}_{4} \mathrm{Br}_{2}$ ber.: 211.8659 gef.: $211.8654[\mathrm{M}]^{+*}(\mathrm{EI}-\mathrm{HRMS})$.

$\mathbf{C}_{4} \mathbf{H}_{4} \mathbf{B r}_{2}$ (211.88). 


\subsubsection{1-Azido-4-brombut-2-in (222d)}

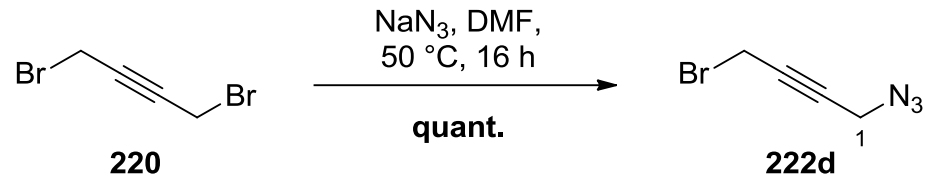

Zu einer Lösung des Dibromids 220 (1.00 g, 4.72 mmol, 1.0 Äq.) in DMF (10 mL) wurde bei Raumtemperatur $\mathrm{NaN}_{3}$ (307 mg, $4.72 \mathrm{mmol}, 1.0$ Äq.) gegeben, auf $50{ }^{\circ} \mathrm{C}$ erhitzt und $16 \mathrm{~h}$ gerührt. Anschließend wurde die Lösung auf Raumtemperatur abgekühlt und mit ges. $\mathrm{NaHCO}_{3}$-Lösung $(10 \mathrm{~mL})$ und ges. $\mathrm{Na}_{2} \mathrm{~S}_{2} \mathrm{O}_{3}$-Lösung $(10 \mathrm{~mL})$ versetzt. Es wurde mit $\mathrm{Et}_{2} \mathrm{O}$ $(3 \times 10 \mathrm{~mL})$ extrahiert, die vereinigten organischen Phasen mit ges. NaCl-Lösung $(20 \mathrm{~mL})$ gewaschen, über $\mathrm{Na}_{2} \mathrm{SO}_{4}$ getrocknet und das Lösungsmittel unter vermindertem Druck entfernt. Da das Zielprodukt 222d zu einer raschen Polymerisation neigt, ist eine direkte Umsetzung erforderlich.

${ }^{1} \mathbf{H}-\mathbf{N M R}\left(300 \mathrm{MHz}, \mathrm{CDCl}_{3}\right): \delta(\mathrm{ppm})=3.93\left(\mathrm{~s}, 2 \mathrm{H}, 4-\mathrm{H}_{2}\right), 3.98\left(\mathrm{~s}, 2 \mathrm{H}, 1-\mathrm{H}_{2}\right)$.

$\mathbf{C}_{4} \mathbf{H}_{4} \mathbf{B r N}_{3}$ (174.00). 


\subsubsection{4-Azidocyclohexanol (225)}

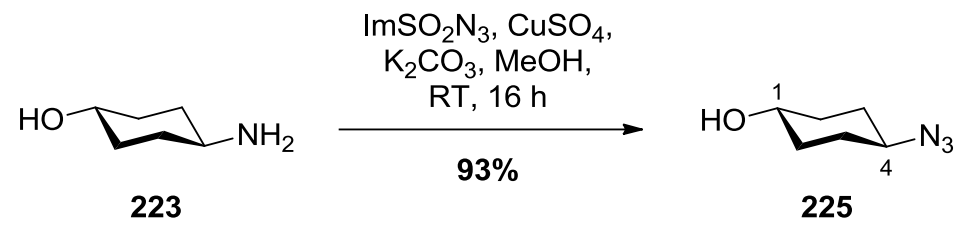

$\mathrm{Zu}$ einer Lösung aus Amin 223 (500 mg, 4.34 mmol, 1.0 Äq.), $\mathrm{CuSO}_{4}$ (10.8 mg, $43.4 \mu \mathrm{mol}$, $1 \mathrm{~mol} \%$ ) und $\mathrm{K}_{2} \mathrm{CO}_{3}$ (660 mg, $4.78 \mathrm{mmol}, 1.1$ Äq.) in $\mathrm{MeOH}$ (20 mL) wurde bei Raumtemperatur das Hydrochlorid von 224 (1.09 g, 5.21 mmol, 1.2 Äq.) gegeben und $16 \mathrm{~h}$ bei dieser Temperatur gerührt. Das Lösungsmittel wurde anschließend unter vermindertem Druck entfernt und $\mathrm{H}_{2} \mathrm{O}(50 \mathrm{~mL})$ sowie $\mathrm{HCl}$ (konz., $2.5 \mathrm{~mL}$ ) zugefügt. Die Mischung wurde mit EtOAc $(3 \times 30 \mathrm{~mL})$ extrahiert, die vereinigten organischen Phasen mit ges. NaCl-Lösung (50 mL) gewaschen, über $\mathrm{Na}_{2} \mathrm{SO}_{4}$ getrocknet und das Lösungsmittel unter vermindertem Druck entfernt. Säulenchromatographische Reinigung an Kieselgel ( $n$ Pentan/EtOAc 4:1) lieferte die Zielverbindung 225 (569 mg, $4.03 \mathrm{mmol}$, 93\%) in Form eines farblosen Feststoffes.

DC: $\mathrm{R}_{f}=0.25(n$ Pentan/EtOAc $4: 1)$.

UV $\left(\mathrm{CH}_{3} \mathrm{CN}\right)$ : keine Absorption.

IR (ATR): $\tilde{v}\left(\mathrm{~cm}^{-1}\right)=3275,2939,2862,2082,1454,1369,1263,1228,1062,917,903,566$.

${ }^{1} \mathbf{H}-\mathbf{N M R}\left(300 \mathrm{MHz}, \mathrm{CDCl}_{3}\right): \delta(\mathrm{ppm})=1.24-1.46\left(\mathrm{~m}, 4 \mathrm{H}, 2-\mathrm{H}_{\mathrm{A}}, 3-\mathrm{H}_{\mathrm{A}}, 5-\mathrm{H}_{\mathrm{A}}, 6-\mathrm{H}_{\mathrm{A}}\right)$, 1.90-2.05 (m, $\left.4 \mathrm{H}, 2-\mathrm{H}_{\mathrm{B}}, 3-\mathrm{H}_{\mathrm{B}}, 5-\mathrm{H}_{\mathrm{B}}, 6-\mathrm{H}_{\mathrm{B}}\right), 3.32$ (tt, $\left.J=10.1,3.4 \mathrm{~Hz}, 1 \mathrm{H}, 4-\mathrm{H}\right), 3.63$ (tt, $J=$ 9.5, 3.5 Hz, $1 \mathrm{H}, 1-\mathrm{H})$.

${ }^{13}$ C-NMR $\left(126 \mathrm{MHz}, \mathrm{CDCl}_{3}\right): \delta(\mathrm{ppm})=28.9(\mathrm{C}-3, \mathrm{C}-5), 32.6(\mathrm{C}-2, \mathrm{C}-6), 58.9(\mathrm{C}-4), 68.8$ (C-1).

MS (EI, $70 \mathrm{eV}): \mathrm{m} / \mathrm{z}(\%)=141.1(7)[\mathrm{M}]^{+\cdot}$.

HRMS von $\mathrm{C}_{6} \mathrm{H}_{11} \mathrm{~N}_{3} \mathrm{O}$ ber.: 141.0902 gef.: $141.0908\left[^{+M}\right]^{+\cdot}($ EI-HRMS).

$\mathbf{C}_{6} \mathbf{H}_{11} \mathbf{N}_{3} \mathrm{O}$ (141.17). 


\subsection{8 (S)-5-(4-Azidobutoxy)-6-((tert-butyldimethylsilyl)oxy)-1-((2-methoxyethoxy)-} methoxy)hexan (226)

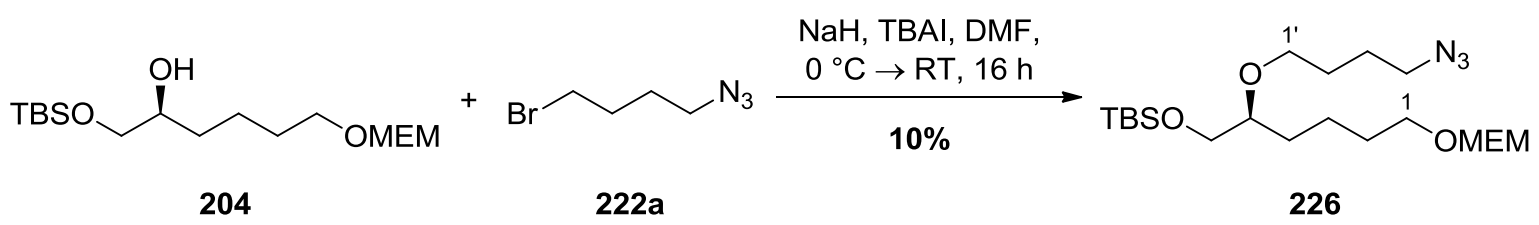

Zu einer Lösung aus Alkohol 204 (150 mg, 446 mol, 1.0 Äq.), Bromid 222a (95.2 mg, $535 \mu \mathrm{mol}, 1.2$ Äq.) und TBAI (16.5 mg, $44.6 \mu \mathrm{mol}, 10 \mathrm{~mol} \%$ ) in DMF (15 mL) wurde bei $0{ }^{\circ} \mathrm{C}$ portionsweise $\mathrm{NaH}$ (60\%ig in Mineralöl, $21.4 \mathrm{mg}, 535 \mu \mathrm{mol}, 1.2 \mathrm{Äq}$.) gegeben und $1 \mathrm{~h}$ bei dieser Temperatur gerührt. Anschließend wurde langsam auf Raumtemperatur erwärmt und weitere $15 \mathrm{~h}$ gerührt. Die Reaktion wurde durch Zugabe von ges. $\mathrm{NaHCO}_{3}$-Lösung $(10 \mathrm{~mL})$ beendet und mit $\mathrm{Et}_{2} \mathrm{O}(3 \times 15 \mathrm{~mL})$ extrahiert. Die vereinigten organischen Phasen wurden mit ges. NaCl-Lösung $(30 \mathrm{~mL})$ gewaschen, über $\mathrm{Na}_{2} \mathrm{SO}_{4}$ getrocknet und das Lösungsmittel unter vermindertem Druck entfernt. Säulenchromatographische Reinigung an Kieselgel ( $n$ Pentan/EtOAc 5:1 $\rightarrow 2: 1$ ) lieferte die Zielverbindung 226 (19.0 mg, $43.8 \mu \mathrm{mol}$, 10\%) in Form einer farblosen Flüssigkeit.

DC: $\mathrm{R}_{f}=0.48(n$ Pentan/EtOAc 5:1).

${ }^{1} \mathbf{H}-\mathbf{N M R}\left(300 \mathrm{MHz}, \mathrm{CDCl}_{3}\right): \delta(\mathrm{ppm})=0.02\left(\mathrm{~s}, 6 \mathrm{H}, 2 \times \mathrm{SiCH}_{3}\right), 0.85\left(\mathrm{~s}, 9 \mathrm{H}, \mathrm{SiC}\left(\mathrm{CH}_{3}\right)_{3}\right)$, 1.30-1.50 (m, 4 H, 3- $\left.\mathrm{H}_{2}, 4-\mathrm{H}_{2}\right), 1.50-1.74$ (m, $\left.6 \mathrm{H}, 2-\mathrm{H}_{2}, 2^{\prime}-\mathrm{H}_{2}, 3^{\prime}-\mathrm{H}_{2}\right), 3.20-3.33$ (m, $2 \mathrm{H}$, 4'- $\left.\mathrm{H}_{2}\right), 3.36\left(\mathrm{~s}, 3 \mathrm{H}, \mathrm{OCH}_{3}\right), 3.39\left(\mathrm{t}, J=6.6 \mathrm{~Hz}, 2 \mathrm{H}, 1^{\prime}-\mathrm{H}_{2}\right), 3.46-3.55\left(\mathrm{~m}, 6 \mathrm{H}, 1-\mathrm{H}_{2}, 6-\mathrm{H}_{2}\right.$, $\mathrm{OCH}_{2} \mathrm{CH}_{2} \mathrm{OMe}$ ), 3.62-3.69 (m, $\left.2 \mathrm{H}, \mathrm{OCH}_{2} \mathrm{CH}_{2} \mathrm{OMe}\right), 3.69-3.77\left(\mathrm{~m}_{\mathrm{c}}, 1 \mathrm{H}, 5-\mathrm{H}\right), 4.67$ (s, $2 \mathrm{H}$, $\left.\mathrm{OCH}_{2} \mathrm{O}\right)$.

${ }^{13}$ C-NMR $\left(126 \mathrm{MHz}, \mathrm{CDCl}_{3}\right): \delta(\mathrm{ppm})=-4.8,-4.4\left(\mathrm{Si}\left(\mathrm{CH}_{3}\right)_{2}\right), 18.1\left(\mathrm{Si} \underline{\mathrm{C}}\left(\mathrm{CH}_{3}\right)_{3}\right), 21.9(\mathrm{C}-3)$, $25.8\left(\mathrm{SiC}\left(\underline{\mathrm{CH}}_{3}\right)_{3}\right), 26.8\left(\mathrm{C}-3^{\prime}\right), 28.2\left(\mathrm{C}-2^{\prime}\right), 29.8(\mathrm{C}-2), 34.5(\mathrm{C}-4), 51.3\left(\mathrm{C}-4{ }^{\prime}\right), 58.9\left(\mathrm{OCH}_{3}\right)$, 65.6 (C-6), $66.6\left(\mathrm{OCH}_{2} \mathrm{CH}_{2} \mathrm{OMe}\right), 67.8$ (C-1), 70.5 (C-1'), 71.3 (C-5), $71.8\left(\mathrm{OCH}_{2} \mathrm{CH}_{2} \mathrm{OMe}\right)$, $95.4\left(\mathrm{OCH}_{2} \mathrm{O}\right)$.

MS (ESI): $\mathrm{m} / \mathrm{z}(\%)=456.3(100)[\mathrm{M}+\mathrm{Na}]^{+}$.

HRMS von $\mathrm{C}_{20} \mathrm{H}_{43} \mathrm{~N}_{3} \mathrm{O}_{5} \mathrm{Si}$ ber.: $\quad 456.2864$

gef.: $\quad 456.2864[\mathrm{M}+\mathrm{Na}]^{+}(\mathrm{ESI}-\mathrm{HRMS})$.

$\mathrm{C}_{20} \mathrm{H}_{43} \mathrm{~N}_{3} \mathrm{O}_{5} \mathrm{Si}$ (433.67). 


\subsection{Glykosylierung des Spinosyn-Analogons 156}

4.6.1 (3a $S, 5 \mathrm{a} R, 5 \mathrm{~b} S, 9 S, 13 S, 14 R, 16 \mathrm{a} S, 16 \mathrm{~b} R)-13-(2,3,4,6$-Tetradesoxy-4-azido- $\alpha / \beta-\mathrm{D}-$ glucopyranosyl)-9-ethyl-14-methyl-3,3a,-5b,6,9,10,11,12,13,14-decahydro- $1 \mathrm{H}$-asindaceno[3,2-d][1] ]xacyclododecin-2,7,15(5aH,16aH,16bH)trion $((\alpha / \beta)-156)$

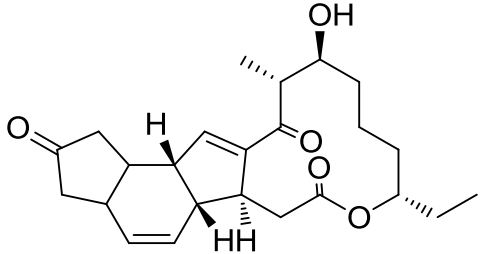

157

$+$

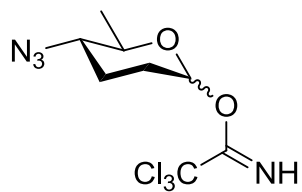

197

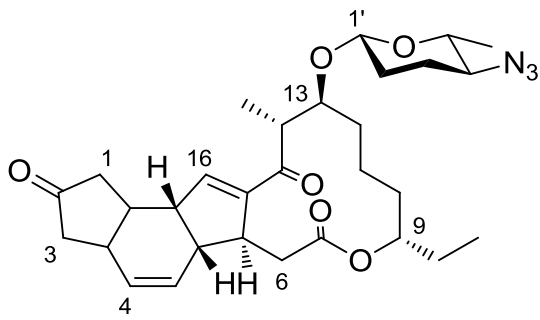

( $\beta)-156$

$\mathrm{Et}_{3} \mathrm{SiH}, \mathrm{I}_{2}$, MS $5 \AA, \mathrm{CH}_{2} \mathrm{Cl}_{2}$, $-87^{\circ} \mathrm{C}, 8 \mathrm{~h}$ $51 \%$ $\alpha / \beta=1: 1.5$

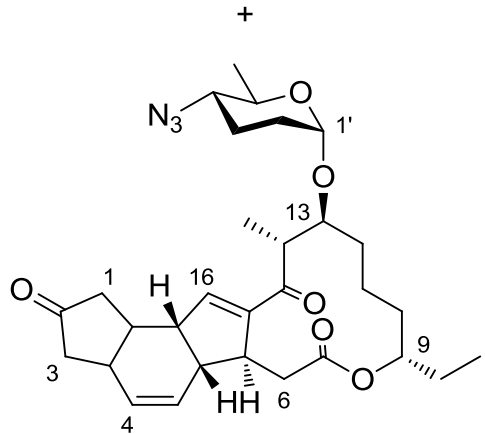

$(\alpha)-156$

Eine Lösung aus Alkohol 157 (15.0 mg, $37.5 \mu \mathrm{mol}, 1.0$ Äq.) und frisch hergestelltem Azid 197 (16.9 mg, $56.2 \mu \mathrm{mol}, 1.5$ Äq.) in $\mathrm{CH}_{2} \mathrm{Cl}_{2}(5 \mathrm{~mL})$ wurde bei Raumtemperatur 30 min über fein gemörsertem, aktiviertem Molekularsieb $5 \AA$ gerührt. Anschließend wurde die Reaktionslösung auf $-87^{\circ} \mathrm{C}$ gekühlt, langsam $\mathrm{Et}_{3} \mathrm{SiH}(11.2 \mu \mathrm{L}, 8.23 \mathrm{mg}, 70.4 \mu \mathrm{mol}, 1.9 \mathrm{Äq}$.) und eine Lösung aus $\mathrm{I}_{2}(14.3 \mathrm{mg}, 56.2 \mu \mathrm{mol}, 1.5$ Äq.) in Toluol ( $3 \mathrm{~mL})$ zugegeben und $8 \mathrm{~h}$ bei dieser Temperatur gerührt. Die Reaktion wurde durch Zugabe von $\mathrm{NEt}_{3}(1 \mathrm{~mL})$ beendet und ges. $\mathrm{NaHCO}_{3}$-Lösung $(5 \mathrm{~mL})$ sowie ges. $\mathrm{Na}_{2} \mathrm{~S}_{2} \mathrm{O}_{3}$-Lösung $(5 \mathrm{~mL})$ zugegeben. Es wurde mit $\mathrm{CH}_{2} \mathrm{Cl}_{2}(3 \times 5 \mathrm{~mL})$ extrahiert, die vereinigten organischen Phasen mit ges. NaCl-Lösung $(10 \mathrm{~mL})$ gewaschen und über $\mathrm{Na}_{2} \mathrm{SO}_{4}$ getrocknet. Säulenchromatographische Reinigung an Kieselgel (Petrolether/EtOAc 7:1) lieferte die Zielverbindung ( $\beta$ )-156 (6.12 mg, $11.3 \mu \mathrm{mol}$, 30\%) und das Anomer $(\alpha)$-156 (4.21 mg, $7.78 \mu \mathrm{mol}, 21 \%)$ jeweils in Form eines farblosen Feststoffes.

Analytische Daten für $(\alpha)-\mathbf{1 5 6}$ :

DC: $\mathbf{R}_{f}=0.64$ (Petrolether/EtOAc 2:1).

Drehwert: $[\alpha]_{\mathrm{D}}^{20}=+96.4^{\circ}\left(c=0.055, \mathrm{CHCl}_{3}\right)$. 
UV $\left(\mathrm{CH}_{3} \mathrm{CN}\right): \lambda_{\max }(\lg \varepsilon)=241 \mathrm{~nm}(3.8910)$

IR (ATR): $\tilde{v}\left(\mathrm{~cm}^{-1}\right)=2932,2096,1745,1717,1659,1458,1374,1260,1226,992,734$.

${ }^{1} \mathbf{H}-\mathbf{N M R}\left(600 \mathrm{MHz}, \mathrm{CDCl}_{3}\right): \delta(\mathrm{ppm})=0.79\left(\mathrm{t}, J=7.4 \mathrm{~Hz}, 3 \mathrm{H}, 9-\mathrm{CH}_{2} \mathrm{CH}_{3}\right), 0.97-1.08(\mathrm{~m}$, $\left.1 \mathrm{H}, 11-\mathrm{H}_{\mathrm{a}}\right), 1.15-1.29\left(\mathrm{~m}, 8 \mathrm{H}, 10-\mathrm{H}_{\mathrm{A}}, 14-\mathrm{CH}_{3}, 3-\mathrm{H}_{\mathrm{A}}, 6-\mathrm{H}_{3}\right), 1.36-1.48\left(\mathrm{~m}, 3 \mathrm{H}, 12-\mathrm{H}_{\mathrm{A}}\right.$, $\left.11-\mathrm{H}_{\mathrm{B}}, 9-\mathrm{CH}_{\mathrm{A}} \mathrm{H}_{\mathrm{B}} \mathrm{CH}_{3}\right), 1.47-1.61\left(\mathrm{~m}, 3 \mathrm{H}, 12-\mathrm{H}_{\mathrm{B}}, 9-\mathrm{CH}_{\mathrm{A}} \mathrm{H}_{\mathrm{B}} \mathrm{CH}_{3}, 2^{\prime}-\mathrm{H}_{\mathrm{A}}\right), 1.62-1.69(\mathrm{~m}, 1 \mathrm{H}$, $\left.10-\mathrm{H}_{\mathrm{B}}\right), 1.69-2.03(\mathrm{~m}, 5 \mathrm{H}), 2.35-2.52(\mathrm{~m}, 5 \mathrm{H})\left(6-\mathrm{H}_{\mathrm{A}}, 3-\mathrm{H}_{\mathrm{B}}, 1-\mathrm{H}_{2}, 2^{\prime}-\mathrm{H}_{\mathrm{B}}, 3^{\prime}-\mathrm{H}_{2}, 4^{\prime}-\mathrm{H}\right)$, 2.93-3.02 (m, 2 H, 16b-H, 16a-H), 3.04 (dd, $J=13.6,4.9$ Hz, 1 H, 6-H $)$, 3.09-3.14 (m, 1 H, 5b-H), 3.28-3.33 (m, $1 \mathrm{H}, 14-\mathrm{H}), 3.54-3.56(\mathrm{~m}, 1 \mathrm{H}, 5 \mathrm{a}-\mathrm{H}), 3.62-3.65(\mathrm{~m}, 1 \mathrm{H}, 3 \mathrm{a}-\mathrm{H})$, 3.78-3.81 (m, $1 \mathrm{H}, 13-\mathrm{H}), 4.08$ (q, $J=7.1 \mathrm{~Hz}, 1 \mathrm{H}, 5 '-\mathrm{H}), 4.61-4.66$ (m, $1 \mathrm{H}, 9-\mathrm{H}), 4.84$ (t, $\left.J=2.3 \mathrm{~Hz}, 1 \mathrm{H}, 1^{\prime}-\mathrm{H}\right), 5.83(\mathrm{dt}, J=9.5,3.0 \mathrm{~Hz}, 1 \mathrm{H}, 5-\mathrm{H}), 5.91(\mathrm{dt}, J=9.5,2.2 \mathrm{~Hz}, 1 \mathrm{H}, 4-\mathrm{H})$, $6.75(\mathrm{~s}, 1 \mathrm{H}, 16-\mathrm{H})$.

${ }^{13}$ C-NMR $\left(126 \mathrm{MHz}, \mathrm{CDCl}_{3}\right): \delta(\mathrm{ppm})=9.5\left(9-\mathrm{CH}_{2} \underline{\mathrm{CH}}_{3}\right), 18.3,18.7\left(14-\mathrm{CH}_{3}, \mathrm{C}-6\right.$ '), 19.8 (C-11), 23.9 (C-2'), 27.9 (9- $\left.\mathrm{CH}_{2} \mathrm{CH}_{3}\right), 29.6,30.5,30.9$ (C-12, C-5', C-4'), 34.8 (C-6), 40.5 (C10), 41.1 (C-5a), 43.2, 43.9, 44.9 (C-1, C-3, C-3'), 46.2 (C-14), 47.8 (C-5b), 49.5 (C-16a), 62.5 (C-16b), 67.9 (C-3a), 75.8 (C-13), 76.1 (C-9), 91.7 (C-1'), 128.1 (C-4), 129.7 (C-5), 144.1 (C-15a), 145.7 (C-16), 172.2 (C-7), 202.2 (C-15), 215.7 (C-2).

MS (ESI): $m / z(\%)=562.3(100)[\mathrm{M}+\mathrm{Na}]^{+}$.

HRMS von $\mathrm{C}_{30} \mathrm{H}_{41} \mathrm{~N}_{3} \mathrm{O}_{6}$ ber.: $\quad 562.2888$ gef.: $\quad 562.2890[\mathrm{M}+\mathrm{Na}]^{+}(\mathrm{ESI}-\mathrm{HRMS})$.

$\mathrm{C}_{30} \mathrm{H}_{41} \mathbf{N}_{3} \mathbf{O}_{6}$ (539.66).

Analytische Daten für $(\beta)-\mathbf{1 5 6}$ :

DC: $\mathbf{R}_{f}=0.57$ (Pentrolether/EtOAc 2:1).

Drehwert: $[\alpha]_{\mathrm{D}}^{20}=-191.5^{\circ}\left(c=0.051, \mathrm{CHCl}_{3}\right)$.

UV $\left(\mathrm{CH}_{3} \mathrm{CN}\right): \lambda_{\max }(\lg \varepsilon)=235 \mathrm{~nm}(3.715)$.

IR (ATR): $\tilde{v}\left(\mathrm{~cm}^{-1}\right)=2926,2100,1743,1717,1657,1457,1371,1259,1226,1160,1064$, 989, 735.

${ }^{1} \mathbf{H}-\mathbf{N M R}\left(600 \mathrm{MHz}, \mathrm{CDCl}_{3}\right): \delta(\mathrm{ppm})=0.80\left(\mathrm{t}, J=7.5 \mathrm{~Hz}, 3 \mathrm{H}, 9-\mathrm{CH}_{2} \mathrm{CH}_{3}\right), 1.10-1.19(\mathrm{~m}$, $\left.4 \mathrm{H}, 6{ }^{\prime}-\mathrm{H}_{3}, 12-\mathrm{H}_{\mathrm{A}}\right), 1.19-1.30\left(\mathrm{~m}, 5 \mathrm{H}, 14-\mathrm{CH}_{3}, 11-\mathrm{H}_{2}\right), 1.35-1.79(\mathrm{~m}, 9 \mathrm{H}), 1.87-2.00$ (m, $3 \mathrm{H})\left(1-\mathrm{H}_{2}, 3-\mathrm{H}_{\mathrm{A}}, 9-\mathrm{CH}_{2} \mathrm{CH}_{3}, 10-\mathrm{H}_{2}, 12-\mathrm{H}_{\mathrm{B}}, 2^{\prime}-\mathrm{H}_{2}, 3-\mathrm{H}_{2}\right), 2.11-2.18(\mathrm{~m}, 1 \mathrm{H}, 16 \mathrm{a}-\mathrm{H})$, 2.33-2.45 (m, $\left.2 \mathrm{H}, 6-\mathrm{H}_{\mathrm{A}}, 4^{\prime}-\mathrm{H}\right), 2.50\left(\mathrm{dd}, J=17.6,7.1 \mathrm{~Hz}, 1 \mathrm{H}, 3-\mathrm{H}_{\mathrm{B}}\right), 2.94-3.01(\mathrm{~m}, 2 \mathrm{H}$, 16b-H, 3a-H), 3.04-3.14 (m, 2 H, 6-H $\left.\mathrm{H}_{\mathrm{B}}, 5 \mathrm{~b}-\mathrm{H}\right), 3.23-3.31$ (m, $\left.2 \mathrm{H}, 14-\mathrm{H}, 51-\mathrm{H}\right), 3.50-3.58$ $\left(\mathrm{m}_{\mathrm{c}}, 1 \mathrm{H}, 5 \mathrm{a}-\mathrm{H}\right), 3.62(\mathrm{dt}, J=9.3,4.4 \mathrm{~Hz}, 1 \mathrm{H}, 13-\mathrm{H}), 4.41-4.47$ (m, $\left.1 \mathrm{H}, 1^{\prime}-\mathrm{H}\right), 4.62-4.68$ (m, 
$1 \mathrm{H}, 9-\mathrm{H}), 5.86(\mathrm{dt}, J=9.8,2.9 \mathrm{~Hz}, 1 \mathrm{H}, 5-\mathrm{H}), 5.92(\mathrm{dt}, J=9.8,2.1 \mathrm{~Hz}, 1 \mathrm{H}, 4-\mathrm{H}), 6.72$ (s, $1 \mathrm{H}, 16-\mathrm{H})$.

${ }^{13}$ C-NMR $\left(126 \mathrm{MHz}, \mathrm{CDCl}_{3}\right): \delta(\mathrm{ppm})=9.5\left(9-\mathrm{CH}_{2} \mathrm{CH}_{3}\right), 16.3\left(\mathrm{C}-6\right.$ '), $18.8\left(14-\mathrm{CH}_{3}\right), 21.5$ (C-12), 28.0 (C-16a), $28.5\left(9-\mathrm{CH}_{2} \mathrm{CH}_{3}\right), 30.3^{*}, 30.3^{*}, 34.3$ (C-6), 34.4* (* = C-11, C-2', C-3'), 40.7 (C-4'), 41.2 (C-5a), 43.2 (C-1), 43.9 (C-3), 44.6 (C-10), 47.6 (C-5'), 47.8 (C-5b), 49.3 (C-3a), 62.0 (C-16b), 74.3 (C-14), 77.2 (C-9), 81.0 (C-13), 103.0 (C-1'), 128.1 (C-4), 129.4 (C-5), 144.6 (C-15a), 146.0 (C-16), 172.3 (C-7), 202.3 (C-15), 215.7 (C-2).

MS (ESI): $m / z(\%)=562.3(100)[\mathrm{M}+\mathrm{Na}]^{+}$.

HRMS von $\mathrm{C}_{30} \mathrm{H}_{41} \mathrm{~N}_{3} \mathrm{O}_{6}$ ber.: 562.2888 gef.: $562.2879[\mathrm{M}+\mathrm{Na}]^{+}(\mathrm{ESI}-\mathrm{HRMS})$.

$\mathrm{C}_{30} \mathbf{H}_{41} \mathbf{N}_{3} \mathbf{O}_{6}$ (539.66). 


\section{Enantioselektive Synthese von (+)-Linoxepin}

\subsection{Synthese des Benzylbromids 165}

\subsubsection{5-Iodbenzo[d][1,3]dioxol (240)}

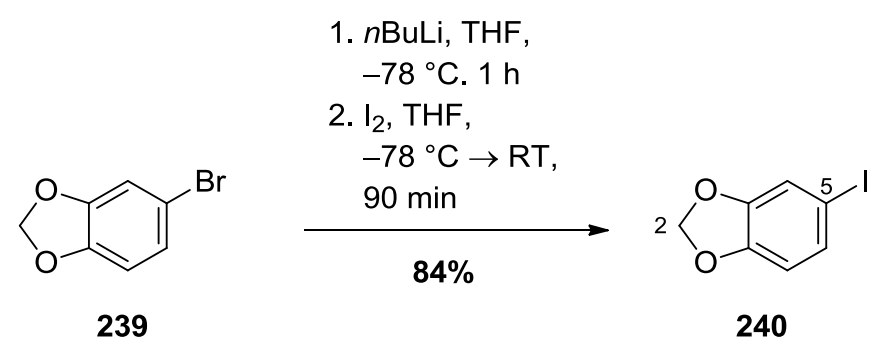

Zu einer Lösung aus 5-Brombenzo[d][1,3]dioxol (239) (3.00 mL, 5.01 g, 24.9 mmol, 1.0 Äq.) in THF $(50 \mathrm{~mL})$ wurde bei $-78^{\circ} \mathrm{C}$ langsam $n \operatorname{BuLi}(2.5 \mathrm{M}$ in $n$ Hexan, $11.0 \mathrm{~mL}, 27.5 \mathrm{mmol}$, 1.1 Äq.) getropft und $1 \mathrm{~h}$ bei dieser Temperatur gerührt. Anschließend wurde eine Lösung aus Iod (9.50 g, 37.4 mmol, 1.5 Äq.) in THF (20 mL) zugegeben und das Reaktionsgemisch über $5 \mathrm{~h}$ auf Raumtemperatur erwärmt. Die Reaktion wurde durch Zugabe von ges. $\mathrm{NH}_{4} \mathrm{Cl}$-Lösung (75 mL) und ges. $\mathrm{Na}_{2} \mathrm{~S}_{2} \mathrm{O}_{3}$-Lösung $(100 \mathrm{~mL})$ beendet und das Gemisch mit $\mathrm{Et}_{2} \mathrm{O}(3 \times 100 \mathrm{~mL})$ extrahiert. Die vereinten organischen Phasen wurden mit ges. $\mathrm{NaHCO}_{3}$-Lösung $(2 \times 50 \mathrm{~mL})$ gewaschen, über $\mathrm{Na}_{2} \mathrm{SO}_{4}$ getrocknet und das Lösungsmittel im Vakuum entfernt. Nach säulenchromatographischer Reinigung an Kieselgel (nPentan) wurde das Produkt 240 (5.22 g, $21.0 \mathrm{mmol}, 84 \%$ ) als hellgelbes Öl isoliert.

DC: $\mathbf{R}_{f}=0.24$ ( $n$ Pentan).

UV $\left(\mathrm{CH}_{3} \mathrm{CN}\right): \lambda_{\max }(\lg \varepsilon)=206 \mathrm{~nm}$ (4.4682), 242 (3.8308), 292 (3.5516).

IR (ATR): $\tilde{v}\left(\mathrm{~cm}^{-1}\right)=2889,1469,1224,1030,931,863,794,567$.

${ }^{1} \mathbf{H}-\mathbf{N M R}\left(300 \mathrm{MHz}, \mathrm{CDCl}_{3}\right): \delta(\mathrm{ppm})=5.95\left(\mathrm{~s}, 2 \mathrm{H}, 2-\mathrm{H}_{2}\right), 6.59(\mathrm{dd}, J=7.9,0.5 \mathrm{~Hz}, 1 \mathrm{H}$, 7-H), 7.12 (dd, $J=1.8,0.8 \mathrm{~Hz}, 1 \mathrm{H}, 4-\mathrm{H}), 7.14$ (dd, $J=7.9,1.7 \mathrm{~Hz}, 1 \mathrm{H}, 6-\mathrm{H})$.

${ }^{13}$ C-NMR $\left(126 \mathrm{MHz}, \mathrm{CDCl}_{3}\right): \delta(\mathrm{ppm})=82.2(\mathrm{C}-5), 101.4(\mathrm{C}-2), 110.4(\mathrm{C}-7), 117.6(\mathrm{C}-4)$, 130.6 (C-6), 147.8 (C-7a), 148.6 (C-3a).

MS (EI, $70 \mathrm{eV}): \mathrm{m} / \mathrm{z}(\%)=121.0(28)[\mathrm{M}-\mathrm{I}]^{+}, 247.9(100)[\mathrm{M}]^{+.}$.

HRMS von $\mathrm{C}_{7} \mathrm{H}_{5} \mathrm{IO}_{2}$

ber.: 247.9334

gef.: $247.9340[\mathrm{M}]^{+\cdot}(\mathrm{EI}-\mathrm{HRMS})$.

$\mathbf{C}_{7} \mathbf{H}_{5} \mathbf{I O}_{2}$ (248.01). 


\subsubsection{5-Iodbenzo $[d][1,3]$ dioxol-4-carbaldehyd (241)}

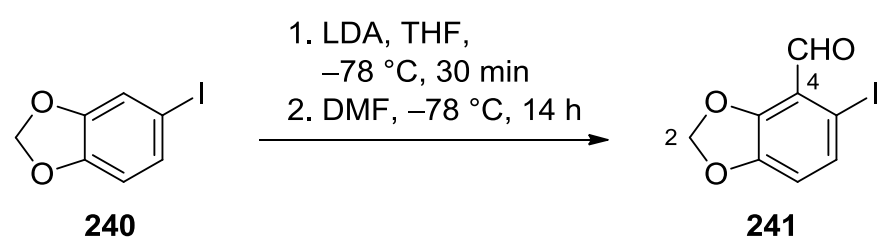

Eine frisch hergestellte Lösung von LDA (266) (61.8 mmol, 3.0 Äq.) in THF (60 mL) wurde bei $-78{ }^{\circ} \mathrm{C}$ langsam zu einer Lösung des Iodids 240 (5.10 g, $20.6 \mathrm{mmol}, 1.0$ Äq.) in THF $(250 \mathrm{~mL})$ gegeben und $30 \mathrm{~min}$ bei dieser Temperatur gerührt. DMF $(15.9 \mathrm{~mL}, 15.1 \mathrm{~g}$, $206 \mathrm{mmol}, 10.0$ Äq.) wurde hinzugegeben und das Gemisch weitere $18 \mathrm{~h}$ bei $-78{ }^{\circ} \mathrm{C}$ gerührt. Nach Zugabe von ges. $\mathrm{NH}_{4} \mathrm{Cl}$-Lösung $(250 \mathrm{~mL})$ wurde mit $\mathrm{CH}_{2} \mathrm{Cl}_{2}(3 \times 200 \mathrm{~mL})$ extrahiert, die vereinten organischen Phasen über $\mathrm{Na}_{2} \mathrm{SO}_{4}$ getrocknet und das Lösungsmittel unter vermindertem Druck entfernt. Das Rohprodukt 241 wurde ohne weitere Aufreinigung zum Alkohol 242 umgesetzt.

Analytische Daten des Rohprodukts:

MS (ESI): m/z (\%) = $298.9(41)[\mathrm{M}+\mathrm{Na}]^{+}, 330.9(55)[\mathrm{M}+\mathrm{MeOH}+\mathrm{Na}]^{+}$.

HRMS von $\mathrm{C}_{8} \mathrm{H}_{5} \mathrm{IO}_{3}$ ber.: 298.9176 gef.: $298.9182[\mathrm{M}+\mathrm{Na}]^{+}(\mathrm{ESI}-\mathrm{HRMS})$.

$\mathrm{C}_{8} \mathrm{H}_{5} \mathrm{IO}_{3}$ (276.03). 


\subsubsection{4-Hydroxymethyl-5-iodbenzo[d][1,3]dioxol (242)}<smiles>O=Cc1c(I)ccc2c1OCO2</smiles>

241
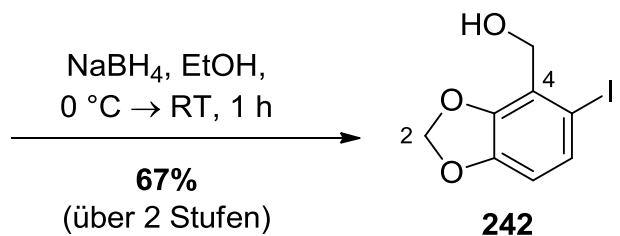

242

$\mathrm{Zu}$ einer Suspension des Aldehyds 241 ( 61.8 mmol, 1.0 Äq.) in EtOH (45 mL) wurde bei $0{ }^{\circ} \mathrm{C}$ portionsweise $\mathrm{NaBH}_{4}(780 \mathrm{mg}, 20.6 \mathrm{mmol}, 1.0$ Äq.) gegeben und das Gemisch nach beendeter Zugabe innerhalb von $1 \mathrm{~h}$ auf Raumtemperatur erwärmt. Anschließend wurde ges. $\mathrm{NH}_{4} \mathrm{Cl}$-Lösung $(80 \mathrm{~mL})$ zugefügt und das Gemisch mit $\mathrm{CH}_{2} \mathrm{Cl}_{2}(3 \times 60 \mathrm{~mL})$ extrahiert. Die vereinigten organischen Phasen wurden über $\mathrm{Na}_{2} \mathrm{SO}_{4}$ getrocknet und das Rohprodukt mittels Säulenchromatographie an Kieselgel ( $n$ Pentan/EtOAc 3:1) gereinigt. Die Zielverbindung 242 ( $3.83 \mathrm{~g}, 13.8 \mathrm{mmol}, 67 \%$ über 2 Stufen) wurde in Form eines farblosen Feststoffs erhalten.

DC: $\mathbf{R}_{f}=0.43(n$ Pentan/EtOAc $=3: 1)$.

UV $\left(\mathrm{CH}_{3} \mathrm{CN}\right): \lambda_{\max }(\lg \varepsilon)=209 \mathrm{~nm}$ (4.5285), 244 (3.8411), 296 (3.6278).

IR (ATR): $\tilde{v}\left(\mathrm{~cm}^{-1}\right)=3357,1447,1235,1047,926,795$.

${ }^{1} \mathbf{H}$-NMR $\left(300 \mathrm{MHz}, \mathrm{CDCl}_{3}\right): \delta(\mathrm{ppm})=2.15(\mathrm{~s}, 1 \mathrm{H}, \mathrm{OH}), 4.70\left(\mathrm{~s}, 2 \mathrm{H}, \mathrm{CH}_{2} \mathrm{OH}\right), 6.00(\mathrm{~s}$, $\left.2 \mathrm{H}, 2-\mathrm{H}_{2}\right), 6.54(\mathrm{~d}, J=8.2 \mathrm{~Hz}, 1 \mathrm{H}, 7-\mathrm{H}), 7.28(\mathrm{~d}, J=8.2 \mathrm{~Hz}, 1 \mathrm{H}, 6-\mathrm{H})$.

${ }^{13} \mathrm{C}-\mathrm{NMR}\left(126 \mathrm{MHz}, \mathrm{CDCl}_{3}\right): \delta(\mathrm{ppm})=63.3\left(\mathrm{CH}_{2} \mathrm{OH}\right), 88.2(\mathrm{C}-5), 101.6(\mathrm{C}-2), 110.1$ (C-7), 124.2 (C-4), 132.0 (C-6), 146.5 (C-3a), 148.5 (C-7a).

MS (ESI): $\mathrm{m} / \mathrm{z}(\%)=301.0(100)[\mathrm{M}+\mathrm{Na}]^{+}, 578.9(30)[2 \mathrm{M}+\mathrm{Na}]^{+}$.

HRMS von $\mathrm{C}_{8} \mathrm{H}_{7} \mathrm{IO}_{3}$ ber.: 300.9332 gef.: $300.9336[\mathrm{M}+\mathrm{Na}]^{+}(\mathrm{ESI}-\mathrm{HRMS})$.

$\mathrm{C}_{8} \mathrm{H}_{7} \mathrm{IO}_{3}(278.05)$. 


\subsubsection{4-(Brommethyl)-5-iodbenzo[d] $[1,3]$ dioxol (165)}<smiles>OCc1c(I)ccc2c1OCO2</smiles>

242

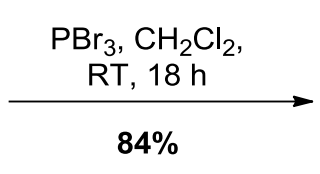

$84 \%$

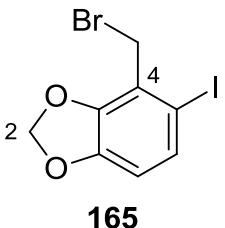

165

$\mathrm{Zu}$ einer Lösung aus Benzylalkohol 242 (3.76 g, $13.5 \mathrm{mmol}, 1.0$ Äq.) in $\mathrm{CH}_{2} \mathrm{Cl}_{2}$ (100 mL) wurde bei Raumtemperatur langsam $\mathrm{PBr}_{3}(2.54 \mathrm{~mL}, 7.32 \mathrm{~g}, 27.0 \mathrm{mmol}, 2.0$ Äq.) getropft und $18 \mathrm{~h}$ gerührt. Das Lösungsmittel wurde unter vermindertem Druck entfernt und das Rohprodukt langsam mit $0{ }^{\circ} \mathrm{C}$ kalter ges. $\mathrm{NaHCO}_{3}$-Lösung $(220 \mathrm{~mL})$ versetzt. Anschließend wurde mit $\mathrm{CH}_{2} \mathrm{Cl}_{2}(3 \times 200 \mathrm{~mL})$ extrahiert, die vereinten organischen Phasen über $\mathrm{Na}_{2} \mathrm{SO}_{4}$ getrocknet und das Lösungsmittel unter vermindertem Druck entfernt. Säulenchromatographische Reinigung an Kieselgel (nPentan/EtOAc 30:1) lieferte das Benzylbromid 165 (3.87 g, $11.3 \mathrm{mmol}, 84 \%)$ als farbloses Öl.

DC: $\mathrm{R}_{f}=0.46(n$ Pentan/EtOAc $=30: 1)$.

UV $\left(\mathrm{CH}_{3} \mathrm{CN}\right): \lambda_{\max }(\lg \varepsilon): 205 \mathrm{~nm}(4.3387), 312$ (3.5798).

IR (ATR): $\tilde{v}\left(\mathrm{~cm}^{-1}\right)=2900,1454,1251,1050,924,804,564$.

${ }^{1} \mathbf{H}-\mathbf{N M R}\left(300 \mathrm{MHz}, \mathrm{CDCl}_{3}\right): \delta(\mathrm{ppm})=4.55\left(\mathrm{~s}, 2 \mathrm{H}, \mathrm{CH}_{2} \mathrm{Br}\right), 6.05\left(\mathrm{~s}, 2 \mathrm{H}, 2-\mathrm{H}_{2}\right), 6.54(\mathrm{~d}, J=$ $8.2 \mathrm{~Hz}, 1 \mathrm{H}, 7-\mathrm{H}), 7.31$ (d, $J=8.2 \mathrm{~Hz}, 1 \mathrm{H}, 6-\mathrm{H})$.

${ }^{13}$ C-NMR $\left(126 \mathrm{MHz}, \mathrm{CDCl}_{3}\right): \delta(\mathrm{ppm})=31.5\left(\mathrm{CH}_{2} \mathrm{Br}\right), 88.9(\mathrm{C}-5), 102.1(\mathrm{C}-2), 110.5(\mathrm{C}-7)$, 121.8 (C-4), 132.3 (C-6), 146.6 (C-7a), 148.2 (C-3a).

MS (EI, $70 \mathrm{eV}): \mathrm{m} / \mathrm{z}(\%)=260.9(100)[\mathrm{M}-\mathrm{Br}]^{+}, 339.9,341.9(21)[\mathrm{M}]^{+*}$.

HRMS von $\mathrm{C}_{8} \mathrm{H}_{6} \mathrm{BrIO}_{2}$ ber.: $\quad 339.8596,341.8577$ gef.: $\quad 339.8594,341.8577[\mathrm{M}]^{+\cdot}($ EI-HRMS).

$\mathrm{C}_{8} \mathrm{H}_{6} \mathrm{BrIO}_{2}$ (340.94). 


\subsection{Synthese des Vinylsilans 164}

\subsubsection{2-Brom-3-acetoxy-4-methoxybenzaldehyd (244)}<smiles>COc1ccc(C=O)c(Br)c1O</smiles>

243

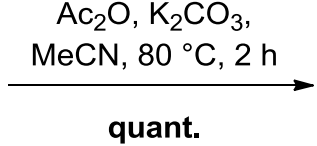

quant.

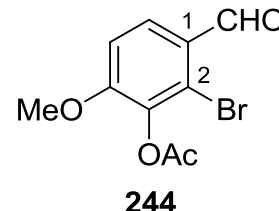

244

$\mathrm{Zu}$ einer Lösung aus Isovanillin (243) (5.00 g, $21.6 \mathrm{mmol}, 1.0$ Äq.) in MeCN (150 mL) wurden $\mathrm{K}_{2} \mathrm{CO}_{3}$ (6.57 g, $47.5 \mathrm{mmol}, 2.2$ Äq.) und $\mathrm{Ac}_{2} \mathrm{O}$ (2.40 mL, $2.61 \mathrm{~g}, 25.6 \mathrm{mmol}, 1.2$ Äq.) gegeben und die Reaktionsmischung $2 \mathrm{~h}$ bei $80{ }^{\circ} \mathrm{C}$ gerührt. Nach dem Abkühlen auf Raumtemperatur wurde die Reaktion durch Zugabe von ges. $\mathrm{NH}_{4} \mathrm{Cl}$-Lösung $(100 \mathrm{~mL})$ beendet und die Reaktionslösung anschließend mit $\mathrm{CH}_{2} \mathrm{Cl}_{2}(3 \times 100 \mathrm{~mL})$ extrahiert. Die vereinigten organischen Phasen wurden mit ges. $\mathrm{NaHCO}_{3}$-Lösung $(100 \mathrm{~mL})$ und ges. $\mathrm{NaCl}$ Lösung (100 mL) gewaschen, über $\mathrm{Na}_{2} \mathrm{SO}_{4}$ getrocknet und das Lösungsmittel unter vermindertem Druck entfernt. Säulenchromatographische Reinigung an Kieselgel ( $n$ Pentan/EtOAc 6:1 $\rightarrow 4: 1)$ lieferte die Zielverbindung 244 (6.15 g, 22.5 mmol, quant.) in Form von farblosen Kristallen.

DC: $\mathrm{R}_{f}=0.31(n$ Pentan/EtOAc 6:1).

UV $\left(\mathrm{CH}_{3} \mathrm{CN}\right): \lambda_{\max }(\lg \varepsilon)=201 \mathrm{~nm}(4.3663), 278$ (3.7762).

IR (ATR): $\tilde{v}\left(\mathrm{~cm}^{-1}\right)=1774,1686,1585,1487,1278,1252,1188,1167,1139,1021,880,811$, 515.

${ }^{1} \mathbf{H}-\mathbf{N M R}\left(300 \mathrm{MHz}, \mathrm{CDCl}_{3}\right): \delta(\mathrm{ppm})=2.40\left(\mathrm{~s}, 3 \mathrm{H}, \mathrm{OC}(\mathrm{O}) \mathrm{CH}_{3}\right), 3.92\left(\mathrm{~s}, 3 \mathrm{H}, \mathrm{OCH}_{3}\right), 7.02$ (d, $J=8.8 \mathrm{~Hz}, 1 \mathrm{H}, 5-\mathrm{H}), 7.87$ (d, $J=8.8 \mathrm{~Hz}, 1 \mathrm{H}, 6-\mathrm{H}), 10.22$ (d, $J=0.7 \mathrm{~Hz}, \mathrm{CHO})$.

${ }^{13}$ C-NMR $\left(126 \mathrm{MHz}, \mathrm{CDCl}_{3}\right): \delta(\mathrm{ppm})=20.3\left(\mathrm{OC}(\mathrm{O}) \underline{\mathrm{CH}}_{3}\right), 56.5\left(\mathrm{OCH}_{3}{ }^{\prime}\right), 110.9(\mathrm{C}-5), 122.5$ (C-2), 127.1 (C-3), 128.7 (C-4), 138.0 (C-1), 157.1 (C-6), 167.7 (C=O), 190.2 (CHO).

MS (ESI): $m / z(\%)=273.0,275.0(12)[\mathrm{M}+\mathrm{H}]^{+}, 295.0,297.0(79)[\mathrm{M}+\mathrm{Na}]^{+}, 327.0,329.0$ (100) $[\mathrm{M}+\mathrm{MeOH}+\mathrm{Na}]^{+}$.

HRMS von $\mathrm{C}_{10} \mathrm{H}_{9} \mathrm{BrO}_{4}$ ber.: $294.9576,296.9556$ gef.: $\quad 294.9576,296.9559[\mathrm{M}+\mathrm{Na}]^{+}(\mathrm{ESI}-\mathrm{HRMS})$.

$\mathrm{C}_{10} \mathrm{H}_{9} \mathrm{BrO}_{4}(273.08)$. 


\subsubsection{1-(2-Brom-3-hydroxy-4-methoxyphenyl)-3-(dimethyl(phenyl)-silyl)-prop-2-in-1-} yl-acetat (246)<smiles>COc1ccc(C=O)c(OC(C)=O)c1Br</smiles>

244

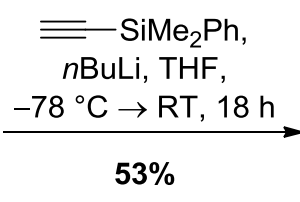

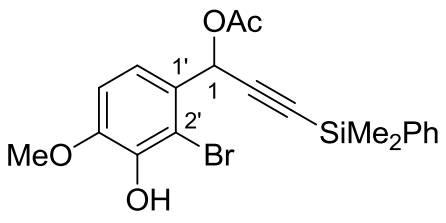

246

$\mathrm{Zu}$ einer Lösung aus Dimethylphenylsilylacetylen (1.00 g, $1.10 \mathrm{~mL}, 6.24 \mathrm{mmol}, 1.2$ Äq.) in THF (20 mL) wurde bei $-50{ }^{\circ} \mathrm{C}$ langsam eine Lösung aus $n$ BuLi $(2.5 \mathrm{M}$ in Hexan, $2.29 \mathrm{~mL}$, $5.72 \mathrm{mmol}, 1.1$ Äq.) gegeben. Die Reaktionsmischung wurde $30 \mathrm{~min}$ bei $-50{ }^{\circ} \mathrm{C}$ gerührt und anschließend auf $-78{ }^{\circ} \mathrm{C}$ abgekühlt. Anschließend wurde eine Lösung des Aldehyds 244 (1.42 g, $5.20 \mathrm{mmol}, 1.0$ Äq.) in THF (10 mL) zugegeben und die Reaktionsmischung über Nacht auf Raumtemperatur erwärmt. Die Reaktion wurde durch Zugabe von ges. $\mathrm{NH}_{4} \mathrm{Cl}$ Lösung $(60 \mathrm{~mL})$ beendet, die organische Phase abgetrennt und die wässrige Phase mit $\mathrm{CH}_{2} \mathrm{Cl}_{2}$ $(3 \times 65 \mathrm{~mL})$ extrahiert. Die vereinigten organischen Phasen wurden mit ges. NaCl-Lösung $(100 \mathrm{~mL})$ gewaschen, über $\mathrm{Na}_{2} \mathrm{SO}_{4}$ getrocknet und das Lösungsmittel unter vermindertem Druck entfernt. Die Zielverbindung 246 (1.18 g, $2.73 \mathrm{mmol}, 53 \%)$ wurde nach säulenchromatographischer Reinigung an Kieselgel ( $n$ Pentan/EtOAc 5:1 $\rightarrow 3: 1$ ) als farbloses Öl erhalten.

DC: $\mathbf{R}_{f}=0.49(n$ Pentan/EtOAc 3:1).

$\mathbf{U V}\left(\mathrm{CH}_{3} \mathrm{CN}\right): \lambda_{\max }(\lg \varepsilon)=208 \mathrm{~nm}$ (4.6211), 287 (3.5208).

IR (ATR): $\tilde{v}\left(\mathrm{~cm}^{-1}\right)=3490,2180,1746,1604,1489,1218,1031,816,781,731,698$.

${ }^{1} \mathbf{H}-\mathbf{N M R}\left(300 \mathrm{MHz}, \mathrm{CDCl}_{3}\right): \delta(\mathrm{ppm})=0.45\left(\mathrm{~s}, 6 \mathrm{H}, \mathrm{Si}\left(\mathrm{CH}_{3}\right)_{2}\right), 2.11\left(\mathrm{~s}, 3 \mathrm{H}, \mathrm{OC}(\mathrm{O}) \mathrm{CH}_{3}\right)$, $3.92\left(\mathrm{~s}, 3 \mathrm{H}, \mathrm{OCH}_{3}\right), 6.08$ (s, $\left.1 \mathrm{H}, \mathrm{OH}\right), 6.72(\mathrm{~s}, 1 \mathrm{H}, 1-\mathrm{H}), 6.86\left(\mathrm{~d}, J=8.6 \mathrm{~Hz}, 1 \mathrm{H}, 5^{\prime}-\mathrm{H}\right)$, 7.35-7.42 (m, 4 H, 6'-H, 3×SiPh-H), 7.60-7.66 (m, 2 H, 2×SiPh-H).

${ }^{13}$ C-NMR $\left(126 \mathrm{MHz}, \mathrm{CDCl}_{3}\right): \delta(\mathrm{ppm})=-1.1\left(\mathrm{Si}\left(\mathrm{CH}_{3}\right)_{2}\right), 20.8\left(\mathrm{OC}(\mathrm{O}) \underline{\mathrm{CH}}_{3}\right), 56.3\left(\mathrm{OCH}_{3}\right)$, 65.4 (C-1), 90.8 (C-2), 102.4 (C-3), 109.3 (C-5'), 109.9 (C-2'), 120.8 (C-4'), 127.9 (2×SiPh-C), 128.5 (SiPh-C), 129.5 (SiPh-C), 133.7 (2×SiPh-C), 136.3 (C-3'), 143.0 (C-1'), $147.6\left(\mathrm{C}^{-6}\right), 169.4(\mathrm{C}=\mathrm{O})$.

MS (ESI): $m / z(\%)=457.1(44)[\mathrm{M}+\mathrm{Na}]^{+}, 889.1(86)[2 \mathrm{M}+\mathrm{Na}]^{+} ; 433.0(100)[\mathrm{M}-\mathrm{H}]^{-}$.

HRMS von $\mathrm{C}_{20} \mathrm{H}_{21} \mathrm{BrO}_{4} \mathrm{Si} \quad$ ber.: $431.0320,433.0292$ $455.0285,457.0265$

gef.: $\quad 431.0306,433.0300[\mathrm{M}-\mathrm{H}]^{-}$ 455.0273, 457.0256 [M+Na ${ }^{+}$(ESI-HRMS). 
$\mathrm{C}_{20} \mathrm{H}_{21} \mathrm{BrO}_{4} \mathrm{Si}$ (433.37). 


\subsubsection{2-Brom-3-(3-(dimethyl(phenyl)silyl)prop-2-yn-1-yl)-6-methoxyphenol (247)}<smiles>COc1ccc(C(C#CSc2ccccc2)OC(C)=O)c(Br)c1O</smiles>

246

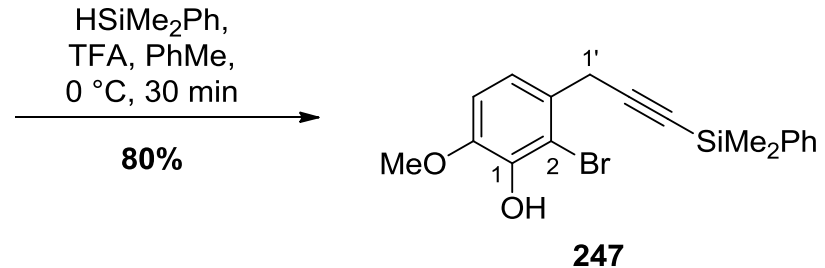

247

$\mathrm{Zu}$ einer Lösung aus Verbindung 246 (980 mg, $2.26 \mathrm{mmol}, 1.0$ Äq.) in Toluol (15 mL) wurden bei $0{ }^{\circ} \mathrm{C}$ langsam TFA $(526 \mu \mathrm{L}, \quad 772 \mathrm{mg}, \quad 6.75 \mathrm{mmol}, \quad 3.0$ Äq. $)$ und Dimethylphenylsilan (1.04 mL, $923 \mathrm{mg}, 6.75 \mathrm{mmol}, 3.0$ Äq.) gegeben. Es wurde für $30 \mathrm{~min}$ bei $0{ }^{\circ} \mathrm{C}$ gerührt, die Reaktion durch Zugabe von ges. NaCl-Lösung $(50 \mathrm{~mL})$ beendet und die organische Phase abgetrennt. Nach dem Extrahieren der wässrigen Phase mit $\mathrm{CH}_{2} \mathrm{Cl}_{2}$ $(1 \times 100 \mathrm{~mL}, 2 \times 50 \mathrm{~mL})$ wurden die vereinigten organischen Phasen über $\mathrm{Na}_{2} \mathrm{SO}_{4}$ getrocknet und das Lösungsmittel unter vermindertem Druck entfernt. Die Zielverbindung 247 (677 mg, $1.80 \mathrm{mmol}, 80 \%)$ wurde mittels Säulenchromatographie an Kieselgel ( $n$ Pentan/EtOAc 9:1) als farbloses Öl erhalten.

DC: $\mathbf{R}_{f}=0.33(n$ Pentan/EtOAc 9:1).

UV $\left(\mathrm{CH}_{3} \mathrm{CN}\right): \lambda_{\max }(\lg \varepsilon) 204 \mathrm{~nm}$ (4.6884), 286 (3.6023).

IR (ATR): $\tilde{v}\left(\mathrm{~cm}^{-1}\right)=3490,2174,1605,1487,1283,1248,1114,1031,948,815,779,730$, 697.

${ }^{1} \mathbf{H}-\mathbf{N M R}\left(300 \mathrm{MHz}, \mathrm{CDCl}_{3}\right): \delta(\mathrm{ppm})=0.45\left(\mathrm{~s}, 6 \mathrm{H}, \mathrm{Si}\left(\mathrm{CH}_{3}\right)_{2}\right), 3.73\left(\mathrm{~s}, 2 \mathrm{H}, 1^{\prime}-\mathrm{H}_{2}\right), 3.90(\mathrm{~s}$, $\left.3 \mathrm{H}, \mathrm{OCH}_{3}\right), 6.01(\mathrm{~s}, 1 \mathrm{H}, \mathrm{OH}), 6.83(\mathrm{~d}, J=8.4 \mathrm{~Hz}, 1 \mathrm{H}, 5-\mathrm{H}), 7.17(\mathrm{dt}, J=8.4,0.7 \mathrm{~Hz}, 1 \mathrm{H}$, 4-H), 7.36-7.41 (m, 3 H, 3×SiPh-H), 7.64-7.70 (m, 2 H, 2×SiPh-H).

${ }^{13}$ C-NMR $\left(126 \mathrm{MHz}, \mathrm{CDCl}_{3}\right): \delta(\mathrm{ppm})=-0.7\left(\mathrm{Si}\left(\mathrm{CH}_{3}\right)_{2}\right), 26.7\left(\mathrm{C}-1^{\prime}\right), 56.3\left(\mathrm{OCH}_{3}\right), 85.8(\mathrm{C}-$ 2'), 105.3 (C-3'), 109.5 (C-5), 110.0 (C-2), 119.8 (C-4), 127.8 (2×SiPh-C), 128.5 (SiPh-C), 129.3 (SiPh-C), 133.7 (2×SiPh-C), 137.2 (C-3), 143.1 (C-1), 145.9 (C-6).

MS (ESI): $m / z(\%)=375.0(71)[\mathrm{M}]^{+}, 399.0(22)[\mathrm{M}+\mathrm{Na}]^{+}, 415.0(41)[\mathrm{M}+\mathrm{K}]^{+}$.

HRMS von $\mathrm{C}_{18} \mathrm{H}_{19} \mathrm{BrO}_{2} \mathrm{Si} \quad$ ber.: $373.0254,375.0234$ gef.: $\quad 373.0251,375.0233[\mathrm{M}-\mathrm{H}]^{-}(\mathrm{ESI}-\mathrm{HRMS})$.

$\mathrm{C}_{18} \mathrm{H}_{19} \mathrm{BrO} \mathrm{O}_{2} \mathrm{Si}$ (375.34). 


\subsection{4 (Z)-2-Brom-3-(3-(dimethyl(phenyl)silyl)allyl)-6-methoxyphenol (164)}

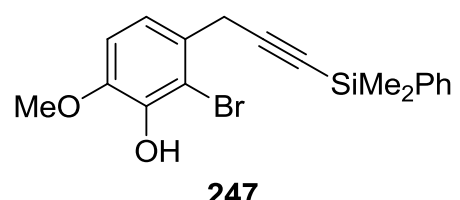

247

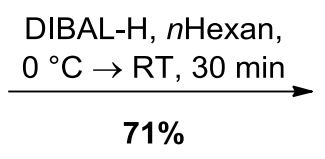

$71 \%$

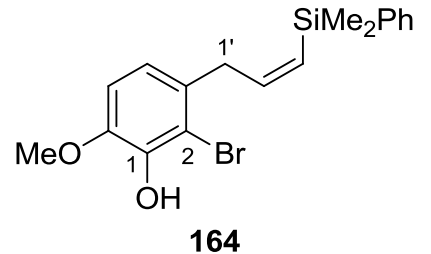

164

Zu einer Lösung des Alkins 247 (632 mg, 1.68 mmol, 1.0 Äq.) in $n$ Hexan (15 mL) wurde bei $0{ }^{\circ} \mathrm{C}$ langsam DIBAL-H ( $1 \mathrm{M}$ in Toluol, $5.02 \mathrm{~mL}, 5.02 \mathrm{mmol}, 3.0$ Äq. $)$ gegeben und die Reaktionslösung über $1 \mathrm{~h}$ auf Raumtemperatur erwärmt. Anschließend wurden bei $0{ }^{\circ} \mathrm{C} 3 \mathrm{M}$ $\mathrm{NaOH}$-Lösung (3.5 mL) und ges. Kaliumnatriumtartrat-Lösung $(25 \mathrm{~mL})$ zugegeben, $\mathrm{mit}_{\mathrm{Et}} \mathrm{O}$ $(3 \times 20 \mathrm{~mL})$ extrahiert und die vereinigten organischen Phasen mit ges. NaCl-Lösung $(25 \mathrm{~mL})$ gewaschen und über $\mathrm{Na}_{2} \mathrm{SO}_{4}$ getrocknet. Das Lösungsmittel wurde unter vermindertem Druck entfernt und das Rohprodukt mittels Säulenchromatographie an Kieselgel ( $n$ Pentan/EtOAc 9:1) gereinigt. Die Zielverbindung 164 (453 mg, $1.20 \mathrm{mmol}, 71 \%)$ wurde als farbloses Öl erhalten.

DC: $\mathrm{R}_{f}=0.41(n$ Pentan/EtOAc 9:1).

UV $\left(\mathrm{CH}_{3} \mathrm{CN}\right): \lambda_{\max }(\lg \varepsilon)=204 \mathrm{~nm}$ (4.7841), 285 (3.5443).

IR (ATR): $\tilde{v}\left(\mathrm{~cm}^{-1}\right)=3499,1605,1485,1281,1246,1111,1034,816,781,729,699$.

${ }^{1} \mathbf{H}-\mathbf{N M R}\left(300 \mathrm{MHz}, \mathrm{CDCl}_{3}\right): \delta(\mathrm{ppm})=0.45\left(\mathrm{~s}, 6 \mathrm{H}, \mathrm{Si}\left(\mathrm{CH}_{3}\right)_{2}\right), 3.47(\mathrm{dd}, J=7.2,1.4 \mathrm{~Hz}$, $\left.2 \mathrm{H}, 1^{\prime}-\mathrm{H}_{2}\right), 3.88\left(\mathrm{~s}, 3 \mathrm{H}, \mathrm{OCH}_{3}\right), 5.83$ (dt, $\left.J=13.9,1.5 \mathrm{~Hz}, 1 \mathrm{H}, 3^{\prime}-\mathrm{H}\right), 5.95$ (s, $\left.1 \mathrm{H}, \mathrm{OH}\right), 6.51$ $\left(\mathrm{dt}, J=13.9,7.1 \mathrm{~Hz}, 1 \mathrm{H}, 2^{\prime}-\mathrm{H}\right), 6.53$ (d, $\left.J=8.4 \mathrm{~Hz}, 1 \mathrm{H}, 4-\mathrm{H}\right), 6.71(\mathrm{~d}, J=8.4 \mathrm{~Hz}, 1 \mathrm{H}, 5-\mathrm{H})$, 7.34-7.39 (m, 3 H, 3×SiPh-H), 7.57-7.63 (m, 2 H, 2×SiPh-H).

${ }^{13}$ C-NMR $\left(126 \mathrm{MHz}, \mathrm{CDCl}_{3}\right): \delta(\mathrm{ppm})=-1.0\left(\mathrm{Si}\left(\mathrm{CH}_{3}\right)_{2}\right), 39.0\left(\mathrm{C}-1^{\prime}\right), 56.3\left(\mathrm{OCH}_{3}\right), 109.5$ (C-5), 110.8 (C-2), 120.1 (C-4), 127.8 (2×SiPh-C), 128.6 (SiPh-C), 129.0 (SiPh-C), 132.6 (C-3'), 133.8 (2×SiPh-C), 139.3 (C-3), 143.1 (C-1), 145.4 (C-6), 147.2 (C-2').

MS (ESI): $m / z(\%)=401.0(100)[\mathrm{M}+\mathrm{Na}]^{+}$.

HRMS von $\mathrm{C}_{17} \mathrm{H}_{21} \mathrm{BrO}_{2} \mathrm{Si} \quad$ ber.: $375.0421,377.0402$ 399.0386, 401.0367

gef.: $\quad 375.0419,377.0413\left[\mathrm{M}-\mathrm{H}^{-}\right.$ 399.0396, 401.0407 [M+Na] ${ }^{+}$(ESI-HRMS).

$\mathrm{C}_{18} \mathrm{H}_{21} \mathrm{BrO}_{2} \mathrm{Si}$ (377.35). 


\subsection{Synthese des Domino-Vorläufers 249}

\subsection{1 (Z)-(3-(2-Brom-3-((5-iodbenzo[d] [1,3]dioxol-4-yl)methoxy)-4-methoxyphenyl)-} prop-1-en-1-yl)dimethyl(phenyl)silan (162)
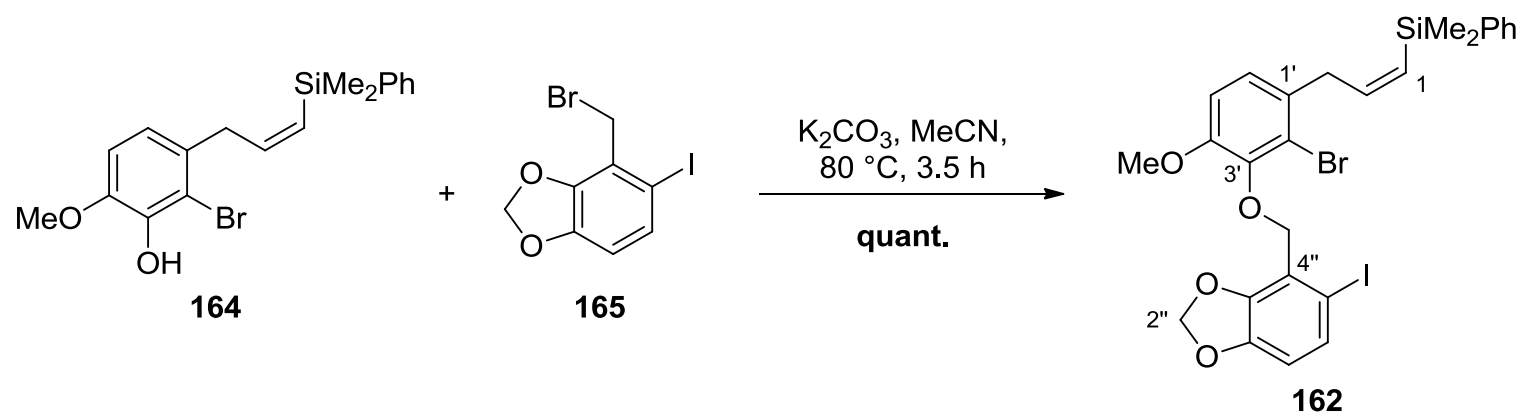

Zu einer Lösung aus Phenol 164 (265 mg, $702 \mu \mathrm{mol}, 1.0$ Äq) und Bromid 165 (287 mg, $843 \mu \mathrm{mol}$, 1.2 $\mathrm{Äq}$.) in $\mathrm{MeCN}(10 \mathrm{~mL})$ wurde bei Raumtemperatur $\mathrm{K}_{2} \mathrm{CO}_{3}$ (218 mg, 1.58 mmol, 2.2 Äq.) gegeben, die Reaktionslösung auf $80{ }^{\circ} \mathrm{C}$ erhitzt und $3.5 \mathrm{~h}$ gerührt. Nach dem Abkühlen auf Raumtemperatur wurde die Reaktion durch Zugabe von ges. NaCl-Lösung (20 mL) beendet, mit $\mathrm{CH}_{2} \mathrm{Cl}_{2}(3 \times 40 \mathrm{~mL})$ extrahiert und die vereinigten organischen Phasen über $\mathrm{Na}_{2} \mathrm{SO}_{4}$ getrocknet. Säulenchromatographische Reinigung an Kieselgel ( $n$ Pentan/EtOAc 30:1 $\rightarrow 15: 1)$ lieferte die Zielverbindung 162 (447 mg, $701 \mu \mathrm{mol}$, quant.) in Form eines farblosen Öls. Der überschüssige Anteil des Bromids 165 konnte dabei vollständig reisoliert werden.

DC: $\mathbf{R}_{f}=0.29(n$ Pentan/EtOAc 15:1).

UV $\left(\mathrm{CH}_{3} \mathrm{CN}\right): \lambda_{\max }(\lg \varepsilon)=203 \mathrm{~nm}$ (4.8215), 247 (4.0594), 304 (3.9061).

IR (ATR): $\tilde{v}\left(\mathrm{~cm}^{-1}\right)=2953,1593,1450,1246,1025,797,699$.

${ }^{1} \mathbf{H}-\mathbf{N M R}\left(300 \mathrm{MHz}, \mathrm{CDCl}_{3}\right): \delta(\mathrm{ppm})=0.41\left(\mathrm{~s}, 6 \mathrm{H}, \mathrm{Si}\left(\mathrm{CH}_{3}\right)_{2}\right), 3.43(\mathrm{dd}, J=7.1,1.4 \mathrm{~Hz}$, $\left.2 \mathrm{H}, 3-\mathrm{H}_{2}\right), 3.75\left(\mathrm{~s}, 3 \mathrm{H}, \mathrm{OCH}_{3}\right), 5.14\left(\mathrm{~s}, 2 \mathrm{H}, \mathrm{ArOCH}_{2}\right), 5.79(\mathrm{dt}, J=13.9,1.4 \mathrm{~Hz}, 1 \mathrm{H}, 1-\mathrm{H})$, 5.90 (s, 2 H, 2"-H $), 6.47$ (dt, $J=13.9,7.1 \mathrm{~Hz}, 1 \mathrm{H}, 2-\mathrm{H}), 6.53$ (d, J= 8.1 Hz, 1 H, 7"-H), 6.66 $\left(\mathrm{d}, J=8.5 \mathrm{~Hz}, 1 \mathrm{H}, 5^{\prime}-\mathrm{H}\right), 6.71\left(\mathrm{~d}, J=8.5 \mathrm{~Hz}, 1 \mathrm{H}, 66^{\prime}-\mathrm{H}\right), 7.29\left(\mathrm{~d}, J=8.2 \mathrm{~Hz}, 1 \mathrm{H}, 6^{\prime \prime}-\mathrm{H}\right)$, 7.32-7.37 (m, 3 H, 3×SiPh-H), 7.54-7.60 (m, 2 H, 2×SiPh-H).

${ }^{13}$ C-NMR $\left(126 \mathrm{MHz}, \mathrm{CDCl}_{3}\right): \delta(\mathrm{ppm})=-1.0\left(\mathrm{Si}\left(\mathrm{CH}_{3}\right)_{2}\right), 39.3(\mathrm{C}-3), 56.1\left(\mathrm{OCH}_{3}\right), 72.3$ $\left(\mathrm{ArOCH}_{2}\right), 90.3$ (C-5"), 101.6 (C-2"), 110.4 (C-7"), 111.2 (C-5'), 120.6 (C-2'), 121.5 (C-4"), 124.7 (C-6'), 127.8 (2×SiPh-C), 128.4 (C-1), 128.9 (2×SiPh-C), 131.9 (C-6"), 132.5 (SiPh-C), 133.8 (C-1'), 139.3 (SiPh-C), 145.1 (C-7a"), 147.4 (C-2), 147.8, 147.9 (C-3', C-3a"), 152.1 (C-4').

MS (ESI): $m / z(\%)=656.0(100)\left[\mathrm{M}+\mathrm{NH}_{4}\right]^{+}, 1297.0(100)[2 \mathrm{M}+\mathrm{Na}]^{+}$. 
HRMS von $\mathrm{C}_{26} \mathrm{H}_{26} \mathrm{BrIO}_{4} \mathrm{Si} \quad$ ber.: $\quad 658.9721,660.9702$

gef.: $\quad 658.9724,660.9704[\mathrm{M}+\mathrm{H}]^{+}($ESI-HRMS).

$\mathrm{C}_{26} \mathrm{H}_{26} \mathrm{BrIO}{ }_{4} \mathrm{Si}$ (637.38). 


\subsection{2 (Z)-((3-(4-((2-Brom-6-methoxy-3-(3-(dimethyl(phenyl)silyl)allyl)-phenoxy)-} methyl)benzo[d][1,3]dioxol-5-yl)prop-2-in-1-yl)oxy)triisopropylsilan (249)
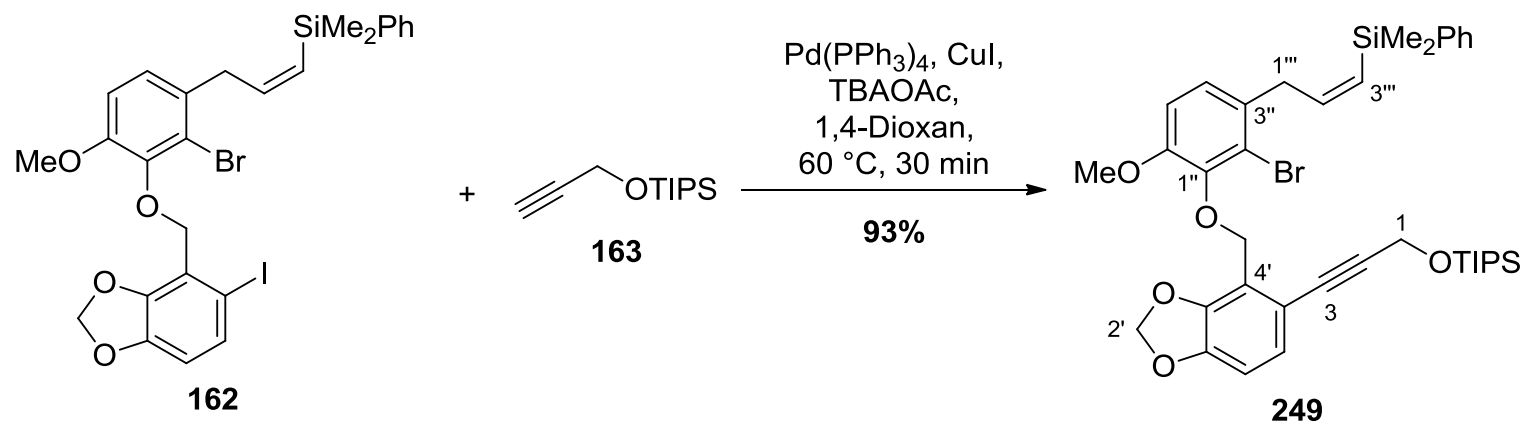

$\mathrm{Zu}$ einer Lösung aus Iodid 162 (420 mg, $659 \mu \mathrm{mol}, 1.0$ Äq) und Alkin 163 (168 mg, $791 \mu \mathrm{mol}, 1.2$ Äq.) in 1,4-Dioxan (5 mL) wurden bei Raumtemperatur $\mathrm{Pd}\left(\mathrm{PPh}_{3}\right)_{4}(38.1 \mathrm{mg}$, $32.9 \mu \mathrm{mol}, 5 \mathrm{~mol} \%)$, CuI (18.8 mg, $98.8 \mu \mathrm{mol}, 15 \mathrm{~mol} \%)$ und TBAOAc (596 mg, $1.98 \mathrm{mmol}$, 3.0 Äq.) gegeben, auf $60{ }^{\circ} \mathrm{C}$ erhitzt und $30 \mathrm{~min}$ gerührt. Nach dem Abkühlen auf Raumtemperatur wurde die Reaktion durch Zugabe von ges. $\mathrm{NH}_{4} \mathrm{Cl}$-Lösung (10 mL) beendet, mit $\mathrm{Et}_{2} \mathrm{O}(3 \times 10 \mathrm{~mL})$ extrahiert, die vereinigten organischen Phasen mit ges. NaCl-Lösung gewaschen und über $\mathrm{Na}_{2} \mathrm{SO}_{4}$ getrocknet. Säulenchromatographische Reinigung an Kieselgel ( $n$ Pentan/EtOAc 30:1 $\rightarrow 20: 1)$ lieferte die Zielverbindung 249 (444 mg, $615 \mu \mathrm{mol}, 93 \%$ ) in Form eines farblosen Öls.

DC: $\mathrm{R}_{f}=0.40$ (Petrolether/EtOAc 9:1).

UV $\left(\mathrm{CH}_{3} \mathrm{CN}\right): \lambda_{\max }(\lg \varepsilon)=267 \mathrm{~nm}$ (4.1301), 288 (3.7698), 308 (3.8100).

IR (ATR): $\tilde{v}\left(\mathrm{~cm}^{-1}\right)=2942,1597,1459,1246,1093,1037,813,681$.

${ }^{1} \mathbf{H}-\mathbf{N M R}\left(300 \mathrm{MHz}, \mathrm{CDCl}_{3}\right): \delta(\mathrm{ppm})=0.41\left(\mathrm{~s}, 6 \mathrm{H}, \mathrm{Si}\left(\mathrm{CH}_{3}\right)_{2}\right), 1.02-1.17(\mathrm{~m}, 21 \mathrm{H}$, $\left.\mathrm{Si}\left(\mathrm{CH}\left(\mathrm{CH}_{3}\right)_{2}\right)_{3}\right), 3.42$ (dd, $\left.J=7.1,1.2 \mathrm{~Hz}, 2 \mathrm{H}, 1{ }^{\prime \prime}-\mathrm{H}_{2}\right), 3.75\left(\mathrm{~s}, 3 \mathrm{H}, \mathrm{OCH}_{3}\right), 4.48(\mathrm{~s}, 2 \mathrm{H}$, 1-H $\mathrm{H}_{2}$ ), 5.17 (s, $2 \mathrm{H}, \mathrm{ArOCH}_{2}$ ), 5.77 (dt, $\left.J=13.9,1.3 \mathrm{~Hz}, 1 \mathrm{H}, 3^{\prime \prime \prime}-\mathrm{H}\right), 5.96$ (s, $\left.2 \mathrm{H}, 2^{\prime}-\mathrm{H}\right), 6.46$ (dt, $\left.J=14.1,7.1 \mathrm{~Hz}, 1 \mathrm{H}, 2^{\prime \prime}-\mathrm{H}\right), 6.62$ (d, $\left.J=8.5 \mathrm{~Hz}, 1 \mathrm{H}, 4 "-\mathrm{H}\right), 6.69$ (d, $J=8.5 \mathrm{~Hz}, 1 \mathrm{H}$, 5"-H), 6.71 (d, $\left.J=8.1 \mathrm{~Hz}, 1 \mathrm{H}, 7^{\prime}-\mathrm{H}\right), 6.96$ (d, $J=8.1 \mathrm{~Hz}, 1 \mathrm{H}, 6$ '-H), 7.31-7.37 (m, $3 \mathrm{H}$, $3 \times \mathrm{SiPh}-\mathrm{H})$, 7.53-7.59 (m, $2 \mathrm{H}, 3 \times \mathrm{SiPh}-\mathrm{H})$.

${ }^{13} \mathbf{C}-\mathbf{N M R}\left(126 \mathrm{MHz}, \mathrm{CDCl}_{3}\right): \delta(\mathrm{ppm})=-0.9\left(\mathrm{Si}\left(\mathrm{CH}_{3}\right)_{2}\right), 12.1\left(\mathrm{Si}\left(\underline{\mathrm{CH}}\left(\mathrm{CH}_{3}\right)_{2}\right)_{3}\right), 18.0$ $\left(\mathrm{Si}\left(\mathrm{CH}\left(\mathrm{CH}_{3}\right)_{2}\right)_{3}\right), 39.3\left(\mathrm{C}-1{ }^{\prime \prime \prime}\right), 52.5(\mathrm{C}-1), 56.2\left(\mathrm{OCH}_{3}\right), 67.4\left(\mathrm{ArOCH}_{2}\right), 82.1(\mathrm{C}-3), 89.9$ (C-2), 101.6 (C-2'), 108.4 (C-7'), 111.3 (C-5"), 117.5 (C-5'), 120.0 (C-4'), 120.6 (C-2"), 124.6 (C-4"), 126.6 (C-6'), 127.8 (2×SiPh-C), 128.3 (C-3"'), 128.9 (2×SiPh-C), 132.5 (SiPh-C), 133.8 (C-3"), 139.4 (SiPh-C), 145.7 (C-1"), 147.4 (C-3a'), 147.5 (C-2'"), 147.7 (C-7a'), 152.2 (C-6"). 
MS (ESI): $m / z(\%)=740.5(100)\left[\mathrm{M}+\mathrm{NH}_{4}\right]^{+}$.

HRMS von $\mathrm{C}_{38} \mathrm{H}_{49} \mathrm{BrO}_{5} \mathrm{Si}_{2} \quad$ ber.: $738.2640,740.2622$ gef.: $\quad 738.2635,740.2622\left[\mathrm{M}+\mathrm{NH}_{4}\right]^{+}(\mathrm{ESI}-\mathrm{HRMS})$.

$\mathrm{C}_{38} \mathrm{H}_{49} \mathrm{BrO}_{5} \mathrm{Si}_{2}$ (721.87). 


\subsection{Enantioselektive Synthese von (+)-Linoxepin (69)}

\subsection{1 (Z)-((7-((Dimethyl(phenyl)silyl)methylen)-11-methoxy-8,13-dihydro-7H-[1,3]-} dioxolo[4',5':3,4]benzo[1,2-e]naphtho[1,8-bc]oxepin-6-yl)methoxy)triisopropylsilan (161)

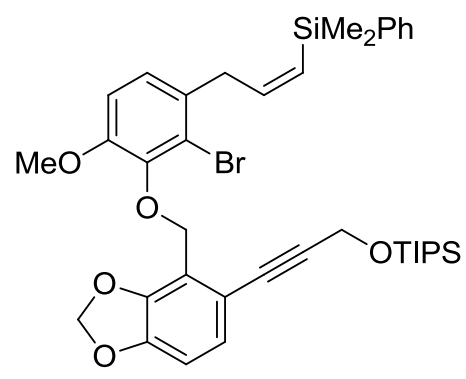

249

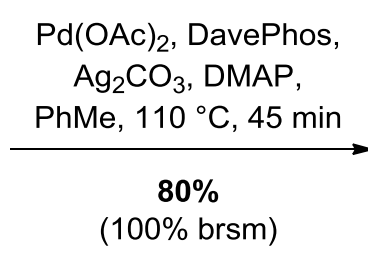

o brsm)

$\mathrm{Zu}$ einer Lösung des Dominovorläufers 249 (200 mg, $277 \mu \mathrm{mol}, 1.0$ Äq.), $\mathrm{Ag}_{2} \mathrm{CO}_{3}$ (107 mg, $388 \mu \mathrm{mol}, 1.4$ Äq.) und DMAP (33.8 mg, $277 \mu \mathrm{mol}, 1.0$ Äq.) in Toluol (15 mL) wurde bei $110{ }^{\circ} \mathrm{C}$ eine Lösung aus $\mathrm{Pd}(\mathrm{OAc})_{2}(6.22 \mathrm{mg}, 27.7 \mu \mathrm{mol}, 10 \mathrm{~mol} \%)$ und DavePhos $(54.5 \mathrm{mg}$, $139 \mu \mathrm{mol}, 50 \mathrm{~mol} \%)$ in Toluol $(4 \mathrm{~mL})$ gegeben und $45 \mathrm{~min}$ bei dieser Temperatur gerührt. Nach dem Abkühlen auf Raumtemperatur wurde das Lösungsmittel unter vermindertem Druck entfernt und das Rohprodukt mittels Säulenchromatographie an Kieselgel ( $n$ Pentan/EtOAc 30:1 $\rightarrow 10: 1)$ gereinigt. Die Zielverbindung 161 (142 mg, $222 \mu \mathrm{mol}, 80 \%$, $100 \%$ brsm) wurde in Form eines farblosen Schaums isoliert.

DC $\mathbf{R}_{f}=0.27$ ( $n$ Pentan/EtOAc 10:1).

UV $\left(\mathrm{CH}_{3} \mathrm{CN}\right): \lambda_{\max }(\lg \varepsilon)=314 \mathrm{~nm}(4.1962)$.

IR (ATR): $\tilde{v}\left(\mathrm{~cm}^{-1}\right)=2938,1608,1458,1231,1084,1034,841,733,687$.

${ }^{1} \mathbf{H}-\mathbf{N M R}\left(300 \mathrm{MHz}, \mathrm{CDCl}_{3}\right): \delta(\mathrm{ppm})=0.27\left(\mathrm{~d}, J=11.4 \mathrm{~Hz}, 6 \mathrm{H}, \mathrm{Si}\left(\mathrm{CH}_{3}\right)_{2}\right), 0.94(\mathrm{dd}, J=$ 8.7, $\left.6.8 \mathrm{~Hz}, 21 \mathrm{H}, \mathrm{Si}\left(\mathrm{CH}\left(\mathrm{CH}_{3}\right)_{2}\right)_{3}\right), 3.25\left(\mathrm{~d}, J=14.3 \mathrm{~Hz}, 1 \mathrm{H}, 8^{\prime}-\mathrm{H}_{\mathrm{A}}\right), 3.68(\mathrm{dt}, J=14.2$, $\left.1.8 \mathrm{~Hz}, 1 \mathrm{H}, 8 \mathrm{\prime}-\mathrm{H}_{\mathrm{B}}\right), 3.81\left(\mathrm{~s}, 3 \mathrm{H}, \mathrm{OCH}_{3}\right), 3.88$ (d, $\left.J=9.4 \mathrm{~Hz}, 1 \mathrm{H}, 1-\mathrm{H}_{\mathrm{A}}\right), 4.12(\mathrm{~d}, J=9.4 \mathrm{~Hz}$, $\left.1 \mathrm{H}, 1-\mathrm{H}_{\mathrm{B}}\right), 5.04\left(\mathrm{~d}, J=12.1 \mathrm{~Hz}, 1 \mathrm{H}, 13^{\prime}-\mathrm{H}_{\mathrm{A}}\right), 5.31\left(\mathrm{~d}, J=12.1 \mathrm{~Hz}, 1 \mathrm{H}, 13^{\prime}-\mathrm{H}_{\mathrm{B}}\right), 5.71(\mathrm{~d}, J=$ $1.9 \mathrm{~Hz}, 1 \mathrm{H}, 1 "-\mathrm{H}), 5.98$ (dd, $\left.J=13.7,1.5 \mathrm{~Hz}, 2 \mathrm{H}, 2^{\prime}-\mathrm{H}_{2}\right), 6.62$ (d, $\left.J=8.0 \mathrm{~Hz}, 1 \mathrm{H}, 10^{\prime}-\mathrm{H}\right)$, $6.65\left(\mathrm{~d}, J=8.0 \mathrm{~Hz}, 1 \mathrm{H}, 4^{\prime}-\mathrm{H}\right), 6.75$ (dd, $\left.J=8.1,1.2 \mathrm{~Hz}, 1 \mathrm{H}, 9^{\prime}-\mathrm{H}\right), 7.21-7.28$ (m, $3 \mathrm{H}$, $3 \times \mathrm{SiPh}-\mathrm{H}), 7.40-7.47$ (m, 3 H, 5'-H, 2×SiPh-H).

${ }^{13}$ C-NMR $\left(126 \mathrm{MHz}, \mathrm{CDCl}_{3}\right): \delta(\mathrm{ppm})=-1.7,-0.9\left(\mathrm{Si}\left(\mathrm{CH}_{3}\right)_{2}\right), 12.0\left(\mathrm{Si}\left(\underline{\mathrm{CH}}\left(\mathrm{CH}_{3}\right)_{2}\right)_{3}\right), 18.0$ $\left(\mathrm{Si}\left(\mathrm{CH}\left(\mathrm{CH}_{3}\right)_{2}\right)_{3}\right), 46.2\left(\mathrm{C}-8^{\prime}\right), 56.0\left(\mathrm{OCH}_{3}\right), 63.9(\mathrm{C}-13 '), 64.2(\mathrm{C}-1), 101.4\left(\mathrm{C}-2^{\prime}\right), 107.5(\mathrm{C}-$ 4'), 109.3 (C-10'), 117.1 (C-13a'), 117.9 (C-9'), 122.3 (5b $\left.{ }^{1 \prime}\right), 123.0$ (C-5'), 123.4 (C-1"), 127.6 (2×SiPh-C), 128.8 (SiPh-C), 131.1 (C-8a'), 133.0 (C-5a'), 133.7 (2×SiPh-C), 135.3 (SiPh-C), 
138.8 (C-5b'), 139.0 (C-6'), 144.5 (C-13b'), 146.3 (C-11a'), 147.4 (C-3a'), 148.5 (C-11'), 156.5 (C-7').

MS (ESI): $m / z(\%)=641.3(33)[\mathrm{M}+\mathrm{H}]^{+}, 663.3(100)[\mathrm{M}+\mathrm{Na}]^{+}$.

HRMS von $\mathrm{C}_{38} \mathrm{H}_{48} \mathrm{O}_{5} \mathrm{Si}_{2}$ ber.: 663.2932 gef.: $663.2905[\mathrm{M}+\mathrm{Na}]^{+}(\mathrm{ESI}-\mathrm{HRMS})$.

$\mathrm{C}_{38} \mathrm{H}_{48} \mathrm{O}_{5} \mathrm{Si}_{2}(640.96)$. 


\subsection{2 (R)-(Dimethyl(phenyl)silyl)((R)-11-methoxy-6-(((triisopropylsilyl)oxy)methyl)-}

8,13-dihydro-7H-[1,3]dioxolo[4',5':3,4]benzo[1,2-e]naphtho[1,8-bc]oxepin-7-yl)methanol (258)
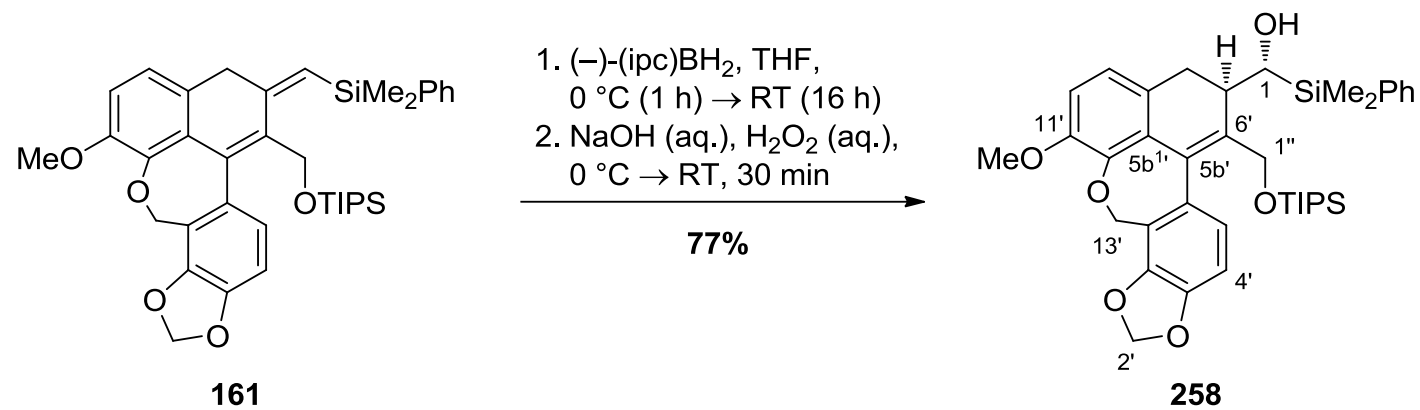

Zu einer Lösung des Alkens 161 (137 mg, $214 \mu \mathrm{mol}, 1.0$ Äq.) in THF (10 mL) wurde bei $0{ }^{\circ} \mathrm{C}$ langsam das in situ hergestellte (-)-(ipc)BH $\mathrm{BH}_{2}(128 \mathrm{mg}, 855 \mu \mathrm{mol}, 4.0$ Äq.) in THF $(10 \mathrm{~mL})$ gegeben und das Reaktionsgemisch über $16 \mathrm{~h}$ auf Raumtemperatur erwärmt. Anschließend wurde bei $0{ }^{\circ} \mathrm{C}$ sehr langsam eine Mischung aus wässriger $\mathrm{NaOH}$-Lösung ( $2 \mathrm{M}$, $2 \mathrm{~mL}$ ) und $\mathrm{H}_{2} \mathrm{O}_{2}(15 \mathrm{wt} \%, 2 \mathrm{~mL})$ zugetropft, $30 \mathrm{~min}$ bei $0{ }^{\circ} \mathrm{C}$ und $30 \mathrm{~min}$ bei Raumtemperatur gerührt. Die Reaktion wurde durch Zugabe von ges. $\mathrm{NH}_{4} \mathrm{Cl}$-Lösung $(20 \mathrm{~mL})$ beendet und mit EtOAc $(3 \times 20 \mathrm{~mL})$ extrahiert. Die vereinigten organischen Phasen wurden mit ges. NaClLösung (40 mL) gewaschen und über $\mathrm{Na}_{2} \mathrm{SO}_{4}$ getrocknet. Säulenchromatographische Reinigung an Kieselgel ( $n$ Pentan/EtOAc 10:1 $\rightarrow 4: 1)$ lieferte die Zielverbindung 258 (108 mg, $164 \mu \mathrm{mol}, 77 \%)$ in Form eines farblosen Schaums.

DC $\mathrm{R}_{f}=0.17(n$ Pentan/EtOAc 5:1).

UV $\left(\mathrm{CH}_{3} \mathrm{CN}\right): \lambda_{\max }(\lg \varepsilon)=290 \mathrm{~nm}(3.7562)$.

IR (ATR): $\tilde{v}\left(\mathrm{~cm}^{-1}\right)=2941,2864,1459,1250,1098,1032,881,796,681$.

${ }^{1} \mathbf{H}-\mathbf{N M R}\left(600 \mathrm{MHz}, \mathrm{CDCl}_{3}\right): \delta(\mathrm{ppm})=0.33,0.35\left(2 \mathrm{~s}, 6 \mathrm{H}, \mathrm{Si}\left(\mathrm{CH}_{3}\right)_{2}\right), 0.85-1.10(\mathrm{~m}, 21 \mathrm{H}$, $\left.\mathrm{Si}\left(\mathrm{CH}\left(\mathrm{CH}_{3}\right)_{2}\right)_{3}\right), 2.86-2.99\left(\mathrm{~m}, 3 \mathrm{H}, 7^{\prime}-\mathrm{H}, 8^{\prime}-\mathrm{H}_{2}\right), 3.75$ (s, $\left.1 \mathrm{H}, 1-\mathrm{H}\right), 3.77$ (s, $\left.3 \mathrm{H}, \mathrm{OCH}_{3}\right), 4.05$ $\left(\mathrm{d}, J=10.7 \mathrm{~Hz}, 1 \mathrm{H}, 1 "-\mathrm{H}_{\mathrm{A}}\right), 4.12\left(\mathrm{~d}, J=10.7 \mathrm{~Hz}, 1 \mathrm{H}, 1 "-\mathrm{H}_{\mathrm{B}}\right), 5.11(\mathrm{~d}, J=12.4 \mathrm{~Hz}, 1 \mathrm{H}$, $\left.13^{\prime}-\mathrm{H}_{\mathrm{A}}\right), 5.28\left(\mathrm{~d}, J=12.4 \mathrm{~Hz}, 1 \mathrm{H}, 13^{\prime}-\mathrm{H}_{\mathrm{B}}\right), 5.95\left(\mathrm{~d}, J=1.5 \mathrm{~Hz}, 1 \mathrm{H}, 2^{\prime}-\mathrm{H}_{\mathrm{A}}\right), 6.00(\mathrm{~d}, J=$ $\left.1.5 \mathrm{~Hz}, 1 \mathrm{H}, 2^{\prime}-\mathrm{H}_{\mathrm{B}}\right), 6.37$ (d, J = 7.9 Hz, $\left.1 \mathrm{H}, 9^{\prime}-\mathrm{H}\right), 6.54$ (d, $\left.J=8.2 \mathrm{~Hz}, 1 \mathrm{H}, 10^{\prime}-\mathrm{H}\right), 6.65$ (d, $\left.J=7.9 \mathrm{~Hz}, 1 \mathrm{H}, 4^{\prime}-\mathrm{H}\right), 6.99$ (d, $\left.J=7.9 \mathrm{~Hz}, 1 \mathrm{H}, 5^{\prime}-\mathrm{H}\right), 7.28-7.39$ (m, $\left.3 \mathrm{H}, 3 \times \mathrm{SiPh}-\mathrm{H}\right), 7.55$ (dd, $J=7.9,1.4 \mathrm{~Hz}, 2 \mathrm{H}, 2 \times \mathrm{SiPh}-\mathrm{H}$ ).

${ }^{13}$ C-NMR $\left(126 \mathrm{MHz}, \mathrm{CDCl}_{3}\right): \delta(\mathrm{ppm})=-4.4,-3.8\left(\mathrm{Si}\left(\mathrm{CH}_{3}\right)_{2}\right), 12.0\left(\mathrm{Si}\left(\underline{\mathrm{CH}}\left(\mathrm{CH}_{3}\right)_{2}\right)_{3}\right), 18.1$ $\left(\mathrm{Si}\left(\mathrm{CH}\left(\mathrm{CH}_{3}\right)_{2}\right)_{3}\right), 32.8\left(\mathrm{C}-8^{\prime}\right), 38.7$ (C-7'), $55.9\left(\mathrm{OCH}_{3}\right), 62.7\left(\mathrm{C}-1^{\prime \prime}\right), 64.1\left(\mathrm{C}-13^{\prime}\right), 67.2(\mathrm{C}-1)$, $101.4\left(\mathrm{C}-2^{\prime}\right), 107.6\left(\mathrm{C}-4^{\prime}\right), 109.6\left(\mathrm{C}-10^{\prime}\right), 117.3\left(\mathrm{C}-13 \mathrm{a}^{\prime}\right), 119.5\left(\mathrm{C}-9^{\prime}\right), 122.6\left(\mathrm{C}^{\prime}-5 \mathrm{~b}^{1 /}\right), 123.1$ 
(C-5'), 127.9 (2×SiPh-C), 128.9 (C-8a'), 129.3 (SiPh-C), 134.1 (2×SiPh-C), 137.2 (C-5b'), 137.2 (C-5a'), 139.5 (C-6'), 141.9 (SiPh-C), 144.6 (C-13b'), 145.8 (C-11a'), 147.3 (C-3a'), $148.8\left(11^{\prime}\right)$.

MS (ESI): $m / z(\%)=681.3(29)[\mathrm{M}+\mathrm{Na}]^{+}$.

HRMS von $\mathrm{C}_{38} \mathrm{H}_{50} \mathrm{O}_{6} \mathrm{Si}_{2}$

ber.: $\quad 681.3038$

gef.: $681.3025[\mathrm{M}+\mathrm{Na}]^{+}(\mathrm{ESI}-\mathrm{HRMS})$.

$\mathrm{C}_{38} \mathrm{H}_{50} \mathrm{O}_{6} \mathrm{Si}_{2}$ (658.97). 


\subsection{3 (R)-(11-Methoxy-8,13-dihydro-7H-[1,3]dioxolo[ $\left[4^{\prime}, 5^{\prime}: 3,4\right]$ benzo[1,2-e $]$ naphtho[1,8-} bc]oxepin-6,7-diyl)dimethanol (160)

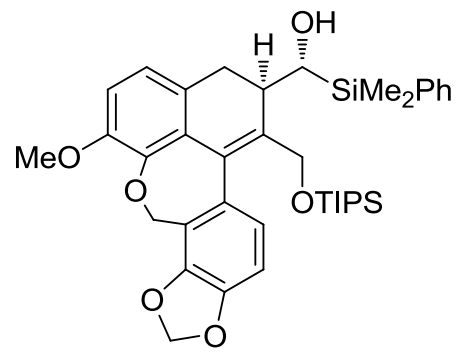

258

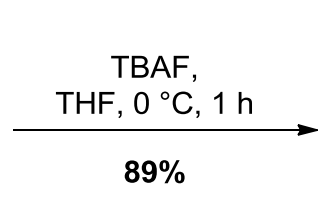

$89 \%$

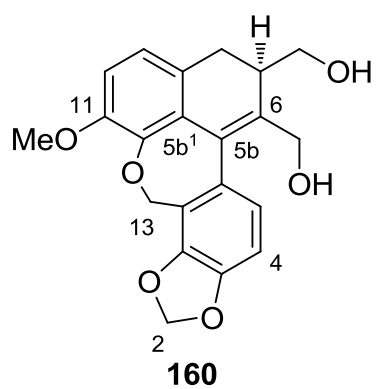

Zu einer Lösung des Silylethers 258 (93.0 mg, $141 \mu \mathrm{mol}, 1.0$ Äq.) in THF (3 mL) wurde bei $0{ }^{\circ} \mathrm{C}$ TBAF ( $1 \mathrm{M}$ in THF, $353 \mu \mathrm{L}, 353 \mu \mathrm{mol}, 2.5$ Äq.) gegeben, langsam auf Raumtemperatur erwärmt und $1 \mathrm{~h}$ gerührt. Die Reaktion wurde durch Zugabe von ges. $\mathrm{NH}_{4} \mathrm{Cl}$-Lösung $(5 \mathrm{~mL})$ beendet und mit $\mathrm{CH}_{2} \mathrm{Cl}_{2}(3 \times 5 \mathrm{~mL})$ extrahiert. Die vereinigten organischen Phasen wurden mit ges. NaCl-Lösung $(10 \mathrm{~mL})$ gewaschen und über $\mathrm{Na}_{2} \mathrm{SO}_{4}$ getrocknet. Nach säulenchromatographischer Reinigung an Kieselgel $\left(\mathrm{CH}_{2} \mathrm{Cl}_{2} / \mathrm{MeOH} 100: 1 \rightarrow 25: 1\right)$ wurde die Zielverbindung 160 (45.9 mg, $125 \mu \mathrm{mol}, 89 \%$, $52 \%$ ee) in Form eines farblosen Schaums isoliert. Das Gemisch beider Enantiomere wurde mittels präparativer HPLC getrennt und beide Verbindungen in reiner Form erhalten.

DC $\mathrm{R}_{f}=0.36\left(\mathrm{CH}_{2} \mathrm{Cl}_{2} / \mathrm{MeOH} 19: 1\right)$.

Drehwert: $\quad(+)-(R)-160: \quad[\alpha]_{\mathrm{D}}^{20}=+1.2^{\circ}\left(c=0.50, \mathrm{CHCl}_{3}\right)$,

$$
(-)-(S)-160: \quad[\alpha]_{\mathrm{D}}^{20}=-1.0^{\circ}\left(c=0.50, \mathrm{CHCl}_{3}\right) \text {. }
$$

UV $\left(\mathrm{CH}_{3} \mathrm{CN}\right): \lambda_{\max }(\lg \varepsilon)=287 \mathrm{~nm}(4.0472)$.

IR (ATR): $\tilde{v}\left(\mathrm{~cm}^{-1}\right)=3313,2930,1573,1461,1255,1098,1031$.

${ }^{1} \mathbf{H}-\mathbf{N M R}\left(600 \mathrm{MHz}, \mathrm{CDCl}_{3}\right): \delta(\mathrm{ppm})=2.67\left(\mathrm{~d}, J=15.4 \mathrm{~Hz}, 1 \mathrm{H}, 8-\mathrm{H}_{\mathrm{A}}\right), 2.75-2.79(\mathrm{~m}, 1 \mathrm{H}$, 7-H), $2.90\left(\mathrm{dd}, J=15.4,5.5 \mathrm{~Hz}, 1 \mathrm{H}, 8-\mathrm{H}_{\mathrm{B}}\right), 3.47\left(\mathrm{dd}, J=9.8,5.9 \mathrm{~Hz}, 1 \mathrm{H}, 7-\mathrm{CH}_{\mathrm{A}} \mathrm{H}_{\mathrm{B}} \mathrm{OH}\right)$, $3.57\left(\mathrm{dd}, J=9.5,9.5 \mathrm{~Hz}, 1 \mathrm{H}, 7-\mathrm{CH}_{\mathrm{A}} H_{\mathrm{B}} \mathrm{OH}\right), 3.77\left(\mathrm{~s}, 3 \mathrm{H}, \mathrm{OCH}_{3}\right), 4.19$ (d, $J=12.5 \mathrm{~Hz}, 1 \mathrm{H}$, 6- $\left.\mathrm{CH}_{\mathrm{A}} \mathrm{H}_{\mathrm{B}} \mathrm{OH}\right), 4.35\left(\mathrm{~d}, J=12.5 \mathrm{~Hz}, 1 \mathrm{H}, 6-\mathrm{CH}_{\mathrm{A}} H_{\mathrm{B}} \mathrm{OH}\right), 5.12\left(\mathrm{~d}, J=12.3 \mathrm{~Hz}, 1 \mathrm{H}, 13-\mathrm{H}_{\mathrm{A}}\right)$, $5.26\left(\mathrm{~d}, J=12.3 \mathrm{~Hz}, 1 \mathrm{H}, 13-\mathrm{H}_{\mathrm{B}}\right), 5.96\left(\mathrm{dd}, J=14.9,1.5 \mathrm{~Hz}, 2 \mathrm{H}, 2-\mathrm{H}_{2}\right), 6.53(\mathrm{~d}, J=7.9 \mathrm{~Hz}$, $1 \mathrm{H}, 9-\mathrm{H}), 6.62(\mathrm{~d}, J=8.1 \mathrm{~Hz}, 1 \mathrm{H}, 4-\mathrm{H}), 6.67(\mathrm{~d}, J=7.7 \mathrm{~Hz}, 1 \mathrm{H}, 10-\mathrm{H}), 6.68(\mathrm{~d}, J=7.8 \mathrm{~Hz}$, $1 \mathrm{H}, 5-\mathrm{H})$.

${ }^{13}$ C-NMR $\left(126 \mathrm{MHz}, \mathrm{CDCl}_{3}\right): \delta(\mathrm{ppm})=32.4(\mathrm{C}-8), 38.2(\mathrm{C}-7), 55.9\left(\mathrm{OCH}_{3}\right), 62.1$ (6- $\left.\mathrm{CH}_{2} \mathrm{OH}\right), 63.1$ (7- $\left.\mathrm{CH}_{2} \mathrm{OH}\right), 63.9$ (C-13), 101.5 (C-2), 107.4 (C-10), 109.6 (C-4), 117.5 
(C-13a), 119.7 (C-5), $121.5\left(\mathrm{C}-5 \mathrm{~b}^{1}\right), 123.0$ (C-9), 127.5 (C-8a), 132.8 (C-5b), 132.9 (C-5a), 139.2 (C-6), 144.7 (C-13b), 145.9 (C-11a), 147.4 (C-3a), 148.7 (C-11).

MS (ESI): $m / z(\%)=351.1(100)\left[\mathrm{M}-\mathrm{H}_{2} \mathrm{O}+\mathrm{H}\right]^{+}, 391.1(74)[\mathrm{M}+\mathrm{Na}]^{+}, 759.3(66)[2 \mathrm{M}+\mathrm{Na}]^{+}$.

HRMS von $\mathrm{C}_{21} \mathrm{H}_{20} \mathrm{O}_{6}$ ber.: 391.1152 gef.: $391.1151[\mathrm{M}+\mathrm{Na}]^{+}(\mathrm{ESI}-\mathrm{HRMS})$.

$\mathrm{C}_{21} \mathrm{H}_{20} \mathrm{O}_{6}$ (368.38).

HPLC (analytisch): Säule: $\quad$ Chiralpak $^{\circledR}$ IA, $250 \times 4.6 \mathrm{~mm}, 5 \mu \mathrm{m}$

Eluens: $\quad n$ Hexan/iPrOH 88:12

Fluß: $\quad 0.8 \mathrm{~mL} / \mathrm{min}$

$\lambda: \quad 226 \mathrm{~nm}$

$t_{\mathrm{R}}: \quad(+)-(R)-160: 23.8 \mathrm{~min},(-)-(S)-160: 29.7 \mathrm{~min}$.

HPLC (präparativ): Säule: $\quad$ Chiralpak $^{\circledR}$ IA, $250 \times 20 \mathrm{~mm}, 5 \mu \mathrm{m}$

Eluens: $\quad n$ Hexan/iPrOH 90:10

Fluß: $\quad 18 \mathrm{~mL} / \mathrm{min}$

$\lambda$ : $\quad 226 \mathrm{~nm}$

$t_{\mathrm{R}}: \quad(+)-(R)-160: 30.0 \mathrm{~min},(-)-(S)-160: 41.2 \mathrm{~min}$. 


\subsection{4 (+)-Linoxepin (69)}

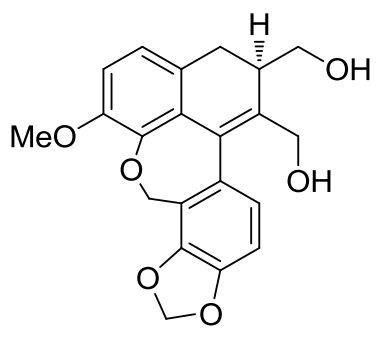

160

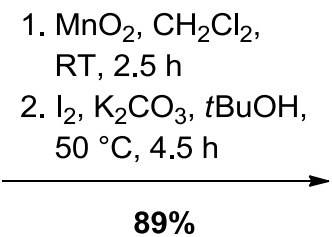

$89 \%$

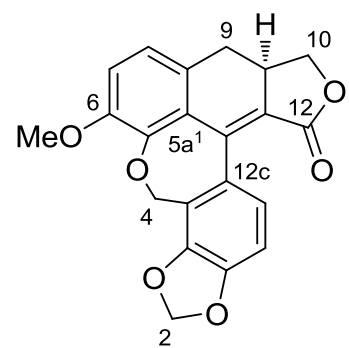

$(+)$-Linoxepin (69)

$\mathrm{Zu}$ einer Lösung des Diols (+)-160 (21.0mg, $57.0 \mu \mathrm{mol}, 1.0$ Äq.) in $\mathrm{CH}_{2} \mathrm{Cl}_{2}(2 \mathrm{~mL})$ wurde bei Raumtemperatur portionsweise $\mathrm{MnO}_{2}(49.6 \mathrm{mg}, 570 \mu \mathrm{mol}, 10.0$ Äq.) gegeben und $2.5 \mathrm{~h}$ gerührt. Die Lösung wurde anschließend durch Celite ${ }^{\circledR}$ filtriert, mit $\mathrm{CH}_{2} \mathrm{Cl}_{2}(3 \times 5 \mathrm{~mL})$ nachgewaschen und das Lösungsmittel unter vermindertem Druck entfernt. Der entstandene Aldehyd wurde direkt in $t \mathrm{BuOH}(2 \mathrm{~mL})$ gelöst, $\mathrm{K}_{2} \mathrm{CO}_{3}\left(27.6 \mathrm{mg}, 200 \mu \mathrm{mol}, 3.5\right.$ Äq.) und $\mathrm{I}_{2}$ (21.7 mg, $85.5 \mu \mathrm{mol}, 1.5$ Äq.) zugegeben und $4.5 \mathrm{~h}$ bei $50^{\circ} \mathrm{C}$ gerührt. Nach dem Abkühlen auf Raumtemperatur wurde EtOAc $(15 \mathrm{~mL})$ zugegeben und mit ges. $\mathrm{Na}_{2} \mathrm{~S}_{2} \mathrm{O}_{3}$-Lösung $(2 \times 15 \mathrm{~mL})$ und ges. NaCl-Lösung $(15 \mathrm{~mL})$ gewaschen. Die wässrigen Phasen wurden vereinigt und mit EtOAc $(2 \times 15 \mathrm{~mL})$ extrahiert. Die vereinigten organischen Phasen wurden über $\mathrm{Na}_{2} \mathrm{SO}_{4}$ getrocknet und das Lösungsmittel unter vermindertem Druck entfernt. Säulenchromatographische Reinigung an Kieselgel (Petrolether/EtOAc 2:1) lieferte die Zielverbindung (+)-69 (18.5 mg, $50.8 \mu \mathrm{mol}, 89 \%)$ in Form eines gelben Feststoffes.

Analog zu dieser Vorschrift wurde (-)-160 (10.5 mg, $28.5 \mu \mathrm{mol}, 1.0$ Äq) mit $\mathrm{MnO}_{2}(24.8 \mathrm{mg}$, $285 \mu \mathrm{mol}, 10.0$ Äq.), $\mathrm{K}_{2} \mathrm{CO}_{3}$ (13.8 mg, $100 \mu \mathrm{mol}, 3.5$ Äq.) und $\mathrm{I}_{2}$ (10.9 mg, $42.8 \mu \mathrm{mol}$, 1.5 Äq.) zu (-)-160 (9.30 mg, 25.5 $\mu \mathrm{mol}, 89 \%)$ umgesetzt.

DC: $\mathrm{R}_{f}=0.23$ (Petrolether/EtOAc 2:1).

Drehwert: $[\alpha]_{\mathrm{D}}^{20}=+96.1^{\circ}\left(\mathrm{c}=0.61, \mathrm{CHCl}_{3}\right)$.

$\mathbf{U V}\left(\mathrm{CH}_{3} \mathrm{CN}\right): \lambda_{\max }(\lg \varepsilon)=287 \mathrm{~nm}(4.0470)$.

IR (ATR): $\tilde{v}\left(\mathrm{~cm}^{-1}\right)=3313,2930,1573,1461,1255,1098,1031$.

${ }^{1} \mathbf{H}-\mathbf{N M R}\left(600 \mathrm{MHz}, \mathrm{CDCl}_{3}\right): \delta(\mathrm{ppm})=2.64\left(\mathrm{dt}, J=14.9,1.1 \mathrm{~Hz}, 1 \mathrm{H}, 9-\mathrm{H}_{\mathrm{A}}\right), 2.98(\mathrm{dd}, J=$ 14.6, $5.7 \mathrm{~Hz}, 1 \mathrm{H}, 9-\mathrm{H}_{\mathrm{B}}$ ), 3.26 (ddt, $\left.J=14.6,8.8,5.7 \mathrm{~Hz}, 1 \mathrm{H}, 9 \mathrm{a}-\mathrm{H}\right), 3.83\left(\mathrm{~s}, 3 \mathrm{H}, \mathrm{OCH}_{3}\right), 4.01$ (t, $\left.J=8.7 \mathrm{~Hz}, 1 \mathrm{H}, 10-\mathrm{H}_{\mathrm{A}}\right), 4.66\left(\mathrm{t}, J=8.9 \mathrm{~Hz}, 1 \mathrm{H}, 10-\mathrm{H}_{\mathrm{B}}\right), 5.12\left(\mathrm{~d}, J=12.5 \mathrm{~Hz}, 1 \mathrm{H}, 4-\mathrm{H}_{\mathrm{A}}\right)$, $5.37\left(\mathrm{~d}, J=12.5 \mathrm{~Hz}, 1 \mathrm{H}, 4-\mathrm{H}_{\mathrm{B}}\right), 6.01\left(\mathrm{~d}, J=1.9 \mathrm{~Hz}, 1 \mathrm{H}, 2-\mathrm{H}_{\mathrm{A}}\right), 6.01(\mathrm{~d}, J=1.9 \mathrm{~Hz}, 1 \mathrm{H}$, $\left.2-\mathrm{H}_{\mathrm{B}}\right), 6.72(\mathrm{~d}, J=8.0 \mathrm{~Hz}, 1 \mathrm{H}, 14-\mathrm{H}), 6.78(\mathrm{~d}, J=8.2 \mathrm{~Hz}, 1 \mathrm{H}, 7-\mathrm{H}), 6.83(\mathrm{~d}, J=8.2 \mathrm{~Hz}, 1 \mathrm{H}$, $8-\mathrm{H}), 6.85(\mathrm{~d}, J=8.0 \mathrm{~Hz}, 1 \mathrm{H}, 13-\mathrm{H})$. 
${ }^{13}$ C-NMR $\left(126 \mathrm{MHz}, \mathrm{CDCl}_{3}\right): \delta(\mathrm{ppm})=34.5(\mathrm{C}-9), 36.9(\mathrm{C}-9 \mathrm{a}), 56.2\left(\mathrm{OCH}_{3}\right), 64.7(\mathrm{C}-4)$, 70.0 (C-10), 101.8 (C-2), 108.1 (C-14), 111.8 (C-7), 116.5 (C-3b), 119.8 (C-8), 122.2 (C-5a $\left.{ }^{1}\right)$, 124.1 (C-13), 124.3 (C-12a), 128.1 (C-8a), 129.4 (C-12c), 144.7 (C-3a), 145.6 (C-12b), 148.5 (C-5a), 149.0 (C-14a), 149.4 (C-6), 168.7 (C-12).

MS (ESI): m/z (\%) = $365.1(46)[\mathrm{M}+\mathrm{H}]^{+}, 387.1(98)[\mathrm{M}+\mathrm{Na}]^{+}, 751.2(100)[2 \mathrm{M}+\mathrm{Na}]^{+}, 1115.3$ (17) $[3 \mathrm{M}+\mathrm{Na}]^{+}$.

HRMS von $\mathrm{C}_{21} \mathrm{H}_{16} \mathrm{O}_{6}$ ber.: $\quad 365.1020$ gef.: $365.1019[\mathrm{M}+\mathrm{H}]^{+}($ESI-HRMS).

$\mathbf{C}_{21} \mathrm{H}_{16} \mathbf{O}_{6}$ (364.35). 


\section{Synthese von potentiellen Hsp47-Inhibitoren}

\subsection{Synthese des Dihydropyrimidons 166}

\subsubsection{2-Mercapto-6-oxo-4-(thiophen-2-yl)-1,6-dihydropyrimidin-5-carbonitril (166)}

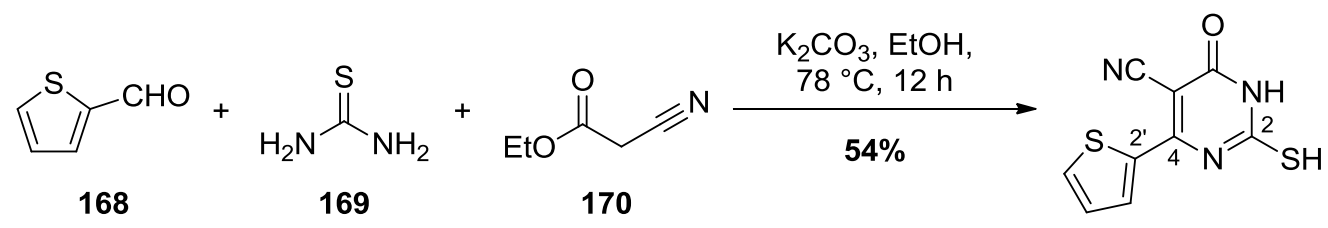

166

Zu einer Lösung aus Thioharnstoff (168) (761 mg, 10.0 mmol, 1.0 Äq.) und $\mathrm{K}_{2} \mathrm{CO}_{3}(1.38 \mathrm{~g}$, $10.0 \mathrm{mmol}, 1.0$ Äq.) in EtOH (10 mL) wurden bei Raumtemperatur Ethylcyanoacetat (170) (1.06 mL, 1.13 g, 10.0 mmol, 1.0 Äq.) und Thiophen-2-carbaldehyd (169) (1.12 g, $935 \mu \mathrm{L}$, 10.0 mmol, 1.0 Äq.) gegeben und die resultierende Suspension $12 \mathrm{~h}$ unter Rückfluss erhitzt. Anschließend wurde das Reaktionsgemisch durch Zugabe von Eisessig ( $2 \mathrm{~mL}$ ) neutralisiert, der Niederschlag abfiltriert und mit auf $0{ }^{\circ} \mathrm{C}$ gekühltem EtOH $(10 \mathrm{~mL})$ gewaschen. Nach Umkristallisation aus $\mathrm{H}_{2} \mathrm{O}$ wurde die Zielverbindung 166 (1.28 g, $5.44 \mathrm{mmol}, 54 \%$ ) in Form von braunen Kristallen isoliert.

DC $\mathrm{R}_{f}=0.17\left(\mathrm{CH}_{2} \mathrm{Cl}_{2} / \mathrm{MeOH} 8: 1\right)$.

Smp.: Zersetzung bei ca. $360{ }^{\circ} \mathrm{C}$.

$\mathbf{U V}\left(\mathrm{CH}_{3} \mathrm{CN}\right): \lambda_{\max }(\lg \varepsilon)=280 \mathrm{~nm}$ (3.5382), 313 (3.2464), 387 (3.2758).

IR (ATR): $\tilde{v}\left(\mathrm{~cm}^{-1}\right)=3403,3038,2875,2211,1638,1444,1408,1241,1117$.

${ }^{1} \mathbf{H}-\mathbf{N M R}\left(300 \mathrm{MHz}, \mathrm{DMSO}-\mathrm{d}_{6}\right): \delta(\mathrm{ppm})=7.25\left(\mathrm{dd}, J=5.0,3.9 \mathrm{~Hz}, 1 \mathrm{H}, 4^{\prime}-\mathrm{H}\right), 7.85(\mathrm{dd}, J=$ 5.0, 1.0 Hz, $\left.1 \mathrm{H}, 3^{\prime}-\mathrm{H}\right), 8.15$ (dd, $\left.J=3.9,1.0 \mathrm{~Hz}, 1 \mathrm{H}, 5^{\prime}-\mathrm{H}\right)$.

${ }^{13}$ C-NMR (126 MHz, DMSO-d $\left.{ }_{6}\right): \delta(\mathrm{ppm})=82.2(\mathrm{C}-5), 120.1(\mathrm{CN}), 129.3(\mathrm{C}-4$ '), 130.3 (C-5'), 132.9 (C-3'), 142.9 (C-2'), 159.3 (C-6), 163.7 (C-4), 183.2 (C-2).

MS (ESI): $m / z(\%)=236.0(24)[\mathrm{M}+\mathrm{H}]^{+}, 258.0(100)[\mathrm{M}+\mathrm{Na}]^{+}, 274.0(81)[\mathrm{M}+\mathrm{K}]^{+}$.

HRMS von $\mathrm{C}_{9} \mathrm{H}_{5} \mathrm{~N}_{3} \mathrm{OS}_{2}$ ber.: 235.9947 gef.: $235.9943[\mathrm{M}+\mathrm{H}]^{+}(\mathrm{ESI}-\mathrm{HRMS})$.

$\mathrm{C}_{9} \mathrm{H}_{5} \mathbf{N}_{\mathbf{3}} \mathbf{O S}_{2}$ (235.28). 


\subsection{Synthese der Acetophenone 261a-g}

\subsubsection{1-(4-Chlor-3-methylphenyl)ethanon (261a)}

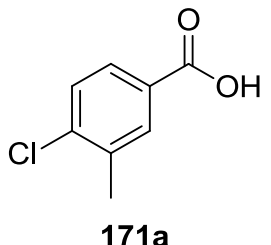

A: CuCN, MeLi, THF, $0{ }^{\circ} \mathrm{C} \rightarrow \mathrm{RT}, 14 \mathrm{~h}, 73 \%$

B: $\mathrm{MeLi}, \mathrm{Et}_{2} \mathrm{O}, \mathrm{THF}$ $0{ }^{\circ} \mathrm{C} \rightarrow \mathrm{RT}, 2.5 \mathrm{~h}, 65 \%$

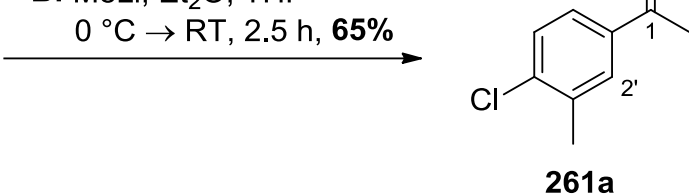

Methode A: CuCN (524 mg, 5.85 mmol, 5.0 Äq.), MeLi (1.6 M in $\mathrm{Et}_{2} \mathrm{O}, 7.31 \mathrm{~mL}, 11.7 \mathrm{mmol}$, 10.0 Äq.) und Benzoesäure 171a (200 mg, 1.17 mmol, 1.0 Äq.) wurden gemäß AAV1 umgesetzt. Säulenchromatographische Reinigung an Kieselgel ( $n$ Pentan $/ \mathrm{CH}_{2} \mathrm{Cl}_{2}$ 2:1) lieferte die Zielverbindung 261a (144 mg, $853 \mu \mathrm{mol}, 73 \%$ ) als farbloses Öl.

Methode B: Zu einer Lösung der Benzoesäure 171a (200 mg, $1.17 \mathrm{mmol}, 1.0$ Äq.) in $\mathrm{Et}_{2} \mathrm{O} / \mathrm{THF}(1: 1,20 \mathrm{~mL})$ wurde bei $0{ }^{\circ} \mathrm{C}$ langsam MeLi (1.6 $\mathrm{M}$ in $\mathrm{Et}_{2} \mathrm{O}, 1.54 \mathrm{~mL}, 2.1$ Äq.) getropft und $2 \mathrm{~h}$ bei dieser Temperatur und $30 \mathrm{~min}$ bei Raumtemperatur gerührt. Anschließend wurde die Reaktion durch Zugabe von ges. $\mathrm{NH}_{4} \mathrm{Cl}$-Lösung $(10 \mathrm{~mL})$ beendet und das Gemisch mit $\mathrm{Et}_{2} \mathrm{O}(3 \times 10 \mathrm{~mL})$ extrahiert. Die vereinigten organischen Phasen wurden über $\mathrm{Na}_{2} \mathrm{SO}_{4}$ getrocknet, das Lösungsmittel unter vermindertem Druck entfernt und der Rückstand über Säulenchromatographie an Kieselgel ( $n$ Pentan/ $\mathrm{CH}_{2} \mathrm{Cl}_{2} 3: 1 \rightarrow 1: 1$ ) gereinigt. Die Zielverbindung 261a (129 mg, $764 \mu \mathrm{mol}$, 65\%) wurde in Form eines farblosen Öls erhalten.

DC: $\mathrm{R}_{f}=0.29\left(n\right.$ Pentan $\left./ \mathrm{CH}_{2} \mathrm{Cl}_{2}=2: 1\right)$.

UV $\left(\mathrm{CH}_{3} \mathrm{CN}\right): \lambda_{\max }(\lg \varepsilon)=195 \mathrm{~nm}$ (4.505), 251 (4.189), 282 (3.200), 401 (1.66), 501 (1.249). IR (ATR): $\tilde{v}\left(\mathrm{~cm}^{-1}\right)=1683,1356,1292,1248,1047,816,710,611$.

${ }^{1}$ H-NMR $\left(300\right.$ MHz, DMSO-d 6 ): $\delta(\mathrm{ppm})=2.41$ (s, $3 \mathrm{H}, 3$ '- $\left.-\mathrm{CH}_{3}\right), 2.56$ (s, $\left.3 \mathrm{H}, 2-\mathrm{H}_{3}\right), 7.39$ (d, $J=8.3 \mathrm{~Hz}, 1 \mathrm{H}, 5 '-\mathrm{H}), 7.61-7.75\left(\mathrm{~m}, 1 \mathrm{H}, 6{ }^{\prime}-\mathrm{H}\right), 7.80\left(\mathrm{dt}, J=2.1,0.5 \mathrm{~Hz}, 1 \mathrm{H}, 2^{\prime}-\mathrm{H}\right)$.

${ }^{13}$ C-NMR (126 MHz, DMSO-d 6$): \delta(\mathrm{ppm})=20.1\left(3^{\prime}-\mathrm{CH}_{3}\right), 26.5$ (C-2), 127.1 (C-6'), 129.3 (C-5'), 130.7 (C-2'), 135.5 (C-1'), 136.5 (C-3'), 139.7 (C-4'), $197.2(\mathrm{C}=\mathrm{O})$.

MS (ESI): m/z (\%) = $169.0(8)[\mathrm{M}+\mathrm{H}]^{+}, 191.0(56)[\mathrm{M}+\mathrm{Na}]^{+}$.

HRMS von $\mathrm{C}_{9} \mathrm{H}_{9} \mathrm{ClO}$

$\mathrm{C}_{9} \mathrm{H}_{9} \mathrm{ClO}$ (168.03). ber.: 191.0234

gef.: $191.0236[\mathrm{M}+\mathrm{Na}]^{+}$(ESI-HRMS). 


\subsubsection{1-(4-Chlor-2-methylphenyl)ethanon (261b)}

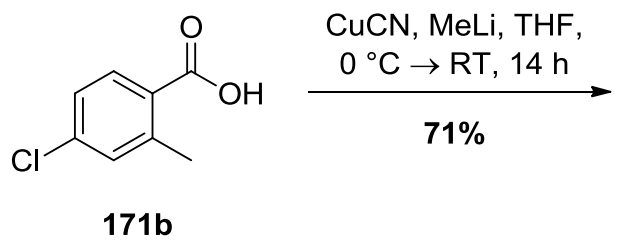<smiles>CC(=O)c1ccc(Cl)cc1C</smiles>

$\mathrm{CuCN}$ (2.62 g, 29.3 mmol, 5.0 Äq.), MeLi (1.6 M in Et $2 \mathrm{O}, 36.6 \mathrm{~mL}, 58.6 \mathrm{mmol}, 10.0$ Äq.) und Benzoesäure 171b (1.00 g, $5.86 \mathrm{mmol}, 1.0$ Äq.) wurden gemäß AAV1 umgesetzt. Säulenchromatographische Reinigung an Kieselgel ( $n$ Pentan/ $\mathrm{CH}_{2} \mathrm{Cl}_{2} \quad 3: 1 \rightarrow 2: 1$ ) lieferte die Zielverbindung 261b (696 mg, 4.13 mmol, 71\%) als farbloser Feststoff.

DC: $\mathrm{R}_{f}=0.41\left(n\right.$ Pentan/ $\left.\mathrm{CH}_{2} \mathrm{Cl}_{2} 2: 1\right)$.

UV $\left(\mathrm{CH}_{3} \mathrm{CN}\right): \lambda_{\max }(\lg \varepsilon)=206 \mathrm{~nm}$ (4.3783), 248 (4.0767), 282 (3.0866).

IR (ATR): $\tilde{v}\left(\mathrm{~cm}^{-1}\right)=3353,3062,3000,1682,1593,1557,1244,1105,955$.

${ }^{1} \mathbf{H}-\mathbf{N M R}\left(300 \mathrm{MHz}, \mathrm{CDCl}_{3}\right): \delta(\mathrm{ppm})=2.50\left(\mathrm{~s}, 3 \mathrm{H}, 2^{\prime}-\mathrm{CH}_{3}\right), 2.55\left(\mathrm{~s}, 3 \mathrm{H}, 2-\mathrm{H}_{3}\right), 7.20-7.24$ (m, 2 H, 3'-H, 5'-H), 7.63 (d, $J=8.9 \mathrm{~Hz}, 1 \mathrm{H}, 6$ '-H).

${ }^{13}$ C-NMR (126 MHz, $\left.\mathrm{CDCl}_{3}\right): \delta(\mathrm{ppm})=21.9\left(2^{\prime}-\mathrm{CH}_{3}\right), 29.7(\mathrm{C}-2), 126.1\left(\mathrm{C}-5{ }^{\prime}\right), 131.1\left(\mathrm{C}-6^{\prime}\right)$, 132.3 (C-3'), 136.1 (C-2'), 137.7 (C-4'), 141.1 (C-1'), $201.0(\mathrm{C}=\mathrm{O})$.

MS (ESI): $m / z(\%)=169.0(33)[\mathrm{M}+\mathrm{H}]^{+}, 191.0(100)[\mathrm{M}+\mathrm{Na}]^{+}$.

HRMS von $\mathrm{C}_{9} \mathrm{H}_{9} \mathrm{ClO}$ ber.: 191.0234 gef.: $191.0240[\mathrm{M}+\mathrm{Na}]^{+}(\mathrm{ESI}-\mathrm{HRMS})$.

$\mathrm{C}_{9} \mathrm{H}_{9} \mathrm{ClO}(168.62)$. 


\subsubsection{1-(2-Chlor-3-methylphenyl)ethanon (261c)}<smiles>Cc1ccccc1C(=O)O</smiles>

$171 c$

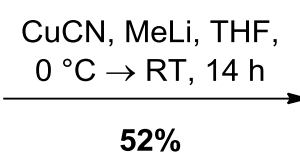

$52 \%$<smiles>CC(=O)c1cccc(C)c1Cl</smiles>

261c

$\mathrm{CuCN}$ (5.25 g, 58.6 mmol, 5.0 Äq.), MeLi (1.6 M in Et $2 \mathrm{O}, 73.3 \mathrm{~mL}, 117 \mathrm{mmol}, 10.0$ Äq.) und Benzoesäure 171c $(2.00 \mathrm{~g}, 11.7 \mathrm{mmol}, 1.0$ Äq.) wurden gemäß AAV1 umgesetzt. Säulenchromatographische Reinigung an Kieselgel ( $n$ Pentan/ $\mathrm{CH}_{2} \mathrm{Cl}_{2} \quad 3: 1 \rightarrow 2: 1$ ) lieferte die Zielverbindung 261c (1.02 g, $6.03 \mathrm{mmol}, 52 \%)$ als farblose, viskose Flüssigkeit.

DC: $\mathrm{R}_{f}=0.41\left(n\right.$ Pentan/ $\left.\mathrm{CH}_{2} \mathrm{Cl}_{2} 2: 1\right)$.

UV $\left(\mathrm{CH}_{3} \mathrm{CN}\right): \lambda_{\max }(\lg \varepsilon)=208 \mathrm{~nm}$ (4.3314), 239 (3.6679), 282 (2.9881).

IR (ATR): $\tilde{v}\left(\mathrm{~cm}^{-1}\right)=3068,3053,2982,1693,1407,1353,1269,1046$.

${ }^{1} \mathbf{H}-\mathbf{N M R}\left(300 \mathrm{MHz}, \mathrm{CDCl}_{3}\right): \delta(\mathrm{ppm})=2.45\left(\mathrm{~s}, 3 \mathrm{H}, 3^{\prime}-\mathrm{CH}_{3}\right), 2.65\left(\mathrm{~s}, 3 \mathrm{H}, 2-\mathrm{H}_{3}\right), 7.24(\mathrm{t}, J=$ $7.5 \mathrm{~Hz}, 1 \mathrm{H}, 5$ '-H), 7.29-7.37 (m, 2 H, 4'-H, 6'-H).

${ }^{13}$ C-NMR (126 MHz, $\left.\mathrm{CDCl}_{3}\right): \delta(\mathrm{ppm})=20.6\left(3^{\prime}-\mathrm{CH}_{3}\right), 31.1(\mathrm{C}-2), 126.5\left(\mathrm{C}-4{ }^{\prime}\right), 126.8\left(\mathrm{C}-5^{\prime}\right)$, 130.9 (C-2'), 133.2 (C-6'), 137.8 (C-3'), 140.7 (C-1'), $202.0(\mathrm{C}=\mathrm{O})$.

MS (ESI): $m / z(\%)=169.0(16)[\mathrm{M}+\mathrm{H}]^{+}, 191.0(100)[\mathrm{M}+\mathrm{Na}]^{+}$.

HRMS von $\mathrm{C}_{9} \mathrm{H}_{9} \mathrm{ClO}$

$\mathrm{C}_{9} \mathrm{H}_{9} \mathrm{ClO}$ (168.62). ber.: 191.0234 gef.: $191.0236[\mathrm{M}+\mathrm{Na}]^{+}(\mathrm{ESI}-\mathrm{HRMS})$. 


\subsubsection{1-(3-Chlor-4-methylphenyl)ethanon (261d)}<smiles>Cc1ccc(C(=O)O)cc1Cl</smiles>

$171 d$

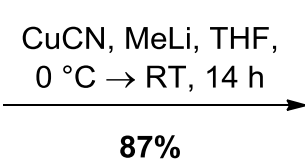

$87 \%$<smiles>CC(=O)c1ccc(C)c(Cl)c1</smiles>

261d

$\mathrm{CuCN}$ (5.25 g, 58.6 mmol, 5.0 Äq.), MeLi (1.6 M in Et $2 \mathrm{O}, 73.3 \mathrm{~mL}, 117 \mathrm{mmol}, 10.0$ Äq.) und Benzoesäure 171d (2.00 g, $11.7 \mathrm{mmol}, 1.0$ Äq.) wurden gemäß AAV1 umgesetzt. Säulenchromatographische Reinigung an Kieselgel ( $n$ Pentan/ $\mathrm{CH}_{2} \mathrm{Cl}_{2} \quad 3: 1 \rightarrow 2: 1$ ) lieferte die Zielverbindung 261d (1.72 mg, 10.2 mmol, 87\%) als farblosen Feststoff.

DC: $\mathrm{R}_{f}=0.39\left(n\right.$ Pentan/ $\left.\mathrm{CH}_{2} \mathrm{Cl}_{2} 2: 1\right)$.

Smp.: $45^{\circ} \mathrm{C}$.

UV $\left(\mathrm{CH}_{3} \mathrm{CN}\right): \lambda_{\max }(\lg \varepsilon)=210 \mathrm{~nm}$ (4.4713), 248 (4.1233), 285 (3.1408).

IR (ATR): $\tilde{v}\left(\mathrm{~cm}^{-1}\right)=3336,2985,2924,1913,1740,1678,1599,1382,1354,1249,819$.

${ }^{1} \mathbf{H}-\mathbf{N M R}\left(300 \mathrm{MHz}, \mathrm{CDCl}_{3}\right): \delta(\mathrm{ppm})=2.39\left(\mathrm{~s}, 3 \mathrm{H}, 4{ }^{\prime}-\mathrm{CH}_{3}\right), 2.53\left(\mathrm{~s}, 3 \mathrm{H}, 2-\mathrm{H}_{3}\right), 7.28(\mathrm{~d}, J=$ $\left.7.9 \mathrm{~Hz}, 1 \mathrm{H}, 5^{\prime}-\mathrm{H}\right), 7.70$ (dd, $\left.J=7.9,1.8 \mathrm{~Hz}, 1 \mathrm{H}, 6^{\prime}-\mathrm{H}\right), 7.88$ (d, $\left.J=1.8 \mathrm{~Hz}, 1 \mathrm{H}, 2^{\prime}-\mathrm{H}\right)$.

${ }^{13}$ C-NMR $\left(126 \mathrm{MHz}, \mathrm{CDCl}_{3}\right): \delta(\mathrm{ppm})=20.6\left(4^{\prime}-\mathrm{CH}_{3}\right), 26.8(\mathrm{C}-2), 126.8\left(\mathrm{C}-6{ }^{\prime}\right), 129.3\left(\mathrm{C}-2^{\prime}\right)$, $131.4\left(\mathrm{C}^{2} 5^{\prime}\right), 135.2$ (C-3'), 136.7 (C-4'), $142.0\left(\mathrm{C}-1^{\prime}\right), 196.8(\mathrm{C}=\mathrm{O})$.

MS (ESI): $m / z(\%)=169.1(84)[\mathrm{M}+\mathrm{H}]^{+}, 191.0(100)[\mathrm{M}+\mathrm{Na}]^{+}$.

HRMS von $\mathrm{C}_{9} \mathrm{H}_{9} \mathrm{ClO}$ ber.: $\quad 169.0415$ gef.: $\quad 169.0418[\mathrm{M}+\mathrm{H}]^{+}(\mathrm{ESI}-\mathrm{HRMS})$.

$\mathrm{C}_{9} \mathrm{H}_{9} \mathrm{ClO}$ (168.62). 


\subsubsection{1-(4-Chlor-3-(trifluormethyl)phenyl)ethanon (261e)}<smiles>O=C(O)c1ccc(Cl)c(C(F)(F)F)c1</smiles>

$171 \mathrm{e}$

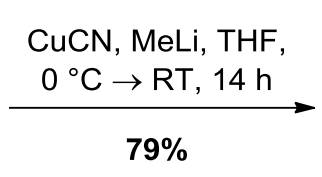

$79 \%$<smiles>CC(=O)c1ccc(Cl)c(C(F)(F)F)c1</smiles>

261e

$\mathrm{CuCN}$ (2.00 g, $22.3 \mathrm{mmol}, 5.0$ Äq.), MeLi (1.6 M in Et $2 \mathrm{O}, 27.9 \mathrm{~mL}, 44.5 \mathrm{mmol}, 10.0$ Äq.) und Benzoesäure 171e (1.00 g, $4.45 \mathrm{mmol}, 1.0$ Äq.) wurden gemäß AAV1 umgesetzt. Säulenchromatographische Reinigung an Kieselgel ( $n$ Pentan $/ \mathrm{CH}_{2} \mathrm{Cl}_{2} \quad 3: 1 \rightarrow 2: 1$ ) lieferte die Zielverbindung 261e (785 mg, $3.52 \mathrm{mmol}, 79 \%$ ) als gelbe, viskose Flüssigkeit.

DC: $\mathrm{R}_{f}=0.50\left(n\right.$ Pentan/ $\left.\mathrm{CH}_{2} \mathrm{Cl}_{2} 2: 1\right)$.

UV $\left(\mathrm{CH}_{3} \mathrm{CN}\right): \lambda_{\max }(\lg \varepsilon)=200 \mathrm{~nm}$ (4.4082), 245 (4.1905), 278 (3.1618), 286 (3.0785).

IR (ATR): $\tilde{v}\left(\mathrm{~cm}^{-1}\right)=3078,3056,3009,1692,1360,1317,1237,1129,1035$.

${ }^{1} \mathbf{H}-\mathbf{N M R}\left(300 \mathrm{MHz}, \mathrm{CDCl}_{3}\right): \delta(\mathrm{ppm})=2.63\left(\mathrm{~s}, 3 \mathrm{H}, 2-\mathrm{H}_{3}\right), 7.62\left(\mathrm{~d}, J=8.3 \mathrm{~Hz}, 1 \mathrm{H}, 5^{\prime}-\mathrm{H}\right)$, 8.04 (dd, $\left.J=8.3,2.2 \mathrm{~Hz}, 1 \mathrm{H}, 6^{\prime}-\mathrm{H}\right), 8.26$ (d, $\left.J=2.3 \mathrm{~Hz}, 1 \mathrm{H}, 2^{\prime}-\mathrm{H}\right)$.

${ }^{13}$ C-NMR $\left(126 \mathrm{MHz}, \mathrm{CDCl}_{3}\right): \delta(\mathrm{ppm})=26.8(\mathrm{C}-2), 122.8\left(\mathrm{q}, J=273.7 \mathrm{~Hz}, 3^{\prime}-\mathrm{CF}_{3}\right), 127.8$ (q, $\left.J=5.3 \mathrm{~Hz}, \mathrm{C}-2^{\prime}\right), 129.3$ (q, $\left.J=32.0 \mathrm{~Hz}, \mathrm{C}-3^{\prime}\right), 132.3\left(\mathrm{C}-5^{\prime}\right), 132.7$ (C-6'), 135.8 (C-1'), 137.7 (q, $J=1.5 \mathrm{~Hz}, \mathrm{C}-4$ '), $195.8(\mathrm{C}=\mathrm{O})$.

${ }^{19}$ F-NMR $\left(282 \mathrm{MHz}, \mathrm{CDCl}_{3}\right): \delta(\mathrm{ppm})=-62.9$.

MS (ESI): $m / z(\%)=221.0(3)[\mathrm{M}-\mathrm{H}]^{-}$.

$\mathrm{C}_{9} \mathrm{H}_{6} \mathrm{ClF}_{3} \mathrm{O}$ (222.59). 


\subsubsection{1-(4-Fluor-3-methylphenyl)ethanon (261f)}<smiles>Cc1cc(C(=O)O)ccc1F</smiles>

$171 f$

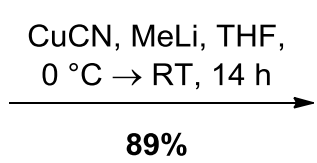

$89 \%$<smiles>CC(=O)c1ccc(F)c(C)c1</smiles>

$261 f$

CuCN (5.81 g, 64.9 mmol, 5.0 Äq.), MeLi (1.6 M in Et 2 O, 81.1 mL, 130 mmol, 10.0 Äq.) und Benzoesäure 171f $(2.00 \mathrm{~g}, 13.0 \mathrm{mmol}, 1.0$ Äq.) wurden gemäß AAV1 umgesetzt. Säulenchromatographische Reinigung an Kieselgel ( $n$ Pentan/ $\mathrm{CH}_{2} \mathrm{Cl}_{2} \quad 3: 1 \rightarrow 2: 1$ ) lieferte die Zielverbindung $261 f$ (1.75 g, 11.5 mmol, 89\%) als farblose, viskose Flüssigkeit.

DC: $\mathrm{R}_{f}=0.37\left(n\right.$ Pentan $\left./ \mathrm{CH}_{2} \mathrm{Cl}_{2} 2: 1\right)$.

UV $\left(\mathrm{CH}_{3} \mathrm{CN}\right): \lambda_{\max }(\lg \varepsilon)=204 \mathrm{~nm}$ (4.6162), 245 (4.2461), 273 (3.2067), 283 (3.0779).

IR (ATR): $\tilde{v}\left(\mathrm{~cm}^{-1}\right)=3353,2959,2927,1681,1589,1498,1356,1256,1174,1124,1114$.

${ }^{1} \mathbf{H}-\mathbf{N M R}\left(300 \mathrm{MHz}, \mathrm{CDCl}_{3}\right): \delta(\mathrm{ppm})=2.31\left(\mathrm{~d}, J=1.9 \mathrm{~Hz}, 3 \mathrm{H}, 3^{\prime}-\mathrm{CH}_{3}\right), 2.56\left(\mathrm{~s}, 3 \mathrm{H}, 2-\mathrm{H}_{3}\right)$, 7.05 (t, $\left.J=8.9 \mathrm{~Hz}, 1 \mathrm{H}, 5^{\prime}-\mathrm{H}\right), 7.74-7.79$ (m, $\left.1 \mathrm{H}, 6^{\prime}-\mathrm{H}\right), 7.79-7.84$ (m, $\left.1 \mathrm{H}, 2^{\prime}-\mathrm{H}\right)$.

${ }^{13}$ C-NMR $\left(126 \mathrm{MHz}, \mathrm{CDCl}_{3}\right): \delta(\mathrm{ppm})=14.9\left(\mathrm{~d}, J=3.6 \mathrm{~Hz}, 3^{\prime}-\mathrm{CH}_{3}\right), 26.8(\mathrm{C}-2), 115.5(\mathrm{~d}$, $\left.J=23.0 \mathrm{~Hz}, \mathrm{C}^{-5} 5^{\prime}\right), 125.6$ (d, $\left.J=17.9 \mathrm{~Hz}, \mathrm{C}-3^{\prime}\right), 128.6\left(\mathrm{~d}, J=9.4 \mathrm{~Hz}, \mathrm{C}-2^{\prime}\right), 132.4$ (d, $J=$ $6.6 \mathrm{~Hz}, \mathrm{C}-6$ '), 133.6 (d, $\left.J=3.3 \mathrm{~Hz}, \mathrm{C}-1^{\prime}\right), 164.7$ (d, $\left.J=253.5 \mathrm{~Hz}, \mathrm{C}-4^{\prime}\right), 197.1$ (C=O).

${ }^{19}$ F-NMR $\left(282 \mathrm{MHz}, \mathrm{CDCl}_{3}\right): \delta=-109.52\left(\mathrm{~m}_{\mathrm{c}}\right)$.

MS (ESI): $m / z(\%)=153.1(100)[\mathrm{M}+\mathrm{H}]^{+}, 175.1(69)[\mathrm{M}+\mathrm{Na}]^{+}$.

HRMS von $\mathrm{C}_{9} \mathrm{H}_{9} \mathrm{FO}$ ber.: 153.0710 gef.: $\quad 153.0709[\mathrm{M}+\mathrm{H}]^{+}(\mathrm{ESI}-\mathrm{HRMS})$.

$\mathrm{C}_{9} \mathrm{H}_{9} \mathrm{FO}$ (152.17). 


\subsubsection{1-(4-Brom-3-methylphenyl)ethanon (261g)}<smiles>O=C(O)c1ccc(Br)c(C(F)(F)Cl)c1</smiles><smiles>CC(=O)c1ccc(Br)c(C(C)(C)C)c1</smiles>

CuCN (2.09 g, 23.3 mmol, 5.0 Äq.), MeLi (1.6 M in Et 2 O, 29.1 mL, 46.5 mmol, 10.0 Äq.) und Benzoesäure $171 \mathrm{~g}(1.00 \mathrm{~g}, 4.65 \mathrm{mmol}, 1.0$ Äq.) wurden gemäß AAV1 umgesetzt. Säulenchromatographische Reinigung an Kieselgel ( $n$ Pentan $/ \mathrm{CH}_{2} \mathrm{Cl}_{2} \quad 3: 1 \rightarrow 2: 1$ ) lieferte die Zielverbindung $\mathbf{2 6 1 g}$ (628 mg, 2.95 mmol, 63\%) als farblose, viskose Flüssigkeit.

DC: $\mathrm{R}_{f}=0.47\left(n\right.$ Pentan/ $\left.\mathrm{CH}_{2} \mathrm{Cl}_{2} 2: 1\right)$.

UV $\left(\mathrm{CH}_{3} \mathrm{CN}\right): \lambda_{\max }(\lg \varepsilon)=205 \mathrm{~nm}$ (4.3124), 255 (4.2056), 284 (3.2542), 293 (3.1138).

IR (ATR): $\tilde{v}\left(\mathrm{~cm}^{-1}\right)=3353,3154,3056,1682,1589,1566,1354,1247,1026$.

${ }^{1} \mathbf{H}-\mathbf{N M R}\left(300 \mathrm{MHz}, \mathrm{CDCl}_{3}\right): \delta(\mathrm{ppm})=2.46\left(\mathrm{~s}, 3 \mathrm{H}, 3{ }^{\prime}-\mathrm{CH}_{3}\right), 2.57\left(\mathrm{~s}, 3 \mathrm{H}, 2-\mathrm{H}_{3}\right), 7.62\left(\mathrm{~s}_{\mathrm{br}}\right.$, $\left.2 \mathrm{H}, 2^{\prime}-\mathrm{H}, 5^{\prime}-\mathrm{H}\right), 7.81$ ( $\mathrm{s}_{\mathrm{br}}, 1 \mathrm{H}, 6$ '-H).

${ }^{13}$ C-NMR (126 MHz, $\left.\mathrm{CDCl}_{3}\right): \delta(\mathrm{ppm})=23.3\left(3^{\prime}-\mathrm{CH}_{3}\right), 26.9(\mathrm{C}-2), 127.4\left(\mathrm{C}-5{ }^{\prime}\right), 130.7\left(\mathrm{C}-6^{\prime}\right)$, 131.0 (C-4'), 133.0 (C-2'), 136.5 (C-3'), 138.8 (C-1'), $197.6(\mathrm{C}=\mathrm{O})$.

MS (ESI): $m / z(\%)=213.0(19)[\mathrm{M}+\mathrm{H}]^{+}, 235.0(100)[\mathrm{M}+\mathrm{Na}]^{+}$.

HRMS von $\mathrm{C}_{9} \mathrm{H}_{9} \mathrm{BrO}$

$\mathrm{C}_{9} \mathrm{H}_{9} \mathrm{BrO}$ (213.07) ber.: 234.9729 gef.: $234.9732[\mathrm{M}+\mathrm{Na}]^{+}(\mathrm{ESI}-\mathrm{HRMS})$. 


\subsection{Synthese der $\alpha$-Bromacetophenone 167a-g}

\subsubsection{2-Brom-1-(4-chlor-3-methylphenyl)ethanon (167a)}
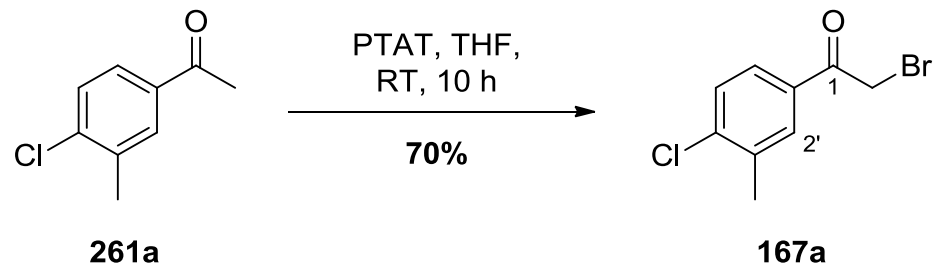

Das Methylketon 261a (2.06 g, 12.2 mmol, 1.0 Äq.) wurde gemäß AAV2 mit PTAT (4.59 g, $12.2 \mathrm{mmol}$, 1.0 Äq.) umgesetzt. Säulenchromatographische Reinigung an Kieselgel $(n$ Pentan/EtOAc 100:1 $\rightarrow 25: 1)$ lieferte die Zielverbindung 167a $(2.11 \mathrm{~g}, 8.54 \mathrm{mmol}, 70 \%)$ als farbloser Feststoff.

DC: $\mathrm{R}_{f}=0.31(n$ Pentan/EtOAc $=25: 1)$.

Smp.: $57^{\circ} \mathrm{C}$.

UV $\left(\mathrm{CH}_{3} \mathrm{CN}\right): \lambda_{\max }(\lg \varepsilon)=194 \mathrm{~nm}$ (5.0031), 260 (4.1154), 370 (2.2767), 401 (1.9545), 501 (1.4082).

IR (ATR): $\tilde{v}\left(\mathrm{~cm}^{-1}\right)=1634,1592,1297,1266,1218,1049,810,736,701$.

${ }^{1} \mathbf{H}-\mathbf{N M R}\left(300 \mathrm{MHz}, \mathrm{DMSO}-\mathrm{d}_{6}\right): \delta(\mathrm{ppm})=2.42\left(\mathrm{~s}, 3 \mathrm{H}, 3^{\prime}-\mathrm{CH}_{3}\right), 4.38$ (s, $\left.2 \mathrm{H}, 2-\mathrm{H}_{2}\right), 7.43(\mathrm{~d}$, $\left.J=8.0 \mathrm{~Hz}, 1 \mathrm{H}, 5^{\prime}-\mathrm{H}\right), 7.67-7.78$ (m, $\left.1 \mathrm{H}, 6^{\prime}-\mathrm{H}\right), 7.79-7.87$ (m, 1 H, 2'-H).

13C-NMR (126 MHz, DMSO-d $\left.\mathrm{d}_{6}\right): \delta(\mathrm{ppm})=20.1\left(3^{\prime}-\mathrm{CH}_{3}\right), 30.5(\mathrm{C}-2), 127.6\left(\mathrm{C}^{\prime} 6^{\prime}\right), 129.6$ (C-5'), 131.3 (C-2'), 132.3 (C-1'), 137.0 (C-3'), 140.7 (C-4'), 190.5 (C=O).

MS (ESI): $\mathrm{m} / \mathrm{z}(\%)=246.9(4)[\mathrm{M}+\mathrm{H}]^{+}, 268.9(52)[\mathrm{M}+\mathrm{Na}]^{+}$.

HRMS von $\mathrm{C}_{9} \mathrm{H}_{8} \mathrm{BrClO}$ ber.: 268.9339 gef.: $268.9339[\mathrm{M}+\mathrm{Na}]^{+}(\mathrm{ESI}-\mathrm{HRMS})$.

$\mathrm{C}_{9} \mathrm{H}_{8} \mathrm{BrClO}$ (245.94). 


\subsubsection{2-Brom-1-(4-chlor-2-methylphenyl)ethanon (167b)}
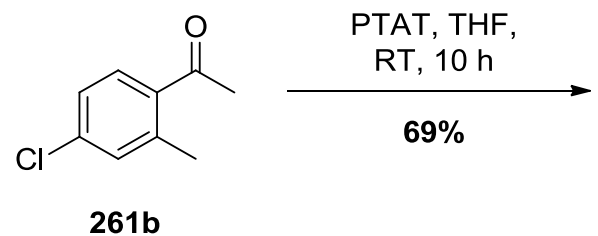<smiles>Cc1cc(Cl)ccc1C(=O)CBr</smiles>

Das Methylketon 261b (603 mg, 3.58 mmol, 1.0 Äq.) wurde gemäß AAV2 mit PTAT (1.35 g, $3.58 \mathrm{mmol}$, 1.0 Äq.) umgesetzt. Säulenchromatographische Reinigung an Kieselgel ( $n$ Pentan/EtOAc 100:1 $\rightarrow 25: 1)$ lieferte die Zielverbindung 167b (611 mg, 2.47 mmol, 69\%) als farbloses Öl.

DC: $\mathrm{R}_{f}=0.42(n$ Pentan/EtOAc 25:1).

UV $\left(\mathrm{CH}_{3} \mathrm{CN}\right): \lambda_{\max }(\lg \varepsilon)=256 \mathrm{~nm}$ (4.0272).

IR (ATR): $\tilde{v}\left(\mathrm{~cm}^{-1}\right)=2969,2930,2856,1681,1592,1556,1256,1093,977$.

${ }^{1} \mathbf{H}-\mathbf{N M R}\left(300 \mathrm{MHz}, \mathrm{CDCl}_{3}\right): \delta(\mathrm{ppm})=2.51\left(\mathrm{~s}, 3 \mathrm{H}, 2^{\prime}-\mathrm{CH}_{3}\right), 4.37$ (s, $\left.2 \mathrm{H}, 2-\mathrm{H}_{2}\right), 7.24-7.29$ (m, 2 H, 3'-H, 5'-H), 7.62 (d, J = 8.1 Hz, 1 H, 6'-H).

${ }^{13}$ C-NMR (126 MHz, $\left.\mathrm{CDCl}_{3}\right): \delta(\mathrm{ppm})=21.7\left(2^{\prime}-\mathrm{CH}_{3}\right), 33.5(\mathrm{C}-2), 126.3\left(\mathrm{C}-5{ }^{\prime}\right), 130.8\left(\mathrm{C}-6^{\prime}\right)$, 132.7 (C-3'), 132.9 (C-2'), 138.7 (C-4'), $142.4\left(\mathrm{C}-1^{\prime}\right), 193.4(\mathrm{C}=\mathrm{O})$.

MS (ESI): $m / z(\%)=249.0(24)[\mathrm{M}+\mathrm{H}]^{+}, 270.9(40)[\mathrm{M}+\mathrm{Na}]^{+}$.

HRMS von $\mathrm{C}_{9} \mathrm{H}_{8} \mathrm{BrClO}$ ber.: 270.9318 gef.: $270.9317[\mathrm{M}+\mathrm{Na}]^{+}(\mathrm{ESI}-\mathrm{HRMS})$.

$\mathrm{C}_{9} \mathrm{H}_{8} \mathrm{BrClO}(247.52)$. 


\subsubsection{2-Brom-1-(2-chlor-3-methylphenyl)ethanon (167c)}<smiles>CC(=O)c1cccc(C)c1Cl</smiles>

261c

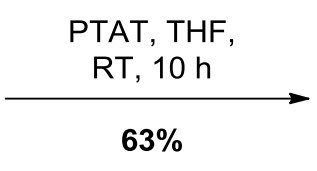

$63 \%$<smiles>Cc1cccc(C(=O)CBr)c1Cl</smiles>

$167 c$

Das Methylketon 261c (600 mg, 3.56 mmol, 1.0 Äq.) wurde gemäß AAV2 mit PTAT (1.34 g, $3.56 \mathrm{mmol}$, 1.0 Äq.) umgesetzt. Säulenchromatographische Reinigung an Kieselgel $(n$ Pentan/EtOAc 100:1 $\rightarrow 25: 1)$ lieferte die Zielverbindung 167c (522 mg, $2.11 \mathrm{mmol}, 59 \%$ ) als farbloses Öl.

DC: $\mathbf{R}_{f}=0.42(n$ Pentan/EtOAc 25:1).

UV $\left(\mathrm{CH}_{3} \mathrm{CN}\right): \lambda_{\max }(\lg \varepsilon)=208 \mathrm{~nm}(4.2461), 246$ (3.6481).

IR (ATR): $\tilde{v}\left(\mathrm{~cm}^{-1}\right)=3070,3051,2955,1698,1575,1285,1047$.

${ }^{1} \mathbf{H}-\mathbf{N M R}\left(300 \mathrm{MHz}, \mathrm{CDCl}_{3}\right): \delta(\mathrm{ppm})=2.42\left(\mathrm{~s}, 3 \mathrm{H}, 3^{\prime}-\mathrm{CH}_{3}\right), 4.48\left(\mathrm{~s}, 2 \mathrm{H}, 2-\mathrm{H}_{2}\right), 7.24(\mathrm{t}, J=$ $\left.7.4 \mathrm{~Hz}, 1 \mathrm{H}, 5^{\prime}-\mathrm{H}\right), 7.25-7.39$ (m, 2 H, 4'-H, 6'-H).

${ }^{13}$ C-NMR $\left(126 \mathrm{MHz}, \mathrm{CDCl}_{3}\right): \delta(\mathrm{ppm})=20.6\left(3^{\prime}-\mathrm{CH}_{3}\right), 35.0(\mathrm{C}-2), 127.1\left(\mathrm{C}-4{ }^{\prime}\right), 127.5\left(\mathrm{C}-5^{\prime}\right)$, $131.0\left(\mathrm{C}-2^{\prime}\right), 134.0\left(\mathrm{C}^{\prime} 6^{\prime}\right), 137.6\left(\mathrm{C}-3^{\prime}\right), 138.0\left(\mathrm{C}-1^{\prime}\right), 195.3(\mathrm{C}=\mathrm{O})$.

MS (ESI): $m / z(\%)=270.9(45)[\mathrm{M}+\mathrm{Na}]^{+}$.

HRMS von $\mathrm{C}_{9} \mathrm{H}_{8} \mathrm{BrClO}$

$\mathrm{C}_{9} \mathrm{H}_{8} \mathrm{BrClO}(247.52)$. ber.: $\quad 270.9318$

gef.: $270.9314[\mathrm{M}+\mathrm{Na}]^{+}(\mathrm{ESI}-\mathrm{HRMS})$. 
6.3.4 2-Brom-1-(3-chlor-4-methylphenyl)ethanon (167d)<smiles>CC(=O)c1ccc(C)c(Cl)c1</smiles>

261d

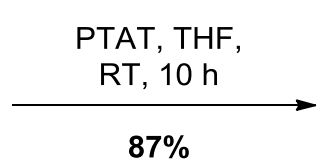

$87 \%$<smiles>Cc1ccc(C(=O)CBr)cc1Cl</smiles>

$167 d$

Das Methylketon 261d (800 mg, 4.74 mmol, 1.0 Äq.) wurde gemäß AAV2 mit PTAT (1.78 g, $4.74 \mathrm{mmol}$, 1.0 Äq.) umgesetzt. Säulenchromatographische Reinigung an Kieselgel $(n$ Pentan/EtOAc 100:1 $\rightarrow 25: 1)$ lieferte die Zielverbindung 167d (632 mg, $2.16 \mathrm{mmol}, 76 \%$ ) als farblosen Feststoff.

DC: $\mathrm{R}_{f}: 0.5(n$ Pentan/EtOAc 25:1).

Smp: $29{ }^{\circ} \mathrm{C}$.

UV $\left(\mathrm{CH}_{3} \mathrm{CN}\right): \lambda_{\max }(\lg \varepsilon)=212 \mathrm{~nm}$ (4.3174), $256 \mathrm{~nm}$ (4.0413).

IR (ATR): $\tilde{v}\left(\mathrm{~cm}^{-1}\right)=3372,3062,3038,3007,2953,1915,1688,1598,1439,1282,1190$.

${ }^{1} \mathbf{H}-\mathbf{N M R}\left(300 \mathrm{MHz}, \mathrm{CDCl}_{3}\right): \delta(\mathrm{ppm})=2.44\left(\mathrm{~s}, 3 \mathrm{H}, 4{ }^{\prime}-\mathrm{CH}_{3}\right), 4.40\left(\mathrm{~s}, 2 \mathrm{H}, 2-\mathrm{H}_{2}\right), 7.35(\mathrm{~d}, J=$ $\left.7.9 \mathrm{~Hz}, 1 \mathrm{H}, 5^{\prime}-\mathrm{H}\right), 7.76$ (dd, $\left.J=7.9,1.8 \mathrm{~Hz}, 1 \mathrm{H}, 6^{\prime}-\mathrm{H}\right), 7.95$ (d, $\left.J=1.8 \mathrm{~Hz}, 1 \mathrm{H}, 2^{\prime}-\mathrm{H}\right)$.

${ }^{13}$ C-NMR $\left(126 \mathrm{MHz}, \mathrm{CDCl}_{3}\right): \delta(\mathrm{ppm})=20.8\left(4^{\prime}-\mathrm{CH}_{3}\right), 30.8(\mathrm{C}-2), 127.4\left(\mathrm{C}-6{ }^{\prime}\right), 129.8\left(\mathrm{C}-2^{\prime}\right)$, $131.6\left(\mathrm{C}^{2} \mathbf{5}^{\prime}\right), 133.5$ (C-3'), $135.6\left(\mathrm{C}-4^{\prime}\right), 143.2\left(\mathrm{C}-1^{\prime}\right), 190.2(\mathrm{C}=\mathrm{O})$.

MS (ESI): $m / z(\%)=270.9(100)[\mathrm{M}+\mathrm{Na}]^{+}$.

HRMS von $\mathrm{C}_{9} \mathrm{H}_{8} \mathrm{BrClO}$ ber.: $\quad 270.9318$ gef.: $270.9318[\mathrm{M}+\mathrm{Na}]+(\mathrm{ESI}-\mathrm{HRMS})$.

$\mathrm{C}_{9} \mathrm{H}_{8} \mathrm{BrClO}(247.52)$. 


\subsubsection{2-Brom-1-(4-chlor-3-(trifluormethyl)phenyl)ethanon (167e)}<smiles>CC(=O)c1ccc(Cl)c(C(F)(F)F)c1</smiles>

261e

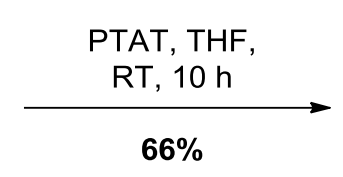<smiles>O=C(CBr)c1ccc(Cl)c(C(F)(F)F)c1</smiles>

167 e

Das Methylketon 261e (760 mg, 3.41 mmol, 1.0 Äq.) wurde gemäß AAV2 mit PTAT (1.28 g, $3.41 \mathrm{mmol}$, 1.0 Äq.) umgesetzt. Säulenchromatographische Reinigung an Kieselgel ( $n$ Pentan/EtOAc 100:1 $\rightarrow 25: 1$ ) lieferte die Zielverbindung 167e (684 mg, $2.27 \mathrm{mmol}, 67 \%$ ) als farbloses Öl.

DC: $\mathrm{R}_{f}=0.57(n$ Penan/EtOAc 25:1).

UV $\left(\mathrm{CH}_{3} \mathrm{CN}\right): \lambda_{\max }(\lg \varepsilon)=251 \mathrm{~nm}(4.1138)$.

IR (ATR): $\tilde{v}\left(\mathrm{~cm}^{-1}\right)=3104,3080,2946,1689,1602,1252,1130,1109,1034$.

${ }^{1} \mathbf{H}-\mathbf{N M R}\left(300 \mathrm{MHz}, \mathrm{CDCl}_{3}\right): \delta(\mathrm{ppm})=4.41\left(\mathrm{~s}, 2 \mathrm{H}, 2-\mathrm{H}_{2}\right), 7.66\left(\mathrm{~d}, J=8.4 \mathrm{~Hz}, 1 \mathrm{H}, 5^{\prime}-\mathrm{H}\right)$, 8.09 (dd, $\left.J=8.4,2.2 \mathrm{~Hz}, 1 \mathrm{H}, 6^{\prime}-\mathrm{H}\right), 8.31$ (d, $\left.J=2.2 \mathrm{~Hz}, 1 \mathrm{H}, 2^{\prime}-\mathrm{H}\right)$.

${ }^{13} \mathbf{C}-\mathbf{N M R}\left(126 \mathrm{MHz}, \mathrm{CDCl}_{3}\right): \delta(\mathrm{ppm})=30.1(\mathrm{C}-2), 122.6\left(\mathrm{q}, J=273.7 \mathrm{~Hz}, 3^{\prime}-\mathrm{CF}_{3}\right), 128.5$ (q, $\left.J=5.3 \mathrm{~Hz}, \mathrm{C}^{\prime} 2^{\prime}\right), 129.7$ (q, $\left.J=32.1 \mathrm{~Hz}, \mathrm{C}-3^{\prime}\right), 132.6\left(\mathrm{C}-5^{\prime}\right), 132.7\left(\mathrm{C}-1^{\prime}\right), 133.3$ (C-6'), $138.6(\mathrm{q}, J=1.5 \mathrm{~Hz}, \mathrm{C}-4$ '), $189.5(\mathrm{C}=\mathrm{O})$.

${ }^{19}$ F-NMR $\left(282 \mathrm{MHz}, \mathrm{CDCl}_{3}\right): \delta(\mathrm{ppm})=-63.0$.

MS (ESI): $m / z(\%)=300.9(68)[\mathrm{M}-\mathrm{H}]^{-}$.

HRMS von $\mathrm{C}_{9} \mathrm{H}_{5} \mathrm{BrClF}_{3} \mathrm{O}$ ber.: $\quad 300.9070$

gef.: $300.9074[\mathrm{M}-\mathrm{H}]^{-}$(ESI-HRMS).

$\mathrm{C}_{9} \mathrm{H}_{5} \mathrm{BrClF}_{3} \mathrm{O}$ (301.49). 


\subsubsection{2-Brom-1-(4-Fluor-3-methylphenyl)ethanon (167f)}<smiles>CC(=O)c1ccc(F)c(C)c1</smiles>

$261 f$

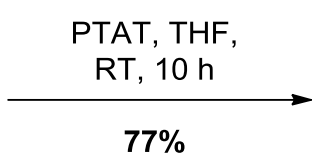

$77 \%$<smiles>Cc1cc(C(=O)CBr)ccc1F</smiles>

$167 f$

Das Methylketon $261 f$ (800 mg, 5.26 mmol, 1.0 Äq.) wurde gemäß AAV2 mit PTAT (1.98 g, $5.26 \mathrm{mmol}$, 1.0 Äq.) umgesetzt. Säulenchromatographische Reinigung an Kieselgel $(n$ Pentan/EtOAc 100:1 $\rightarrow 25: 1)$ lieferte die Zielverbindung 167f (933 mg, 4.04 mmol, 77\%) als farblosen Feststoff.

DC: $\mathbf{R}_{f}=0.45(n$ Pentan/EtOAc 25:1).

Smp.: $47^{\circ} \mathrm{C}$.

UV $\left(\mathrm{CH}_{3} \mathrm{CN}\right): \lambda_{\max }(\lg \varepsilon)=203 \mathrm{~nm}$ (4.3128), 253 (4.0018).

IR (ATR): $\tilde{v}\left(\mathrm{~cm}^{-1}\right)=3096,3068,2947,2931,1914,1776,1683,1608,1238,1122,821$.

${ }^{1} \mathbf{H}-\mathbf{N M R}\left(300 \mathrm{MHz}, \mathrm{CDCl}_{3}\right): \delta(\mathrm{ppm})=2.34\left(\mathrm{~d}, J=2.0 \mathrm{~Hz}, 3 \mathrm{H}, 3^{\prime}-\mathrm{CH}_{3}\right), 4.40\left(\mathrm{~s}, 2 \mathrm{H}, 2-\mathrm{H}_{2}\right)$, 7.09 (t, $\left.J=8.8 \mathrm{~Hz}, 1 \mathrm{H}, 5^{\prime}-\mathrm{H}\right), 7.78-7.83$ (m, 1 H, 6'-H), 7.83-7.88 (m, 1 H, 2'-H).

${ }^{13} \mathrm{C}-\mathrm{NMR}\left(126 \mathrm{MHz}, \mathrm{CDCl}_{3}\right): \delta(\mathrm{ppm})=14.9\left(\mathrm{~d}, J=3.3 \mathrm{~Hz}, 3^{\prime}-\mathrm{CH}_{3}\right), 30.9(\mathrm{C}-2), 115.9(\mathrm{~d}$, $\left.J=23.4 \mathrm{~Hz}, \mathrm{C}-5^{\prime}\right), 126.3$ (d, $\left.J=18.2 \mathrm{~Hz}, \mathrm{C}-3^{\prime}\right), 129.4$ (d, $\left.J=9.4 \mathrm{~Hz}, \mathrm{C}-2^{\prime}\right), 130.4$ (d, $J=$ $\left.3.3 \mathrm{~Hz}, \mathrm{C}-1^{\prime}\right), 138.1$ (d, $\left.J=6.9 \mathrm{~Hz}, \mathrm{C}^{\prime} 6^{\prime}\right), 165.1$ (d, $\left.J=255.3 \mathrm{~Hz}, \mathrm{C}-4^{\prime}\right), 190.4$ (C=O).

${ }^{19}$ F-NMR $\left(282 \mathrm{MHz}, \mathrm{CDCl}_{3}\right): \delta(\mathrm{ppm})=-107.4\left(\mathrm{~m}_{\mathrm{c}}\right)$.

MS (EI, $70 \mathrm{eV}): m / z(\%)=230.0(5)[\mathrm{M}]^{+*}$.

HRMS von $\mathrm{C}_{9} \mathrm{H}_{8} \mathrm{BrFO}$ ber.: $\quad 229.9743$

gef.: $229.9746[\mathrm{M}]^{+*}(\mathrm{EI}-\mathrm{HRMS})$.

$\mathrm{C}_{9} \mathrm{H}_{8} \mathrm{BrFO}$ (231.06). 


\subsubsection{2-Brom-1-(4-brom-3-methylphenyl)ethanon (167g)}<smiles>CC(=O)c1ccc(Br)c(C)c1</smiles>

$261 \mathrm{~g}$

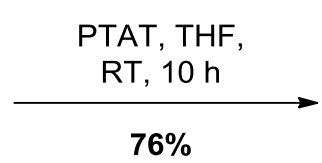

$76 \%$<smiles>Cc1cc(C(=O)CBr)ccc1Br</smiles>

$167 g$

Das Methylketon 261g (602 mg, 2.83 mmol, 1.0 Äq.) wurde gemäß AAV2 mit PTAT (1.06 g $2.83 \mathrm{mmol}$, 1.0 Äq.) umgesetzt. Säulenchromatographische Reinigung an Kieselgel ( $n$ Pentan/EtOAc 100:1 $\rightarrow 25: 1$ ) lieferte die Zielverbindung 167g (632 mg, 2.16 mmol, 76\%) als farblosen Feststoff.

DC: $\mathbf{R}_{f}=0.64(n$ Pentan/EtOAc 25:1).

Smp.: $76^{\circ} \mathrm{C}$.

UV $\left(\mathrm{CH}_{3} \mathrm{CN}\right): \lambda_{\max }(\lg \varepsilon)=263 \mathrm{~nm}(4.1213)$.

IR (ATR): $\tilde{v}\left(\mathrm{~cm}^{-1}\right)=3368,3088,3072,2946,1692,1564,1384,1216,1028$.

${ }^{1} \mathbf{H}-\mathbf{N M R}\left(300 \mathrm{MHz}, \mathrm{CDCl}_{3}\right): \delta(\mathrm{ppm})=2.46\left(\mathrm{~s}, 3 \mathrm{H}, 3^{\prime}-\mathrm{CH}_{3}\right), 4.40\left(\mathrm{~s}, 2 \mathrm{H}, 2-\mathrm{H}_{2}\right), 7.60-7.67$ (m, 2 H, 2'-H, 5'-H), 7.82-7.84 (m, 1 H, 6'-H).

${ }^{13}$ C-NMR (126 MHz, $\left.\mathrm{CDCl}_{3}\right): \delta(\mathrm{ppm})=23.3\left(3^{\prime}-\mathrm{CH}_{3}\right), 30.9$ (C-2), $127.9\left(\mathrm{C}-5{ }^{\prime}\right), 131.2\left(\mathrm{C}-6^{\prime}\right)$, 132.0 (C-4'), 133.3, 133.3 (C-2', C-3'), 139.3 (C-1'), $191.0(\mathrm{C}=\mathrm{O})$.

MS (ESI): $m / z(\%)=312.9(34)[\mathrm{M}+\mathrm{Na}]^{+}$.

HRMS von $\mathrm{C}_{9} \mathrm{H}_{8} \mathrm{Br}_{2} \mathrm{O}$ ber.: $\quad 312.8834$ gef.: $312.8820[\mathrm{M}+\mathrm{Na}]^{+}(\mathrm{ESI}-\mathrm{HRMS})$.

$\mathbf{C}_{9} \mathbf{H}_{8} \mathrm{Br}_{2} \mathbf{O}$ (291.97). 


\subsection{Synthese der potentiellen Hsp47-Inhibitoren 48a-g}

6.4.1 2-((2-(4-Chlor-3-methylphenyl)-2-oxoethyl)thio)-6-oxo-4-(thiophen-2-yl)-1,6dihydropyrimidin-5-carbonitril (48a)<smiles>N#Cc1c(-c2cccs2)nc(S)[nH]c1=O</smiles>

166<smiles>Cc1cc(C(=O)CBr)ccc1Cl</smiles>

$167 a$
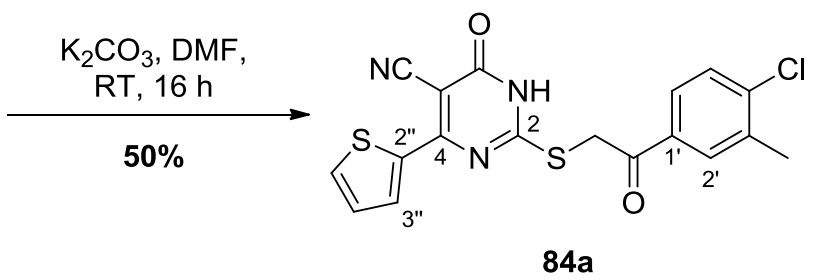

Dihydropyrimidon 166 (235 mg, 1.00 mmol, 1.0 Äq.), $\alpha$-Bromacetophenon 167b (248 mg, $1.00 \mathrm{mmol}, 1.0$ Äq.) und $\mathrm{K}_{2} \mathrm{CO}_{3}$ (138 mg, $1.00 \mathrm{mmol}, 1.0$ Äq.) wurden gemäß AAV3 umgesetzt und das Rohprodukt aus MeOH umkristallisiert. Die Zielverbindung 84b (199 mg, $500 \mu \mathrm{mol}, 50 \%)$ wurde in Form eines braunen Feststoffs isoliert.

DC: $\mathrm{R}_{f}=0.24\left(\mathrm{CH}_{2} \mathrm{Cl}_{2} / \mathrm{MeOH}=12: 1\right)$.

Smp.: $280{ }^{\circ} \mathrm{C}$.

UV $\left(\mathrm{CH}_{3} \mathrm{CN}\right): \lambda_{\max }(\lg \varepsilon)=194 \mathrm{~nm}$ (5.2419), 258 (4.4767), 319 (4.1842).

IR (ATR): $\tilde{v}\left(\mathrm{~cm}^{-1}\right)=1666,1544,1531,1510,1415,1253,1051,983,726$.

${ }^{1} \mathbf{H}-\mathbf{N M R}\left(600 \mathrm{MHz}, \mathrm{DMSO}-\mathrm{d}_{6}\right): \delta(\mathrm{ppm})=2.42\left(\mathrm{~s}, 3 \mathrm{H}, 3^{\prime}-\mathrm{CH}_{3}\right), 4.96\left(\mathrm{~s}, 2 \mathrm{H}, \mathrm{CH}_{2}\right), 7.22$ (dd, $\left.J=5.0,3.9 \mathrm{~Hz}, 1 \mathrm{H}, 4^{\prime \prime}-\mathrm{H}\right), 7.63$ (d, $\left.J=8.3 \mathrm{~Hz}, 1 \mathrm{H}, 5 '-\mathrm{H}\right), 7.85-7.94$ (m, $2 \mathrm{H}, 6$ '-H, 3"-H), 8.06 (d, J = 2.1 Hz, 1 H, 2'-H), 8.12 (dd, $J=4.0,1.0 \mathrm{~Hz}, 1 \mathrm{H}, 5$ "-H).

${ }^{13}$ C-NMR $\left(126 \mathrm{MHz}\right.$, DMSO-d $\left.{ }_{6}\right): \delta(\mathrm{ppm})=19.5\left(3^{\prime}-\mathrm{CH}_{3}\right), 38.6\left(\mathrm{CH}_{2}\right), 88.1(\mathrm{C}-5), 115.9$ (CN), 127.5 (C-6'), 129.2 (C-4"), 129.3 (C-5'), 130.9 (C-2'), 131.5 (C-5"), 134.2 (C-1'), 134.6 (C-3"), 136.2 (C-3'), 138.9 (C-2", C-4'), 158.5 (C-6), 160.7 (C-4), 164.8 (C-2), 191.2 (C=O). MS (ESI): $\mathrm{m} / \mathrm{z}(\%)=402.0(5)[\mathrm{M}+\mathrm{H}]^{+}, 424.0(100)[\mathrm{M}+\mathrm{Na}]^{+}, 440.0(53)[\mathrm{M}+\mathrm{K}]^{+} ; 400.0$ (100) $[\mathrm{M}-\mathrm{H}]^{-}, 801.0$ (4) [2M-H]'.

HRMS von $\mathrm{C}_{18} \mathrm{H}_{12} \mathrm{ClN}_{3} \mathrm{O}_{2} \mathrm{~S}_{2}$ ber.: 423.9952 gef.: $423.9933[\mathrm{M}+\mathrm{Na}]^{+}(\mathrm{ESI}-\mathrm{HRMS})$.

$\mathrm{C}_{18} \mathrm{H}_{12} \mathrm{ClN}_{3} \mathrm{O}_{2} \mathbf{S}_{2}$ (401.01). 


\subsubsection{2-((2-(4-Chlor-2-methylphenyl)-2-oxoethyl)thio)-6-oxo-4-(thiophen-2-yl)-1,6-} dihydropyrimidin-5-carbonitril (84b)<smiles>N#Cc1c(-c2cccs2)nc(S)[nH]c1=O</smiles>

166<smiles>Cc1cc(Cl)ccc1C(=O)CBr</smiles>

$167 b$

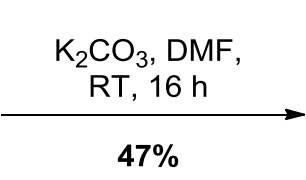<smiles>Cc1cc(Cl)ccc1C(=O)CSc1nc(-c2cccs2)c(C#N)c(=O)[nH]1</smiles>

84b

Dihydropyrimidon 166 (333 mg, 1.41 mmol, 1.0 Äq.), $\alpha$-Bromacetophenon 167b (350 mg, $1.41 \mathrm{mmol}, 1.0$ Äq.) und $\mathrm{K}_{2} \mathrm{CO}_{3}$ (195 mg, $1.41 \mathrm{mmol}, 1.0$ Äq.) wurden gemäß AAV3 umgesetzt und das Rohprodukt aus MeOH umkristallisiert. Die Zielverbindung 84b (268 mg, $666 \mu \mathrm{mol}, 47 \%$ ) wurde in Form eines braunen Feststoffs isoliert.

DC: $\mathrm{R}_{f}=0.50\left(\mathrm{CH}_{2} \mathrm{Cl}_{2} / \mathrm{MeOH} 8: 1\right)$.

Smp.: $232{ }^{\circ} \mathrm{C}$.

UV $\left(\mathrm{CH}_{3} \mathrm{CN}\right): \lambda_{\max }(\lg \varepsilon)=205 \mathrm{~nm}$ (4.2516), 256 (4.0564), 297 (3.7531), 347 (3.5576), 366 (3.3172).

IR (ATR): $\tilde{v}\left(\mathrm{~cm}^{-1}\right)=2911,2710,2217,1693,1654,1545,1471,1413,1259,1201,977$.

${ }^{1} \mathbf{H}-\mathbf{N M R}\left(600 \mathrm{MHz}, \mathrm{DMSO}-\mathrm{d}_{6}\right): \delta(\mathrm{ppm})=2.34\left(\mathrm{~s}, 3 \mathrm{H}, 2^{\prime}-\mathrm{CH}_{3}\right), 4.90\left(\mathrm{~s}, 2 \mathrm{H}, \mathrm{CH}_{2}\right), 7.28$ (dd, $\left.J=4.8,4.0 \mathrm{~Hz}, 1 \mathrm{H}, 4^{\prime \prime}-\mathrm{H}\right), 7.44$ (d, $\left.J=1.8 \mathrm{~Hz}, 1 \mathrm{H}, 3^{\prime}-\mathrm{H}\right), 7.50$ (dd, $J=8.3,1.8 \mathrm{~Hz}, 1 \mathrm{H}$, 5'-H), 7.97 (dd, $\left.J=4.8,0.7 \mathrm{~Hz}, 1 \mathrm{H}, 3^{\prime \prime}-\mathrm{H}\right), 8.04$ (d, $\left.J=8.3 \mathrm{~Hz}, 1 \mathrm{H}, 6^{\prime}-\mathrm{H}\right), 8.18$ (dd, $J=4.0$, $\left.0.7 \mathrm{~Hz}, 1 \mathrm{H}, 5^{\prime \prime}-\mathrm{H}\right)$.

${ }^{13}$ C-NMR $\left(126 \mathrm{MHz}\right.$, DMSO-d 6 ): $\delta(\mathrm{ppm})=20.2\left(2^{\prime}-\mathrm{CH}_{3}\right), 40.4\left(\mathrm{CH}_{2}\right), 88.1(\mathrm{C}-5), 115.7$ (CN), 125.7 (C-5'), 129.0 (C-4"), 130.8 (C-6'), 131.2 (C-3'), 131.5 (C-5"), 134.4 (C-3"), 134.5 (C-1'), 136.4 (C-4'), 138.7 (C-2'), 140.5 (C-2"), 158.5 (C-6), 160.6 (C-4), 164.7 (C-2), 194.2 $(\mathrm{C}=\mathrm{O})$.

MS (ESI): $m / z(\%)=400.0(100)[\mathrm{M}-\mathrm{H}]^{-}$.

HRMS von $\mathrm{C}_{18} \mathrm{H}_{12} \mathrm{ClN}_{3} \mathrm{O}_{2} \mathrm{~S}_{2}$ ber.: 399.9987 gef.: $399.9982[\mathrm{M}-\mathrm{H}]^{-}$(ESI-HRMS).

$\mathrm{C}_{18} \mathrm{H}_{12} \mathrm{ClN}_{3} \mathrm{O}_{2} \mathrm{~S}_{2}$ (401.88). 


\subsubsection{2-((2-(2-Chlor-3-methylphenyl)-2-oxoethyl)thio)-6-oxo-4-(thiophen-2-yl)-1,6-} dihydropyrimidin-5-carbonitril (84c)<smiles>Cc1cccc(C(=O)CBr)c1Cl</smiles>

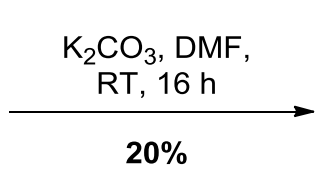

$167 c$

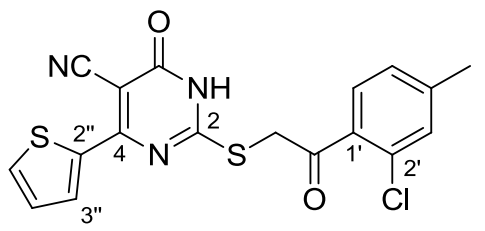

84c

Dihydropyrimidon 166 (344 mg, 1.46 mmol, 1.0 Äq.), $\alpha$-Bromacetophenon 167c (361 mg, $1.46 \mathrm{mmol}, 1.0$ Äq.) und $\mathrm{K}_{2} \mathrm{CO}_{3}(180 \mathrm{mg}, 1.46 \mathrm{mmol}, 1.0$ Äq.) wurden gemäß AAV3 umgesetzt und das Rohprodukt aus MeOH umkristallisiert. Die Zielverbindung 84c (119 mg, $297 \mu \mathrm{mol}, 20 \%$ ) wurde in Form eines braunen Feststoffs isoliert.

DC: $\mathrm{R}_{f}=0.73\left(\mathrm{CH}_{2} \mathrm{Cl}_{2} / \mathrm{MeOH} 8: 1\right)$.

Smp.: Zersetzung bei ca. $190{ }^{\circ} \mathrm{C}$.

UV $\left(\mathrm{CH}_{3} \mathrm{CN}\right): \lambda_{\max }(\lg \varepsilon)=256 \mathrm{~nm}$ (4.1976), 298 (3.8969), 341 (3.6658).

IR (ATR): $\tilde{v}\left(\mathrm{~cm}^{-1}\right)=3277,3079,2910,2198,1685,1547,1531,1465,1287,1101,983$.

${ }^{1} \mathbf{H}-\mathbf{N M R}\left(600 \mathrm{MHz}, \mathrm{DMSO}-\mathrm{d}_{6}\right): \delta(\mathrm{ppm})=2.36\left(\mathrm{~s}, 3 \mathrm{H}, 3{ }^{\prime}-\mathrm{CH}_{3}\right), 4.60\left(\mathrm{~s}, 2 \mathrm{H}, \mathrm{CH}_{2}\right), 7.23$ $\left(\mathrm{dd}, J=5.0,3.9 \mathrm{~Hz}, 1 \mathrm{H}, 4^{\prime \prime}-\mathrm{H}\right), 7.41-7.44$ (m, $\left.2 \mathrm{H}, 4^{\prime}-\mathrm{H}, 5 '-\mathrm{H}\right), 7.80(\mathrm{dd}, J=5.0,0.9 \mathrm{~Hz}, 1 \mathrm{H}$, 3"-H), 7.99-8.01 (m, 1 H, 6'-H), 8.10 (dd, J = 3.9, 0.9 Hz, 1 H, 5"-H).

${ }^{13}$ C-NMR $\left(126 \mathrm{MHz}, \mathrm{DMSO}-\mathrm{d}_{6}\right): \delta(\mathrm{ppm})=20.0\left(3^{\prime}-\mathrm{CH}_{3}\right), 39.5\left(\mathrm{CH}_{2}\right), 86.0(\mathrm{C}-5), 119.0$ (CN), 125.5 (C-4'), 128.3 (C-4"), 129.1 (C-5"), 130.5 (C-5'), 131.0 (C-6'), 131.3 (C-3"), 135.7 (C-2"), 135.8 (C-2'), 140.0 (C-3'), 141.0 (C-1'), 158.6 (C-6), 167.4 (C-2), 168.9 (C-4), 196.7 $(\mathrm{C}=\mathrm{O})$.

MS (ESI): $m / z(\%)=400.0(100)[\mathrm{M}-\mathrm{H}]^{-}$.

HRMS von $\mathrm{C}_{18} \mathrm{H}_{12} \mathrm{ClN}_{3} \mathrm{O}_{2} \mathrm{~S}_{2}$ ber.: 399.9987

gef.: $399.9986[\mathrm{M}-\mathrm{H}]^{-}$(ESI-HRMS).

$\mathrm{C}_{18} \mathrm{H}_{12} \mathrm{ClN}_{3} \mathrm{O}_{2} \mathrm{~S}_{2}$ (401.88). 


\subsubsection{2-((2-(3-Chlor-4-methylphenyl)-2-oxoethyl)thio)-6-oxo-4-(thiophen-2-yl)-1,6-} dihydropyrimidin-5-carbonitril (84d)<smiles>Cc1ccc(C(=O)CBr)cc1Cl</smiles><smiles>Cc1ccc(C(=O)CSc2nc(-c3cccs3)c(C#N)c(=O)[nH]2)cc1Cl</smiles>

Dihydropyrimidon 166 (478 mg, 2.03 mmol, 1.0 Äq.), $\alpha$-Bromacetophenon 167d (500 mg, $2.03 \mathrm{mmol}, 1.0$ Äq.) und $\mathrm{K}_{2} \mathrm{CO}_{3}(281 \mathrm{mg}, 2.03 \mathrm{mmol}, 1.0$ Äq.) wurden gemäß AAV3 umgesetzt und das Rohprodukt aus $\mathrm{MeOH}$ umkristallisiert. Die Zielverbindung 84d (444 mg, $1.10 \mathrm{mmol}, 54 \%$ ) wurde in Form eines orangenen Feststoffs isoliert.

DC: $\mathrm{R}_{f}=0.48\left(\mathrm{CH}_{2} \mathrm{Cl}_{2} / \mathrm{MeOH} 8: 1\right)$.

Smp.: Zersetzung bei ca. $251{ }^{\circ} \mathrm{C}$.

UV $\left(\mathrm{CH}_{3} \mathrm{CN}\right): \lambda_{\max }(\lg \varepsilon)=211 \mathrm{~nm}$ (4.3442), 256 (4.1985), 296 (3.8313), 346 (3.4210), 371 (2.8150).

IR (ATR): $\tilde{v}\left(\mathrm{~cm}^{-1}\right)=2966,2918,2194,1701,1564,1450,1281,1204,1144,1047,981$.

${ }^{1} \mathbf{H}-\mathbf{N M R}\left(600 \mathrm{MHz}, \mathrm{DMSO}-\mathrm{d}_{6}\right): \delta(\mathrm{ppm})=2.44\left(\mathrm{~s}, 3 \mathrm{H}, 4^{\prime}-\mathrm{CH}_{3}\right), 4.66\left(\mathrm{~s}, 2 \mathrm{H}, \mathrm{CH}_{2}\right), 7.20$ $\left(\mathrm{dd}, J=5.1,3.9 \mathrm{~Hz}, 1 \mathrm{H}, 4^{\prime \prime}-\mathrm{H}\right), 7.55$ (d, $\left.J=7.9 \mathrm{~Hz}, 1 \mathrm{H}, 5^{\prime}-\mathrm{H}\right), 7.71(\mathrm{dd}, J=5.1,1.0 \mathrm{~Hz}, 1 \mathrm{H}$, 3"-H), 7.95 (dd, $\left.J=7.9,1.7 \mathrm{~Hz}, 1 \mathrm{H}, 6{ }^{\prime}-\mathrm{H}\right), 8.06$ (d, $\left.J=1.7 \mathrm{~Hz}, 1 \mathrm{H}, 2^{\prime}-\mathrm{H}\right), 8.07$ (dd, $J=3.9$, $\left.1.0 \mathrm{~Hz}, 1 \mathrm{H}, 5^{\prime \prime}-\mathrm{H}\right)$.

${ }^{13}$ C-NMR $(126 \mathrm{MHz}$, DMSO-d 6$): \delta(\mathrm{ppm})=20.7\left(4^{\prime}-\mathrm{CH}_{3}\right), 38.2\left(\mathrm{CH}_{2}\right), 86.3(\mathrm{C}-5), 121.1$ (CN), 127.9 (C-6'), 129.0 (C-4"), 129.1 (C-5"), 129.4 (C-2'), 131.1 (C-3"), 132.4 (C-5'), 134.6 (C-3'), 136.4 (C-4'), 142.1 (C-1'), 142.7 (C-2"), 159.4 (C-6), 170.9 (C-4), 171.2 (C-2), 194.2 $(\mathrm{C}=\mathrm{O})$.

MS (ESI): $m / z(\%)=440.0(100)[\mathrm{M}+\mathrm{K}]^{+} ; 400.0(100)[\mathrm{M}-\mathrm{H}]^{-}$.

HRMS von $\mathrm{C}_{18} \mathrm{H}_{12} \mathrm{ClN}_{3} \mathrm{O}_{2} \mathrm{~S}_{2}$ ber.: 399.9987 gef.: $399.9991[\mathrm{M}-\mathrm{H}]^{-}$(ESI-HRMS).

$\mathrm{C}_{18} \mathrm{H}_{12} \mathrm{ClN}_{3} \mathrm{O}_{2} \mathrm{~S}_{2}$ (401.88). 


\subsubsection{2-((2-(4-Chlor-3-(trifluormethyl)phenyl)-2-oxoethyl)thi-o)-6-oxo-4-(thiophen-2-} yl)-1,6-dihydropyrimidin-5-carbonitril (84e)<smiles>N#Cc1c(-c2cccs2)nc(S)[nH]c1=O</smiles>

166<smiles>O=C(CBr)c1ccc(Cl)c(C(F)(F)F)c1</smiles>

$167 e$
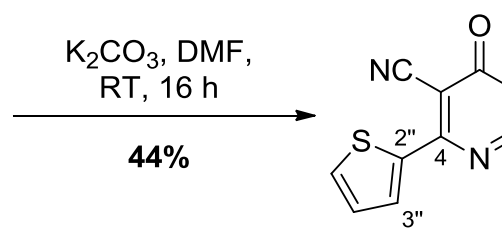

$84 e$

Dihydropyrimidon 166 (156 mg, $663 \mu \mathrm{mol}, 1.0$ Äq.), $\alpha$-Bromacetophenon 167e (200 mg, $663 \mu \mathrm{mol}, 1.0$ Äq.) und $\mathrm{K}_{2} \mathrm{CO}_{3}(91.6 \mathrm{mg}, 663 \mu \mathrm{mol}, 1.0$ Äq.) wurden gemäß AAV3 umgesetzt und das Rohprodukt aus $\mathrm{MeOH}$ umkristallisiert. Die Zielverbindung 84e (134 mg, $294 \mu \mathrm{mol}, 44 \%$ ) wurde in Form eines gelben Feststoffs isoliert.

DC: $\mathrm{R}_{f}=0.61\left(\mathrm{CH}_{2} \mathrm{Cl}_{2} / \mathrm{MeOH} 8: 1\right)$.

Smp.: Zersetzung bei ca. $200{ }^{\circ} \mathrm{C}$.

UV $\left(\mathrm{CH}_{3} \mathrm{CN}\right): \lambda_{\max }(\lg \varepsilon)=253 \mathrm{~nm}$ (4.3750), 302 (4.0463), 341 (3.8260).

IR (ATR): $\tilde{v}\left(\mathrm{~cm}^{-1}\right)=3093,2920,1663,1530,1476,1410,1388,1176,1134,983$.

${ }^{1} \mathbf{H}-\mathbf{N M R}\left(300 \mathrm{MHz}, \mathrm{CD}_{3} \mathrm{OD}, 50{ }^{\circ} \mathrm{C}\right): \delta(\mathrm{ppm})=4.96\left(\mathrm{~s}_{\mathrm{br}}, 2 \mathrm{H}, \mathrm{CH}_{2}\right.$; in DMSO-d 6$), 7.14(\mathrm{dd}$, $J=5.1,3.9 \mathrm{~Hz}, 1 \mathrm{H}, 4 "-\mathrm{H}), 7.54\left(\mathrm{dd}, J=5.1,1.0 \mathrm{~Hz}, 1 \mathrm{H}, 3^{\prime \prime}-\mathrm{H}\right), 7.78(\mathrm{~d}, J=8.4 \mathrm{~Hz}, 1 \mathrm{H}$, 5'-H), $8.15\left(\mathrm{dd}, J=8.4,2.1 \mathrm{~Hz}, 1 \mathrm{H}, 5^{\prime \prime}-\mathrm{H}\right), 8.35$ (dd, $J=3.9,1.0 \mathrm{~Hz}, 1 \mathrm{H}, 6$ '-H), 8.42 (d, $J=$ $\left.2.1 \mathrm{~Hz}, 1 \mathrm{H}, 2^{\prime}-\mathrm{H}\right)$.

${ }^{13} \mathrm{C}$-NMR $\left(126 \mathrm{MHz}, \mathrm{CD}_{3} \mathrm{OD}, 50{ }^{\circ} \mathrm{C}\right): \delta(\mathrm{ppm})=39.1\left(\mathrm{CH}_{2}\right.$; in DMSO-d 6 ), $88.3(\mathrm{C}-5), 121.0$ $(\mathrm{CN}), 124.8$ (q, $\left.J=273.6 \mathrm{~Hz}, 3^{\prime}-\mathrm{CF}_{3}\right), 129.6$ (q, $\left.J=5.4 \mathrm{~Hz}, \mathrm{C}-2^{\prime}\right), 129.7$ (C-4"), 130.4 (q, $J=$ 31.8 Hz, C-3'), 131.2 (C-5"), 132.0 (C-3"), 134.0 (C-5'), 135.3 (C-6'), 137.3 (C-2"), 138.7 (q, $J=1.6 \mathrm{~Hz}, \mathrm{C}-4$ '), 143.4 (C-1'), 162.1 (C-6), $173.6(\mathrm{C}-4), 175.1$ (C-2), $195.3(\mathrm{C}=\mathrm{O})$.

${ }^{19}$ F-NMR (283 MHz, CD $\left.3 \mathrm{OD}\right): \delta(\mathrm{ppm})=-60.2\left(\mathrm{~m}_{\mathrm{c}}\right)$.

MS (ESI): $m / z(\%)=456.0(26)[\mathrm{M}+\mathrm{H}]^{+}, 478.0(61)[\mathrm{M}+\mathrm{Na}]^{+}$.

HRMS von $\mathrm{C}_{18} \mathrm{H}_{9} \mathrm{ClF}_{3} \mathrm{~N}_{3} \mathrm{O}_{2} \mathrm{~S}_{2}$ ber.: $\quad 453.9704$ gef.: $\quad 453.9707[\mathrm{M}-\mathrm{H}]^{-}$(ESI-HRMS).

$\mathrm{C}_{18} \mathrm{H}_{9} \mathrm{ClF}_{3} \mathrm{~N}_{3} \mathrm{O}_{2} \mathrm{~S}_{2}$ (455.85). 


\subsubsection{2-((2-(4-Fluor-3-methylphenyl)-2-oxoethyl)thio)-6-oxo-4-(thiophen-2-yl)-1,6-} dihydropyrimidin-5-carbonitril (84f)<smiles>N#Cc1c(-c2cccs2)nc(S)[nH]c1=O</smiles>

166<smiles>Cc1cc(C(=O)CBr)ccc1F</smiles>

$167 f$
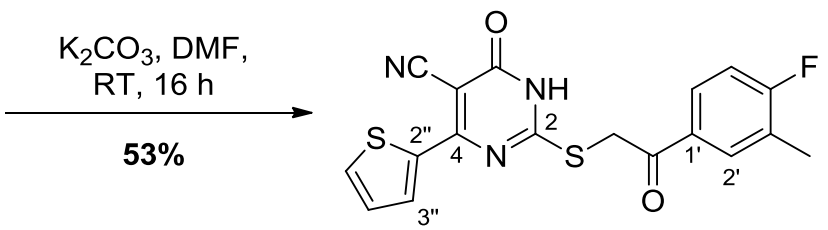

$84 f$

Dihydropyrimidon 166 (358 mg, $1.52 \mathrm{mmol}, 1.0$ Äq.), $\alpha$-Bromacetophenon $167 f(350 \mathrm{mg}$, $1.52 \mathrm{mmol}$, 1.0 Äq.) und $\mathrm{K}_{2} \mathrm{CO}_{3}$ (138 mg, $1.52 \mathrm{mmol}, 1.0$ Äq.) wurden gemäß AAV3 umgesetzt und das Rohprodukt aus $\mathrm{MeOH}$ umkristallisiert. Die Zielverbindung 84f (307 mg, $800 \mu \mathrm{mol}, 53 \%$ ) wurde in Form eines braunen Feststoffs isoliert.

DC: $\mathrm{R}_{f}=0.63\left(\mathrm{CH}_{2} \mathrm{Cl}_{2} / \mathrm{MeOH} 8: 1\right)$.

Smp.: Zersetzung bei ca. $257^{\circ} \mathrm{C}$.

UV ( $\left.\mathrm{CH}_{3} \mathrm{CN}\right): \lambda_{\max }(\lg \varepsilon)=201 \mathrm{~nm}$ (4.5351), 255 (4.3675), 297 (4.0805), 343 (3.7434).

IR (ATR): $\tilde{v}\left(\mathrm{~cm}^{-1}\right)=2968,2919,2194,1698,1561,1450,1282,1242,1109,981$.

${ }^{1} \mathbf{H}-\mathbf{N M R}\left(600 \mathrm{MHz}, \mathrm{DMSO}-\mathrm{d}_{6}\right): \delta(\mathrm{ppm})=2.32\left(\mathrm{~s}, 3 \mathrm{H}, 3^{\prime}-\mathrm{CH}_{3}\right), 4.65\left(\mathrm{~s}, 2 \mathrm{H}, \mathrm{CH}_{2}\right), 7.20$ (dd, $J=4.9,4.0 \mathrm{~Hz}, 1 \mathrm{H}, 4$ "-H), 7.32 (t, $\left.J=9.1 \mathrm{~Hz}, 1 \mathrm{H}, 5^{\prime}-\mathrm{H}\right), 7.72(\mathrm{dd}, J=4.9,0.7 \mathrm{~Hz}, 1 \mathrm{H}$, 3"-H), 7.98 (m, $1 \mathrm{H}, 6$ '-H), 8.07 (dd, $J=4.0,0.7 \mathrm{~Hz}, 1 \mathrm{H}, 5 "-\mathrm{H}), 8.09$ (dd, $J=7.8,2.0 \mathrm{~Hz}$, $\left.1 \mathrm{H}, 2^{\prime}-\mathrm{H}\right)$.

${ }^{13}$ C-NMR $\left(126 \mathrm{MHz}, \mathrm{DMSO}-\mathrm{d}_{6}\right): \delta(\mathrm{ppm})=14.9\left(3^{\prime}-\mathrm{CH}_{3}\right), 38.2\left(\mathrm{CH}_{2}\right), 86.2(\mathrm{C}-5), 116.2(\mathrm{~d}$, $\left.J=22.9 \mathrm{~Hz}, \mathrm{C}-5^{\prime}\right), 121.1(\mathrm{CN}), 125.7$ (d, $\left.J=17.8 \mathrm{~Hz}, \mathrm{C}-3^{\prime}\right), 128.9,129.1$ (C-2', C-4"), 129.5 (d, $J=9.4$ Hz, C-6'), 131.2 (C-3"), 133.2, 133.4 (C-1', C-5"), 142.8 (C-2"), 159.5 (C-6), 164.6 (d, $J=251.3 \mathrm{~Hz}, \mathrm{C}-4$ '), $171.0(\mathrm{C}-4), 171.3(\mathrm{C}-2), 194.3$ (C=O).

${ }^{19}$ F-NMR $\left(282 \mathrm{MHz}\right.$, DMSO-d $\left.\mathrm{d}_{6}\right): \delta(\mathrm{ppm})=-105.3\left(\mathrm{~m}_{\mathrm{c}}\right)$.

MS (ESI): $m / z(\%)=386.0(6.3)[\mathrm{M}+\mathrm{H}]^{+}, 424.0(100)[\mathrm{M}+\mathrm{K}]^{+}$.

HRMS von $\mathrm{C}_{18} \mathrm{H}_{12} \mathrm{FN}_{3} \mathrm{O}_{2} \mathrm{~S}_{2}$ ber.: 384.0282 gef.: $384.0285[\mathrm{M}-\mathrm{H}]^{-}$(ESI-HRMS).

$\mathrm{C}_{18} \mathrm{H}_{12} \mathrm{FN}_{3} \mathrm{O}_{2} \mathrm{~S}_{2}$ (385.43). 


\subsubsection{2-((2-(4-Brom-3-methylphenyl)-2-oxoethyl)thio)-6-oxo-4-(thiophen-2-yl)-1,6-} dihydropyrimidin-5-carbonitril $(84 \mathrm{~g})$<smiles>N#Cc1c(-c2cccs2)nc(S)[nH]c1=O</smiles>

166<smiles>Cc1cc(C(=O)CBr)ccc1Br</smiles>

$167 \mathrm{~g}$
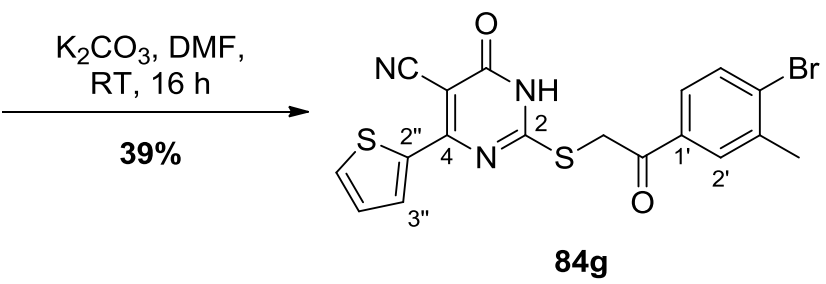

Dihydropyrimidon 166 (161 mg, $685 \mu \mathrm{mol}, 1.0$ Äq.), $\alpha$-Bromacetophenon 167g (200 mg, $685 \mu \mathrm{mol}, 1.0$ Äq.) und $\mathrm{K}_{2} \mathrm{CO}_{3}(94.7 \mathrm{mg}, 685 \mu \mathrm{mol}, 1.0$ Äq.) wurden gemäß AAV3 umgesetzt und das Rohprodukt aus $\mathrm{MeOH}$ umkristallisiert. Die Zielverbindung 84g (118 mg, $265 \mu \mathrm{mol}, 39 \%$ ) wurde in Form eines braunen Feststoffs isoliert.

DC: $\mathrm{R}_{f}=0.56\left(\mathrm{CH}_{2} \mathrm{Cl}_{2} / \mathrm{MeOH} 8: 1\right)$.

Smp.: Zersetzung bei ca. $240{ }^{\circ} \mathrm{C}$.

UV $\left(\mathrm{CH}_{3} \mathrm{CN}\right): \lambda_{\max }(\lg \varepsilon)=260 \mathrm{~nm}$ (4.0897), 292 (3.6656), 343 (3.2247), 371 (2.5540).

IR (ATR): $\tilde{v}\left(\mathrm{~cm}^{-1}\right)=2963,2918,1700,1555,1452,1224,1145,1014,982$.

${ }^{1} \mathbf{H}-\mathbf{N M R}\left(600 \mathrm{MHz}, \mathrm{DMSO}-\mathrm{d}_{6}\right): \delta(\mathrm{ppm})=2.48\left(\mathrm{~s}, 3 \mathrm{H}, 3^{\prime}-\mathrm{CH}_{3}\right), 5.00\left(\mathrm{~s}, 2 \mathrm{H}, \mathrm{CH}_{2}\right)$, 7.24-7.27 (m, 1 H, 4"-H), 7.84 ( $\mathrm{s}_{\mathrm{br}}, 2 \mathrm{H}, 2^{\prime}-\mathrm{H}, 5$ '-H), 7.92-7.96 (m, $1 \mathrm{H}, 3$ "'-H), 8.09 ( $\mathrm{s}_{\mathrm{br}}, 1 \mathrm{H}$, 6'-H), 8.15-8.17 (m, 1 H, 5"-H).

${ }^{13}$ C-NMR $\left(126 \mathrm{MHz}, \mathrm{DMSO}-\mathrm{d}_{6}\right): \delta(\mathrm{ppm})=23.2\left(3^{\prime}-\mathrm{CH}_{3}\right), 39.5\left(\mathrm{CH}_{2}\right), 89.1(\mathrm{C}-5), 116.9$ (CN), 128.4 (C-5'), 130.1 (C-4"), 131.2 (C-4'), 131.6 (C-6'), 132.5 (C-5"), 133.6 (C-2'), 135.6 (C-3"), 135.7 (C-3'), 139.0 (C-2"), 139.8 (C-1'), 159.4 (C-6), 161.6 (C-4), 165.7 (C-2), 192.3 $(\mathrm{C}=\mathrm{O})$.

MS (ESI): $m / z(\%)=445.9(100)[\mathrm{M}-\mathrm{H}]^{-}$.

HRMS von $\mathrm{C}_{18} \mathrm{H}_{12} \mathrm{BrN}_{3} \mathrm{O}_{2} \mathrm{~S}_{2}$ ber.: 445.9461 gef.: $445.9466[\mathrm{M}-\mathrm{H}]^{-}$(ESI-HRMS).

$\mathrm{C}_{18} \mathrm{H}_{12} \mathrm{BrN}_{3} \mathrm{O}_{2} \mathbf{S}_{2}$ (446.34). 


\section{E. ANHANG}

\section{Abkürzungsverzeichnis}
AAV
Allgemeine Arbeitsvorschrift
Ac
Acetyl
aq.
wässrig
Äq.
Äquivalent
ber.
berechnet
BINAP
2,2'-Bis-diphenylphosphino1,1'-binaphthyl
brsm
based on recovered starting material
$\mathrm{Bu}$
Butyl
$\mathrm{Bz}$
Benzoyl
$\mathrm{c}$
Konzentration
CD
circular dichroismus
COSY
correlated spectroscopy
d
$\operatorname{Tag}(\mathrm{e})$
DavePhos 2-Dicyclohexylphosphino-2'-(N,N-dimethylamino)biphenyl
DBU Diaza(1,3)bicyclo[5.4.0]undecan
DC Dünnschichtchromatographie
DCC $\quad N, N^{\prime}$-Dicyclohexylcarbodiimid
DIBAL-H Diisobutylaluminiumhydrid
DIPA Di-iso-propylamin
DIPEA Di-iso-propylethylamin
DMAP 4-(Dimethyl)-aminopyridin
DMF Dimethylformamid
DMI Demethylierungsinhibitoren
DMP Dess-Martin Periodinan
DMSO Dimethylsulfoxid
EA Evans-Auxiliar
EDC 1,2-Dichlorethan
EDCI 1-Ethyl-3-(3-dimethylaminopropyl)carbodiimid
ee Enantiomeric Excess, Enantiomerenüberschuß
EI Elektronenionosation 
ER Endoplasmatisches Retikulum

ESI Elektronsprayionsiation

Et Ethyl

EXSY Exchange Spectroscopy

GC Gaschromatographie

gef gefunden

ges. gesättigt

h Stunde(n)

HBK Herrmann-Beller-Katalysator

HMBC Heteronuclear Multiple Bond Correlation

HMDS Hexamethyldisilylazid

HOBt 1-Hydroxybenzotriazol

HPLC High Performance Liquid Chromatography

HRMS High Resolution Mass Spectrometry

Hsp Hitzeschockprotein

HSQC Heteronuclear Single Quantum Correlation

$\mathrm{Hz} \quad$ Hertz

i ipso, iso

$i \operatorname{Pr} \quad$ Isopropyl

IR Infrarotspektroskopie

L Ligand

LDA Lithiumdiisopropylamid

m meta

M Molar

$m / z \quad$ Verhältnis zwischen Masse und Ladung in der Massenspektrometrie

Me Methyl

MEM (2-Methoxyethoxy)methoxy

$\min \quad$ Minute(n)

Ms Methansulfonyl

MS Massenspektrometrie

MS Molsieb

MTBE Methyl-tert-butylether

$n \quad$ normal, unverzweigt

NMR Nuclear Magnetic Resonance 


\begin{tabular}{|c|c|}
\hline NOESY & Nuclear-Overhauser-Effect Spectroscopy \\
\hline$o$ & ortho \\
\hline$p$ & para \\
\hline p.a. & pro analysi \\
\hline $\mathrm{Ph}$ & Phenyl \\
\hline $\mathrm{PhMe}$ & Toluol \\
\hline ppm & parts per million, Einheit der chemischen Verschiebung \\
\hline $\operatorname{Pr}$ & Propyl \\
\hline PTAT & Phenyltrimethylammoniumtribromid \\
\hline py & Pyridin \\
\hline quant. & quantitativ \\
\hline $\mathrm{R}$ & Rest \\
\hline rac & racemisch \\
\hline $\mathrm{R}_{f}$ & Retentionswert für Dünnschichtchromatographie \\
\hline RT & Raumtemperatur \\
\hline Smp. & Schmelzpunkt \\
\hline$t$, tert & tertiär \\
\hline $\mathrm{T}$ & Temperatur \\
\hline TBA & Tetrabutylammonium \\
\hline TBAF & Tetra- $n$-butylammoniumfluorid \\
\hline TBS & tert-Butyldimethylsilyl \\
\hline Tf & Triflat \\
\hline TFA & Trifluoressigsäure \\
\hline THF & Tetrahydrofuran \\
\hline TIPS & Triisopropylsilyl \\
\hline tol & Tolyl \\
\hline Ts & $p$-Toluolsulfonyl \\
\hline$t_{\mathrm{R}}$ & Retentionszeit \\
\hline UV & Ultraviolett \\
\hline vgl. & vergleiche \\
\hline Vis & Visueller Bereich (380-750 nm) \\
\hline $\mathrm{X}$ & Halogenid \\
\hline z.B. & zum Beispiel \\
\hline
\end{tabular}




\section{Literatur}

${ }^{1}$ W. Krämer, U. Schirmer, Modern Crop Protection Compounds, Wiley-VCH Verlag GmbH \& Co. KGaA, Weinheim, 2007.

${ }^{2}$ J. Stetter, F. Lieb, Angew. Chem. Int. Ed. 2000, 39, 1724-1744; Ó. López, J. G. FernándezBolaños, M. V. Gil, Green Chem. 2005, 7, 431-442.

${ }_{3}$ C. Fest, K. J. Schmidt; Chemie der Pflanzenschutz- und Schädlingsbekämpfungsmittel, Bd. 1 (Hrsg.: R. Wegler), Springer, Berlin, 1970, S. 246-453; M. Eto, Organophosphorous Pesticides: Organic and Biological Chemistry, CRC, Cleveland, Ohio, 1974.

${ }_{4}$ K. Mellanby, The DDT Story, The British Crop Protection Council, Farnham, Surrey, Großbritannien, 1992; R. G. Beatty, The DDT Myth, John Day, New York, 1973; H. Rochling; Chemie der Pflanzenschutz- und Schadlingsbekampfungsmittel, Bd. 1 (Hrsg.: R. Wegler), Springer, Berlin, 1970, S. 121-129.

${ }_{5}$ R. Wegler, L. Eue; Chemie der Pflanzenschutz- und Schadlingsbekampfungsmittel, Bd. 5 (Hrsg.: R. Wegler), Springer, Berlin, 1977, S. 180-191.

${ }^{6}$ W. Kramer; Chemie der Pflanzenschutz- und Schadlingsbekampfungsmittel (Hrsg.: K. H. Buschel), Springer, Stuttgart, 1977, S. 137.

${ }^{7}$ W. B. Wheeler, J. Agric. Food. Chem. 2002, 50, 4151-4155; M. Güven, M. Sungur, B. Eser, I. Sari, F. Altuntas, J. Toxicol., Clin.Toxicol. 2004, 42, 617-623; F. Kamel, J. A. Hoppin, Environ. Health Perspect. 2004, 112, 950-958; B. Walker, Jr., J. Nidiry, Inhalation Toxicol. 2002, 14, 975-990; G. W. Ware, D. M. Whitacre, The Pesticide Book, 6th edn., Thomson Publications, Fresno, Californien, 2004.

${ }^{8}$ M. A. Sogorb, E. Vilanova, Toxicol. Lett. 2002, 128, 215-228.

${ }^{9}$ B. van Wendel de Joode, C. Wesseling, H. Kromhout, P. Monge, M. García, D. Mergler, Lancet 2001, 357, 1014-1016.

${ }^{10}$ B. Weiss, Neurotoxicology 2000, 21, 67-74.

${ }^{11}$ M. Zumbado, M. Goethals, E. E. Álvarez-León, O. P. Luzardo, F. Cabrera, L. SerraMajem, L. Dominguez-Boada, Sci. Total Environ. 2005, 339, 49-62.

${ }^{12}$ J. Stetter, F. Lieb, Angew. Chem. Int. Ed. 2000, 39, 1724-11744; W. Schwab, Pharm. uns. Zeit 2000, 2, 107-114.

${ }^{13}$ H. Börner, Pflanzenkrankheiten und Pflanzenschutz, Springer-Verlag, Berlin Heidelberg, 2009.

${ }^{14}$ M. Hennigsen, Chem. Unserer Zeit 2003, 37, 98-111. 
${ }^{15}$ http://www.umweltbundesamt.de/daten/land-forstwirtschaft/landwirtschaft/pflanzenschutzmittel-einsatz-in-der-landwirtschaft (aufgerufen am 13.05.15).

${ }^{16}$ T. Seitz, M. G. Hoffmann, H. Krähmer, Chem. Unserer Zeit 2003, 37, 112-126.

${ }^{17}$ J. Davies, J. C. Casaley, Pestic. Sci. 1999, 55, 1043-1058.

${ }^{18}$ M. Beckmann, K.-J. Haack, Chem. uns. Zeit 2003, 37, 88-97.

${ }^{19}$ M. Zumbado, M. Goethals, E. E. Álvarez-León, O. P. Luzardo, F. Cabrera, L. SerraMajem, L. Dominguez-Boada, Sci. Total Environ. 2005, 339, 49-62.

${ }^{20}$ H. A. Kirst, J. Antibiot (Tokyo), 2010, 63, 101-111.

${ }^{21}$ M. Bertasso, M. Holzenkampfer, A. Zeeck, E. Stackebrandt, W. Beil, H.-P. Fiedler, J. Antibiot. 2003, 56, 365-371.

${ }^{22}$ H. Seto, H. Yonehara, S. Aizawa, H. Akutsu, J. Clardy, E. Arnold, M. Tanabe, S. Urano, Chem. Abstr. 1980, 92, 211459u.

${ }^{23}$ P. Lewer, D. R. Hahn, ～L. L. Karr, D. O. Duebelbeis, J. R. Gilbert, G. D. Crouse, T. Worden, T. C. Sparks, P. McKamey, R. Edwards, P. R. Graupner, Bioorg. Med. Chem. 2009, 17, 4185-4196; H. J. Kim, R. Pongdee, Q. Wu, L. Hong, H.-W. Liu, J. Am. Chem. Soc. 2007, 129, 14582-14584.

${ }^{24}$ V. L. Salgado, Pestic. Biochem. Physiol. 1998, 60, 91-102; V. L. Salgado, J. J. Sheets, G. B. Watson, A. L. Schmidt, Pestic. Biochem. Physiol. 1998, 60, 103-110; G. D. Thompson, R. Dutton, T. C. Sparks, Pest. Manag. Sci. 2000, 56, 696-702; T. C. Sparks, G. D. Crouse, G. Durst, Pest. Manag. Sci. 2001, 57, 896-905.

${ }^{25}$ L. R. Kent (Eli Lilly), EP 1744768 B1, 2010.

${ }^{26}$ S. Liu, Q. X. Li, Chemosphere 2004, 56, 1121-1127.

27 G. D. Thompson, R. Dutton, T. C. Sparks, Pest. Manag. Sci. 2000, 56, 696-702;

T. C. Sparks, G. D. Crouse, G. Durst, Pest, Manag. Sci. 2001, 57, 896-905.

${ }^{28}$ C. B. Cleveland, M. A. Mayes, S. A. Cryer, Pest. Manag. Sci. 2001, 58, 70-84.

${ }^{29}$ N. Orr, A. J. Shaffner, K. Richey, G. D. Crouse, Pestic. Biochem. Physiol, 2009, 95, 1-5, BienSchV 1992; Bienenschutzverordnung vom 22. Juli 1992 (BGBI. I S.1420).

${ }^{30}$ E. I. Rabea, H. M. Nasr, M. E. I. Badaway, Arch. Environ. Contam. Toxicol. 2010, 58, $722-732$.

${ }^{31}$ M. Miles, Bull. Insectol. 2003, 56, 119-124.

${ }^{32}$ T. Perry, J. A. McKenzie, P. Batterham, Insect. Biochem. Molec. Biol. 2007, 37, 184-188.

${ }^{33}$ G. B. Watson, Pest. Biochem. Physiol. 2009, 71, 20-28. 
${ }^{34}$ N. Orr, A. J. Shaffner, K. Richey, G. D. Crouse, Pest. Biochem. Physiol. 2009, 95, 1-5;

T. Perry, J. A. McKenzie, P. Batterham, Insect Biochem. Molec. Biol. 2007, 37, 184-188.

${ }^{35}$ M. D. K. Markussen, M. Kristensen, Pest. Manag. Sci. 2012, 68, 75-82; T. Shono, J. G. Scott, Pestic. Biochem. Physiol. 2003, 75, 1-7; H. P. Young, W. B. Bailey, R. M. Roe, T. Iwasa, T. C. Sparks, Proc. Beltwide Cotton Production Conf. 2001, S. 1167-1171; C. F. Wyuss, H. P. Young, J. Shukla, R. M. Roe, Crop. Protect. 2003, 22, 307-314; W. D. Bailey, H. P. Young, R. M. Roe, Proc. Beltwide Cotton Production Conf. 1999, S. 1221-1224; L. C. Creemer, H. A. Kirst, J. W. Paschal, J. Antibiot. 1998, 51, 795-800.

${ }^{36}$ J. K. Moulton, D. A. Pepper, T. J. Dennehy, Proc. Beltwide Cotton Production Conf. 1999, S. 884-889; J. K. Moulton, D. A. Pepper, T. J. Dennehy, Pest Manag. Sci. 2000, 56, 842-848; J.-Z. Zhao, Y.-X. Li, H. L. Collins, L. Gusukuma-Minuto, R. F. L. Mau, G. D. Thompson, A. M. Shelton, J. Econ. Entomol. 2002, 95, 430-436;A. H. Sayyed, D. Omar, D. J. Wright, Pest. Manag. Sci. 2004, 60, 827-832; J. Dripps, B. Olson, T. C. Sparks, G. D. Crouse, Plant Health Progress 2008.

${ }^{37}$ T. C. Sparks, P. B. Anzeveno, J. G. Martynow, J. Gifford, M. B. Hertlein, T. V. Worden, H. A. Kirst, Pest. Biochem. Physiol. 2000, 67, 103-110.

${ }^{38}$ H. A. Kirst, L. C. Creemer, S. A. Naylor, P. T. Pugh, D. E. Snyder, J. R. Lowe, J. Z. Rothwell, T. C. Sparks, T. V. Worden, Curr. Topics Med. Chem. 2002, 2, 675-699; L. C. Creemer, H. A. Kirst, J. W. Paschal, J. Antibiot. 1998, 51, 795-800.

${ }^{39}$ P. B. Anzeveno, F. R. Green, III, ACS Symposium Series 2002, 800, 262-276; C. V. De Amicis, P. R. Graupner, J. A. Erickson, J. W. Paschal, H. A. Kirst, L. C. Creemer, P. E. Fanwick, J. Org. Chem. 2001， 66, 8431-8435. T. C. Sparks, P. B. Anzeveno, J. G. Martynow, J. Gifford, M. B. Hertlein, T. V. Worden, H. A. Kirst, Pest. Biochem. Physiol. 2000, 67, 103-110.

${ }^{40}$ J. E. Dripps, B. Olson, T. C. Sparks, G. D. Crouse, Plant Health Progress 2008, (22. August 2008); D. R. Hahn, G. Gustafson, C. Waldron, B. Bullard, J. D. Jackson, J. Mitchell, J. Ind. Microbiol. Biotechnol. 2006, 33, 94-104; L. Besard, V. Mommaerts, G. Abdu-Alla, G. Smagghe, Pest. Manag. Sci. 2011, 67, 541-547.

${ }^{41}$ J. Daeble, T. C. Sparks, P. Johnson, P. R. Graupner, Bioorg. Med. Chem. 2009, 17, 4197-4205.

${ }^{42}$ T. C. Sparks, P. B. Anzeveno, J. G. Martynow, J. M. Gifford, M. B. Hertlein, T. V. Worden, H. A. Kirst, Pestic. Biochem. Physiol 2000, 67, 187-197. 
${ }^{43}$ H. J. Kim, R. Pongdee, Q. Wu, L. Hong, H.-W. Liu, J. Am. Chem. Soc. 2007, 129, 14582-14584; Y. Chen, Y. Lin, K. Tsai, H. Chiu, J. Biol. Chem. 2009, 284, 7352 7363; H. J. Kim, J. A. White-Phillip, Y. Ogasawara, N. Shin, E. A. Isiorho, H.-W. Liu, J. Am. Chem. Soc. 2010, 132, 2901-2903; L. Hong, Z. Zhao, H.-W. Liu, J. Am. Chem. Soc. 2006, $128,14262-14263$.

${ }^{44}$ H. J. Kim, M. W. Ruszczycky， S.-H. Choi，Y.-N. Liu， H.-W. Liu，Nature，2011，473, $109-112$.

${ }^{45}$ C. E. Aroyan, A. Dermenci, S. J. Miller, Tetrahedron 2009, 65, 4069-4084.

${ }^{46}$ D. A. Evans, W. C. Black, J. Am. Chem. Soc. 1993, 115, 4497-4513.

${ }^{47}$ L. A. Paquette, Z. Gao, Z. Ni, G. F. Smith, J. Am. Chem. Soc. 1998, 120, 2543-2552;

L. A. Paquette, I. Collado, M. Purdie, J. Am. Chem. Soc. 1998, 120, 2553-2562;

L. A. Paquette, Z. Gao, Z. Ni, G. F. Smith, Tetrahedron Lett. 1997, 38, 1271-1274

${ }^{48}$ W. R. Cunha, M. L. Andrade e Silva, R. C. S. Veneziani, S. R. Ambrósio and J. K. Bastos, Lignans: Chemical and Biological Properties In Phytochemicals - A Global Perspective of Their Role in Nutrition and Health (Ed. V. Rao), Intech, 2012, 213-234.

${ }^{49}$ R. D. Haworth, Annu. Rep. Prog. Chem. 1936, 33, 266-279.

${ }^{50}$ O. R. Gottlieb, Fortschr. Chem. Org. Naturstoff, 1978, 35, 1-72.

${ }^{51}$ D. C. Ayres, J. D. Loike, Lignans: Chemical, Biological and Clinical Properties, Cambridge University Press, Cambridge 1990.

${ }^{52}$ P. M. Dewick, Medicinal Natural Products - A Biosynthetic Approach, Vol.3, Wiley, Chichester 2009.

${ }^{53}$ T. J. Schmidt, S. Vößing, M. Klaes, S. Grimme, Planta Med 2007, 73, 1574-1580.

${ }^{54}$ L. F. Tietze, S.-C. Düfert, J. Clerc, M. Bischoff, C. Maaß, D. Stalke, Angew. Chem Int Ed. 2013, 52, 3191-3194.

${ }^{55}$ H. Weinstabl, M. Suhartono, Z. Qureshi, M. Lautens, Angew. Chem. Int. Ed. 2013, 52, $5305-5308$.

${ }^{56}$ Statistisches Bundesamt, Todesursachen in Deutschland - Fachserie 12, Reihe 4 - 2012, 2013, S. 4.

${ }^{57}$ C. W. Hamm, Z. Kardiol. 2004, 93, 324-341.

${ }^{58}$ M. Shawkat Razzaque T. Taguchi, Trends. Mol. Med. 2007, 13, 45-53.

${ }^{59}$ S. W. Li, A. L. Sieron, A. Fertala, Y. Hojima, W. V. Arnold, D. J. Prockop, Proc. Natl. Acad. Sci. 1996, 93, 5127-5130. 
${ }^{60}$ S. R. Lammande, J. F. Bateman, Semin. Cell Dev. Biol. 1999, 10, 455-464.

${ }^{61}$ A. Rita, K. Gordon, Cell Tissue Res. 2010, 339, 247-257.

${ }^{62}$ K. Hauser J. Rassow, Biochemie, Thieme-Verlag, Stuttgart 2006.

${ }^{63}$ J. Weissmann, A. Horwich, Cell 2006, 125, 443-451.

${ }^{64}$ S. P. Goldberg, W.D. Jordan, J. Vasc. Surg. 1999, 29, 748-751.

${ }^{65}$ E. Leikina, M. V. Mertts, Proc. Natl. Acad. Sci. USA 2002, 99, 1314-1318.

${ }^{66}$ Y. Ishada, K. Nagata, Methods in Enzymology 2011, 499, 167-178.

${ }^{67}$ Y. Sato, K. Murase, J. Kato, M. Kobune, T. Sato, Y. Kawano, R. Takimoto, K. Takada, K. Miyanishi, T. Matsunaga, T. Takayama, Y. Niitsu, Nat. Biotechnol. 2008, 26, 431-442.

${ }^{68}$ M. Satoh, K. Hirayoshi, S. Yokota, N. Hosokawa, K. Nagata, J. Cell. Biol. 1996, 2, $469-483$.

${ }^{69}$ A. Nakai, M. Satoh, K. Hirayoshi, K. Nagata, J. Cell. Biol. 1992, 4, 903-914.

${ }^{70}$ M. S. Razzaque, T. Taguchi, J. Pathol. 1997, 183, 24-29; M. Sunamoto, K. Kuze, H. Tsuji, N. Ohishi, K. Yagi, K. Nagata, T. Kita, T. Doi, Lab. Invest. 1998, 78, 967-972.

${ }^{71}$ C. A. Thomson, H. M. Atkinson, V. S. Ananthanarayanan, J. Med. Chem. 2005, 48, $1680-1684$.

72 "The Nobel Prize in Chemistry 2010", Nobelprize.org, Nobel Media AB 2013; http://www.nobelprize.org/nobel_prizes/chemistry/laureates/2010/ (aufgerufen am 13.05.15). ${ }^{73}$ T. Mizoroki, K. Mori, A. Ozaki, Bull. Chem. Soc. Jpn. 1971, 44, 581; R. F. Heck, J. P. Nolley, Jr., J. Org. Chem. 1972, 34, 2320-2322.

${ }^{74}$ I. P. Beletskaya, A. V. Cheprakov, Chem. Rev. 2000, 100, 3009-3066; W. Cabri, I. Candiani, Acc. Chem. Res. 1995, 28, 2-7.

${ }^{75}$ L. F. Tietze, H. Ila, H. P. Bell, Chem. Rev. 2004, 104, 3453-3516.

${ }^{76}$ Y. Sato, M. Sodeoka, M. Shibasaki, J. Org. Chem. 1989, 54, 4738-4739.

${ }^{77}$ N. E. Carpenter, D. J. Kucera, L. E. Overman, J. Org. Chem. 1989, 54, 5846-5848.

${ }^{78}$ D. Mc Cartney, P. J. Guiry, Chem. Soc. Rev. 2011, 40, 5122-5150; A. B. Dounay, L. E. Overman, Chem. Rev. 2003, 103, 2945-2963; I. P. Beletskaya, A. V. Cheprakov, Chem. Rev. 2000, 100, 3009-3066; A. de Meijere, F. E. Meyer, Angew. Chem. Int. Ed. Engl. 1994, 33, 2379-2411.

79 J. Dupont, C. S. Consorti, J. Spencer, Chem. Rev. 2005, 105, 2527-2571.

${ }^{80}$ F. Ozawa, A. Kubo, T. Hayashi, Chem. Lett. 1992, 2177-2180.

${ }^{81}$ C. Amatore, A. Jutand, Acc. Chem. Res. 2000, 33, 314-321. 
${ }^{82}$ C. Amatore, A. Jutand, M. A. M'Barki, Organometallics 1992, 11, 3009-3013; F. Ozawa, A. Kubo, T. Hayashi, Chem. Lett. 1992, 21, 2177-2180.

${ }^{83}$ L. S. Santos, G. B. Rosso, R. A. Pilli, M. N. Eberlin, J. Org. Chem. 2007, 72, 5809-5812.

${ }^{84}$ J. F. Hartwig, F. Paul, J. Am. Chem. Soc. 1995, 117, 5373-5374.

${ }^{85}$ M. R. Biscoe, B. P. Fors, S. L. Buchwald, J. Am. Chem. Soc. 2008, 130, 6686-6687; E. R. Strieter, D. G. Blackmond， S. L. Buchwald， J. Am. Chem. Soc. 2003, 125, 13978-13980; M. W. Hooper, M. Utsunomiya， J. F. Hartwig，J. Org. Chem. 2003， 68, 2861-2873; A. F. Littke，C. Dai，G. C. Fu，J. Am. Chem. Soc. 2000，122，4020-4028; A. F. Littke, G. C. Fu, J. Org. Chem. 1999, 64, 10-11.

${ }^{86}$ C. Amatore, E. Carré, A. Jutand, M. A. M'Barki, Organometallics 1995, 14, 1818-1826.

${ }^{87}$ S. Kozuch, S. Shaik, A. Jutand, C. Amatore, Chem. Eur. J. 2005, 10, 3072-3080.

${ }^{88}$ C. Amatore, E. Carré, A. Jutand, M. A. M’Barki, G. Meyer, Organometallics 1995, 14, $5605-5614$.

${ }^{89}$ C. Amatore, E. Carré, A. Jutand, Y. Medjour, Pure Appl. Chem. 2002, 76, 565-576.

${ }^{90}$ Die cis-/trans-Isomerisierung wäre theoretisch auch nach der Koordination des Alkens möglich.

${ }^{91}$ M. J. S. Dewar, Bull. Soc. Chim. Fr. 1951, 18, C79; J. Chatt, L. A. Duncanson, J. Chem. Soc. 1953, 2939-2947; J. Chatt, L. A. Duncanson, L. M. Venanzi, J. Chem. Soc. 1955, 4456-4460.

${ }^{92}$ Christoph Elschenbroich, Organometallchemie, 6. Aufl., Teubner-Verlag, Wiesbaden 2008.

${ }^{93}$ I. P. Beletskaya, A. V. Cheprakov, Chem. Rev. 2000, 100, 3009-3066.

${ }^{94}$ A. B. Dounay, L. E. Overman, Chem. Rev. 2003, 103, 2945-2963.

${ }^{95}$ S. E. Gibson, N. Guillo, R. J. Middleton, A. Thuilliez, M. J. Tozer, J. Chem. Soc., Perkin Trans. 1 1997, 447-456.

${ }^{96}$ K. Albert, P. Gisdakis, N. Rösch, Organometallics 1998, 17, 1608-1616; R. J. Deeth, A. Smith, K. K. Hii, J. M. Brown, Tetrahedron Lett. 1998, 39, 3229-3232.

${ }^{97}$ IUPAC Compendium of Chemical Terminology, 2nd Ed., Blackwell Scientific Publications, Oxford 1997; J. I. Seeman, J. Chem. Educ. 1986, 63, 42-48.

${ }^{98}$ A. B. Dounay, P. G. Humphreys, L. E. Overman, A. D. Wrobleski, J. Am. Chem. Soc. 2008, $130,5368-5377$. 
${ }^{99}$ L. F. Tietze, R. Schimpf, Angew. Chem. Int. Ed. 1994, 33, 1089-1091; L. F. Tietze, O. Burkhardt, Liebigs Ann. Chem. 1995, 1153-1157; L. F. Tietze, T. Raschke, Synlett 1995, 597-598; L. F. Tietze, T. Raschke, Liebigs Ann. Chem. 1996, 1981-1987.

${ }^{100}$ L. F. Tietze, G. Brasche, K. Gericke, Domino Reactions in Organic Synthesis, WileyVCH, Weinheim 2006.

${ }^{101}$ K. C. Nicolaou, J. S. Chen, Chem. Soc. Rev. 2009, 38, 2993-3009; K. C. Nicolaou, D. J. Edmonds, P. G. Bulger, Angew. Chem. Int. Ed. 2006, 45, 7134-7186.

${ }^{102}$ K. U. Wendt, G. E. Schulz, E. J. Corey, D. R. Liu, Angew. Chem. Int. Ed. 2000, 39, 2812-2833.

${ }^{103}$ H. P. Pepper, H. C. Lam, W. M. Bloch, J. H. George, Org. Lett. 2012, 14, 5162-5164.

${ }^{104}$ L. F. Tietze, S. Jackenkroll, J. Hierold, L. Ma, B. Waldecker, Chem. Eur. J. 2014, 20, $8628-8635$.

${ }^{105}$ M. Leibeling, D. B. Werz, Chem. Eur. J. 2012, 18, 6138-6141.

${ }^{106}$ L. F. Tietze, M. Spescha, T. Nöbel, J. Am. Chem. Soc. 1998, 120, 8971-8977; L. F. Tietze, M. Spescha, T. Nöbel, Angew. Chem. Int. Ed. Engl. 1996, 35, 2259-2261; L. F. Tietze, J. M. Wiegand, C. Vock, J. Organomet. Chem. 2003, 687, 346-352; L. F. Tietze, W.-R. Krahnert, Synlett 2001, 560-562; L. F. Tietze, W.-R. Krahnert, Chem. Eur. J. 2002, 8, 2116-2125; L. F. Tietze, S. Petersen, Eur. J. Org. Chem. 2001, 9, 1619-1624; L. F. Tietze, S. Petersen, Eur. J. Org. Chem. 2000, 1827-1830; L. F. Tietze, L. P. Lücke, F. Major, P. Müller, Aust. J. Chem. 2004, 57, 635-640.

${ }^{107}$ S. Dietz, Dissertation, Universität Göttingen, 2011.

${ }^{108}$ L. F. Tietze, R. Schimpf, Angew. Chem. Int. Ed. Engl. 1994, 33, 1089-1091; L. F. Tietze, K. Kahle, T. Raschke, Chem. Eur. J. 2002, 8, 401-407; L. F. Tietze, K. Thede, R. Schimpf, F. Sannicolo, Chem. Commun. 2000, 583-584; L. F. Tietze, A. Modi, Eur. J. Org. Chem. 2000, 1959-1964.

${ }^{109}$ Diese Ergebnisse basieren auf einer unveröffentlichten Forschungsarbeit von J. Clerc, Universität Göttingen, 2012.

${ }^{110}$ H. B. Hansen, Bachelorarbeit, Universität Göttingen, 2014.

${ }^{111}$ G. Brasche, Dissertation, Universität Göttingen, 2005; A. Grube, Dissertation, Universität Göttingen, 2007; T. Scheffer, Dissertation, Universität Göttingen, 2012; N. Schützenmeister, Dissertation, Universität Göttingen, 2012;

${ }^{112}$ Y. Foricher, J. Mann, Tetrahedron Lett. 1999, 41, 2007-2009. 
${ }^{113}$ H. J. Schuster, Disseration, Universität Göttingen, 2008.

${ }^{114}$ J. C. Conway, P. Quayle, A. C. Reagan, C. J. Urch, Tetrahedron 2005, 61, 11910-11923.

${ }^{115}$ Die Verbindung rac-182 wurde im Rahmen der Doktorarbeit von T. Scheffer hergestellt.

${ }^{116}$ S. P. Brown, M. P. Brochu, C. J. Sinz, D. W. C. MacMillan, J. Am. Chem. Soc. 2003, 125, 10808-10809.

${ }^{117}$ M. Movassaghi, M. Tjandra, J. Qi, J. Am. Chem. Soc. 2009, 131, 9648-9650.

${ }^{118}$ E. N. Jacobsen, I. Markó, W. S. Mungall, G. Schröder, K. B. Sharpless, J. Am. Chem. Soc. 1988, 110, 1968-1970; K. B. Sharpless, W. Amberg, Y. L. Bennani, G. A. Crispino, J. Hartung, K. S. Jeong, H. L. Kwong, K. Morikawa, Z.-M. Wang, D. Xu, X.-L. Zhang, J. Org. Chem. 1992, 57, 2786-2771; H. C. Kolb, M. S. VanNieuwenhze, K. B. Sharpless, Chem. Rev. 1994, 94, 2483-2547.

${ }^{119}$ Diese Ergebnisse basieren auf einer unveröffentlichten Forschungsarbeit von T. A. Khan, Universität Göttingen, 2015.

${ }^{120}$ Die Verbindung 157 wurde von der Bayer AG bereitgestellt.

${ }^{121}$ S.-C. Düfert, Dissertation, Universität Göttingen, 2014.

${ }^{122}$ Iodbenzo[d][1,3]dioxol (240) ist kommerziell erhältlich, kann aber auf die hier beschriebene Weise kosteneffizienter hergestellt werden.

${ }^{123}$ A. M. Sajith, A. Muralidharan, Tetrahedron Lett. 2012, 53, 5206-5210; S. Urgaonkar, J. G. Verkade, J. Org. Chem. 2004, 69, 5752-5755.

${ }^{124}$ E. R. Strieter, D. G. Blackmond, S. L. Buchwald, J. Am. Chem. Soc. 2003, 125, 13978-13980; E. R. Strieter, S. L. Buchwald, Angew. Chem. Int. Ed. 2006, 45, 925-928.

${ }^{125}$ M. V. Lakshmikantham, A. F. Garito, A. P. Cava, J. Org. Chem. 1978, 22, 4395-4397;

H. C. Brown, P. K. Jadhav, A. K. Mandal, J. Org. Chem. 1982, 47, 5074-5083.

${ }^{126}$ S. Masamune, B. M. Kim, J. S. Petersen, T. Sato, S. J. Veenstra, T. Imai, J. Am. Chem. Soc. 1985, 107, 4549-4551.

${ }^{127}$ Diese Ergebnisse basieren auf einer unveröffentlichten Forschungsarbeit von S.-C. Düfert, Universität Göttingen, 2014.

128 J. Jeener, B. H. Meier, P. Bachmann, R. R. Ernst, J. Chem. Phys. 1979, 71, 4546-4553;

Y. Shvo, E. C. Taylor, K. Mislow, M. Raban, J. Am. Chem. Soc. 1967, 89, 4910-4917.

${ }^{129}$ Die Definition besagt, dass Atropisomeren mit physikalischen Methoden trennbare Substanzen sind, welche mindestens eine Halbwertszeit von $\tau=1000 \mathrm{~s}$ (16.7 min) aufweisen. K. Oki, Topics in Stereochemistry, Vol. 14 (Eds.: N. L. Allinger, E. E. Eliel, S. H. Wilen), 
Wiley Interscience, New York 1983, 1-76; G. Bringmann, A. J. P. Mortimer, P. A. Keller, M. J. Gresser, J. Garner, M. Breuning, Angew. Chem. Int. Ed. 2005, 44, 5384-5427.

${ }^{130}$ M. Hesse, H. Meier, B. Zeeh, Spektroskopische Methoden in der organischen Chemie, 7th ed., Thieme, Stuttgart, New York 2005, 16-17.

${ }^{131}$ H. Gilman, P. R. van Ess, J. Am. Chem. Soc. 1933, 55, 1258-1261.

132 D. T. Genna, G. H. Posner, Organic Letters 2011, 13, 5358-5361.

${ }^{133}$ J. Jaques A., M. Marquet, Tetrahedron Lett. 1959, 9, 24-26.

${ }^{134}$ G. Brasche, Dissertation, Universität Göttingen, 2005. 


\section{Danksagung}

Zum Abschluss möchte ich mich an dieser Stelle bei allen bedanken, die auf die eine oder andere Weise ihren Anteil an dem Gelingen dieser Arbeit beigetragen haben.

Zuallererst gilt mein Dank den Mitarbeitern der analytischen Abteilungen der Fakultät für Chemie. Ich danke Reinhard Machinek, Michael John, Christiane Siebert, Martin Weitemeyer, Carola Zolke und Ralf Schöne für das überaus schnelle und zuverlässige Messen von unzähligen NMR-Spektren. Ganz besonders möchte ich Reinhard Machinek und Michael John danken, die auch bei schwierigsten NMR-Problemen mit Rat und Tat zur Seite standen. Des Weiteren danke ich Holm Frauendorf, Frank Hambloch, Györgi Sommer-Udvarnoki, Ulrike Bode und Gabriele Krökel für das zügige Messen der Massenspektren. Besonderer Dank gilt dabei Holm Frauendorf für seine wichtigen Ratschläge, seine Unterstützung bei Problemen und die interessanten und lehrreichen Gespräche. Ebenfalls danken möchte ich Olaf Senge für seinen Einsatz und seine Bemühungen an der chiralen GC, sowie sein fundiertes Wissen an der HPLC und die stete Diskussionsbereitschaft. Weiterhin bedanke ich mich bei Evelyn Pfeil und Katja Grube für das unverzügliche Messen von UV/Vis- und IRSpektren sowie das Messen der Drehwerte.

Ebenfalls bedanken möchte ich mich bei Gabi Schmidt von der Firma Jasco, die jederzeit mit viel Kompetenz und großem Engagement Hilfe und Ratschläge rund um das Thema HPLC erteilen konnte, bei technischen Problemen stets erreichbar war und zeitnah Abhilfe schaffen konnte. Arne Visscher aus der Arbeitsgruppe von Prof. Dietmar Stalke sei für das Messen der Fluoreszenzspektren und Sven Ole Reichmann für die Bemühungen zur Kristallisation einiger Substanzen gedankt. Mein Dank gilt außerdem Martina Pretor für ihre schnelle und kompetente Hilfe bei EDV-Problemen jeglicher Art. Sabine Schacht möchte ich für die unermüdliche Verwaltungsarbeit danken, die den Arbeitskreis am Laufen hielt. Gleiches gilt für Isabel Trzeciok und Berta Hampel, die im Dekanat bei Problemen mit etwaigen bürokratischen Hürden stets hilfsbereit waren. Außerdem danke ich Holger Tucholla und Rupert Schrommek für die vorbildliche Versorgung mit Chemikalien und anderen Laborutensilien sowie die kleinen Scherze zwischendurch. Ein Dank gebührt auch den Hausmeistern Frank Peters und Wolfram Matthies sowie den Mitarbeitern der Werkstätten für die schnelle Reparatur jeglicher Laborgeräte.

Ich möchte mich außerdem bei Prof. Zimmermann für die interessante Einführung in das pharmakologische Institut und die synthetisch erfolgreiche Kooperation hinsichtlich der potentiellen Hsp47-Inhibitoren danken. 
Dem Spinosyn-Team um Timo Scheffer, Nina Schützenmeister, Simone Dietz, Niels Böhnke, Christian Stadler, Gordon Brasche und Alexander Grube danke ich für die hervorragende und unermüdliche Vorarbeit auf diesem umfangreichen Thema. Ebenso sei Jérôme Clerc, Svenia Düfert und Matthias Bischoff für die exzellenten Studien zum Thema Linoxepin gedankt. Mein Dank gilt außerdem meinem Bachelorstudenten Frederik Kramm für seine hervorragenden Leistungen auf dem Thema der Hsp47-Inhibitoren. Des Weiteren danke ich meinen Laborpraktikanten Lennard Krause, Sandra Lange und Svenja Neumann, die mir durch ihre Vorläufer-Synthesen eine Menge Arbeit erspart haben.

Ich danke außerdem allen Mitarbeitern der Abteilung Tietze, die auch die frustrierenden und ermüdenden Episoden dieser Arbeit zu einem Vergnügen gemacht haben. Besonders hervorheben möchte ich dabei Jérôme Clerc, Christoph Eichhorst, Sven Heidemann, Judith Hierold, Stefan Jackenkroll, Tobias Nack, Timo Scheffer, Kianga Schmuck und Bernd Waldecker. Meinen Langzeit-Labormitarbeitern Jérôme, Christoph, Stefan, und Bernd sei für die überaus entspannte, teils chaotische, aber immer wieder auch intellektuelle und stets angenehme Atmosphäre gedankt.

Für das überaus zügige und sogfältige Korrekturlesen dieser Arbeit und die konstruktiven Kritiken bedanke ich mich ganz herzlich bei Christoph Eichhorst, Judith Hierold und Sven Heidemann. Alle verbliebenen Fehler sind allein mir zuzuschreiben.

Verschiedene Menschen haben meinen Weg in Göttingen begleitet und diesen zu einer angenehmen Episode in meinem Leben gemacht. Ich möchte mich besonders bei meinen Kommilitonen Sven Ole Reichmann, Arne Visscher, Lennard Krause, Thilo Baronsky, Benjamin Gerdes und Corinna Kramer bedanken, die mir während meines kompletten Studiums Gesellschaft geleistet haben. Abseits der chemischen Fakultät gebührt auch Jan Seiker, Till Nolting, Fritz Wilhelms, Lukas Seiker, Till Hartmann und Julian Theilen großer Dank für die schönen Abende sowie die unzähligen Unternehmungen in und rund um Göttingen. Sönke Hägerbäumer, Elmar Lodwig, Benjamin Möhlmeier, Stefan Solinski, Bastian Lütkemeier, Jan Christoph Obermeier, Julian Uphoff, Tobias Schlusen, Achim Diekmeyer und Carina Stallmann danke ich stellvertretend für die große Unterstützung aus meiner Heimat.

Meinen Eltern und meinem Bruder danke ich für ihre bedingungslose Unterstützung, die mir dieses Studium ermöglicht hat und ohne die diese Arbeit wohl nicht zustande gekommen wäre. Danke für die schönen Stunden in der Heimat und den nötigen Rückhalt. Ohne euch wäre ich nicht da, wo ich bin. 
Der größte Dank gebührt allerdings Jana für ihre Liebe, ihre Unterstützung und ihre ungeahnte Fähigkeit mich immer wieder an die wirklich wertvollen Dinge im Leben zu erinnern.

Herzlichsten Dank!

Meine akademischen Lehrer waren unter anderem:

L. Ackermann, G. Beuermann, P. Botschwina, M. Buback, U. Diederichsen, C. Ducho, G. Eckold, A. El-Armouche, H. Frauendorf, C. Griesinger, W. Hack, R. Machinek, J. Magull, S. Mayr， F. Meyer， G. M. Sheldrick， L. F. Tietze， S. Schmatz， J. Schröder， D. Stalke, C. Steinem, H. J. Steinfelder, M. Suhm, P. Vana, D. B. Werz, W.-H. Zimmermann. 


\section{Lebenslauf}

\section{Persönliche Daten}

Name:

Simon Biller

Geburtsdatum:

24.03.1986

Geburtsort:

Lübbecke

Staatsangehörigkeit:

deutsch

Familenstand:

ledig

\section{Schulausbildung}

08/1992-07/1996

08/1996-07/2005

$07 / 2005$

Grundschule Oberbauerschaft, Hüllhorst

Wittekind-Gymnasium, Lübbecke

Allgemeine Hochschulreife

\section{Hochschulausbildung}

10/2006-07/2009

$08 / 2009$

$10 / 2009-08 / 2011$

09/2011

$11 / 2011-05 / 2015$

Studium der Chemie mit Bachelorarbeit am Institut für Organische und Biomolekulare Chemie der Georg-AugustUniversität Göttingen unter der Leitung von Prof. Dr. Dr. h.c. L. F. Tietze mit dem Thema: „Totalsynthese von Orizaterpenylbenzoat" (1.0)

Abschluss als Bachelor of Science in Chemie (1.7)

Studium der Chemie mit Masterarbeit am Institut für Organische und Biomolekulare Chemie der Georg-August-Universität Göttingen unter der Leitung von Prof. Dr. Dr. h.c. L. F. Tietze mit dem Thema: „Untersuchungen zur Totalsynthese von Angucyclinon $C^{\text {" }}(1.0)$

Abschluss als Master of Science in Chemie (mit Auszeichnung)

Dissertation am Institut für Organische und Biomolekulare Chemie der Georg-August-Universität Göttingen unter der Leitung von Prof. Dr. Dr. h.c. L. F. Tietze mit dem Thema: „Zugang zu Agrar- \& Pharmawirkstoffen durch organische Synthese - Design und Synthese von Spinosynanaloga und Hsp47-Inhibitoren sowie enantioselektive Totalsynthese des Naturstoffes Linoxepin“"

Disputation 